\title{
Cross-Metathesis of Allenes. Mechanistic Analysis and Identification of a Ru-CAAC as the Most Effective Catalyst
}

\author{
Stella A. Gonsales, ${ }^{\ddagger}$ Zoé C. Mueller, ${ }^{\ddagger}$ Fengyue Zhao ${ }^{\perp}$ Paulo H. S. Paioti, ${ }^{\star}$ Lydia Karmazin, ${ }^{\ddagger}$ Jing Wan, ${ }^{\S}$ \\ Fang Liu* ${ }^{* \perp}$, K. N. Houk ${ }^{* \dagger}$, and Amir H. Hoveyda*,,,+

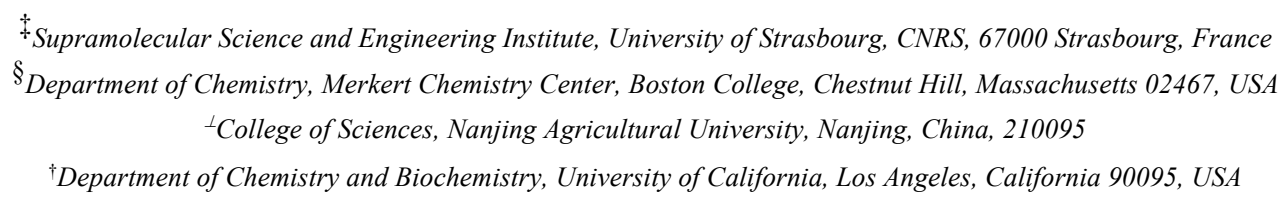

email: amir.hoveyda@bc.edu or ahoveyda@unistra.fr;

for computational studies: acialiu@njau.edu.cn or houk@chem.ucla.edu

\section{SUPPORTING INFORMATION}

Table of Contents

1. General.... 2

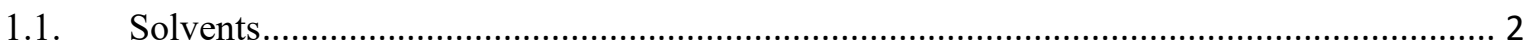

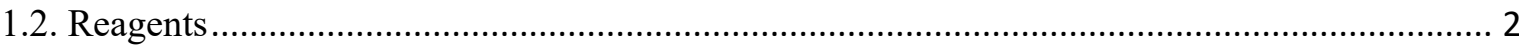

2. Synthesis and Characterization of the Starting Materials................................................... 4

3. Synthesis and Characterization of Cross-Metathesis Products............................................ 4

3.2 Analytical data for 1,3-disubstituted allene products ............................................................. 5

4. Synthesis and Characterization of Ru-Vinylidene Complexes...............................................

4.1. Synthesis and characterization of Ru-vinyl-1 ................................................................. 7

4.2. Synthesis and Characterization of Ru-vinyl-2 and Ru-vinyl-3 ............................................. 8

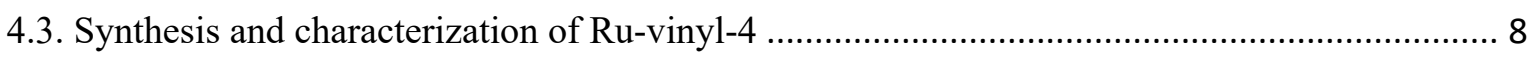

4.4. Synthesis and characterization of Ru-carbide .................................................................... 9

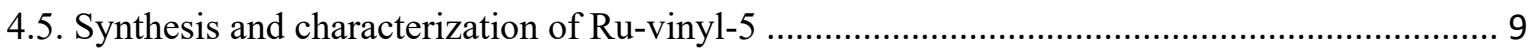

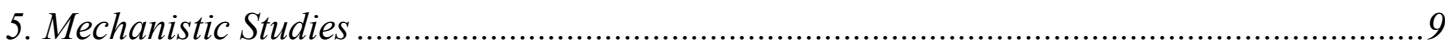

5.1. Reaction of Ru-I and Ru-II with allenyl-B(pin) to generate Ru-vinyl-1. ............................... 9

5.2. Energy barriers for the loss of a phosphine ligand from various Ru complexes ..................... 10

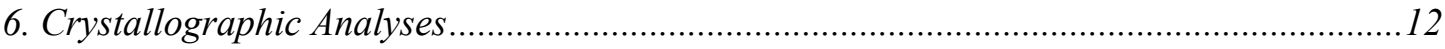

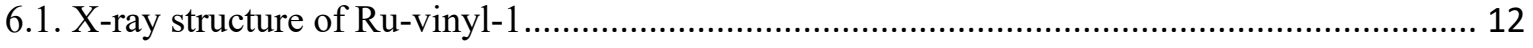

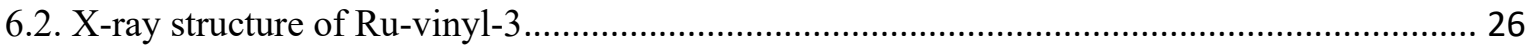

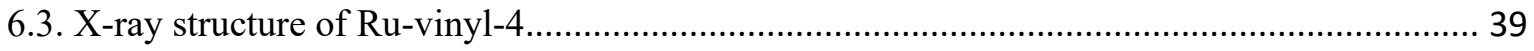


6.4. X-ray structure of Ru-carbide 57

6.5. X-ray structure of Ru-vinyl-5. 64

7. Computational (DFT) Studies .77

7.1. For complexes where $\mathrm{L}=\mathrm{PCy}_{3}$ 77

7.2. For complexes where $\mathrm{L}=\mathrm{NHC}$ 126

7.3. For complexes where $\mathrm{L}=\mathrm{CAAC}$ 169

8. NMR Spectra. .210

8.1. Allenyl substrates 210

8.2. Cross-metathesis products. 218

8.3. NMR spectra of Ru complexes 244

9. References 257

\section{General}

Unless otherwise noted, transformations were performed with distilled and degassed solvents under an atmosphere of dry $\mathrm{N}_{2}$, in oven $\left(135^{\circ} \mathrm{C}\right)$ or flame-dried glassware with standard dry box or vacuum line techniques. NMR spectra were acquired on the $400 \mathrm{MHz}$ or $500 \mathrm{MHz}$ Bruker instruments at Institute of Science Supramolecular Engineering (ISIS), University of Strasbourg. Chemical shifts for ${ }^{1} \mathrm{H}$ NMR spectra are reported in ppm from tetramethylsilane with the solvent resonance resulting from incomplete deuterium incorporation as the internal standard $\left(\mathrm{CDCl}_{3}: \delta 7.26 \mathrm{ppm}, \mathrm{CD}_{2} \mathrm{Cl}_{2}: \delta 5.32 \mathrm{ppm}, \mathrm{C}_{6} \mathrm{D}_{6}: \delta 7.16 \mathrm{ppm}\right.$, DMSO$\left.d_{6}: \delta 2.50 \mathrm{ppm}\right)$. Data are reported as follows: chemical shift, multiplicity $(\mathrm{s}=$ singlet, $\mathrm{d}=$ doublet, $\mathrm{t}=$ triplet, $\mathrm{q}=$ quartet, $\mathrm{p}=$ pentet, $\mathrm{br}=$ broad, $\mathrm{m}=$ multiplet $)$, coupling constants $(\mathrm{Hz})$, and integration. ${ }^{13} \mathrm{C} \mathrm{NMR}$ spectra were recorded with complete proton decoupling. Chemical shifts are reported in ppm from tetramethylsilane with the solvent resonance as the internal standard $\left(\mathrm{CDCl}_{3}: \delta 77.16 \mathrm{ppm}, \mathrm{CD}_{2} \mathrm{Cl}_{2}: \delta 53.84\right.$ ppm, $\mathrm{C}_{6} \mathrm{D}_{6}: \delta 128.06 \mathrm{ppm}$; DMSO- $\left.d_{6}: \delta 39.52 \mathrm{ppm}\right)$. High-resolution mass spectrometry was performed on a UHPLC + Ultimate 300 from Thermo equipped with a PDA detector coupled with a Q Exactive extended Mass HRMS from ThermoFisher Scientific. Infrared (IR) spectra were recorded on a Bruker FTIR Alpha (ATR Mode) spectrometer, $v_{\max }$ in $\mathrm{cm}^{-1}$. Bands are characterized as broad (br), strong (s), medium (m), or weak (w).

\subsection{Solvents}

THF, $\mathrm{CH}_{2} \mathrm{Cl}_{2}, \mathrm{MeCN}$ and $\mathrm{Et}_{2} \mathrm{O}$ were purified under a positive pressure of dry Ar by a modified Innovative Technologies purification system. Anhydrous DMF, DMSO, MeOH and 1,4-dioxane were purchased from Acros with sure-seal and molecule sieves. $\mathrm{CDCl}_{3}, \mathrm{C}_{6} \mathrm{D}_{6}, \mathrm{CD}_{2} \mathrm{Cl}_{2}$ and DMSO- $d_{6}$ were purchased from Eurisotop and stored over activated $4 \AA$ molecular sieves prior to use. Purification of products was carried out with reagent grade solvents (Fisher).

\subsection{Reagents}

But-3-yn-1-ol: purchased from TCI and used as received.

Formaldehyde: purchased from TCI and used as received.

CuI: purchased from Strem and used as received.

Dicyclohexylamine: purchased from Fluorochem and used as received. 
Diisopropylamine: purchased from Fluorochem and used as received.

tert-Butyldimethylsilyl chloride: purchased from Fluorochem and used as received.

Imidazole: purchased from Aldrich and used as received.

4,4,5,5-Tetramethyl-2-(propa-1,2-dien-1-yl)-1,3,2-dioxaborolane: purchased from TCI and used as received.

First-generation phosphine-free Ru complex (HG-I): purchased from Aldrich and used as received.

Methanesulfonyl chloride (MsCl): purchased from Aldrich and used as received.

Triethylamine: purchased from Fluorochem and used as received.

Lithium bromide: purchased from Fluorochem and used as received.

But-3-yn-1-ylbenzene: purchased from TCI and used as received.

Hex-5-ynoic acid: purchased from TCI and used as received.

Prop-2-yn-1-amine: purchased from TCI and used as received.

Di-tert-butyl dicarbonate $\left((\mathbf{B o c})_{2} \mathbf{O}\right)$ : purchased from Fluorochem and used as received.

3-Chlorobenzo[b]thiophene-2-carboxylic acid: purchased from TCI and used as received.

Indomethacin: purchased from TCI and used as received.

Hex-5-yn-1-ol: purchased from TCI and used as received.

Hept-6-yn-1-ol: purchased from TCI and used as received.

4,4,5,5-Tetramethyl-1,3,2-dioxaborolane ( $\mathrm{HB}($ pin)): purchased from TCI and used as received.

Bis(2,4,6-triisopropylphenyl)borane (HB(trip) $\left.)_{2}\right)$ : purchased from TCI and used as received.

(Diacetoxyiodo)benzene: purchased from Fluorochem and used as received.

2,2,6,6-Tetramethylpiperidine 1-oxyl (TEMPO): purchased from TCI and used as received.

Sodium bicarbonate: purchased from Ficher and used as received.

Pent-4-enoic acid: purchased from TCI and used as received.

Hex-5-ynoic acid: purchased from TCI and used as received.

Potassium tert-butoxide: purchased from Strem and used as received.

$\mathrm{N}, \mathrm{N}$-Dimethylpyridin-4-amine: purchased from Fluorochem and used as received.

$N, N^{\prime}$-Diisopropylcarbodiimide: purchased from TCI and used as received.

Ethyl buta-2,3-dienoate: purchased from Aldrich and used as received.

Benylbromide: purchased from TCI and used as received.

Prop-2-yn-1-ol: purchased from TCI and used as received.

Sodium hydride ( $\mathrm{NaH}, \mathbf{6 0} \% \mathrm{wt}$ in mineral oil): purchased from TCI and used as received.

3-Octyn-1-ol: purchased from TCI and used as received.

Ethane-1,2-diamine: purchased from Aldrich and used as received.

Dec-9-yn-1-ol: purchased from TCI and used as received.

Undec-10-yn-1-ol: purchased from TCI and used as received.

Dodec-11-yn-1-ol: purchased from Fluorochem and used as received.

1-bromododecane: purchased from Aldrich and used as received. 
Bis(pinacolato)diboron: purchased from Fluorochem and used as received.

Potassium carbonate: purchased from Fluorochem and used as received.

Cyclohexylallene: purchased from Aldrich and used as received.

2-(2,6-Diisopropylphenyl)-3,3-dimethyl-2-azaspiro[4.5]dec-1-en-2-ium

hydrogen

dichloride

(Cyclohexyl CAAC): purchased from TCI and used as received.

\section{Synthesis and Characterization of the Starting Materials}

Allenes were prepared according to a reported procedure. ${ }^{1} \mathbf{R u}-\mathbf{V}$ complexes were prepared according to a previously reported procedure. ${ }^{2}$

8-(4,4,5,5-Tetramethyl-1,3,2-dioxaborolan-2-yl)octa-6,7-dien-1-yl-3-chlorobenzo[b]thio phene-2carboxylate (2f) ${ }^{1} \mathbf{H}$ NMR (400 MHz, $\mathbf{C D C l}_{3}$ ): $\delta$ 8.00-7.96 (m, 1H), 7.83-7.81 (m, 1H), 7.55-7.48 (m, $2 \mathrm{H}), 5.11(\mathrm{p}, J=6.7 \mathrm{~Hz}, 1 \mathrm{H}), 4.66(\mathrm{dt}, J=6.6,3.2 \mathrm{~Hz}, 2 \mathrm{H}), 4.38(\mathrm{t}, J=6.6 \mathrm{~Hz}, 2 \mathrm{H}), 2.08-2.02(\mathrm{~m}, 2 \mathrm{H})$, 1.85-1.78 (m, 2H), 1.56-1.48 (m, 4H); ${ }^{13} \mathbf{C}$ NMR (101 MHz, $\left.\mathbf{C D C l}_{3}\right): \delta 208.7,161.5,138.8,137.3,128.3$, 127.3, 126.3, 125.6, 124.0, 122.9, 89.9, 75.0, 65.9, 28.7, 28.5, 28.2, 25.6; IR (neat): 2924 (m), 2854 (m), 1954 (w), 1723 (s), 1228 (s), 753 (s), 728 (m) cm ${ }^{-1}$; HRMS[M+Na] $]^{+}$: Calcd for $\mathrm{C}_{17} \mathrm{H}_{27} \mathrm{O}_{2} \mathrm{NaClS}: 343.0530$, Found: 343.0547.

Hepta-5,6-dien-1-yl 2-(1-(4-chlorobenzoyl)-5-methoxy-2-methyl-1H-indol-3-yl)acetate (2g) ${ }^{\mathbf{1}} \mathrm{H}$ NMR $\left(400 \mathrm{MHz}, \mathbf{C D C l}_{3}\right): \delta 7.68-7.64(\mathrm{~m}, 2 \mathrm{H}), 7.48-7.45(\mathrm{~m}, 2 \mathrm{H}), 6.97(\mathrm{~d}, J=2.4 \mathrm{~Hz}, 1 \mathrm{H}), 6.86(\mathrm{~d}, J=9.0 \mathrm{~Hz}$, $1 \mathrm{H}), 6.67(\mathrm{dd}, J=9.0,2.6 \mathrm{~Hz}, 1 \mathrm{H}), 5.03(\mathrm{p}, J=6.7 \mathrm{~Hz}, 1 \mathrm{H}), 4.64(\mathrm{dt}, J=6.6,3.2 \mathrm{~Hz}, 2 \mathrm{H}), 4.11(\mathrm{t}, J=6.6$ $\mathrm{Hz}, 2 \mathrm{H}), 3.83$ (s, 3H), 3.66 (s, 2H), 2.39 (s, 3H), 2.02-1.95 (m, 2H), 1.70-1.63 (m, 2H), 1.47-1.40 (m, 2H); ${ }^{13}$ C NMR (101 MHz, CDCl $)$ ): $\delta$ 208.6, 171.5, 168.4, 156.2, 139.4, 136.0, 134.1, 131.3, 130.9, 130.8, 129.3, 115.1, 112.8, 111.8, 101.4, 89.6, 75.1, 65.1, 55.8, 30.6, 28.1, 27.8, 25.5, 13.5; IR (neat): 3055 (w), 2930 (m), 1953 (w), 1730 (s), 1680 (s), 1312 (s), 732 (s) cm ${ }^{-1}$; HRMS[M+H] $]^{+}$: Calcd for $\mathrm{C}_{29} \mathrm{H}_{27} \mathrm{O}_{4} \mathrm{NCl}_{\text {: }}$ 452.1623, Found: 452.1623.

Octa-6,7-dien-1-yl pent-4-enoate (2h) ${ }^{1} \mathbf{H}$ NMR (400 MHz, $\mathbf{C D C l}_{3}$ ): $\delta 5.87-5.77$ (m, 1H), 5.12-4.98 (m, $3 \mathrm{H}), 4.65(\mathrm{dt}, J=6.6,3.2 \mathrm{~Hz}, 2 \mathrm{H}), 4.07(\mathrm{t}, J=6.7 \mathrm{~Hz}, 2 \mathrm{H}), 2.43-2.34(\mathrm{~m}, 4 \mathrm{H}), 2.04-1.97(\mathrm{~m}, 2 \mathrm{H}), 1.66-$ 1.59 (m, 2H), 1.48-1.34 (m, 4H); ${ }^{13} \mathbf{C}$ NMR (101 MHz, $\left.\mathbf{C D C l}_{3}\right): \delta 208.6,173.3,136.9,115.6,89.9,74.9$, 64.6, 33.7, 29.0, 28.8, 28.6, 28.2, 25.5; IR (neat): 3079 (w), 2934 (m), 2858 (m), 1954 (m), 1167(s), 841 (s) $\mathrm{cm}^{-1} ;$ HRMS[M+H] $]^{+}$: Calcd for $\mathrm{C}_{13} \mathrm{H}_{21} \mathrm{O}_{2}: 209.1536$, Found: 209.1536 .

Octa-6,7-dien-1-yl hex-4-ynoate (2j) ${ }^{1} \mathbf{H}$ NMR (400 MHz, $\left.\mathbf{C D C l}_{3}\right): \delta 5.09$ (p, $\left.J=6.8 \mathrm{~Hz}, 1 \mathrm{H}\right), 4.66$ (dt, $J$ $=6.5,3.2 \mathrm{~Hz}, 2 \mathrm{H}), 4.09(\mathrm{t}, J=6.7 \mathrm{~Hz}, 2 \mathrm{H}), 2.51-2.41(\mathrm{~m}, 4 \mathrm{H}), 2.04-1.97(\mathrm{~m}, 2 \mathrm{H}), 1.76(\mathrm{t}, J=2.4 \mathrm{~Hz}, 3 \mathrm{H})$, $1.68-1.61(\mathrm{~m}, 2 \mathrm{H}), 1.49-1.35(\mathrm{~m}, 4 \mathrm{H}) ;{ }^{13} \mathbf{C}$ NMR (101 MHz, $\left.\mathbf{C D C l}_{3}\right): \delta 208.7,171.1,89.9,81.7,77.4$, 76.4 , 74.9, 64.7, 34.2, 28.8, 28.6, 28.2, 25.5, 14.9, 3.6; IR (neat): 2921 (m), 2855 (w), 1953 (w), 1733 (s), 1162 (s), $842(\mathrm{~s}) \mathrm{cm}^{-1}$; HRMS[M+H] ${ }^{+}$: Calcd for $\mathrm{C}_{14} \mathrm{H}_{21} \mathrm{O}_{2}: 221.1536$, Found: 221.1536 .

\section{Synthesis and Characterization of Cross-Metathesis Products}

3.1. General cross-metathesis procedure. In a $\mathrm{N}_{2}$-filled glovebox, a $7 \mathrm{~mL}$ oven-dried vial, equipped with a magnetic stir bar, was charged with allene $\mathbf{2 a}(19.8 \mathrm{mg}, 0.10 \mathrm{mmol}), 4,4,5,5$-tetramethyl-2-(propa-1,2dien-1-yl)-1,3,2-dioxaborolane 1 (49.8 $\mathrm{mg}, 0.30 \mathrm{mmol})$ and $1.0 \mathrm{~mL} \mathrm{CH}_{2} \mathrm{Cl}_{2}$. The mixture was then charged with Ru-Va (100 $\mu \mathrm{L}$ of a $0.05 \mathrm{M}$ stock solution in $\mathrm{CH}_{2} \mathrm{Cl}_{2} ; 3.2 \mathrm{mg}, 0.005 \mathrm{mmol}$ ). The vessel was loosely capped and the mixture was allowed to stir in the glovebox at $22{ }^{\circ} \mathrm{C}$ for $16 \mathrm{~h}$. The vessel was then removed from of glovebox, after which the reaction was quenched by the addition of $2.0 \mathrm{~mL}$ of wet (undistilled) 
diethyl ether. The volatiles were removed in vacuo, leaving behind dark brown oil, which was purified by silica gel chromatography (100:0-20:1 pentane:ethyl acetate) to afford 1,3-disubstituted allene 3a as paleyellow oil ( $21.7 \mathrm{mg}, 0.067 \mathrm{mmol}, 67 \%$ yield). Reaction at $1.0 \mathrm{mmol}$ scale was performed for substrate $\mathbf{3 b}$, giving the same conversion as at $0.1 \mathrm{mmol}(85 \%$ conv), and a slightly lower yield $(65 \%)$ due to purification complications.

\subsection{Analytical data for 1,3-disubstituted allene products}

tert-Butyldimethyl((5-(4,4,5,5-tetramethyl-1,3,2-dioxaborolan-2-yl)penta-3,4-dien-1-yl)oxy)silane

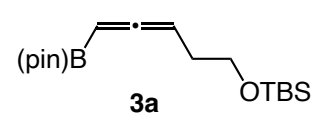

(3a). ${ }^{1}$ H NMR (400 MHz, $\left.\mathbf{C D C l}_{3}\right): \delta 5.08$ (q, $\left.J=7.0 \mathrm{~Hz}, 1 \mathrm{H}\right), 4.89$ (dt, $J=6.6,3.3$ $\mathrm{Hz}, 1 \mathrm{H}), 3.67(\mathrm{t}, J=6.9 \mathrm{~Hz}, 2 \mathrm{H}), 2.28-2.22(\mathrm{~m}, 2 \mathrm{H}), 1.27(\mathrm{~s}, 6 \mathrm{H}), 1.26(\mathrm{~s}, 6 \mathrm{H}), 0.89$ (s, 9H), 0.05 (s, 6H). ${ }^{13} \mathbf{C}$ NMR (101 MHz, $\left.\mathbf{C D C l}_{3}\right): \delta 216.7,83.7,82.6,63.1,31.2$, 26.1, 24.9, 24.8, 18.5, -5.1; IR (neat): 2951 (m), 2920 (s), 1945 (m), 1377(m), 727 (m) cm ${ }^{-1}$; HRMS $[\mathbf{M}+\mathbf{H}]^{+}$: Calcd for $\mathrm{C}_{17} \mathrm{H}_{34} \mathrm{BO}_{3} \mathrm{Si}$ : 325.23648, Found: 325.237141 .

4,4,5,5-Tetramethyl-2-(5-phenylpenta-1,2-dien-1-yl)-1,3,2-dioxaborolane (3b). Following the general

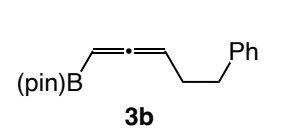
procedure, the dark brown oil residue was purified by silica gel chromatography (100:050:1 petroleum ether: $\left.\mathrm{Et}_{2} \mathrm{O}\right)$ to afford $\mathbf{3 b}$ as colorless oil $(19.2 \mathrm{mg}, 0.071 \mathrm{mmol}, 71 \%$ yield). ${ }^{1} \mathbf{H}$ NMR (400 MHz, $\left.\mathbf{C D C l}_{3}\right): \delta 7.30-7.26(\mathrm{~m}, 2 \mathrm{H}), 7.21-7.16(\mathrm{~m}, 3 \mathrm{H}), 5.14(\mathrm{q}$, $\mathrm{J}=6.7 \mathrm{~Hz}, 1 \mathrm{H}), 4.93(\mathrm{dt}, \mathrm{J}=7.0,3.7 \mathrm{~Hz}, 1 \mathrm{H}), 2.76-2.70(\mathrm{~m}, 2 \mathrm{H}), 2.36-2.31(\mathrm{~m}, 2 \mathrm{H}), 1.28(\mathrm{~s}, 6 \mathrm{H}), 1.27(\mathrm{~s}$, 6H). ${ }^{13} \mathbf{C}$ NMR (101 MHz, $\left.\mathbf{C D C l}_{3}\right): \delta 216.4,141.9,128.6,128.4,126.0,85.6,83.7,35.6,29.1,25.0,24.8$; IR (neat): 2920 (s), 2852 (s), 1454 (m), 1377 (w), 1144 (w), 698 (w) cm $\mathrm{cm}^{-1}$ HRMS [M+Na] ${ }^{+}$: Calcd for $\mathrm{C}_{17} \mathrm{H}_{23} \mathrm{BNaO}_{2}$ : 293.1684, found : 293.1676 .

4,4,5,5-Tetramethyl-2-(4-phenylbuta-1,2-dien-1-yl)-1,3,2-dioxaborolane (3c). Following the general

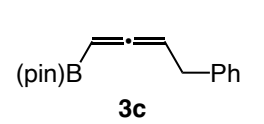
procedure, the dark brown oil residue was purified by filtration through a silica gel plug using $\mathrm{Et}_{2} \mathrm{O}$ as the eluent, followed by Kugelrohr distillation which was used to remove remaining allenyl B(pin) $\mathbf{1}$ and 2-isopropoxystyrene, affording $\mathbf{3 c}$ as pale-yellow oil (12.8 mg, 0.050 mmol, 50 \% yield). ${ }^{1} \mathbf{H}$ NMR (400 MHz, $\left.\mathbf{C D C l}_{3}\right): 7.29-7.25(\mathrm{~m}, 3 \mathrm{H}), 7.23-7.17(\mathrm{~m}, 2 \mathrm{H}), 5.24$ (q, $J=7.2 \mathrm{~Hz}, 1 \mathrm{H}), 4.93(\mathrm{dt}, J=6.6,3.3 \mathrm{~Hz}, 1 \mathrm{H}), 3.44-3.31(\mathrm{~m}, 2 \mathrm{H}), 1.28(\mathrm{~s}, 12 \mathrm{H}) .{ }^{13} \mathbf{C}$ NMR (101 MHz, $\left.\mathbf{C D C l}_{3}\right) \delta 217.0,140.4,128.7,128.4,126.3,83.8,83.7,83.3,75.2,34.3,24.7$. HRMS [M+Na] $]^{+}$: Calcd for $\mathrm{C}_{16} \mathrm{H}_{21} \mathrm{BNaO}_{2}$ : 279.1527, found : 279.1523 .

Methyl 7-(4,4,5,5-tetramethyl-1,3,2-dioxaborolan-2-yl)hepta-5,6-dienoate (3d). Following the general

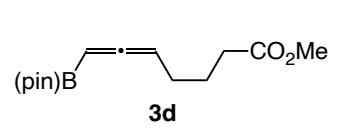
procedure, the dark brown oil residue was purified by silica gel chromatography (100:0-20:1 petroleum ether:ethyl acetate) to afford $\mathbf{3 d}$ as pale-yellow oil (18.9 $\mathrm{mg}$, $0.071 \mathrm{mmol}, 71 \%$ yield). ${ }^{1} \mathbf{H}$ NMR (400 $\left.\mathbf{~ M H z}, \mathbf{C D C l}_{3}\right): \delta 5.04(\mathrm{q}, J=6.8 \mathrm{~Hz}, 1 \mathrm{H})$, $4.91(\mathrm{dt}, J=6.8,3.5 \mathrm{~Hz}, 1 \mathrm{H}), 3.66(\mathrm{~s}, 3 \mathrm{H}), 2.42-2.38(\mathrm{~m}, 2 \mathrm{H}), 2.10-2.04(\mathrm{~m}, 2 \mathrm{H}), 1.80-1.74(\mathrm{~m}, 2 \mathrm{H}), 1.27$ (s, 6H), 1.26 (s, 6H); ${ }^{13} \mathbf{C}$ NMR (101 MHz, $\left.\mathbf{C D C l}_{3}\right): \delta 216.6,174.3,85.1,83.7,51.6,33.2,26.8,25.0,24.7$, 24.3. IR (neat): 2923 (s), 2850 (m), 1975 (w), 1738 (s), 1327 (m), 1145 (s), 848 (w) $\mathrm{cm}^{-1}$; HRMS [M+H] $]^{+}$: Calcd for $\mathrm{C}_{14} \mathrm{H}_{24} \mathrm{BO}_{4}: 267.1763$, found: 267.1752 .

tert-Butyl(4-(4,4,5,5-tetramethyl-1,3,2-dioxaborolan-2-yl)buta-2,3-dien-1-yl)carbamate

(3e).

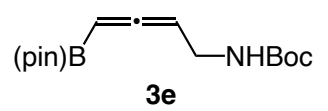

Following the general procedure, the dark brown oil residue was purified by silica gel chromatography (50:1-10:1 petroleum ether:ethyl acetate) to afford $\mathbf{3 e}$ as paleyellow oil (14.8 mg, $0.050 \mathrm{mmol}, 50 \%$ yield). ${ }^{1} \mathbf{H}$ NMR (400 $\left.\mathbf{~ M H z}, \mathbf{C D C l}_{3}\right): \delta 5.16$ (q, $J=6.4 \mathrm{~Hz}, 1 \mathrm{H}), 5.03$ (dt, $J=6.7,3.5 \mathrm{~Hz}, 1 \mathrm{H}), 4.64$ (brs, 1H), 3.80-3.69 (m, 2H), 1.43 (s, 9H), 1.27 (s, 6H), 1.27 (s, 6H); ${ }^{13} \mathbf{C}$ NMR (101 MHz, $\left.\mathbf{C D C l}_{3}\right): \delta 215.8,155.8,84.7,83.9,79.5,38.5,28.5,24.9,24.8$; 
IR (neat): 3351 (w), 2977 (m), 2925 (m), 1949 (w), 1699 (s), 1142 (s), 847 (m) cm ${ }^{-1}$; HRMS[M+H] : Calcd for $\mathrm{C}_{15} \mathrm{H}_{27} \mathrm{BNO}_{4}$ : 296.2028, found: 296.2023.

8-(4,4,5,5-Tetramethyl-1,3,2-dioxaborolan-2-yl)octa-6,7-dien-1-yl 3-chlorobenzo[b]thio phenne-2-

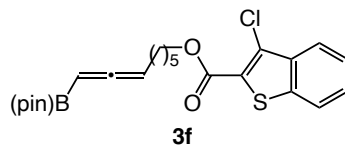

carboxylate (3f). Following the general procedure, the dark brown oil residue was purified by silica gel chromatography (100:0-25:1 petroleum ether:ethyl acetate) to afford $3 f$ as pale-yellow oil (27.7 $\mathrm{mg}, 0.062 \mathrm{mmol}, 62 \%$ yield). ${ }^{1}$ H NMR (400 MHz, $\left.\mathbf{C D C l}_{3}\right): \delta 8.02-7.93(\mathrm{~m}, 1 \mathrm{H}), 7.86-7.77(\mathrm{~m}, 1 \mathrm{H}), 7.52(\mathrm{p}, J=7.2,2 \mathrm{H}), 5.08(\mathrm{q}, J=6.8 \mathrm{~Hz}, 1 \mathrm{H})$, $4.91(\mathrm{dt}, J=6.8,3.5 \mathrm{~Hz}, 1 \mathrm{H}), 4.37(\mathrm{t}, J=6.7 \mathrm{~Hz}, 2 \mathrm{H}), 2.07(\mathrm{~m}, 2 \mathrm{H}), 1.80(\mathrm{~m}, 2 \mathrm{H}), 1.55-1.48(\mathrm{~m}, 4 \mathrm{H})$, 1.27 (s, 6H), 1.26 (s, 6H); ${ }^{13} \mathbf{C}$ NMR (101 MHz, $\left.\mathbf{C D C l}_{3}\right): \delta 216.6,161.5,138.8,137.3,128.3,127.3,126.3$, 125.6, 124.0, 122.9, 85.8, 83.7, 66.0, 28.7, 28.5, 27.2, 25.4, 25.0, 24.8; IR (neat): 2922 (m), 2852 (m), 1945 (w), $1723(\mathrm{~m}), 1512(\mathrm{~m}), 1226(\mathrm{~s}), 752(\mathrm{~m}) \mathrm{cm}^{-1}$; HRMS[M+H] ${ }^{+}$: Calcd for $\mathrm{C}_{23} \mathrm{H}_{29} \mathrm{BClO}_{4} \mathrm{~S}:$ : 447.1563, found: 447.1553 .

8-(4,4,5,5-Tetramethyl-1,3,2-dioxaborolan-2-yl)octa-6,7-dien-1-yl 2-(1-((4-chlorophenyl) (ג3-

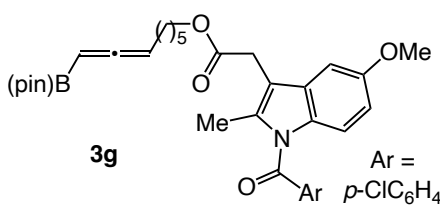
oxidaneylidene)methyl)-5-methoxy-2-methyl-1H-indol-3-yl)acetate

(3g). Following the general procedure, the dark brown oil residue was purified by silica gel chromatography (100:0-10:1 petroleum ether:ethyl acetate) to afford $3 \mathrm{~g}$ as pale-yellow oil (40.0 $\mathrm{mg}, 0.069 \mathrm{mmol}, 69 \%$ yield). ${ }^{1}$ H NMR (400 MHz,CDCl $)$ ): $\delta \delta 7.66(\mathrm{~d}, J=8.4 \mathrm{~Hz}, 2 \mathrm{H}), 7.47(\mathrm{~d}, J=8.6$ $\mathrm{Hz}, 2 \mathrm{H}), 6.96(\mathrm{~d}, J=2.6 \mathrm{~Hz}, 1 \mathrm{H}), 6.86(\mathrm{~d}, J=9.0 \mathrm{~Hz}, 1 \mathrm{H}), 6.66(\mathrm{dd}, J=9.0,2.6 \mathrm{~Hz}, 1 \mathrm{H}), 5.02(\mathrm{q}, J=6.8$ $\mathrm{Hz}, 1 \mathrm{H}), 4.88(\mathrm{dt}, J=6.8,3.6 \mathrm{~Hz}, 1 \mathrm{H}), 4.09(\mathrm{t}, J=6.7 \mathrm{~Hz}, 2 \mathrm{H}), 3.83(\mathrm{~s}, 3 \mathrm{H}), 3.65(\mathrm{~s}, 2 \mathrm{H}), 2.38(\mathrm{~s}, 3 \mathrm{H}), 2.04$ - $1.94(\mathrm{~m}, 2 \mathrm{H}), 1.62(\mathrm{p}, J=6.8 \mathrm{~Hz}, 3 \mathrm{H}), 1.45-1.31(\mathrm{~m}, 5 \mathrm{H}), 1.27(\mathrm{~s}, 6 \mathrm{H}), 1.26(\mathrm{~s}, 6 \mathrm{H}) ;{ }^{13} \mathrm{C}$ NMR (101 MHz, $\left.\mathbf{C D C l}_{3}\right): \delta 216.5,171.1,168.4,156.2,139.4,136.0,134.1,131.3,131.0,130.8,129.3,115.09,112.9$, 111.8, 101.5, 85.7, 83.7, 65.3, 55.8, 30.6, 28.7, 28.5, 27.2, 25.3, 25.0, 24.8, 13.5; IR (neat): 2921 (s), 2852 (m), 1944 (m), 1732 (m), 1682 (m), 1314 (s), 1141 (s), 847 (s), 754 (m) cm ${ }^{-1}$; HRMS[M+H] ${ }^{+}$: Calcd for $\mathrm{C}_{33} \mathrm{H}_{40} \mathrm{BClO}_{6} \mathrm{~N}: 592.2632$, Found: 592.2617.

8-(4,4,5,5-Tetramethyl-1,3,2-dioxaborolan-2-yl)octa-6,7-dien-1-yl pent-4-enoate $(3 \mathrm{~h})$. Following the general procedure, the dark brown oil residue was purified by silica gel chromatography (40:1-20:1

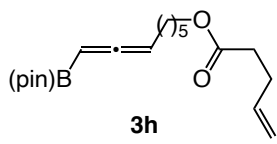
petroleum ether:ethyl acetate) to afford $\mathbf{3 h}$ as pale-yellow oil (19.4 $\mathrm{mg}, 0.058 \mathrm{mmol}, 58$ \% yield). ${ }^{1} \mathbf{H}$ NMR (400 MHz, $\left.\mathbf{C D C l}_{3}\right): \delta 5.87-5.77(\mathrm{~m}, 1 \mathrm{H}), 5.08-4.98(\mathrm{~m}, 3 \mathrm{H}), 4.89$ $(\mathrm{dt}, \mathrm{J}=6.8,3.5 \mathrm{~Hz}, 1 \mathrm{H}), 4.07(\mathrm{t}, \mathrm{J}=6.8 \mathrm{~Hz}, 3 \mathrm{H}), 2.42-2.35(\mathrm{~m}, 4 \mathrm{H}), 2.07-2.01(\mathrm{~m}$, 2H), 1.66-1.59 (m, 2H), 1.46-1.39 (m, 4H), 1.27 (s, 6H), 1.26 (s, 6H); ${ }^{13}$ C NMR (101 MHz, CDCl $)$ : $\delta 216.6,173.3,136.9,115.6,85.8,83.7,64.6,33.7,29.1,28.8,28.6,27.2,25.3,25.0,24.7$; IR (neat): 2977 (m), 2924 (m), 2855 (m), 1945 (m), 1735 (s), $1327(\mathrm{~m}), 1142$ (s), 847 (s), 727 (m) cm ${ }^{-1}$; HRMS[M+H] Calcd for $\mathrm{C}_{19} \mathrm{H}_{32} \mathrm{BO}_{4}$ : 335.2389, Found: 335.2377.

8-(4,4,5,5-Tetramethyl-1,3,2-dioxaborolan-2-yl)octa-6,7-dien-1-yl hex-4-ynoate (3j). Following the general procedure, the dark brown oil residue was purified by silica gel chromatography (98:2-96:4

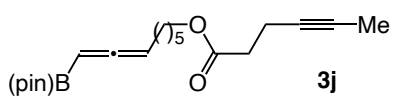
petroleum ether:ethyl acetate) to afford $\mathbf{3} \mathbf{j}$ as pale-yellow oil $(23.9 \mathrm{mg}, 0.069$ mmol, 69 \% yield). ${ }^{1} \mathbf{H}$ NMR (400 $\left.\mathbf{M H z}, \mathbf{C D C l}_{3}\right): \delta 5.05$ (q, $\left.J=6.8 \mathrm{~Hz}, 1 \mathrm{H}\right)$, $4.89(\mathrm{dt}, J=6.8,3.5 \mathrm{~Hz}, 1 \mathrm{H}), 4.09(\mathrm{t}, J=6.8 \mathrm{~Hz}, 2 \mathrm{H}), 2.51-2.41(\mathrm{~m}, 4 \mathrm{H}), 2.07-$ $2.01(\mathrm{~m}, 2 \mathrm{H}), 1.76(\mathrm{t}, \mathrm{J}=2.4 \mathrm{~Hz}, 3 \mathrm{H}), 1.67-1.62(\mathrm{~m}, 2 \mathrm{H}), 1.46-1.40(\mathrm{~m}, 4 \mathrm{H}), 1.27(\mathrm{~d}, J=4.0 \mathrm{~Hz}, 12 \mathrm{H})$; ${ }^{13} \mathbf{C}$ NMR (101 MHz, $\left.\mathbf{C D C l}_{3}\right)$ : 216.6, 172.4, 85.8, 83.68, 77.4, 76.4, 65.4, 34.2, 28.8, 28.6, 27.2 25.3, 25.0, 
24.8, 14.9, 3.6; IR (neat): 2921 (m), 2854 (m), 1945 (m), 1735 (s), 1143 (s), 847 (m) cm $\mathrm{cm}^{-1}$; HRMS[M+H]+: Calcd for $\mathrm{C}_{20} \mathrm{H}_{32} \mathrm{BO}_{4}$ : 347.2389, Found: 347.2386.

9-(4,4,5,5-Tetramethyl-1,3,2-dioxaborolan-2-yl)nona-7,8-dien-1-ol (3k). Following the general (pin)B $=\square-L_{\mathrm{OH}}$ procedure, the dark brown oil residue was purified by silica gel chromatography $3 \mathrm{k}$ (50:1-0:100 petroleum ether:Et $\left.t_{2} \mathrm{O}\right)$ to afford $3 \mathbf{k}$ as pale-yellow oil $(10.1 \mathrm{mg}, 0.040$ mmol, $40 \%$ yield, obtained as mixture of $\mathbf{3 k}$ and its boronate. Fraction of pure $\mathbf{3 k}$ used for full characterization). ${ }^{1} \mathbf{H}$ NMR (400 $\left.\mathbf{M H z}, \mathbf{C D C l}_{3}\right): \delta 5.05$ (q, $\left.J=6.8 \mathrm{~Hz}, 1 \mathrm{H}\right), 4.90(\mathrm{dt}, J=6.8,3.5 \mathrm{~Hz}, 1 \mathrm{H})$, $3.64(\mathrm{q}, J=5.9 \mathrm{~Hz}, 2 \mathrm{H}), 2.06-2.02(\mathrm{~m}, 2 \mathrm{H}), 1.60-1.54(\mathrm{~m}, 2 \mathrm{H}), 1.47-1.41(\mathrm{~m}, 4 \mathrm{H}), 1.28(\mathrm{~s}, 6 \mathrm{H}), 1.27(\mathrm{~s}$, 6H); ${ }^{13} \mathbf{C}$ NMR (101 MHz, $\left.\mathbf{C D C l}_{3}\right): \delta 216.6,85.8,83.7,63.1,32.6,28.8,27.2,24.9,24.9,24.8$; IR (neat): 3356 (w), 2977 (m), 2927 (s), 2856 (m), 1945 (m), 1414 (s), 1331 (s), 1144 (s), 847 (m) cm ; HRMS[M+H] ${ }^{+}$: Calcd for $\mathrm{C}_{14} \mathrm{H}_{26} \mathrm{BO}_{3}: 253.1970$, Found: 253.1965 .

8-(4,4,5,5-Tetramethyl-1,3,2-dioxaborolan-2-yl)octa-6,7-dienoic acid (31). Following the general procedure, the dark brown oil residue was purified by silica gel chromatography $($ pin) $\mathrm{B}=\underbrace{=}_{31} \mathrm{CO}_{2} \mathrm{H}$ (95:5-90:10 petroleum ether:ethyl acetate) to afford $\mathbf{3 I}$ as pale-yellow oil (16.8 $\mathrm{mg}$, 0.063 mmol, 63 \% yield). ${ }^{1} \mathbf{H}$ NMR (400 MHz, $\left.\mathbf{C D C l}_{3}\right): \delta 5.05$ (q, $\left.J=6.8 \mathrm{~Hz}, 1 \mathrm{H}\right)$, $4.90(\mathrm{dt}, J=7.0,3.6 \mathrm{~Hz}, 1 \mathrm{H}), 2.35(\mathrm{t}, J=7.5 \mathrm{~Hz}, 2 \mathrm{H}), 2.10-2.01(\mathrm{~m}, 2 \mathrm{H}), 1.75-1.65(\mathrm{~m}, 2 \mathrm{H}), 1.53-1.43$ (m, 2H), 1.27 (s, 6H), 1.26 (s, 6H); ${ }^{13} \mathbf{C}$ NMR (101 MHz, $\left.\mathbf{C D C l}_{3}\right): \delta 216.56,179.07,85.54,83.71,83.35$, 33.83, 28.47, 26.91, 24.98, 24.95, 24.72, 24.67, 24.24, 24.04. IR (neat): 3427 (w), 2977 (w), 2924 (m), 2854 (s), 1945 (m), 1321 (s), 1141 (s), 1331 (s), 1144 (s), 847 (m), 731 (m) cm ${ }^{-1}$; HRMS [M+H] ${ }^{+}$: Calcd for $\mathrm{C}_{14} \mathrm{H}_{24} \mathrm{BO}_{4}: 267.1762$, found: 267.1756 .

2-(3-Cyclohexylpropa-1,2-dien-1-yl)-4,4,5,5-tetramethyl-1,3,2-dioxaborolane (3m). Following the =- general procedure, the dark brown oil residue was purified by silica gel chromatography $3 \mathrm{~m}$ (100:1-25:1 petroleum ether: $\left.\mathrm{Et}_{2} \mathrm{O}\right)$ to afford $\mathbf{3 m}$ as pale-yellow oil $(5.2 \mathrm{mg}, 0.021 \mathrm{mmol}, 21$ \% yield, isolated with unidentified byproducts). ${ }^{1} \mathbf{H}$ NMR (400 $\left.\mathbf{M H z}, \mathbf{C D C l}_{3}\right): \delta 5.07$ (t, $J$ $=6.2 \mathrm{~Hz}, 1 \mathrm{H}), 4.93-4.91(\mathrm{~m}, 1 \mathrm{H}), 2.05(\mathrm{~m}, 1 \mathrm{H}), 1.80-1.57(\mathrm{~m}, 10 \mathrm{H}), 1.27(\mathrm{~s}, 6 \mathrm{H}), 1.26(\mathrm{~s}, 6 \mathrm{H})$. This compound has been synthesized previously and its full chracterization has been reported. ${ }^{3}$ The $1 \mathrm{H}$ NMR data are consistent.

tert-Butyldimethyl((7-phenylhepta-3,4-dien-1-yl)oxy)silane (6). Following the general procedure, the dark brown oil residue was purified by silica gel chromatography (100:0-20:1 petroleum ether:ethyl Ph $\square=$ OTBS acetate) to afford 6 as pale yellow oil $(8.2 \mathrm{mg}, 0.027 \mathrm{mmol}, 27 \%$ yield, isolated with $6 \quad 5-10 \%$ of homo-metathesis impurity). ${ }^{1} \mathbf{H}$ NMR (400 $\left.\mathbf{~ M H z}, \mathbf{C D C l}_{3}\right): \delta{ }^{1} \mathbf{H}$ NMR (400 MHz, CDCl $): \delta 7.29-7.28(\mathrm{~m}, 2 \mathrm{H}), 7.21-7.15(\mathrm{~m}, 3 \mathrm{H}), 5.17-5.04(\mathrm{~m}, 2 \mathrm{H}), 3.65(\mathrm{dt}, J=9.8,6.9 \mathrm{~Hz}, 3 \mathrm{H})$, $2.72(\mathrm{t}, J=7.6 \mathrm{~Hz}, 2 \mathrm{H}), 2.35-2.26(\mathrm{~m}, 2 \mathrm{H}), 2.25-2.12(\mathrm{~m}, 3 \mathrm{H}), 0.90(\mathrm{~s}, 12 \mathrm{H}), 0.06(\mathrm{~s}, 9 \mathrm{H}) .{ }^{13} \mathbf{C}$ NMR (101 MHz, $\left.\mathbf{C D C l}_{3}\right) \delta 204.8,142.0,128.6,128.4,125.9,90.3,88.2,63.2,35.6,32.8,32.7,30.7,26.1,-5.1$. HRMS [M+H] ${ }^{+}$: Calcd for $\mathrm{C}_{19} \mathrm{H}_{31} \mathrm{OSi}: 303.2139$, Found: 303.2132 .

\section{Synthesis and Characterization of Ru-Vinylidene Complexes}

4.1. Synthesis and characterization of Ru-vinyl-1. In a glovebox, an oven-dried $7 \mathrm{~mL}$ vial equipped with a magnetic stir bar was charged with complex Ru-I (40 mg, $49 \mu \mathrm{mol}), \mathrm{CH}_{2} \mathrm{Cl}_{2}(1 \mathrm{~mL})$, followed by allenyl-B(pin) $(13.1 \mu \mathrm{L}, 12.1 \mathrm{mg}, 73 \mu \mathrm{mol})$. The resulting red solution was allowed to stir at $22{ }^{\circ} \mathrm{C}$ for $1.5 \mathrm{~h}$, after which the volatiles were removed in vacuo to afford dark red solid. Pentane $(2 \mathrm{~mL})$ was added and the suspension was filtered through short plug of celite $(2 \times 1 \mathrm{~cm}$ pipette). The resulting orange solid residue remained at the top of the celite plug, being further washed with pentane $(2 \mathrm{~mL})$ 
and then eluted through the plug with $\mathrm{CH}_{2} \mathrm{Cl}_{2}(1 \mathrm{~mL})$ into an oven-dried $7 \mathrm{~mL}$ vial. The $\mathrm{CH}_{2} \mathrm{Cl}_{2}$ was removed in vacuo, affording complex Ru-vinyl-1 as pale orange solid (39.4 mg, $45 \mu \mathrm{mol}, 91 \%$ yield). Crystals suitable for $\mathrm{X}$-ray analysis were obtained by allowing a $\mathrm{CH}_{2} \mathrm{Cl}_{2}$ solution of $\mathbf{R u}$-vinyl-1 to stand for seven days at $-40{ }^{\circ} \mathrm{C}$.

Ru-vinyl-1: ${ }^{1} \mathbf{H}$ NMR ( $\left.\mathbf{C D}_{\mathbf{2}} \mathbf{C l}_{2}, \mathbf{4 0 0} \mathbf{M H z}\right): \delta 3.21(1 \mathrm{H}, \mathrm{t}, J=1.9 \mathrm{~Hz}, \mathrm{RuCC} H), 2.64(6 \mathrm{H}, \mathrm{tt}, J=12.0 \mathrm{~Hz}$, $3.6 \mathrm{~Hz}), 2.08(12 \mathrm{H}, \mathrm{bd}, J=12.6 \mathrm{~Hz}), 1.86-1.75(12 \mathrm{H}, \mathrm{m}), 1.75-1.54(18 \mathrm{H}, \mathrm{m}), 1.34-1.19(20 \mathrm{H}, \mathrm{m}), 1.17$ $\operatorname{ppm}(12 \mathrm{H}, \mathrm{s}) .{ }^{13} \mathbf{C} \mathbf{N M R}\left(\mathbf{C D}_{2} \mathbf{C l}_{\mathbf{2}}, \mathbf{1 0 1} \mathbf{M H z}\right): \delta 331.0(\mathrm{t}, J=12.2 \mathrm{~Hz}), 82.6,33.7$ (t, $\left.J=9.1 \mathrm{~Hz}\right), 30.4,28.3$ $(\mathrm{t}, J=5.1 \mathrm{~Hz}), 27.0,25.0,24.8(\mathrm{~d}, J=14.8 \mathrm{~Hz}) .{ }^{31} \mathbf{P} \mathbf{N M R}\left(\mathbf{C D}_{2} \mathbf{C l}_{2}, \mathbf{1 6 2} \mathbf{~ M H z}\right): \delta 24.4 \mathrm{ppm}(2 \mathrm{P}, \mathrm{s}) .{ }^{11} \mathbf{B}$ NMR (CD $\mathbf{C D}_{2}, 128 \mathrm{MHz}$ ): $\delta 30.1 \mathrm{ppm}(\mathrm{s}, 1 \mathrm{~B})$. HRMS (ESI) m/z: [M-Cl] ${ }^{+}$Calcd for $\mathrm{C}_{44} \mathrm{H}_{79} \mathrm{BClP}_{2} \mathrm{O}_{2} \mathrm{Ru}^{+}$: 849.4390, Found: 849.4495 .

4.2. Synthesis and Characterization of Ru-vinyl-2 and Ru-vinyl-3. In a glove box, an oven-dried $7 \mathrm{~mL}$ vial, equipped with a magnetic stir bar, was charged with Ru-I (100.0 mg, $0.122 \mathrm{mmol})$ and $\mathrm{CH}_{2} \mathrm{Cl}_{2}(2 \mathrm{~mL})$, followed by penta-3,4-dien-1-ylbenzene $\mathbf{2 b}(22.8 \mathrm{mg}, 0.158 \mathrm{mmol})$. The mixture was then allowed to stir at $22{ }^{\circ} \mathrm{C}$ for $1.5 \mathrm{~h}$, after which the red solution was subjected to vacuum to remove volatiles, leaving behind a brownish solid. Pentane $(2 \mathrm{~mL})$ was added and the suspension was filtered through short plug of celite (2 $\mathrm{x} 1 \mathrm{~cm}$ pipette). The resulting dark red solid residue remained at the top of the celite plug, being further washed with pentane $(2 \mathrm{~mL})$ and then eluted through the plug with $\mathrm{CH}_{2} \mathrm{Cl}_{2}(1 \mathrm{~mL})$ into an oven-dried $7 \mathrm{~mL}$ vial. The volatiles were subsequently removed in vacuo, affording a 0.3:1 mixture of Ru-vinyl-2 and Ruvinyl-3 as red solid (Ru-vinyl-2: $12.9 \mathrm{mg}, 0.017 \mathrm{mmol}, 14 \%$ yield; Ru-vinyl-3: $59.0 \mathrm{mg}, 0.068 \mathrm{mmol}, 56 \%$ yield). Crystals of Ru-vinyl-3 suitable for X-ray analysis were obtained by allowing a concentrated $\mathrm{CH}_{2} \mathrm{Cl}_{2}$ solution of Ru-vinyl-2/Ru-vinyl-3 mixture to stand for one day at $-40^{\circ} \mathrm{C}$.

Ru-vinyl-2: ${ }^{1} \mathbf{H}$ NMR ( $\mathbf{C D}_{2} \mathbf{C l}_{2}, 400$ MHz): $\delta 3.64$ (s, 2H, RuCCH$)_{2}, 2.71-2.54(\mathrm{~m}, 6 \mathrm{H}), 2.07-2.04$ (m, 12H), 1.80-1.50 (m, 30H), $1.25(\mathrm{~m}, 18 \mathrm{H}) .{ }^{13} \mathbf{C}$ NMR (CD $\left.\mathbf{C l}_{2}, \mathbf{1 0 1} \mathbf{M H z}\right): \delta 325.7(\mathrm{t}, J=10.1 \mathrm{~Hz}$, $\left.\mathrm{Ru}=C=\mathrm{CH}_{2}\right), 100.7\left(\mathrm{~s}, \mathrm{Ru}=\mathrm{C}=C \mathrm{H}_{2}\right), 32.8(\mathrm{t}, J=10.1 \mathrm{~Hz}), 30.4,28.3,27.0 .{ }^{31} \mathrm{P} \mathrm{NMR}\left(\mathrm{CD}_{2} \mathrm{Cl}_{2}, 162 \mathrm{MHz}\right): \delta$ $35.1 \mathrm{ppm}(2 \mathrm{P}, \mathrm{s})$.

Ru-vinyl-3: ${ }^{1} \mathbf{H}$ NMR (CD $\mathbf{C C l}_{2}, 400$ MHz): $\delta 7.27-7.13(\mathrm{~m}, 5 \mathrm{H}), 3.39\left(\mathrm{tt},{ }^{3} J_{\mathrm{HH}}=7.2 \mathrm{~Hz},{ }^{4} J_{\mathrm{PH}}=2.4 \mathrm{~Hz}, 1 \mathrm{H}\right.$, $\left.\mathrm{RuCCHCH}_{2} \mathrm{CH}_{2} \mathrm{Ph}\right), 2.71-2.54\left(\mathrm{~m}, 10 \mathrm{H}, \mathrm{RuCCHCH}_{2} \mathrm{CH}_{2} \mathrm{Ph}\right), 2.07-2.04(\mathrm{~m}, 12 \mathrm{H}), 1.80-1.50$ (m, 30H), 1.25 (m, 18H) ${ }^{13} \mathbf{C}$ NMR ( $\mathbf{C D}_{2} \mathbf{C l}_{2}, 101$ MHz): $\delta 334.7$ (t, $\left.J=13.1 \mathrm{~Hz}, \mathrm{Ru}=C=\mathrm{CH}(\mathrm{CH} 2)_{2} \mathrm{Ph}\right), 142.3,128.9$, 128.6, 126.1, 105.7 (t, $\left.J=4.0 \mathrm{~Hz}, \mathrm{Ru}=\mathrm{C}=\mathrm{CH}\left(\mathrm{CH}_{2}\right)_{2} \mathrm{Ph}\right), 37.9,33.2(\mathrm{t}, J=9.1 \mathrm{~Hz}), 30.4,28.3$, 27.0, 22.5 . ${ }^{31} \mathrm{P}$ NMR $\left(\mathrm{CD}_{2} \mathrm{Cl}_{2}, 162 \mathrm{MHz}\right): \delta 24.2 \mathrm{ppm}(2 \mathrm{P}, \mathrm{s})$.

4.3. Synthesis and characterization of Ru-vinyl-4. In a glove box, an oven-dried $7 \mathrm{~mL}$ vial, equipped with a magnetic stir bar, was charged with Ru-III (25.0 mg, $0.029 \mathrm{mmol})$ and $\mathrm{CH}_{2} \mathrm{Cl}_{2}(0.5 \mathrm{~mL})$, followed by of allenyl-B(pin) $(1 ; 21.2 \mu \mathrm{L}, 19.6 \mathrm{mg}, 0.118 \mathrm{mmol})$. The solution was allowed to stir at $22{ }^{\circ} \mathrm{C}$ for $24 \mathrm{~h}$, after which the red solution was subjected to vacuum, affording a dark brown solid. Pentane $(2 \mathrm{~mL})$ was added and a light brown precipitate formed. The solid was filtered off by passing the resulting suspension through a short plug of celite $(2 \times 1 \mathrm{~cm}$ pipette). The pentane filtrate was dried in vacuo to furnish Ruvinyl-4 as red-brown solid (24.3 mg, 0.027 mmol, 92\% yield). Crystals of Ru-vinyl-4 suitable for X-ray analysis were obtained by allowing a concentrated pentane solution of Ru-vinyl-4 to stand for three days at $-40^{\circ} \mathrm{C}$.

Ru-vinyl-4: ${ }^{1} \mathbf{H}$ NMR (CD $\mathbf{C l}_{2}, 400$ MHz): $\delta 6.91$ (s, 2H), 6.89 (s, 2H), 3.88 (m, 4H), 2.53 (s, 6H), 2.44 (s, 6H), 2.35-2.29 (m, 3H), 2.28 (s, 3H), 2.25 (s, 3H), 1.85 (s, 1H, Ru=C=CHB(pin)), 1.84-1.55 (m, $16 \mathrm{H})$, 
1.31-1.07 (m, $20 \mathrm{H}), 1.18(\mathrm{~s}, 12 \mathrm{H}) .{ }^{13} \mathbf{C}$ NMR (CD $\left.\mathbf{C l}_{2}, \mathbf{1 0 1} \mathbf{M H z}\right): \delta 330.0(\mathrm{~d}, J=15.2 \mathrm{~Hz}$, $\mathrm{Ru}=C=\mathrm{CHB}($ pin) $), 211.4(\mathrm{~d}, 105.0 \mathrm{~Hz}), 139.1,138.8,138.2,137.8,129.9,129.6,82.2,32.8$ (d, $J=17.2$ $\mathrm{Hz}), 29.8,28.3,28.2,26.7,25.1,22.7,21.2,20.2,18.9,14.2 .{ }^{31} \mathrm{P} \mathrm{NMR}\left(\mathrm{CD}_{2} \mathrm{Cl}_{2}, 162 \mathrm{MHz}\right): \delta 21.4 \mathrm{ppm}(\mathrm{s}$, 1P). ${ }^{11} \mathrm{~B} \mathrm{NMR}\left(\mathrm{CD}_{2} \mathrm{Cl}_{2}, 128 \mathrm{MHz}\right): \delta 22.6(\mathrm{~s}, 1 \mathrm{~B})$

4.4. Synthesis and characterization of Ru-carbide. In a glove box, an oven-dried $7 \mathrm{~mL}$ vial was charged with Ru-IV (24.6 mg, $0.039 \mathrm{mmol})$, thf- $d_{8}(0.5 \mathrm{~mL})$, followed by allenyl-B(pin) $(\mathbf{1} ; 10.6 \mu \mathrm{L}, 9.8 \mathrm{mg}, 0.059$ mmol). The mixture was then transferred to a J-young tube and reaction progress was followed through ${ }^{1} \mathrm{H}$ NMR spectroscopy for five days $\left(22^{\circ} \mathrm{C}\right)$. During this time, orange crystals of Ru-carbide that were suitable for X-ray analysis formed. The solvents were carefully decanted, and the dark-brown solid was washed with pentane and dried in vacuo, affording a dark-brown powder ( $9.6 \mathrm{mg}, 0.010 \mathrm{mmol}, 50 \%$ yield). The insolubility of Ru-carbide in various organic solvents and its facile decomposition in $\mathrm{CH}_{2} \mathrm{Cl}_{2}$ did not allow spectroscopic analysis.

4.5. Synthesis and characterization of Ru-vinyl-5. In a glove box, an oven-dried $7 \mathrm{~mL}$ vial was charged with complex Ru-Va (10.0 mg, $0.016 \mathrm{mmol}), \mathrm{CH}_{2} \mathrm{Cl}_{2}(0.5 \mathrm{~mL})$, followed by $5.5 \mu \mathrm{L}$ of allenyl-B(pin) (1; $5.5 \mu \mathrm{L}, 5.1 \mathrm{mg}, 0.031 \mathrm{mmol})$, and phenanthroline $(4 ; 4.2 \mathrm{mg}, 0.023 \mathrm{mmol})$. The brown solution was then transferred to a J-young NMR tube, removed from the glovebox, and allowed to warm to $40{ }^{\circ} \mathrm{C}$ by being placed in an oil bath at this temperature. After $40 \mathrm{~h}$ at $40{ }^{\circ} \mathrm{C}$, the mixture was brought back into the glovebox and the volatiles were removed in vacuo to afford red solid. Pentane $(4 \mathrm{~mL})$ was added, causing the solution to turn light-yellow and a red precipitate was formed. The solid was filtered through a short celite column ( 2 x $1 \mathrm{~cm}$ pipette) and washed extensively with pentane $(\sim 15 \mathrm{~mL})$. The resulting dark red residue remained at the top of the celite plug, and it was then eluted through the plug with $\mathrm{CH}_{2} \mathrm{Cl}_{2}(1 \mathrm{~mL})$ into an oven-dried $7 \mathrm{~mL}$ vial. Removal of the volatiles in vacuo afforded Ru-vinyl-5 as red-brown solid $(9.0 \mathrm{mg}, 0.013 \mathrm{mmol}$, $82 \%$ yield). Crystals of Ru-vinyl-5 suitable for X-ray analysis were obtained by allowing a concentrated pentane solution of to stand for one day at $-40{ }^{\circ} \mathrm{C}$.

Ru-vinyl-5: ${ }^{1} \mathbf{H}$ NMR (CD $\mathbf{C l}_{2}$, 400 MHz): $\delta 9.62$ (d, 3.6 Hz, 1H), 8.93 (d, 3.6 Hz, 1H), 8.41 (dd, 8.2 Hz, $1.4 \mathrm{~Hz}, 1 \mathrm{H}), 8.35(\mathrm{dd}, 8.2 \mathrm{~Hz}, 1.4 \mathrm{~Hz}, 1 \mathrm{H}), 7.93(\mathrm{~m}, 2 \mathrm{H}), 7.83(\mathrm{~m}, 1 \mathrm{H}), 7.58(\mathrm{~m}, 1 \mathrm{H}), 7.37-7.27(\mathrm{~m}, 2 \mathrm{H})$, 7.08 (dd, 7.3 Hz, 2.0 Hz, 1H), 4.51-4.35 (m, 2H), 3.20 (p, $6.4 \mathrm{~Hz}, 1 \mathrm{H}), 2.77$ (p, 6.4 Hz, 1H), 2.42-2.17 (m, 2H), 1.95-1.53 (m, 7H), 1.49 (d, $6.4 \mathrm{~Hz}, 3 \mathrm{H}), 1.47$ (s, 3H), 1.35 (d, $6.4 \mathrm{~Hz}, 3 \mathrm{H}), 1.27$ (d, $6.4 \mathrm{~Hz}, 3 \mathrm{H}), 1.26$ (s, 3H), 1.24 (s, 2H), 1.15 (s, 2H), 0.34 (d, $6.4 \mathrm{~Hz}, 3 \mathrm{H}) .{ }^{13} \mathbf{C}$ NMR $\left(\mathbf{C D}_{2} \mathbf{C l}_{2}, \mathbf{1 0 1} \mathbf{M H z}\right): \delta 337.44$ $\left(\mathrm{Ru}=C=\mathrm{CH}_{2}\right), 275.9,156.4,150.8,149.2,146,7,146.3,138.5,136.8,136.6,130.8,130.2,129.4,127.7$, $127.1,126.9,125.6,125.2,124.1,86.8,79.0,65.2$, 49.2, 37.5, 35.1, 32.5, 31.0, 30.6, 29.3, 28.9, 27.9, 26.21, $25.5,24.5,24.0,23.5$.

\section{Mechanistic Studies}

5.1. Reaction of Ru-I and Ru-II with allenyl-B(pin) to generate Ru-vinyl-1. Spectroscopic studies $\left({ }^{1} \mathrm{H}\right.$ and ${ }^{31} \mathrm{P}$ NMR) indicated that reaction of either Ru-I or Ru-II with allenyl-B(pin) (1) leads to the formation of the same vinylidene species, namely Ru-vinyl-1. 
Scheme S1. Spectroscopic ( ${ }^{1}$ H NMR) Analysis of the Reactions Between Ru-I and Ru-II with Allenyl-B(pin)

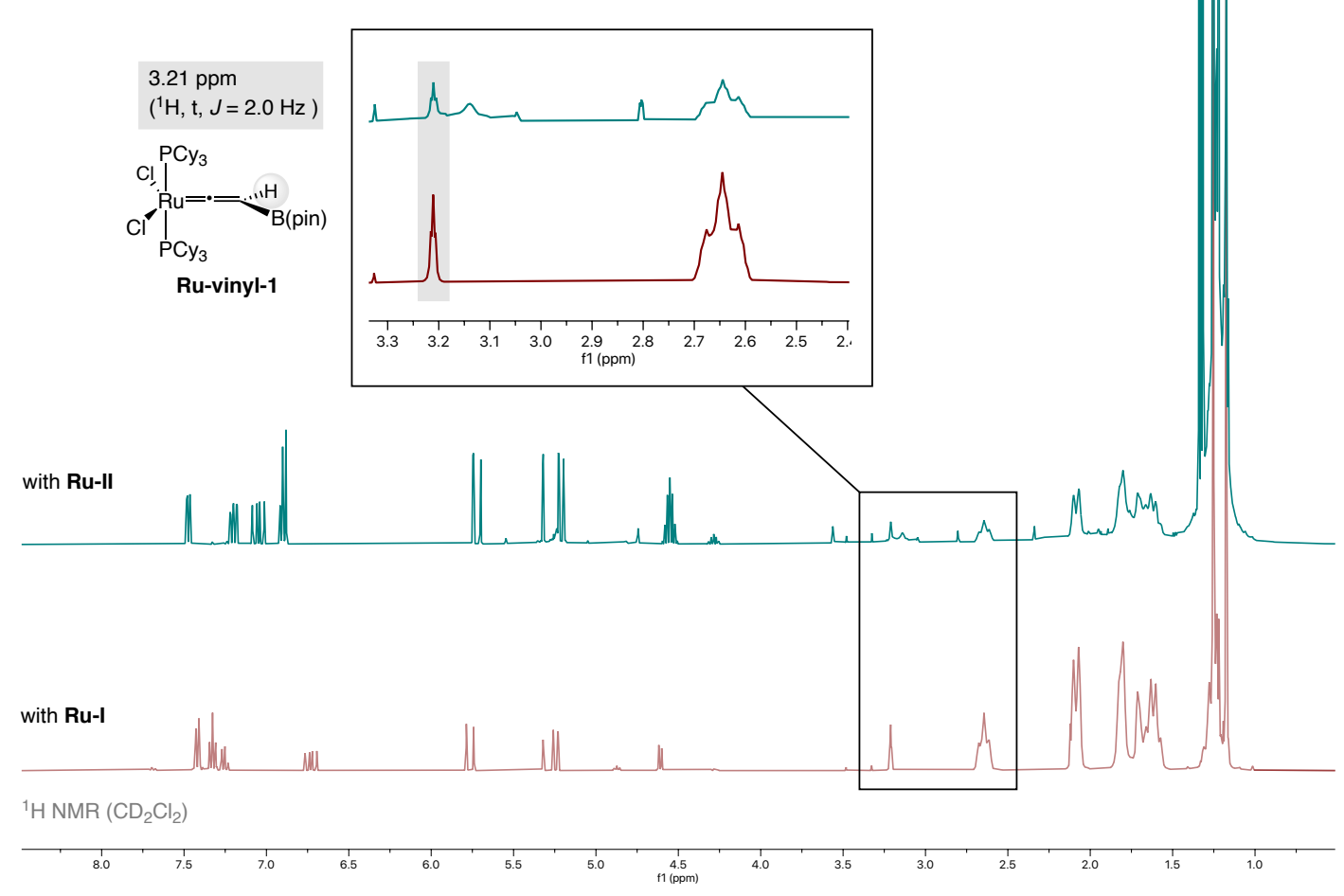

Scheme S2. Spectroscopic ( ${ }^{31}$ P NMR) Analysis of the Reactions Between Ru-I and Ru-II with Allenyl-B(pin)
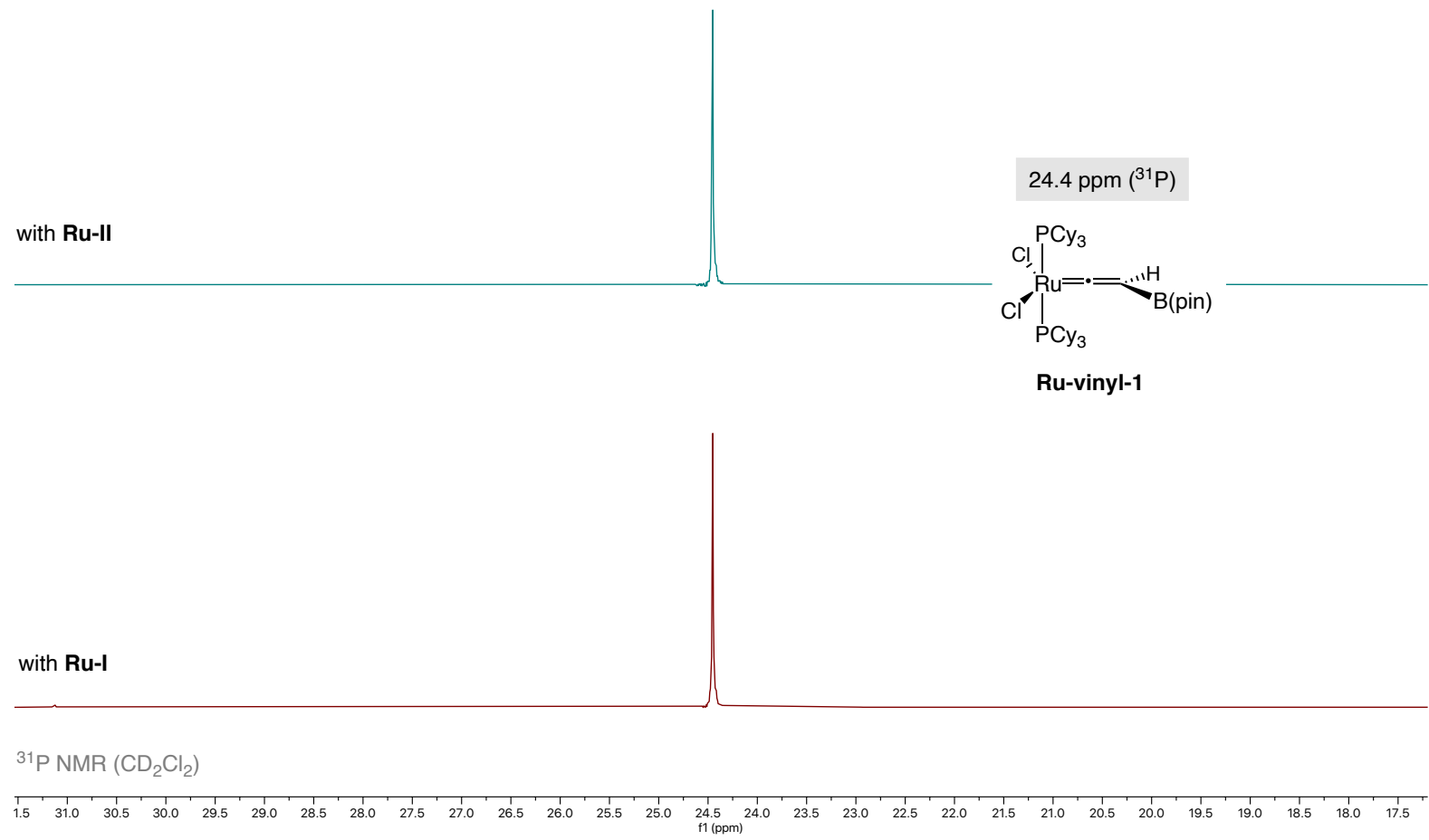

5.2. Energy barriers for the loss of a phosphine ligand from various Ru complexes. DFT studies indicated that loss of a phosphine ligand from a well-defined Ru complex is energetically prohibitive and thus unlikely. 
Scheme S3. Calculated (DFT) Energetics for Dissociation of $\mathrm{PCy}_{3}$ From Various Ru Complexes ${ }^{a}$

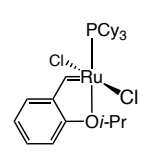

Ru-II

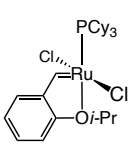

Ru-II

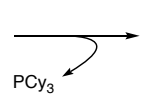

$\Delta \mathrm{G}=36.6 \mathrm{kcal} / \mathrm{mol}$

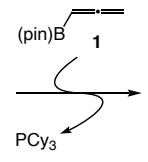

$\Delta \mathrm{G}=24.0 \mathrm{kcal} / \mathrm{mo}$
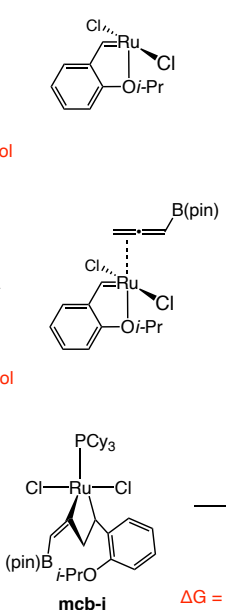

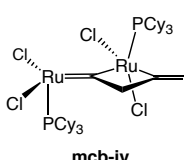

mcb-iv

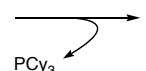

(1)
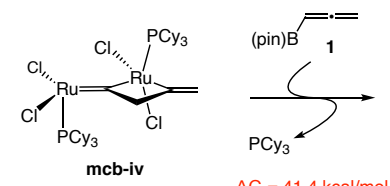

$\Delta \mathrm{G}=41.4 \mathrm{kcal} / \mathrm{mol}$
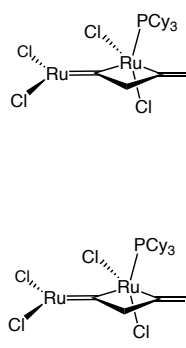

Scheme S4. Calculated (DFT) Energetics for Intramolecular Transfer of a $\mathrm{PCy}_{3} \mathrm{Ligand}^{a}$

a. Through $\mathrm{Cl}$-bridged complexes:

$\mathrm{Cl}_{1, \ldots}^{\mathrm{PC}}$

$\mathrm{PCy}_{3}$

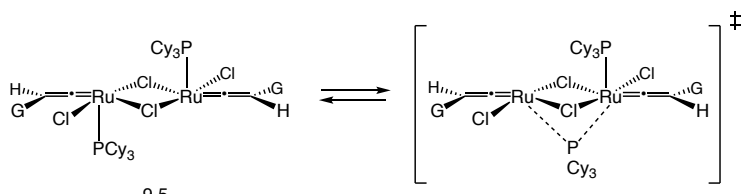

\section{$\mathrm{G}=\mathrm{B}(\mathrm{pin}) \quad 0.0$}

$$
\begin{array}{cll}
G=\mathrm{H} & 0.0
\end{array}
$$

$\mathrm{G}=\left(\mathrm{CH}_{2}\right)_{2} \mathrm{OTBS} \quad 0.0$

$-9.5$

$-5.9$

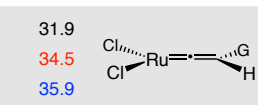

or

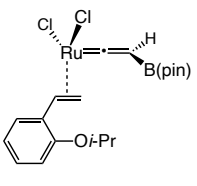

or

$25.3+2{ }_{29.2}^{\mathrm{G}}$

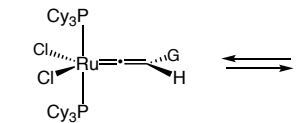

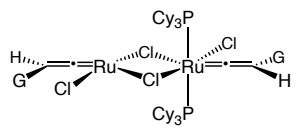

$G=B($ pin) Ru-vinyl-1

20.7
24.8

b. Through Ru-substituted ruthenacyclobutane intermediates:

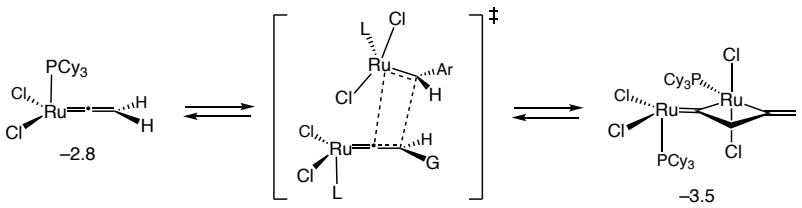

2.2

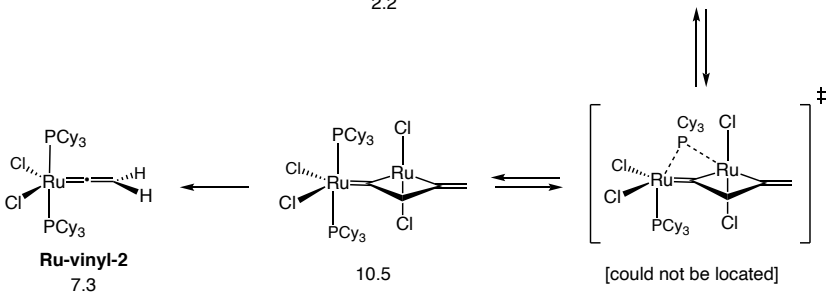

aPerformed with $\omega B 97 X-D / 6-311++G(d, p)-S D D(R u), S M D\left(C H_{2} C l_{2}\right) / / B 3 L Y P-D 3 / 6-31 G(d)-S D D(R u)$. 


\section{Crystallographic Analyses}

The crystals were placed in oil in an Ar filled glovebox, and brought out to be analyzed. A single crystal was selected, mounted on a glass fiber and placed in a low-temperature $\mathrm{N}_{2}$ stream. X-ray diffraction data collection was carried out on a Bruker PHOTON III DUO CPAD diffractometer equipped with an Oxford Cryosystem liquid $\mathrm{N}_{2}$ device, through the use of Mo-K $\alpha$ radiation $(\lambda=0.71073 \AA)$. The crystal-detector distance was $37 \mathrm{~mm}$. The cell parameters were determined (APEX3 software) ${ }^{4}$ from reflections taken from 1 set of 180 frames at $1 \mathrm{~s}$ exposure. The structures were solved by the use of the program SHELXT-2014. ${ }^{5}$ The refinement and all further calculations were carried out with SHELXL-2014 or SHELXL-2018. ${ }^{6}$ The $\mathrm{H}$-atoms were included in calculated positions and treated as riding atoms through the use of SHELXL default parameters. The non-H atoms were refined anisotropically through the use of weighted full-matrix least-squares on F2. A semi-empirical absorption correction was applied with SADABS in APEX3. ${ }^{4}$

\subsection{X-ray structure of Ru-vinyl-1}

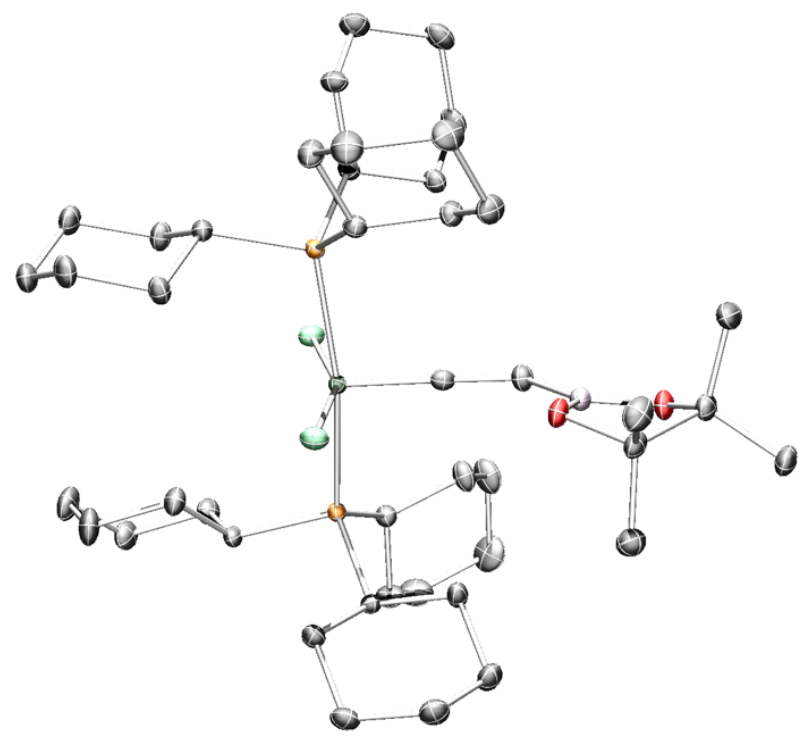

Table S1. Sample and crystal data for $\mathrm{C}_{46} \mathrm{H}_{83} \mathrm{BCl}_{6} \mathrm{O}_{2} \mathrm{P}_{2} \mathrm{Ru}$

Identification code

Chemical formula

Formula weight

Temperature

Wavelength

Crystal size

Crystal system

Space group

Unit cell dimensions ahsg200520

$\mathrm{C}_{46} \mathrm{H}_{83} \mathrm{BCl}_{6} \mathrm{O}_{2} \mathrm{P}_{2} \mathrm{Ru}$

$1054.64 \mathrm{~g} / \mathrm{mol}$

120 (2) K

$0.71073 \AA$

$0.250 \times 0.220 \times 0.200 \mathrm{~mm}$

Monoclinic

P 21/c

$\mathrm{a}=11.7963(5) \AA \quad \alpha=90^{\circ}$ 


\begin{tabular}{lll} 
& $\mathrm{b}=19.8341(10) \AA$ & $\beta=97.263(2)^{\circ}$ \\
& $\mathrm{c}=22.5597(12) \AA$ & $\gamma=90^{\circ}$ \\
Volume & $5235.9(4) \AA^{3}$ & \\
$\mathrm{Z}$ & 4 \\
Density (calculated) & $1.338 \mathrm{~g} / \mathrm{cm}^{3}$ & \\
Absorption coefficient & $0.701 \mathrm{~mm}^{-1}$ \\
$\mathrm{~F}(000)$ & 2224 \\
\hline
\end{tabular}

\section{Table S2. Data collection and structure refinement for $\mathrm{C}_{46} \mathrm{H}_{83} \mathrm{BCl}_{6} \mathrm{O}_{2} \mathrm{P}_{2} \mathrm{Ru}$}

Theta range for data collectior 2.021 to $29.159^{\circ}$

\begin{tabular}{|c|c|c|}
\hline Index ranges & \multicolumn{2}{|c|}{$-16<=\mathrm{h}<=15,-27<=\mathrm{k}<=27,-30<=1<=30$} \\
\hline Reflections collected & \multicolumn{2}{|l|}{190208} \\
\hline Independent reflections & \multicolumn{2}{|c|}{$14116[\mathrm{R}(\mathrm{int})=0.0360]$} \\
\hline \multicolumn{3}{|c|}{ Completeness to theta $=25.24100 .0 \%$} \\
\hline Absorption correction & \multicolumn{2}{|c|}{ Semi-empirical from equivalents } \\
\hline Max. and min. transmission & \multicolumn{2}{|c|}{0.7458 and 0.7210} \\
\hline Refinement method & \multicolumn{2}{|c|}{ Full-matrix least-squares on $\mathrm{F}^{2}$} \\
\hline Data / restraints / parameters & \multicolumn{2}{|c|}{$14116 / 0 / 527$} \\
\hline Goodness-of-fit on $\mathrm{F}^{2}$ & \multicolumn{2}{|l|}{1.043} \\
\hline \multirow[t]{2}{*}{ Final $\mathrm{R}$ indices } & $\mathrm{I}>2 \sigma(\mathrm{I})$ & $\mathrm{R} 1=0.0219, \mathrm{wR} 2=0.0515$ \\
\hline & all data & $\mathrm{R} 1=0.0261, \mathrm{wR} 2=0.0544$ \\
\hline Largest diff. peak and hole & \multicolumn{2}{|c|}{0.816 and $-0.738 \mathrm{e}^{-3}$} \\
\hline
\end{tabular}

Table S3. Atomic coordinates $\left(\mathrm{x}^{10^{4}}\right)$ and equivalent isotropic atomic displacement parameters $\left(\AA^{2} \times 10^{3}\right)$ for $\mathrm{C}_{46} \mathrm{H}_{83} \mathrm{BCl}_{6} \mathrm{O}_{2} \mathrm{P}_{2} \mathrm{Ru}$

$\mathrm{U}(\mathrm{eq})$ is defined as one third of the trace of the orthogonalized $\mathrm{U}_{\mathrm{ij}}$ tensor.

\begin{tabular}{lllll}
\hline $\mathrm{C}(1)$ & $6601(1)$ & $7492(1)$ & $5504(1)$ & $14(1)$ \\
$\mathrm{C}(2)$ & $5596(1)$ & $7458(1)$ & $5171(1)$ & $17(1)$ \\
$\mathrm{C}(3)$ & $4288(1)$ & $7854(1)$ & $3618(1)$ & $17(1)$ \\
$\mathrm{C}(4)$ & $5585(1)$ & $7902(1)$ & $3547(1)$ & $17(1)$ \\
$\mathrm{C}(5)$ & $3753(1)$ & $8540(1)$ & $3708(1)$ & $24(1)$ \\
$\mathrm{C}(6)$ & $3568(1)$ & $7456(1)$ & $3132(1)$ & $22(1)$ \\
$\mathrm{C}(7)$ & $5916(1)$ & $8524(1)$ & $3216(1)$ & $25(1)$ \\
$\mathrm{C}(8)$ & $6043(1)$ & $7267(1)$ & $3277(1)$ & $23(1)$
\end{tabular}




\begin{tabular}{|c|c|c|c|c|}
\hline$C(9)$ & 7183(1) & $5758(1)$ & $6124(1)$ & $14(1)$ \\
\hline $\mathrm{C}(10)$ & $7376(1)$ & $5012(1)$ & $5987(1)$ & $21(1)$ \\
\hline $\mathrm{C}(11)$ & $6616(1)$ & $4557(1)$ & $6316(1)$ & $29(1)$ \\
\hline $\mathrm{C}(12)$ & $5363(1)$ & $4746(1)$ & $6164(1)$ & $34(1)$ \\
\hline$C(13)$ & $5166(1)$ & $5490(1)$ & $6290(1)$ & $29(1)$ \\
\hline$C(14)$ & 5923(1) & 5951(1) & $5958(1)$ & $21(1)$ \\
\hline$C(15)$ & $9612(1)$ & $6105(1)$ & $6334(1)$ & $14(1)$ \\
\hline$C(16)$ & $10435(1)$ & $6710(1)$ & $6363(1)$ & $19(1)$ \\
\hline $\mathrm{C}(17)$ & 11599(1) & $6534(1)$ & $6708(1)$ & $27(1)$ \\
\hline $\mathrm{C}(18)$ & 11479(1) & $6284(1)$ & $7334(1)$ & $28(1)$ \\
\hline$C(19)$ & 10651(1) & $5695(1)$ & $7316(1)$ & $23(1)$ \\
\hline$C(20)$ & $9483(1)$ & $5870(1)$ & 6972(1) & $19(1)$ \\
\hline $\mathrm{C}(21)$ & $8495(1)$ & $5964(1)$ & $5115(1)$ & $13(1)$ \\
\hline$C(22)$ & $9648(1)$ & $6149(1)$ & $4906(1)$ & $17(1)$ \\
\hline$C(23)$ & 9793(1) & $5794(1)$ & $4320(1)$ & $21(1)$ \\
\hline$C(24)$ & $8807(1)$ & $5948(1)$ & $3833(1)$ & $22(1)$ \\
\hline$C(25)$ & $7666(1)$ & $5770(1)$ & $4043(1)$ & $23(1)$ \\
\hline$C(26)$ & 7511(1) & $6137(1)$ & $4625(1)$ & $18(1)$ \\
\hline $\mathrm{C}(27)$ & $6740(1)$ & $8914(1)$ & $6676(1)$ & $14(1)$ \\
\hline $\mathrm{C}(28)$ & $5544(1)$ & $8643(1)$ & 6441(1) & $17(1)$ \\
\hline $\mathrm{C}(29)$ & $4695(1)$ & $8781(1)$ & $6887(1)$ & $20(1)$ \\
\hline$C(30)$ & $4655(1)$ & $9525(1)$ & $7052(1)$ & $23(1)$ \\
\hline $\mathrm{C}(31)$ & $5845(1)$ & $9779(1)$ & $7296(1)$ & $23(1)$ \\
\hline$C(32)$ & $6686(1)$ & $9664(1)$ & 6841(1) & $18(1)$ \\
\hline$C(33)$ & $9213(1)$ & $8946(1)$ & $6651(1)$ & $14(1)$ \\
\hline$C(34)$ & $9423(1)$ & $8578(1)$ & $7255(1)$ & $19(1)$ \\
\hline$C(35)$ & $10547(1)$ & $8803(1)$ & $7618(1)$ & $21(1)$ \\
\hline$C(36)$ & $11556(1)$ & $8709(1)$ & $7264(1)$ & $22(1)$ \\
\hline$C(37)$ & $11347(1)$ & $9087(1)$ & $6674(1)$ & $22(1)$ \\
\hline $\mathrm{C}(38)$ & $10244(1)$ & $8845(1)$ & $6305(1)$ & $18(1)$ \\
\hline$C(39)$ & $7755(1)$ & $9285(1)$ & $5544(1)$ & $14(1)$ \\
\hline$C(40)$ & $6541(1)$ & $9306(1)$ & 5201(1) & $17(1)$ \\
\hline $\mathrm{C}(41)$ & $6525(1)$ & $9694(1)$ & $4614(1)$ & $21(1)$ \\
\hline $\mathrm{C}(42)$ & $6986(1)$ & $10407(1)$ & $4725(1)$ & $23(1)$ \\
\hline $\mathrm{C}(43)$ & $8187(1)$ & $10392(1)$ & $5068(1)$ & $24(1)$ \\
\hline$C(44)$ & $8211(1)$ & $10007(1)$ & $5659(1)$ & $19(1)$ \\
\hline $\mathrm{C}(45)$ & $2308(1)$ & $7259(1)$ & $5054(1)$ & $39(1)$ \\
\hline$C(46)$ & $9075(1)$ & $8112(1)$ & $3653(1)$ & $30(1)$ \\
\hline $\mathrm{B}(1)$ & $5361(1)$ & $7629(1)$ & $4505(1)$ & $16(1)$ \\
\hline $\mathrm{O}(1)$ & $6112(1)$ & $7931(1)$ & $4169(1)$ & $18(1)$ \\
\hline
\end{tabular}




\begin{tabular}{lllll}
$\mathrm{O}(2)$ & $4309(1)$ & $7495(1)$ & $4183(1)$ & $18(1)$ \\
$\mathrm{P}(1)$ & $8255(1)$ & $6326(1)$ & $5848(1)$ & $11(1)$ \\
$\mathrm{P}(2)$ & $7851(1)$ & $8699(1)$ & $6192(1)$ & $12(1)$ \\
$\mathrm{Cl}(1)$ & $9046(1)$ & $7803(1)$ & $5188(1)$ & $17(1)$ \\
$\mathrm{Cl}(2)$ & $7449(1)$ & $7252(1)$ & $6908(1)$ & $16(1)$ \\
$\mathrm{Cl}(3)$ & $2703(1)$ & $7787(1)$ & $5676(1)$ & $49(1)$ \\
$\mathrm{Cl}(4)$ & $2781(1)$ & $6422(1)$ & $5201(1)$ & $43(1)$ \\
$\mathrm{Cl}(5)$ & $10455(1)$ & $7749(1)$ & $3749(1)$ & $48(1)$ \\
$\mathrm{Cl}(6)$ & $9132(1)$ & $8996(1)$ & $3715(1)$ & $49(1)$ \\
$\mathrm{Ru}(1)$ & $7933(1)$ & $7514(1)$ & $5947(1)$ & $10(1)$ \\
\hline
\end{tabular}

\begin{tabular}{|c|c|c|c|}
\hline \multicolumn{4}{|c|}{ Table S4. Bond lengths $(\AA)$ for $\mathrm{C}_{46} \mathrm{H}_{83} \mathrm{BCl}_{6} \mathrm{O}_{2} \mathrm{P}_{2} \mathrm{Ru}$} \\
\hline $\mathrm{C}(1)-\mathrm{C}(2)$ & $1.3219(16)$ & $\mathrm{C}(25)-\mathrm{H}(25 \mathrm{~A})$ & 0.99 \\
\hline $\mathrm{C}(1)-\mathrm{Ru}(1)$ & $1.7536(12)$ & $\mathrm{C}(25)-\mathrm{H}(25 \mathrm{~B})$ & 0.99 \\
\hline $\mathrm{C}(2)-\mathrm{B}(1)$ & $1.5304(17)$ & $\mathrm{C}(26)-\mathrm{H}(26 \mathrm{~A})$ & 0.99 \\
\hline $\mathrm{C}(2)-\mathrm{H}(2)$ & 0.95 & $\mathrm{C}(26)-\mathrm{H}(26 \mathrm{~B})$ & 0.99 \\
\hline $\mathrm{C}(3)-\mathrm{O}(2)$ & $1.4592(14)$ & $\mathrm{C}(27)-\mathrm{C}(32)$ & $1.5361(16)$ \\
\hline$C(3)-C(6)$ & $1.5202(16)$ & $\mathrm{C}(27)-\mathrm{C}(28)$ & $1.5401(16)$ \\
\hline $\mathrm{C}(3)-\mathrm{C}(5)$ & $1.5234(18)$ & $\mathrm{C}(27)-\mathrm{P}(2)$ & $1.8572(11)$ \\
\hline $\mathrm{C}(3)-\mathrm{C}(4)$ & $1.5610(17)$ & $\mathrm{C}(27)-\mathrm{H}(27)$ & 1 \\
\hline $\mathrm{C}(4)-\mathrm{O}(1)$ & $1.4629(14)$ & $\mathrm{C}(28)-\mathrm{C}(29)$ & $1.5315(16)$ \\
\hline $\mathrm{C}(4)-\mathrm{C}(7)$ & $1.5181(17)$ & $\mathrm{C}(28)-\mathrm{H}(28 \mathrm{~A})$ & 0.99 \\
\hline$C(4)-C(8)$ & $1.5255(18)$ & $\mathrm{C}(28)-\mathrm{H}(28 \mathrm{~B})$ & 0.99 \\
\hline $\mathrm{C}(5)-\mathrm{H}(5 \mathrm{~A})$ & 0.98 & $\mathrm{C}(29)-\mathrm{C}(30)$ & $1.5247(19)$ \\
\hline $\mathrm{C}(5)-\mathrm{H}(5 \mathrm{~B})$ & 0.98 & C(29)-H(29A) & 0.99 \\
\hline $\mathrm{C}(5)-\mathrm{H}(5 \mathrm{C})$ & 0.98 & C(29)-H(29B) & 0.99 \\
\hline $\mathrm{C}(6)-\mathrm{H}(6 \mathrm{~A})$ & 0.98 & $\mathrm{C}(30)-\mathrm{C}(31)$ & $1.5270(19)$ \\
\hline $\mathrm{C}(6)-\mathrm{H}(6 \mathrm{~B})$ & 0.98 & $\mathrm{C}(30)-\mathrm{H}(30 \mathrm{~A})$ & 0.99 \\
\hline $\mathrm{C}(6)-\mathrm{H}(6 \mathrm{C})$ & 0.98 & $\mathrm{C}(30)-\mathrm{H}(30 \mathrm{~B})$ & 0.99 \\
\hline $\mathrm{C}(7)-\mathrm{H}(7 \mathrm{~A})$ & 0.98 & $C(31)-C(32)$ & $1.5323(17)$ \\
\hline $\mathrm{C}(7)-\mathrm{H}(7 \mathrm{~B})$ & 0.98 & $\mathrm{C}(31)-\mathrm{H}(31 \mathrm{~A})$ & 0.99 \\
\hline $\mathrm{C}(7)-\mathrm{H}(7 \mathrm{C})$ & 0.98 & $\mathrm{C}(31)-\mathrm{H}(31 \mathrm{~B})$ & 0.99 \\
\hline $\mathrm{C}(8)-\mathrm{H}(8 \mathrm{~A})$ & 0.98 & $\mathrm{C}(32)-\mathrm{H}(32 \mathrm{~A})$ & 0.99 \\
\hline $\mathrm{C}(8)-\mathrm{H}(8 \mathrm{~B})$ & 0.98 & $\mathrm{C}(32)-\mathrm{H}(32 \mathrm{~B})$ & 0.99 \\
\hline $\mathrm{C}(8)-\mathrm{H}(8 \mathrm{C})$ & 0.98 & $\mathrm{C}(33)-\mathrm{C}(34)$ & $1.5388(16)$ \\
\hline C(9)-C(10) & $1.5343(17)$ & $\mathrm{C}(33)-\mathrm{C}(38)$ & $1.5393(16)$ \\
\hline C(9)-C(14) & $1.5346(16)$ & $\mathrm{C}(33)-\mathrm{P}(2)$ & $1.8636(11)$ \\
\hline $\mathrm{C}(9)-\mathrm{P}(1)$ & $1.8594(12)$ & $\mathrm{C}(33)-\mathrm{H}(33)$ & 1 \\
\hline C(9)-H(9) & 1 & $C(34)-C(35)$ & $1.5344(16)$ \\
\hline
\end{tabular}




\begin{tabular}{|c|c|c|c|}
\hline$C(10)-C(11)$ & $1.5292(18)$ & $\mathrm{C}(34)-\mathrm{H}(34 \mathrm{~A})$ & 0.99 \\
\hline $\mathrm{C}(10)-\mathrm{H}(10 \mathrm{~A})$ & 0.99 & $\mathrm{C}(34)-\mathrm{H}(34 \mathrm{~B})$ & 0.99 \\
\hline $\mathrm{C}(10)-\mathrm{H}(10 \mathrm{~B})$ & 0.99 & $C(35)-C(36)$ & $1.5249(18)$ \\
\hline$C(11)-C(12)$ & $1.521(2)$ & $\mathrm{C}(35)-\mathrm{H}(35 \mathrm{~A})$ & 0.99 \\
\hline $\mathrm{C}(11)-\mathrm{H}(11 \mathrm{~A})$ & 0.99 & $\mathrm{C}(35)-\mathrm{H}(35 \mathrm{~B})$ & 0.99 \\
\hline $\mathrm{C}(11)-\mathrm{H}(11 \mathrm{~B})$ & 0.99 & $C(36)-C(37)$ & $1.5220(18)$ \\
\hline$C(12)-C(13)$ & $1.526(2)$ & $\mathrm{C}(36)-\mathrm{H}(36 \mathrm{~A})$ & 0.99 \\
\hline $\mathrm{C}(12)-\mathrm{H}(12 \mathrm{~A})$ & 0.99 & $\mathrm{C}(36)-\mathrm{H}(36 \mathrm{~B})$ & 0.99 \\
\hline $\mathrm{C}(12)-\mathrm{H}(12 \mathrm{~B})$ & 0.99 & $\mathrm{C}(37)-\mathrm{C}(38)$ & $1.5295(17)$ \\
\hline$C(13)-C(14)$ & $1.5369(18)$ & $\mathrm{C}(37)-\mathrm{H}(37 \mathrm{~A})$ & 0.99 \\
\hline $\mathrm{C}(13)-\mathrm{H}(13 \mathrm{~A})$ & 0.99 & $\mathrm{C}(37)-\mathrm{H}(37 \mathrm{~B})$ & 0.99 \\
\hline $\mathrm{C}(13)-\mathrm{H}(13 \mathrm{~B})$ & 0.99 & $\mathrm{C}(38)-\mathrm{H}(38 \mathrm{~A})$ & 0.99 \\
\hline $\mathrm{C}(14)-\mathrm{H}(14 \mathrm{~A})$ & 0.99 & $\mathrm{C}(38)-\mathrm{H}(38 \mathrm{~B})$ & 0.99 \\
\hline $\mathrm{C}(14)-\mathrm{H}(14 \mathrm{~B})$ & 0.99 & $\mathrm{C}(39)-\mathrm{C}(40)$ & $1.5397(16)$ \\
\hline$C(15)-C(20)$ & $1.5384(16)$ & $C(39)-C(44)$ & $1.5407(16)$ \\
\hline$C(15)-C(16)$ & $1.5396(16)$ & $\mathrm{C}(39)-\mathrm{P}(2)$ & $1.8599(12)$ \\
\hline $\mathrm{C}(15)-\mathrm{P}(1)$ & $1.8739(11)$ & C(39)-H(39) & 1 \\
\hline $\mathrm{C}(15)-\mathrm{H}(15)$ & 1 & $\mathrm{C}(40)-\mathrm{C}(41)$ & $1.5304(16)$ \\
\hline$C(16)-C(17)$ & $1.5298(17)$ & $\mathrm{C}(40)-\mathrm{H}(40 \mathrm{~A})$ & 0.99 \\
\hline $\mathrm{C}(16)-\mathrm{H}(16 \mathrm{~A})$ & 0.99 & $\mathrm{C}(40)-\mathrm{H}(40 \mathrm{~B})$ & 0.99 \\
\hline $\mathrm{C}(16)-\mathrm{H}(16 \mathrm{~B})$ & 0.99 & $\mathrm{C}(41)-\mathrm{C}(42)$ & $1.5251(18)$ \\
\hline $\mathrm{C}(17)-\mathrm{C}(18)$ & $1.521(2)$ & $\mathrm{C}(41)-\mathrm{H}(41 \mathrm{~A})$ & 0.99 \\
\hline $\mathrm{C}(17)-\mathrm{H}(17 \mathrm{~A})$ & 0.99 & $\mathrm{C}(41)-\mathrm{H}(41 \mathrm{~B})$ & 0.99 \\
\hline $\mathrm{C}(17)-\mathrm{H}(17 \mathrm{~B})$ & 0.99 & $C(42)-C(43)$ & $1.5256(18)$ \\
\hline$C(18)-C(19)$ & $1.5213(19)$ & $\mathrm{C}(42)-\mathrm{H}(42 \mathrm{~A})$ & 0.99 \\
\hline $\mathrm{C}(18)-\mathrm{H}(18 \mathrm{~A})$ & 0.99 & $\mathrm{C}(42)-\mathrm{H}(42 \mathrm{~B})$ & 0.99 \\
\hline $\mathrm{C}(18)-\mathrm{H}(18 \mathrm{~B})$ & 0.99 & $\mathrm{C}(43)-\mathrm{C}(44)$ & $1.5347(17)$ \\
\hline$C(19)-C(20)$ & $1.5335(17)$ & $\mathrm{C}(43)-\mathrm{H}(43 \mathrm{~A})$ & 0.99 \\
\hline C(19)-H(19A) & 0.99 & $\mathrm{C}(43)-\mathrm{H}(43 \mathrm{~B})$ & 0.99 \\
\hline $\mathrm{C}(19)-\mathrm{H}(19 \mathrm{~B})$ & 0.99 & $\mathrm{C}(44)-\mathrm{H}(44 \mathrm{~A})$ & 0.99 \\
\hline $\mathrm{C}(20)-\mathrm{H}(20 \mathrm{~A})$ & 0.99 & $\mathrm{C}(44)-\mathrm{H}(44 \mathrm{~B})$ & 0.99 \\
\hline $\mathrm{C}(20)-\mathrm{H}(20 \mathrm{~B})$ & 0.99 & $\mathrm{C}(45)-\mathrm{Cl}(3)$ & $1.765(2)$ \\
\hline$C(21)-C(26)$ & $1.5369(15)$ & $\mathrm{C}(45)-\mathrm{Cl}(4)$ & $1.7697(19)$ \\
\hline$C(21)-C(22)$ & $1.5381(15)$ & $\mathrm{C}(45)-\mathrm{H}(45 \mathrm{~A})$ & 0.99 \\
\hline $\mathrm{C}(21)-\mathrm{P}(1)$ & $1.8568(11)$ & $\mathrm{C}(45)-\mathrm{H}(45 \mathrm{~B})$ & 0.99 \\
\hline $\mathrm{C}(21)-\mathrm{H}(21)$ & 1 & $\mathrm{C}(46)-\mathrm{Cl}(6)$ & $1.7599(16)$ \\
\hline$C(22)-C(23)$ & $1.5274(16)$ & $\mathrm{C}(46)-\mathrm{Cl}(5)$ & $1.7680(16)$ \\
\hline $\mathrm{C}(22)-\mathrm{H}(22 \mathrm{~A})$ & 0.99 & $\mathrm{C}(46)-\mathrm{H}(46 \mathrm{~A})$ & 0.99 \\
\hline $\mathrm{C}(22)-\mathrm{H}(22 \mathrm{~B})$ & 0.99 & $\mathrm{C}(46)-\mathrm{H}(46 \mathrm{~B})$ & 0.99 \\
\hline$C(23)-C(24)$ & $1.5257(18)$ & $\mathrm{B}(1)-\mathrm{O}(1)$ & $1.3750(16)$ \\
\hline
\end{tabular}




\begin{tabular}{llll}
$\mathrm{C}(23)-\mathrm{H}(23 \mathrm{~A})$ & 0.99 & $\mathrm{~B}(1)-\mathrm{O}(2)$ & $1.3822(15)$ \\
$\mathrm{C}(23)-\mathrm{H}(23 \mathrm{~B})$ & 0.99 & $\mathrm{P}(1)-\mathrm{Ru}(1)$ & $2.4023(3)$ \\
$\mathrm{C}(24)-\mathrm{C}(25)$ & $1.5232(18)$ & $\mathrm{P}(2)-\mathrm{Ru}(1)$ & $2.4192(3)$ \\
$\mathrm{C}(24)-\mathrm{H}(24 \mathrm{~A})$ & 0.99 & $\mathrm{Cl}(1)-\mathrm{Ru}(1)$ & $2.3581(3)$ \\
$\mathrm{C}(24)-\mathrm{H}(24 \mathrm{~B})$ & 0.99 & $\mathrm{Cl}(2)-\mathrm{Ru}(1)$ & $2.3667(3)$ \\
$\mathrm{C}(25)-\mathrm{C}(26)$ & $1.5322(17)$ & & \\
\hline
\end{tabular}

\section{Table S5. Bond angles $\left({ }^{\circ}\right)$ for $\mathrm{C}_{46} \mathrm{H}_{83} \mathrm{BCl}_{6} \mathrm{O}_{2} \mathrm{P}_{2} \mathrm{Ru}$}

Symmetry transformations used to generate equivalent atoms: $\# 1-\mathrm{x}+1,-\mathrm{y}+1,-\mathrm{z}+1$

\begin{tabular}{|c|c|c|c|}
\hline $\mathrm{C}(2)-\mathrm{C}(1)-\mathrm{Ru}(1)$ & $178.55(10)$ & $\mathrm{C}(32)-\mathrm{C}(27)-\mathrm{C}(28)$ & $110.86(9)$ \\
\hline $\mathrm{C}(1)-\mathrm{C}(2)-\mathrm{B}(1)$ & $125.74(11)$ & $\mathrm{C}(32)-\mathrm{C}(27)-\mathrm{P}(2)$ & 114.81(8) \\
\hline $\mathrm{C}(1)-\mathrm{C}(2)-\mathrm{H}(2)$ & 117.1 & $\mathrm{C}(28)-\mathrm{C}(27)-\mathrm{P}(2)$ & $113.71(8)$ \\
\hline $\mathrm{B}(1)-\mathrm{C}(2)-\mathrm{H}(2)$ & 117.1 & $\mathrm{C}(32)-\mathrm{C}(27)-\mathrm{H}(27)$ & 105.5 \\
\hline $\mathrm{O}(2)-\mathrm{C}(3)-\mathrm{C}(6)$ & $108.79(10)$ & $\mathrm{C}(28)-\mathrm{C}(27)-\mathrm{H}(27)$ & 105.5 \\
\hline $\mathrm{O}(2)-\mathrm{C}(3)-\mathrm{C}(5)$ & $106.25(10)$ & $\mathrm{P}(2)-\mathrm{C}(27)-\mathrm{H}(27)$ & 105.5 \\
\hline$C(6)-C(3)-C(5)$ & $110.93(10)$ & $\mathrm{C}(29)-\mathrm{C}(28)-\mathrm{C}(27)$ & $111.06(10)$ \\
\hline $\mathrm{O}(2)-\mathrm{C}(3)-\mathrm{C}(4)$ & $102.13(9)$ & $\mathrm{C}(29)-\mathrm{C}(28)-\mathrm{H}(28 \mathrm{~A})$ & 109.4 \\
\hline$C(6)-C(3)-C(4)$ & $115.06(10)$ & $\mathrm{C}(27)-\mathrm{C}(28)-\mathrm{H}(28 \mathrm{~A})$ & 109.4 \\
\hline$C(5)-C(3)-C(4)$ & $112.86(10)$ & $\mathrm{C}(29)-\mathrm{C}(28)-\mathrm{H}(28 \mathrm{~B})$ & 109.4 \\
\hline $\mathrm{O}(1)-\mathrm{C}(4)-\mathrm{C}(7)$ & $109.55(10)$ & $\mathrm{C}(27)-\mathrm{C}(28)-\mathrm{H}(28 \mathrm{~B})$ & 109.4 \\
\hline $\mathrm{O}(1)-\mathrm{C}(4)-\mathrm{C}(8)$ & $106.67(10)$ & $\mathrm{H}(28 \mathrm{~A})-\mathrm{C}(28)-\mathrm{H}(28 \mathrm{~B})$ & 108 \\
\hline$C(7)-C(4)-C(8)$ & $110.37(11)$ & $\mathrm{C}(30)-\mathrm{C}(29)-\mathrm{C}(28)$ & $112.06(10)$ \\
\hline $\mathrm{O}(1)-\mathrm{C}(4)-\mathrm{C}(3)$ & $102.00(9)$ & $\mathrm{C}(30)-\mathrm{C}(29)-\mathrm{H}(29 \mathrm{~A})$ & 109.2 \\
\hline$C(7)-C(4)-C(3)$ & $114.51(10)$ & $\mathrm{C}(28)-\mathrm{C}(29)-\mathrm{H}(29 \mathrm{~A})$ & 109.2 \\
\hline$C(8)-C(4)-C(3)$ & $113.09(10)$ & $\mathrm{C}(30)-\mathrm{C}(29)-\mathrm{H}(29 \mathrm{~B})$ & 109.2 \\
\hline $\mathrm{C}(3)-\mathrm{C}(5)-\mathrm{H}(5 \mathrm{~A})$ & 109.5 & $\mathrm{C}(28)-\mathrm{C}(29)-\mathrm{H}(29 \mathrm{~B})$ & 109.2 \\
\hline $\mathrm{C}(3)-\mathrm{C}(5)-\mathrm{H}(5 \mathrm{~B})$ & 109.5 & $\mathrm{H}(29 \mathrm{~A})-\mathrm{C}(29)-\mathrm{H}(29 \mathrm{~B})$ & 107.9 \\
\hline $\mathrm{H}(5 \mathrm{~A})-\mathrm{C}(5)-\mathrm{H}(5 \mathrm{~B})$ & 109.5 & $\mathrm{C}(29)-\mathrm{C}(30)-\mathrm{C}(31)$ & $110.58(10)$ \\
\hline $\mathrm{C}(3)-\mathrm{C}(5)-\mathrm{H}(5 \mathrm{C})$ & 109.5 & $\mathrm{C}(29)-\mathrm{C}(30)-\mathrm{H}(30 \mathrm{~A})$ & 109.5 \\
\hline $\mathrm{H}(5 \mathrm{~A})-\mathrm{C}(5)-\mathrm{H}(5 \mathrm{C})$ & 109.5 & $\mathrm{C}(31)-\mathrm{C}(30)-\mathrm{H}(30 \mathrm{~A})$ & 109.5 \\
\hline $\mathrm{H}(5 \mathrm{~B})-\mathrm{C}(5)-\mathrm{H}(5 \mathrm{C})$ & 109.5 & $\mathrm{C}(29)-\mathrm{C}(30)-\mathrm{H}(30 \mathrm{~B})$ & 109.5 \\
\hline$C(3)-C(6)-H(6 A)$ & 109.5 & $\mathrm{C}(31)-\mathrm{C}(30)-\mathrm{H}(30 \mathrm{~B})$ & 109.5 \\
\hline $\mathrm{C}(3)-\mathrm{C}(6)-\mathrm{H}(6 \mathrm{~B})$ & 109.5 & $\mathrm{H}(30 \mathrm{~A})-\mathrm{C}(30)-\mathrm{H}(30 \mathrm{~B})$ & 108.1 \\
\hline $\mathrm{H}(6 \mathrm{~A})-\mathrm{C}(6)-\mathrm{H}(6 \mathrm{~B})$ & 109.5 & $\mathrm{C}(30)-\mathrm{C}(31)-\mathrm{C}(32)$ & $110.66(10)$ \\
\hline $\mathrm{C}(3)-\mathrm{C}(6)-\mathrm{H}(6 \mathrm{C})$ & 109.5 & $\mathrm{C}(30)-\mathrm{C}(31)-\mathrm{H}(31 \mathrm{~A})$ & 109.5 \\
\hline $\mathrm{H}(6 \mathrm{~A})-\mathrm{C}(6)-\mathrm{H}(6 \mathrm{C})$ & 109.5 & $\mathrm{C}(32)-\mathrm{C}(31)-\mathrm{H}(31 \mathrm{~A})$ & 109.5 \\
\hline $\mathrm{H}(6 \mathrm{~B})-\mathrm{C}(6)-\mathrm{H}(6 \mathrm{C})$ & 109.5 & $\mathrm{C}(30)-\mathrm{C}(31)-\mathrm{H}(31 \mathrm{~B})$ & 109.5 \\
\hline $\mathrm{C}(4)-\mathrm{C}(7)-\mathrm{H}(7 \mathrm{~A})$ & 109.5 & $\mathrm{C}(32)-\mathrm{C}(31)-\mathrm{H}(31 \mathrm{~B})$ & 109.5 \\
\hline $\mathrm{C}(4)-\mathrm{C}(7)-\mathrm{H}(7 \mathrm{~B})$ & 109.5 & $H(31 A)-C(31)-H(31 B)$ & 108.1 \\
\hline
\end{tabular}




\begin{tabular}{|c|c|c|c|}
\hline $\mathrm{H}(7 \mathrm{~A})-\mathrm{C}(7)-\mathrm{H}(7 \mathrm{~B})$ & 109.5 & $\mathrm{C}(31)-\mathrm{C}(32)-\mathrm{C}(27)$ & $110.95(10)$ \\
\hline $\mathrm{C}(4)-\mathrm{C}(7)-\mathrm{H}(7 \mathrm{C})$ & 109.5 & $\mathrm{C}(31)-\mathrm{C}(32)-\mathrm{H}(32 \mathrm{~A})$ & 109.4 \\
\hline $\mathrm{H}(7 \mathrm{~A})-\mathrm{C}(7)-\mathrm{H}(7 \mathrm{C})$ & 109.5 & $\mathrm{C}(27)-\mathrm{C}(32)-\mathrm{H}(32 \mathrm{~A})$ & 109.4 \\
\hline H(7B)-C(7)-H(7C) & 109.5 & $\mathrm{C}(31)-\mathrm{C}(32)-\mathrm{H}(32 \mathrm{~B})$ & 109.4 \\
\hline $\mathrm{C}(4)-\mathrm{C}(8)-\mathrm{H}(8 \mathrm{~A})$ & 109.5 & $\mathrm{C}(27)-\mathrm{C}(32)-\mathrm{H}(32 \mathrm{~B})$ & 109.4 \\
\hline $\mathrm{C}(4)-\mathrm{C}(8)-\mathrm{H}(8 \mathrm{~B})$ & 109.5 & $\mathrm{H}(32 \mathrm{~A})-\mathrm{C}(32)-\mathrm{H}(32 \mathrm{~B})$ & 108 \\
\hline $\mathrm{H}(8 \mathrm{~A})-\mathrm{C}(8)-\mathrm{H}(8 \mathrm{~B})$ & 109.5 & $\mathrm{C}(34)-\mathrm{C}(33)-\mathrm{C}(38)$ & $109.75(9)$ \\
\hline $\mathrm{C}(4)-\mathrm{C}(8)-\mathrm{H}(8 \mathrm{C})$ & 109.5 & $\mathrm{C}(34)-\mathrm{C}(33)-\mathrm{P}(2)$ & $113.48(8)$ \\
\hline $\mathrm{H}(8 \mathrm{~A})-\mathrm{C}(8)-\mathrm{H}(8 \mathrm{C})$ & 109.5 & $\mathrm{C}(38)-\mathrm{C}(33)-\mathrm{P}(2)$ & $111.42(8)$ \\
\hline $\mathrm{H}(8 \mathrm{~B})-\mathrm{C}(8)-\mathrm{H}(8 \mathrm{C})$ & 109.5 & $\mathrm{C}(34)-\mathrm{C}(33)-\mathrm{H}(33)$ & 107.3 \\
\hline$C(10)-C(9)-C(14)$ & $110.79(10)$ & $\mathrm{C}(38)-\mathrm{C}(33)-\mathrm{H}(33)$ & 107.3 \\
\hline $\mathrm{C}(10)-\mathrm{C}(9)-\mathrm{P}(1)$ & $113.07(8)$ & $\mathrm{P}(2)-\mathrm{C}(33)-\mathrm{H}(33)$ & 107.3 \\
\hline $\mathrm{C}(14)-\mathrm{C}(9)-\mathrm{P}(1)$ & $116.47(8)$ & $C(35)-C(34)-C(33)$ & $111.39(10)$ \\
\hline $\mathrm{C}(10)-\mathrm{C}(9)-\mathrm{H}(9)$ & 105.1 & $\mathrm{C}(35)-\mathrm{C}(34)-\mathrm{H}(34 \mathrm{~A})$ & 109.3 \\
\hline $\mathrm{C}(14)-\mathrm{C}(9)-\mathrm{H}(9)$ & 105.1 & $\mathrm{C}(33)-\mathrm{C}(34)-\mathrm{H}(34 \mathrm{~A})$ & 109.3 \\
\hline $\mathrm{P}(1)-\mathrm{C}(9)-\mathrm{H}(9)$ & 105.1 & $\mathrm{C}(35)-\mathrm{C}(34)-\mathrm{H}(34 \mathrm{~B})$ & 109.3 \\
\hline$C(11)-C(10)-C(9)$ & $111.06(10)$ & $\mathrm{C}(33)-\mathrm{C}(34)-\mathrm{H}(34 \mathrm{~B})$ & 109.3 \\
\hline $\mathrm{C}(11)-\mathrm{C}(10)-\mathrm{H}(10 \mathrm{~A})$ & 109.4 & $\mathrm{H}(34 \mathrm{~A})-\mathrm{C}(34)-\mathrm{H}(34 \mathrm{~B})$ & 108 \\
\hline $\mathrm{C}(9)-\mathrm{C}(10)-\mathrm{H}(10 \mathrm{~A})$ & 109.4 & $C(36)-C(35)-C(34)$ & $111.37(10)$ \\
\hline $\mathrm{C}(11)-\mathrm{C}(10)-\mathrm{H}(10 \mathrm{~B})$ & 109.4 & $\mathrm{C}(36)-\mathrm{C}(35)-\mathrm{H}(35 \mathrm{~A})$ & 109.4 \\
\hline $\mathrm{C}(9)-\mathrm{C}(10)-\mathrm{H}(10 \mathrm{~B})$ & 109.4 & $\mathrm{C}(34)-\mathrm{C}(35)-\mathrm{H}(35 \mathrm{~A})$ & 109.4 \\
\hline $\mathrm{H}(10 \mathrm{~A})-\mathrm{C}(10)-\mathrm{H}(10 \mathrm{~B})$ & 108 & $\mathrm{C}(36)-\mathrm{C}(35)-\mathrm{H}(35 \mathrm{~B})$ & 109.4 \\
\hline$C(12)-C(11)-C(10)$ & $110.91(12)$ & $\mathrm{C}(34)-\mathrm{C}(35)-\mathrm{H}(35 \mathrm{~B})$ & 109.4 \\
\hline $\mathrm{C}(12)-\mathrm{C}(11)-\mathrm{H}(11 \mathrm{~A})$ & 109.5 & $\mathrm{H}(35 \mathrm{~A})-\mathrm{C}(35)-\mathrm{H}(35 \mathrm{~B})$ & 108 \\
\hline $\mathrm{C}(10)-\mathrm{C}(11)-\mathrm{H}(11 \mathrm{~A})$ & 109.5 & $C(37)-C(36)-C(35)$ & $110.28(10)$ \\
\hline $\mathrm{C}(12)-\mathrm{C}(11)-\mathrm{H}(11 \mathrm{~B})$ & 109.5 & $\mathrm{C}(37)-\mathrm{C}(36)-\mathrm{H}(36 \mathrm{~A})$ & 109.6 \\
\hline $\mathrm{C}(10)-\mathrm{C}(11)-\mathrm{H}(11 \mathrm{~B})$ & 109.5 & $\mathrm{C}(35)-\mathrm{C}(36)-\mathrm{H}(36 \mathrm{~A})$ & 109.6 \\
\hline $\mathrm{H}(11 \mathrm{~A})-\mathrm{C}(11)-\mathrm{H}(11 \mathrm{~B})$ & 108 & $\mathrm{C}(37)-\mathrm{C}(36)-\mathrm{H}(36 \mathrm{~B})$ & 109.6 \\
\hline $\mathrm{C}(11)-\mathrm{C}(12)-\mathrm{C}(13)$ & $111.28(12)$ & $\mathrm{C}(35)-\mathrm{C}(36)-\mathrm{H}(36 \mathrm{~B})$ & 109.6 \\
\hline $\mathrm{C}(11)-\mathrm{C}(12)-\mathrm{H}(12 \mathrm{~A})$ & 109.4 & $\mathrm{H}(36 \mathrm{~A})-\mathrm{C}(36)-\mathrm{H}(36 \mathrm{~B})$ & 108.1 \\
\hline $\mathrm{C}(13)-\mathrm{C}(12)-\mathrm{H}(12 \mathrm{~A})$ & 109.4 & $\mathrm{C}(36)-\mathrm{C}(37)-\mathrm{C}(38)$ & $110.68(10)$ \\
\hline $\mathrm{C}(11)-\mathrm{C}(12)-\mathrm{H}(12 \mathrm{~B})$ & 109.4 & $\mathrm{C}(36)-\mathrm{C}(37)-\mathrm{H}(37 \mathrm{~A})$ & 109.5 \\
\hline $\mathrm{C}(13)-\mathrm{C}(12)-\mathrm{H}(12 \mathrm{~B})$ & 109.4 & $\mathrm{C}(38)-\mathrm{C}(37)-\mathrm{H}(37 \mathrm{~A})$ & 109.5 \\
\hline $\mathrm{H}(12 \mathrm{~A})-\mathrm{C}(12)-\mathrm{H}(12 \mathrm{~B})$ & 108 & $\mathrm{C}(36)-\mathrm{C}(37)-\mathrm{H}(37 \mathrm{~B})$ & 109.5 \\
\hline $\mathrm{C}(12)-\mathrm{C}(13)-\mathrm{C}(14)$ & $111.85(12)$ & $\mathrm{C}(38)-\mathrm{C}(37)-\mathrm{H}(37 \mathrm{~B})$ & 109.5 \\
\hline $\mathrm{C}(12)-\mathrm{C}(13)-\mathrm{H}(13 \mathrm{~A})$ & 109.2 & $\mathrm{H}(37 \mathrm{~A})-\mathrm{C}(37)-\mathrm{H}(37 \mathrm{~B})$ & 108.1 \\
\hline $\mathrm{C}(14)-\mathrm{C}(13)-\mathrm{H}(13 \mathrm{~A})$ & 109.2 & $\mathrm{C}(37)-\mathrm{C}(38)-\mathrm{C}(33)$ & $110.90(10)$ \\
\hline $\mathrm{C}(12)-\mathrm{C}(13)-\mathrm{H}(13 \mathrm{~B})$ & 109.2 & $\mathrm{C}(37)-\mathrm{C}(38)-\mathrm{H}(38 \mathrm{~A})$ & 109.5 \\
\hline $\mathrm{C}(14)-\mathrm{C}(13)-\mathrm{H}(13 \mathrm{~B})$ & 109.2 & $\mathrm{C}(33)-\mathrm{C}(38)-\mathrm{H}(38 \mathrm{~A})$ & 109.5 \\
\hline $\mathrm{H}(13 \mathrm{~A})-\mathrm{C}(13)-\mathrm{H}(13 \mathrm{~B})$ & 107.9 & $\mathrm{C}(37)-\mathrm{C}(38)-\mathrm{H}(38 \mathrm{~B})$ & 109.5 \\
\hline
\end{tabular}




\begin{tabular}{|c|c|c|c|}
\hline$C(9)-C(14)-C(13)$ & $109.73(10)$ & $\mathrm{C}(33)-\mathrm{C}(38)-\mathrm{H}(38 \mathrm{~B})$ & 109.5 \\
\hline $\mathrm{C}(9)-\mathrm{C}(14)-\mathrm{H}(14 \mathrm{~A})$ & 109.7 & $\mathrm{H}(38 \mathrm{~A})-\mathrm{C}(38)-\mathrm{H}(38 \mathrm{~B})$ & 108 \\
\hline $\mathrm{C}(13)-\mathrm{C}(14)-\mathrm{H}(14 \mathrm{~A})$ & 109.7 & $C(40)-C(39)-C(44)$ & $110.06(9)$ \\
\hline $\mathrm{C}(9)-\mathrm{C}(14)-\mathrm{H}(14 \mathrm{~B})$ & 109.7 & $\mathrm{C}(40)-\mathrm{C}(39)-\mathrm{P}(2)$ & $111.85(8)$ \\
\hline $\mathrm{C}(13)-\mathrm{C}(14)-\mathrm{H}(14 \mathrm{~B})$ & 109.7 & $\mathrm{C}(44)-\mathrm{C}(39)-\mathrm{P}(2)$ & $117.59(8)$ \\
\hline $\mathrm{H}(14 \mathrm{~A})-\mathrm{C}(14)-\mathrm{H}(14 \mathrm{~B})$ & 108.2 & $\mathrm{C}(40)-\mathrm{C}(39)-\mathrm{H}(39)$ & 105.4 \\
\hline$C(20)-C(15)-C(16)$ & $109.44(9)$ & $\mathrm{C}(44)-\mathrm{C}(39)-\mathrm{H}(39)$ & 105.4 \\
\hline $\mathrm{C}(20)-\mathrm{C}(15)-\mathrm{P}(1)$ & $116.13(8)$ & $\mathrm{P}(2)-\mathrm{C}(39)-\mathrm{H}(39)$ & 105.4 \\
\hline $\mathrm{C}(16)-\mathrm{C}(15)-\mathrm{P}(1)$ & $109.31(8)$ & $C(41)-C(40)-C(39)$ & $110.95(10)$ \\
\hline $\mathrm{C}(20)-\mathrm{C}(15)-\mathrm{H}(15)$ & 107.2 & $\mathrm{C}(41)-\mathrm{C}(40)-\mathrm{H}(40 \mathrm{~A})$ & 109.4 \\
\hline $\mathrm{C}(16)-\mathrm{C}(15)-\mathrm{H}(15)$ & 107.2 & $\mathrm{C}(39)-\mathrm{C}(40)-\mathrm{H}(40 \mathrm{~A})$ & 109.4 \\
\hline $\mathrm{P}(1)-\mathrm{C}(15)-\mathrm{H}(15)$ & 107.2 & $\mathrm{C}(41)-\mathrm{C}(40)-\mathrm{H}(40 \mathrm{~B})$ & 109.4 \\
\hline$C(17)-C(16)-C(15)$ & $111.36(10)$ & $\mathrm{C}(39)-\mathrm{C}(40)-\mathrm{H}(40 \mathrm{~B})$ & 109.4 \\
\hline C(17)-C(16)-H(16A) & 109.4 & $\mathrm{H}(40 \mathrm{~A})-\mathrm{C}(40)-\mathrm{H}(40 \mathrm{~B})$ & 108 \\
\hline $\mathrm{C}(15)-\mathrm{C}(16)-\mathrm{H}(16 \mathrm{~A})$ & 109.4 & $C(42)-C(41)-C(40)$ & $111.04(10)$ \\
\hline $\mathrm{C}(17)-\mathrm{C}(16)-\mathrm{H}(16 \mathrm{~B})$ & 109.4 & $\mathrm{C}(42)-\mathrm{C}(41)-\mathrm{H}(41 \mathrm{~A})$ & 109.4 \\
\hline $\mathrm{C}(15)-\mathrm{C}(16)-\mathrm{H}(16 \mathrm{~B})$ & 109.4 & $\mathrm{C}(40)-\mathrm{C}(41)-\mathrm{H}(41 \mathrm{~A})$ & 109.4 \\
\hline $\mathrm{H}(16 \mathrm{~A})-\mathrm{C}(16)-\mathrm{H}(16 \mathrm{~B})$ & 108 & $\mathrm{C}(42)-\mathrm{C}(41)-\mathrm{H}(41 \mathrm{~B})$ & 109.4 \\
\hline C(18)-C(17)-C(16) & $111.36(12)$ & $\mathrm{C}(40)-\mathrm{C}(41)-\mathrm{H}(41 \mathrm{~B})$ & 109.4 \\
\hline $\mathrm{C}(18)-\mathrm{C}(17)-\mathrm{H}(17 \mathrm{~A})$ & 109.4 & $\mathrm{H}(41 \mathrm{~A})-\mathrm{C}(41)-\mathrm{H}(41 \mathrm{~B})$ & 108 \\
\hline $\mathrm{C}(16)-\mathrm{C}(17)-\mathrm{H}(17 \mathrm{~A})$ & 109.4 & $\mathrm{C}(41)-\mathrm{C}(42)-\mathrm{C}(43)$ & $110.73(11)$ \\
\hline $\mathrm{C}(18)-\mathrm{C}(17)-\mathrm{H}(17 \mathrm{~B})$ & 109.4 & $\mathrm{C}(41)-\mathrm{C}(42)-\mathrm{H}(42 \mathrm{~A})$ & 109.5 \\
\hline $\mathrm{C}(16)-\mathrm{C}(17)-\mathrm{H}(17 \mathrm{~B})$ & 109.4 & $\mathrm{C}(43)-\mathrm{C}(42)-\mathrm{H}(42 \mathrm{~A})$ & 109.5 \\
\hline $\mathrm{H}(17 \mathrm{~A})-\mathrm{C}(17)-\mathrm{H}(17 \mathrm{~B})$ & 108 & $\mathrm{C}(41)-\mathrm{C}(42)-\mathrm{H}(42 \mathrm{~B})$ & 109.5 \\
\hline $\mathrm{C}(17)-\mathrm{C}(18)-\mathrm{C}(19)$ & $111.09(11)$ & $\mathrm{C}(43)-\mathrm{C}(42)-\mathrm{H}(42 \mathrm{~B})$ & 109.5 \\
\hline C(17)-C(18)-H(18A) & 109.4 & $\mathrm{H}(42 \mathrm{~A})-\mathrm{C}(42)-\mathrm{H}(42 \mathrm{~B})$ & 108.1 \\
\hline C(19)-C(18)-H(18A) & 109.4 & $\mathrm{C}(42)-\mathrm{C}(43)-\mathrm{C}(44)$ & $111.35(10)$ \\
\hline C(17)-C(18)-H(18B) & 109.4 & $\mathrm{C}(42)-\mathrm{C}(43)-\mathrm{H}(43 \mathrm{~A})$ & 109.4 \\
\hline C(19)-C(18)-H(18B) & 109.4 & $\mathrm{C}(44)-\mathrm{C}(43)-\mathrm{H}(43 \mathrm{~A})$ & 109.4 \\
\hline $\mathrm{H}(18 \mathrm{~A})-\mathrm{C}(18)-\mathrm{H}(18 \mathrm{~B})$ & 108 & $\mathrm{C}(42)-\mathrm{C}(43)-\mathrm{H}(43 \mathrm{~B})$ & 109.4 \\
\hline $\mathrm{C}(18)-\mathrm{C}(19)-\mathrm{C}(20)$ & $111.85(11)$ & $\mathrm{C}(44)-\mathrm{C}(43)-\mathrm{H}(43 \mathrm{~B})$ & 109.4 \\
\hline C(18)-C(19)-H(19A) & 109.2 & $\mathrm{H}(43 \mathrm{~A})-\mathrm{C}(43)-\mathrm{H}(43 \mathrm{~B})$ & 108 \\
\hline $\mathrm{C}(20)-\mathrm{C}(19)-\mathrm{H}(19 \mathrm{~A})$ & 109.2 & $C(43)-C(44)-C(39)$ & $110.35(10)$ \\
\hline C(18)-C(19)-H(19B) & 109.2 & $\mathrm{C}(43)-\mathrm{C}(44)-\mathrm{H}(44 \mathrm{~A})$ & 109.6 \\
\hline C(20)-C(19)-H(19B) & 109.2 & $\mathrm{C}(39)-\mathrm{C}(44)-\mathrm{H}(44 \mathrm{~A})$ & 109.6 \\
\hline $\mathrm{H}(19 \mathrm{~A})-\mathrm{C}(19)-\mathrm{H}(19 \mathrm{~B})$ & 107.9 & $\mathrm{C}(43)-\mathrm{C}(44)-\mathrm{H}(44 \mathrm{~B})$ & 109.6 \\
\hline C(19)-C(20)-C(15) & $110.74(10)$ & $\mathrm{C}(39)-\mathrm{C}(44)-\mathrm{H}(44 \mathrm{~B})$ & 109.6 \\
\hline $\mathrm{C}(19)-\mathrm{C}(20)-\mathrm{H}(20 \mathrm{~A})$ & 109.5 & $\mathrm{H}(44 \mathrm{~A})-\mathrm{C}(44)-\mathrm{H}(44 \mathrm{~B})$ & 108.1 \\
\hline $\mathrm{C}(15)-\mathrm{C}(20)-\mathrm{H}(20 \mathrm{~A})$ & 109.5 & $\mathrm{Cl}(3)-\mathrm{C}(45)-\mathrm{Cl}(4)$ & $111.29(9)$ \\
\hline C(19)-C(20)-H(20B) & 109.5 & $\mathrm{Cl}(3)-\mathrm{C}(45)-\mathrm{H}(45 \mathrm{~A})$ & 109.4 \\
\hline
\end{tabular}




\begin{tabular}{|c|c|c|c|}
\hline $\mathrm{C}(15)-\mathrm{C}(20)-\mathrm{H}(20 \mathrm{~B})$ & 109.5 & $\mathrm{Cl}(4)-\mathrm{C}(45)-\mathrm{H}(45 \mathrm{~A})$ & 109.4 \\
\hline $\mathrm{H}(20 \mathrm{~A})-\mathrm{C}(20)-\mathrm{H}(20 \mathrm{~B})$ & 108.1 & $\mathrm{Cl}(3)-\mathrm{C}(45)-\mathrm{H}(45 \mathrm{~B})$ & 109.4 \\
\hline $\mathrm{C}(26)-\mathrm{C}(21)-\mathrm{C}(22)$ & $110.13(9)$ & $\mathrm{Cl}(4)-\mathrm{C}(45)-\mathrm{H}(45 \mathrm{~B})$ & 109.4 \\
\hline $\mathrm{C}(26)-\mathrm{C}(21)-\mathrm{P}(1)$ & $111.62(8)$ & $\mathrm{H}(45 \mathrm{~A})-\mathrm{C}(45)-\mathrm{H}(45 \mathrm{~B})$ & 108 \\
\hline $\mathrm{C}(22)-\mathrm{C}(21)-\mathrm{P}(1)$ & $114.88(8)$ & $\mathrm{Cl}(6)-\mathrm{C}(46)-\mathrm{Cl}(5)$ & $111.70(8)$ \\
\hline $\mathrm{C}(26)-\mathrm{C}(21)-\mathrm{H}(21)$ & 106.6 & $\mathrm{Cl}(6)-\mathrm{C}(46)-\mathrm{H}(46 \mathrm{~A})$ & 109.3 \\
\hline $\mathrm{C}(22)-\mathrm{C}(21)-\mathrm{H}(21)$ & 106.6 & $\mathrm{Cl}(5)-\mathrm{C}(46)-\mathrm{H}(46 \mathrm{~A})$ & 109.3 \\
\hline $\mathrm{P}(1)-\mathrm{C}(21)-\mathrm{H}(21)$ & 106.6 & $\mathrm{Cl}(6)-\mathrm{C}(46)-\mathrm{H}(46 \mathrm{~B})$ & 109.3 \\
\hline $\mathrm{C}(23)-\mathrm{C}(22)-\mathrm{C}(21)$ & $110.85(10)$ & $\mathrm{Cl}(5)-\mathrm{C}(46)-\mathrm{H}(46 \mathrm{~B})$ & 109.3 \\
\hline $\mathrm{C}(23)-\mathrm{C}(22)-\mathrm{H}(22 \mathrm{~A})$ & 109.5 & $\mathrm{H}(46 \mathrm{~A})-\mathrm{C}(46)-\mathrm{H}(46 \mathrm{~B})$ & 107.9 \\
\hline $\mathrm{C}(21)-\mathrm{C}(22)-\mathrm{H}(22 \mathrm{~A})$ & 109.5 & $\mathrm{O}(1)-\mathrm{B}(1)-\mathrm{O}(2)$ & $113.12(10)$ \\
\hline $\mathrm{C}(23)-\mathrm{C}(22)-\mathrm{H}(22 \mathrm{~B})$ & 109.5 & $\mathrm{O}(1)-\mathrm{B}(1)-\mathrm{C}(2)$ & $125.99(11)$ \\
\hline $\mathrm{C}(21)-\mathrm{C}(22)-\mathrm{H}(22 \mathrm{~B})$ & 109.5 & $\mathrm{O}(2)-\mathrm{B}(1)-\mathrm{C}(2)$ & $120.87(11)$ \\
\hline $\mathrm{H}(22 \mathrm{~A})-\mathrm{C}(22)-\mathrm{H}(22 \mathrm{~B})$ & 108.1 & $\mathrm{~B}(1)-\mathrm{O}(1)-\mathrm{C}(4)$ & 106.61(9) \\
\hline $\mathrm{C}(24)-\mathrm{C}(23)-\mathrm{C}(22)$ & $111.84(10)$ & $\mathrm{B}(1)-\mathrm{O}(2)-\mathrm{C}(3)$ & $106.29(9)$ \\
\hline C(24)-C(23)-H(23A) & 109.2 & $\mathrm{C}(21)-\mathrm{P}(1)-\mathrm{C}(9)$ & $104.58(5)$ \\
\hline $\mathrm{C}(22)-\mathrm{C}(23)-\mathrm{H}(23 \mathrm{~A})$ & 109.2 & $\mathrm{C}(21)-\mathrm{P}(1)-\mathrm{C}(15)$ & $102.51(5)$ \\
\hline $\mathrm{C}(24)-\mathrm{C}(23)-\mathrm{H}(23 \mathrm{~B})$ & 109.2 & $\mathrm{C}(9)-\mathrm{P}(1)-\mathrm{C}(15)$ & $103.23(5)$ \\
\hline $\mathrm{C}(22)-\mathrm{C}(23)-\mathrm{H}(23 \mathrm{~B})$ & 109.2 & $\mathrm{C}(21)-\mathrm{P}(1)-\mathrm{Ru}(1)$ & $120.37(4)$ \\
\hline $\mathrm{H}(23 \mathrm{~A})-\mathrm{C}(23)-\mathrm{H}(23 \mathrm{~B})$ & 107.9 & $\mathrm{C}(9)-\mathrm{P}(1)-\mathrm{Ru}(1)$ & $116.14(4)$ \\
\hline $\mathrm{C}(25)-\mathrm{C}(24)-\mathrm{C}(23)$ & $110.70(11)$ & $\mathrm{C}(15)-\mathrm{P}(1)-\mathrm{Ru}(1)$ & $107.93(4)$ \\
\hline $\mathrm{C}(25)-\mathrm{C}(24)-\mathrm{H}(24 \mathrm{~A})$ & 109.5 & $\mathrm{C}(27)-\mathrm{P}(2)-\mathrm{C}(39)$ & 109.91(5) \\
\hline $\mathrm{C}(23)-\mathrm{C}(24)-\mathrm{H}(24 \mathrm{~A})$ & 109.5 & $\mathrm{C}(27)-\mathrm{P}(2)-\mathrm{C}(33)$ & $103.63(5)$ \\
\hline $\mathrm{C}(25)-\mathrm{C}(24)-\mathrm{H}(24 \mathrm{~B})$ & 109.5 & $\mathrm{C}(39)-\mathrm{P}(2)-\mathrm{C}(33)$ & $103.61(5)$ \\
\hline $\mathrm{C}(23)-\mathrm{C}(24)-\mathrm{H}(24 \mathrm{~B})$ & 109.5 & $\mathrm{C}(27)-\mathrm{P}(2)-\mathrm{Ru}(1)$ & $114.13(4)$ \\
\hline $\mathrm{H}(24 \mathrm{~A})-\mathrm{C}(24)-\mathrm{H}(24 \mathrm{~B})$ & 108.1 & $\mathrm{C}(39)-\mathrm{P}(2)-\mathrm{Ru}(1)$ & $115.34(4)$ \\
\hline $\mathrm{C}(24)-\mathrm{C}(25)-\mathrm{C}(26)$ & $111.23(10)$ & $\mathrm{C}(33)-\mathrm{P}(2)-\mathrm{Ru}(1)$ & $109.01(4)$ \\
\hline $\mathrm{C}(24)-\mathrm{C}(25)-\mathrm{H}(25 \mathrm{~A})$ & 109.4 & $\mathrm{C}(1)-\mathrm{Ru}(1)-\mathrm{Cl}(1)$ & $97.62(4)$ \\
\hline $\mathrm{C}(26)-\mathrm{C}(25)-\mathrm{H}(25 \mathrm{~A})$ & 109.4 & $\mathrm{C}(1)-\mathrm{Ru}(1)-\mathrm{Cl}(2)$ & $102.38(4)$ \\
\hline $\mathrm{C}(24)-\mathrm{C}(25)-\mathrm{H}(25 \mathrm{~B})$ & 109.4 & $\mathrm{Cl}(1)-\mathrm{Ru}(1)-\mathrm{Cl}(2)$ & $159.988(11)$ \\
\hline $\mathrm{C}(26)-\mathrm{C}(25)-\mathrm{H}(25 \mathrm{~B})$ & 109.4 & $\mathrm{C}(1)-\mathrm{Ru}(1)-\mathrm{P}(1)$ & $93.60(4)$ \\
\hline $\mathrm{H}(25 \mathrm{~A})-\mathrm{C}(25)-\mathrm{H}(25 \mathrm{~B})$ & 108 & $\mathrm{Cl}(1)-\mathrm{Ru}(1)-\mathrm{P}(1)$ & $93.523(10)$ \\
\hline $\mathrm{C}(25)-\mathrm{C}(26)-\mathrm{C}(21)$ & $110.39(10)$ & $\mathrm{Cl}(2)-\mathrm{Ru}(1)-\mathrm{P}(1)$ & $85.968(10)$ \\
\hline $\mathrm{C}(25)-\mathrm{C}(26)-\mathrm{H}(26 \mathrm{~A})$ & 109.6 & $\mathrm{C}(1)-\mathrm{Ru}(1)-\mathrm{P}(2)$ & $95.52(4)$ \\
\hline $\mathrm{C}(21)-\mathrm{C}(26)-\mathrm{H}(26 \mathrm{~A})$ & 109.6 & $\mathrm{Cl}(1)-\mathrm{Ru}(1)-\mathrm{P}(2)$ & $88.375(10)$ \\
\hline $\mathrm{C}(25)-\mathrm{C}(26)-\mathrm{H}(26 \mathrm{~B})$ & 109.6 & $\mathrm{Cl}(2)-\mathrm{Ru}(1)-\mathrm{P}(2)$ & $89.018(10)$ \\
\hline $\mathrm{C}(21)-\mathrm{C}(26)-\mathrm{H}(26 \mathrm{~B})$ & 109.6 & $\mathrm{P}(1)-\mathrm{Ru}(1)-\mathrm{P}(2)$ & $170.361(10)$ \\
\hline $\mathrm{H}(26 \mathrm{~A})-\mathrm{C}(26)-\mathrm{H}(26 \mathrm{~B})$ & 108.1 & & \\
\hline
\end{tabular}




\begin{tabular}{|c|c|c|c|}
\hline \multicolumn{4}{|c|}{ Table S6. Torsion angles $\left({ }^{\circ}\right)$ for $\mathrm{C}_{46} \mathrm{H}_{83} \mathrm{BCl}_{6} \mathrm{O}_{2} \mathrm{P}_{2} \mathrm{Ru}$} \\
\hline $\mathrm{O}(2)-\mathrm{C}(3)-\mathrm{C}(4)-\mathrm{O}(1)$ & $-30.83(11)$ & $\mathrm{C}(41)-\mathrm{C}(42)-\mathrm{C}(43)-\mathrm{C}(44)$ & $-56.59(15)$ \\
\hline $\mathrm{C}(6)-\mathrm{C}(3)-\mathrm{C}(4)-\mathrm{O}(1)$ & $-148.48(10)$ & $\mathrm{C}(42)-\mathrm{C}(43)-\mathrm{C}(44)-\mathrm{C}(39)$ & $57.00(14)$ \\
\hline $\mathrm{C}(5)-\mathrm{C}(3)-\mathrm{C}(4)-\mathrm{O}(1)$ & $82.83(11)$ & $C(40)-C(39)-C(44)-C(43)$ & $-56.70(13)$ \\
\hline $\mathrm{O}(2)-\mathrm{C}(3)-\mathrm{C}(4)-\mathrm{C}(7)$ & $-149.05(10)$ & $\mathrm{P}(2)-\mathrm{C}(39)-\mathrm{C}(44)-\mathrm{C}(43)$ & $173.66(9)$ \\
\hline$C(6)-C(3)-C(4)-C(7)$ & $93.30(13)$ & $\mathrm{C}(1)-\mathrm{C}(2)-\mathrm{B}(1)-\mathrm{O}(1)$ & $-9.8(2)$ \\
\hline$C(5)-C(3)-C(4)-C(7)$ & $-35.39(14)$ & $\mathrm{C}(1)-\mathrm{C}(2)-\mathrm{B}(1)-\mathrm{O}(2)$ & $171.72(12)$ \\
\hline $\mathrm{O}(2)-\mathrm{C}(3)-\mathrm{C}(4)-\mathrm{C}(8)$ & $83.35(11)$ & $\mathrm{O}(2)-\mathrm{B}(1)-\mathrm{O}(1)-\mathrm{C}(4)$ & $-9.60(14)$ \\
\hline $\mathrm{C}(6)-\mathrm{C}(3)-\mathrm{C}(4)-\mathrm{C}(8)$ & $-34.31(14)$ & $\mathrm{C}(2)-\mathrm{B}(1)-\mathrm{O}(1)-\mathrm{C}(4)$ & $171.79(12)$ \\
\hline$C(5)-C(3)-C(4)-C(8)$ & $-163.00(10)$ & $\mathrm{C}(7)-\mathrm{C}(4)-\mathrm{O}(1)-\mathrm{B}(1)$ & $146.58(11)$ \\
\hline $\mathrm{C}(14)-\mathrm{C}(9)-\mathrm{C}(10)-\mathrm{C}(11)$ & $-57.32(14)$ & $\mathrm{C}(8)-\mathrm{C}(4)-\mathrm{O}(1)-\mathrm{B}(1)$ & $-93.96(11)$ \\
\hline$P(1)-C(9)-C(10)-C(11)$ & $169.85(9)$ & $\mathrm{C}(3)-\mathrm{C}(4)-\mathrm{O}(1)-\mathrm{B}(1)$ & $24.87(12)$ \\
\hline$C(9)-C(10)-C(11)-C(12)$ & $56.23(16)$ & $\mathrm{O}(1)-\mathrm{B}(1)-\mathrm{O}(2)-\mathrm{C}(3)$ & $-11.58(14)$ \\
\hline$C(10)-C(11)-C(12)-C(13)$ & $-55.26(17)$ & $\mathrm{C}(2)-\mathrm{B}(1)-\mathrm{O}(2)-\mathrm{C}(3)$ & $167.11(11)$ \\
\hline$C(11)-C(12)-C(13)-C(14)$ & $55.81(17)$ & $\mathrm{C}(6)-\mathrm{C}(3)-\mathrm{O}(2)-\mathrm{B}(1)$ & $148.06(10)$ \\
\hline$C(10)-C(9)-C(14)-C(13)$ & $56.58(14)$ & $\mathrm{C}(5)-\mathrm{C}(3)-\mathrm{O}(2)-\mathrm{B}(1)$ & $-92.45(11)$ \\
\hline $\mathrm{P}(1)-\mathrm{C}(9)-\mathrm{C}(14)-\mathrm{C}(13)$ & $-172.33(10)$ & $\mathrm{C}(4)-\mathrm{C}(3)-\mathrm{O}(2)-\mathrm{B}(1)$ & $26.01(12)$ \\
\hline$C(12)-C(13)-C(14)-C(9)$ & $-56.10(16)$ & $\mathrm{C}(26)-\mathrm{C}(21)-\mathrm{P}(1)-\mathrm{C}(9)$ & $77.14(9)$ \\
\hline$C(20)-C(15)-C(16)-C(17)$ & $57.06(13)$ & $\mathrm{C}(22)-\mathrm{C}(21)-\mathrm{P}(1)-\mathrm{C}(9)$ & $-156.57(8)$ \\
\hline$P(1)-C(15)-C(16)-C(17)$ & $-174.73(9)$ & $\mathrm{C}(26)-\mathrm{C}(21)-\mathrm{P}(1)-\mathrm{C}(15)$ & $-175.40(8)$ \\
\hline$C(15)-C(16)-C(17)-C(18)$ & $-56.52(15)$ & $\mathrm{C}(22)-\mathrm{C}(21)-\mathrm{P}(1)-\mathrm{C}(15)$ & $-49.12(9)$ \\
\hline$C(16)-C(17)-C(18)-C(19)$ & $54.79(16)$ & $\mathrm{C}(26)-\mathrm{C}(21)-\mathrm{P}(1)-\mathrm{Ru}(1)$ & $-55.71(9)$ \\
\hline$C(17)-C(18)-C(19)-C(20)$ & $-55.07(16)$ & $\mathrm{C}(22)-\mathrm{C}(21)-\mathrm{P}(1)-\mathrm{Ru}(1)$ & $70.57(9)$ \\
\hline $\mathrm{C}(18)-\mathrm{C}(19)-\mathrm{C}(20)-\mathrm{C}(15)$ & $56.51(15)$ & $\mathrm{C}(10)-\mathrm{C}(9)-\mathrm{P}(1)-\mathrm{C}(21)$ & $38.81(10)$ \\
\hline$C(16)-C(15)-C(20)-C(19)$ & $-56.69(13)$ & $\mathrm{C}(14)-\mathrm{C}(9)-\mathrm{P}(1)-\mathrm{C}(21)$ & $-91.20(10)$ \\
\hline $\mathrm{P}(1)-\mathrm{C}(15)-\mathrm{C}(20)-\mathrm{C}(19)$ & $178.99(9)$ & $\mathrm{C}(10)-\mathrm{C}(9)-\mathrm{P}(1)-\mathrm{C}(15)$ & $-68.11(9)$ \\
\hline $\mathrm{C}(26)-\mathrm{C}(21)-\mathrm{C}(22)-\mathrm{C}(23)$ & $-56.40(13)$ & $\mathrm{C}(14)-\mathrm{C}(9)-\mathrm{P}(1)-\mathrm{C}(15)$ & $161.87(9)$ \\
\hline $\mathrm{P}(1)-\mathrm{C}(21)-\mathrm{C}(22)-\mathrm{C}(23)$ & $176.55(8)$ & $\mathrm{C}(10)-\mathrm{C}(9)-\mathrm{P}(1)-\mathrm{Ru}(1)$ & $174.01(7)$ \\
\hline $\mathrm{C}(21)-\mathrm{C}(22)-\mathrm{C}(23)-\mathrm{C}(24)$ & $55.72(14)$ & $\mathrm{C}(14)-\mathrm{C}(9)-\mathrm{P}(1)-\mathrm{Ru}(1)$ & $44.00(10)$ \\
\hline$C(22)-C(23)-C(24)-C(25)$ & $-55.29(15)$ & $\mathrm{C}(20)-\mathrm{C}(15)-\mathrm{P}(1)-\mathrm{C}(21)$ & $-139.34(9)$ \\
\hline $\mathrm{C}(23)-\mathrm{C}(24)-\mathrm{C}(25)-\mathrm{C}(26)$ & $56.14(15)$ & $\mathrm{C}(16)-\mathrm{C}(15)-\mathrm{P}(1)-\mathrm{C}(21)$ & $96.28(9)$ \\
\hline$C(24)-C(25)-C(26)-C(21)$ & $-57.63(14)$ & $\mathrm{C}(20)-\mathrm{C}(15)-\mathrm{P}(1)-\mathrm{C}(9)$ & $-30.85(10)$ \\
\hline $\mathrm{C}(22)-\mathrm{C}(21)-\mathrm{C}(26)-\mathrm{C}(25)$ & $57.33(13)$ & $\mathrm{C}(16)-\mathrm{C}(15)-\mathrm{P}(1)-\mathrm{C}(9)$ & $-155.23(8)$ \\
\hline $\mathrm{P}(1)-\mathrm{C}(21)-\mathrm{C}(26)-\mathrm{C}(25)$ & $-173.83(9)$ & $\mathrm{C}(20)-\mathrm{C}(15)-\mathrm{P}(1)-\mathrm{Ru}(1)$ & $92.64(9)$ \\
\hline $\mathrm{C}(32)-\mathrm{C}(27)-\mathrm{C}(28)-\mathrm{C}(29)$ & $-54.15(13)$ & $\mathrm{C}(16)-\mathrm{C}(15)-\mathrm{P}(1)-\mathrm{Ru}(1)$ & $-31.74(9)$ \\
\hline $\mathrm{P}(2)-\mathrm{C}(27)-\mathrm{C}(28)-\mathrm{C}(29)$ & $174.72(8)$ & $\mathrm{C}(32)-\mathrm{C}(27)-\mathrm{P}(2)-\mathrm{C}(39)$ & $-49.27(10)$ \\
\hline $\mathrm{C}(27)-\mathrm{C}(28)-\mathrm{C}(29)-\mathrm{C}(30)$ & $54.60(14)$ & $\mathrm{C}(28)-\mathrm{C}(27)-\mathrm{P}(2)-\mathrm{C}(39)$ & $79.89(9)$ \\
\hline$C(28)-C(29)-C(30)-C(31)$ & $-56.07(14)$ & $\mathrm{C}(32)-\mathrm{C}(27)-\mathrm{P}(2)-\mathrm{C}(33)$ & $60.93(9)$ \\
\hline $\mathrm{C}(29)-\mathrm{C}(30)-\mathrm{C}(31)-\mathrm{C}(32)$ & $57.21(14)$ & $\mathrm{C}(28)-\mathrm{C}(27)-\mathrm{P}(2)-\mathrm{C}(33)$ & $-169.91(8)$ \\
\hline
\end{tabular}




\begin{tabular}{llll}
$\mathrm{C}(30)-\mathrm{C}(31)-\mathrm{C}(32)-\mathrm{C}(27)$ & $-57.64(14)$ & $\mathrm{C}(32)-\mathrm{C}(27)-\mathrm{P}(2)-\mathrm{Ru}(1)$ & $179.34(7)$ \\
$\mathrm{C}(28)-\mathrm{C}(27)-\mathrm{C}(32)-\mathrm{C}(31)$ & $55.99(13)$ & $\mathrm{C}(28)-\mathrm{C}(27)-\mathrm{P}(2)-\mathrm{Ru}(1)$ & $-51.49(9)$ \\
$\mathrm{P}(2)-\mathrm{C}(27)-\mathrm{C}(32)-\mathrm{C}(31)$ & $-173.45(8)$ & $\mathrm{C}(40)-\mathrm{C}(39)-\mathrm{P}(2)-\mathrm{C}(27)$ & $-53.61(9)$ \\
$\mathrm{C}(38)-\mathrm{C}(33)-\mathrm{C}(34)-\mathrm{C}(35)$ & $55.15(13)$ & $\mathrm{C}(44)-\mathrm{C}(39)-\mathrm{P}(2)-\mathrm{C}(27)$ & $75.19(10)$ \\
$\mathrm{P}(2)-\mathrm{C}(33)-\mathrm{C}(34)-\mathrm{C}(35)$ & $-179.48(8)$ & $\mathrm{C}(40)-\mathrm{C}(39)-\mathrm{P}(2)-\mathrm{C}(33)$ & $-163.82(8)$ \\
$\mathrm{C}(33)-\mathrm{C}(34)-\mathrm{C}(35)-\mathrm{C}(36)$ & $-55.72(14)$ & $\mathrm{C}(44)-\mathrm{C}(39)-\mathrm{P}(2)-\mathrm{C}(33)$ & $-35.02(10)$ \\
$\mathrm{C}(34)-\mathrm{C}(35)-\mathrm{C}(36)-\mathrm{C}(37)$ & $56.51(15)$ & $\mathrm{C}(40)-\mathrm{C}(39)-\mathrm{P}(2)-\mathrm{Ru}(1)$ & $77.14(8)$ \\
$\mathrm{C}(35)-\mathrm{C}(36)-\mathrm{C}(37)-\mathrm{C}(38)$ & $-57.91(14)$ & $\mathrm{C}(44)-\mathrm{C}(39)-\mathrm{P}(2)-\mathrm{Ru}(1)$ & $-154.06(8)$ \\
$\mathrm{C}(36)-\mathrm{C}(37)-\mathrm{C}(38)-\mathrm{C}(33)$ & $58.61(14)$ & $\mathrm{C}(34)-\mathrm{C}(33)-\mathrm{P}(2)-\mathrm{C}(27)$ & $59.06(9)$ \\
$\mathrm{C}(34)-\mathrm{C}(33)-\mathrm{C}(38)-\mathrm{C}(37)$ & $-56.65(13)$ & $\mathrm{C}(38)-\mathrm{C}(33)-\mathrm{P}(2)-\mathrm{C}(27)$ & $-176.46(8)$ \\
$\mathrm{P}(2)-\mathrm{C}(33)-\mathrm{C}(38)-\mathrm{C}(37)$ & $176.79(9)$ & $\mathrm{C}(34)-\mathrm{C}(33)-\mathrm{P}(2)-\mathrm{C}(39)$ & $173.84(8)$ \\
$\mathrm{C}(44)-\mathrm{C}(39)-\mathrm{C}(40)-\mathrm{C}(41)$ & $56.92(13)$ & $\mathrm{C}(38)-\mathrm{C}(33)-\mathrm{P}(2)-\mathrm{C}(39)$ & $-61.67(9)$ \\
$\mathrm{P}(2)-\mathrm{C}(39)-\mathrm{C}(40)-\mathrm{C}(41)$ & $-170.42(8)$ & $\mathrm{C}(34)-\mathrm{C}(33)-\mathrm{P}(2)-\mathrm{Ru}(1)$ & $-62.85(9)$ \\
$\mathrm{C}(39)-\mathrm{C}(40)-\mathrm{C}(41)-\mathrm{C}(42)$ & $-56.86(14)$ & $\mathrm{C}(38)-\mathrm{C}(33)-\mathrm{P}(2)-\mathrm{Ru}(1)$ & $61.63(9)$ \\
$\mathrm{C}(40)-\mathrm{C}(41)-\mathrm{C}(42)-\mathrm{C}(43)$ & $56.28(14)$ & & \\
\hline
\end{tabular}

Table S7. Anisotropic atomic displacement parameters $\left(\AA^{2} \times 10^{3}\right)$ for $\mathrm{C}_{46} \mathrm{H}_{83} \mathrm{BCl}_{6} \mathrm{O}_{2} \mathrm{P}_{2} \mathrm{Ru}$

The anisotropic atomic displacement factor exponent takes the form: $-2 \pi^{2}\left[h^{2} a^{* 2} U_{11}+\ldots+2 h k\right.$

\begin{tabular}{lllllll}
$\left.\mathrm{a}^{*} \mathrm{~b}^{*} \mathrm{U}_{12}\right]$ & \multicolumn{7}{c}{} & & \\
$\mathrm{C}(1)$ & $17(1)$ & $14(1)$ & $12(1)$ & $1(1)$ & $5(1)$ & $1(1)$ \\
$\mathrm{C}(2)$ & $15(1)$ & $20(1)$ & $16(1)$ & $2(1)$ & $0(1)$ & $0(1)$ \\
$\mathrm{C}(3)$ & $18(1)$ & $19(1)$ & $14(1)$ & $1(1)$ & $-3(1)$ & $1(1)$ \\
$\mathrm{C}(4)$ & $20(1)$ & $18(1)$ & $13(1)$ & $-1(1)$ & $-2(1)$ & $-2(1)$ \\
$\mathrm{C}(5)$ & $25(1)$ & $23(1)$ & $23(1)$ & $-2(1)$ & $-5(1)$ & $6(1)$ \\
$\mathrm{C}(6)$ & $22(1)$ & $25(1)$ & $18(1)$ & $-3(1)$ & $-6(1)$ & $-2(1)$ \\
$\mathrm{C}(7)$ & $31(1)$ & $24(1)$ & $19(1)$ & $4(1)$ & $-1(1)$ & $-5(1)$ \\
$\mathrm{C}(8)$ & $23(1)$ & $24(1)$ & $23(1)$ & $-4(1)$ & $4(1)$ & $-1(1)$ \\
$\mathrm{C}(9)$ & $14(1)$ & $14(1)$ & $15(1)$ & $1(1)$ & $3(1)$ & $-1(1)$ \\
$\mathrm{C}(10)$ & $24(1)$ & $14(1)$ & $27(1)$ & $-1(1)$ & $10(1)$ & $-4(1)$ \\
$\mathrm{C}(11)$ & $35(1)$ & $17(1)$ & $36(1)$ & $3(1)$ & $14(1)$ & $-5(1)$ \\
$\mathrm{C}(12)$ & $30(1)$ & $34(1)$ & $40(1)$ & $5(1)$ & $9(1)$ & $-16(1)$ \\
$\mathrm{C}(13)$ & $17(1)$ & $36(1)$ & $34(1)$ & $12(1)$ & $8(1)$ & $-4(1)$ \\
$\mathrm{C}(14)$ & $14(1)$ & $27(1)$ & $24(1)$ & $9(1)$ & $3(1)$ & $-1(1)$ \\
$\mathrm{C}(15)$ & $13(1)$ & $14(1)$ & $15(1)$ & $0(1)$ & $-1(1)$ & $2(1)$ \\
$\mathrm{C}(16)$ & $18(1)$ & $20(1)$ & $19(1)$ & $1(1)$ & $-2(1)$ & $-4(1)$ \\
$\mathrm{C}(17)$ & $18(1)$ & $35(1)$ & $27(1)$ & $5(1)$ & $-7(1)$ & $-7(1)$ \\
$\mathrm{C}(18)$ & $27(1)$ & $32(1)$ & $22(1)$ & $1(1)$ & $-10(1)$ & $-4(1)$ \\
$\mathrm{C}(19)$ & $24(1)$ & $24(1)$ & $19(1)$ & $4(1)$ & $-6(1)$ & $2(1)$ \\
$\mathrm{C}(20)$ & $20(1)$ & $20(1)$ & $16(1)$ & $5(1)$ & $-2(1)$ & $2(1)$ \\
& & & & & &
\end{tabular}




\begin{tabular}{|c|c|c|c|c|c|c|}
\hline$C(21)$ & $13(1)$ & $14(1)$ & $13(1)$ & $-2(1)$ & $2(1)$ & $0(1)$ \\
\hline$C(22)$ & $14(1)$ & $20(1)$ & $17(1)$ & $-2(1)$ & $4(1)$ & $1(1)$ \\
\hline$C(23)$ & $20(1)$ & $23(1)$ & $21(1)$ & $-3(1)$ & $8(1)$ & $4(1)$ \\
\hline$C(24)$ & $25(1)$ & $27(1)$ & $15(1)$ & $-3(1)$ & $6(1)$ & $2(1)$ \\
\hline$C(25)$ & $22(1)$ & $30(1)$ & $16(1)$ & $-6(1)$ & $1(1)$ & $-1(1)$ \\
\hline$C(26)$ & $14(1)$ & $26(1)$ & $14(1)$ & $-3(1)$ & $0(1)$ & $2(1)$ \\
\hline$C(27)$ & $13(1)$ & $16(1)$ & $15(1)$ & $-1(1)$ & $3(1)$ & $2(1)$ \\
\hline$C(28)$ & $13(1)$ & $18(1)$ & $19(1)$ & $0(1)$ & $4(1)$ & $1(1)$ \\
\hline$C(29)$ & $15(1)$ & $25(1)$ & $22(1)$ & $5(1)$ & $6(1)$ & $3(1)$ \\
\hline$C(30)$ & $21(1)$ & $28(1)$ & $22(1)$ & $1(1)$ & $9(1)$ & $7(1)$ \\
\hline$C(31)$ & $26(1)$ & $25(1)$ & $19(1)$ & $-5(1)$ & $8(1)$ & $4(1)$ \\
\hline$C(32)$ & $19(1)$ & $17(1)$ & $20(1)$ & $-3(1)$ & $6(1)$ & $2(1)$ \\
\hline$C(33)$ & $13(1)$ & $15(1)$ & $14(1)$ & $-1(1)$ & $1(1)$ & $0(1)$ \\
\hline$C(34)$ & $16(1)$ & $25(1)$ & $14(1)$ & $1(1)$ & $0(1)$ & $-3(1)$ \\
\hline$C(35)$ & $19(1)$ & $28(1)$ & $16(1)$ & $-2(1)$ & $-3(1)$ & $-1(1)$ \\
\hline$C(36)$ & $15(1)$ & $26(1)$ & $22(1)$ & $-1(1)$ & $-4(1)$ & $2(1)$ \\
\hline$C(37)$ & $13(1)$ & $30(1)$ & $24(1)$ & $2(1)$ & $2(1)$ & $-2(1)$ \\
\hline$C(38)$ & $13(1)$ & $26(1)$ & $15(1)$ & $1(1)$ & $2(1)$ & $1(1)$ \\
\hline$C(39)$ & $16(1)$ & $13(1)$ & $15(1)$ & $1(1)$ & $2(1)$ & $1(1)$ \\
\hline $\mathrm{C}(40)$ & $18(1)$ & $15(1)$ & $16(1)$ & $2(1)$ & $-1(1)$ & $0(1)$ \\
\hline$C(41)$ & $26(1)$ & $20(1)$ & $15(1)$ & $3(1)$ & $-1(1)$ & $1(1)$ \\
\hline$C(42)$ & $30(1)$ & $19(1)$ & $19(1)$ & $7(1)$ & $2(1)$ & $1(1)$ \\
\hline$C(43)$ & $26(1)$ & $19(1)$ & $25(1)$ & $7(1)$ & $4(1)$ & $-5(1)$ \\
\hline$C(44)$ & $21(1)$ & $15(1)$ & $20(1)$ & $3(1)$ & $0(1)$ & $-3(1)$ \\
\hline$C(45)$ & $23(1)$ & $55(1)$ & $38(1)$ & $14(1)$ & $4(1)$ & $-3(1)$ \\
\hline$C(46)$ & $27(1)$ & $34(1)$ & $29(1)$ & $1(1)$ & $3(1)$ & $-7(1)$ \\
\hline $\mathrm{B}(1)$ & $15(1)$ & $16(1)$ & $16(1)$ & $-1(1)$ & $-2(1)$ & $1(1)$ \\
\hline $\mathrm{O}(1)$ & $17(1)$ & 21(1) & $14(1)$ & $1(1)$ & $-3(1)$ & $-3(1)$ \\
\hline $\mathrm{O}(2)$ & $15(1)$ & $23(1)$ & $15(1)$ & $3(1)$ & $-2(1)$ & $-1(1)$ \\
\hline $\mathrm{P}(1)$ & $11(1)$ & $12(1)$ & $11(1)$ & $0(1)$ & $1(1)$ & $0(1)$ \\
\hline $\mathrm{P}(2)$ & $11(1)$ & $12(1)$ & $12(1)$ & $0(1)$ & $1(1)$ & $1(1)$ \\
\hline $\mathrm{Cl}(1)$ & $19(1)$ & $16(1)$ & $16(1)$ & $1(1)$ & $7(1)$ & $1(1)$ \\
\hline $\mathrm{Cl}(2)$ & $22(1)$ & $16(1)$ & $12(1)$ & $1(1)$ & $5(1)$ & $1(1)$ \\
\hline $\mathrm{Cl}(3)$ & $46(1)$ & $31(1)$ & $76(1)$ & $-7(1)$ & $25(1)$ & $-5(1)$ \\
\hline $\mathrm{Cl}(4)$ & $35(1)$ & $39(1)$ & $56(1)$ & $-14(1)$ & $13(1)$ & $-8(1)$ \\
\hline $\mathrm{Cl}(5)$ & $36(1)$ & $63(1)$ & $47(1)$ & $7(1)$ & $10(1)$ & $9(1)$ \\
\hline $\mathrm{Cl}(6)$ & $51(1)$ & $34(1)$ & $65(1)$ & $-5(1)$ & $16(1)$ & $-11(1)$ \\
\hline $\mathrm{Ru}(1)$ & $10(1)$ & 11(1) & $9(1)$ & $0(1)$ & $1(1)$ & $1(1)$ \\
\hline
\end{tabular}


Table S8. Hydrogen atomic coordinates $\left(\mathrm{x}^{10^{4}}\right)$ and isotropic atomic displacement parameters $\left(\AA^{2} \times 10^{3}\right)$ for $\mathrm{C}_{46} \mathrm{H}_{83} \mathrm{BCl}_{6} \mathrm{O}_{2} \mathrm{P}_{2} \mathrm{Ru}$

\begin{tabular}{|c|c|c|c|c|}
\hline $\mathrm{H}(2)$ & 4964 & 7316 & 5362 & 21 \\
\hline $\mathrm{H}(5 \mathrm{~A})$ & 2987 & 8477 & 3824 & 36 \\
\hline $\mathrm{H}(5 \mathrm{~B})$ & 3699 & 8798 & 3335 & 36 \\
\hline $\mathrm{H}(5 \mathrm{C})$ & 4229 & 8786 & 4024 & 36 \\
\hline $\mathrm{H}(6 \mathrm{~A})$ & 3853 & 6992 & 3128 & 33 \\
\hline $\mathrm{H}(6 \mathrm{~B})$ & 3617 & 7667 & 2743 & 33 \\
\hline $\mathrm{H}(6 \mathrm{C})$ & 2770 & 7453 & 3212 & 33 \\
\hline $\mathrm{H}(7 \mathrm{~A})$ & 5737 & 8929 & 3435 & 37 \\
\hline $\mathrm{H}(7 \mathrm{~B})$ & 5489 & 8532 & 2815 & 37 \\
\hline $\mathrm{H}(7 \mathrm{C})$ & 6738 & 8512 & 3186 & 37 \\
\hline $\mathrm{H}(8 \mathrm{~A})$ & 6880 & 7271 & 3343 & 35 \\
\hline $\mathrm{H}(8 \mathrm{~B})$ & 5783 & 7255 & 2847 & 35 \\
\hline $\mathrm{H}(8 \mathrm{C})$ & 5760 & 6868 & 3468 & 35 \\
\hline $\mathrm{H}(9)$ & 7324 & 5793 & 6570 & 17 \\
\hline $\mathrm{H}(10 \mathrm{~A})$ & 7204 & 4935 & 5551 & 25 \\
\hline $\mathrm{H}(10 \mathrm{~B})$ & 8188 & 4895 & 6109 & 25 \\
\hline $\mathrm{H}(11 \mathrm{~A})$ & 6727 & 4082 & 6204 & 34 \\
\hline $\mathrm{H}(11 \mathrm{~B})$ & 6839 & 4601 & 6753 & 34 \\
\hline $\mathrm{H}(12 \mathrm{~A})$ & 4894 & 4466 & 6402 & 41 \\
\hline $\mathrm{H}(12 \mathrm{~B})$ & 5117 & 4652 & 5736 & 41 \\
\hline $\mathrm{H}(13 \mathrm{~A})$ & 5331 & 5573 & 6725 & 35 \\
\hline $\mathrm{H}(13 \mathrm{~B})$ & 4353 & 5602 & 6165 & 35 \\
\hline $\mathrm{H}(14 \mathrm{~A})$ & 5709 & 5904 & 5521 & 26 \\
\hline $\mathrm{H}(14 \mathrm{~B})$ & 5807 & 6427 & 6068 & 26 \\
\hline $\mathrm{H}(15)$ & 9978 & 5728 & 6133 & 17 \\
\hline $\mathrm{H}(16 \mathrm{~A})$ & 10538 & 6851 & 5952 & 23 \\
\hline $\mathrm{H}(16 \mathrm{~B})$ & 10099 & 7094 & 6561 & 23 \\
\hline $\mathrm{H}(17 \mathrm{~A})$ & 11968 & 6180 & 6489 & 33 \\
\hline $\mathrm{H}(17 \mathrm{~B})$ & 12095 & 6938 & 6734 & 33 \\
\hline $\mathrm{H}(18 \mathrm{~A})$ & 11201 & 6656 & 7570 & 34 \\
\hline $\mathrm{H}(18 \mathrm{~B})$ & 12237 & 6141 & 7534 & 34 \\
\hline H(19A) & 10550 & 5564 & 7730 & 28 \\
\hline H(19B) & 10977 & 5304 & 7124 & 28 \\
\hline $\mathrm{H}(20 \mathrm{~A})$ & 9121 & 6232 & 7185 & 23 \\
\hline $\mathrm{H}(20 \mathrm{~B})$ & 8980 & 5469 & 6953 & 23 \\
\hline $\mathrm{H}(21)$ & 8490 & 5463 & 5162 & 16 \\
\hline $\mathrm{H}(22 \mathrm{~A})$ & 9689 & 6643 & 4851 & 20 \\
\hline $\mathrm{H}(22 \mathrm{~B})$ & 10277 & 6015 & 5217 & 20 \\
\hline
\end{tabular}




\begin{tabular}{|c|c|c|c|c|}
\hline $\mathrm{H}(23 \mathrm{~A})$ & 10520 & 5941 & 4184 & 25 \\
\hline $\mathrm{H}(23 \mathrm{~B})$ & 9837 & 5301 & 4388 & 25 \\
\hline $\mathrm{H}(24 \mathrm{~A})$ & 8816 & 6433 & 3730 & 27 \\
\hline $\mathrm{H}(24 \mathrm{~B})$ & 8903 & 5685 & 3470 & 27 \\
\hline $\mathrm{H}(25 \mathrm{~A})$ & 7629 & 5277 & 4107 & 27 \\
\hline $\mathrm{H}(25 \mathrm{~B})$ & 7037 & 5896 & 3729 & 27 \\
\hline $\mathrm{H}(26 \mathrm{~A})$ & 6774 & 6003 & 4757 & 22 \\
\hline $\mathrm{H}(26 \mathrm{~B})$ & 7492 & 6630 & 4554 & 22 \\
\hline $\mathrm{H}(27)$ & 6970 & 8673 & 7061 & 17 \\
\hline $\mathrm{H}(28 \mathrm{~A})$ & 5270 & 8862 & 6055 & 20 \\
\hline $\mathrm{H}(28 \mathrm{~B})$ & 5589 & 8152 & 6371 & 20 \\
\hline $\mathrm{H}(29 \mathrm{~A})$ & 3923 & 8633 & 6712 & 24 \\
\hline $\mathrm{H}(29 \mathrm{~B})$ & 4916 & 8513 & 7254 & 24 \\
\hline $\mathrm{H}(30 \mathrm{~A})$ & 4130 & 9588 & 7358 & 28 \\
\hline $\mathrm{H}(30 \mathrm{~B})$ & 4357 & 9790 & 6695 & 28 \\
\hline $\mathrm{H}(31 \mathrm{~A})$ & 6118 & 9538 & 7672 & 27 \\
\hline $\mathrm{H}(31 \mathrm{~B})$ & 5808 & 10266 & 7388 & 27 \\
\hline $\mathrm{H}(32 \mathrm{~A})$ & 7455 & 9820 & 7011 & 22 \\
\hline $\mathrm{H}(32 \mathrm{~B})$ & 6442 & 9931 & 6477 & 22 \\
\hline $\mathrm{H}(33)$ & 9162 & 9439 & 6738 & 17 \\
\hline $\mathrm{H}(34 \mathrm{~A})$ & 8781 & 8670 & 7487 & 22 \\
\hline $\mathrm{H}(34 \mathrm{~B})$ & 9448 & 8085 & 7185 & 22 \\
\hline $\mathrm{H}(35 \mathrm{~A})$ & 10489 & 9285 & 7726 & 26 \\
\hline $\mathrm{H}(35 \mathrm{~B})$ & 10679 & 8538 & 7992 & 26 \\
\hline $\mathrm{H}(36 \mathrm{~A})$ & 12262 & 8879 & 7501 & 26 \\
\hline $\mathrm{H}(36 \mathrm{~B})$ & 11661 & 8223 & 7187 & 26 \\
\hline $\mathrm{H}(37 \mathrm{~A})$ & 11999 & 9013 & 6445 & 27 \\
\hline $\mathrm{H}(37 \mathrm{~B})$ & 11293 & 9576 & 6752 & 27 \\
\hline $\mathrm{H}(38 \mathrm{~A})$ & 10316 & 8361 & 6208 & 22 \\
\hline $\mathrm{H}(38 \mathrm{~B})$ & 10120 & 9099 & 5925 & 22 \\
\hline $\mathrm{H}(39)$ & 8245 & 9081 & 5261 & 17 \\
\hline $\mathrm{H}(40 \mathrm{~A})$ & 6267 & 8840 & 5115 & 20 \\
\hline $\mathrm{H}(40 \mathrm{~B})$ & 6016 & 9525 & 5452 & 20 \\
\hline $\mathrm{H}(41 \mathrm{~A})$ & 6996 & 9453 & 4349 & 25 \\
\hline $\mathrm{H}(41 \mathrm{~B})$ & 5732 & 9716 & 4410 & 25 \\
\hline $\mathrm{H}(42 \mathrm{~A})$ & 6472 & 10664 & 4957 & 27 \\
\hline $\mathrm{H}(42 \mathrm{~B})$ & 7004 & 10640 & 4338 & 27 \\
\hline $\mathrm{H}(43 \mathrm{~A})$ & 8456 & 10859 & 5150 & 28 \\
\hline $\mathrm{H}(43 \mathrm{~B})$ & 8714 & 10173 & 4818 & 28 \\
\hline $\mathrm{H}(44 \mathrm{~A})$ & 7735 & 10247 & 5923 & 23 \\
\hline
\end{tabular}




\begin{tabular}{lllll}
$\mathrm{H}(44 \mathrm{~B})$ & 9004 & 9990 & 5864 & 23 \\
$\mathrm{H}(45 \mathrm{~A})$ & 2644 & 7435 & 4705 & 47 \\
$\mathrm{H}(45 \mathrm{~B})$ & 1466 & 7262 & 4956 & 47 \\
$\mathrm{H}(46 \mathrm{~A})$ & 8680 & 7987 & 3255 & 36 \\
$\mathrm{H}(46 \mathrm{~B})$ & 8626 & 7928 & 3958 & 36 \\
\hline
\end{tabular}

\subsection{X-ray structure of Ru-vinyl-3}

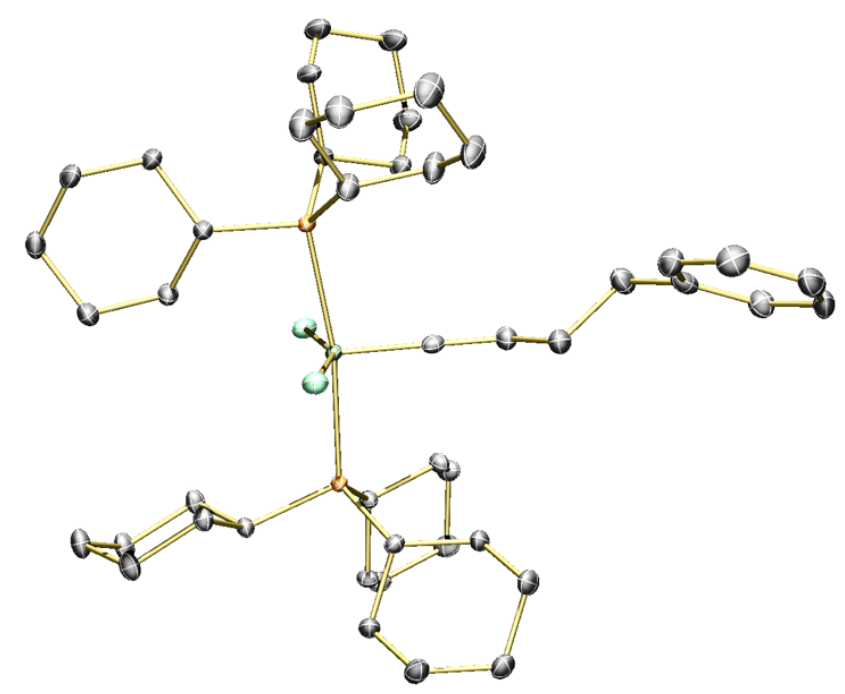

\section{Table S9. Sample and crystal data for $\mathrm{C}_{47} \mathrm{H}_{78} \mathrm{Cl}_{4} \mathrm{P}_{2} \mathrm{Ru}$}

\begin{tabular}{|c|c|c|}
\hline Identification code & \multicolumn{2}{|l|}{ ahsg200520_02 } \\
\hline Chemical formula & \multicolumn{2}{|l|}{$\mathrm{C}_{47} \mathrm{H}_{78} \mathrm{Cl}_{4} \mathrm{P}_{2} \mathrm{Ru}$} \\
\hline Formula weight & \multicolumn{2}{|l|}{$947.90 \mathrm{~g} / \mathrm{mol}$} \\
\hline Temperature & \multicolumn{2}{|l|}{$120(2) \mathrm{K}$} \\
\hline Wavelength & \multicolumn{2}{|l|}{$0.71073 \AA$} \\
\hline Crystal size & \multicolumn{2}{|c|}{$0.250 \times 0.220 \times 0.200 \mathrm{~mm}$} \\
\hline Crystal system & \multicolumn{2}{|l|}{ Triclinic } \\
\hline Space group & \multicolumn{2}{|l|}{$\mathrm{P}-1$} \\
\hline \multirow[t]{3}{*}{ Unit cell dimensions } & $a=13.1023(6) \AA$ & $\alpha=76.999(2)^{\circ}$ \\
\hline & $\mathrm{b}=13.5062(7) \AA$ & $\beta=72.305(2)^{\circ}$ \\
\hline & $\mathrm{c}=15.1308(8) \AA$ & $\gamma=65.888(2)^{\circ}$ \\
\hline Volume & \multicolumn{2}{|l|}{$2312.6(2) \AA^{3}$} \\
\hline Z & \multicolumn{2}{|l|}{2} \\
\hline Density (calculated) & \multicolumn{2}{|l|}{$1.361 \mathrm{~g} / \mathrm{cm}^{3}$} \\
\hline Absorption coefficient & \multicolumn{2}{|l|}{$0.671 \mathrm{~mm}^{-1}$} \\
\hline $\mathrm{F}(000)$ & \multicolumn{2}{|l|}{1004} \\
\hline
\end{tabular}




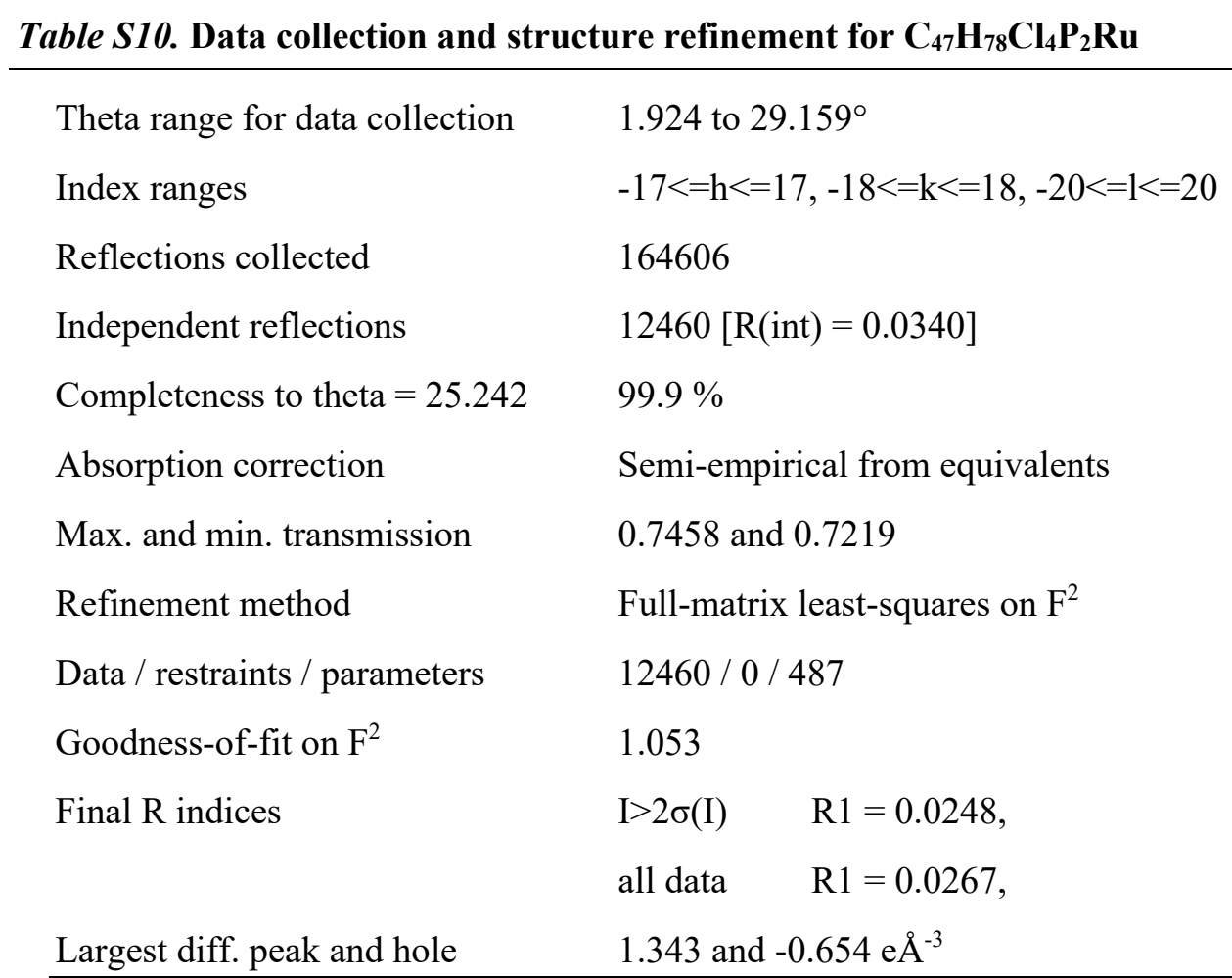

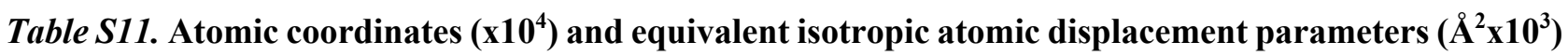
for $\mathrm{C}_{47} \mathrm{H}_{78} \mathrm{Cl}_{4} \mathbf{P}_{2} \mathbf{R u}$

(U(eq) is defined as one third of the trace of the orthogonalized $U_{\mathrm{ij}}$ tensor.)

\begin{tabular}{lllll}
\hline $\mathrm{C}(1)$ & $5775(1)$ & $3282(1)$ & $3811(1)$ & $15(1)$ \\
$\mathrm{C}(2)$ & $5855(1)$ & $3313(1)$ & $4647(1)$ & $19(1)$ \\
$\mathrm{C}(3)$ & $6052(1)$ & $2346(1)$ & $5404(1)$ & $20(1)$ \\
$\mathrm{C}(4)$ & $5286(1)$ & $2696(1)$ & $6360(1)$ & $24(1)$ \\
$\mathrm{C}(5)$ & $5486(1)$ & $1804(1)$ & $7156(1)$ & $22(1)$ \\
$\mathrm{C}(6)$ & $5206(1)$ & $900(1)$ & $7254(1)$ & $25(1)$ \\
$\mathrm{C}(7)$ & $5350(2)$ & $99(1)$ & $8018(1)$ & $28(1)$ \\
$\mathrm{C}(8)$ & $5812(2)$ & $201(1)$ & $8699(1)$ & $28(1)$ \\
$\mathrm{C}(9)$ & $6120(1)$ & $1055(1)$ & $8612(1)$ & $27(1)$ \\
$\mathrm{C}(10)$ & $5957(1)$ & $1880(1)$ & $7848(1)$ & $23(1)$ \\
$\mathrm{C}(11)$ & $8030(1)$ & $4181(1)$ & $1920(1)$ & $13(1)$ \\
$\mathrm{C}(12)$ & $9287(1)$ & $4061(1)$ & $1441(1)$ & $15(1)$ \\
$\mathrm{C}(13)$ & $9421(1)$ & $5170(1)$ & $1253(1)$ & $19(1)$ \\
$\mathrm{C}(14)$ & $9020(1)$ & $5690(1)$ & $2156(1)$ & $21(1)$ \\
$\mathrm{C}(15)$ & $7775(1)$ & $5807(1)$ & $2637(1)$ & $19(1)$ \\
$\mathrm{C}(16)$ & $7622(1)$ & $4707(1)$ & $2832(1)$ & $16(1)$ \\
$\mathrm{C}(17)$ & $8716(1)$ & $1736(1)$ & $2569(1)$ & $14(1)$
\end{tabular}




\begin{tabular}{|c|c|c|c|c|}
\hline$C(18)$ & $8865(1)$ & 1971(1) & $3463(1)$ & $16(1)$ \\
\hline$C(19)$ & $9557(1)$ & $923(1)$ & 3993(1) & $21(1)$ \\
\hline$C(20)$ & $10733(1)$ & $342(1)$ & $3371(1)$ & $22(1)$ \\
\hline$C(21)$ & $10595(1)$ & $115(1)$ & $2481(1)$ & $22(1)$ \\
\hline$C(22)$ & $9910(1)$ & $1162(1)$ & $1946(1)$ & $18(1)$ \\
\hline$C(23)$ & $8149(1)$ & $2670(1)$ & $773(1)$ & $13(1)$ \\
\hline$C(24)$ & $7652(1)$ & $3652(1)$ & $82(1)$ & $17(1)$ \\
\hline$C(25)$ & $8183(1)$ & $3359(1)$ & $-921(1)$ & $20(1)$ \\
\hline$C(26)$ & $8009(1)$ & $2357(1)$ & $-1055(1)$ & $21(1)$ \\
\hline$C(27)$ & $8451(1)$ & $1386(1)$ & $-344(1)$ & $19(1)$ \\
\hline$C(28)$ & $7910(1)$ & $1687(1)$ & $655(1)$ & $15(1)$ \\
\hline$C(29)$ & $2619(1)$ & $4788(1)$ & $3368(1)$ & $16(1)$ \\
\hline $\mathrm{C}(30)$ & $1344(1)$ & $4905(1)$ & $3674(1)$ & $23(1)$ \\
\hline $\mathrm{C}(31)$ & $553(1)$ & $6101(2)$ & $3775(1)$ & $29(1)$ \\
\hline$C(32)$ & $832(1)$ & $6554(2)$ & $4473(1)$ & $29(1)$ \\
\hline$C(33)$ & $2098(1)$ & $6424(1)$ & $4192(1)$ & $26(1)$ \\
\hline$C(34)$ & $2895(1)$ & $5237(1)$ & $4080(1)$ & $20(1)$ \\
\hline$C(35)$ & $3343(1)$ & $2340(1)$ & $4003(1)$ & $22(1)$ \\
\hline$C(36)$ & $3244(2)$ & $2510(1)$ & 4991(1) & $25(1)$ \\
\hline$C(37)$ & $3193(2)$ & $1515(2)$ & $5684(1)$ & $31(1)$ \\
\hline$C(38)$ & $2302(2)$ & $1105(2)$ & $5632(1)$ & $33(1)$ \\
\hline$C(39)$ & $2419(2)$ & $945(1)$ & $4637(1)$ & $26(1)$ \\
\hline $\mathrm{C}(40)$ & $2410(2)$ & $1972(2)$ & $3956(1)$ & $28(1)$ \\
\hline $\mathrm{C}(41)$ & $3578(1)$ & $3088(1)$ & $2016(1)$ & $15(1)$ \\
\hline$C(42)$ & $4424(1)$ & $3402(1)$ & $1156(1)$ & $18(1)$ \\
\hline $\mathrm{C}(43)$ & $4568(1)$ & $2863(1)$ & $317(1)$ & $20(1)$ \\
\hline $\mathrm{C}(44)$ & $3419(1)$ & $3129(1)$ & $94(1)$ & $21(1)$ \\
\hline$C(45)$ & $2527(1)$ & $2909(1)$ & $953(1)$ & $21(1)$ \\
\hline$C(46)$ & $2402(1)$ & $3456(1)$ & $1785(1)$ & $17(1)$ \\
\hline $\mathrm{C}(47)$ & $2366(2)$ & $1089(2)$ & $8473(1)$ & $32(1)$ \\
\hline $\mathrm{P}(1)$ & $7687(1)$ & 2951(1) & $2012(1)$ & $11(1)$ \\
\hline $\mathrm{P}(2)$ & $3691(1)$ & $3416(1)$ & $3104(1)$ & $12(1)$ \\
\hline $\mathrm{Cl}(1)$ & $5167(1)$ & $5072(1)$ & $2034(1)$ & $17(1)$ \\
\hline $\mathrm{Cl}(2)$ & $6146(1)$ & $1313(1)$ & $2777(1)$ & $17(1)$ \\
\hline $\mathrm{Cl}(3)$ & $1896(1)$ & $1469(1)$ & $9612(1)$ & $47(1)$ \\
\hline $\mathrm{Cl}(4)$ & $1202(1)$ & $1287(1)$ & $8018(1)$ & $30(1)$ \\
\hline $\mathrm{Ru}(1)$ & $5681(1)$ & $3217(1)$ & $2680(1)$ & $10(1)$ \\
\hline
\end{tabular}




\begin{tabular}{|c|c|c|c|}
\hline \multicolumn{4}{|c|}{ Table S12. Bond lengths ( $(\AA)$ for $\mathrm{C}_{47} \mathrm{H}_{78} \mathrm{Cl}_{4} \mathrm{P}_{2} \mathrm{Ru}$} \\
\hline$C(1)-C(2)$ & $1.3125(19)$ & $\mathrm{C}(26)-\mathrm{H}(26 \mathrm{~B})$ & 0.99 \\
\hline $\mathrm{C}(1)-\mathrm{Ru}(1)$ & $1.7754(14)$ & $\mathrm{C}(27)-\mathrm{C}(28)$ & $1.5300(19)$ \\
\hline$C(2)-C(3)$ & $1.521(2)$ & $\mathrm{C}(27)-\mathrm{H}(27 \mathrm{~A})$ & 0.99 \\
\hline $\mathrm{C}(2)-\mathrm{H}(2)$ & 0.95 & $\mathrm{C}(27)-\mathrm{H}(27 \mathrm{~B})$ & 0.99 \\
\hline $\mathrm{C}(3)-\mathrm{C}(4)$ & $1.537(2)$ & $\mathrm{C}(28)-\mathrm{H}(28 \mathrm{~A})$ & 0.99 \\
\hline $\mathrm{C}(3)-\mathrm{H}(3 \mathrm{~A})$ & 0.99 & $\mathrm{C}(28)-\mathrm{H}(28 \mathrm{~B})$ & 0.99 \\
\hline $\mathrm{C}(3)-\mathrm{H}(3 \mathrm{~B})$ & 0.99 & $\mathrm{C}(29)-\mathrm{C}(34)$ & $1.5396(19)$ \\
\hline$C(4)-C(5)$ & $1.500(2)$ & $C(29)-C(30)$ & $1.5406(19)$ \\
\hline $\mathrm{C}(4)-\mathrm{H}(4 \mathrm{~A})$ & 0.99 & $\mathrm{C}(29)-\mathrm{P}(2)$ & $1.8523(14)$ \\
\hline $\mathrm{C}(4)-\mathrm{H}(4 \mathrm{~B})$ & 0.99 & $\mathrm{C}(29)-\mathrm{H}(29)$ & 1 \\
\hline$C(5)-C(6)$ & $1.378(2)$ & $\mathrm{C}(30)-\mathrm{C}(31)$ & $1.534(2)$ \\
\hline$C(5)-C(10)$ & $1.408(2)$ & $\mathrm{C}(30)-\mathrm{H}(30 \mathrm{~A})$ & 0.99 \\
\hline$C(6)-C(7)$ & $1.394(2)$ & $\mathrm{C}(30)-\mathrm{H}(30 \mathrm{~B})$ & 0.99 \\
\hline $\mathrm{C}(6)-\mathrm{H}(6)$ & 0.95 & $\mathrm{C}(31)-\mathrm{C}(32)$ & $1.528(2)$ \\
\hline$C(7)-C(8)$ & $1.399(2)$ & $\mathrm{C}(31)-\mathrm{H}(31 \mathrm{~A})$ & 0.99 \\
\hline $\mathrm{C}(7)-\mathrm{H}(7)$ & 0.95 & $\mathrm{C}(31)-\mathrm{H}(31 \mathrm{~B})$ & 0.99 \\
\hline$C(8)-C(9)$ & $1.339(3)$ & $C(32)-C(33)$ & $1.527(2)$ \\
\hline $\mathrm{C}(8)-\mathrm{H}(8)$ & 0.95 & $\mathrm{C}(32)-\mathrm{H}(32 \mathrm{~A})$ & 0.99 \\
\hline $\mathrm{C}(9)-\mathrm{C}(10)$ & $1.413(2)$ & $\mathrm{C}(32)-\mathrm{H}(32 \mathrm{~B})$ & 0.99 \\
\hline $\mathrm{C}(9)-\mathrm{H}(9)$ & 0.95 & $\mathrm{C}(33)-\mathrm{C}(34)$ & $1.529(2)$ \\
\hline $\mathrm{C}(10)-\mathrm{H}(10)$ & 0.95 & $\mathrm{C}(33)-\mathrm{H}(33 \mathrm{~A})$ & 0.99 \\
\hline$C(11)-C(12)$ & $1.5400(18)$ & $\mathrm{C}(33)-\mathrm{H}(33 \mathrm{~B})$ & 0.99 \\
\hline$C(11)-C(16)$ & $1.5409(18)$ & $\mathrm{C}(34)-\mathrm{H}(34 \mathrm{~A})$ & 0.99 \\
\hline $\mathrm{C}(11)-\mathrm{P}(1)$ & $1.8582(13)$ & $\mathrm{C}(34)-\mathrm{H}(34 \mathrm{~B})$ & 0.99 \\
\hline $\mathrm{C}(11)-\mathrm{H}(11)$ & 1 & $C(35)-C(40)$ & $1.521(2)$ \\
\hline$C(12)-C(13)$ & $1.5319(19)$ & $\mathrm{C}(35)-\mathrm{C}(36)$ & $1.523(2)$ \\
\hline $\mathrm{C}(12)-\mathrm{H}(12 \mathrm{~A})$ & 0.99 & $\mathrm{C}(35)-\mathrm{P}(2)$ & $1.8559(15)$ \\
\hline $\mathrm{C}(12)-\mathrm{H}(12 \mathrm{~B})$ & 0.99 & $\mathrm{C}(35)-\mathrm{H}(35)$ & 1 \\
\hline$C(13)-C(14)$ & $1.526(2)$ & $C(36)-C(37)$ & $1.518(2)$ \\
\hline $\mathrm{C}(13)-\mathrm{H}(13 \mathrm{~A})$ & 0.99 & $C(36)-H(36 A)$ & 0.99 \\
\hline $\mathrm{C}(13)-\mathrm{H}(13 \mathrm{~B})$ & 0.99 & $\mathrm{C}(36)-\mathrm{H}(36 \mathrm{~B})$ & 0.99 \\
\hline $\mathrm{C}(14)-\mathrm{C}(15)$ & $1.528(2)$ & $\mathrm{C}(37)-\mathrm{C}(38)$ & $1.510(2)$ \\
\hline $\mathrm{C}(14)-\mathrm{H}(14 \mathrm{~A})$ & 0.99 & $\mathrm{C}(37)-\mathrm{H}(37 \mathrm{~A})$ & 0.99 \\
\hline $\mathrm{C}(14)-\mathrm{H}(14 \mathrm{~B})$ & 0.99 & $\mathrm{C}(37)-\mathrm{H}(37 \mathrm{~B})$ & 0.99 \\
\hline$C(15)-C(16)$ & $1.5322(19)$ & $\mathrm{C}(38)-\mathrm{C}(39)$ & $1.522(2)$ \\
\hline $\mathrm{C}(15)-\mathrm{H}(15 \mathrm{~A})$ & 0.99 & $\mathrm{C}(38)-\mathrm{H}(38 \mathrm{~A})$ & 0.99 \\
\hline $\mathrm{C}(15)-\mathrm{H}(15 \mathrm{~B})$ & 0.99 & $\mathrm{C}(38)-\mathrm{H}(38 \mathrm{~B})$ & 0.99 \\
\hline $\mathrm{C}(16)-\mathrm{H}(16 \mathrm{~A})$ & 0.99 & $C(39)-C(40)$ & $1.528(2)$ \\
\hline
\end{tabular}




\begin{tabular}{|c|c|c|c|}
\hline $\mathrm{C}(16)-\mathrm{H}(16 \mathrm{~B})$ & 0.99 & $\mathrm{C}(39)-\mathrm{H}(39 \mathrm{~A})$ & 0.99 \\
\hline C(17)-C(18) & $1.5398(18)$ & $\mathrm{C}(39)-\mathrm{H}(39 \mathrm{~B})$ & 0.99 \\
\hline $\mathrm{C}(17)-\mathrm{C}(22)$ & $1.5418(19)$ & $\mathrm{C}(40)-\mathrm{H}(40 \mathrm{~A})$ & 0.99 \\
\hline $\mathrm{C}(17)-\mathrm{P}(1)$ & $1.8669(13)$ & $\mathrm{C}(40)-\mathrm{H}(40 \mathrm{~B})$ & 0.99 \\
\hline $\mathrm{C}(17)-\mathrm{H}(17)$ & 1 & $C(41)-C(42)$ & $1.5371(19)$ \\
\hline C(18)-C(19) & $1.5342(19)$ & $\mathrm{C}(41)-\mathrm{C}(46)$ & $1.5392(18)$ \\
\hline $\mathrm{C}(18)-\mathrm{H}(18 \mathrm{~A})$ & 0.99 & $\mathrm{C}(41)-\mathrm{P}(2)$ & $1.8585(14)$ \\
\hline $\mathrm{C}(18)-\mathrm{H}(18 \mathrm{~B})$ & 0.99 & $\mathrm{C}(41)-\mathrm{H}(41)$ & 1 \\
\hline C(19)-C(20) & $1.529(2)$ & $\mathrm{C}(42)-\mathrm{C}(43)$ & $1.528(2)$ \\
\hline $\mathrm{C}(19)-\mathrm{H}(19 \mathrm{~A})$ & 0.99 & $\mathrm{C}(42)-\mathrm{H}(42 \mathrm{~A})$ & 0.99 \\
\hline $\mathrm{C}(19)-\mathrm{H}(19 \mathrm{~B})$ & 0.99 & $\mathrm{C}(42)-\mathrm{H}(42 \mathrm{~B})$ & 0.99 \\
\hline$C(20)-C(21)$ & $1.522(2)$ & $\mathrm{C}(43)-\mathrm{C}(44)$ & $1.524(2)$ \\
\hline $\mathrm{C}(20)-\mathrm{H}(20 \mathrm{~A})$ & 0.99 & $\mathrm{C}(43)-\mathrm{H}(43 \mathrm{~A})$ & 0.99 \\
\hline $\mathrm{C}(20)-\mathrm{H}(20 \mathrm{~B})$ & 0.99 & $\mathrm{C}(43)-\mathrm{H}(43 \mathrm{~B})$ & 0.99 \\
\hline$C(21)-C(22)$ & $1.533(2)$ & $\mathrm{C}(44)-\mathrm{C}(45)$ & $1.527(2)$ \\
\hline $\mathrm{C}(21)-\mathrm{H}(21 \mathrm{~A})$ & 0.99 & $\mathrm{C}(44)-\mathrm{H}(44 \mathrm{~A})$ & 0.99 \\
\hline $\mathrm{C}(21)-\mathrm{H}(21 \mathrm{~B})$ & 0.99 & $\mathrm{C}(44)-\mathrm{H}(44 \mathrm{~B})$ & 0.99 \\
\hline $\mathrm{C}(22)-\mathrm{H}(22 \mathrm{~A})$ & 0.99 & $\mathrm{C}(45)-\mathrm{C}(46)$ & $1.5338(19)$ \\
\hline $\mathrm{C}(22)-\mathrm{H}(22 \mathrm{~B})$ & 0.99 & $\mathrm{C}(45)-\mathrm{H}(45 \mathrm{~A})$ & 0.99 \\
\hline$C(23)-C(24)$ & $1.5355(18)$ & $\mathrm{C}(45)-\mathrm{H}(45 \mathrm{~B})$ & 0.99 \\
\hline$C(23)-C(28)$ & $1.5409(18)$ & $\mathrm{C}(46)-\mathrm{H}(46 \mathrm{~A})$ & 0.99 \\
\hline $\mathrm{C}(23)-\mathrm{P}(1)$ & $1.8622(13)$ & $\mathrm{C}(46)-\mathrm{H}(46 \mathrm{~B})$ & 0.99 \\
\hline $\mathrm{C}(23)-\mathrm{H}(23)$ & 1 & $\mathrm{C}(47)-\mathrm{Cl}(4)$ & $1.7603(19)$ \\
\hline$C(24)-C(25)$ & $1.5319(19)$ & $\mathrm{C}(47)-\mathrm{Cl}(3)$ & $1.7623(19)$ \\
\hline $\mathrm{C}(24)-\mathrm{H}(24 \mathrm{~A})$ & 0.99 & $\mathrm{C}(47)-\mathrm{H}(47 \mathrm{~A})$ & 0.99 \\
\hline $\mathrm{C}(24)-\mathrm{H}(24 \mathrm{~B})$ & 0.99 & $\mathrm{C}(47)-\mathrm{H}(47 \mathrm{~B})$ & 0.99 \\
\hline$C(25)-C(26)$ & $1.526(2)$ & $\mathrm{P}(1)-\mathrm{Ru}(1)$ & $2.4157(4)$ \\
\hline $\mathrm{C}(25)-\mathrm{H}(25 \mathrm{~A})$ & 0.99 & $\mathrm{P}(2)-\mathrm{Ru}(1)$ & $2.4028(4)$ \\
\hline $\mathrm{C}(25)-\mathrm{H}(25 \mathrm{~B})$ & 0.99 & $\mathrm{Cl}(1)-\mathrm{Ru}(1)$ & $2.3674(4)$ \\
\hline$C(26)-C(27)$ & $1.529(2)$ & $\mathrm{Cl}(2)-\mathrm{Ru}(1)$ & $2.3695(4)$ \\
\hline $\mathrm{C}(26)-\mathrm{H}(26 \mathrm{~A})$ & 0.99 & & \\
\hline
\end{tabular}

\section{Table S13. Bond angles $\left({ }^{\circ}\right)$ for $\mathrm{C}_{47} \mathrm{H}_{78} \mathrm{Cl}_{4} \mathrm{P}_{2} \mathrm{Ru}$}

Symmetry transformations used to generate equivalent atoms: $\# 1-\mathrm{x}+1,-\mathrm{y}+1,-\mathrm{z}+1$

\begin{tabular}{llll}
\hline $\mathrm{C}(2)-\mathrm{C}(1)-\mathrm{Ru}(1)$ & $179.06(13)$ & $\mathrm{C}(27)-\mathrm{C}(28)-\mathrm{H}(28 \mathrm{~B})$ & 109.5 \\
$\mathrm{C}(1)-\mathrm{C}(2)-\mathrm{C}(3)$ & $125.01(13)$ & $\mathrm{C}(23)-\mathrm{C}(28)-\mathrm{H}(28 \mathrm{~B})$ & 109.5 \\
$\mathrm{C}(1)-\mathrm{C}(2)-\mathrm{H}(2)$ & 117.5 & $\mathrm{H}(28 \mathrm{~A})-\mathrm{C}(28)-\mathrm{H}(28 \mathrm{~B})$ & 108.1 \\
$\mathrm{C}(3)-\mathrm{C}(2)-\mathrm{H}(2)$ & 117.5 & $\mathrm{C}(34)-\mathrm{C}(29)-\mathrm{C}(30)$ & $109.59(11)$ \\
$\mathrm{C}(2)-\mathrm{C}(3)-\mathrm{C}(4)$ & $110.67(12)$ & $\mathrm{C}(34)-\mathrm{C}(29)-\mathrm{P}(2)$ & $111.81(9)$ \\
$\mathrm{C}(2)-\mathrm{C}(3)-\mathrm{H}(3 \mathrm{~A})$ & 109.5 & $\mathrm{C}(30)-\mathrm{C}(29)-\mathrm{P}(2)$ & $116.55(10)$
\end{tabular}




\begin{tabular}{|c|c|c|c|}
\hline $\mathrm{C}(4)-\mathrm{C}(3)-\mathrm{H}(3 \mathrm{~A})$ & 109.5 & C(34)-C(29)-H(29) & 106 \\
\hline $\mathrm{C}(2)-\mathrm{C}(3)-\mathrm{H}(3 \mathrm{~B})$ & 109.5 & C(30)-C(29)-H(29) & 106 \\
\hline $\mathrm{C}(4)-\mathrm{C}(3)-\mathrm{H}(3 \mathrm{~B})$ & 109.5 & $\mathrm{P}(2)-\mathrm{C}(29)-\mathrm{H}(29)$ & 106 \\
\hline $\mathrm{H}(3 \mathrm{~A})-\mathrm{C}(3)-\mathrm{H}(3 \mathrm{~B})$ & 108.1 & $\mathrm{C}(31)-\mathrm{C}(30)-\mathrm{C}(29)$ & $110.81(13)$ \\
\hline$C(5)-C(4)-C(3)$ & $114.02(13)$ & $\mathrm{C}(31)-\mathrm{C}(30)-\mathrm{H}(30 \mathrm{~A})$ & 109.5 \\
\hline $\mathrm{C}(5)-\mathrm{C}(4)-\mathrm{H}(4 \mathrm{~A})$ & 108.7 & $\mathrm{C}(29)-\mathrm{C}(30)-\mathrm{H}(30 \mathrm{~A})$ & 109.5 \\
\hline $\mathrm{C}(3)-\mathrm{C}(4)-\mathrm{H}(4 \mathrm{~A})$ & 108.7 & $\mathrm{C}(31)-\mathrm{C}(30)-\mathrm{H}(30 \mathrm{~B})$ & 109.5 \\
\hline $\mathrm{C}(5)-\mathrm{C}(4)-\mathrm{H}(4 \mathrm{~B})$ & 108.7 & $\mathrm{C}(29)-\mathrm{C}(30)-\mathrm{H}(30 \mathrm{~B})$ & 109.5 \\
\hline $\mathrm{C}(3)-\mathrm{C}(4)-\mathrm{H}(4 \mathrm{~B})$ & 108.7 & $\mathrm{H}(30 \mathrm{~A})-\mathrm{C}(30)-\mathrm{H}(30 \mathrm{~B})$ & 108.1 \\
\hline $\mathrm{H}(4 \mathrm{~A})-\mathrm{C}(4)-\mathrm{H}(4 \mathrm{~B})$ & 107.6 & $\mathrm{C}(32)-\mathrm{C}(31)-\mathrm{C}(30)$ & $111.05(13)$ \\
\hline$C(6)-C(5)-C(10)$ & $117.38(14)$ & $\mathrm{C}(32)-\mathrm{C}(31)-\mathrm{H}(31 \mathrm{~A})$ & 109.4 \\
\hline$C(6)-C(5)-C(4)$ & $122.51(15)$ & $\mathrm{C}(30)-\mathrm{C}(31)-\mathrm{H}(31 \mathrm{~A})$ & 109.4 \\
\hline$C(10)-C(5)-C(4)$ & $120.10(14)$ & $\mathrm{C}(32)-\mathrm{C}(31)-\mathrm{H}(31 \mathrm{~B})$ & 109.4 \\
\hline$C(5)-C(6)-C(7)$ & $121.99(15)$ & $\mathrm{C}(30)-\mathrm{C}(31)-\mathrm{H}(31 \mathrm{~B})$ & 109.4 \\
\hline $\mathrm{C}(5)-\mathrm{C}(6)-\mathrm{H}(6)$ & 119 & $\mathrm{H}(31 \mathrm{~A})-\mathrm{C}(31)-\mathrm{H}(31 \mathrm{~B})$ & 108 \\
\hline $\mathrm{C}(7)-\mathrm{C}(6)-\mathrm{H}(6)$ & 119 & $\mathrm{C}(33)-\mathrm{C}(32)-\mathrm{C}(31)$ & $110.70(13)$ \\
\hline$C(6)-C(7)-C(8)$ & $119.35(15)$ & $\mathrm{C}(33)-\mathrm{C}(32)-\mathrm{H}(32 \mathrm{~A})$ & 109.5 \\
\hline $\mathrm{C}(6)-\mathrm{C}(7)-\mathrm{H}(7)$ & 120.3 & $\mathrm{C}(31)-\mathrm{C}(32)-\mathrm{H}(32 \mathrm{~A})$ & 109.5 \\
\hline $\mathrm{C}(8)-\mathrm{C}(7)-\mathrm{H}(7)$ & 120.3 & $\mathrm{C}(33)-\mathrm{C}(32)-\mathrm{H}(32 \mathrm{~B})$ & 109.5 \\
\hline $\mathrm{C}(9)-\mathrm{C}(8)-\mathrm{C}(7)$ & $120.18(15)$ & $\mathrm{C}(31)-\mathrm{C}(32)-\mathrm{H}(32 \mathrm{~B})$ & 109.5 \\
\hline $\mathrm{C}(9)-\mathrm{C}(8)-\mathrm{H}(8)$ & 119.9 & $\mathrm{H}(32 \mathrm{~A})-\mathrm{C}(32)-\mathrm{H}(32 \mathrm{~B})$ & 108.1 \\
\hline $\mathrm{C}(7)-\mathrm{C}(8)-\mathrm{H}(8)$ & 119.9 & $\mathrm{C}(32)-\mathrm{C}(33)-\mathrm{C}(34)$ & $111.70(13)$ \\
\hline $\mathrm{C}(8)-\mathrm{C}(9)-\mathrm{C}(10)$ & $120.70(15)$ & $\mathrm{C}(32)-\mathrm{C}(33)-\mathrm{H}(33 \mathrm{~A})$ & 109.3 \\
\hline $\mathrm{C}(8)-\mathrm{C}(9)-\mathrm{H}(9)$ & 119.6 & $\mathrm{C}(34)-\mathrm{C}(33)-\mathrm{H}(33 \mathrm{~A})$ & 109.3 \\
\hline $\mathrm{C}(10)-\mathrm{C}(9)-\mathrm{H}(9)$ & 119.6 & $\mathrm{C}(32)-\mathrm{C}(33)-\mathrm{H}(33 \mathrm{~B})$ & 109.3 \\
\hline$C(5)-C(10)-C(9)$ & $120.36(15)$ & $\mathrm{C}(34)-\mathrm{C}(33)-\mathrm{H}(33 \mathrm{~B})$ & 109.3 \\
\hline $\mathrm{C}(5)-\mathrm{C}(10)-\mathrm{H}(10)$ & 119.8 & $\mathrm{H}(33 \mathrm{~A})-\mathrm{C}(33)-\mathrm{H}(33 \mathrm{~B})$ & 107.9 \\
\hline $\mathrm{C}(9)-\mathrm{C}(10)-\mathrm{H}(10)$ & 119.8 & $\mathrm{C}(33)-\mathrm{C}(34)-\mathrm{C}(29)$ & $111.02(12)$ \\
\hline$C(12)-C(11)-C(16)$ & $110.80(11)$ & $\mathrm{C}(33)-\mathrm{C}(34)-\mathrm{H}(34 \mathrm{~A})$ & 109.4 \\
\hline $\mathrm{C}(12)-\mathrm{C}(11)-\mathrm{P}(1)$ & $115.33(9)$ & $\mathrm{C}(29)-\mathrm{C}(34)-\mathrm{H}(34 \mathrm{~A})$ & 109.4 \\
\hline $\mathrm{C}(16)-\mathrm{C}(11)-\mathrm{P}(1)$ & $115.18(9)$ & $\mathrm{C}(33)-\mathrm{C}(34)-\mathrm{H}(34 \mathrm{~B})$ & 109.4 \\
\hline $\mathrm{C}(12)-\mathrm{C}(11)-\mathrm{H}(11)$ & 104.7 & $\mathrm{C}(29)-\mathrm{C}(34)-\mathrm{H}(34 \mathrm{~B})$ & 109.4 \\
\hline $\mathrm{C}(16)-\mathrm{C}(11)-\mathrm{H}(11)$ & 104.7 & $\mathrm{H}(34 \mathrm{~A})-\mathrm{C}(34)-\mathrm{H}(34 \mathrm{~B})$ & 108 \\
\hline $\mathrm{P}(1)-\mathrm{C}(11)-\mathrm{H}(11)$ & 104.7 & $\mathrm{C}(40)-\mathrm{C}(35)-\mathrm{C}(36)$ & $112.15(13)$ \\
\hline $\mathrm{C}(13)-\mathrm{C}(12)-\mathrm{C}(11)$ & $110.63(11)$ & $\mathrm{C}(40)-\mathrm{C}(35)-\mathrm{P}(2)$ & $119.23(11)$ \\
\hline $\mathrm{C}(13)-\mathrm{C}(12)-\mathrm{H}(12 \mathrm{~A})$ & 109.5 & $\mathrm{C}(36)-\mathrm{C}(35)-\mathrm{P}(2)$ & $113.26(11)$ \\
\hline $\mathrm{C}(11)-\mathrm{C}(12)-\mathrm{H}(12 \mathrm{~A})$ & 109.5 & $\mathrm{C}(40)-\mathrm{C}(35)-\mathrm{H}(35)$ & 103.3 \\
\hline $\mathrm{C}(13)-\mathrm{C}(12)-\mathrm{H}(12 \mathrm{~B})$ & 109.5 & $\mathrm{C}(36)-\mathrm{C}(35)-\mathrm{H}(35)$ & 103.3 \\
\hline $\mathrm{C}(11)-\mathrm{C}(12)-\mathrm{H}(12 \mathrm{~B})$ & 109.5 & $\mathrm{P}(2)-\mathrm{C}(35)-\mathrm{H}(35)$ & 103.3 \\
\hline $\mathrm{H}(12 \mathrm{~A})-\mathrm{C}(12)-\mathrm{H}(12 \mathrm{~B})$ & 108.1 & $\mathrm{C}(37)-\mathrm{C}(36)-\mathrm{C}(35)$ & $111.93(14)$ \\
\hline
\end{tabular}




\begin{tabular}{|c|c|c|c|}
\hline $\mathrm{C}(14)-\mathrm{C}(13)-\mathrm{C}(12)$ & $110.95(12)$ & $\mathrm{C}(37)-\mathrm{C}(36)-\mathrm{H}(36 \mathrm{~A})$ & 109.2 \\
\hline $\mathrm{C}(14)-\mathrm{C}(13)-\mathrm{H}(13 \mathrm{~A})$ & 109.5 & $\mathrm{C}(35)-\mathrm{C}(36)-\mathrm{H}(36 \mathrm{~A})$ & 109.2 \\
\hline $\mathrm{C}(12)-\mathrm{C}(13)-\mathrm{H}(13 \mathrm{~A})$ & 109.5 & $\mathrm{C}(37)-\mathrm{C}(36)-\mathrm{H}(36 \mathrm{~B})$ & 109.2 \\
\hline $\mathrm{C}(14)-\mathrm{C}(13)-\mathrm{H}(13 \mathrm{~B})$ & 109.5 & $\mathrm{C}(35)-\mathrm{C}(36)-\mathrm{H}(36 \mathrm{~B})$ & 109.2 \\
\hline $\mathrm{C}(12)-\mathrm{C}(13)-\mathrm{H}(13 \mathrm{~B})$ & 109.5 & $\mathrm{H}(36 \mathrm{~A})-\mathrm{C}(36)-\mathrm{H}(36 \mathrm{~B})$ & 107.9 \\
\hline $\mathrm{H}(13 \mathrm{~A})-\mathrm{C}(13)-\mathrm{H}(13 \mathrm{~B})$ & 108 & $\mathrm{C}(38)-\mathrm{C}(37)-\mathrm{C}(36)$ & $113.65(14)$ \\
\hline$C(13)-C(14)-C(15)$ & $110.84(12)$ & $\mathrm{C}(38)-\mathrm{C}(37)-\mathrm{H}(37 \mathrm{~A})$ & 108.8 \\
\hline C(13)-C(14)-H(14A) & 109.5 & $\mathrm{C}(36)-\mathrm{C}(37)-\mathrm{H}(37 \mathrm{~A})$ & 108.8 \\
\hline $\mathrm{C}(15)-\mathrm{C}(14)-\mathrm{H}(14 \mathrm{~A})$ & 109.5 & $\mathrm{C}(38)-\mathrm{C}(37)-\mathrm{H}(37 \mathrm{~B})$ & 108.8 \\
\hline $\mathrm{C}(13)-\mathrm{C}(14)-\mathrm{H}(14 \mathrm{~B})$ & 109.5 & $\mathrm{C}(36)-\mathrm{C}(37)-\mathrm{H}(37 \mathrm{~B})$ & 108.8 \\
\hline $\mathrm{C}(15)-\mathrm{C}(14)-\mathrm{H}(14 \mathrm{~B})$ & 109.5 & $\mathrm{H}(37 \mathrm{~A})-\mathrm{C}(37)-\mathrm{H}(37 \mathrm{~B})$ & 107.7 \\
\hline $\mathrm{H}(14 \mathrm{~A})-\mathrm{C}(14)-\mathrm{H}(14 \mathrm{~B})$ & 108.1 & $\mathrm{C}(37)-\mathrm{C}(38)-\mathrm{C}(39)$ & $111.66(14)$ \\
\hline$C(14)-C(15)-C(16)$ & $111.59(12)$ & $\mathrm{C}(37)-\mathrm{C}(38)-\mathrm{H}(38 \mathrm{~A})$ & 109.3 \\
\hline $\mathrm{C}(14)-\mathrm{C}(15)-\mathrm{H}(15 \mathrm{~A})$ & 109.3 & $\mathrm{C}(39)-\mathrm{C}(38)-\mathrm{H}(38 \mathrm{~A})$ & 109.3 \\
\hline $\mathrm{C}(16)-\mathrm{C}(15)-\mathrm{H}(15 \mathrm{~A})$ & 109.3 & $\mathrm{C}(37)-\mathrm{C}(38)-\mathrm{H}(38 \mathrm{~B})$ & 109.3 \\
\hline C(14)-C(15)-H(15B) & 109.3 & $\mathrm{C}(39)-\mathrm{C}(38)-\mathrm{H}(38 \mathrm{~B})$ & 109.3 \\
\hline $\mathrm{C}(16)-\mathrm{C}(15)-\mathrm{H}(15 \mathrm{~B})$ & 109.3 & $\mathrm{H}(38 \mathrm{~A})-\mathrm{C}(38)-\mathrm{H}(38 \mathrm{~B})$ & 107.9 \\
\hline $\mathrm{H}(15 \mathrm{~A})-\mathrm{C}(15)-\mathrm{H}(15 \mathrm{~B})$ & 108 & C(38)-C(39)-C(40) & $112.13(14)$ \\
\hline$C(15)-C(16)-C(11)$ & $110.31(11)$ & $\mathrm{C}(38)-\mathrm{C}(39)-\mathrm{H}(39 \mathrm{~A})$ & 109.2 \\
\hline $\mathrm{C}(15)-\mathrm{C}(16)-\mathrm{H}(16 \mathrm{~A})$ & 109.6 & $\mathrm{C}(40)-\mathrm{C}(39)-\mathrm{H}(39 \mathrm{~A})$ & 109.2 \\
\hline $\mathrm{C}(11)-\mathrm{C}(16)-\mathrm{H}(16 \mathrm{~A})$ & 109.6 & C(38)-C(39)-H(39B) & 109.2 \\
\hline $\mathrm{C}(15)-\mathrm{C}(16)-\mathrm{H}(16 \mathrm{~B})$ & 109.6 & $\mathrm{C}(40)-\mathrm{C}(39)-\mathrm{H}(39 \mathrm{~B})$ & 109.2 \\
\hline $\mathrm{C}(11)-\mathrm{C}(16)-\mathrm{H}(16 \mathrm{~B})$ & 109.6 & H(39A)-C(39)-H(39B) & 107.9 \\
\hline $\mathrm{H}(16 \mathrm{~A})-\mathrm{C}(16)-\mathrm{H}(16 \mathrm{~B})$ & 108.1 & $\mathrm{C}(35)-\mathrm{C}(40)-\mathrm{C}(39)$ & $110.58(14)$ \\
\hline $\mathrm{C}(18)-\mathrm{C}(17)-\mathrm{C}(22)$ & $109.49(11)$ & $\mathrm{C}(35)-\mathrm{C}(40)-\mathrm{H}(40 \mathrm{~A})$ & 109.5 \\
\hline C(18)-C(17)-P(1) & $112.30(9)$ & $\mathrm{C}(39)-\mathrm{C}(40)-\mathrm{H}(40 \mathrm{~A})$ & 109.5 \\
\hline $\mathrm{C}(22)-\mathrm{C}(17)-\mathrm{P}(1)$ & $117.39(9)$ & $\mathrm{C}(35)-\mathrm{C}(40)-\mathrm{H}(40 \mathrm{~B})$ & 109.5 \\
\hline C(18)-C(17)-H(17) & 105.6 & $\mathrm{C}(39)-\mathrm{C}(40)-\mathrm{H}(40 \mathrm{~B})$ & 109.5 \\
\hline $\mathrm{C}(22)-\mathrm{C}(17)-\mathrm{H}(17)$ & 105.6 & $\mathrm{H}(40 \mathrm{~A})-\mathrm{C}(40)-\mathrm{H}(40 \mathrm{~B})$ & 108.1 \\
\hline $\mathrm{P}(1)-\mathrm{C}(17)-\mathrm{H}(17)$ & 105.6 & $C(42)-C(41)-C(46)$ & $109.05(11)$ \\
\hline $\mathrm{C}(19)-\mathrm{C}(18)-\mathrm{C}(17)$ & $111.43(11)$ & $\mathrm{C}(42)-\mathrm{C}(41)-\mathrm{P}(2)$ & $111.38(9)$ \\
\hline C(19)-C(18)-H(18A) & 109.3 & $\mathrm{C}(46)-\mathrm{C}(41)-\mathrm{P}(2)$ & $120.88(10)$ \\
\hline C(17)-C(18)-H(18A) & 109.3 & $\mathrm{C}(42)-\mathrm{C}(41)-\mathrm{H}(41)$ & 104.7 \\
\hline C(19)-C(18)-H(18B) & 109.3 & $\mathrm{C}(46)-\mathrm{C}(41)-\mathrm{H}(41)$ & 104.7 \\
\hline $\mathrm{C}(17)-\mathrm{C}(18)-\mathrm{H}(18 \mathrm{~B})$ & 109.3 & $\mathrm{P}(2)-\mathrm{C}(41)-\mathrm{H}(41)$ & 104.7 \\
\hline $\mathrm{H}(18 \mathrm{~A})-\mathrm{C}(18)-\mathrm{H}(18 \mathrm{~B})$ & 108 & $\mathrm{C}(43)-\mathrm{C}(42)-\mathrm{C}(41)$ & $111.07(12)$ \\
\hline $\mathrm{C}(20)-\mathrm{C}(19)-\mathrm{C}(18)$ & $111.08(12)$ & $\mathrm{C}(43)-\mathrm{C}(42)-\mathrm{H}(42 \mathrm{~A})$ & 109.4 \\
\hline C(20)-C(19)-H(19A) & 109.4 & $\mathrm{C}(41)-\mathrm{C}(42)-\mathrm{H}(42 \mathrm{~A})$ & 109.4 \\
\hline C(18)-C(19)-H(19A) & 109.4 & $\mathrm{C}(43)-\mathrm{C}(42)-\mathrm{H}(42 \mathrm{~B})$ & 109.4 \\
\hline C(20)-C(19)-H(19B) & 109.4 & $\mathrm{C}(41)-\mathrm{C}(42)-\mathrm{H}(42 \mathrm{~B})$ & 109.4 \\
\hline
\end{tabular}




\begin{tabular}{|c|c|c|c|}
\hline $\mathrm{C}(18)-\mathrm{C}(19)-\mathrm{H}(19 \mathrm{~B})$ & 109.4 & $\mathrm{H}(42 \mathrm{~A})-\mathrm{C}(42)-\mathrm{H}(42 \mathrm{~B})$ & 108 \\
\hline $\mathrm{H}(19 \mathrm{~A})-\mathrm{C}(19)-\mathrm{H}(19 \mathrm{~B})$ & 108 & $\mathrm{C}(44)-\mathrm{C}(43)-\mathrm{C}(42)$ & $112.03(12)$ \\
\hline$C(21)-C(20)-C(19)$ & $110.58(12)$ & $\mathrm{C}(44)-\mathrm{C}(43)-\mathrm{H}(43 \mathrm{~A})$ & 109.2 \\
\hline$C(21)-C(20)-H(20 A)$ & 109.5 & $\mathrm{C}(42)-\mathrm{C}(43)-\mathrm{H}(43 \mathrm{~A})$ & 109.2 \\
\hline$C(19)-C(20)-H(20 A)$ & 109.5 & $\mathrm{C}(44)-\mathrm{C}(43)-\mathrm{H}(43 \mathrm{~B})$ & 109.2 \\
\hline $\mathrm{C}(21)-\mathrm{C}(20)-\mathrm{H}(20 \mathrm{~B})$ & 109.5 & $\mathrm{C}(42)-\mathrm{C}(43)-\mathrm{H}(43 \mathrm{~B})$ & 109.2 \\
\hline C(19)-C(20)-H(20B) & 109.5 & $\mathrm{H}(43 \mathrm{~A})-\mathrm{C}(43)-\mathrm{H}(43 \mathrm{~B})$ & 107.9 \\
\hline $\mathrm{H}(20 \mathrm{~A})-\mathrm{C}(20)-\mathrm{H}(20 \mathrm{~B})$ & 108.1 & $C(43)-C(44)-C(45)$ & $112.16(12)$ \\
\hline $\mathrm{C}(20)-\mathrm{C}(21)-\mathrm{C}(22)$ & $111.79(12)$ & $\mathrm{C}(43)-\mathrm{C}(44)-\mathrm{H}(44 \mathrm{~A})$ & 109.2 \\
\hline $\mathrm{C}(20)-\mathrm{C}(21)-\mathrm{H}(21 \mathrm{~A})$ & 109.3 & $\mathrm{C}(45)-\mathrm{C}(44)-\mathrm{H}(44 \mathrm{~A})$ & 109.2 \\
\hline $\mathrm{C}(22)-\mathrm{C}(21)-\mathrm{H}(21 \mathrm{~A})$ & 109.3 & $\mathrm{C}(43)-\mathrm{C}(44)-\mathrm{H}(44 \mathrm{~B})$ & 109.2 \\
\hline $\mathrm{C}(20)-\mathrm{C}(21)-\mathrm{H}(21 \mathrm{~B})$ & 109.3 & $\mathrm{C}(45)-\mathrm{C}(44)-\mathrm{H}(44 \mathrm{~B})$ & 109.2 \\
\hline $\mathrm{C}(22)-\mathrm{C}(21)-\mathrm{H}(21 \mathrm{~B})$ & 109.3 & $\mathrm{H}(44 \mathrm{~A})-\mathrm{C}(44)-\mathrm{H}(44 \mathrm{~B})$ & 107.9 \\
\hline $\mathrm{H}(21 \mathrm{~A})-\mathrm{C}(21)-\mathrm{H}(21 \mathrm{~B})$ & 107.9 & $\mathrm{C}(44)-\mathrm{C}(45)-\mathrm{C}(46)$ & $112.51(12)$ \\
\hline $\mathrm{C}(21)-\mathrm{C}(22)-\mathrm{C}(17)$ & $110.66(12)$ & $\mathrm{C}(44)-\mathrm{C}(45)-\mathrm{H}(45 \mathrm{~A})$ & 109.1 \\
\hline $\mathrm{C}(21)-\mathrm{C}(22)-\mathrm{H}(22 \mathrm{~A})$ & 109.5 & $\mathrm{C}(46)-\mathrm{C}(45)-\mathrm{H}(45 \mathrm{~A})$ & 109.1 \\
\hline $\mathrm{C}(17)-\mathrm{C}(22)-\mathrm{H}(22 \mathrm{~A})$ & 109.5 & $\mathrm{C}(44)-\mathrm{C}(45)-\mathrm{H}(45 \mathrm{~B})$ & 109.1 \\
\hline $\mathrm{C}(21)-\mathrm{C}(22)-\mathrm{H}(22 \mathrm{~B})$ & 109.5 & $\mathrm{C}(46)-\mathrm{C}(45)-\mathrm{H}(45 \mathrm{~B})$ & 109.1 \\
\hline $\mathrm{C}(17)-\mathrm{C}(22)-\mathrm{H}(22 \mathrm{~B})$ & 109.5 & $\mathrm{H}(45 \mathrm{~A})-\mathrm{C}(45)-\mathrm{H}(45 \mathrm{~B})$ & 107.8 \\
\hline $\mathrm{H}(22 \mathrm{~A})-\mathrm{C}(22)-\mathrm{H}(22 \mathrm{~B})$ & 108.1 & $C(45)-C(46)-C(41)$ & $110.04(11)$ \\
\hline $\mathrm{C}(24)-\mathrm{C}(23)-\mathrm{C}(28)$ & $109.59(11)$ & $\mathrm{C}(45)-\mathrm{C}(46)-\mathrm{H}(46 \mathrm{~A})$ & 109.7 \\
\hline $\mathrm{C}(24)-\mathrm{C}(23)-\mathrm{P}(1)$ & $114.79(9)$ & $\mathrm{C}(41)-\mathrm{C}(46)-\mathrm{H}(46 \mathrm{~A})$ & 109.7 \\
\hline $\mathrm{C}(28)-\mathrm{C}(23)-\mathrm{P}(1)$ & $112.33(9)$ & $\mathrm{C}(45)-\mathrm{C}(46)-\mathrm{H}(46 \mathrm{~B})$ & 109.7 \\
\hline $\mathrm{C}(24)-\mathrm{C}(23)-\mathrm{H}(23)$ & 106.5 & $\mathrm{C}(41)-\mathrm{C}(46)-\mathrm{H}(46 \mathrm{~B})$ & 109.7 \\
\hline $\mathrm{C}(28)-\mathrm{C}(23)-\mathrm{H}(23)$ & 106.5 & $\mathrm{H}(46 \mathrm{~A})-\mathrm{C}(46)-\mathrm{H}(46 \mathrm{~B})$ & 108.2 \\
\hline $\mathrm{P}(1)-\mathrm{C}(23)-\mathrm{H}(23)$ & 106.5 & $\mathrm{Cl}(4)-\mathrm{C}(47)-\mathrm{Cl}(3)$ & $111.62(10)$ \\
\hline$C(25)-C(24)-C(23)$ & $110.15(11)$ & $\mathrm{Cl}(4)-\mathrm{C}(47)-\mathrm{H}(47 \mathrm{~A})$ & 109.3 \\
\hline $\mathrm{C}(25)-\mathrm{C}(24)-\mathrm{H}(24 \mathrm{~A})$ & 109.6 & $\mathrm{Cl}(3)-\mathrm{C}(47)-\mathrm{H}(47 \mathrm{~A})$ & 109.3 \\
\hline $\mathrm{C}(23)-\mathrm{C}(24)-\mathrm{H}(24 \mathrm{~A})$ & 109.6 & $\mathrm{Cl}(4)-\mathrm{C}(47)-\mathrm{H}(47 \mathrm{~B})$ & 109.3 \\
\hline $\mathrm{C}(25)-\mathrm{C}(24)-\mathrm{H}(24 \mathrm{~B})$ & 109.6 & $\mathrm{Cl}(3)-\mathrm{C}(47)-\mathrm{H}(47 \mathrm{~B})$ & 109.3 \\
\hline $\mathrm{C}(23)-\mathrm{C}(24)-\mathrm{H}(24 \mathrm{~B})$ & 109.6 & $\mathrm{H}(47 \mathrm{~A})-\mathrm{C}(47)-\mathrm{H}(47 \mathrm{~B})$ & 108 \\
\hline $\mathrm{H}(24 \mathrm{~A})-\mathrm{C}(24)-\mathrm{H}(24 \mathrm{~B})$ & 108.1 & $\mathrm{C}(11)-\mathrm{P}(1)-\mathrm{C}(23)$ & $102.14(6)$ \\
\hline$C(26)-C(25)-C(24)$ & $112.07(12)$ & $\mathrm{C}(11)-\mathrm{P}(1)-\mathrm{C}(17)$ & $110.22(6)$ \\
\hline $\mathrm{C}(26)-\mathrm{C}(25)-\mathrm{H}(25 \mathrm{~A})$ & 109.2 & $\mathrm{C}(23)-\mathrm{P}(1)-\mathrm{C}(17)$ & $102.47(6)$ \\
\hline $\mathrm{C}(24)-\mathrm{C}(25)-\mathrm{H}(25 \mathrm{~A})$ & 109.2 & $\mathrm{C}(11)-\mathrm{P}(1)-\mathrm{Ru}(1)$ & $114.02(4)$ \\
\hline $\mathrm{C}(26)-\mathrm{C}(25)-\mathrm{H}(25 \mathrm{~B})$ & 109.2 & $\mathrm{C}(23)-\mathrm{P}(1)-\mathrm{Ru}(1)$ & $111.95(4)$ \\
\hline $\mathrm{C}(24)-\mathrm{C}(25)-\mathrm{H}(25 \mathrm{~B})$ & 109.2 & $\mathrm{C}(17)-\mathrm{P}(1)-\mathrm{Ru}(1)$ & $114.72(4)$ \\
\hline $\mathrm{H}(25 \mathrm{~A})-\mathrm{C}(25)-\mathrm{H}(25 \mathrm{~B})$ & 107.9 & $\mathrm{C}(29)-\mathrm{P}(2)-\mathrm{C}(35)$ & $110.77(7)$ \\
\hline $\mathrm{C}(25)-\mathrm{C}(26)-\mathrm{C}(27)$ & $111.36(12)$ & $\mathrm{C}(29)-\mathrm{P}(2)-\mathrm{C}(41)$ & $109.88(6)$ \\
\hline $\mathrm{C}(25)-\mathrm{C}(26)-\mathrm{H}(26 \mathrm{~A})$ & 109.4 & $\mathrm{C}(35)-\mathrm{P}(2)-\mathrm{C}(41)$ & $103.21(7)$ \\
\hline
\end{tabular}




\begin{tabular}{llll}
$\mathrm{C}(27)-\mathrm{C}(26)-\mathrm{H}(26 \mathrm{~A})$ & 109.4 & $\mathrm{C}(29)-\mathrm{P}(2)-\mathrm{Ru}(1)$ & $116.55(5)$ \\
$\mathrm{C}(25)-\mathrm{C}(26)-\mathrm{H}(26 \mathrm{~B})$ & 109.4 & $\mathrm{C}(35)-\mathrm{P}(2)-\mathrm{Ru}(1)$ & $114.85(5)$ \\
$\mathrm{C}(27)-\mathrm{C}(26)-\mathrm{H}(26 \mathrm{~B})$ & 109.4 & $\mathrm{C}(41)-\mathrm{P}(2)-\mathrm{Ru}(1)$ & $100.05(4)$ \\
$\mathrm{H}(26 \mathrm{~A})-\mathrm{C}(26)-\mathrm{H}(26 \mathrm{~B})$ & 108 & $\mathrm{C}(1)-\mathrm{Ru}(1)-\mathrm{Cl}(1)$ & $102.05(4)$ \\
$\mathrm{C}(26)-\mathrm{C}(27)-\mathrm{C}(28)$ & $111.14(12)$ & $\mathrm{C}(1)-\mathrm{Ru}(1)-\mathrm{Cl}(2)$ & $99.18(4)$ \\
$\mathrm{C}(26)-\mathrm{C}(27)-\mathrm{H}(27 \mathrm{~A})$ & 109.4 & $\mathrm{Cl}(1)-\mathrm{Ru}(1)-\mathrm{Cl}(2)$ & $158.775(13)$ \\
$\mathrm{C}(28)-\mathrm{C}(27)-\mathrm{H}(27 \mathrm{~A})$ & 109.4 & $\mathrm{C}(1)-\mathrm{Ru}(1)-\mathrm{P}(2)$ & $96.71(4)$ \\
$\mathrm{C}(26)-\mathrm{C}(27)-\mathrm{H}(27 \mathrm{~B})$ & 109.4 & $\mathrm{Cl}(1)-\mathrm{Ru}(1)-\mathrm{P}(2)$ & $89.955(12)$ \\
$\mathrm{C}(28)-\mathrm{C}(27)-\mathrm{H}(27 \mathrm{~B})$ & 109.4 & $\mathrm{Cl}(2)-\mathrm{Ru}(1)-\mathrm{P}(2)$ & $87.365(12)$ \\
$\mathrm{H}(27 \mathrm{~A})-\mathrm{C}(27)-\mathrm{H}(27 \mathrm{~B})$ & 108 & $\mathrm{C}(1)-\mathrm{Ru}(1)-\mathrm{P}(1)$ & $92.56(4)$ \\
$\mathrm{C}(27)-\mathrm{C}(28)-\mathrm{C}(23)$ & $110.57(11)$ & $\mathrm{Cl}(1)-\mathrm{Ru}(1)-\mathrm{P}(1)$ & $89.574(12)$ \\
$\mathrm{C}(27)-\mathrm{C}(28)-\mathrm{H}(28 \mathrm{~A})$ & 109.5 & $\mathrm{Cl}(2)-\mathrm{Ru}(1)-\mathrm{P}(1)$ & $89.688(12)$ \\
$\mathrm{C}(23)-\mathrm{C}(28)-\mathrm{H}(28 \mathrm{~A})$ & 109.5 & $\mathrm{P}(2)-\mathrm{Ru}(1)-\mathrm{P}(1)$ & $170.617(12)$ \\
\hline
\end{tabular}

\section{Table S14. Torsion angles ( ${ }^{\circ}$ ) for $\mathrm{C}_{47} \mathrm{H}_{78} \mathrm{Cl}_{4} \mathrm{P}_{2} \mathrm{Ru}$}

\begin{tabular}{|c|c|c|c|}
\hline $\mathrm{C}(1)-\mathrm{C}(2)-\mathrm{C}(3)-\mathrm{C}(4)$ & $-138.05(15)$ & $\mathrm{C}(37)-\mathrm{C}(38)-\mathrm{C}(39)-\mathrm{C}(40)$ & $-53.4(2)$ \\
\hline $\mathrm{C}(2)-\mathrm{C}(3)-\mathrm{C}(4)-\mathrm{C}(5)$ & $-176.19(13)$ & $\mathrm{C}(36)-\mathrm{C}(35)-\mathrm{C}(40)-\mathrm{C}(39)$ & $-55.03(19)$ \\
\hline$C(3)-C(4)-C(5)-C(6)$ & $-67.8(2)$ & $\mathrm{P}(2)-\mathrm{C}(35)-\mathrm{C}(40)-\mathrm{C}(39)$ & $169.23(12)$ \\
\hline$C(3)-C(4)-C(5)-C(10)$ & $113.32(16)$ & $\mathrm{C}(38)-\mathrm{C}(39)-\mathrm{C}(40)-\mathrm{C}(35)$ & $55.5(2)$ \\
\hline$C(10)-C(5)-C(6)-C(7)$ & $1.7(2)$ & $C(46)-C(41)-C(42)-C(43)$ & $-59.47(15)$ \\
\hline $\mathrm{C}(4)-\mathrm{C}(5)-\mathrm{C}(6)-\mathrm{C}(7)$ & $-177.23(15)$ & $\mathrm{P}(2)-\mathrm{C}(41)-\mathrm{C}(42)-\mathrm{C}(43)$ & $164.60(10)$ \\
\hline$C(5)-C(6)-C(7)-C(8)$ & $-1.2(3)$ & $\mathrm{C}(41)-\mathrm{C}(42)-\mathrm{C}(43)-\mathrm{C}(44)$ & $55.57(16)$ \\
\hline $\mathrm{C}(6)-\mathrm{C}(7)-\mathrm{C}(8)-\mathrm{C}(9)$ & $-0.6(3)$ & $\mathrm{C}(42)-\mathrm{C}(43)-\mathrm{C}(44)-\mathrm{C}(45)$ & $-50.97(17)$ \\
\hline $\mathrm{C}(7)-\mathrm{C}(8)-\mathrm{C}(9)-\mathrm{C}(10)$ & $1.7(3)$ & $\mathrm{C}(43)-\mathrm{C}(44)-\mathrm{C}(45)-\mathrm{C}(46)$ & $51.55(17)$ \\
\hline$C(6)-C(5)-C(10)-C(9)$ & $-0.5(2)$ & $\mathrm{C}(44)-\mathrm{C}(45)-\mathrm{C}(46)-\mathrm{C}(41)$ & $-55.93(16)$ \\
\hline $\mathrm{C}(4)-\mathrm{C}(5)-\mathrm{C}(10)-\mathrm{C}(9)$ & $178.42(14)$ & $\mathrm{C}(42)-\mathrm{C}(41)-\mathrm{C}(46)-\mathrm{C}(45)$ & $59.18(15)$ \\
\hline $\mathrm{C}(8)-\mathrm{C}(9)-\mathrm{C}(10)-\mathrm{C}(5)$ & $-1.2(2)$ & $\mathrm{P}(2)-\mathrm{C}(41)-\mathrm{C}(46)-\mathrm{C}(45)$ & $-169.83(10)$ \\
\hline $\mathrm{C}(16)-\mathrm{C}(11)-\mathrm{C}(12)-\mathrm{C}(13)$ & $-56.67(15)$ & $\mathrm{C}(12)-\mathrm{C}(11)-\mathrm{P}(1)-\mathrm{C}(23)$ & $-55.39(11)$ \\
\hline $\mathrm{P}(1)-\mathrm{C}(11)-\mathrm{C}(12)-\mathrm{C}(13)$ & $170.21(9)$ & $\mathrm{C}(16)-\mathrm{C}(11)-\mathrm{P}(1)-\mathrm{C}(23)$ & $173.56(10)$ \\
\hline $\mathrm{C}(11)-\mathrm{C}(12)-\mathrm{C}(13)-\mathrm{C}(14)$ & $56.89(15)$ & $\mathrm{C}(12)-\mathrm{C}(11)-\mathrm{P}(1)-\mathrm{C}(17)$ & $52.95(11)$ \\
\hline $\mathrm{C}(12)-\mathrm{C}(13)-\mathrm{C}(14)-\mathrm{C}(15)$ & $-56.61(16)$ & $\mathrm{C}(16)-\mathrm{C}(11)-\mathrm{P}(1)-\mathrm{C}(17)$ & $-78.10(11)$ \\
\hline $\mathrm{C}(13)-\mathrm{C}(14)-\mathrm{C}(15)-\mathrm{C}(16)$ & $56.48(16)$ & $\mathrm{C}(12)-\mathrm{C}(11)-\mathrm{P}(1)-\mathrm{Ru}(1)$ & $-176.36(8)$ \\
\hline $\mathrm{C}(14)-\mathrm{C}(15)-\mathrm{C}(16)-\mathrm{C}(11)$ & $-56.04(15)$ & $\mathrm{C}(16)-\mathrm{C}(11)-\mathrm{P}(1)-\mathrm{Ru}(1)$ & $52.59(10)$ \\
\hline $\mathrm{C}(12)-\mathrm{C}(11)-\mathrm{C}(16)-\mathrm{C}(15)$ & $56.00(14)$ & $\mathrm{C}(24)-\mathrm{C}(23)-\mathrm{P}(1)-\mathrm{C}(11)$ & $-51.94(11)$ \\
\hline $\mathrm{P}(1)-\mathrm{C}(11)-\mathrm{C}(16)-\mathrm{C}(15)$ & $-170.81(9)$ & $\mathrm{C}(28)-\mathrm{C}(23)-\mathrm{P}(1)-\mathrm{C}(11)$ & $-178.02(9)$ \\
\hline $\mathrm{C}(22)-\mathrm{C}(17)-\mathrm{C}(18)-\mathrm{C}(19)$ & $-56.82(15)$ & $\mathrm{C}(24)-\mathrm{C}(23)-\mathrm{P}(1)-\mathrm{C}(17)$ & $-166.12(10)$ \\
\hline $\mathrm{P}(1)-\mathrm{C}(17)-\mathrm{C}(18)-\mathrm{C}(19)$ & $170.90(10)$ & $\mathrm{C}(28)-\mathrm{C}(23)-\mathrm{P}(1)-\mathrm{C}(17)$ & $67.80(10)$ \\
\hline $\mathrm{C}(17)-\mathrm{C}(18)-\mathrm{C}(19)-\mathrm{C}(20)$ & $56.71(16)$ & $\mathrm{C}(24)-\mathrm{C}(23)-\mathrm{P}(1)-\mathrm{Ru}(1)$ & $70.45(10)$ \\
\hline $\mathrm{C}(18)-\mathrm{C}(19)-\mathrm{C}(20)-\mathrm{C}(21)$ & $-55.48(17)$ & $\mathrm{C}(28)-\mathrm{C}(23)-\mathrm{P}(1)-\mathrm{Ru}(1)$ & $-55.62(10)$ \\
\hline
\end{tabular}




\begin{tabular}{|c|c|c|c|}
\hline $\mathrm{C}(19)-\mathrm{C}(20)-\mathrm{C}(21)-\mathrm{C}(22)$ & $56.12(17)$ & $\mathrm{C}(18)-\mathrm{C}(17)-\mathrm{P}(1)-\mathrm{C}(11)$ & $47.85(11)$ \\
\hline $\mathrm{C}(20)-\mathrm{C}(21)-\mathrm{C}(22)-\mathrm{C}(17)$ & $-57.19(16)$ & $\mathrm{C}(22)-\mathrm{C}(17)-\mathrm{P}(1)-\mathrm{C}(11)$ & $-80.37(11)$ \\
\hline $\mathrm{C}(18)-\mathrm{C}(17)-\mathrm{C}(22)-\mathrm{C}(21)$ & $56.60(15)$ & $\mathrm{C}(18)-\mathrm{C}(17)-\mathrm{P}(1)-\mathrm{C}(23)$ & $155.97(9)$ \\
\hline $\mathrm{P}(1)-\mathrm{C}(17)-\mathrm{C}(22)-\mathrm{C}(21)$ & $-173.85(10)$ & $\mathrm{C}(22)-\mathrm{C}(17)-\mathrm{P}(1)-\mathrm{C}(23)$ & $27.75(11)$ \\
\hline$C(28)-C(23)-C(24)-C(25)$ & $-58.21(15)$ & $\mathrm{C}(18)-\mathrm{C}(17)-\mathrm{P}(1)-\mathrm{Ru}(1)$ & $-82.47(10)$ \\
\hline $\mathrm{P}(1)-\mathrm{C}(23)-\mathrm{C}(24)-\mathrm{C}(25)$ & $174.30(10)$ & $\mathrm{C}(22)-\mathrm{C}(17)-\mathrm{P}(1)-\mathrm{Ru}(1)$ & $149.31(9)$ \\
\hline $\mathrm{C}(23)-\mathrm{C}(24)-\mathrm{C}(25)-\mathrm{C}(26)$ & $56.31(16)$ & $\mathrm{C}(34)-\mathrm{C}(29)-\mathrm{P}(2)-\mathrm{C}(35)$ & $83.03(11)$ \\
\hline $\mathrm{C}(24)-\mathrm{C}(25)-\mathrm{C}(26)-\mathrm{C}(27)$ & $-54.11(17)$ & $\mathrm{C}(30)-\mathrm{C}(29)-\mathrm{P}(2)-\mathrm{C}(35)$ & $-44.11(12)$ \\
\hline$C(25)-C(26)-C(27)-C(28)$ & $54.18(17)$ & $\mathrm{C}(34)-\mathrm{C}(29)-\mathrm{P}(2)-\mathrm{C}(41)$ & $-163.58(10)$ \\
\hline $\mathrm{C}(26)-\mathrm{C}(27)-\mathrm{C}(28)-\mathrm{C}(23)$ & $-57.06(16)$ & $\mathrm{C}(30)-\mathrm{C}(29)-\mathrm{P}(2)-\mathrm{C}(41)$ & $69.28(12)$ \\
\hline $\mathrm{C}(24)-\mathrm{C}(23)-\mathrm{C}(28)-\mathrm{C}(27)$ & $59.06(14)$ & $\mathrm{C}(34)-\mathrm{C}(29)-\mathrm{P}(2)-\mathrm{Ru}(1)$ & $-50.74(11)$ \\
\hline $\mathrm{P}(1)-\mathrm{C}(23)-\mathrm{C}(28)-\mathrm{C}(27)$ & $-172.09(9)$ & $\mathrm{C}(30)-\mathrm{C}(29)-\mathrm{P}(2)-\mathrm{Ru}(1)$ & $-177.88(9)$ \\
\hline$C(34)-C(29)-C(30)-C(31)$ & $57.35(16)$ & $\mathrm{C}(40)-\mathrm{C}(35)-\mathrm{P}(2)-\mathrm{C}(29)$ & $79.43(14)$ \\
\hline $\mathrm{P}(2)-\mathrm{C}(29)-\mathrm{C}(30)-\mathrm{C}(31)$ & $-174.43(11)$ & $\mathrm{C}(36)-\mathrm{C}(35)-\mathrm{P}(2)-\mathrm{C}(29)$ & $-55.86(13)$ \\
\hline $\mathrm{C}(29)-\mathrm{C}(30)-\mathrm{C}(31)-\mathrm{C}(32)$ & $-57.50(18)$ & $\mathrm{C}(40)-\mathrm{C}(35)-\mathrm{P}(2)-\mathrm{C}(41)$ & $-38.12(15)$ \\
\hline $\mathrm{C}(30)-\mathrm{C}(31)-\mathrm{C}(32)-\mathrm{C}(33)$ & $55.9(2)$ & $\mathrm{C}(36)-\mathrm{C}(35)-\mathrm{P}(2)-\mathrm{C}(41)$ & $-173.41(11)$ \\
\hline $\mathrm{C}(31)-\mathrm{C}(32)-\mathrm{C}(33)-\mathrm{C}(34)$ & $-55.5(2)$ & $\mathrm{C}(40)-\mathrm{C}(35)-\mathrm{P}(2)-\mathrm{Ru}(1)$ & $-145.95(12)$ \\
\hline $\mathrm{C}(32)-\mathrm{C}(33)-\mathrm{C}(34)-\mathrm{C}(29)$ & $56.36(18)$ & $\mathrm{C}(36)-\mathrm{C}(35)-\mathrm{P}(2)-\mathrm{Ru}(1)$ & $78.76(12)$ \\
\hline $\mathrm{C}(30)-\mathrm{C}(29)-\mathrm{C}(34)-\mathrm{C}(33)$ & $-56.68(16)$ & $\mathrm{C}(42)-\mathrm{C}(41)-\mathrm{P}(2)-\mathrm{C}(29)$ & $93.62(11)$ \\
\hline $\mathrm{P}(2)-\mathrm{C}(29)-\mathrm{C}(34)-\mathrm{C}(33)$ & $172.51(11)$ & $\mathrm{C}(46)-\mathrm{C}(41)-\mathrm{P}(2)-\mathrm{C}(29)$ & $-36.37(13)$ \\
\hline $\mathrm{C}(40)-\mathrm{C}(35)-\mathrm{C}(36)-\mathrm{C}(37)$ & $52.69(19)$ & $\mathrm{C}(42)-\mathrm{C}(41)-\mathrm{P}(2)-\mathrm{C}(35)$ & $-148.21(10)$ \\
\hline$P(2)-C(35)-C(36)-C(37)$ & $-168.83(12)$ & $\mathrm{C}(46)-\mathrm{C}(41)-\mathrm{P}(2)-\mathrm{C}(35)$ & $81.80(12)$ \\
\hline $\mathrm{C}(35)-\mathrm{C}(36)-\mathrm{C}(37)-\mathrm{C}(38)$ & $-50.8(2)$ & $\mathrm{C}(42)-\mathrm{C}(41)-\mathrm{P}(2)-\mathrm{Ru}(1)$ & $-29.53(10)$ \\
\hline $\mathrm{C}(36)-\mathrm{C}(37)-\mathrm{C}(38)-\mathrm{C}(39)$ & $51.1(2)$ & $\mathrm{C}(46)-\mathrm{C}(41)-\mathrm{P}(2)-\mathrm{Ru}(1)$ & $-159.52(10)$ \\
\hline
\end{tabular}

\begin{tabular}{|c|c|c|c|c|c|c|}
\hline Table $S$ & sotrop & ic disp & ent par & $\operatorname{rss}(\AA$ & or $\mathrm{C}_{47} \mathrm{H}$ & ${ }_{2} \mathrm{Ru}$ \\
\hline The anis & tomic d & nent fac & onent tal & form: & $\mathrm{a}^{* 2} \mathrm{U}_{11}$ & $\mathrm{~h} \mathrm{k} \mathrm{a}^{*} \mathrm{~b}^{*} \mathrm{U}_{12}$ \\
\hline $\mathrm{C}(1)$ & $12(1)$ & $16(1)$ & $17(1)$ & $0(1)$ & $-3(1)$ & $-7(1)$ \\
\hline $\mathrm{C}(2)$ & $19(1)$ & $22(1)$ & $16(1)$ & $-4(1)$ & $-5(1)$ & $-9(1)$ \\
\hline$C(3)$ & $20(1)$ & $24(1)$ & $17(1)$ & $-2(1)$ & $-5(1)$ & $-7(1)$ \\
\hline $\mathrm{C}(4)$ & $24(1)$ & $24(1)$ & $17(1)$ & $-2(1)$ & $-3(1)$ & $-5(1)$ \\
\hline$C(5)$ & $17(1)$ & $20(1)$ & $18(1)$ & $-2(1)$ & $1(1)$ & $-2(1)$ \\
\hline$C(6)$ & $24(1)$ & $30(1)$ & $24(1)$ & $-6(1)$ & $-9(1)$ & $-8(1)$ \\
\hline $\mathrm{C}(7)$ & $29(1)$ & $23(1)$ & $33(1)$ & $-2(1)$ & $-8(1)$ & $-11(1)$ \\
\hline $\mathrm{C}(8)$ & $26(1)$ & $30(1)$ & $19(1)$ & $0(1)$ & $-5(1)$ & $-4(1)$ \\
\hline $\mathrm{C}(9)$ & $25(1)$ & $35(1)$ & $17(1)$ & $-8(1)$ & $-8(1)$ & $-3(1)$ \\
\hline $\mathrm{C}(10)$ & $26(1)$ & $23(1)$ & $21(1)$ & $-3(1)$ & $-4(1)$ & $-11(1)$ \\
\hline $\mathrm{C}(11)$ & $12(1)$ & $14(1)$ & $13(1)$ & $-2(1)$ & $-2(1)$ & $-7(1)$ \\
\hline $\mathrm{C}(12)$ & $13(1)$ & $16(1)$ & $17(1)$ & $-4(1)$ & $-1(1)$ & $-7(1)$ \\
\hline
\end{tabular}




\begin{tabular}{|c|c|c|c|c|c|c|}
\hline$C(13)$ & $16(1)$ & $18(1)$ & $23(1)$ & $-2(1)$ & $-2(1)$ & $-10(1)$ \\
\hline$C(14)$ & $19(1)$ & $19(1)$ & $30(1)$ & $-6(1)$ & $-5(1)$ & $-10(1)$ \\
\hline$C(15)$ & $19(1)$ & $17(1)$ & $23(1)$ & $-7(1)$ & $-4(1)$ & $-7(1)$ \\
\hline$C(16)$ & $16(1)$ & $17(1)$ & $15(1)$ & $-5(1)$ & $-2(1)$ & $-8(1)$ \\
\hline $\mathrm{C}(17)$ & $12(1)$ & $14(1)$ & $15(1)$ & $-1(1)$ & $-5(1)$ & $-4(1)$ \\
\hline $\mathrm{C}(18)$ & $15(1)$ & $19(1)$ & $13(1)$ & $-2(1)$ & $-4(1)$ & $-3(1)$ \\
\hline$C(19)$ & $21(1)$ & $23(1)$ & $16(1)$ & $2(1)$ & $-8(1)$ & $-5(1)$ \\
\hline $\mathrm{C}(20)$ & $19(1)$ & $22(1)$ & $22(1)$ & $-1(1)$ & $-11(1)$ & $-2(1)$ \\
\hline $\mathrm{C}(21)$ & $20(1)$ & $19(1)$ & $24(1)$ & $-6(1)$ & $-10(1)$ & $0(1)$ \\
\hline $\mathrm{C}(22)$ & $14(1)$ & $20(1)$ & $16(1)$ & $-5(1)$ & $-4(1)$ & $-1(1)$ \\
\hline$C(23)$ & $14(1)$ & $14(1)$ & $11(1)$ & $-2(1)$ & $-3(1)$ & $-6(1)$ \\
\hline$C(24)$ & $23(1)$ & $14(1)$ & $13(1)$ & $-1(1)$ & $-5(1)$ & $-6(1)$ \\
\hline$C(25)$ & $31(1)$ & $19(1)$ & $12(1)$ & $0(1)$ & $-5(1)$ & $-10(1)$ \\
\hline$C(26)$ & $30(1)$ & $22(1)$ & $14(1)$ & $-3(1)$ & $-6(1)$ & $-11(1)$ \\
\hline$C(27)$ & $26(1)$ & $16(1)$ & $16(1)$ & $-4(1)$ & $-4(1)$ & $-9(1)$ \\
\hline $\mathrm{C}(28)$ & $19(1)$ & $15(1)$ & $14(1)$ & $-2(1)$ & $-4(1)$ & $-8(1)$ \\
\hline$C(29)$ & $12(1)$ & $21(1)$ & $15(1)$ & $-4(1)$ & $-3(1)$ & $-5(1)$ \\
\hline $\mathrm{C}(30)$ & $12(1)$ & $34(1)$ & $26(1)$ & $-14(1)$ & $-3(1)$ & $-6(1)$ \\
\hline $\mathrm{C}(31)$ & $14(1)$ & $37(1)$ & $33(1)$ & $-17(1)$ & $-6(1)$ & $0(1)$ \\
\hline$C(32)$ & $16(1)$ & $35(1)$ & $36(1)$ & $-21(1)$ & $-2(1)$ & $-4(1)$ \\
\hline$C(33)$ & $18(1)$ & $26(1)$ & $35(1)$ & $-14(1)$ & $-4(1)$ & $-6(1)$ \\
\hline $\mathrm{C}(34)$ & $16(1)$ & $24(1)$ & $21(1)$ & $-7(1)$ & $-5(1)$ & $-7(1)$ \\
\hline$C(35)$ & $26(1)$ & $29(1)$ & $17(1)$ & $3(1)$ & $-6(1)$ & $-18(1)$ \\
\hline$C(36)$ & $33(1)$ & $33(1)$ & $18(1)$ & $4(1)$ & $-11(1)$ & $-21(1)$ \\
\hline$C(37)$ & $36(1)$ & $45(1)$ & $20(1)$ & $10(1)$ & $-12(1)$ & $-26(1)$ \\
\hline $\mathrm{C}(38)$ & $39(1)$ & $40(1)$ & $27(1)$ & $11(1)$ & $-13(1)$ & $-26(1)$ \\
\hline$C(39)$ & $33(1)$ & $29(1)$ & $23(1)$ & $3(1)$ & $-7(1)$ & $-22(1)$ \\
\hline$C(40)$ & $35(1)$ & $36(1)$ & $22(1)$ & $4(1)$ & $-10(1)$ & $-24(1)$ \\
\hline $\mathrm{C}(41)$ & $13(1)$ & $20(1)$ & $13(1)$ & $-3(1)$ & $-3(1)$ & $-7(1)$ \\
\hline $\mathrm{C}(42)$ & $17(1)$ & $26(1)$ & $14(1)$ & $-2(1)$ & $-3(1)$ & $-11(1)$ \\
\hline $\mathrm{C}(43)$ & $19(1)$ & $27(1)$ & $14(1)$ & $-5(1)$ & $-2(1)$ & $-9(1)$ \\
\hline$C(44)$ & $24(1)$ & $23(1)$ & $16(1)$ & $-4(1)$ & $-8(1)$ & $-7(1)$ \\
\hline$C(45)$ & $18(1)$ & $26(1)$ & $22(1)$ & $-9(1)$ & $-7(1)$ & $-7(1)$ \\
\hline$C(46)$ & $14(1)$ & $22(1)$ & $18(1)$ & $-5(1)$ & $-5(1)$ & $-6(1)$ \\
\hline$C(47)$ & $30(1)$ & $37(1)$ & $33(1)$ & $-13(1)$ & $-1(1)$ & $-16(1)$ \\
\hline $\mathrm{P}(1)$ & $10(1)$ & $12(1)$ & $10(1)$ & $-2(1)$ & $-2(1)$ & $-5(1)$ \\
\hline $\mathrm{P}(2)$ & $10(1)$ & $17(1)$ & $11(1)$ & $-1(1)$ & $-3(1)$ & $-6(1)$ \\
\hline $\mathrm{Cl}(1)$ & $15(1)$ & $14(1)$ & $21(1)$ & $2(1)$ & $-5(1)$ & $-5(1)$ \\
\hline $\mathrm{Cl}(2)$ & $16(1)$ & $14(1)$ & $21(1)$ & $-2(1)$ & $-3(1)$ & $-7(1)$ \\
\hline $\mathrm{Cl}(3)$ & $71(1)$ & $52(1)$ & $32(1)$ & $-9(1)$ & $-10(1)$ & $-35(1)$ \\
\hline
\end{tabular}




\begin{tabular}{lllllll}
$\mathrm{Cl}(4)$ & $29(1)$ & $30(1)$ & $29(1)$ & $-6(1)$ & $-3(1)$ & $-12(1)$ \\
$\mathrm{Ru}(1)$ & $9(1)$ & $12(1)$ & $10(1)$ & $-1(1)$ & $-3(1)$ & $-5(1)$ \\
\hline
\end{tabular}

Table S16. Hydrogen atomic coordinates $\left(\mathrm{x}^{4} 0^{4}\right)$ and isotropic atomic displacement parameters $\left(\AA^{2} \times 10^{3}\right)$ for $\mathrm{C}_{47} \mathrm{H}_{78} \mathrm{Cl}_{4} \mathrm{P}_{2} \mathrm{Ru}$

\begin{tabular}{|c|c|c|c|c|}
\hline $\mathrm{H}(2)$ & 5784 & 3990 & 4792 & 22 \\
\hline $\mathrm{H}(3 \mathrm{~A})$ & 6871 & 2041 & 5429 & 24 \\
\hline $\mathrm{H}(3 \mathrm{~B})$ & 5875 & 1767 & 5250 & 24 \\
\hline $\mathrm{H}(4 \mathrm{~A})$ & 5426 & 3314 & 6483 & 29 \\
\hline $\mathrm{H}(4 \mathrm{~B})$ & 4470 & 2960 & 6336 & 29 \\
\hline $\mathrm{H}(6)$ & 4906 & 820 & 6788 & 30 \\
\hline $\mathrm{H}(7)$ & 5137 & -509 & 8075 & 33 \\
\hline $\mathrm{H}(8)$ & 5906 & -338 & 9224 & 33 \\
\hline $\mathrm{H}(9)$ & 6453 & 1108 & 9069 & 32 \\
\hline $\mathrm{H}(10)$ & 6167 & 2488 & 7802 & 27 \\
\hline $\mathrm{H}(11)$ & 7569 & 4740 & 1492 & 15 \\
\hline $\mathrm{H}(12 \mathrm{~A})$ & 9797 & 3549 & 1845 & 18 \\
\hline $\mathrm{H}(12 \mathrm{~B})$ & 9522 & 3753 & 844 & 18 \\
\hline $\mathrm{H}(13 \mathrm{~A})$ & 10238 & 5072 & 968 & 22 \\
\hline $\mathrm{H}(13 \mathrm{~B})$ & 8961 & 5661 & 806 & 22 \\
\hline $\mathrm{H}(14 \mathrm{~A})$ & 9081 & 6419 & 2013 & 25 \\
\hline $\mathrm{H}(14 \mathrm{~B})$ & 9525 & 5232 & 2581 & 25 \\
\hline $\mathrm{H}(15 \mathrm{~A})$ & 7263 & 6322 & 2235 & 23 \\
\hline $\mathrm{H}(15 \mathrm{~B})$ & 7547 & 6115 & 3233 & 23 \\
\hline $\mathrm{H}(16 \mathrm{~A})$ & 6800 & 4816 & 3110 & 19 \\
\hline $\mathrm{H}(16 \mathrm{~B})$ & 8071 & 4213 & 3285 & 19 \\
\hline $\mathrm{H}(17)$ & 8343 & 1180 & 2780 & 16 \\
\hline $\mathrm{H}(18 \mathrm{~A})$ & 9266 & 2494 & 3295 & 20 \\
\hline $\mathrm{H}(18 \mathrm{~B})$ & 8097 & 2313 & 3873 & 20 \\
\hline $\mathrm{H}(19 \mathrm{~A})$ & 9665 & 1106 & 4548 & 25 \\
\hline $\mathrm{H}(19 \mathrm{~B})$ & 9121 & 427 & 4213 & 25 \\
\hline $\mathrm{H}(20 \mathrm{~A})$ & 11202 & 804 & 3208 & 26 \\
\hline $\mathrm{H}(20 \mathrm{~B})$ & 11141 & -355 & 3715 & 26 \\
\hline $\mathrm{H}(21 \mathrm{~A})$ & 10195 & -407 & 2643 & 26 \\
\hline $\mathrm{H}(21 \mathrm{~B})$ & 11366 & -226 & 2075 & 26 \\
\hline $\mathrm{H}(22 \mathrm{~A})$ & 9814 & 980 & 1385 & 21 \\
\hline $\mathrm{H}(22 \mathrm{~B})$ & 10340 & 1662 & 1737 & 21 \\
\hline $\mathrm{H}(23)$ & 9004 & 2464 & 583 & 15 \\
\hline $\mathrm{H}(24 \mathrm{~A})$ & 6806 & 3866 & 223 & 20 \\
\hline
\end{tabular}




\begin{tabular}{|c|c|c|c|c|}
\hline $\mathrm{H}(24 \mathrm{~B})$ & 7819 & 4280 & 149 & 20 \\
\hline $\mathrm{H}(25 \mathrm{~A})$ & 7829 & 3988 & -1355 & 24 \\
\hline $\mathrm{H}(25 \mathrm{~B})$ & 9018 & 3216 & -1078 & 24 \\
\hline $\mathrm{H}(26 \mathrm{~A})$ & 8422 & 2159 & -1694 & 25 \\
\hline $\mathrm{H}(26 \mathrm{~B})$ & 7179 & 2534 & -986 & 25 \\
\hline $\mathrm{H}(27 \mathrm{~A})$ & 9299 & 1144 & -469 & 23 \\
\hline $\mathrm{H}(27 \mathrm{~B})$ & 8265 & 771 & -413 & 23 \\
\hline $\mathrm{H}(28 \mathrm{~A})$ & 8232 & 1054 & 1099 & 18 \\
\hline $\mathrm{H}(28 \mathrm{~B})$ & 7068 & 1869 & 798 & 18 \\
\hline H(29) & 2698 & 5284 & 2773 & 19 \\
\hline $\mathrm{H}(30 \mathrm{~A})$ & 1162 & 4638 & 3205 & 28 \\
\hline $\mathrm{H}(30 \mathrm{~B})$ & 1209 & 4452 & 4278 & 28 \\
\hline $\mathrm{H}(31 \mathrm{~A})$ & 646 & 6544 & 3160 & 34 \\
\hline $\mathrm{H}(31 \mathrm{~B})$ & -259 & 6154 & 3989 & 34 \\
\hline $\mathrm{H}(32 \mathrm{~A})$ & 342 & 7337 & 4499 & 35 \\
\hline $\mathrm{H}(32 \mathrm{~B})$ & 662 & 6161 & 5103 & 35 \\
\hline $\mathrm{H}(33 \mathrm{~A})$ & 2271 & 6681 & 4674 & 31 \\
\hline $\mathrm{H}(33 \mathrm{~B})$ & 2245 & 6886 & 3595 & 31 \\
\hline $\mathrm{H}(34 \mathrm{~A})$ & 2805 & 4786 & 4691 & 23 \\
\hline $\mathrm{H}(34 \mathrm{~B})$ & 3705 & 5190 & 3868 & 23 \\
\hline $\mathrm{H}(35)$ & 4060 & 1678 & 3878 & 26 \\
\hline $\mathrm{H}(36 \mathrm{~A})$ & 3914 & 2668 & 5000 & 30 \\
\hline $\mathrm{H}(36 \mathrm{~B})$ & 2540 & 3149 & 5184 & 30 \\
\hline $\mathrm{H}(37 \mathrm{~A})$ & 3959 & 921 & 5567 & 37 \\
\hline $\mathrm{H}(37 \mathrm{~B})$ & 3021 & 1700 & 6323 & 37 \\
\hline $\mathrm{H}(38 \mathrm{~A})$ & 1523 & 1635 & 5872 & 39 \\
\hline $\mathrm{H}(38 \mathrm{~B})$ & 2389 & 402 & 6035 & 39 \\
\hline $\mathrm{H}(39 \mathrm{~A})$ & 1776 & 746 & 4623 & 31 \\
\hline $\mathrm{H}(39 \mathrm{~B})$ & 3147 & 334 & 4434 & 31 \\
\hline $\mathrm{H}(40 \mathrm{~A})$ & 2539 & 1818 & 3313 & 34 \\
\hline $\mathrm{H}(40 \mathrm{~B})$ & 1648 & 2563 & 4113 & 34 \\
\hline $\mathrm{H}(41)$ & 3871 & 2272 & 2081 & 18 \\
\hline $\mathrm{H}(42 \mathrm{~A})$ & 4136 & 4205 & 998 & 22 \\
\hline $\mathrm{H}(42 \mathrm{~B})$ & 5182 & 3172 & 1301 & 22 \\
\hline $\mathrm{H}(43 \mathrm{~A})$ & 5080 & 3114 & -234 & 24 \\
\hline $\mathrm{H}(43 \mathrm{~B})$ & 4940 & 2061 & 450 & 24 \\
\hline $\mathrm{H}(44 \mathrm{~A})$ & 3539 & 2683 & -392 & 25 \\
\hline $\mathrm{H}(44 \mathrm{~B})$ & 3122 & 3907 & -161 & 25 \\
\hline $\mathrm{H}(45 \mathrm{~A})$ & 2753 & 2111 & 1134 & 25 \\
\hline $\mathrm{H}(45 \mathrm{~B})$ & 1772 & 3182 & 793 & 25 \\
\hline
\end{tabular}




\begin{tabular}{lllll}
$\mathrm{H}(46 \mathrm{~A})$ & 1851 & 3255 & 2335 & 21 \\
$\mathrm{H}(46 \mathrm{~B})$ & 2095 & 4260 & 1633 & 21 \\
$\mathrm{H}(47 \mathrm{~A})$ & 2814 & 1528 & 8061 & 39 \\
$\mathrm{H}(47 \mathrm{~B})$ & 2881 & 311 & 8484 & 39 \\
\hline
\end{tabular}

\subsection{X-ray structure of Ru-vinyl-4}

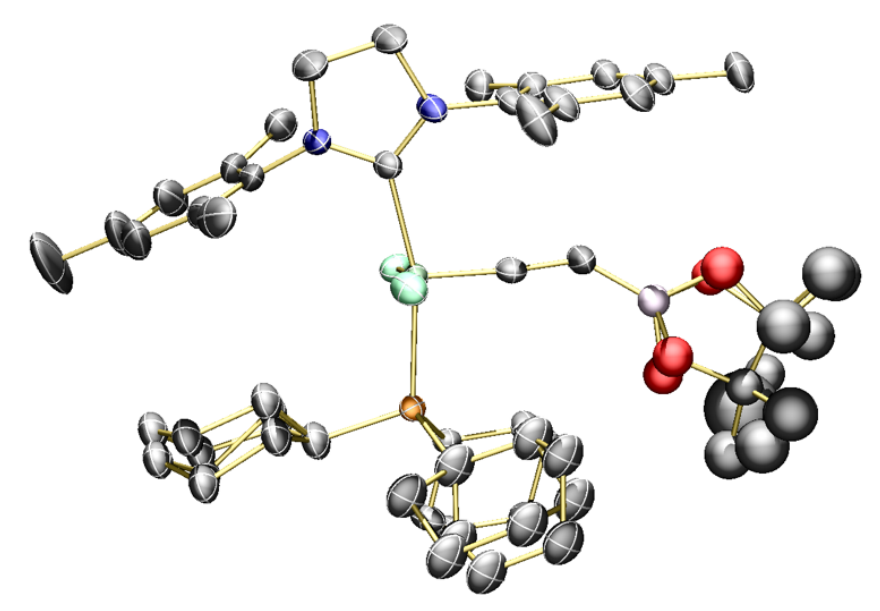

Table S17. Sample and crystal data for $\mathrm{C}_{47} \mathrm{H}_{72} \mathrm{BCl}_{2} \mathrm{~N}_{2} \mathrm{O}_{2} \mathrm{PRu}$

\begin{tabular}{lll}
\hline Identification code & ahsg200908 & \\
Chemical formula & $\mathrm{C}_{47} \mathrm{H}_{72} \mathrm{BCl}_{2} \mathrm{~N}_{2} \mathrm{O}_{2} \mathrm{PRu}$ & \\
Formula weight & $910.81 \mathrm{~g} / \mathrm{mol}$ & \\
Temperature & $120(2) \mathrm{K}$ & \\
Wavelength & $0.71073 \AA$ & \\
Crystal size & $0.150 \times 0.120 \times 0.100 \mathrm{~mm}$ & \\
Crystal system & Tetragonal & \\
Space group & $\mathrm{I} 41 / \mathrm{a}$ & $\alpha=90^{\circ}$ \\
Unit cell dimensions & $\mathrm{a}=39.3935(13) \AA$ & $\beta=90^{\circ}$ \\
& $\mathrm{b}=39.3935(13) \AA$ & $\gamma=90^{\circ}$ \\
& $\mathrm{c}=11.8614(7) \AA$ & \\
Volume & $18407.1(16) \AA^{3}$ & \\
$Z$ & 16 & \\
Density (calculated) & $1.315 \mathrm{~g} / \mathrm{cm}^{3}$ & \\
Absorption coefficient & $0.530 \mathrm{~mm}^{-1}$ & \\
F(000) & 7712 & \\
\hline
\end{tabular}

Table S18. Data collection and structure refinement for $\mathrm{C}_{47} \mathrm{H}_{72} \mathrm{BCl}_{2} \mathrm{~N}_{2} \mathrm{O}_{2} \mathrm{PRu}$

Theta range for data collection 2.068 to $27.914^{\circ}$

Index ranges $\quad-51<=\mathrm{h}<=51,-51<=\mathrm{k}<=51,-15<=1<=15$ 


\begin{tabular}{|c|c|}
\hline Reflections collected & 137608 \\
\hline Independent reflections & $11008[\mathrm{R}(\mathrm{int})=0.1083]$ \\
\hline Completeness to theta $=25.242$ & $100.0 \%$ \\
\hline Absorption correction & Semi-empirical from equivalents \\
\hline Max. and min. transmission & 0.7456 and 0.7142 \\
\hline Refinement method & Full-matrix least-squares on $\mathrm{F}^{2}$ \\
\hline Data / restraints / parameters & $11008 / 5 / 492$ \\
\hline Goodness-of-fit on $\mathrm{F}^{2}$ & 1.022 \\
\hline Final $\mathrm{R}$ indices & $\mathrm{R} 1=0.0513, \mathrm{wR} 2=0.10 \mathrm{t}$ \\
\hline & $\mathrm{R} 1=0.0817, \mathrm{wR} 2=0.12 \mathrm{~s}$ \\
\hline
\end{tabular}

\section{Table S19. Atomic coordinates $\left(\mathrm{x}^{10^{4}}\right)$ and equivalent isotropic atomic displacement parameters} $\left(\AA^{2}\right)$ for $\mathrm{C}_{47} \mathrm{H}_{72} \mathrm{BCl}_{2} \mathrm{~N}_{2} \mathrm{O}_{2} \mathrm{PRu}$

$\mathrm{U}(\mathrm{eq})$ is defined as one third of the trace of the orthogonalized $\mathrm{U}_{\mathrm{ij}}$ tensor.

\begin{tabular}{lllll}
\hline & \multicolumn{1}{c}{$\mathbf{x} / \mathbf{a}$} & $\mathbf{y} / \mathbf{b}$ & $\mathbf{z} / \mathbf{c}$ & $\mathbf{U}(\mathbf{q})$ \\
\hline $\mathrm{C}(1)$ & $6208(1)$ & $1729(1)$ & $4171(3)$ & $31(1)$ \\
$\mathrm{C}(2)$ & $6471(1)$ & $2218(1)$ & $4909(5)$ & $53(1)$ \\
$\mathrm{C}(3)$ & $6268(1)$ & $2318(1)$ & $3902(4)$ & $49(1)$ \\
$\mathrm{C}(4)$ & $6467(1)$ & $1689(1)$ & $6086(4)$ & $43(1)$ \\
$\mathrm{C}(5)$ & $6802(1)$ & $1583(1)$ & $6270(4)$ & $53(1)$ \\
$\mathrm{C}(6)$ & $6877(2)$ & $1451(2)$ & $7334(5)$ & $79(2)$ \\
$\mathrm{C}(7)$ & $6641(2)$ & $1425(2)$ & $8174(5)$ & $90(2)$ \\
$\mathrm{C}(8)$ & $6314(2)$ & $1539(1)$ & $7975(4)$ & $72(2)$ \\
$\mathrm{C}(9)$ & $6225(1)$ & $1679(1)$ & $6939(4)$ & $49(1)$ \\
$\mathrm{C}(10)$ & $7072(1)$ & $1622(1)$ & $5391(5)$ & $65(2)$ \\
$\mathrm{C}(11)$ & $6736(3)$ & $1282(2)$ & $9329(6)$ & $155(5)$ \\
$\mathrm{C}(12)$ & $5879(1)$ & $1833(1)$ & $6787(4)$ & $50(1)$ \\
$\mathrm{C}(13)$ & $5911(1)$ & $1997(1)$ & $2542(4)$ & $34(1)$ \\
$\mathrm{C}(14)$ & $5565(1)$ & $2013(1)$ & $2792(3)$ & $33(1)$ \\
$\mathrm{C}(15)$ & $5340(1)$ & $2042(1)$ & $1891(3)$ & $35(1)$ \\
$\mathrm{C}(16)$ & $5448(1)$ & $2048(1)$ & $788(4)$ & $38(1)$ \\
$\mathrm{C}(17)$ & $5794(1)$ & $2037(1)$ & $577(4)$ & $43(1)$ \\
$\mathrm{C}(18)$ & $6031(1)$ & $2015(1)$ & $1446(4)$ & $42(1)$ \\
$\mathrm{C}(19)$ & $5438(1)$ & $1982(1)$ & $3981(3)$ & $40(1)$ \\
& & &
\end{tabular}




\begin{tabular}{|c|c|c|c|c|}
\hline & $\mathbf{x} / \mathbf{a}$ & $\mathbf{y} / \mathbf{b}$ & $\mathbf{z} / \mathbf{c}$ & $\mathrm{U}(\mathbf{e q})$ \\
\hline$C(20)$ & $5196(1)$ & $2053(1)$ & $-165(4)$ & $49(1)$ \\
\hline $\mathrm{C}(21)$ & $6405(1)$ & 2001(2) & $1195(4)$ & $60(1)$ \\
\hline$C(22)$ & $5895(1)$ & $1204(1)$ & $2687(3)$ & $32(1)$ \\
\hline$C(23)$ & $5672(1)$ & $1225(1)$ & $1860(4)$ & $50(1)$ \\
\hline$C(24)$ & $5221(2)$ & $901(2)$ & $-690(6)$ & $49(1)$ \\
\hline$C(25)$ & $5527(2)$ & $650(2)$ & $-627(6)$ & $49(1)$ \\
\hline$C(26)$ & $4889(3)$ & $755(3)$ & $-763(10)$ & $98(2)$ \\
\hline$C(27)$ & $5280(3)$ & 1141(3) & $-1700(9)$ & $98(2)$ \\
\hline$C(28)$ & $5718(3)$ & $589(3)$ & $-1676(10)$ & $98(2)$ \\
\hline$C(29)$ & $5418(3)$ & 291(3) & $-177(10)$ & $98(2)$ \\
\hline$C(24 B)$ & $5341(4)$ & $592(4)$ & $-206(12)$ & $49(1)$ \\
\hline$C(25 B)$ & $5353(4)$ & $900(4)$ & $-955(13)$ & $49(1)$ \\
\hline$C(26 B)$ & $4964(5)$ & $544(6)$ & $-40(20)$ & $97(4)$ \\
\hline$C(27 B)$ & $5604(6)$ & $400(6)$ & $-960(20)$ & $97(4)$ \\
\hline $\mathrm{C}(28 \mathrm{~B})$ & $5697(5)$ & $991(6)$ & $-1570(20)$ & $97(4)$ \\
\hline$C(29 B)$ & $5026(6)$ & 1071(6) & $-1350(20)$ & $97(4)$ \\
\hline$C(30)$ & $5842(1)$ & $341(1)$ & 4081(3) & $27(1)$ \\
\hline$C(31)$ & $5595(1)$ & $371(1)$ & $3101(4)$ & $39(1)$ \\
\hline$C(32)$ & $5254(1)$ & $205(1)$ & $3379(4)$ & $44(1)$ \\
\hline$C(33)$ & $5299(1)$ & $-165(1)$ & $3725(4)$ & $42(1)$ \\
\hline$C(34)$ & $5550(1)$ & $-193(1)$ & $4706(4)$ & $39(1)$ \\
\hline$C(35)$ & $5891(1)$ & $-34(1)$ & $4400(4)$ & $37(1)$ \\
\hline$C(36)$ & $6472(1)$ & $412(1)$ & $2711(4)$ & $52(1)$ \\
\hline$C(37)$ & $6351(1)$ & $541(1)$ & $1577(4)$ & $52(1)$ \\
\hline $\mathrm{C}(38)$ & $6510(2)$ & $323(2)$ & $623(7)$ & $52(1)$ \\
\hline C(39) & $6889(2)$ & $305(2)$ & $723(7)$ & $52(1)$ \\
\hline$C(40)$ & $7003(2)$ & $155(2)$ & $1868(7)$ & $52(1)$ \\
\hline $\mathrm{C}(41)$ & $6851(2)$ & $360(2)$ & $2820(7)$ & $52(1)$ \\
\hline$C(38 B)$ & $6611(3)$ & $547(3)$ & $598(10)$ & $52(1)$ \\
\hline$C(39 B)$ & $6692(3)$ & $180(3)$ & $496(10)$ & $52(1)$ \\
\hline $\mathrm{C}(40 \mathrm{~B})$ & $6828(3)$ & $46(3)$ & $1550(10)$ & $52(1)$ \\
\hline$C(41 B)$ & $6562(3)$ & $87(3)$ & $2561(10)$ & $52(1)$ \\
\hline
\end{tabular}




\begin{tabular}{|c|c|c|c|c|}
\hline & $\mathbf{x} / \mathbf{a}$ & $\mathbf{y} / \mathbf{b}$ & $\mathbf{z} / \mathbf{c}$ & $\mathrm{U}(\mathrm{eq})$ \\
\hline $\mathrm{C}(42)$ & $6493(1)$ & $471(1)$ & $5131(4)$ & $43(1)$ \\
\hline$C(43)$ & $6785(2)$ & $719(2)$ & $5349(6)$ & $43(1)$ \\
\hline$C(44)$ & $7026(2)$ & $583(2)$ & $6274(6)$ & $43(1)$ \\
\hline$C(45)$ & $6828(2)$ & $543(2)$ & $7375(6)$ & $43(1)$ \\
\hline$C(46)$ & $6535(3)$ & $370(2)$ & $7204(9)$ & $43(1)$ \\
\hline $\mathrm{C}(47)$ & $6290(2)$ & $457(2)$ & $6281(7)$ & $43(1)$ \\
\hline$C(43 B)$ & $6874(3)$ & $583(3)$ & $5001(10)$ & $43(1)$ \\
\hline$C(44 B)$ & $7082(3)$ & $391(3)$ & $5899(9)$ & $43(1)$ \\
\hline$C(45 B)$ & $6938(3)$ & $439(3)$ & $7057(9)$ & $43(1)$ \\
\hline$C(46 B)$ & $6541(4)$ & $274(3)$ & 7113(13) & $43(1)$ \\
\hline$C(47 B)$ & $6355(3)$ & $556(3)$ & $6213(11)$ & $43(1)$ \\
\hline $\mathrm{B}(1)$ & $5562(1)$ & $1028(1)$ & $819(4)$ & $37(1)$ \\
\hline $\mathrm{N}(1)$ & $6382(1)$ & $1855(1)$ & $5049(3)$ & $37(1)$ \\
\hline $\mathrm{N}(2)$ & $6148(1)$ & 1989(1) & $3461(3)$ & $35(1)$ \\
\hline $\mathrm{O}(1)$ & $5242(1)$ & $1088(1)$ & $363(4)$ & $56(1)$ \\
\hline $\mathrm{O}(2)$ & $5740(1)$ & $813(1)$ & $224(4)$ & $56(1)$ \\
\hline $\mathrm{O}(1 \mathrm{~B})$ & $5533(4)$ & $686(3)$ & $756(10)$ & $83(4)$ \\
\hline $\mathrm{O}(2 \mathrm{~B})$ & $5477(4)$ & $1168(3)$ & $-205(10)$ & $83(4)$ \\
\hline $\mathrm{P}(1)$ & $6233(1)$ & $596(1)$ & $3895(1)$ & $25(1)$ \\
\hline $\mathrm{Cl}(1)$ & $5697(1)$ & $1130(1)$ & $5149(1)$ & $35(1)$ \\
\hline $\mathrm{Cl}(2)$ & $6683(1)$ & $1268(1)$ & $2936(1)$ & $38(1)$ \\
\hline $\mathrm{Ru}(1)$ & $6156(1)$ & $1203(1)$ & $3878(1)$ & $25(1)$ \\
\hline
\end{tabular}

Table S20. Bond lengths ( $\AA$ ) for $\mathrm{C}_{47} \mathrm{H}_{72} \mathrm{BCl}_{2} \mathrm{~N}_{2} \mathrm{O}_{2} \mathrm{PRu}$

\begin{tabular}{llll}
\hline $\mathrm{C}(1)-\mathrm{N}(1)$ & $1.343(5)$ & $\mathrm{C}(30)-\mathrm{C}(31)$ & $1.520(5)$ \\
$\mathrm{C}(1)-\mathrm{N}(2)$ & $1.347(5)$ & $\mathrm{C}(30)-\mathrm{C}(35)$ & $1.539(5)$ \\
$\mathrm{C}(1)-\mathrm{Ru}(1)$ & $2.113(4)$ & $\mathrm{C}(30)-\mathrm{P}(1)$ & $1.852(4)$ \\
$\mathrm{C}(2)-\mathrm{N}(1)$ & $1.482(5)$ & $\mathrm{C}(30)-\mathrm{H}(30)$ & 1 \\
$\mathrm{C}(2)-\mathrm{C}(3)$ & $1.491(6)$ & $\mathrm{C}(31)-\mathrm{C}(32)$ & $1.529(5)$ \\
$\mathrm{C}(2)-\mathrm{H}(2 \mathrm{~A})$ & 0.99 & $\mathrm{C}(31)-\mathrm{H}(31 \mathrm{~A})$ & 0.99 \\
$\mathrm{C}(2)-\mathrm{H}(2 \mathrm{~B})$ & 0.99 & $\mathrm{C}(31)-\mathrm{H}(31 \mathrm{~B})$ & 0.99 \\
$\mathrm{C}(3)-\mathrm{N}(2)$ & $1.476(5)$ & $\mathrm{C}(32)-\mathrm{C}(33)$ & $1.523(6)$
\end{tabular}




\begin{tabular}{|c|c|c|c|}
\hline $\mathrm{C}(3)-\mathrm{H}(3 \mathrm{~A})$ & 0.99 & $\mathrm{C}(32)-\mathrm{H}(32 \mathrm{~A})$ & 0.99 \\
\hline $\mathrm{C}(3)-\mathrm{H}(3 \mathrm{~B})$ & 0.99 & $\mathrm{C}(32)-\mathrm{H}(32 \mathrm{~B})$ & 0.99 \\
\hline $\mathrm{C}(4)-\mathrm{C}(9)$ & $1.392(7)$ & $C(33)-C(34)$ & $1.528(6)$ \\
\hline$C(4)-C(5)$ & $1.401(6)$ & $\mathrm{C}(33)-\mathrm{H}(33 \mathrm{~A})$ & 0.99 \\
\hline $\mathrm{C}(4)-\mathrm{N}(1)$ & $1.434(5)$ & $\mathrm{C}(33)-\mathrm{H}(33 \mathrm{~B})$ & 0.99 \\
\hline$C(5)-C(6)$ & $1.397(7)$ & $C(34)-C(35)$ & $1.526(5)$ \\
\hline$C(5)-C(10)$ & $1.497(8)$ & $\mathrm{C}(34)-\mathrm{H}(34 \mathrm{~A})$ & 0.99 \\
\hline$C(6)-C(7)$ & $1.367(10)$ & $\mathrm{C}(34)-\mathrm{H}(34 \mathrm{~B})$ & 0.99 \\
\hline $\mathrm{C}(6)-\mathrm{H}(6)$ & 0.95 & $\mathrm{C}(35)-\mathrm{H}(35 \mathrm{~A})$ & 0.99 \\
\hline $\mathrm{C}(7)-\mathrm{C}(8)$ & $1.384(8)$ & $\mathrm{C}(35)-\mathrm{H}(35 \mathrm{~B})$ & 0.99 \\
\hline $\mathrm{C}(7)-\mathrm{C}(11)$ & $1.526(8)$ & $\mathrm{C}(36)-\mathrm{C}(41 \mathrm{~B})$ & $1.338(13)$ \\
\hline $\mathrm{C}(8)-\mathrm{C}(9)$ & $1.393(6)$ & $C(36)-C(41)$ & $1.514(9)$ \\
\hline $\mathrm{C}(8)-\mathrm{H}(8)$ & 0.95 & $C(36)-C(37)$ & $1.516(7)$ \\
\hline $\mathrm{C}(9)-\mathrm{C}(12)$ & $1.504(6)$ & $\mathrm{C}(36)-\mathrm{P}(1)$ & $1.841(4)$ \\
\hline $\mathrm{C}(10)-\mathrm{H}(10 \mathrm{~A})$ & 0.98 & $\mathrm{C}(36)-\mathrm{H}(36)$ & 1 \\
\hline $\mathrm{C}(10)-\mathrm{H}(10 \mathrm{~B})$ & 0.98 & $\mathrm{C}(36)-\mathrm{H}(36 \mathrm{~B})$ & 1 \\
\hline $\mathrm{C}(10)-\mathrm{H}(10 \mathrm{C})$ & 0.98 & $\mathrm{C}(37)-\mathrm{C}(38 \mathrm{~B})$ & $1.548(12)$ \\
\hline $\mathrm{C}(11)-\mathrm{H}(11 \mathrm{~A})$ & 0.98 & $\mathrm{C}(37)-\mathrm{C}(38)$ & $1.553(9)$ \\
\hline $\mathrm{C}(11)-\mathrm{H}(11 \mathrm{~B})$ & 0.98 & $\mathrm{C}(37)-\mathrm{H}(37 \mathrm{~A})$ & 0.99 \\
\hline $\mathrm{C}(11)-\mathrm{H}(11 \mathrm{C})$ & 0.98 & $\mathrm{C}(37)-\mathrm{H}(37 \mathrm{~B})$ & 0.99 \\
\hline $\mathrm{C}(12)-\mathrm{H}(12 \mathrm{~A})$ & 0.98 & $\mathrm{C}(37)-\mathrm{H}(37 \mathrm{C})$ & 0.99 \\
\hline $\mathrm{C}(12)-\mathrm{H}(12 \mathrm{~B})$ & 0.98 & $\mathrm{C}(37)-\mathrm{H}(37 \mathrm{D})$ & 0.99 \\
\hline $\mathrm{C}(12)-\mathrm{H}(12 \mathrm{C})$ & 0.98 & $\mathrm{C}(38)-\mathrm{C}(39)$ & $1.500(11)$ \\
\hline $\mathrm{C}(13)-\mathrm{C}(18)$ & $1.385(6)$ & $\mathrm{C}(38)-\mathrm{H}(38 \mathrm{~A})$ & 0.99 \\
\hline$C(13)-C(14)$ & $1.398(5)$ & $\mathrm{C}(38)-\mathrm{H}(38 \mathrm{~B})$ & 0.99 \\
\hline $\mathrm{C}(13)-\mathrm{N}(2)$ & $1.435(5)$ & $C(39)-C(40)$ & $1.547(12)$ \\
\hline $\mathrm{C}(14)-\mathrm{C}(15)$ & $1.391(5)$ & $\mathrm{C}(39)-\mathrm{H}(39 \mathrm{~A})$ & 0.99 \\
\hline $\mathrm{C}(14)-\mathrm{C}(19)$ & $1.501(6)$ & $\mathrm{C}(39)-\mathrm{H}(39 \mathrm{~B})$ & 0.99 \\
\hline $\mathrm{C}(15)-\mathrm{C}(16)$ & $1.375(6)$ & $\mathrm{C}(40)-\mathrm{C}(41)$ & $1.510(10)$ \\
\hline $\mathrm{C}(15)-\mathrm{H}(15)$ & 0.95 & $\mathrm{C}(40)-\mathrm{H}(40 \mathrm{~A})$ & 0.99 \\
\hline$C(16)-C(17)$ & $1.387(6)$ & $\mathrm{C}(40)-\mathrm{H}(40 \mathrm{~B})$ & 0.99 \\
\hline$C(16)-C(20)$ & $1.505(5)$ & $\mathrm{C}(41)-\mathrm{H}(41 \mathrm{~A})$ & 0.99 \\
\hline $\mathrm{C}(17)-\mathrm{C}(18)$ & $1.394(6)$ & $\mathrm{C}(41)-\mathrm{H}(41 \mathrm{~B})$ & 0.99 \\
\hline $\mathrm{C}(17)-\mathrm{H}(17)$ & 0.95 & $\mathrm{C}(38 \mathrm{~B})-\mathrm{C}(39 \mathrm{~B})$ & $1.485(18)$ \\
\hline
\end{tabular}




\begin{tabular}{|c|c|c|c|}
\hline $\mathrm{C}(18)-\mathrm{C}(21)$ & $1.504(6)$ & $\mathrm{C}(38 \mathrm{~B})-\mathrm{H}(38 \mathrm{C})$ & 0.99 \\
\hline C(19)-H(19A) & 0.98 & $\mathrm{C}(38 \mathrm{~B})-\mathrm{H}(38 \mathrm{D})$ & 0.99 \\
\hline $\mathrm{C}(19)-\mathrm{H}(19 \mathrm{~B})$ & 0.98 & $C(39 B)-C(40 B)$ & $1.458(18)$ \\
\hline C(19)-H(19C) & 0.98 & C(39B)-H(39C) & 0.99 \\
\hline $\mathrm{C}(20)-\mathrm{H}(20 \mathrm{~A})$ & 0.98 & C(39B)-H(39D) & 0.99 \\
\hline $\mathrm{C}(20)-\mathrm{H}(20 \mathrm{~B})$ & 0.98 & $\mathrm{C}(40 \mathrm{~B})-\mathrm{C}(41 \mathrm{~B})$ & $1.601(15)$ \\
\hline $\mathrm{C}(20)-\mathrm{H}(20 \mathrm{C})$ & 0.98 & $\mathrm{C}(40 \mathrm{~B})-\mathrm{H}(40 \mathrm{C})$ & 0.99 \\
\hline $\mathrm{C}(21)-\mathrm{H}(21 \mathrm{~A})$ & 0.98 & $\mathrm{C}(40 \mathrm{~B})-\mathrm{H}(40 \mathrm{D})$ & 0.99 \\
\hline $\mathrm{C}(21)-\mathrm{H}(21 \mathrm{~B})$ & 0.98 & $\mathrm{C}(41 \mathrm{~B})-\mathrm{H}(41 \mathrm{C})$ & 0.99 \\
\hline $\mathrm{C}(21)-\mathrm{H}(21 \mathrm{C})$ & 0.98 & $\mathrm{C}(41 \mathrm{~B})-\mathrm{H}(41 \mathrm{D})$ & 0.99 \\
\hline$C(22)-C(23)$ & $1.319(5)$ & $\mathrm{C}(42)-\mathrm{C}(47 \mathrm{~B})$ & $1.434(14)$ \\
\hline$C(22)-R u(1)$ & $1.748(4)$ & $C(42)-C(43)$ & $1.531(8)$ \\
\hline $\mathrm{C}(23)-\mathrm{B}(1)$ & $1.523(6)$ & $\mathrm{C}(42)-\mathrm{C}(43 \mathrm{~B})$ & $1.571(12)$ \\
\hline $\mathrm{C}(23)-\mathrm{H}(23)$ & 0.95 & $C(42)-C(47)$ & $1.582(9)$ \\
\hline$C(24)-C(26)$ & $1.433(13)$ & $\mathrm{C}(42)-\mathrm{P}(1)$ & $1.856(4)$ \\
\hline $\mathrm{C}(24)-\mathrm{O}(1)$ & $1.452(8)$ & $\mathrm{C}(42)-\mathrm{H}(42)$ & 1 \\
\hline$C(24)-C(27)$ & $1.542(12)$ & $\mathrm{C}(42)-\mathrm{H}(42 \mathrm{~B})$ & 1 \\
\hline$C(24)-C(25)$ & $1.559(9)$ & $C(43)-C(44)$ & $1.547(9)$ \\
\hline $\mathrm{C}(25)-\mathrm{O}(2)$ & $1.461(7)$ & $\mathrm{C}(43)-\mathrm{H}(43 \mathrm{~A})$ & 0.99 \\
\hline$C(25)-C(28)$ & $1.473(12)$ & $\mathrm{C}(43)-\mathrm{H}(43 \mathrm{~B})$ & 0.99 \\
\hline$C(25)-C(29)$ & $1.572(12)$ & $C(44)-C(45)$ & $1.530(10)$ \\
\hline $\mathrm{C}(26)-\mathrm{H}(26 \mathrm{~A})$ & 0.98 & $\mathrm{C}(44)-\mathrm{H}(44 \mathrm{~A})$ & 0.99 \\
\hline $\mathrm{C}(26)-\mathrm{H}(26 \mathrm{~B})$ & 0.98 & $\mathrm{C}(44)-\mathrm{H}(44 \mathrm{~B})$ & 0.99 \\
\hline $\mathrm{C}(26)-\mathrm{H}(26 \mathrm{C})$ & 0.98 & $C(45)-C(46)$ & $1.354(12)$ \\
\hline $\mathrm{C}(27)-\mathrm{H}(27 \mathrm{~A})$ & 0.98 & $\mathrm{C}(45)-\mathrm{H}(45 \mathrm{~A})$ & 0.99 \\
\hline $\mathrm{C}(27)-\mathrm{H}(27 \mathrm{~B})$ & 0.98 & $\mathrm{C}(45)-\mathrm{H}(45 \mathrm{~B})$ & 0.99 \\
\hline $\mathrm{C}(27)-\mathrm{H}(27 \mathrm{C})$ & 0.98 & $C(46)-C(47)$ & $1.498(13)$ \\
\hline $\mathrm{C}(28)-\mathrm{H}(28 \mathrm{~A})$ & 0.98 & $\mathrm{C}(46)-\mathrm{H}(46 \mathrm{~A})$ & 0.99 \\
\hline $\mathrm{C}(28)-\mathrm{H}(28 \mathrm{~B})$ & 0.98 & $\mathrm{C}(46)-\mathrm{H}(46 \mathrm{~B})$ & 0.99 \\
\hline $\mathrm{C}(28)-\mathrm{H}(28 \mathrm{C})$ & 0.98 & $\mathrm{C}(47)-\mathrm{H}(47 \mathrm{~A})$ & 0.99 \\
\hline $\mathrm{C}(29)-\mathrm{H}(29 \mathrm{~A})$ & 0.98 & $\mathrm{C}(47)-\mathrm{H}(47 \mathrm{~B})$ & 0.99 \\
\hline C(29)-H(29B) & 0.98 & $\mathrm{C}(43 \mathrm{~B})-\mathrm{C}(44 \mathrm{~B})$ & $1.541(14)$ \\
\hline C(29)-H(29C) & 0.98 & $\mathrm{C}(43 \mathrm{~B})-\mathrm{H}(43 \mathrm{C})$ & 0.99 \\
\hline $\mathrm{C}(24 \mathrm{~B})-\mathrm{O}(1 \mathrm{~B})$ & $1.418(17)$ & $\mathrm{C}(43 \mathrm{~B})-\mathrm{H}(43 \mathrm{D})$ & 0.99 \\
\hline
\end{tabular}




\begin{tabular}{llll}
$\mathrm{C}(24 \mathrm{~B})-\mathrm{C}(25 \mathrm{~B})$ & $1.505(16)$ & $\mathrm{C}(44 \mathrm{~B})-\mathrm{C}(45 \mathrm{~B})$ & $1.498(16)$ \\
$\mathrm{C}(24 \mathrm{~B})-\mathrm{C}(26 \mathrm{~B})$ & $1.510(17)$ & $\mathrm{C}(44 \mathrm{~B})-\mathrm{H}(44 \mathrm{C})$ & 0.99 \\
$\mathrm{C}(24 \mathrm{~B})-\mathrm{C}(27 \mathrm{~B})$ & $1.564(16)$ & $\mathrm{C}(44 \mathrm{~B})-\mathrm{H}(44 \mathrm{D})$ & 0.99 \\
$\mathrm{C}(25 \mathrm{~B})-\mathrm{O}(2 \mathrm{~B})$ & $1.46(2)$ & $\mathrm{C}(45 \mathrm{~B})-\mathrm{C}(46 \mathrm{~B})$ & $1.695(19)$ \\
$\mathrm{C}(25 \mathrm{~B})-\mathrm{C}(29 \mathrm{~B})$ & $1.527(17)$ & $\mathrm{C}(45 \mathrm{~B})-\mathrm{H}(45 \mathrm{C})$ & 0.99 \\
$\mathrm{C}(25 \mathrm{~B})-\mathrm{C}(28 \mathrm{~B})$ & $1.578(17)$ & $\mathrm{C}(45 \mathrm{~B})-\mathrm{H}(45 \mathrm{D})$ & 0.99 \\
$\mathrm{C}(26 \mathrm{~B})-\mathrm{H}(26 \mathrm{D})$ & 0.98 & $\mathrm{C}(46 \mathrm{~B})-\mathrm{C}(47 \mathrm{~B})$ & $1.705(19)$ \\
$\mathrm{C}(26 \mathrm{~B})-\mathrm{H}(26 \mathrm{E})$ & 0.98 & $\mathrm{C}(46 \mathrm{~B})-\mathrm{H}(46 \mathrm{C})$ & 0.99 \\
$\mathrm{C}(26 \mathrm{~B})-\mathrm{H}(26 \mathrm{~F})$ & 0.98 & $\mathrm{C}(46 \mathrm{~B})-\mathrm{H}(46 \mathrm{D})$ & 0.99 \\
$\mathrm{C}(27 \mathrm{~B})-\mathrm{H}(27 \mathrm{D})$ & 0.98 & $\mathrm{C}(47 \mathrm{~B})-\mathrm{H}(47 \mathrm{C})$ & 0.99 \\
$\mathrm{C}(27 \mathrm{~B})-\mathrm{H}(27 \mathrm{E})$ & 0.98 & $\mathrm{C}(47 \mathrm{~B})-\mathrm{H}(47 \mathrm{D})$ & 0.99 \\
$\mathrm{C}(27 \mathrm{~B})-\mathrm{H}(27 \mathrm{~F})$ & 0.98 & $\mathrm{~B}(1)-\mathrm{O}(2)$ & $1.305(7)$ \\
$\mathrm{C}(28 \mathrm{~B})-\mathrm{H}(28 \mathrm{D})$ & 0.98 & $\mathrm{~B}(1)-\mathrm{O}(1 \mathrm{~B})$ & $1.354(13)$ \\
$\mathrm{C}(28 \mathrm{~B})-\mathrm{H}(28 \mathrm{E})$ & 0.98 & $\mathrm{~B}(1)-\mathrm{O}(2 \mathrm{~B})$ & $1.375(12)$ \\
$\mathrm{C}(28 \mathrm{~B})-\mathrm{H}(28 \mathrm{~F})$ & 0.98 & $\mathrm{~B}(1)-\mathrm{O}(1)$ & $1.391(6)$ \\
$\mathrm{C}(29 \mathrm{~B})-\mathrm{H}(29 \mathrm{D})$ & 0.98 & $\mathrm{P}(1)-\mathrm{Ru}(1)$ & $2.4092(9)$ \\
$\mathrm{C}(29 \mathrm{~B})-\mathrm{H}(29 \mathrm{E})$ & 0.98 & $\mathrm{Cl}(1)-\mathrm{Ru}(1)$ & $2.3706(10)$ \\
$\mathrm{C}(29 \mathrm{~B})-\mathrm{H}(29 \mathrm{~F})$ & 0.98 & $\mathrm{Cl}(2)-\mathrm{Ru}(1)$ & $2.3714(10)$ \\
\hline
\end{tabular}

\section{Table S21. Bond angles $\left({ }^{\circ}\right)$ for $\mathrm{C}_{47} \mathrm{H}_{72} \mathrm{BCl}_{2} \mathrm{~N}_{2} \mathrm{O}_{2} \mathrm{PRu}$}

Symmetry transformations used to generate equivalent atoms: $\# 1-\mathrm{x}+1,-\mathrm{y}+1,-\mathrm{z}+1$

\begin{tabular}{llll}
\hline $\mathrm{N}(1)-\mathrm{C}(1)-\mathrm{N}(2)$ & $107.1(3)$ & $\mathrm{C}(35)-\mathrm{C}(34)-\mathrm{C}(33)$ & $110.9(3)$ \\
$\mathrm{N}(1)-\mathrm{C}(1)-\mathrm{Ru}(1)$ & $122.6(3)$ & $\mathrm{C}(35)-\mathrm{C}(34)-\mathrm{H}(34 \mathrm{~A})$ & 109.5 \\
$\mathrm{~N}(2)-\mathrm{C}(1)-\mathrm{Ru}(1)$ & $128.8(3)$ & $\mathrm{C}(33)-\mathrm{C}(34)-\mathrm{H}(34 \mathrm{~A})$ & 109.5 \\
$\mathrm{~N}(1)-\mathrm{C}(2)-\mathrm{C}(3)$ & $102.5(3)$ & $\mathrm{C}(35)-\mathrm{C}(34)-\mathrm{H}(34 \mathrm{~B})$ & 109.5 \\
$\mathrm{~N}(1)-\mathrm{C}(2)-\mathrm{H}(2 \mathrm{~A})$ & 111.3 & $\mathrm{C}(33)-\mathrm{C}(34)-\mathrm{H}(34 \mathrm{~B})$ & 109.5 \\
$\mathrm{C}(3)-\mathrm{C}(2)-\mathrm{H}(2 \mathrm{~A})$ & 111.3 & $\mathrm{H}(34 \mathrm{~A})-\mathrm{C}(34)-\mathrm{H}(34 \mathrm{~B})$ & 108 \\
$\mathrm{~N}(1)-\mathrm{C}(2)-\mathrm{H}(2 \mathrm{~B})$ & 111.3 & $\mathrm{C}(34)-\mathrm{C}(35)-\mathrm{C}(30)$ & $110.0(3)$ \\
$\mathrm{C}(3)-\mathrm{C}(2)-\mathrm{H}(2 \mathrm{~B})$ & 111.3 & $\mathrm{C}(34)-\mathrm{C}(35)-\mathrm{H}(35 \mathrm{~A})$ & 109.7 \\
$\mathrm{H}(2 \mathrm{~A})-\mathrm{C}(2)-\mathrm{H}(2 \mathrm{~B})$ & 109.2 & $\mathrm{C}(30)-\mathrm{C}(35)-\mathrm{H}(35 \mathrm{~A})$ & 109.7 \\
$\mathrm{~N}(2)-\mathrm{C}(3)-\mathrm{C}(2)$ & $103.0(3)$ & $\mathrm{C}(34)-\mathrm{C}(35)-\mathrm{H}(35 \mathrm{~B})$ & 109.7 \\
$\mathrm{~N}(2)-\mathrm{C}(3)-\mathrm{H}(3 \mathrm{~A})$ & 111.2 & $\mathrm{C}(30)-\mathrm{C}(35)-\mathrm{H}(35 \mathrm{~B})$ & 109.7 \\
$\mathrm{C}(2)-\mathrm{C}(3)-\mathrm{H}(3 \mathrm{~A})$ & 111.2 & $\mathrm{H}(35 \mathrm{~A})-\mathrm{C}(35)-\mathrm{H}(35 \mathrm{~B})$ & 108.2 \\
$\mathrm{~N}(2)-\mathrm{C}(3)-\mathrm{H}(3 \mathrm{~B})$ & 111.2 & $\mathrm{C}(41 \mathrm{~B})-\mathrm{C}(36)-\mathrm{C}(37)$ & $106.7(7)$ \\
$\mathrm{C}(2)-\mathrm{C}(3)-\mathrm{H}(3 \mathrm{~B})$ & 111.2 & $\mathrm{C}(41)-\mathrm{C}(36)-\mathrm{C}(37)$ & $115.6(5)$ \\
$\mathrm{H}(3 \mathrm{~A})-\mathrm{C}(3)-\mathrm{H}(3 \mathrm{~B})$ & 109.1 & $\mathrm{C}(41 \mathrm{~B})-\mathrm{C}(36)-\mathrm{P}(1)$ & $127.8(6)$
\end{tabular}




\begin{tabular}{|c|c|c|c|}
\hline$C(9)-C(4)-C(5)$ & $121.6(4)$ & $\mathrm{C}(41)-\mathrm{C}(36)-\mathrm{P}(1)$ & $119.6(4)$ \\
\hline $\mathrm{C}(9)-\mathrm{C}(4)-\mathrm{N}(1)$ & $118.4(4)$ & $\mathrm{C}(37)-\mathrm{C}(36)-\mathrm{P}(1)$ & $112.5(3)$ \\
\hline $\mathrm{C}(5)-\mathrm{C}(4)-\mathrm{N}(1)$ & $119.4(5)$ & $\mathrm{C}(41)-\mathrm{C}(36)-\mathrm{H}(36)$ & 101.8 \\
\hline$C(6)-C(5)-C(4)$ & $116.8(5)$ & $\mathrm{C}(37)-\mathrm{C}(36)-\mathrm{H}(36)$ & 101.8 \\
\hline$C(6)-C(5)-C(10)$ & $121.2(5)$ & $\mathrm{P}(1)-\mathrm{C}(36)-\mathrm{H}(36)$ & 101.8 \\
\hline$C(4)-C(5)-C(10)$ & $122.0(5)$ & $\mathrm{C}(41 \mathrm{~B})-\mathrm{C}(36)-\mathrm{H}(36 \mathrm{~B})$ & 102 \\
\hline$C(7)-C(6)-C(5)$ & $123.0(5)$ & $\mathrm{C}(37)-\mathrm{C}(36)-\mathrm{H}(36 \mathrm{~B})$ & 102 \\
\hline$C(7)-C(6)-H(6)$ & 118.5 & $\mathrm{P}(1)-\mathrm{C}(36)-\mathrm{H}(36 \mathrm{~B})$ & 102 \\
\hline $\mathrm{C}(5)-\mathrm{C}(6)-\mathrm{H}(6)$ & 118.5 & $C(36)-C(37)-C(38 B)$ & $117.5(6)$ \\
\hline$C(6)-C(7)-C(8)$ & $118.9(5)$ & $\mathrm{C}(36)-\mathrm{C}(37)-\mathrm{C}(38)$ & $109.5(5)$ \\
\hline$C(6)-C(7)-C(11)$ & $121.1(6)$ & $\mathrm{C}(36)-\mathrm{C}(37)-\mathrm{H}(37 \mathrm{~A})$ & 109.8 \\
\hline $\mathrm{C}(8)-\mathrm{C}(7)-\mathrm{C}(11)$ & $120.0(7)$ & $\mathrm{C}(38)-\mathrm{C}(37)-\mathrm{H}(37 \mathrm{~A})$ & 109.8 \\
\hline$C(7)-C(8)-C(9)$ & $121.0(6)$ & $\mathrm{C}(36)-\mathrm{C}(37)-\mathrm{H}(37 \mathrm{~B})$ & 109.8 \\
\hline $\mathrm{C}(7)-\mathrm{C}(8)-\mathrm{H}(8)$ & 119.5 & $\mathrm{C}(38)-\mathrm{C}(37)-\mathrm{H}(37 \mathrm{~B})$ & 109.8 \\
\hline $\mathrm{C}(9)-\mathrm{C}(8)-\mathrm{H}(8)$ & 119.5 & $\mathrm{H}(37 \mathrm{~A})-\mathrm{C}(37)-\mathrm{H}(37 \mathrm{~B})$ & 108.2 \\
\hline$C(4)-C(9)-C(8)$ & $118.6(5)$ & $C(36)-C(37)-H(37 C)$ & 107.9 \\
\hline $\mathrm{C}(4)-\mathrm{C}(9)-\mathrm{C}(12)$ & $121.6(4)$ & $\mathrm{C}(38 \mathrm{~B})-\mathrm{C}(37)-\mathrm{H}(37 \mathrm{C})$ & 107.9 \\
\hline $\mathrm{C}(8)-\mathrm{C}(9)-\mathrm{C}(12)$ & $119.6(5)$ & $\mathrm{C}(36)-\mathrm{C}(37)-\mathrm{H}(37 \mathrm{D})$ & 107.9 \\
\hline $\mathrm{C}(5)-\mathrm{C}(10)-\mathrm{H}(10 \mathrm{~A})$ & 109.5 & $\mathrm{C}(38 \mathrm{~B})-\mathrm{C}(37)-\mathrm{H}(37 \mathrm{D})$ & 107.9 \\
\hline $\mathrm{C}(5)-\mathrm{C}(10)-\mathrm{H}(10 \mathrm{~B})$ & 109.5 & $\mathrm{H}(37 \mathrm{C})-\mathrm{C}(37)-\mathrm{H}(37 \mathrm{D})$ & 107.2 \\
\hline $\mathrm{H}(10 \mathrm{~A})-\mathrm{C}(10)-\mathrm{H}(10 \mathrm{~B})$ & 109.5 & $\mathrm{C}(39)-\mathrm{C}(38)-\mathrm{C}(37)$ & $111.7(6)$ \\
\hline $\mathrm{C}(5)-\mathrm{C}(10)-\mathrm{H}(10 \mathrm{C})$ & 109.5 & $\mathrm{C}(39)-\mathrm{C}(38)-\mathrm{H}(38 \mathrm{~A})$ & 109.3 \\
\hline $\mathrm{H}(10 \mathrm{~A})-\mathrm{C}(10)-\mathrm{H}(10 \mathrm{C})$ & 109.5 & $\mathrm{C}(37)-\mathrm{C}(38)-\mathrm{H}(38 \mathrm{~A})$ & 109.3 \\
\hline $\mathrm{H}(10 \mathrm{~B})-\mathrm{C}(10)-\mathrm{H}(10 \mathrm{C})$ & 109.5 & $\mathrm{C}(39)-\mathrm{C}(38)-\mathrm{H}(38 \mathrm{~B})$ & 109.3 \\
\hline $\mathrm{C}(7)-\mathrm{C}(11)-\mathrm{H}(11 \mathrm{~A})$ & 109.5 & $\mathrm{C}(37)-\mathrm{C}(38)-\mathrm{H}(38 \mathrm{~B})$ & 109.3 \\
\hline $\mathrm{C}(7)-\mathrm{C}(11)-\mathrm{H}(11 \mathrm{~B})$ & 109.5 & $\mathrm{H}(38 \mathrm{~A})-\mathrm{C}(38)-\mathrm{H}(38 \mathrm{~B})$ & 107.9 \\
\hline $\mathrm{H}(11 \mathrm{~A})-\mathrm{C}(11)-\mathrm{H}(11 \mathrm{~B})$ & 109.5 & $\mathrm{C}(38)-\mathrm{C}(39)-\mathrm{C}(40)$ & $112.1(7)$ \\
\hline $\mathrm{C}(7)-\mathrm{C}(11)-\mathrm{H}(11 \mathrm{C})$ & 109.5 & $\mathrm{C}(38)-\mathrm{C}(39)-\mathrm{H}(39 \mathrm{~A})$ & 109.2 \\
\hline $\mathrm{H}(11 \mathrm{~A})-\mathrm{C}(11)-\mathrm{H}(11 \mathrm{C})$ & 109.5 & $\mathrm{C}(40)-\mathrm{C}(39)-\mathrm{H}(39 \mathrm{~A})$ & 109.2 \\
\hline $\mathrm{H}(11 \mathrm{~B})-\mathrm{C}(11)-\mathrm{H}(11 \mathrm{C})$ & 109.5 & $\mathrm{C}(38)-\mathrm{C}(39)-\mathrm{H}(39 \mathrm{~B})$ & 109.2 \\
\hline $\mathrm{C}(9)-\mathrm{C}(12)-\mathrm{H}(12 \mathrm{~A})$ & 109.5 & $\mathrm{C}(40)-\mathrm{C}(39)-\mathrm{H}(39 \mathrm{~B})$ & 109.2 \\
\hline $\mathrm{C}(9)-\mathrm{C}(12)-\mathrm{H}(12 \mathrm{~B})$ & 109.5 & H(39A)-C(39)-H(39B) & 107.9 \\
\hline $\mathrm{H}(12 \mathrm{~A})-\mathrm{C}(12)-\mathrm{H}(12 \mathrm{~B})$ & 109.5 & $C(41)-C(40)-C(39)$ & $109.8(7)$ \\
\hline $\mathrm{C}(9)-\mathrm{C}(12)-\mathrm{H}(12 \mathrm{C})$ & 109.5 & $\mathrm{C}(41)-\mathrm{C}(40)-\mathrm{H}(40 \mathrm{~A})$ & 109.7 \\
\hline $\mathrm{H}(12 \mathrm{~A})-\mathrm{C}(12)-\mathrm{H}(12 \mathrm{C})$ & 109.5 & $\mathrm{C}(39)-\mathrm{C}(40)-\mathrm{H}(40 \mathrm{~A})$ & 109.7 \\
\hline $\mathrm{H}(12 \mathrm{~B})-\mathrm{C}(12)-\mathrm{H}(12 \mathrm{C})$ & 109.5 & $\mathrm{C}(41)-\mathrm{C}(40)-\mathrm{H}(40 \mathrm{~B})$ & 109.7 \\
\hline $\mathrm{C}(18)-\mathrm{C}(13)-\mathrm{C}(14)$ & $122.0(4)$ & $\mathrm{C}(39)-\mathrm{C}(40)-\mathrm{H}(40 \mathrm{~B})$ & 109.7 \\
\hline $\mathrm{C}(18)-\mathrm{C}(13)-\mathrm{N}(2)$ & $119.5(4)$ & $\mathrm{H}(40 \mathrm{~A})-\mathrm{C}(40)-\mathrm{H}(40 \mathrm{~B})$ & 108.2 \\
\hline $\mathrm{C}(14)-\mathrm{C}(13)-\mathrm{N}(2)$ & $118.3(4)$ & $\mathrm{C}(40)-\mathrm{C}(41)-\mathrm{C}(36)$ & $113.5(6)$ \\
\hline$C(15)-C(14)-C(13)$ & $117.4(4)$ & $\mathrm{C}(40)-\mathrm{C}(41)-\mathrm{H}(41 \mathrm{~A})$ & 108.9 \\
\hline
\end{tabular}




\begin{tabular}{|c|c|c|c|}
\hline$C(15)-C(14)-C(19)$ & $121.2(4)$ & $\mathrm{C}(36)-\mathrm{C}(41)-\mathrm{H}(41 \mathrm{~A})$ & 108.9 \\
\hline$C(13)-C(14)-C(19)$ & $121.4(3)$ & $\mathrm{C}(40)-\mathrm{C}(41)-\mathrm{H}(41 \mathrm{~B})$ & 108.9 \\
\hline$C(16)-C(15)-C(14)$ & $122.5(4)$ & $\mathrm{C}(36)-\mathrm{C}(41)-\mathrm{H}(41 \mathrm{~B})$ & 108.9 \\
\hline $\mathrm{C}(16)-\mathrm{C}(15)-\mathrm{H}(15)$ & 118.8 & $\mathrm{H}(41 \mathrm{~A})-\mathrm{C}(41)-\mathrm{H}(41 \mathrm{~B})$ & 107.7 \\
\hline $\mathrm{C}(14)-\mathrm{C}(15)-\mathrm{H}(15)$ & 118.8 & $\mathrm{C}(39 \mathrm{~B})-\mathrm{C}(38 \mathrm{~B})-\mathrm{C}(37)$ & $100.9(10)$ \\
\hline$C(15)-C(16)-C(17)$ & $118.3(4)$ & $\mathrm{C}(39 \mathrm{~B})-\mathrm{C}(38 \mathrm{~B})-\mathrm{H}(38 \mathrm{C})$ & 111.6 \\
\hline$C(15)-C(16)-C(20)$ & $120.8(4)$ & $\mathrm{C}(37)-\mathrm{C}(38 \mathrm{~B})-\mathrm{H}(38 \mathrm{C})$ & 111.6 \\
\hline$C(17)-C(16)-C(20)$ & $120.8(4)$ & $\mathrm{C}(39 \mathrm{~B})-\mathrm{C}(38 \mathrm{~B})-\mathrm{H}(38 \mathrm{D})$ & 111.6 \\
\hline$C(16)-C(17)-C(18)$ & $121.8(4)$ & $\mathrm{C}(37)-\mathrm{C}(38 \mathrm{~B})-\mathrm{H}(38 \mathrm{D})$ & 111.6 \\
\hline $\mathrm{C}(16)-\mathrm{C}(17)-\mathrm{H}(17)$ & 119.1 & $\mathrm{H}(38 \mathrm{C})-\mathrm{C}(38 \mathrm{~B})-\mathrm{H}(38 \mathrm{D})$ & 109.4 \\
\hline $\mathrm{C}(18)-\mathrm{C}(17)-\mathrm{H}(17)$ & 119.1 & $\mathrm{C}(40 \mathrm{~B})-\mathrm{C}(39 \mathrm{~B})-\mathrm{C}(38 \mathrm{~B})$ & $111.2(11)$ \\
\hline$C(13)-C(18)-C(17)$ & $117.9(4)$ & $\mathrm{C}(40 \mathrm{~B})-\mathrm{C}(39 \mathrm{~B})-\mathrm{H}(39 \mathrm{C})$ & 109.4 \\
\hline$C(13)-C(18)-C(21)$ & $121.2(4)$ & $\mathrm{C}(38 \mathrm{~B})-\mathrm{C}(39 \mathrm{~B})-\mathrm{H}(39 \mathrm{C})$ & 109.4 \\
\hline $\mathrm{C}(17)-\mathrm{C}(18)-\mathrm{C}(21)$ & $120.9(4)$ & $\mathrm{C}(40 \mathrm{~B})-\mathrm{C}(39 \mathrm{~B})-\mathrm{H}(39 \mathrm{D})$ & 109.4 \\
\hline $\mathrm{C}(14)-\mathrm{C}(19)-\mathrm{H}(19 \mathrm{~A})$ & 109.5 & $\mathrm{C}(38 \mathrm{~B})-\mathrm{C}(39 \mathrm{~B})-\mathrm{H}(39 \mathrm{D})$ & 109.4 \\
\hline $\mathrm{C}(14)-\mathrm{C}(19)-\mathrm{H}(19 \mathrm{~B})$ & 109.5 & $\mathrm{H}(39 \mathrm{C})-\mathrm{C}(39 \mathrm{~B})-\mathrm{H}(39 \mathrm{D})$ & 108 \\
\hline H(19A)-C(19)-H(19B) & 109.5 & $\mathrm{C}(39 \mathrm{~B})-\mathrm{C}(40 \mathrm{~B})-\mathrm{C}(41 \mathrm{~B})$ & $111.5(10)$ \\
\hline $\mathrm{C}(14)-\mathrm{C}(19)-\mathrm{H}(19 \mathrm{C})$ & 109.5 & $\mathrm{C}(39 \mathrm{~B})-\mathrm{C}(40 \mathrm{~B})-\mathrm{H}(40 \mathrm{C})$ & 109.3 \\
\hline H(19A)-C(19)-H(19C) & 109.5 & $\mathrm{C}(41 \mathrm{~B})-\mathrm{C}(40 \mathrm{~B})-\mathrm{H}(40 \mathrm{C})$ & 109.3 \\
\hline H(19B)-C(19)-H(19C) & 109.5 & $\mathrm{C}(39 \mathrm{~B})-\mathrm{C}(40 \mathrm{~B})-\mathrm{H}(40 \mathrm{D})$ & 109.3 \\
\hline $\mathrm{C}(16)-\mathrm{C}(20)-\mathrm{H}(20 \mathrm{~A})$ & 109.5 & $\mathrm{C}(41 \mathrm{~B})-\mathrm{C}(40 \mathrm{~B})-\mathrm{H}(40 \mathrm{D})$ & 109.3 \\
\hline $\mathrm{C}(16)-\mathrm{C}(20)-\mathrm{H}(20 \mathrm{~B})$ & 109.5 & $\mathrm{H}(40 \mathrm{C})-\mathrm{C}(40 \mathrm{~B})-\mathrm{H}(40 \mathrm{D})$ & 108 \\
\hline $\mathrm{H}(20 \mathrm{~A})-\mathrm{C}(20)-\mathrm{H}(20 \mathrm{~B})$ & 109.5 & $\mathrm{C}(36)-\mathrm{C}(41 \mathrm{~B})-\mathrm{C}(40 \mathrm{~B})$ & $111.6(10)$ \\
\hline $\mathrm{C}(16)-\mathrm{C}(20)-\mathrm{H}(20 \mathrm{C})$ & 109.5 & $\mathrm{C}(36)-\mathrm{C}(41 \mathrm{~B})-\mathrm{H}(41 \mathrm{C})$ & 109.3 \\
\hline$H(20 A)-C(20)-H(20 C)$ & 109.5 & $\mathrm{C}(40 \mathrm{~B})-\mathrm{C}(41 \mathrm{~B})-\mathrm{H}(41 \mathrm{C})$ & 109.3 \\
\hline $\mathrm{H}(20 \mathrm{~B})-\mathrm{C}(20)-\mathrm{H}(20 \mathrm{C})$ & 109.5 & $\mathrm{C}(36)-\mathrm{C}(41 \mathrm{~B})-\mathrm{H}(41 \mathrm{D})$ & 109.3 \\
\hline $\mathrm{C}(18)-\mathrm{C}(21)-\mathrm{H}(21 \mathrm{~A})$ & 109.5 & $\mathrm{C}(40 \mathrm{~B})-\mathrm{C}(41 \mathrm{~B})-\mathrm{H}(41 \mathrm{D})$ & 109.3 \\
\hline $\mathrm{C}(18)-\mathrm{C}(21)-\mathrm{H}(21 \mathrm{~B})$ & 109.5 & $\mathrm{H}(41 \mathrm{C})-\mathrm{C}(41 \mathrm{~B})-\mathrm{H}(41 \mathrm{D})$ & 108 \\
\hline $\mathrm{H}(21 \mathrm{~A})-\mathrm{C}(21)-\mathrm{H}(21 \mathrm{~B})$ & 109.5 & $\mathrm{C}(47 \mathrm{~B})-\mathrm{C}(42)-\mathrm{C}(43 \mathrm{~B})$ & $112.6(7)$ \\
\hline $\mathrm{C}(18)-\mathrm{C}(21)-\mathrm{H}(21 \mathrm{C})$ & 109.5 & $\mathrm{C}(43)-\mathrm{C}(42)-\mathrm{C}(47)$ & $104.8(5)$ \\
\hline $\mathrm{H}(21 \mathrm{~A})-\mathrm{C}(21)-\mathrm{H}(21 \mathrm{C})$ & 109.5 & $\mathrm{C}(47 \mathrm{~B})-\mathrm{C}(42)-\mathrm{P}(1)$ & $115.8(6)$ \\
\hline $\mathrm{H}(21 \mathrm{~B})-\mathrm{C}(21)-\mathrm{H}(21 \mathrm{C})$ & 109.5 & $\mathrm{C}(43)-\mathrm{C}(42)-\mathrm{P}(1)$ & $112.2(4)$ \\
\hline$C(23)-C(22)-R u(1)$ & $173.2(4)$ & $\mathrm{C}(43 \mathrm{~B})-\mathrm{C}(42)-\mathrm{P}(1)$ & $112.1(5)$ \\
\hline $\mathrm{C}(22)-\mathrm{C}(23)-\mathrm{B}(1)$ & $139.5(4)$ & $\mathrm{C}(47)-\mathrm{C}(42)-\mathrm{P}(1)$ & $114.4(4)$ \\
\hline $\mathrm{C}(22)-\mathrm{C}(23)-\mathrm{H}(23)$ & 110.3 & $\mathrm{C}(43)-\mathrm{C}(42)-\mathrm{H}(42)$ & 108.4 \\
\hline $\mathrm{B}(1)-\mathrm{C}(23)-\mathrm{H}(23)$ & 110.3 & $\mathrm{C}(47)-\mathrm{C}(42)-\mathrm{H}(42)$ & 108.4 \\
\hline $\mathrm{C}(26)-\mathrm{C}(24)-\mathrm{O}(1)$ & $107.8(7)$ & $\mathrm{P}(1)-\mathrm{C}(42)-\mathrm{H}(42)$ & 108.4 \\
\hline$C(26)-C(24)-C(27)$ & $109.6(8)$ & $\mathrm{C}(47 \mathrm{~B})-\mathrm{C}(42)-\mathrm{H}(42 \mathrm{~B})$ & 105.1 \\
\hline $\mathrm{O}(1)-\mathrm{C}(24)-\mathrm{C}(27)$ & $110.5(7)$ & $\mathrm{C}(43 \mathrm{~B})-\mathrm{C}(42)-\mathrm{H}(42 \mathrm{~B})$ & 105.1 \\
\hline$C(26)-C(24)-C(25)$ & $116.9(8)$ & $\mathrm{P}(1)-\mathrm{C}(42)-\mathrm{H}(42 \mathrm{~B})$ & 105.1 \\
\hline
\end{tabular}




\begin{tabular}{|c|c|c|c|}
\hline $\mathrm{O}(1)-\mathrm{C}(24)-\mathrm{C}(25)$ & $103.7(5)$ & $C(42)-C(43)-C(44)$ & $111.1(5)$ \\
\hline $\mathrm{C}(27)-\mathrm{C}(24)-\mathrm{C}(25)$ & $108.1(7)$ & $\mathrm{C}(42)-\mathrm{C}(43)-\mathrm{H}(43 \mathrm{~A})$ & 109.4 \\
\hline $\mathrm{O}(2)-\mathrm{C}(25)-\mathrm{C}(28)$ & $111.3(7)$ & $\mathrm{C}(44)-\mathrm{C}(43)-\mathrm{H}(43 \mathrm{~A})$ & 109.4 \\
\hline $\mathrm{O}(2)-\mathrm{C}(25)-\mathrm{C}(24)$ & $101.4(5)$ & $\mathrm{C}(42)-\mathrm{C}(43)-\mathrm{H}(43 \mathrm{~B})$ & 109.4 \\
\hline$C(28)-C(25)-C(24)$ & $117.3(7)$ & $\mathrm{C}(44)-\mathrm{C}(43)-\mathrm{H}(43 \mathrm{~B})$ & 109.4 \\
\hline $\mathrm{O}(2)-\mathrm{C}(25)-\mathrm{C}(29)$ & $108.4(7)$ & $\mathrm{H}(43 \mathrm{~A})-\mathrm{C}(43)-\mathrm{H}(43 \mathrm{~B})$ & 108 \\
\hline $\mathrm{C}(28)-\mathrm{C}(25)-\mathrm{C}(29)$ & $106.1(8)$ & $C(45)-C(44)-C(43)$ & $109.1(6)$ \\
\hline $\mathrm{C}(24)-\mathrm{C}(25)-\mathrm{C}(29)$ & $112.2(7)$ & $\mathrm{C}(45)-\mathrm{C}(44)-\mathrm{H}(44 \mathrm{~A})$ & 109.9 \\
\hline $\mathrm{C}(24)-\mathrm{C}(26)-\mathrm{H}(26 \mathrm{~A})$ & 109.5 & $\mathrm{C}(43)-\mathrm{C}(44)-\mathrm{H}(44 \mathrm{~A})$ & 109.9 \\
\hline $\mathrm{C}(24)-\mathrm{C}(26)-\mathrm{H}(26 \mathrm{~B})$ & 109.5 & $\mathrm{C}(45)-\mathrm{C}(44)-\mathrm{H}(44 \mathrm{~B})$ & 109.9 \\
\hline$H(26 A)-C(26)-H(26 B)$ & 109.5 & $\mathrm{C}(43)-\mathrm{C}(44)-\mathrm{H}(44 \mathrm{~B})$ & 109.9 \\
\hline $\mathrm{C}(24)-\mathrm{C}(26)-\mathrm{H}(26 \mathrm{C})$ & 109.5 & $\mathrm{H}(44 \mathrm{~A})-\mathrm{C}(44)-\mathrm{H}(44 \mathrm{~B})$ & 108.3 \\
\hline$H(26 A)-C(26)-H(26 C)$ & 109.5 & $\mathrm{C}(46)-\mathrm{C}(45)-\mathrm{C}(44)$ & $111.0(7)$ \\
\hline $\mathrm{H}(26 \mathrm{~B})-\mathrm{C}(26)-\mathrm{H}(26 \mathrm{C})$ & 109.5 & $\mathrm{C}(46)-\mathrm{C}(45)-\mathrm{H}(45 \mathrm{~A})$ & 109.4 \\
\hline $\mathrm{C}(24)-\mathrm{C}(27)-\mathrm{H}(27 \mathrm{~A})$ & 109.5 & $\mathrm{C}(44)-\mathrm{C}(45)-\mathrm{H}(45 \mathrm{~A})$ & 109.4 \\
\hline $\mathrm{C}(24)-\mathrm{C}(27)-\mathrm{H}(27 \mathrm{~B})$ & 109.5 & $\mathrm{C}(46)-\mathrm{C}(45)-\mathrm{H}(45 \mathrm{~B})$ & 109.4 \\
\hline $\mathrm{H}(27 \mathrm{~A})-\mathrm{C}(27)-\mathrm{H}(27 \mathrm{~B})$ & 109.5 & $\mathrm{C}(44)-\mathrm{C}(45)-\mathrm{H}(45 \mathrm{~B})$ & 109.4 \\
\hline $\mathrm{C}(24)-\mathrm{C}(27)-\mathrm{H}(27 \mathrm{C})$ & 109.5 & $\mathrm{H}(45 \mathrm{~A})-\mathrm{C}(45)-\mathrm{H}(45 \mathrm{~B})$ & 108 \\
\hline $\mathrm{H}(27 \mathrm{~A})-\mathrm{C}(27)-\mathrm{H}(27 \mathrm{C})$ & 109.5 & $C(45)-C(46)-C(47)$ & $122.8(8)$ \\
\hline $\mathrm{H}(27 \mathrm{~B})-\mathrm{C}(27)-\mathrm{H}(27 \mathrm{C})$ & 109.5 & $\mathrm{C}(45)-\mathrm{C}(46)-\mathrm{H}(46 \mathrm{~A})$ & 106.6 \\
\hline $\mathrm{C}(25)-\mathrm{C}(28)-\mathrm{H}(28 \mathrm{~A})$ & 109.5 & $\mathrm{C}(47)-\mathrm{C}(46)-\mathrm{H}(46 \mathrm{~A})$ & 106.6 \\
\hline $\mathrm{C}(25)-\mathrm{C}(28)-\mathrm{H}(28 \mathrm{~B})$ & 109.5 & $\mathrm{C}(45)-\mathrm{C}(46)-\mathrm{H}(46 \mathrm{~B})$ & 106.6 \\
\hline $\mathrm{H}(28 \mathrm{~A})-\mathrm{C}(28)-\mathrm{H}(28 \mathrm{~B})$ & 109.5 & $\mathrm{C}(47)-\mathrm{C}(46)-\mathrm{H}(46 \mathrm{~B})$ & 106.6 \\
\hline $\mathrm{C}(25)-\mathrm{C}(28)-\mathrm{H}(28 \mathrm{C})$ & 109.5 & $\mathrm{H}(46 \mathrm{~A})-\mathrm{C}(46)-\mathrm{H}(46 \mathrm{~B})$ & 106.6 \\
\hline $\mathrm{H}(28 \mathrm{~A})-\mathrm{C}(28)-\mathrm{H}(28 \mathrm{C})$ & 109.5 & $C(46)-C(47)-C(42)$ & $108.2(7)$ \\
\hline $\mathrm{H}(28 \mathrm{~B})-\mathrm{C}(28)-\mathrm{H}(28 \mathrm{C})$ & 109.5 & $\mathrm{C}(46)-\mathrm{C}(47)-\mathrm{H}(47 \mathrm{~A})$ & 110 \\
\hline $\mathrm{C}(25)-\mathrm{C}(29)-\mathrm{H}(29 \mathrm{~A})$ & 109.5 & $\mathrm{C}(42)-\mathrm{C}(47)-\mathrm{H}(47 \mathrm{~A})$ & 110 \\
\hline $\mathrm{C}(25)-\mathrm{C}(29)-\mathrm{H}(29 \mathrm{~B})$ & 109.5 & $\mathrm{C}(46)-\mathrm{C}(47)-\mathrm{H}(47 \mathrm{~B})$ & 110 \\
\hline H(29A)-C(29)-H(29B) & 109.5 & $\mathrm{C}(42)-\mathrm{C}(47)-\mathrm{H}(47 \mathrm{~B})$ & 110 \\
\hline $\mathrm{C}(25)-\mathrm{C}(29)-\mathrm{H}(29 \mathrm{C})$ & 109.5 & $\mathrm{H}(47 \mathrm{~A})-\mathrm{C}(47)-\mathrm{H}(47 \mathrm{~B})$ & 108.4 \\
\hline $\mathrm{H}(29 \mathrm{~A})-\mathrm{C}(29)-\mathrm{H}(29 \mathrm{C})$ & 109.5 & $\mathrm{C}(44 \mathrm{~B})-\mathrm{C}(43 \mathrm{~B})-\mathrm{C}(42)$ & $107.6(8)$ \\
\hline H(29B)-C(29)-H(29C) & 109.5 & $\mathrm{C}(44 \mathrm{~B})-\mathrm{C}(43 \mathrm{~B})-\mathrm{H}(43 \mathrm{C})$ & 110.2 \\
\hline $\mathrm{O}(1 \mathrm{~B})-\mathrm{C}(24 \mathrm{~B})-\mathrm{C}(25 \mathrm{~B})$ & $104.4(13)$ & $\mathrm{C}(42)-\mathrm{C}(43 \mathrm{~B})-\mathrm{H}(43 \mathrm{C})$ & 110.2 \\
\hline $\mathrm{O}(1 \mathrm{~B})-\mathrm{C}(24 \mathrm{~B})-\mathrm{C}(26 \mathrm{~B})$ & $116.7(16)$ & $\mathrm{C}(44 \mathrm{~B})-\mathrm{C}(43 \mathrm{~B})-\mathrm{H}(43 \mathrm{D})$ & 110.2 \\
\hline $\mathrm{C}(25 \mathrm{~B})-\mathrm{C}(24 \mathrm{~B})-\mathrm{C}(26 \mathrm{~B})$ & $102.2(14)$ & $\mathrm{C}(42)-\mathrm{C}(43 \mathrm{~B})-\mathrm{H}(43 \mathrm{D})$ & 110.2 \\
\hline $\mathrm{O}(1 \mathrm{~B})-\mathrm{C}(24 \mathrm{~B})-\mathrm{C}(27 \mathrm{~B})$ & $103.5(15)$ & $H(43 C)-C(43 B)-H(43 D)$ & 108.5 \\
\hline $\mathrm{C}(25 \mathrm{~B})-\mathrm{C}(24 \mathrm{~B})-\mathrm{C}(27 \mathrm{~B})$ & $91.7(14)$ & $C(45 B)-C(44 B)-C(43 B)$ & $111.7(10)$ \\
\hline $\mathrm{C}(26 \mathrm{~B})-\mathrm{C}(24 \mathrm{~B})-\mathrm{C}(27 \mathrm{~B})$ & $131.9(18)$ & $\mathrm{C}(45 \mathrm{~B})-\mathrm{C}(44 \mathrm{~B})-\mathrm{H}(44 \mathrm{C})$ & 109.3 \\
\hline $\mathrm{O}(2 \mathrm{~B})-\mathrm{C}(25 \mathrm{~B})-\mathrm{C}(24 \mathrm{~B})$ & $103.4(12)$ & $\mathrm{C}(43 \mathrm{~B})-\mathrm{C}(44 \mathrm{~B})-\mathrm{H}(44 \mathrm{C})$ & 109.3 \\
\hline $\mathrm{O}(2 \mathrm{~B})-\mathrm{C}(25 \mathrm{~B})-\mathrm{C}(29 \mathrm{~B})$ & $98.7(15)$ & $\mathrm{C}(45 \mathrm{~B})-\mathrm{C}(44 \mathrm{~B})-\mathrm{H}(44 \mathrm{D})$ & 109.3 \\
\hline
\end{tabular}




\begin{tabular}{|c|c|c|c|}
\hline $\mathrm{C}(24 \mathrm{~B})-\mathrm{C}(25 \mathrm{~B})-\mathrm{C}(29 \mathrm{~B})$ & $120.7(16)$ & $\mathrm{C}(43 \mathrm{~B})-\mathrm{C}(44 \mathrm{~B})-\mathrm{H}(44 \mathrm{D})$ & 109.3 \\
\hline $\mathrm{O}(2 \mathrm{~B})-\mathrm{C}(25 \mathrm{~B})-\mathrm{C}(28 \mathrm{~B})$ & $80.2(14)$ & $\mathrm{H}(44 \mathrm{C})-\mathrm{C}(44 \mathrm{~B})-\mathrm{H}(44 \mathrm{D})$ & 107.9 \\
\hline $\mathrm{C}(24 \mathrm{~B})-\mathrm{C}(25 \mathrm{~B})-\mathrm{C}(28 \mathrm{~B})$ & $118.8(14)$ & $\mathrm{C}(44 \mathrm{~B})-\mathrm{C}(45 \mathrm{~B})-\mathrm{C}(46 \mathrm{~B})$ & $109.6(9)$ \\
\hline $\mathrm{C}(29 \mathrm{~B})-\mathrm{C}(25 \mathrm{~B})-\mathrm{C}(28 \mathrm{~B})$ & $118.8(18)$ & $\mathrm{C}(44 \mathrm{~B})-\mathrm{C}(45 \mathrm{~B})-\mathrm{H}(45 \mathrm{C})$ & 109.8 \\
\hline$C(24 B)-C(26 B)-H(26 D)$ & 109.5 & $\mathrm{C}(46 \mathrm{~B})-\mathrm{C}(45 \mathrm{~B})-\mathrm{H}(45 \mathrm{C})$ & 109.8 \\
\hline$C(24 B)-C(26 B)-H(26 E)$ & 109.5 & $\mathrm{C}(44 \mathrm{~B})-\mathrm{C}(45 \mathrm{~B})-\mathrm{H}(45 \mathrm{D})$ & 109.8 \\
\hline$H(26 D)-C(26 B)-H(26 E)$ & 109.5 & $\mathrm{C}(46 \mathrm{~B})-\mathrm{C}(45 \mathrm{~B})-\mathrm{H}(45 \mathrm{D})$ & 109.8 \\
\hline $\mathrm{C}(24 \mathrm{~B})-\mathrm{C}(26 \mathrm{~B})-\mathrm{H}(26 \mathrm{~F})$ & 109.5 & $\mathrm{H}(45 \mathrm{C})-\mathrm{C}(45 \mathrm{~B})-\mathrm{H}(45 \mathrm{D})$ & 108.2 \\
\hline $\mathrm{H}(26 \mathrm{D})-\mathrm{C}(26 \mathrm{~B})-\mathrm{H}(26 \mathrm{~F})$ & 109.5 & $\mathrm{C}(45 \mathrm{~B})-\mathrm{C}(46 \mathrm{~B})-\mathrm{C}(47 \mathrm{~B})$ & $97.1(9)$ \\
\hline $\mathrm{H}(26 \mathrm{E})-\mathrm{C}(26 \mathrm{~B})-\mathrm{H}(26 \mathrm{~F})$ & 109.5 & $\mathrm{C}(45 \mathrm{~B})-\mathrm{C}(46 \mathrm{~B})-\mathrm{H}(46 \mathrm{C})$ & 112.3 \\
\hline $\mathrm{C}(24 \mathrm{~B})-\mathrm{C}(27 \mathrm{~B})-\mathrm{H}(27 \mathrm{D})$ & 109.5 & $\mathrm{C}(47 \mathrm{~B})-\mathrm{C}(46 \mathrm{~B})-\mathrm{H}(46 \mathrm{C})$ & 112.3 \\
\hline $\mathrm{C}(24 \mathrm{~B})-\mathrm{C}(27 \mathrm{~B})-\mathrm{H}(27 \mathrm{E})$ & 109.5 & $\mathrm{C}(45 \mathrm{~B})-\mathrm{C}(46 \mathrm{~B})-\mathrm{H}(46 \mathrm{D})$ & 112.3 \\
\hline $\mathrm{H}(27 \mathrm{D})-\mathrm{C}(27 \mathrm{~B})-\mathrm{H}(27 \mathrm{E})$ & 109.5 & $\mathrm{C}(47 \mathrm{~B})-\mathrm{C}(46 \mathrm{~B})-\mathrm{H}(46 \mathrm{D})$ & 112.3 \\
\hline $\mathrm{C}(24 \mathrm{~B})-\mathrm{C}(27 \mathrm{~B})-\mathrm{H}(27 \mathrm{~F})$ & 109.5 & $\mathrm{H}(46 \mathrm{C})-\mathrm{C}(46 \mathrm{~B})-\mathrm{H}(46 \mathrm{D})$ & 109.9 \\
\hline $\mathrm{H}(27 \mathrm{D})-\mathrm{C}(27 \mathrm{~B})-\mathrm{H}(27 \mathrm{~F})$ & 109.5 & $\mathrm{C}(42)-\mathrm{C}(47 \mathrm{~B})-\mathrm{C}(46 \mathrm{~B})$ & $104.2(10)$ \\
\hline $\mathrm{H}(27 \mathrm{E})-\mathrm{C}(27 \mathrm{~B})-\mathrm{H}(27 \mathrm{~F})$ & 109.5 & $\mathrm{C}(42)-\mathrm{C}(47 \mathrm{~B})-\mathrm{H}(47 \mathrm{C})$ & 110.9 \\
\hline $\mathrm{C}(25 \mathrm{~B})-\mathrm{C}(28 \mathrm{~B})-\mathrm{H}(28 \mathrm{D})$ & 109.5 & $\mathrm{C}(46 \mathrm{~B})-\mathrm{C}(47 \mathrm{~B})-\mathrm{H}(47 \mathrm{C})$ & 110.9 \\
\hline $\mathrm{C}(25 \mathrm{~B})-\mathrm{C}(28 \mathrm{~B})-\mathrm{H}(28 \mathrm{E})$ & 109.5 & $\mathrm{C}(42)-\mathrm{C}(47 \mathrm{~B})-\mathrm{H}(47 \mathrm{D})$ & 110.9 \\
\hline $\mathrm{H}(28 \mathrm{D})-\mathrm{C}(28 \mathrm{~B})-\mathrm{H}(28 \mathrm{E})$ & 109.5 & $\mathrm{C}(46 \mathrm{~B})-\mathrm{C}(47 \mathrm{~B})-\mathrm{H}(47 \mathrm{D})$ & 110.9 \\
\hline $\mathrm{C}(25 \mathrm{~B})-\mathrm{C}(28 \mathrm{~B})-\mathrm{H}(28 \mathrm{~F})$ & 109.5 & $\mathrm{H}(47 \mathrm{C})-\mathrm{C}(47 \mathrm{~B})-\mathrm{H}(47 \mathrm{D})$ & 108.9 \\
\hline $\mathrm{H}(28 \mathrm{D})-\mathrm{C}(28 \mathrm{~B})-\mathrm{H}(28 \mathrm{~F})$ & 109.5 & $\mathrm{O}(1 \mathrm{~B})-\mathrm{B}(1)-\mathrm{O}(2 \mathrm{~B})$ & $109.3(7)$ \\
\hline $\mathrm{H}(28 \mathrm{E})-\mathrm{C}(28 \mathrm{~B})-\mathrm{H}(28 \mathrm{~F})$ & 109.5 & $\mathrm{O}(2)-\mathrm{B}(1)-\mathrm{O}(1)$ & $112.8(4)$ \\
\hline $\mathrm{C}(25 \mathrm{~B})-\mathrm{C}(29 \mathrm{~B})-\mathrm{H}(29 \mathrm{D})$ & 109.5 & $\mathrm{O}(2)-\mathrm{B}(1)-\mathrm{C}(23)$ & $128.1(4)$ \\
\hline $\mathrm{C}(25 \mathrm{~B})-\mathrm{C}(29 \mathrm{~B})-\mathrm{H}(29 \mathrm{E})$ & 109.5 & $\mathrm{O}(1 \mathrm{~B})-\mathrm{B}(1)-\mathrm{C}(23)$ & $125.2(6)$ \\
\hline$H(29 D)-C(29 B)-H(29 E)$ & 109.5 & $\mathrm{O}(2 \mathrm{~B})-\mathrm{B}(1)-\mathrm{C}(23)$ & $125.5(6)$ \\
\hline $\mathrm{C}(25 \mathrm{~B})-\mathrm{C}(29 \mathrm{~B})-\mathrm{H}(29 \mathrm{~F})$ & 109.5 & $\mathrm{O}(1)-\mathrm{B}(1)-\mathrm{C}(23)$ & $119.0(4)$ \\
\hline $\mathrm{H}(29 \mathrm{D})-\mathrm{C}(29 \mathrm{~B})-\mathrm{H}(29 \mathrm{~F})$ & 109.5 & $\mathrm{C}(1)-\mathrm{N}(1)-\mathrm{C}(4)$ & $128.1(3)$ \\
\hline $\mathrm{H}(29 \mathrm{E})-\mathrm{C}(29 \mathrm{~B})-\mathrm{H}(29 \mathrm{~F})$ & 109.5 & $\mathrm{C}(1)-\mathrm{N}(1)-\mathrm{C}(2)$ & $113.0(3)$ \\
\hline$C(31)-C(30)-C(35)$ & $110.0(3)$ & $\mathrm{C}(4)-\mathrm{N}(1)-\mathrm{C}(2)$ & $118.8(3)$ \\
\hline $\mathrm{C}(31)-\mathrm{C}(30)-\mathrm{P}(1)$ & $113.5(2)$ & $\mathrm{C}(1)-\mathrm{N}(2)-\mathrm{C}(13)$ & $127.3(3)$ \\
\hline $\mathrm{C}(35)-\mathrm{C}(30)-\mathrm{P}(1)$ & $116.5(2)$ & $\mathrm{C}(1)-\mathrm{N}(2)-\mathrm{C}(3)$ & $112.9(3)$ \\
\hline $\mathrm{C}(31)-\mathrm{C}(30)-\mathrm{H}(30)$ & 105.2 & $\mathrm{C}(13)-\mathrm{N}(2)-\mathrm{C}(3)$ & $117.4(3)$ \\
\hline $\mathrm{C}(35)-\mathrm{C}(30)-\mathrm{H}(30)$ & 105.2 & $\mathrm{~B}(1)-\mathrm{O}(1)-\mathrm{C}(24)$ & $107.3(5)$ \\
\hline $\mathrm{P}(1)-\mathrm{C}(30)-\mathrm{H}(30)$ & 105.2 & $\mathrm{~B}(1)-\mathrm{O}(2)-\mathrm{C}(25)$ & $110.5(4)$ \\
\hline$C(30)-C(31)-C(32)$ & $111.3(3)$ & $\mathrm{B}(1)-\mathrm{O}(1 \mathrm{~B})-\mathrm{C}(24 \mathrm{~B})$ & $110.4(10)$ \\
\hline $\mathrm{C}(30)-\mathrm{C}(31)-\mathrm{H}(31 \mathrm{~A})$ & 109.4 & $\mathrm{~B}(1)-\mathrm{O}(2 \mathrm{~B})-\mathrm{C}(25 \mathrm{~B})$ & $109.3(10)$ \\
\hline $\mathrm{C}(32)-\mathrm{C}(31)-\mathrm{H}(31 \mathrm{~A})$ & 109.4 & $\mathrm{C}(36)-\mathrm{P}(1)-\mathrm{C}(30)$ & $107.6(2)$ \\
\hline $\mathrm{C}(30)-\mathrm{C}(31)-\mathrm{H}(31 \mathrm{~B})$ & 109.4 & $\mathrm{C}(36)-\mathrm{P}(1)-\mathrm{C}(42)$ & $102.4(2)$ \\
\hline $\mathrm{C}(32)-\mathrm{C}(31)-\mathrm{H}(31 \mathrm{~B})$ & 109.4 & $\mathrm{C}(30)-\mathrm{P}(1)-\mathrm{C}(42)$ & $102.76(18)$ \\
\hline $\mathrm{H}(31 \mathrm{~A})-\mathrm{C}(31)-\mathrm{H}(31 \mathrm{~B})$ & 108 & $\mathrm{C}(36)-\mathrm{P}(1)-\mathrm{Ru}(1)$ & $116.74(17)$ \\
\hline
\end{tabular}




\begin{tabular}{llll}
$\mathrm{C}(33)-\mathrm{C}(32)-\mathrm{C}(31)$ & $111.4(3)$ & $\mathrm{C}(30)-\mathrm{P}(1)-\mathrm{Ru}(1)$ & $115.74(11)$ \\
$\mathrm{C}(33)-\mathrm{C}(32)-\mathrm{H}(32 \mathrm{~A})$ & 109.3 & $\mathrm{C}(42)-\mathrm{P}(1)-\mathrm{Ru}(1)$ & $109.86(15)$ \\
$\mathrm{C}(31)-\mathrm{C}(32)-\mathrm{H}(32 \mathrm{~A})$ & 109.3 & $\mathrm{C}(22)-\mathrm{Ru}(1)-\mathrm{C}(1)$ & $100.81(15)$ \\
$\mathrm{C}(33)-\mathrm{C}(32)-\mathrm{H}(32 \mathrm{~B})$ & 109.3 & $\mathrm{C}(22)-\mathrm{Ru}(1)-\mathrm{Cl}(1)$ & $93.74(13)$ \\
$\mathrm{C}(31)-\mathrm{C}(32)-\mathrm{H}(32 \mathrm{~B})$ & 109.3 & $\mathrm{C}(1)-\mathrm{Ru}(1)-\mathrm{Cl}(1)$ & $95.01(11)$ \\
$\mathrm{H}(32 \mathrm{~A})-\mathrm{C}(32)-\mathrm{H}(32 \mathrm{~B})$ & 108 & $\mathrm{C}(22)-\mathrm{Ru}(1)-\mathrm{Cl}(2)$ & $97.72(13)$ \\
$\mathrm{C}(32)-\mathrm{C}(33)-\mathrm{C}(34)$ & $110.5(3)$ & $\mathrm{C}(1)-\mathrm{Ru}(1)-\mathrm{Cl}(2)$ & $83.47(11)$ \\
$\mathrm{C}(32)-\mathrm{C}(33)-\mathrm{H}(33 \mathrm{~A})$ & 109.6 & $\mathrm{Cl}(1)-\mathrm{Ru}(1)-\mathrm{Cl}(2)$ & $168.52(4)$ \\
$\mathrm{C}(34)-\mathrm{C}(33)-\mathrm{H}(33 \mathrm{~A})$ & 109.6 & $\mathrm{C}(22)-\mathrm{Ru}(1)-\mathrm{P}(1)$ & $94.75(12)$ \\
$\mathrm{C}(32)-\mathrm{C}(33)-\mathrm{H}(33 \mathrm{~B})$ & 109.6 & $\mathrm{C}(1)-\mathrm{Ru}(1)-\mathrm{P}(1)$ & $163.81(10)$ \\
$\mathrm{C}(34)-\mathrm{C}(33)-\mathrm{H}(33 \mathrm{~B})$ & 109.6 & $\mathrm{Cl}(1)-\mathrm{Ru}(1)-\mathrm{P}(1)$ & $88.35(3)$ \\
$\mathrm{H}(33 \mathrm{~A})-\mathrm{C}(33)-\mathrm{H}(33 \mathrm{~B})$ & 108.1 & $\mathrm{Cl}(2)-\mathrm{Ru}(1)-\mathrm{P}(1)$ & $90.09(3)$ \\
\hline
\end{tabular}

\section{Table S22. Torsion angles $\left({ }^{\circ}\right)$ for $\mathrm{C}_{47} \mathrm{H}_{72} \mathrm{BCl}_{2} \mathrm{~N}_{2} \mathrm{O}_{2} \mathrm{PRu}$}

\begin{tabular}{llll}
\hline $\mathrm{N}(1)-\mathrm{C}(2)-\mathrm{C}(3)-\mathrm{N}(2)$ & $-11.3(5)$ & $\mathrm{C}(43)-\mathrm{C}(42)-\mathrm{C}(47)-\mathrm{C}(46)$ & $-53.9(7)$ \\
$\mathrm{C}(9)-\mathrm{C}(4)-\mathrm{C}(5)-\mathrm{C}(6)$ & $3.2(6)$ & $\mathrm{P}(1)-\mathrm{C}(42)-\mathrm{C}(47)-\mathrm{C}(46)$ & $-177.2(5)$ \\
$\mathrm{N}(1)-\mathrm{C}(4)-\mathrm{C}(5)-\mathrm{C}(6)$ & $174.3(4)$ & $\mathrm{C}(47 \mathrm{~B})-\mathrm{C}(42)-\mathrm{C}(43 \mathrm{~B})-\mathrm{C}(44 \mathrm{~B})$ & $61.6(11)$ \\
$\mathrm{C}(9)-\mathrm{C}(4)-\mathrm{C}(5)-\mathrm{C}(10)$ & $-174.4(4)$ & $\mathrm{P}(1)-\mathrm{C}(42)-\mathrm{C}(43 \mathrm{~B})-\mathrm{C}(44 \mathrm{~B})$ & $-165.7(7)$ \\
$\mathrm{N}(1)-\mathrm{C}(4)-\mathrm{C}(5)-\mathrm{C}(10)$ & $-3.4(6)$ & $\mathrm{C}(42)-\mathrm{C}(43 \mathrm{~B})-\mathrm{C}(44 \mathrm{~B})-\mathrm{C}(45 \mathrm{~B})$ & $-53.5(12)$ \\
$\mathrm{C}(4)-\mathrm{C}(5)-\mathrm{C}(6)-\mathrm{C}(7)$ & $-0.3(8)$ & $\mathrm{C}(43 \mathrm{~B})-\mathrm{C}(44 \mathrm{~B})-\mathrm{C}(45 \mathrm{~B})-\mathrm{C}(46 \mathrm{~B})$ & $62.9(12)$ \\
$\mathrm{C}(10)-\mathrm{C}(5)-\mathrm{C}(6)-\mathrm{C}(7)$ & $177.3(6)$ & $\mathrm{C}(44 \mathrm{~B})-\mathrm{C}(45 \mathrm{~B})-\mathrm{C}(46 \mathrm{~B})-\mathrm{C}(47 \mathrm{~B})$ & $-66.7(11)$ \\
$\mathrm{C}(5)-\mathrm{C}(6)-\mathrm{C}(7)-\mathrm{C}(8)$ & $-1.2(10)$ & $\mathrm{C}(43 \mathrm{~B})-\mathrm{C}(42)-\mathrm{C}(47 \mathrm{~B})-\mathrm{C}(46 \mathrm{~B})$ & $-73.0(10)$ \\
$\mathrm{C}(5)-\mathrm{C}(6)-\mathrm{C}(7)-\mathrm{C}(11)$ & $-179.3(6)$ & $\mathrm{P}(1)-\mathrm{C}(42)-\mathrm{C}(47 \mathrm{~B})-\mathrm{C}(46 \mathrm{~B})$ & $156.2(7)$ \\
$\mathrm{C}(6)-\mathrm{C}(7)-\mathrm{C}(8)-\mathrm{C}(9)$ & $-0.1(9)$ & $\mathrm{C}(45 \mathrm{~B})-\mathrm{C}(46 \mathrm{~B})-\mathrm{C}(47 \mathrm{~B})-\mathrm{C}(42)$ & $70.2(10)$ \\
$\mathrm{C}(11)-\mathrm{C}(7)-\mathrm{C}(8)-\mathrm{C}(9)$ & $178.0(6)$ & $\mathrm{C}(22)-\mathrm{C}(23)-\mathrm{B}(1)-\mathrm{O}(2)$ & $-24.4(10)$ \\
$\mathrm{C}(5)-\mathrm{C}(4)-\mathrm{C}(9)-\mathrm{C}(8)$ & $-4.5(7)$ & $\mathrm{C}(22)-\mathrm{C}(23)-\mathrm{B}(1)-\mathrm{O}(1 \mathrm{~B})$ & $40.4(14)$ \\
$\mathrm{N}(1)-\mathrm{C}(4)-\mathrm{C}(9)-\mathrm{C}(8)$ & $-175.6(4)$ & $\mathrm{C}(22)-\mathrm{C}(23)-\mathrm{B}(1)-\mathrm{O}(2 \mathrm{~B})$ & $-139.6(11)$ \\
$\mathrm{C}(5)-\mathrm{C}(4)-\mathrm{C}(9)-\mathrm{C}(12)$ & $172.1(4)$ & $\mathrm{C}(22)-\mathrm{C}(23)-\mathrm{B}(1)-\mathrm{O}(1)$ & $159.9(6)$ \\
$\mathrm{N}(1)-\mathrm{C}(4)-\mathrm{C}(9)-\mathrm{C}(12)$ & $1.0(6)$ & $\mathrm{N}(2)-\mathrm{C}(1)-\mathrm{N}(1)-\mathrm{C}(4)$ & $171.4(4)$ \\
$\mathrm{C}(7)-\mathrm{C}(8)-\mathrm{C}(9)-\mathrm{C}(4)$ & $2.9(8)$ & $\mathrm{Ru}(1)-\mathrm{C}(1)-\mathrm{N}(1)-\mathrm{C}(4)$ & $-21.3(6)$ \\
$\mathrm{C}(7)-\mathrm{C}(8)-\mathrm{C}(9)-\mathrm{C}(12)$ & $-173.8(5)$ & $\mathrm{N}(2)-\mathrm{C}(1)-\mathrm{N}(1)-\mathrm{C}(2)$ & $-4.4(5)$ \\
$\mathrm{C}(18)-\mathrm{C}(13)-\mathrm{C}(14)-\mathrm{C}(15)$ & $1.0(6)$ & $\mathrm{Ru}(1)-\mathrm{C}(1)-\mathrm{N}(1)-\mathrm{C}(2)$ & $162.9(3)$ \\
$\mathrm{N}(2)-\mathrm{C}(13)-\mathrm{C}(14)-\mathrm{C}(15)$ & $176.0(3)$ & $\mathrm{C}(9)-\mathrm{C}(4)-\mathrm{N}(1)-\mathrm{C}(1)$ & $-82.5(5)$ \\
$\mathrm{C}(18)-\mathrm{C}(13)-\mathrm{C}(14)-\mathrm{C}(19)$ & $177.9(4)$ & $\mathrm{C}(5)-\mathrm{C}(4)-\mathrm{N}(1)-\mathrm{C}(1)$ & $106.2(5)$ \\
$\mathrm{N}(2)-\mathrm{C}(13)-\mathrm{C}(14)-\mathrm{C}(19)$ & $-7.1(5)$ & $\mathrm{C}(9)-\mathrm{C}(4)-\mathrm{N}(1)-\mathrm{C}(2)$ & $93.1(5)$ \\
$\mathrm{C}(13)-\mathrm{C}(14)-\mathrm{C}(15)-\mathrm{C}(16)$ & $1.5(6)$ & $\mathrm{C}(5)-\mathrm{C}(4)-\mathrm{N}(1)-\mathrm{C}(2)$ & $-78.2(5)$ \\
$\mathrm{C}(19)-\mathrm{C}(14)-\mathrm{C}(15)-\mathrm{C}(16)$ & $-175.3(4)$ & $\mathrm{C}(3)-\mathrm{C}(2)-\mathrm{N}(1)-\mathrm{C}(1)$ & $-165.8(4)$ \\
$\mathrm{C}(14)-\mathrm{C}(15)-\mathrm{C}(16)-\mathrm{C}(17)$ & $-2.5(6)$ & $\mathrm{C}(3)-\mathrm{C}(2)-\mathrm{N}(1)-\mathrm{C}(4)$ & $-165.4(4)$ \\
$\mathrm{C}(14)-\mathrm{C}(15)-\mathrm{C}(16)-\mathrm{C}(20)$ & $175.2(4)$ & $\mathrm{N}(1)-\mathrm{C}(1)-\mathrm{N}(2)-\mathrm{C}(13)$ & \\
& & & \\
& & &
\end{tabular}




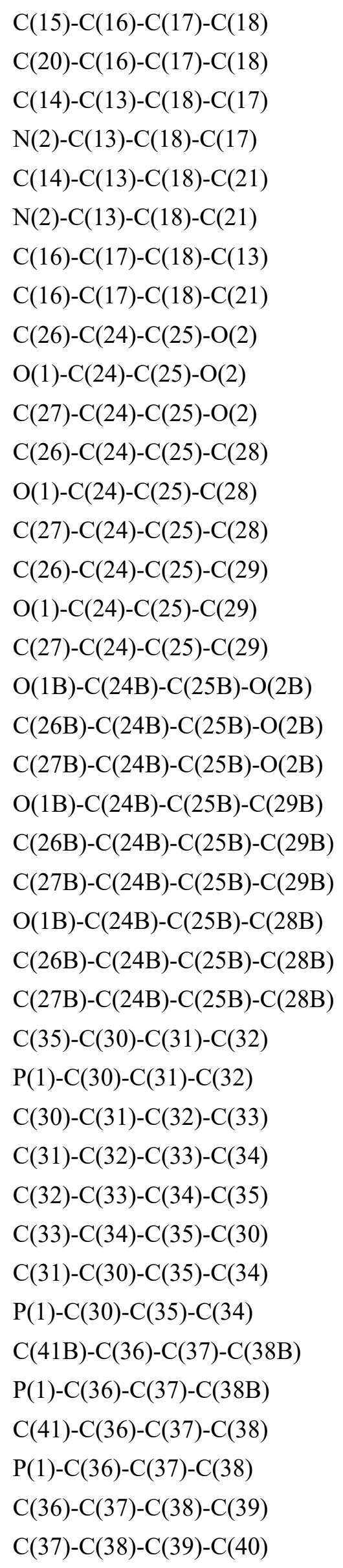

\begin{tabular}{|c|c|}
\hline $1.0(6)$ & $\mathrm{Ru}(1)-\mathrm{C}(1)-\mathrm{N}(2)-\mathrm{C}(13)$ \\
\hline$-176.7(4)$ & $\mathrm{N}(1)-\mathrm{C}(1)-\mathrm{N}(2)-\mathrm{C}(3)$ \\
\hline$-2.4(6)$ & $\mathrm{Ru}(1)-\mathrm{C}(1)-\mathrm{N}(2)-\mathrm{C}(3)$ \\
\hline$-177.4(4)$ & $\mathrm{C}(18)-\mathrm{C}(13)-\mathrm{N}(2)-\mathrm{C}(1)$ \\
\hline $179.4(4)$ & $\mathrm{C}(14)-\mathrm{C}(13)-\mathrm{N}(2)-\mathrm{C}(1)$ \\
\hline $4.4(6)$ & $\mathrm{C}(18)-\mathrm{C}(13)-\mathrm{N}(2)-\mathrm{C}(3)$ \\
\hline $1.4(6)$ & $\mathrm{C}(14)-\mathrm{C}(13)-\mathrm{N}(2)-\mathrm{C}(3)$ \\
\hline $179.6(4)$ & $C(2)-C(3)-N(2)-C(1)$ \\
\hline$-138.3(8)$ & $\mathrm{C}(2)-\mathrm{C}(3)-\mathrm{N}(2)-\mathrm{C}(13)$ \\
\hline$-19.8(8)$ & $\mathrm{O}(2)-\mathrm{B}(1)-\mathrm{O}(1)-\mathrm{C}(24)$ \\
\hline $97.5(7)$ & $\mathrm{C}(23)-\mathrm{B}(1)-\mathrm{O}(1)-\mathrm{C}(24)$ \\
\hline $100.3(10)$ & $\mathrm{C}(26)-\mathrm{C}(24)-\mathrm{O}(1)-\mathrm{B}(1)$ \\
\hline$-141.1(8)$ & $\mathrm{C}(27)-\mathrm{C}(24)-\mathrm{O}(1)-\mathrm{B}(1)$ \\
\hline$-23.8(11)$ & $\mathrm{C}(25)-\mathrm{C}(24)-\mathrm{O}(1)-\mathrm{B}(1)$ \\
\hline$-22.9(10)$ & $\mathrm{O}(1)-\mathrm{B}(1)-\mathrm{O}(2)-\mathrm{C}(25)$ \\
\hline $95.7(8)$ & $\mathrm{C}(23)-\mathrm{B}(1)-\mathrm{O}(2)-\mathrm{C}(25)$ \\
\hline$-147.0(8)$ & $\mathrm{C}(28)-\mathrm{C}(25)-\mathrm{O}(2)-\mathrm{B}(1)$ \\
\hline$-14.4(17)$ & $\mathrm{C}(24)-\mathrm{C}(25)-\mathrm{O}(2)-\mathrm{B}(1)$ \\
\hline $107.6(16)$ & $\mathrm{C}(29)-\mathrm{C}(25)-\mathrm{O}(2)-\mathrm{B}(1)$ \\
\hline$-118.8(16)$ & $\mathrm{O}(2 \mathrm{~B})-\mathrm{B}(1)-\mathrm{O}(1 \mathrm{~B})-\mathrm{C}(24 \mathrm{~B})$ \\
\hline$-123.2(18)$ & $\mathrm{C}(23)-\mathrm{B}(1)-\mathrm{O}(1 \mathrm{~B})-\mathrm{C}(24 \mathrm{~B})$ \\
\hline$-1(2)$ & $\mathrm{C}(25 \mathrm{~B})-\mathrm{C}(24 \mathrm{~B})-\mathrm{O}(1 \mathrm{~B})-\mathrm{B}(1)$ \\
\hline $132.4(19)$ & $\mathrm{C}(26 \mathrm{~B})-\mathrm{C}(24 \mathrm{~B})-\mathrm{O}(1 \mathrm{~B})-\mathrm{B}(1)$ \\
\hline $72(2)$ & $\mathrm{C}(27 \mathrm{~B})-\mathrm{C}(24 \mathrm{~B})-\mathrm{O}(1 \mathrm{~B})-\mathrm{B}(1)$ \\
\hline$-166.3(18)$ & $\mathrm{O}(1 \mathrm{~B})-\mathrm{B}(1)-\mathrm{O}(2 \mathrm{~B})-\mathrm{C}(25 \mathrm{~B})$ \\
\hline$-33(2)$ & $\mathrm{C}(23)-\mathrm{B}(1)-\mathrm{O}(2 \mathrm{~B})-\mathrm{C}(25 \mathrm{~B})$ \\
\hline $56.8(4)$ & $\mathrm{C}(24 \mathrm{~B})-\mathrm{C}(25 \mathrm{~B})-\mathrm{O}(2 \mathrm{~B})-\mathrm{B}(1)$ \\
\hline$-170.7(3)$ & $\mathrm{C}(29 \mathrm{~B})-\mathrm{C}(25 \mathrm{~B})-\mathrm{O}(2 \mathrm{~B})-\mathrm{B}(1)$ \\
\hline$-55.8(5)$ & $\mathrm{C}(28 \mathrm{~B})-\mathrm{C}(25 \mathrm{~B})-\mathrm{O}(2 \mathrm{~B})-\mathrm{B}(1)$ \\
\hline $55.1(5)$ & $\mathrm{C}(41 \mathrm{~B})-\mathrm{C}(36)-\mathrm{P}(1)-\mathrm{C}(30)$ \\
\hline$-56.9(5)$ & $\mathrm{C}(41)-\mathrm{C}(36)-\mathrm{P}(1)-\mathrm{C}(30)$ \\
\hline $58.4(5)$ & $\mathrm{C}(37)-\mathrm{C}(36)-\mathrm{P}(1)-\mathrm{C}(30)$ \\
\hline$-58.1(4)$ & $\mathrm{C}(41 \mathrm{~B})-\mathrm{C}(36)-\mathrm{P}(1)-\mathrm{C}(42)$ \\
\hline $170.9(3)$ & $\mathrm{C}(41)-\mathrm{C}(36)-\mathrm{P}(1)-\mathrm{C}(42)$ \\
\hline$-62.5(9)$ & $\mathrm{C}(37)-\mathrm{C}(36)-\mathrm{P}(1)-\mathrm{C}(42)$ \\
\hline $152.7(6)$ & $\mathrm{C}(41 \mathrm{~B})-\mathrm{C}(36)-\mathrm{P}(1)-\mathrm{Ru}(1)$ \\
\hline 49.4(7) & $\mathrm{C}(41)-\mathrm{C}(36)-\mathrm{P}(1)-\mathrm{Ru}(1)$ \\
\hline$-168.4(4)$ & $\mathrm{C}(37)-\mathrm{C}(36)-\mathrm{P}(1)-\mathrm{Ru}(1)$ \\
\hline$-53.4(8)$ & $\mathrm{C}(31)-\mathrm{C}(30)-\mathrm{P}(1)-\mathrm{C}(36)$ \\
\hline $58.4(10)$ & $\mathrm{C}(35)-\mathrm{C}(30)-\mathrm{P}(1)-\mathrm{C}(36)$ \\
\hline
\end{tabular}

28.3(6)

$-3.9(5)$

$-170.2(3)$

$-112.7(5)$

$72.2(5)$

$86.5(5)$

$-88.6(4)$

10.1(5)

173.6(4)

$-3.5(7)$

172.8(5)

$139.5(7)$

$-100.8(7)$

14.9(7)

$-10.7(7)$

173.4(5)

144.4(7)

18.9(7)

$-99.3(7)$

$-15.8(17)$

164.2(9)

$18.9(17)$

$-93.0(18)$

114.2(17)

$5.6(17)$

$-174.4(9)$

$5.8(17)$

130.4(16)

$-111.6(16)$

$-51.5(8)$

$-135.2(5)$

84.2(4)

56.4(8)

$-27.3(6)$

$-167.9(4)$

176.4(8)

92.7(5)

$-47.9(4)$

$-66.7(3)$

62.7(3) 


\begin{tabular}{llll}
$\mathrm{C}(38)-\mathrm{C}(39)-\mathrm{C}(40)-\mathrm{C}(41)$ & $-56.0(10)$ & $\mathrm{C}(31)-\mathrm{C}(30)-\mathrm{P}(1)-\mathrm{C}(42)$ & $-174.3(3)$ \\
$\mathrm{C}(39)-\mathrm{C}(40)-\mathrm{C}(41)-\mathrm{C}(36)$ & $50.6(9)$ & $\mathrm{C}(35)-\mathrm{C}(30)-\mathrm{P}(1)-\mathrm{C}(42)$ & $-44.9(3)$ \\
$\mathrm{C}(37)-\mathrm{C}(36)-\mathrm{C}(41)-\mathrm{C}(40)$ & $-50.0(9)$ & $\mathrm{C}(31)-\mathrm{C}(30)-\mathrm{P}(1)-\mathrm{Ru}(1)$ & $66.0(3)$ \\
$\mathrm{P}(1)-\mathrm{C}(36)-\mathrm{C}(41)-\mathrm{C}(40)$ & $170.5(5)$ & $\mathrm{C}(35)-\mathrm{C}(30)-\mathrm{P}(1)-\mathrm{Ru}(1)$ & $-164.7(2)$ \\
$\mathrm{C}(36)-\mathrm{C}(37)-\mathrm{C}(38 \mathrm{~B})-\mathrm{C}(39 \mathrm{~B})$ & $61.9(10)$ & $\mathrm{C}(47 \mathrm{~B})-\mathrm{C}(42)-\mathrm{P}(1)-\mathrm{C}(36)$ & $-175.0(6)$ \\
$\mathrm{C}(37)-\mathrm{C}(38 \mathrm{~B})-\mathrm{C}(39 \mathrm{~B})-\mathrm{C}(40 \mathrm{~B})$ & $-58.8(12)$ & $\mathrm{C}(43)-\mathrm{C}(42)-\mathrm{P}(1)-\mathrm{C}(36)$ & $84.6(5)$ \\
$\mathrm{C}(38 \mathrm{~B})-\mathrm{C}(39 \mathrm{~B})-\mathrm{C}(40 \mathrm{~B})-\mathrm{C}(41 \mathrm{~B})$ & $60.1(14)$ & $\mathrm{C}(43 \mathrm{~B})-\mathrm{C}(42)-\mathrm{P}(1)-\mathrm{C}(36)$ & $53.9(6)$ \\
$\mathrm{C}(37)-\mathrm{C}(36)-\mathrm{C}(41 \mathrm{~B})-\mathrm{C}(40 \mathrm{~B})$ & $53.4(10)$ & $\mathrm{C}(47)-\mathrm{C}(42)-\mathrm{P}(1)-\mathrm{C}(36)$ & $-156.2(4)$ \\
$\mathrm{P}(1)-\mathrm{C}(36)-\mathrm{C}(41 \mathrm{~B})-\mathrm{C}(40 \mathrm{~B})$ & $-169.0(6)$ & $\mathrm{C}(47 \mathrm{~B})-\mathrm{C}(42)-\mathrm{P}(1)-\mathrm{C}(30)$ & $-63.5(6)$ \\
$\mathrm{C}(39 \mathrm{~B})-\mathrm{C}(40 \mathrm{~B})-\mathrm{C}(41 \mathrm{~B})-\mathrm{C}(36)$ & $-57.6(14)$ & $\mathrm{C}(43)-\mathrm{C}(42)-\mathrm{P}(1)-\mathrm{C}(30)$ & $-163.8(4)$ \\
$\mathrm{C}(47)-\mathrm{C}(42)-\mathrm{C}(43)-\mathrm{C}(44)$ & $63.2(7)$ & $\mathrm{C}(43 \mathrm{~B})-\mathrm{C}(42)-\mathrm{P}(1)-\mathrm{C}(30)$ & $165.5(6)$ \\
$\mathrm{P}(1)-\mathrm{C}(42)-\mathrm{C}(43)-\mathrm{C}(44)$ & $-172.1(5)$ & $\mathrm{C}(47)-\mathrm{C}(42)-\mathrm{P}(1)-\mathrm{C}(30)$ & $-44.6(4)$ \\
$\mathrm{C}(42)-\mathrm{C}(43)-\mathrm{C}(44)-\mathrm{C}(45)$ & $-61.3(8)$ & $\mathrm{C}(47 \mathrm{~B})-\mathrm{C}(42)-\mathrm{P}(1)-\mathrm{Ru}(1)$ & $60.3(6)$ \\
$\mathrm{C}(43)-\mathrm{C}(44)-\mathrm{C}(45)-\mathrm{C}(46)$ & $49.6(9)$ & $\mathrm{C}(43)-\mathrm{C}(42)-\mathrm{P}(1)-\mathrm{Ru}(1)$ & $-40.1(5)$ \\
$\mathrm{C}(44)-\mathrm{C}(45)-\mathrm{C}(46)-\mathrm{C}(47)$ & $-49.3(12)$ & $\mathrm{C}(43 \mathrm{~B})-\mathrm{C}(42)-\mathrm{P}(1)-\mathrm{Ru}(1)$ & $-70.8(6)$ \\
$\mathrm{C}(45)-\mathrm{C}(46)-\mathrm{C}(47)-\mathrm{C}(42)$ & $52.4(11)$ & $\mathrm{C}(47)-\mathrm{C}(42)-\mathrm{P}(1)-\mathrm{Ru}(1)$ & $79.2(4)$ \\
\hline
\end{tabular}

\section{Table S23. Anisotropic atomic displacement parameters $\left(\AA^{2} \times 10^{3}\right)$ for $\mathrm{C}_{47} \mathrm{H}_{72} \mathrm{BCl}_{2} \mathrm{~N}_{2} \mathrm{O}_{2} \mathrm{PRu}$}

\begin{tabular}{|c|c|c|c|c|c|c|}
\hline \multicolumn{7}{|c|}{ The anisotropic atomic displacement factor exponent takes the form: $-2 \pi^{2}\left[\mathrm{~h}^{2} \mathrm{a}^{* 2} \mathrm{U}_{11}+\ldots+2 \mathrm{~h} \mathrm{k} \mathrm{a}^{*} \mathrm{~b}^{*} \mathrm{U}_{12}\right]$} \\
\hline $\mathrm{C}(1)$ & $28(2)$ & $27(2)$ & $39(2)$ & $2(2)$ & $-7(2)$ & $-1(1)$ \\
\hline $\mathrm{C}(2)$ & $57(3)$ & $29(2)$ & $72(3)$ & $1(2)$ & $-25(2)$ & $-13(2)$ \\
\hline$C(3)$ & $49(2)$ & $25(2)$ & $73(3)$ & $6(2)$ & $-26(2)$ & $-13(2)$ \\
\hline $\mathrm{C}(4)$ & $55(3)$ & $28(2)$ & $45(2)$ & $-10(2)$ & $-28(2)$ & $7(2)$ \\
\hline $\mathrm{C}(5)$ & $58(3)$ & $41(2)$ & $61(3)$ & $-19(2)$ & $-32(2)$ & $12(2)$ \\
\hline$C(6)$ & $96(5)$ & $72(4)$ & $70(4)$ & $-27(3)$ & $-52(4)$ & $44(3)$ \\
\hline$C(7)$ & $129(6)$ & $96(5)$ & $44(3)$ & $-24(3)$ & $-40(4)$ & $66(4)$ \\
\hline$C(8)$ & $117(5)$ & $63(3)$ & $35(3)$ & $-13(2)$ & $-20(3)$ & $41(3)$ \\
\hline $\mathrm{C}(9)$ & $74(3)$ & $38(2)$ & $36(2)$ & $-12(2)$ & $-22(2)$ & $14(2)$ \\
\hline$C(10)$ & $42(3)$ & $56(3)$ & $97(4)$ & $-15(3)$ & $-31(3)$ & $1(2)$ \\
\hline$C(11)$ & $224(11)$ & 189(9) & $51(4)$ & $-17(5)$ & $-59(6)$ & $136(8)$ \\
\hline$C(12)$ & $65(3)$ & $46(2)$ & $37(2)$ & $-10(2)$ & $-7(2)$ & $12(2)$ \\
\hline$C(13)$ & $33(2)$ & $25(2)$ & $44(2)$ & $12(2)$ & $-12(2)$ & $-5(1)$ \\
\hline$C(14)$ & $34(2)$ & $26(2)$ & $39(2)$ & $3(2)$ & $-6(2)$ & $-6(2)$ \\
\hline$C(15)$ & $33(2)$ & $32(2)$ & $40(2)$ & $7(2)$ & $-7(2)$ & $-3(2)$ \\
\hline$C(16)$ & $41(2)$ & $34(2)$ & $40(2)$ & $13(2)$ & $-9(2)$ & $0(2)$ \\
\hline $\mathrm{C}(17)$ & $46(2)$ & $46(2)$ & $38(2)$ & $17(2)$ & $1(2)$ & $1(2)$ \\
\hline $\mathrm{C}(18)$ & $36(2)$ & $42(2)$ & $49(3)$ & $20(2)$ & $-2(2)$ & $-1(2)$ \\
\hline$C(19)$ & $37(2)$ & $43(2)$ & $39(2)$ & $0(2)$ & $-7(2)$ & $-2(2)$ \\
\hline$C(20)$ & $50(3)$ & $56(3)$ & $41(2)$ & $15(2)$ & $-15(2)$ & $0(2)$ \\
\hline
\end{tabular}




\begin{tabular}{|c|c|c|c|c|c|c|}
\hline $\mathrm{C}(21)$ & $38(2)$ & $85(4)$ & $58(3)$ & $31(3)$ & $0(2)$ & $6(2)$ \\
\hline $\mathrm{C}(22)$ & $37(2)$ & $23(2)$ & $37(2)$ & $3(2)$ & $-3(2)$ & $-1(1)$ \\
\hline$C(23)$ & $65(3)$ & $30(2)$ & $54(3)$ & $-2(2)$ & $-33(2)$ & $4(2)$ \\
\hline $\mathrm{C}(24)$ & $38(3)$ & $73(3)$ & $35(3)$ & $-18(2)$ & $-9(2)$ & $15(2)$ \\
\hline$C(25)$ & $38(3)$ & $73(3)$ & $35(3)$ & $-18(2)$ & $-9(2)$ & $15(2)$ \\
\hline$C(26)$ & $93(4)$ & $110(4)$ & $90(4)$ & $12(3)$ & $12(3)$ & $10(3)$ \\
\hline$C(27)$ & $93(4)$ & $110(4)$ & $90(4)$ & $12(3)$ & $12(3)$ & $10(3)$ \\
\hline$C(28)$ & $93(4)$ & $110(4)$ & $90(4)$ & $12(3)$ & $12(3)$ & $10(3)$ \\
\hline$C(29)$ & $93(4)$ & $110(4)$ & $90(4)$ & $12(3)$ & $12(3)$ & $10(3)$ \\
\hline $\mathrm{C}(24 \mathrm{~B})$ & $38(3)$ & $73(3)$ & $35(3)$ & $-18(2)$ & $-9(2)$ & $15(2)$ \\
\hline$C(25 B)$ & $38(3)$ & $73(3)$ & $35(3)$ & $-18(2)$ & $-9(2)$ & $15(2)$ \\
\hline$C(26 B)$ & $112(10)$ & $82(8)$ & $97(10)$ & $-4(7)$ & $11(8)$ & $28(7)$ \\
\hline $\mathrm{C}(27 \mathrm{~B})$ & $112(10)$ & $82(8)$ & $97(10)$ & $-4(7)$ & $11(8)$ & $28(7)$ \\
\hline $\mathrm{C}(28 \mathrm{~B})$ & $112(10)$ & $82(8)$ & $97(10)$ & $-4(7)$ & $11(8)$ & $28(7)$ \\
\hline$C(29 B)$ & $112(10)$ & $82(8)$ & $97(10)$ & $-4(7)$ & $11(8)$ & $28(7)$ \\
\hline$C(30)$ & $31(2)$ & $22(2)$ & $29(2)$ & $2(1)$ & $2(1)$ & $1(1)$ \\
\hline$C(31)$ & $38(2)$ & $39(2)$ & $39(2)$ & $11(2)$ & $-3(2)$ & $-7(2)$ \\
\hline$C(32)$ & $33(2)$ & $45(2)$ & $52(3)$ & $16(2)$ & $-8(2)$ & $-6(2)$ \\
\hline$C(33)$ & $38(2)$ & $33(2)$ & $55(3)$ & $3(2)$ & $-1(2)$ & $-7(2)$ \\
\hline$C(34)$ & $41(2)$ & $28(2)$ & $48(2)$ & $9(2)$ & $2(2)$ & $-4(2)$ \\
\hline$C(35)$ & $33(2)$ & $26(2)$ & $51(2)$ & $7(2)$ & $-1(2)$ & $1(2)$ \\
\hline$C(36)$ & $49(1)$ & $67(2)$ & $41(1)$ & $-14(1)$ & $13(1)$ & $1(1)$ \\
\hline$C(37)$ & $49(1)$ & $67(2)$ & $41(1)$ & $-14(1)$ & $13(1)$ & $1(1)$ \\
\hline$C(38)$ & $49(1)$ & $67(2)$ & $41(1)$ & $-14(1)$ & $13(1)$ & $1(1)$ \\
\hline C(39) & $49(1)$ & $67(2)$ & $41(1)$ & $-14(1)$ & $13(1)$ & $1(1)$ \\
\hline $\mathrm{C}(40)$ & $49(1)$ & $67(2)$ & $41(1)$ & $-14(1)$ & $13(1)$ & $1(1)$ \\
\hline $\mathrm{C}(41)$ & $49(1)$ & $67(2)$ & $41(1)$ & $-14(1)$ & $13(1)$ & $1(1)$ \\
\hline $\mathrm{C}(38 \mathrm{~B})$ & $49(1)$ & $67(2)$ & $41(1)$ & $-14(1)$ & $13(1)$ & $1(1)$ \\
\hline C(39B) & $49(1)$ & $67(2)$ & $41(1)$ & $-14(1)$ & $13(1)$ & $1(1)$ \\
\hline $\mathrm{C}(40 \mathrm{~B})$ & $49(1)$ & $67(2)$ & $41(1)$ & $-14(1)$ & $13(1)$ & $1(1)$ \\
\hline $\mathrm{C}(41 \mathrm{~B})$ & $49(1)$ & $67(2)$ & $41(1)$ & $-14(1)$ & $13(1)$ & $1(1)$ \\
\hline$C(42)$ & $48(1)$ & $47(2)$ & $34(1)$ & $9(1)$ & $-9(1)$ & $-2(1)$ \\
\hline$C(43)$ & $48(1)$ & $47(2)$ & $34(1)$ & $9(1)$ & $-9(1)$ & $-2(1)$ \\
\hline$C(44)$ & $48(1)$ & $47(2)$ & $34(1)$ & $9(1)$ & $-9(1)$ & $-2(1)$ \\
\hline$C(45)$ & $48(1)$ & $47(2)$ & $34(1)$ & $9(1)$ & $-9(1)$ & $-2(1)$ \\
\hline$C(46)$ & $48(1)$ & $47(2)$ & $34(1)$ & $9(1)$ & $-9(1)$ & $-2(1)$ \\
\hline $\mathrm{C}(47)$ & $48(1)$ & $47(2)$ & $34(1)$ & $9(1)$ & $-9(1)$ & $-2(1)$ \\
\hline $\mathrm{C}(43 \mathrm{~B})$ & $48(1)$ & $47(2)$ & $34(1)$ & $9(1)$ & $-9(1)$ & $-2(1)$ \\
\hline $\mathrm{C}(44 \mathrm{~B})$ & $48(1)$ & $47(2)$ & $34(1)$ & $9(1)$ & $-9(1)$ & $-2(1)$ \\
\hline $\mathrm{C}(45 \mathrm{~B})$ & $48(1)$ & $47(2)$ & $34(1)$ & $9(1)$ & $-9(1)$ & $-2(1)$ \\
\hline
\end{tabular}




\begin{tabular}{lllllll}
$\mathrm{C}(46 \mathrm{~B})$ & $48(1)$ & $47(2)$ & $34(1)$ & $9(1)$ & $-9(1)$ & $-2(1)$ \\
$\mathrm{C}(47 \mathrm{~B})$ & $48(1)$ & $47(2)$ & $34(1)$ & $9(1)$ & $-9(1)$ & $-2(1)$ \\
$\mathrm{B}(1)$ & $40(2)$ & $42(2)$ & $30(2)$ & $6(2)$ & $-11(2)$ & $0(2)$ \\
$\mathrm{N}(1)$ & $41(2)$ & $23(2)$ & $47(2)$ & $0(1)$ & $-19(2)$ & $-3(1)$ \\
$\mathrm{N}(2)$ & $34(2)$ & $25(2)$ & $48(2)$ & $9(1)$ & $-14(2)$ & $-8(1)$ \\
$\mathrm{O}(1)$ & $47(2)$ & $70(2)$ & $50(2)$ & $-16(2)$ & $-17(2)$ & $10(2)$ \\
$\mathrm{O}(2)$ & $47(2)$ & $70(2)$ & $50(2)$ & $-16(2)$ & $-17(2)$ & $10(2)$ \\
$\mathrm{O}(1 \mathrm{~B})$ & $147(10)$ & $58(5)$ & $44(5)$ & $20(4)$ & $-48(6)$ & $-57(6)$ \\
$\mathrm{O}(2 \mathrm{~B})$ & $147(10)$ & $58(5)$ & $44(5)$ & $20(4)$ & $-48(6)$ & $-57(6)$ \\
$\mathrm{P}(1)$ & $27(1)$ & $24(1)$ & $22(1)$ & $2(1)$ & $2(1)$ & $-1(1)$ \\
$\mathrm{Cl}(1)$ & $43(1)$ & $31(1)$ & $33(1)$ & $-2(1)$ & $9(1)$ & $-1(1)$ \\
$\mathrm{Cl}(2)$ & $28(1)$ & $35(1)$ & $49(1)$ & $8(1)$ & $1(1)$ & $-7(1)$ \\
$\mathrm{Ru}(1)$ & $27(1)$ & $23(1)$ & $25(1)$ & $3(1)$ & $-4(1)$ & $-4(1)$ \\
\hline
\end{tabular}

Table S24. Hydrogen atomic coordinates $\left(\mathrm{x}^{4} 0^{4}\right)$ and isotropic atomic displacement parameters $\left(\AA^{2} \times 10^{3}\right)$ for $\mathrm{C}_{47} \mathrm{H}_{72} \mathrm{BCl}_{2} \mathrm{~N}_{2} \mathrm{O}_{2} \mathrm{PRu}$

$\begin{array}{lllll}\mathrm{H}(2 \mathrm{~A}) & 6718 & 2247 & 4771 & 63 \\ \mathrm{H}(2 \mathrm{~B}) & 6406 & 2352 & 5580 & 63 \\ \mathrm{H}(3 \mathrm{~A}) & 6075 & 2466 & 4117 & 59 \\ \mathrm{H}(3 \mathrm{~B}) & 6410 & 2438 & 3340 & 59 \\ \mathrm{H}(6) & 7102 & 1376 & 7481 & 95 \\ \mathrm{H}(8) & 6148 & 1521 & 8553 & 86 \\ \mathrm{H}(10 \mathrm{~A}) & 7166 & 1852 & 5425 & 98 \\ \mathrm{H}(10 \mathrm{~B}) & 6974 & 1582 & 4643 & 98 \\ \mathrm{H}(10 \mathrm{C}) & 7253 & 1456 & 5528 & 98 \\ \mathrm{H}(11 \mathrm{~A}) & 6813 & 1047 & 9246 & 232 \\ \mathrm{H}(11 \mathrm{~B}) & 6537 & 1289 & 9824 & 232 \\ \mathrm{H}(11 \mathrm{C}) & 6918 & 1420 & 9657 & 232 \\ \mathrm{H}(12 \mathrm{~A}) & 5902 & 2071 & 6560 & 74 \\ \mathrm{H}(12 \mathrm{~B}) & 5753 & 1820 & 7499 & 74 \\ \mathrm{H}(12 \mathrm{C}) & 5755 & 1708 & 6203 & 74 \\ \mathrm{H}(15) & 5104 & 2059 & 2044 & 42 \\ \mathrm{H}(17) & 5871 & 2044 & -181 & 52 \\ \mathrm{H}(19 \mathrm{~A}) & 5204 & 2065 & 4024 & 59 \\ \mathrm{H}(19 \mathrm{~B}) & 5582 & 2116 & 4483 & 59 \\ \mathrm{H}(19 \mathrm{C}) & 5445 & 1743 & 4212 & 59 \\ \mathrm{H}(20 \mathrm{~A}) & 5289 & 2184 & -795 & 74 \\ \mathrm{H}(20 \mathrm{~B}) & 4984 & 2157 & 92 & 74 \\ \mathrm{H}(20 \mathrm{C}) & 5151 & 1820 & -415 & 74\end{array}$




\begin{tabular}{|c|c|c|c|c|}
\hline $\mathrm{H}(21 \mathrm{~A})$ & 6514 & 2209 & 1469 & 90 \\
\hline $\mathrm{H}(21 \mathrm{~B})$ & 6439 & 1981 & 379 & 90 \\
\hline $\mathrm{H}(21 \mathrm{C})$ & 6505 & 1804 & 1573 & 90 \\
\hline $\mathrm{H}(23)$ & 5539 & 1425 & 1939 & 60 \\
\hline $\mathrm{H}(26 \mathrm{~A})$ & 4849 & 609 & -106 & 146 \\
\hline $\mathrm{H}(26 \mathrm{~B})$ & 4872 & 619 & -1453 & 146 \\
\hline $\mathrm{H}(26 \mathrm{C})$ & 4719 & 936 & -778 & 146 \\
\hline $\mathrm{H}(27 \mathrm{~A})$ & 5073 & 1273 & -1840 & 146 \\
\hline $\mathrm{H}(27 \mathrm{~B})$ & 5335 & 1007 & -2371 & 146 \\
\hline $\mathrm{H}(27 \mathrm{C})$ & 5468 & 1295 & -1530 & 146 \\
\hline $\mathrm{H}(28 \mathrm{~A})$ & 5833 & 798 & -1906 & 146 \\
\hline $\mathrm{H}(28 \mathrm{~B})$ & 5561 & 517 & -2271 & 146 \\
\hline $\mathrm{H}(28 \mathrm{C})$ & 5887 & 410 & -1547 & 146 \\
\hline H(29A) & 5622 & 156 & -19 & 146 \\
\hline $\mathrm{H}(29 \mathrm{~B})$ & 5280 & 176 & -747 & 146 \\
\hline $\mathrm{H}(29 \mathrm{C})$ & 5286 & 318 & 517 & 146 \\
\hline $\mathrm{H}(26 \mathrm{D})$ & 4925 & 382 & 578 & 145 \\
\hline $\mathrm{H}(26 \mathrm{E})$ & 4863 & 456 & -732 & 145 \\
\hline $\mathrm{H}(26 \mathrm{~F})$ & 4860 & 762 & 154 & 145 \\
\hline $\mathrm{H}(27 \mathrm{D})$ & 5815 & 531 & -996 & 145 \\
\hline $\mathrm{H}(27 \mathrm{E})$ & 5512 & 374 & -1724 & 145 \\
\hline $\mathrm{H}(27 \mathrm{~F})$ & 5650 & 176 & -640 & 145 \\
\hline $\mathrm{H}(28 \mathrm{D})$ & 5753 & 1229 & -1425 & 145 \\
\hline $\mathrm{H}(28 \mathrm{E})$ & 5672 & 954 & -2380 & 145 \\
\hline $\mathrm{H}(28 \mathrm{~F})$ & 5879 & 846 & -1276 & 145 \\
\hline H(29D) & 4844 & 1025 & -811 & 145 \\
\hline $\mathrm{H}(29 \mathrm{E})$ & 4962 & 981 & -2093 & 145 \\
\hline $\mathrm{H}(29 \mathrm{~F})$ & 5062 & 1316 & -1408 & 145 \\
\hline $\mathrm{H}(30)$ & 5722 & 446 & 4739 & 33 \\
\hline $\mathrm{H}(31 \mathrm{~A})$ & 5694 & 261 & 2427 & 47 \\
\hline $\mathrm{H}(31 \mathrm{~B})$ & 5558 & 614 & 2923 & 47 \\
\hline $\mathrm{H}(32 \mathrm{~A})$ & 5144 & 331 & 4000 & 52 \\
\hline $\mathrm{H}(32 \mathrm{~B})$ & 5104 & 216 & 2711 & 52 \\
\hline $\mathrm{H}(33 \mathrm{~A})$ & 5385 & -298 & 3076 & 50 \\
\hline $\mathrm{H}(33 \mathrm{~B})$ & 5077 & -261 & 3953 & 50 \\
\hline $\mathrm{H}(34 \mathrm{~A})$ & 5455 & -76 & 5375 & 47 \\
\hline $\mathrm{H}(34 \mathrm{~B})$ & 5583 & -435 & 4901 & 47 \\
\hline $\mathrm{H}(35 \mathrm{~A})$ & 5992 & -158 & 3758 & 44 \\
\hline $\mathrm{H}(35 \mathrm{~B})$ & 6048 & -52 & 5050 & 44 \\
\hline $\mathrm{H}(36)$ & 6387 & 172 & 2705 & 63 \\
\hline
\end{tabular}




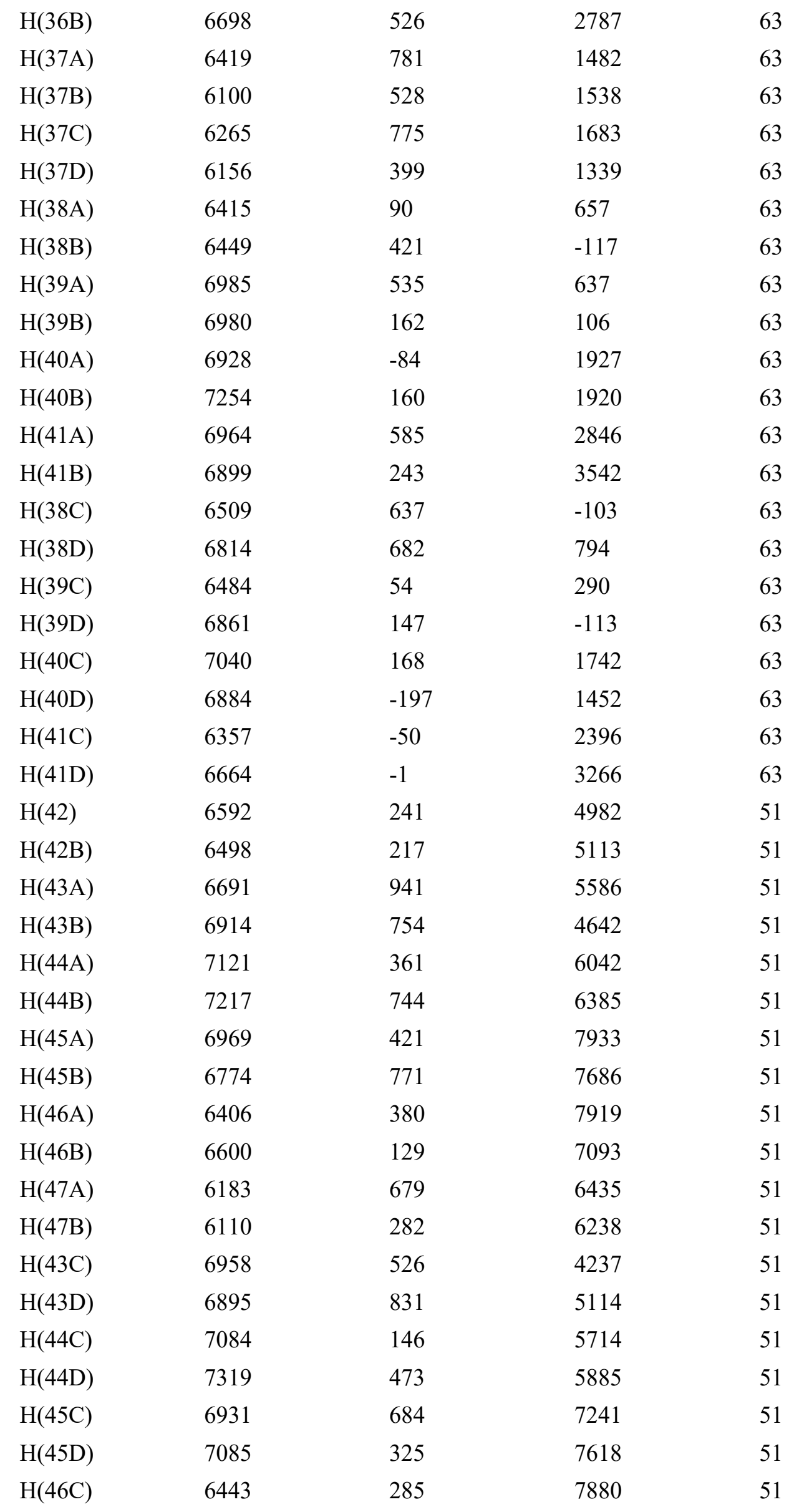




\begin{tabular}{lllll}
$\mathrm{H}(46 \mathrm{D})$ & 6532 & 38 & 6826 & 51 \\
$\mathrm{H}(47 \mathrm{C})$ & 6105 & 529 & 6217 & 51 \\
$\mathrm{H}(47 \mathrm{D})$ & 6412 & 792 & 6422 & 51 \\
\hline
\end{tabular}

\subsection{X-ray structure of Ru-carbide}

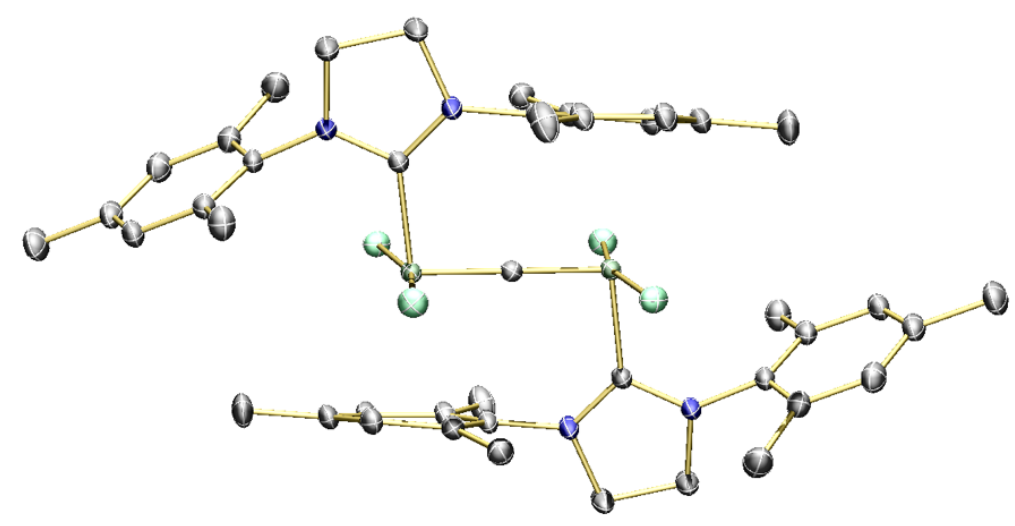

\begin{tabular}{|c|c|c|}
\hline Identification code & \multicolumn{2}{|l|}{ ahsg200701 } \\
\hline Chemical formula & \multicolumn{2}{|l|}{$\mathrm{C}_{43} \mathrm{H}_{52} \mathrm{Cl}_{4} \mathrm{~N}_{4} \mathrm{Ru}_{2}$} \\
\hline Formula weight & \multicolumn{2}{|l|}{$968.82 \mathrm{~g} / \mathrm{mol}$} \\
\hline Temperature & \multicolumn{2}{|l|}{$120(2) \mathrm{K}$} \\
\hline Wavelength & \multicolumn{2}{|l|}{$0.71073 \AA$} \\
\hline Crystal size & \multicolumn{2}{|c|}{$0.200 \times 0.180 \times 0.150 \mathrm{~mm}$} \\
\hline Crystal system & \multicolumn{2}{|l|}{ monoclinic } \\
\hline Space group & \multicolumn{2}{|l|}{ P $21 / c$} \\
\hline \multirow[t]{3}{*}{ Unit cell dimensions } & $a=10.8319(5) \AA$ & $\alpha=90^{\circ}$ \\
\hline & $\mathrm{b}=13.4429(6) \AA$ & $\beta=107.249(2)^{\circ}$ \\
\hline & $\mathrm{c}=14.6488(7) \AA$ & $\gamma=90^{\circ}$ \\
\hline Volume & \multicolumn{2}{|l|}{ 2037.11(16) $\AA^{3}$} \\
\hline $\mathrm{Z}$ & \multicolumn{2}{|l|}{2} \\
\hline Density (calculated) & \multicolumn{2}{|l|}{$1.579 \mathrm{~g} / \mathrm{cm}^{3}$} \\
\hline Absorption coefficient & \multicolumn{2}{|l|}{$1.040 \mathrm{~mm}^{-1}$} \\
\hline $\mathrm{F}(000)$ & \multicolumn{2}{|l|}{988} \\
\hline
\end{tabular}

Table S26. Data collection and structure refinement for $\mathrm{C}_{43} \mathrm{H}_{52} \mathrm{Cl}_{4} \mathrm{~N}_{4} \mathrm{Ru} \mathrm{u}_{2}$

Theta range for data collection

Index ranges

Reflections collected

Independent reflections
1.969 to $30.061^{\circ}$

$-15<=\mathrm{h}<=15,-18<=\mathrm{k}<=18,-20<=1<=20$

77207

$5975[\mathrm{R}(\mathrm{int})=0.0257]$ 
Completeness to theta $=25.242 \quad 100.0 \%$

Absorption correction

Semi-empirical from equivalents

Max. and min. transmission

0.7460 and 0.7140

Refinement method

Full-matrix least-squares on $\mathrm{F}^{2}$

Data / restraints / parameters 5975 / 0 / 247

Goodness-of-fit on $\mathrm{F}^{2}$

1.128

Final R indices

$\mathrm{I}>2 \sigma(\mathrm{I})$

$\mathrm{R} 1=0.0259, \mathrm{wR} 2=0.070 \mathrm{c}$

all data

$\mathrm{R} 1=0.0270, \mathrm{wR} 2=0.070$

Largest diff. peak and hole

2.341 and $-0.720 \mathrm{e}^{-3}$

Table $S 27$. Atomic coordinates $\left(\mathrm{x10}^{4}\right)$ and equivalent isotropic atomic displacement parameters $\left(\AA^{2}\right)$ for for $\mathrm{C}_{43} \mathrm{H}_{52} \mathrm{Cl}_{4} \mathrm{~N}_{4} \mathrm{Ru}_{2}$

$\mathrm{U}(\mathrm{eq})$ is defined as one third of the trace of the orthogonalized $\mathrm{U}_{\mathrm{ij}}$ tensor.

\begin{tabular}{|c|c|c|c|c|}
\hline & $\mathbf{x} / \mathbf{a}$ & $\mathbf{y} / \mathbf{b}$ & $\mathbf{z} / \mathbf{c}$ & $\mathrm{U}(\mathrm{eq})$ \\
\hline$C(1)$ & $6446(2)$ & $3910(1)$ & $6621(1)$ & $16(1)$ \\
\hline$C(2)$ & $7181(2)$ & $2875(2)$ & $7966(2)$ & $29(1)$ \\
\hline$C(3)$ & $5735(2)$ & $2708(2)$ & $7518(2)$ & $32(1)$ \\
\hline$C(4)$ & $8666(2)$ & $4216(1)$ & $7719(1)$ & $19(1)$ \\
\hline$C(5)$ & $9733(2)$ & $3897(1)$ & $7431(1)$ & $20(1)$ \\
\hline$C(6)$ & 10901(2) & $4407(2)$ & $7797(1)$ & $23(1)$ \\
\hline$C(7)$ & $11040(2)$ & $5180(1)$ & $8445(1)$ & $24(1)$ \\
\hline$C(8)$ & $9985(2)$ & $5442(2)$ & $8752(1)$ & $26(1)$ \\
\hline$C(9)$ & $8794(2)$ & $4966(2)$ & $8404(1)$ & $23(1)$ \\
\hline$C(10)$ & $9699(2)$ & $3003(2)$ & $6813(2)$ & $28(1)$ \\
\hline $\mathrm{C}(11)$ & $12320(2)$ & $5708(2)$ & $8827(2)$ & $31(1)$ \\
\hline$C(12)$ & $7704(2)$ & $5253(2)$ & $8795(2)$ & $32(1)$ \\
\hline$C(13)$ & $4169(2)$ & $3334(1)$ & $6002(1)$ & $19(1)$ \\
\hline$C(14)$ & $3702(2)$ & $2530(1)$ & $5402(1)$ & $22(1)$ \\
\hline$C(15)$ & $2404(2)$ & $2542(1)$ & $4844(1)$ & $22(1)$ \\
\hline$C(16)$ & $1608(2)$ & $3352(2)$ & $4831(1)$ & $22(1)$ \\
\hline$C(17)$ & $2118(2)$ & $4171(1)$ & $5417(1)$ & $22(1)$ \\
\hline$C(18)$ & $3384(2)$ & $4154(1)$ & $6050(1)$ & $20(1)$ \\
\hline$C(19)$ & $4563(2)$ & $1686(2)$ & $5310(2)$ & $33(1)$ \\
\hline
\end{tabular}




\begin{tabular}{lcccc}
\hline & $\mathbf{x} / \mathbf{a}$ & $\mathbf{y} / \mathbf{b}$ & $\mathbf{z} / \mathbf{c}$ & $\mathbf{U}(\mathbf{e q})$ \\
\hline $\mathrm{C}(20)$ & $253(2)$ & $3379(2)$ & $4166(2)$ & $31(1)$ \\
$\mathrm{C}(21)$ & $3823(2)$ & $4910(2)$ & $6839(1)$ & $24(1)$ \\
$\mathrm{C}(22)$ & 5000 & 5000 & 5000 & $16(1)$ \\
$\mathrm{N}(1)$ & $7450(1)$ & $3701(1)$ & $7393(1)$ & $20(1)$ \\
$\mathrm{N}(2)$ & $5459(1)$ & $3306(1)$ & $6635(1)$ & $21(1)$ \\
$\mathrm{Cl}(1)$ & $6724(1)$ & $6233(1)$ & $6637(1)$ & $22(1)$ \\
$\mathrm{Cl}(2)$ & $7103(1)$ & $3433(1)$ & $4843(1)$ & $23(1)$ \\
$\mathrm{Ru}(1)$ & $6642(1)$ & $4854(1)$ & $5629(1)$ & $15(1)$ \\
\hline
\end{tabular}

Table S28. Bond lengths ( $(\AA)$ for $\mathrm{C}_{43} \mathrm{H}_{52} \mathrm{Cl}_{4} \mathrm{~N}_{4} \mathrm{Ru}_{2}$

\begin{tabular}{|c|c|c|c|}
\hline $\mathrm{C}(1)-\mathrm{N}(1)$ & $1.345(2)$ & $\mathrm{C}(12)-\mathrm{H}(12 \mathrm{~A})$ & 0.98 \\
\hline $\mathrm{C}(1)-\mathrm{N}(2)$ & $1.347(2)$ & $\mathrm{C}(12)-\mathrm{H}(12 \mathrm{~B})$ & 0.98 \\
\hline $\mathrm{C}(1)-\mathrm{Ru}(1)$ & $1.9880(17)$ & $\mathrm{C}(12)-\mathrm{H}(12 \mathrm{C})$ & 0.98 \\
\hline $\mathrm{C}(2)-\mathrm{N}(1)$ & $1.473(2)$ & $C(13)-C(14)$ & $1.389(3)$ \\
\hline$C(2)-C(3)$ & $1.524(3)$ & $C(13)-C(18)$ & $1.406(3)$ \\
\hline $\mathrm{C}(2)-\mathrm{H}(2 \mathrm{~A})$ & 0.99 & $\mathrm{C}(13)-\mathrm{N}(2)$ & $1.431(2)$ \\
\hline $\mathrm{C}(2)-\mathrm{H}(2 \mathrm{~B})$ & 0.99 & $C(14)-C(15)$ & $1.401(2)$ \\
\hline$C(3)-N(2)$ & $1.476(2)$ & $C(14)-C(19)$ & $1.500(3)$ \\
\hline $\mathrm{C}(3)-\mathrm{H}(3 \mathrm{~A})$ & 0.99 & $C(15)-C(16)$ & $1.386(3)$ \\
\hline $\mathrm{C}(3)-\mathrm{H}(3 \mathrm{~B})$ & 0.99 & $\mathrm{C}(15)-\mathrm{H}(15)$ & 0.95 \\
\hline$C(4)-C(9)$ & $1.400(3)$ & $C(16)-C(17)$ & $1.405(3)$ \\
\hline$C(4)-C(5)$ & $1.409(2)$ & $C(16)-C(20)$ & $1.503(3)$ \\
\hline $\mathrm{C}(4)-\mathrm{N}(1)$ & $1.439(2)$ & $C(17)-C(18)$ & $1.409(2)$ \\
\hline$C(5)-C(6)$ & $1.398(2)$ & $\mathrm{C}(17)-\mathrm{H}(17)$ & 0.95 \\
\hline$C(5)-C(10)$ & $1.499(3)$ & $\mathrm{C}(18)-\mathrm{C}(21)$ & $1.508(3)$ \\
\hline$C(6)-C(7)$ & $1.385(3)$ & $\mathrm{C}(19)-\mathrm{H}(19 \mathrm{~A})$ & 0.98 \\
\hline $\mathrm{C}(6)-\mathrm{H}(6)$ & 0.95 & $\mathrm{C}(19)-\mathrm{H}(19 \mathrm{~B})$ & 0.98 \\
\hline$C(7)-C(8)$ & $1.392(3)$ & $\mathrm{C}(19)-\mathrm{H}(19 \mathrm{C})$ & 0.98 \\
\hline$C(7)-C(11)$ & $1.510(3)$ & $C(20)-H(20 A)$ & 0.98 \\
\hline $\mathrm{C}(8)-\mathrm{C}(9)$ & $1.394(3)$ & $\mathrm{C}(20)-\mathrm{H}(20 \mathrm{~B})$ & 0.98 \\
\hline $\mathrm{C}(8)-\mathrm{H}(8)$ & 0.95 & $\mathrm{C}(20)-\mathrm{H}(20 \mathrm{C})$ & 0.98 \\
\hline
\end{tabular}




\begin{tabular}{llll}
$\mathrm{C}(9)-\mathrm{C}(12)$ & $1.506(3)$ & $\mathrm{C}(21)-\mathrm{H}(21 \mathrm{~A})$ & 0.98 \\
$\mathrm{C}(10)-\mathrm{H}(10 \mathrm{~A})$ & 0.98 & $\mathrm{C}(21)-\mathrm{H}(21 \mathrm{~B})$ & 0.98 \\
$\mathrm{C}(10)-\mathrm{H}(10 \mathrm{~B})$ & 0.98 & $\mathrm{C}(21)-\mathrm{H}(21 \mathrm{C})$ & 0.98 \\
$\mathrm{C}(10)-\mathrm{H}(10 \mathrm{C})$ & 0.98 & $\mathrm{C}(22)-\mathrm{Ru}(1) \# 1$ & $1.75440(15)$ \\
$\mathrm{C}(11)-\mathrm{H}(11 \mathrm{~A})$ & 0.98 & $\mathrm{C}(22)-\mathrm{Ru}(1)$ & $1.75441(15)$ \\
$\mathrm{C}(11)-\mathrm{H}(11 \mathrm{~B})$ & 0.98 & $\mathrm{Cl}(1)-\mathrm{Ru}(1)$ & $2.3540(4)$ \\
$\mathrm{C}(11)-\mathrm{H}(11 \mathrm{C})$ & 0.98 & $\mathrm{Cl}(2)-\mathrm{Ru}(1)$ & $2.3603(5)$ \\
\hline
\end{tabular}

Table S29. Bond angles $\left({ }^{\circ}\right.$ ) for $\mathrm{C}_{43} \mathrm{H}_{52} \mathrm{Cl}_{4} \mathrm{~N}_{4} \mathrm{Ru}_{2}$

\begin{tabular}{|c|c|c|c|}
\hline $\mathrm{N}(1)-\mathrm{C}(1)-\mathrm{N}(2)$ & $108.02(15)$ & $\mathrm{H}(12 \mathrm{~B})-\mathrm{C}(12)-\mathrm{H}(12 \mathrm{C})$ & 109.5 \\
\hline $\mathrm{N}(1)-\mathrm{C}(1)-\mathrm{Ru}(1)$ & $120.85(12)$ & $\mathrm{C}(14)-\mathrm{C}(13)-\mathrm{C}(18)$ & $121.93(16)$ \\
\hline $\mathrm{N}(2)-\mathrm{C}(1)-\mathrm{Ru}(1)$ & $130.73(12)$ & $\mathrm{C}(14)-\mathrm{C}(13)-\mathrm{N}(2)$ & $119.45(16)$ \\
\hline $\mathrm{N}(1)-\mathrm{C}(2)-\mathrm{C}(3)$ & $102.49(14)$ & $\mathrm{C}(18)-\mathrm{C}(13)-\mathrm{N}(2)$ & $118.50(16)$ \\
\hline $\mathrm{N}(1)-\mathrm{C}(2)-\mathrm{H}(2 \mathrm{~A})$ & 111.3 & $C(13)-C(14)-C(15)$ & $118.32(17)$ \\
\hline $\mathrm{C}(3)-\mathrm{C}(2)-\mathrm{H}(2 \mathrm{~A})$ & 111.3 & $C(13)-C(14)-C(19)$ & $121.73(17)$ \\
\hline $\mathrm{N}(1)-\mathrm{C}(2)-\mathrm{H}(2 \mathrm{~B})$ & 111.3 & $\mathrm{C}(15)-\mathrm{C}(14)-\mathrm{C}(19)$ & $119.90(18)$ \\
\hline $\mathrm{C}(3)-\mathrm{C}(2)-\mathrm{H}(2 \mathrm{~B})$ & 111.3 & $\mathrm{C}(16)-\mathrm{C}(15)-\mathrm{C}(14)$ & $121.92(18)$ \\
\hline $\mathrm{H}(2 \mathrm{~A})-\mathrm{C}(2)-\mathrm{H}(2 \mathrm{~B})$ & 109.2 & $\mathrm{C}(16)-\mathrm{C}(15)-\mathrm{H}(15)$ & 119 \\
\hline $\mathrm{N}(2)-\mathrm{C}(3)-\mathrm{C}(2)$ & $102.30(14)$ & $\mathrm{C}(14)-\mathrm{C}(15)-\mathrm{H}(15)$ & 119 \\
\hline $\mathrm{N}(2)-\mathrm{C}(3)-\mathrm{H}(3 \mathrm{~A})$ & 111.3 & $\mathrm{C}(15)-\mathrm{C}(16)-\mathrm{C}(17)$ & $118.55(16)$ \\
\hline $\mathrm{C}(2)-\mathrm{C}(3)-\mathrm{H}(3 \mathrm{~A})$ & 111.3 & $C(15)-C(16)-C(20)$ & $120.76(18)$ \\
\hline $\mathrm{N}(2)-\mathrm{C}(3)-\mathrm{H}(3 \mathrm{~B})$ & 111.3 & $\mathrm{C}(17)-\mathrm{C}(16)-\mathrm{C}(20)$ & $120.60(18)$ \\
\hline $\mathrm{C}(2)-\mathrm{C}(3)-\mathrm{H}(3 \mathrm{~B})$ & 111.3 & $\mathrm{C}(16)-\mathrm{C}(17)-\mathrm{C}(18)$ & $121.20(17)$ \\
\hline $\mathrm{H}(3 \mathrm{~A})-\mathrm{C}(3)-\mathrm{H}(3 \mathrm{~B})$ & 109.2 & $\mathrm{C}(16)-\mathrm{C}(17)-\mathrm{H}(17)$ & 119.4 \\
\hline $\mathrm{C}(9)-\mathrm{C}(4)-\mathrm{C}(5)$ & $121.22(16)$ & $\mathrm{C}(18)-\mathrm{C}(17)-\mathrm{H}(17)$ & 119.4 \\
\hline $\mathrm{C}(9)-\mathrm{C}(4)-\mathrm{N}(1)$ & $118.05(16)$ & $\mathrm{C}(13)-\mathrm{C}(18)-\mathrm{C}(17)$ & $117.72(17)$ \\
\hline $\mathrm{C}(5)-\mathrm{C}(4)-\mathrm{N}(1)$ & $120.38(17)$ & $\mathrm{C}(13)-\mathrm{C}(18)-\mathrm{C}(21)$ & $120.60(16)$ \\
\hline$C(6)-C(5)-C(4)$ & $117.76(17)$ & $\mathrm{C}(17)-\mathrm{C}(18)-\mathrm{C}(21)$ & $121.16(17)$ \\
\hline$C(6)-C(5)-C(10)$ & $118.75(17)$ & $\mathrm{C}(14)-\mathrm{C}(19)-\mathrm{H}(19 \mathrm{~A})$ & 109.5 \\
\hline$C(4)-C(5)-C(10)$ & $123.35(16)$ & $\mathrm{C}(14)-\mathrm{C}(19)-\mathrm{H}(19 \mathrm{~B})$ & 109.5 \\
\hline$C(7)-C(6)-C(5)$ & $122.12(17)$ & $\mathrm{H}(19 \mathrm{~A})-\mathrm{C}(19)-\mathrm{H}(19 \mathrm{~B})$ & 109.5 \\
\hline $\mathrm{C}(7)-\mathrm{C}(6)-\mathrm{H}(6)$ & 118.9 & $\mathrm{C}(14)-\mathrm{C}(19)-\mathrm{H}(19 \mathrm{C})$ & 109.5 \\
\hline $\mathrm{C}(5)-\mathrm{C}(6)-\mathrm{H}(6)$ & 118.9 & $\mathrm{H}(19 \mathrm{~A})-\mathrm{C}(19)-\mathrm{H}(19 \mathrm{C})$ & 109.5 \\
\hline
\end{tabular}




\begin{tabular}{|c|c|c|c|}
\hline $\mathrm{C}(6)-\mathrm{C}(7)-\mathrm{C}(8)$ & $118.63(17)$ & $\mathrm{H}(19 \mathrm{~B})-\mathrm{C}(19)-\mathrm{H}(19 \mathrm{C})$ & 109.5 \\
\hline$C(6)-C(7)-C(11)$ & $120.52(19)$ & $\mathrm{C}(16)-\mathrm{C}(20)-\mathrm{H}(20 \mathrm{~A})$ & 109.5 \\
\hline$C(8)-C(7)-C(11)$ & $120.83(19)$ & $\mathrm{C}(16)-\mathrm{C}(20)-\mathrm{H}(20 \mathrm{~B})$ & 109.5 \\
\hline$C(7)-C(8)-C(9)$ & $121.64(19)$ & $\mathrm{H}(20 \mathrm{~A})-\mathrm{C}(20)-\mathrm{H}(20 \mathrm{~B})$ & 109.5 \\
\hline $\mathrm{C}(7)-\mathrm{C}(8)-\mathrm{H}(8)$ & 119.2 & $C(16)-C(20)-H(20 C)$ & 109.5 \\
\hline $\mathrm{C}(9)-\mathrm{C}(8)-\mathrm{H}(8)$ & 119.2 & $\mathrm{H}(20 \mathrm{~A})-\mathrm{C}(20)-\mathrm{H}(20 \mathrm{C})$ & 109.5 \\
\hline $\mathrm{C}(8)-\mathrm{C}(9)-\mathrm{C}(4)$ & $118.46(18)$ & $\mathrm{H}(20 \mathrm{~B})-\mathrm{C}(20)-\mathrm{H}(20 \mathrm{C})$ & 109.5 \\
\hline $\mathrm{C}(8)-\mathrm{C}(9)-\mathrm{C}(12)$ & $119.16(19)$ & $\mathrm{C}(18)-\mathrm{C}(21)-\mathrm{H}(21 \mathrm{~A})$ & 109.5 \\
\hline $\mathrm{C}(4)-\mathrm{C}(9)-\mathrm{C}(12)$ & $122.36(18)$ & $\mathrm{C}(18)-\mathrm{C}(21)-\mathrm{H}(21 \mathrm{~B})$ & 109.5 \\
\hline $\mathrm{C}(5)-\mathrm{C}(10)-\mathrm{H}(10 \mathrm{~A})$ & 109.5 & $\mathrm{H}(21 \mathrm{~A})-\mathrm{C}(21)-\mathrm{H}(21 \mathrm{~B})$ & 109.5 \\
\hline $\mathrm{C}(5)-\mathrm{C}(10)-\mathrm{H}(10 \mathrm{~B})$ & 109.5 & $\mathrm{C}(18)-\mathrm{C}(21)-\mathrm{H}(21 \mathrm{C})$ & 109.5 \\
\hline $\mathrm{H}(10 \mathrm{~A})-\mathrm{C}(10)-\mathrm{H}(10 \mathrm{~B})$ & 109.5 & $\mathrm{H}(21 \mathrm{~A})-\mathrm{C}(21)-\mathrm{H}(21 \mathrm{C})$ & 109.5 \\
\hline $\mathrm{C}(5)-\mathrm{C}(10)-\mathrm{H}(10 \mathrm{C})$ & 109.5 & $\mathrm{H}(21 \mathrm{~B})-\mathrm{C}(21)-\mathrm{H}(21 \mathrm{C})$ & 109.5 \\
\hline $\mathrm{H}(10 \mathrm{~A})-\mathrm{C}(10)-\mathrm{H}(10 \mathrm{C})$ & 109.5 & $\mathrm{Ru}(1) \# 1-\mathrm{C}(22)-\mathrm{Ru}(1)$ & 180 \\
\hline $\mathrm{H}(10 \mathrm{~B})-\mathrm{C}(10)-\mathrm{H}(10 \mathrm{C})$ & 109.5 & $\mathrm{C}(1)-\mathrm{N}(1)-\mathrm{C}(4)$ & $127.69(15)$ \\
\hline $\mathrm{C}(7)-\mathrm{C}(11)-\mathrm{H}(11 \mathrm{~A})$ & 109.5 & $\mathrm{C}(1)-\mathrm{N}(1)-\mathrm{C}(2)$ & $112.92(14)$ \\
\hline $\mathrm{C}(7)-\mathrm{C}(11)-\mathrm{H}(11 \mathrm{~B})$ & 109.5 & $\mathrm{C}(4)-\mathrm{N}(1)-\mathrm{C}(2)$ & $119.30(14)$ \\
\hline $\mathrm{H}(11 \mathrm{~A})-\mathrm{C}(11)-\mathrm{H}(11 \mathrm{~B})$ & 109.5 & $\mathrm{C}(1)-\mathrm{N}(2)-\mathrm{C}(13)$ & $127.02(15)$ \\
\hline $\mathrm{C}(7)-\mathrm{C}(11)-\mathrm{H}(11 \mathrm{C})$ & 109.5 & $\mathrm{C}(1)-\mathrm{N}(2)-\mathrm{C}(3)$ & $112.65(15)$ \\
\hline $\mathrm{H}(11 \mathrm{~A})-\mathrm{C}(11)-\mathrm{H}(11 \mathrm{C})$ & 109.5 & $\mathrm{C}(13)-\mathrm{N}(2)-\mathrm{C}(3)$ & $119.17(14)$ \\
\hline $\mathrm{H}(11 \mathrm{~B})-\mathrm{C}(11)-\mathrm{H}(11 \mathrm{C})$ & 109.5 & $\mathrm{C}(22)-\mathrm{Ru}(1)-\mathrm{C}(1)$ & $98.29(5)$ \\
\hline $\mathrm{C}(9)-\mathrm{C}(12)-\mathrm{H}(12 \mathrm{~A})$ & 109.5 & $\mathrm{C}(22)-\mathrm{Ru}(1)-\mathrm{Cl}(1)$ & $94.909(12)$ \\
\hline $\mathrm{C}(9)-\mathrm{C}(12)-\mathrm{H}(12 \mathrm{~B})$ & 109.5 & $\mathrm{C}(1)-\mathrm{Ru}(1)-\mathrm{Cl}(1)$ & $92.11(5)$ \\
\hline $\mathrm{H}(12 \mathrm{~A})-\mathrm{C}(12)-\mathrm{H}(12 \mathrm{~B})$ & 109.5 & $\mathrm{C}(22)-\mathrm{Ru}(1)-\mathrm{Cl}(2)$ & $99.342(13)$ \\
\hline $\mathrm{C}(9)-\mathrm{C}(12)-\mathrm{H}(12 \mathrm{C})$ & 109.5 & $\mathrm{C}(1)-\mathrm{Ru}(1)-\mathrm{Cl}(2)$ & $85.61(5)$ \\
\hline $\mathrm{H}(12 \mathrm{~A})-\mathrm{C}(12)-\mathrm{H}(12 \mathrm{C})$ & 109.5 & $\mathrm{Cl}(1)-\mathrm{Ru}(1)-\mathrm{Cl}(2)$ & $165.747(17)$ \\
\hline
\end{tabular}

Symmetry transformations used to generate equivalent atoms: \#1 -x+1,-y+1,-z+1

Table S30. Torsion angles ( ${ }^{\circ}$ ) for $\mathrm{C}_{43} \mathrm{H}_{52} \mathrm{Cl}_{4} \mathrm{~N}_{4} \mathrm{Ru}_{2}$

\begin{tabular}{llll}
\hline $\mathrm{N}(1)-\mathrm{C}(2)-\mathrm{C}(3)-\mathrm{N}(2)$ & $11.9(2)$ & $\mathrm{C}(14)-\mathrm{C}(13)-\mathrm{C}(18)-\mathrm{C}(17)$ & $-4.4(3)$ \\
$\mathrm{C}(9)-\mathrm{C}(4)-\mathrm{C}(5)-\mathrm{C}(6)$ & $-4.9(3)$ & $\mathrm{N}(2)-\mathrm{C}(13)-\mathrm{C}(18)-\mathrm{C}(17)$ & $179.63(15)$ \\
$\mathrm{N}(1)-\mathrm{C}(4)-\mathrm{C}(5)-\mathrm{C}(6)$ & $-177.95(16)$ & $\mathrm{C}(14)-\mathrm{C}(13)-\mathrm{C}(18)-\mathrm{C}(21)$ & $167.51(17)$ \\
$\mathrm{C}(9)-\mathrm{C}(4)-\mathrm{C}(5)-\mathrm{C}(10)$ & $170.79(18)$ & $\mathrm{N}(2)-\mathrm{C}(13)-\mathrm{C}(18)-\mathrm{C}(21)$ & $-8.5(3)$ \\
$\mathrm{N}(1)-\mathrm{C}(4)-\mathrm{C}(5)-\mathrm{C}(10)$ & $-2.3(3)$ & $\mathrm{C}(16)-\mathrm{C}(17)-\mathrm{C}(18)-\mathrm{C}(13)$ & $6.8(3)$
\end{tabular}




\begin{tabular}{|c|c|c|c|}
\hline$C(4)-C(5)-C(6)-C(7)$ & $2.1(3)$ & $C(16)-C(17)-C(18)-C(21)$ & $-165.04(17)$ \\
\hline $\mathrm{C}(10)-\mathrm{C}(5)-\mathrm{C}(6)-\mathrm{C}(7)$ & $-173.79(18)$ & $\mathrm{N}(2)-\mathrm{C}(1)-\mathrm{N}(1)-\mathrm{C}(4)$ & $-174.02(17)$ \\
\hline $\mathrm{C}(5)-\mathrm{C}(6)-\mathrm{C}(7)-\mathrm{C}(8)$ & $1.2(3)$ & $\mathrm{Ru}(1)-\mathrm{C}(1)-\mathrm{N}(1)-\mathrm{C}(4)$ & $12.5(3)$ \\
\hline$C(5)-C(6)-C(7)-C(11)$ & $179.67(18)$ & $\mathrm{N}(2)-\mathrm{C}(1)-\mathrm{N}(1)-\mathrm{C}(2)$ & $2.5(2)$ \\
\hline $\mathrm{C}(6)-\mathrm{C}(7)-\mathrm{C}(8)-\mathrm{C}(9)$ & $-1.9(3)$ & $\mathrm{Ru}(1)-\mathrm{C}(1)-\mathrm{N}(1)-\mathrm{C}(2)$ & $-170.97(14)$ \\
\hline $\mathrm{C}(11)-\mathrm{C}(7)-\mathrm{C}(8)-\mathrm{C}(9)$ & $179.69(19)$ & $\mathrm{C}(9)-\mathrm{C}(4)-\mathrm{N}(1)-\mathrm{C}(1)$ & $93.9(2)$ \\
\hline$C(7)-C(8)-C(9)-C(4)$ & $-0.8(3)$ & $\mathrm{C}(5)-\mathrm{C}(4)-\mathrm{N}(1)-\mathrm{C}(1)$ & $-92.8(2)$ \\
\hline $\mathrm{C}(7)-\mathrm{C}(8)-\mathrm{C}(9)-\mathrm{C}(12)$ & $177.46(19)$ & $\mathrm{C}(9)-\mathrm{C}(4)-\mathrm{N}(1)-\mathrm{C}(2)$ & $-82.4(2)$ \\
\hline $\mathrm{C}(5)-\mathrm{C}(4)-\mathrm{C}(9)-\mathrm{C}(8)$ & $4.3(3)$ & $\mathrm{C}(5)-\mathrm{C}(4)-\mathrm{N}(1)-\mathrm{C}(2)$ & $90.9(2)$ \\
\hline $\mathrm{N}(1)-\mathrm{C}(4)-\mathrm{C}(9)-\mathrm{C}(8)$ & $177.52(17)$ & $\mathrm{C}(3)-\mathrm{C}(2)-\mathrm{N}(1)-\mathrm{C}(1)$ & $-9.6(2)$ \\
\hline $\mathrm{C}(5)-\mathrm{C}(4)-\mathrm{C}(9)-\mathrm{C}(12)$ & $-173.96(18)$ & $\mathrm{C}(3)-\mathrm{C}(2)-\mathrm{N}(1)-\mathrm{C}(4)$ & $167.29(18)$ \\
\hline $\mathrm{N}(1)-\mathrm{C}(4)-\mathrm{C}(9)-\mathrm{C}(12)$ & $-0.7(3)$ & $\mathrm{N}(1)-\mathrm{C}(1)-\mathrm{N}(2)-\mathrm{C}(13)$ & $173.74(18)$ \\
\hline $\mathrm{C}(18)-\mathrm{C}(13)-\mathrm{C}(14)-\mathrm{C}(15)$ & $-0.9(3)$ & $\mathrm{Ru}(1)-\mathrm{C}(1)-\mathrm{N}(2)-\mathrm{C}(13)$ & $-13.7(3)$ \\
\hline $\mathrm{N}(2)-\mathrm{C}(13)-\mathrm{C}(14)-\mathrm{C}(15)$ & $175.06(16)$ & $\mathrm{N}(1)-\mathrm{C}(1)-\mathrm{N}(2)-\mathrm{C}(3)$ & $6.3(2)$ \\
\hline$C(18)-C(13)-C(14)-C(19)$ & $176.28(19)$ & $\mathrm{Ru}(1)-\mathrm{C}(1)-\mathrm{N}(2)-\mathrm{C}(3)$ & $178.89(15)$ \\
\hline $\mathrm{N}(2)-\mathrm{C}(13)-\mathrm{C}(14)-\mathrm{C}(19)$ & $-7.7(3)$ & $\mathrm{C}(14)-\mathrm{C}(13)-\mathrm{N}(2)-\mathrm{C}(1)$ & $116.0(2)$ \\
\hline$C(13)-C(14)-C(15)-C(16)$ & $4.1(3)$ & $\mathrm{C}(18)-\mathrm{C}(13)-\mathrm{N}(2)-\mathrm{C}(1)$ & $-67.9(3)$ \\
\hline$C(19)-C(14)-C(15)-C(16)$ & $-173.17(19)$ & $\mathrm{C}(14)-\mathrm{C}(13)-\mathrm{N}(2)-\mathrm{C}(3)$ & $-77.2(2)$ \\
\hline $\mathrm{C}(14)-\mathrm{C}(15)-\mathrm{C}(16)-\mathrm{C}(17)$ & $-1.7(3)$ & $\mathrm{C}(18)-\mathrm{C}(13)-\mathrm{N}(2)-\mathrm{C}(3)$ & $98.9(2)$ \\
\hline$C(14)-C(15)-C(16)-C(20)$ & $174.93(18)$ & $\mathrm{C}(2)-\mathrm{C}(3)-\mathrm{N}(2)-\mathrm{C}(1)$ & $-11.8(2)$ \\
\hline$C(15)-C(16)-C(17)-C(18)$ & $-3.8(3)$ & $\mathrm{C}(2)-\mathrm{C}(3)-\mathrm{N}(2)-\mathrm{C}(13)$ & $179.65(18)$ \\
\hline $\mathrm{C}(20)-\mathrm{C}(16)-\mathrm{C}(17)-\mathrm{C}(18)$ & $179.49(18)$ & & \\
\hline
\end{tabular}

\section{Table S31. Anisotropic atomic displacement parameters $\left(\AA^{2} \times 10^{3}\right)$ for $\mathrm{C}_{43} \mathrm{H}_{52} \mathrm{Cl}_{4} \mathrm{~N}_{4} \mathrm{Ru}_{2}$}

The anisotropic atomic displacement factor exponent takes the form: $-2 \pi^{2}\left[\mathrm{~h}^{2} \mathrm{a}^{* 2} \mathrm{U}_{11}+\ldots+2 \mathrm{~h} \mathrm{k} \mathrm{a}^{*} \mathrm{~b}^{*} \mathrm{U}_{12}\right]$

\begin{tabular}{lcccccc}
\hline & $\mathbf{U}_{\mathbf{1 1}}$ & $\mathbf{U}_{\mathbf{2 2}}$ & $\mathbf{U}_{\mathbf{3 3}}$ & $\mathbf{U}_{\mathbf{2 3}}$ & $\mathbf{U}_{\mathbf{1 3}}$ & $\mathbf{U}_{\mathbf{1 2}}$ \\
\hline $\mathrm{C}(1)$ & $13(1)$ & $18(1)$ & $18(1)$ & $2(1)$ & $4(1)$ & $-1(1)$ \\
$\mathrm{C}(2)$ & $19(1)$ & $34(1)$ & $29(1)$ & $17(1)$ & $1(1)$ & $-4(1)$ \\
$\mathrm{C}(3)$ & $17(1)$ & $39(1)$ & $35(1)$ & $23(1)$ & $2(1)$ & $-5(1)$ \\
$\mathrm{C}(4)$ & $13(1)$ & $22(1)$ & $19(1)$ & $4(1)$ & $2(1)$ & $-2(1)$ \\
$\mathrm{C}(5)$ & $16(1)$ & $22(1)$ & $21(1)$ & $4(1)$ & $4(1)$ & $0(1)$ \\
$\mathrm{C}(6)$ & $14(1)$ & $26(1)$ & $26(1)$ & $4(1)$ & $5(1)$ & $-1(1)$ \\
$\mathrm{C}(7)$ & $19(1)$ & $24(1)$ & $26(1)$ & $4(1)$ & $1(1)$ & $-5(1)$
\end{tabular}




\begin{tabular}{|c|c|c|c|c|c|c|}
\hline & $\mathrm{U}_{11}$ & $\mathbf{U}_{22}$ & $\mathbf{U}_{33}$ & $\mathbf{U}_{23}$ & $\mathrm{U}_{13}$ & $\mathrm{U}_{12}$ \\
\hline$C(8)$ & $23(1)$ & $26(1)$ & $25(1)$ & $-2(1)$ & $2(1)$ & $-3(1)$ \\
\hline$C(9)$ & $19(1)$ & $29(1)$ & $20(1)$ & $2(1)$ & $4(1)$ & $1(1)$ \\
\hline$C(10)$ & $22(1)$ & $29(1)$ & $31(1)$ & $-4(1)$ & $5(1)$ & $2(1)$ \\
\hline $\mathrm{C}(11)$ & $22(1)$ & $31(1)$ & $37(1)$ & $0(1)$ & $3(1)$ & $-8(1)$ \\
\hline$C(12)$ & $26(1)$ & $49(1)$ & $24(1)$ & $-2(1)$ & $9(1)$ & $5(1)$ \\
\hline$C(13)$ & $12(1)$ & $21(1)$ & $23(1)$ & $6(1)$ & $3(1)$ & $-2(1)$ \\
\hline $\mathrm{C}(14)$ & $15(1)$ & $18(1)$ & $30(1)$ & $4(1)$ & $5(1)$ & $-2(1)$ \\
\hline$C(15)$ & $16(1)$ & $22(1)$ & $28(1)$ & $1(1)$ & $4(1)$ & $-4(1)$ \\
\hline$C(16)$ & $13(1)$ & $27(1)$ & $24(1)$ & $3(1)$ & $4(1)$ & $-2(1)$ \\
\hline$C(17)$ & $15(1)$ & $25(1)$ & $24(1)$ & $2(1)$ & $5(1)$ & $2(1)$ \\
\hline $\mathrm{C}(18)$ & $17(1)$ & $24(1)$ & $20(1)$ & $2(1)$ & $5(1)$ & $-1(1)$ \\
\hline C(19) & $22(1)$ & $21(1)$ & $53(1)$ & $-1(1)$ & $6(1)$ & $2(1)$ \\
\hline$C(20)$ & $15(1)$ & $41(1)$ & $32(1)$ & $-1(1)$ & $0(1)$ & $0(1)$ \\
\hline $\mathrm{C}(21)$ & $23(1)$ & $30(1)$ & $21(1)$ & $-2(1)$ & $7(1)$ & $-2(1)$ \\
\hline$C(22)$ & $15(1)$ & $17(1)$ & $16(1)$ & $1(1)$ & $5(1)$ & $-1(1)$ \\
\hline $\mathrm{N}(1)$ & $14(1)$ & $25(1)$ & $20(1)$ & $7(1)$ & $2(1)$ & $-4(1)$ \\
\hline $\mathrm{N}(2)$ & $14(1)$ & $22(1)$ & $23(1)$ & $9(1)$ & $2(1)$ & $-3(1)$ \\
\hline $\mathrm{Cl}(1)$ & $26(1)$ & $19(1)$ & 21(1) & $-3(1)$ & $8(1)$ & $-2(1)$ \\
\hline $\mathrm{Cl}(2)$ & $26(1)$ & $21(1)$ & $25(1)$ & $-3(1)$ & $10(1)$ & $4(1)$ \\
\hline $\mathrm{Ru}(1)$ & $13(1)$ & $15(1)$ & $16(1)$ & $1(1)$ & $5(1)$ & $0(1)$ \\
\hline
\end{tabular}

Table S32. Hydrogen atomic coordinates $\left(\mathrm{x10}^{4}\right)$ and isotropic atomic displacement parameters $\left(\AA^{2} \times 10^{3}\right)$ for $\mathrm{C}_{43} \mathrm{H}_{52} \mathrm{Cl}_{4} \mathrm{~N}_{4} \mathrm{Ru}_{2}$

\begin{tabular}{lllll}
\hline & $\mathbf{x} / \mathbf{a}$ & $\mathbf{y} / \mathbf{b}$ & $\mathbf{z} / \mathbf{c}$ & $\mathbf{U}(\mathbf{e q})$ \\
$\mathrm{H}(2 \mathrm{~A})$ & 7382 & 3062 & 8648 & 34 \\
$\mathrm{H}(2 \mathrm{~B})$ & 7682 & 2274 & 7910 & 34 \\
$\mathrm{H}(3 \mathrm{~A})$ & 5538 & 1996 & 7373 & 38 \\
$\mathrm{H}(3 \mathrm{~B})$ & 5237 & 2951 & 7941 & 38 \\
$\mathrm{H}(6)$ & 11623 & 4216 & 7594 & 27 \\
$\mathrm{H}(8)$ & 10079 & 5958 & 9211 & 31 \\
$\mathrm{H}(10 \mathrm{~A})$ & 9900 & 3205 & 6230 & 42 \\
$\mathrm{H}(10 B)$ & 8836 & 2704 & 6642 & 42 \\
$\mathrm{H}(10 \mathrm{C})$ & 10339 & 2517 & 7162 & 42
\end{tabular}




\begin{tabular}{lllll} 
& $\mathbf{x} / \mathbf{a}$ & $\mathbf{y} / \mathbf{b}$ & $\mathbf{z} / \mathbf{c}$ & $\mathbf{U}(\mathbf{e q})$ \\
$\mathrm{H}(11 \mathrm{~A})$ & 12993 & 5224 & 9130 & 47 \\
$\mathrm{H}(11 \mathrm{~B})$ & 12255 & 6206 & 9299 & 47 \\
$\mathrm{H}(11 \mathrm{C})$ & 12542 & 6037 & 8300 & 47 \\
$\mathrm{H}(12 \mathrm{~A})$ & 7948 & 5104 & 9480 & 49 \\
$\mathrm{H}(12 \mathrm{~B})$ & 6927 & 4876 & 8463 & 49 \\
$\mathrm{H}(12 \mathrm{C})$ & 7530 & 5967 & 8696 & 49 \\
$\mathrm{H}(15)$ & 2059 & 1977 & 4463 & 27 \\
$\mathrm{H}(17)$ & 1600 & 4748 & 5386 & 26 \\
$\mathrm{H}(19 \mathrm{~A})$ & 5377 & 1952 & 5248 & 49 \\
$\mathrm{H}(19 B)$ & 4132 & 1292 & 4742 & 49 \\
$\mathrm{H}(19 \mathrm{C})$ & 4742 & 1264 & 5879 & 49 \\
$\mathrm{H}(20 \mathrm{~A})$ & 223 & 3806 & 3618 & 46 \\
$\mathrm{H}(20 \mathrm{~B})$ & -331 & 3643 & 4505 & 46 \\
$\mathrm{H}(20 \mathrm{C})$ & -18 & 2704 & 3941 & 46 \\
$\mathrm{H}(21 \mathrm{~A})$ & 3131 & 5395 & 6792 & 37 \\
$\mathrm{H}(21 \mathrm{~B})$ & 4593 & 5255 & 6779 & 37 \\
$\mathrm{H}(21 \mathrm{C})$ & 4032 & 4574 & 7460 & 37 \\
\hline
\end{tabular}

\subsection{X-ray structure of Ru-vinyl-5}

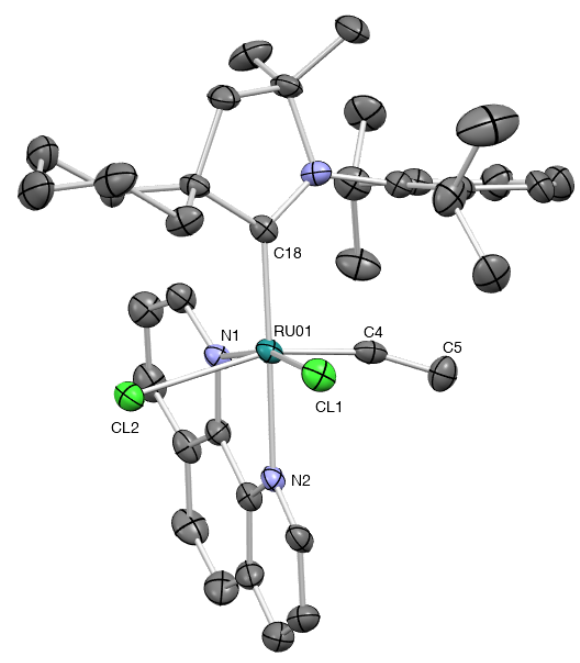

Table S33. Sample and crystal data for $\mathrm{C}_{40} \mathrm{H}_{51} \mathrm{Cl}_{8} \mathrm{~N}_{3} \mathrm{Ru}$

Identification code

Chemical formula

$$
\begin{aligned}
& \text { HSG210330 } \\
& \mathrm{C}_{40} \mathrm{H}_{51} \mathrm{Cl}_{8} \mathrm{~N}_{3} \mathrm{Ru}
\end{aligned}
$$




$\begin{array}{lll}\text { Formula weight } & 958.51 \mathrm{~g} / \mathrm{mol} & \\ \text { Temperature } & 173(2) \mathrm{K} & \\ \text { Wavelength } & 0.71073 \AA & \\ \text { Crystal size } & 0.090 \times 0.090 \times 0.200 \mathrm{~mm} \\ \text { Crystal system } & \text { monoclinic } & \\ \text { Space group } & \mathrm{P} 121 / \mathrm{n} 1 & \\ \text { Unit cell dimensions } & \mathrm{a}=10.7151(10) \AA & \alpha=90^{\circ} \\ & \mathrm{b}=17.6613(19) \AA & \beta=101.613(5)^{\circ} \\ & \mathrm{c}=22.860(3) \AA & \gamma=90^{\circ} \\ \text { Volume } & 4237.5(8) \AA^{3} & \\ \text { Z } & 4 \\ \text { Density (calculated) } & 1.502{\mathrm{~g} / \mathrm{cm}^{3}}^{-1} \\ \text { Absorption coefficient } & 0.908 \mathrm{~mm}^{-1} \\ \text { F(000) } & 1968\end{array}$

\section{Table S34. Data collection and structure refinement for $\mathrm{C}_{40} \mathrm{H}_{51} \mathrm{Cl}_{8} \mathrm{~N}_{3} \mathrm{Ru}$}

Theta range for data collection 2.48 to $27.96^{\circ}$

Index ranges

$-14<=\mathrm{h}<=12,-23<=\mathrm{k}<=23,-30<=1<=29$

Reflections collected

46664

Independent reflections

$9534[\mathrm{R}($ int $)=0.1210]$

Max. and min. transmission

0.8410 and 0.7670

Structure solution technique

direct methods

Structure solution program

SHELXT 2014/5 (Sheldrick, 2014)

Refinement method

Full-matrix least-squares on $\mathrm{F}^{2}$

Refinement program

SHELXL-2018/3 (Sheldrick, 2018)

Function minimized

$\Sigma \mathrm{w}\left(\mathrm{F}_{\mathrm{o}}{ }^{2}-\mathrm{F}_{\mathrm{c}}{ }^{2}\right)^{2}$

Data / restraints / parameters

9534 / 0 / 475

Goodness-of-fit on $\mathrm{F}^{2}$

1.020

$\Delta / \sigma_{\max }$

0.001

Final R indices

5941 data;

$\mathrm{I}>2 \sigma(\mathrm{I}) \quad \mathrm{R} 1=0.0592, \mathrm{wR} 2=0.1149$

all data $\quad \mathrm{R} 1=0.1159, \mathrm{wR} 2=0.1383$

Weighting scheme

$\mathrm{W}=1 /\left[\sigma^{2}\left(\mathrm{~F}_{\mathrm{o}}^{2}\right)+(0.0478 \mathrm{P})^{2}+6.7106 \mathrm{P}\right]$

where $\mathrm{P}=\left(\mathrm{F}_{\mathrm{o}}^{2}+2 \mathrm{~F}_{\mathrm{c}}^{2}\right) / 3$

Largest diff. peak and hole

0.971 and $-0.924 \mathrm{e}^{-3}$

R.M.S. deviation from mean $0.112 \mathrm{e}^{-3}$ 
Table S35. Atomic coordinates and equivalent isotropic atomic displacement parameters $\left(\AA^{2}\right)$ for for $\mathrm{C}_{40} \mathrm{H}_{51} \mathrm{Cl}_{8} \mathrm{~N}_{3} \mathrm{Ru}$

( $\mathrm{U}(\mathrm{eq})$ is defined as one third of the trace of the orthogonalized $\mathrm{U}_{\mathrm{ij}}$ tensor.)

\begin{tabular}{|c|c|c|c|c|}
\hline & $\mathbf{x} / \mathbf{a}$ & $\mathbf{y} / \mathbf{b}$ & $\mathbf{z} / \mathbf{c}$ & $\mathrm{U}(\mathbf{e q})$ \\
\hline $\mathrm{Ru} 1$ & $0.63481(3)$ & $0.65709(2)$ & $0.85258(2)$ & $0.01826(10)$ \\
\hline $\mathrm{Cl1}$ & $0.85404(11)$ & $0.61977(7)$ & $0.87070(6)$ & $0.0279(3)$ \\
\hline $\mathrm{Cl} 2$ & $0.66346(12)$ & $0.70013(7)$ & $0.96167(5)$ & $0.0273(3)$ \\
\hline N1 & $0.5863(3)$ & $0.5548(2)$ & $0.89590(16)$ & $0.0186(8)$ \\
\hline $\mathrm{C} 1$ & $0.6618(5)$ & $0.4972(3)$ & $0.9183(2)$ & $0.0249(11)$ \\
\hline $\mathrm{C} 2$ & $0.6131(5)$ & $0.4321(3)$ & $0.9411(2)$ & $0.0300(12)$ \\
\hline $\mathrm{C} 3$ & $0.4875(5)$ & $0.4270(3)$ & $0.9417(2)$ & $0.0319(12)$ \\
\hline $\mathrm{C} 4$ & $0.4046(5)$ & $0.4867(3)$ & $0.9191(2)$ & $0.0253(11)$ \\
\hline $\mathrm{C} 5$ & $0.2723(5)$ & $0.4881(3)$ & $0.9188(2)$ & $0.0339(13)$ \\
\hline C6 & $0.2001(5)$ & $0.5492(3)$ & $0.8995(2)$ & $0.0327(13)$ \\
\hline $\mathrm{C} 7$ & $0.2545(4)$ & $0.6148(3)$ & $0.8771(2)$ & $0.0232(10)$ \\
\hline $\mathrm{C} 8$ & $0.1841(5)$ & $0.6814(3)$ & $0.8600(2)$ & $0.0317(12)$ \\
\hline C9 & $0.2458(5)$ & $0.7418(3)$ & $0.8424(2)$ & $0.0303(12)$ \\
\hline $\mathrm{C} 10$ & $0.3730(5)$ & $0.7365(3)$ & $0.8385(2)$ & $0.0262(11)$ \\
\hline N2 & $0.4424(3)$ & $0.6740(2)$ & $0.85277(16)$ & $0.0191(8)$ \\
\hline $\mathrm{C} 11$ & $0.3834(4)$ & $0.6139(3)$ & $0.8743(2)$ & $0.0205(10)$ \\
\hline $\mathrm{C} 12$ & $0.4601(4)$ & $0.5497(3)$ & $0.8962(2)$ & $0.0209(10)$ \\
\hline $\mathrm{C} 13$ & $0.6674(4)$ & $0.7553(3)$ & $0.8100(2)$ & $0.0191(10)$ \\
\hline N3 & $0.6480(4)$ & $0.7689(2)$ & $0.75146(17)$ & $0.0206(9)$ \\
\hline $\mathrm{C} 14$ & $0.6633(5)$ & $0.8525(3)$ & $0.7351(2)$ & $0.0268(11)$ \\
\hline $\mathrm{C} 15$ & $0.7442(5)$ & $0.8811(3)$ & $0.7921(2)$ & $0.0273(11)$ \\
\hline $\mathrm{C} 16$ & $0.7216(4)$ & $0.8287(2)$ & $0.8433(2)$ & $0.0219(10)$ \\
\hline $\mathrm{C} 17$ & $0.6234(5)$ & $0.7146(3)$ & $0.7019(2)$ & $0.0222(10)$ \\
\hline $\mathrm{C} 18$ & $0.7301(5)$ & $0.6812(3)$ & $0.6844(2)$ & $0.0250(11)$ \\
\hline
\end{tabular}




\begin{tabular}{|c|c|c|c|c|}
\hline & $\mathbf{x} / \mathbf{a}$ & $\mathbf{y} / \mathbf{b}$ & $\mathbf{z} / \mathbf{c}$ & $\mathbf{U}(\mathbf{e q})$ \\
\hline C19 & $0.7077(6)$ & $0.6384(3)$ & $0.6320(2)$ & $0.0332(13)$ \\
\hline $\mathrm{C} 20$ & $0.5884(6)$ & $0.6266(3)$ & $0.5994(2)$ & $0.0390(14)$ \\
\hline $\mathrm{C} 21$ & $0.4854(5)$ & $0.6575(3)$ & $0.6178(2)$ & $0.0355(13)$ \\
\hline $\mathrm{C} 22$ & $0.4996(5)$ & $0.7026(3)$ & $0.6690(2)$ & $0.0259(11)$ \\
\hline $\mathrm{C} 23$ & $0.8667(5)$ & $0.6856(3)$ & $0.7184(3)$ & $0.0349(13)$ \\
\hline $\mathrm{C} 24$ & $0.9205(5)$ & $0.6063(3)$ & $0.7346(3)$ & $0.0390(14)$ \\
\hline $\mathrm{C} 25$ & $0.9543(6)$ & $0.7242(4)$ & $0.6823(4)$ & $0.072(2)$ \\
\hline $\mathrm{C} 26$ & $0.3782(5)$ & $0.7309(3)$ & $0.6862(2)$ & $0.0294(12)$ \\
\hline $\mathrm{C} 27$ & $0.3034(5)$ & $0.6636(3)$ & $0.7041(2)$ & $0.0367(13)$ \\
\hline $\mathrm{C} 28$ & $0.2924(5)$ & $0.7748(3)$ & $0.6348(2)$ & $0.0385(14)$ \\
\hline $\mathrm{C} 29$ & $0.5320(5)$ & $0.8914(3)$ & $0.7214(3)$ & $0.0392(14)$ \\
\hline $\mathrm{C} 30$ & $0.7270(6)$ & $0.8630(3)$ & $0.6824(2)$ & $0.0447(16)$ \\
\hline C31 & $0.8480(5)$ & $0.8119(3)$ & $0.8859(2)$ & $0.0299(12)$ \\
\hline C32 & $0.9047(6)$ & $0.8827(3)$ & $0.9202(3)$ & $0.0429(15)$ \\
\hline C33 & $0.8113(6)$ & $0.9147(3)$ & $0.9559(3)$ & $0.0502(17)$ \\
\hline $\mathrm{C} 34$ & $0.6852(6)$ & $0.9339(3)$ & $0.9157(2)$ & $0.0400(14)$ \\
\hline $\mathrm{C} 35$ & $0.6282(5)$ & $0.8659(3)$ & $0.8789(2)$ & $0.0288(12)$ \\
\hline C36 & $0.6047(4)$ & $0.5998(3)$ & $0.7865(2)$ & $0.0222(10)$ \\
\hline C37 & $0.5852(5)$ & $0.5435(3)$ & $0.7487(2)$ & $0.0298(12)$ \\
\hline C38 & $0.2790(7)$ & $0.4701(4)$ & $0.7492(4)$ & $0.070(2)$ \\
\hline $\mathrm{Cl} 3$ & $0.3683(3)$ & $0.39244(12)$ & $0.77298(10)$ & $0.0995(8)$ \\
\hline $\mathrm{Cl} 4$ & $0.2004(2)$ & $0.46328(13)$ & $0.67540(11)$ & $0.1008(8)$ \\
\hline C39 & $0.0479(8)$ & $0.3346(4)$ & $0.4853(3)$ & $0.079(2)$ \\
\hline $\mathrm{Cl} 5$ & $0.1690(2)$ & $0.38029(15)$ & $0.53215(11)$ & $0.1040(8)$ \\
\hline $\mathrm{Cl} 6$ & $0.9441(2)$ & $0.39933(13)$ & $0.44070(9)$ & $0.0813(6)$ \\
\hline $\mathrm{C} 40$ & $0.9941(5)$ & $0.6801(3)$ & $0.0150(3)$ & $0.0396(14)$ \\
\hline $\mathrm{Cl} 7$ & $0.01806(15)$ & $0.58590(9)$ & $0.03816(8)$ & $0.0502(4)$ \\
\hline $\mathrm{Cl} 8$ & $0.13436(15)$ & $0.72162(9)$ & $0.00039(8)$ & $0.0518(4)$ \\
\hline
\end{tabular}


Table S36. Bond lengths ( $(\AA)$ for $\mathrm{C}_{40} \mathrm{H}_{51} \mathrm{Cl}_{8} \mathrm{~N}_{3} \mathrm{Ru}$

\begin{tabular}{|c|c|c|c|}
\hline Ru1-C36 & $1.792(5)$ & Ru1-C13 & $2.053(4)$ \\
\hline Ru1-N2 & $2.084(4)$ & Ru1-N1 & $2.174(4)$ \\
\hline Ru1-C11 & $2.3942(12)$ & $\mathrm{Ru} 1-\mathrm{Cl} 2$ & $2.5658(13)$ \\
\hline $\mathrm{N} 1-\mathrm{C} 1$ & $1.335(6)$ & $\mathrm{N} 1-\mathrm{C} 12$ & $1.356(6)$ \\
\hline $\mathrm{C} 1-\mathrm{C} 2$ & $1.406(7)$ & $\mathrm{C} 1-\mathrm{H} 1$ & 0.95 \\
\hline $\mathrm{C} 2-\mathrm{C} 3$ & $1.352(7)$ & $\mathrm{C} 2-\mathrm{H} 2$ & 0.95 \\
\hline $\mathrm{C} 3-\mathrm{C} 4$ & $1.407(7)$ & $\mathrm{C} 3-\mathrm{H} 3$ & 0.95 \\
\hline $\mathrm{C} 4-\mathrm{C} 12$ & $1.411(6)$ & $\mathrm{C} 4-\mathrm{C} 5$ & $1.417(7)$ \\
\hline C5-C6 & $1.349(7)$ & $\mathrm{C} 5-\mathrm{H} 5$ & 0.95 \\
\hline C6-C7 & $1.437(7)$ & C6-H6 & 0.95 \\
\hline C7-C11 & $1.396(6)$ & $\mathrm{C} 7-\mathrm{C} 8$ & $1.409(7)$ \\
\hline C8-C9 & $1.359(7)$ & C8-H8 & 0.95 \\
\hline C9-C10 & $1.387(7)$ & C9-H9 & 0.95 \\
\hline $\mathrm{C} 10-\mathrm{N} 2$ & $1.335(6)$ & $\mathrm{C} 10-\mathrm{H} 10$ & 0.95 \\
\hline N2-C11 & $1.376(5)$ & $\mathrm{C} 11-\mathrm{C} 12$ & $1.430(6)$ \\
\hline C13-N3 & $1.335(6)$ & C13-C16 & $1.555(6)$ \\
\hline N3-C17 & $1.468(6)$ & N3-C14 & $1.539(6)$ \\
\hline $\mathrm{C} 14-\mathrm{C} 15$ & $1.500(7)$ & $\mathrm{C} 14-\mathrm{C} 30$ & $1.512(7)$ \\
\hline C14-C29 & $1.540(7)$ & $\mathrm{C} 15-\mathrm{C} 16$ & $1.548(6)$ \\
\hline $\mathrm{C} 15-\mathrm{H} 15 \mathrm{~A}$ & 0.99 & $\mathrm{C} 15-\mathrm{H} 15 \mathrm{~B}$ & 0.99 \\
\hline C16-C31 & $1.531(7)$ & $\mathrm{C} 16-\mathrm{C} 35$ & $1.558(7)$ \\
\hline $\mathrm{C} 17-\mathrm{C} 22$ & $1.403(7)$ & $\mathrm{C} 17-\mathrm{C} 18$ & $1.414(7)$ \\
\hline C18-C19 & $1.396(7)$ & $\mathrm{C} 18-\mathrm{C} 23$ & $1.515(7)$ \\
\hline C19-C20 & $1.361(8)$ & C19-H19 & 0.95 \\
\hline $\mathrm{C} 20-\mathrm{C} 21$ & $1.371(8)$ & $\mathrm{C} 20-\mathrm{H} 20$ & 0.95 \\
\hline $\mathrm{C} 21-\mathrm{C} 22$ & $1.397(7)$ & $\mathrm{C} 21-\mathrm{H} 21$ & 0.95 \\
\hline $\mathrm{C} 22-\mathrm{C} 26$ & $1.518(7)$ & $\mathrm{C} 23-\mathrm{C} 25$ & $1.530(8)$ \\
\hline $\mathrm{C} 23-\mathrm{C} 24$ & $1.532(7)$ & $\mathrm{C} 23-\mathrm{H} 23$ & 1.0 \\
\hline $\mathrm{C} 24-\mathrm{H} 24 \mathrm{~A}$ & 0.98 & $\mathrm{C} 24-\mathrm{H} 24 \mathrm{~B}$ & 0.98 \\
\hline $\mathrm{C} 24-\mathrm{H} 24 \mathrm{C}$ & 0.98 & $\mathrm{C} 25-\mathrm{H} 25 \mathrm{~A}$ & 0.98 \\
\hline C25-H25B & 0.98 & $\mathrm{C} 25-\mathrm{H} 25 \mathrm{C}$ & 0.98 \\
\hline
\end{tabular}




\begin{tabular}{llll} 
C26-C27 & $1.533(7)$ & C26-C28 & $1.548(7)$ \\
C26-H26 & 1.0 & C27-H27A & 0.98 \\
C27-H27B & 0.98 & C27-H27C & 0.98 \\
C28-H28A & 0.98 & C28-H28B & 0.98 \\
C28-H28C & 0.98 & C29-H29A & 0.98 \\
C29-H29B & 0.98 & C29-H29C & 0.98 \\
C30-H30A & 0.98 & C30-H30B & 0.98 \\
C30-H30C & 0.98 & C31-C32 & $1.534(7)$ \\
C31-H31A & 0.99 & C31-H31B & 0.99 \\
C32-C33 & $1.523(9)$ & C32-H32A & 0.99 \\
C32-H32B & 0.99 & C33-C34 & $1.512(8)$ \\
C33-H33A & 0.99 & C33-H33B & 0.99 \\
C34-C35 & $1.521(7)$ & C34-H34A & 0.99 \\
C34-H34B & 0.99 & C35-H35A & 0.99 \\
C35-H35B & 0.99 & C36-C37 & $1.306(7)$ \\
C37-H37A & 0.95 & C37-H37B & 0.95 \\
C38-Cl3 & $1.697(8)$ & C38-Cl4 & $1.732(8)$ \\
C38-H38A & 0.99 & C38-H38B & 0.99 \\
C39-C15 & $1.710(8)$ & C39-Cl6 & $1.769(8)$ \\
C39-H39A & 0.99 & C39-H39B & 0.99 \\
C40-C17 & $1.749(6)$ & C40-C18 & $1.763(6)$ \\
C40-H40A & 0.99 & C40-H40B & 0.99 \\
\hline
\end{tabular}

\section{Table S37. Bond angles $\left({ }^{\circ}\right)$ for $\mathrm{C}_{40} \mathrm{H}_{51} \mathrm{Cl}_{8} \mathrm{~N}_{3} \mathrm{Ru}$}

\begin{tabular}{llll}
\hline C36-Ru1-C13 & $95.55(19)$ & C36-Ru1-N2 & $94.12(18)$ \\
C13-Ru1-N2 & $98.22(16)$ & C36-Ru1-N1 & $84.02(17)$ \\
C13-Ru1-N1 & $176.04(16)$ & N2-Ru1-N1 & $77.90(14)$ \\
C36-Ru1-C11 & $89.69(15)$ & C13-Ru1-Cl1 & $93.45(13)$ \\
N2-Ru1-Cl1 & $167.31(10)$ & N1-Ru1-Cl1 & $90.49(10)$ \\
C36-Ru1-C12 & $162.24(14)$ & C13-Ru1-Cl2 & $102.22(13)$ \\
N2-Ru1-C12 & $83.13(11)$ & N1-Ru1-Cl2 & $78.24(10)$ \\
C11-Ru1-Cl2 & $89.55(4)$ & C1-N1-C12 & $118.4(4)$ \\
C1-N1-Ru1 & $128.7(3)$ & C12-N1-Ru1 & $112.8(3)$
\end{tabular}




\begin{tabular}{|c|c|c|c|}
\hline $\mathrm{N} 1-\mathrm{C} 1-\mathrm{C} 2$ & $121.5(4)$ & N1-C1-H1 & 119.2 \\
\hline C2-C1-H1 & 119.2 & $\mathrm{C} 3-\mathrm{C} 2-\mathrm{C} 1$ & $120.2(5)$ \\
\hline $\mathrm{C} 3-\mathrm{C} 2-\mathrm{H} 2$ & 119.9 & $\mathrm{C} 1-\mathrm{C} 2-\mathrm{H} 2$ & 119.9 \\
\hline $\mathrm{C} 2-\mathrm{C} 3-\mathrm{C} 4$ & $120.2(5)$ & $\mathrm{C} 2-\mathrm{C} 3-\mathrm{H} 3$ & 119.9 \\
\hline C4-C3-H3 & 119.9 & $\mathrm{C} 3-\mathrm{C} 4-\mathrm{C} 12$ & $116.4(5)$ \\
\hline $\mathrm{C} 3-\mathrm{C} 4-\mathrm{C} 5$ & $124.9(5)$ & $\mathrm{C} 12-\mathrm{C} 4-\mathrm{C} 5$ & $118.7(5)$ \\
\hline C6-C5-C4 & $121.5(5)$ & C6-C5-H5 & 119.2 \\
\hline C4-C5-H5 & 119.2 & $\mathrm{C} 5-\mathrm{C} 6-\mathrm{C} 7$ & $121.0(5)$ \\
\hline C5-C6-H6 & 119.5 & C7-C6-H6 & 119.5 \\
\hline $\mathrm{C} 11-\mathrm{C} 7-\mathrm{C} 8$ & $118.4(5)$ & C11-C7-C6 & $118.9(4)$ \\
\hline C8-C7-C6 & $122.6(5)$ & C9-C8-C7 & $118.2(5)$ \\
\hline C9-C8-H8 & 120.9 & C7-C8-H8 & 120.9 \\
\hline C8-C9-C10 & $120.6(5)$ & C8-C9-H9 & 119.7 \\
\hline C10-C9-H9 & 119.7 & N2-C10-C9 & $123.2(5)$ \\
\hline N2-C10-H10 & 118.4 & C9-C10-H10 & 118.4 \\
\hline C10-N2-C11 & $116.8(4)$ & C10-N2-Ru1 & $128.4(3)$ \\
\hline C11-N2-Ru1 & 114.6(3) & N2-C11-C7 & $122.6(4)$ \\
\hline N2-C11-C12 & $117.6(4)$ & C7-C11-C12 & $119.8(4)$ \\
\hline $\mathrm{N} 1-\mathrm{C} 12-\mathrm{C} 4$ & $123.2(4)$ & N1-C12-C11 & $116.7(4)$ \\
\hline C4-C12-C11 & $120.0(4)$ & N3-C13-C16 & $107.9(4)$ \\
\hline N3-C13-Ru1 & $128.4(3)$ & C16-C13-Ru1 & $123.8(3)$ \\
\hline C13-N3-C17 & $128.6(4)$ & C13-N3-C14 & $114.4(4)$ \\
\hline C17-N3-C14 & $116.8(4)$ & $\mathrm{C} 15-\mathrm{C} 14-\mathrm{C} 30$ & $112.1(4)$ \\
\hline C15-C14-N3 & $100.6(3)$ & $\mathrm{C} 30-\mathrm{C} 14-\mathrm{N} 3$ & $113.5(4)$ \\
\hline C15-C14-C29 & $111.8(4)$ & C30-C14-C29 & $108.9(4)$ \\
\hline N3-C14-C29 & $109.9(4)$ & C14-C15-C16 & $107.7(4)$ \\
\hline C14-C15-H15A & 110.2 & C16-C15-H15A & 110.2 \\
\hline C14-C15-H15B & 110.2 & C16-C15-H15B & 110.2 \\
\hline H15A-C15-H15B & 108.5 & C31-C16-C15 & $110.2(4)$ \\
\hline C31-C16-C13 & $110.2(4)$ & $\mathrm{C} 15-\mathrm{C} 16-\mathrm{C} 13$ & $103.2(4)$ \\
\hline C31-C16-C35 & 109.1(4) & C15-C16-C35 & $111.0(4)$ \\
\hline C13-C16-C35 & $113.0(4)$ & $\mathrm{C} 22-\mathrm{C} 17-\mathrm{C} 18$ & $121.1(5)$ \\
\hline C22-C17-N3 & 121.1(4) & C18-C17-N3 & $117.4(4)$ \\
\hline C19-C18-C17 & $117.4(5)$ & C19-C18-C23 & $116.7(5)$ \\
\hline
\end{tabular}




\begin{tabular}{|c|c|c|c|}
\hline $\mathrm{C} 17-\mathrm{C} 18-\mathrm{C} 23$ & $125.9(5)$ & C20-C19-C18 & $122.2(5)$ \\
\hline C20-C19-H19 & 118.9 & C18-C19-H19 & 118.9 \\
\hline $\mathrm{C} 19-\mathrm{C} 20-\mathrm{C} 21$ & $119.7(5)$ & $\mathrm{C} 19-\mathrm{C} 20-\mathrm{H} 20$ & 120.1 \\
\hline $\mathrm{C} 21-\mathrm{C} 20-\mathrm{H} 20$ & 120.1 & $\mathrm{C} 20-\mathrm{C} 21-\mathrm{C} 22$ & $121.7(5)$ \\
\hline $\mathrm{C} 20-\mathrm{C} 21-\mathrm{H} 21$ & 119.2 & $\mathrm{C} 22-\mathrm{C} 21-\mathrm{H} 21$ & 119.2 \\
\hline $\mathrm{C} 21-\mathrm{C} 22-\mathrm{C} 17$ & $117.8(5)$ & $\mathrm{C} 21-\mathrm{C} 22-\mathrm{C} 26$ & $116.8(5)$ \\
\hline $\mathrm{C} 17-\mathrm{C} 22-\mathrm{C} 26$ & $125.3(5)$ & $\mathrm{C} 18-\mathrm{C} 23-\mathrm{C} 25$ & $112.3(5)$ \\
\hline $\mathrm{C} 18-\mathrm{C} 23-\mathrm{C} 24$ & $110.8(4)$ & $\mathrm{C} 25-\mathrm{C} 23-\mathrm{C} 24$ & $107.2(5)$ \\
\hline $\mathrm{C} 18-\mathrm{C} 23-\mathrm{H} 23$ & 108.8 & $\mathrm{C} 25-\mathrm{C} 23-\mathrm{H} 23$ & 108.8 \\
\hline $\mathrm{C} 24-\mathrm{C} 23-\mathrm{H} 23$ & 108.8 & C23-C24-H24A & 109.5 \\
\hline $\mathrm{C} 23-\mathrm{C} 24-\mathrm{H} 24 \mathrm{~B}$ & 109.5 & H24A-C24-H24B & 109.5 \\
\hline $\mathrm{C} 23-\mathrm{C} 24-\mathrm{H} 24 \mathrm{C}$ & 109.5 & $\mathrm{H} 24 \mathrm{~A}-\mathrm{C} 24-\mathrm{H} 24 \mathrm{C}$ & 109.5 \\
\hline H24B-C24-H24C & 109.5 & $\mathrm{C} 23-\mathrm{C} 25-\mathrm{H} 25 \mathrm{~A}$ & 109.5 \\
\hline $\mathrm{C} 23-\mathrm{C} 25-\mathrm{H} 25 \mathrm{~B}$ & 109.5 & $\mathrm{H} 25 \mathrm{~A}-\mathrm{C} 25-\mathrm{H} 25 \mathrm{~B}$ & 109.5 \\
\hline $\mathrm{C} 23-\mathrm{C} 25-\mathrm{H} 25 \mathrm{C}$ & 109.5 & $\mathrm{H} 25 \mathrm{~A}-\mathrm{C} 25-\mathrm{H} 25 \mathrm{C}$ & 109.5 \\
\hline $\mathrm{H} 25 \mathrm{~B}-\mathrm{C} 25-\mathrm{H} 25 \mathrm{C}$ & 109.5 & $\mathrm{C} 22-\mathrm{C} 26-\mathrm{C} 27$ & $109.6(4)$ \\
\hline $\mathrm{C} 22-\mathrm{C} 26-\mathrm{C} 28$ & $112.0(4)$ & $\mathrm{C} 27-\mathrm{C} 26-\mathrm{C} 28$ & $109.2(4)$ \\
\hline $\mathrm{C} 22-\mathrm{C} 26-\mathrm{H} 26$ & 108.6 & $\mathrm{C} 27-\mathrm{C} 26-\mathrm{H} 26$ & 108.6 \\
\hline $\mathrm{C} 28-\mathrm{C} 26-\mathrm{H} 26$ & 108.6 & C26-C27-H27A & 109.5 \\
\hline $\mathrm{C} 26-\mathrm{C} 27-\mathrm{H} 27 \mathrm{~B}$ & 109.5 & $\mathrm{H} 27 \mathrm{~A}-\mathrm{C} 27-\mathrm{H} 27 \mathrm{~B}$ & 109.5 \\
\hline $\mathrm{C} 26-\mathrm{C} 27-\mathrm{H} 27 \mathrm{C}$ & 109.5 & $\mathrm{H} 27 \mathrm{~A}-\mathrm{C} 27-\mathrm{H} 27 \mathrm{C}$ & 109.5 \\
\hline H27B-C27-H27C & 109.5 & C26-C28-H28A & 109.5 \\
\hline $\mathrm{C} 26-\mathrm{C} 28-\mathrm{H} 28 \mathrm{~B}$ & 109.5 & H28A-C28-H28B & 109.5 \\
\hline $\mathrm{C} 26-\mathrm{C} 28-\mathrm{H} 28 \mathrm{C}$ & 109.5 & $\mathrm{H} 28 \mathrm{~A}-\mathrm{C} 28-\mathrm{H} 28 \mathrm{C}$ & 109.5 \\
\hline H28B-C28-H28C & 109.5 & C14-C29-H29A & 109.5 \\
\hline C14-C29-H29B & 109.5 & H29A-C29-H29B & 109.5 \\
\hline C14-C29-H29C & 109.5 & H29A-C29-H29C & 109.5 \\
\hline H29B-C29-H29C & 109.5 & C14-C30-H30A & 109.5 \\
\hline C14-C30-H30B & 109.5 & H30A-C30-H30B & 109.5 \\
\hline C14-C30-H30C & 109.5 & $\mathrm{H} 30 \mathrm{~A}-\mathrm{C} 30-\mathrm{H} 30 \mathrm{C}$ & 109.5 \\
\hline H30B-C30-H30C & 109.5 & $\mathrm{C} 16-\mathrm{C} 31-\mathrm{C} 32$ & $112.1(4)$ \\
\hline C16-C31-H31A & 109.2 & C32-C31-H31A & 109.2 \\
\hline C16-C31-H31B & 109.2 & C32-C31-H31B & 109.2 \\
\hline H31A-C31-H31B & 107.9 & C33-C32-C31 & $109.9(5)$ \\
\hline
\end{tabular}




\begin{tabular}{|c|c|c|c|}
\hline C33-C32-H32A & 109.7 & C31-C32-H32A & 109.7 \\
\hline C33-C32-H32B & 109.7 & C31-C32-H32B & 109.7 \\
\hline H32A-C32-H32B & 108.2 & C34-C33-C32 & $111.1(5)$ \\
\hline C34-C33-H33A & 109.4 & C32-C33-H33A & 109.4 \\
\hline C34-C33-H33B & 109.4 & C32-C33-H33B & 109.4 \\
\hline H33A-C33-H33B & 108.0 & C33-C34-C35 & $111.6(5)$ \\
\hline C33-C34-H34A & 109.3 & C35-C34-H34A & 109.3 \\
\hline C33-C34-H34B & 109.3 & C35-C34-H34B & 109.3 \\
\hline H34A-C34-H34B & 108.0 & C34-C35-C16 & $113.2(4)$ \\
\hline C34-C35-H35A & 108.9 & C16-C35-H35A & 108.9 \\
\hline C34-C35-H35B & 108.9 & C16-C35-H35B & 108.9 \\
\hline H35A-C35-H35B & 107.8 & C37-C36-Ru1 & $164.7(4)$ \\
\hline C36-C37-H37A & 120.0 & C36-C37-H37B & 120.0 \\
\hline H37A-C37-H37B & 120.0 & $\mathrm{Cl3}-\mathrm{C} 38-\mathrm{Cl} 4$ & $112.9(4)$ \\
\hline $\mathrm{Cl} 3-\mathrm{C} 38-\mathrm{H} 38 \mathrm{~A}$ & 109.0 & Cl4-C38-H38A & 109.0 \\
\hline $\mathrm{Cl} 3-\mathrm{C} 38-\mathrm{H} 38 \mathrm{~B}$ & 109.0 & Cl4-C38-H38B & 109.0 \\
\hline H38A-C38-H38B & 107.8 & $\mathrm{Cl5}-\mathrm{C} 39-\mathrm{Cl} 6$ & $111.4(4)$ \\
\hline C15-C39-H39A & 109.3 & Cl6-C39-H39A & 109.3 \\
\hline C15-C39-H39B & 109.3 & Cl6-C39-H39B & 109.3 \\
\hline H39A-C39-H39B & 108.0 & $\mathrm{Cl} 7-\mathrm{C} 40-\mathrm{Cl} 8$ & $112.0(3)$ \\
\hline $\mathrm{Cl} 7-\mathrm{C} 40-\mathrm{H} 40 \mathrm{~A}$ & 109.2 & $\mathrm{Cl} 8-\mathrm{C} 40-\mathrm{H} 40 \mathrm{~A}$ & 109.2 \\
\hline C17-C40-H40B & 109.2 & $\mathrm{Cl} 8-\mathrm{C} 40-\mathrm{H} 40 \mathrm{~B}$ & 109.2 \\
\hline H40A-C40-H40B & 107.9 & & \\
\hline
\end{tabular}

Table S38. Torsion angles ( ${ }^{\circ}$ ) for $\mathrm{C}_{40} \mathrm{H}_{51} \mathrm{Cl}_{8} \mathrm{~N}_{3} \mathrm{Ru}$

\begin{tabular}{llll}
\hline C12-N1-C1-C2 & $0.7(7)$ & Ru1-N1-C1-C2 & $-175.2(3)$ \\
N1-C1-C2-C3 & $-1.1(8)$ & C1-C2-C3-C4 & $0.4(8)$ \\
C2-C3-C4-C12 & $0.5(7)$ & C2-C3-C4-C5 & $-178.5(5)$ \\
C3-C4-C5-C6 & $176.2(5)$ & C12-C4-C5-C6 & $-2.8(8)$ \\
C4-C5-C6-C7 & $1.4(8)$ & C5-C6-C7-C11 & $1.4(8)$ \\
C5-C6-C7-C8 & $-176.0(5)$ & C11-C7-C8-C9 & $-0.8(7)$ \\
C6-C7-C8-C9 & $176.6(5)$ & C7-C8-C9-C10 & $3.3(8)$ \\
C8-C9-C10-N2 & $-1.7(8)$ & C9-C10-N2-C11 & $-2.4(7)$
\end{tabular}




\begin{tabular}{|c|c|c|c|}
\hline C9-C10-N2-Ru1 & $-177.6(4)$ & C10-N2-C11-C7 & $5.0(7)$ \\
\hline Ru1-N2-C11-C7 & $-179.2(4)$ & C10-N2-C11-C12 & $-173.2(4)$ \\
\hline Ru1-N2-C11-C12 & $2.7(5)$ & $\mathrm{C} 8-\mathrm{C} 7-\mathrm{C} 11-\mathrm{N} 2$ & $-3.4(7)$ \\
\hline C6-C7-C11-N2 & 179.1(4) & C8-C7-C11-C12 & $174.7(4)$ \\
\hline C6-C7-C11-C12 & $-2.8(7)$ & C1-N1-C12-C4 & $0.2(7)$ \\
\hline Ru1-N1-C12-C4 & $176.8(4)$ & C1-N1-C12-C11 & $177.2(4)$ \\
\hline Ru1-N1-C12-C11 & $-6.2(5)$ & $\mathrm{C} 3-\mathrm{C} 4-\mathrm{C} 12-\mathrm{N} 1$ & $-0.9(7)$ \\
\hline C5-C4-C12-N1 & $178.2(4)$ & C3-C4-C12-C11 & $-177.7(4)$ \\
\hline C5-C4-C12-C11 & $1.3(7)$ & N2-C11-C12-N1 & $2.6(6)$ \\
\hline C7-C11-C12-N1 & $-175.6(4)$ & N2-C11-C12-C4 & $179.7(4)$ \\
\hline C7-C11-C12-C4 & $1.5(7)$ & C16-C13-N3-C17 & $166.2(4)$ \\
\hline Ru1-C13-N3-C17 & $-13.7(7)$ & C16-C13-N3-C14 & $-9.1(5)$ \\
\hline Ru1-C13-N3-C14 & $171.0(3)$ & C13-N3-C14-C15 & $21.6(5)$ \\
\hline C17-N3-C14-C15 & $-154.3(4)$ & C13-N3-C14-C30 & $141.4(5)$ \\
\hline C17-N3-C14-C30 & $-34.5(6)$ & C13-N3-C14-C29 & $-96.4(5)$ \\
\hline C17-N3-C14-C29 & $87.7(5)$ & C30-C14-C15-C16 & $-145.4(4)$ \\
\hline N3-C14-C15-C16 & $-24.5(5)$ & C29-C14-C15-C16 & $92.1(5)$ \\
\hline C14-C15-C16-C31 & $138.2(4)$ & C14-C15-C16-C13 & $20.5(5)$ \\
\hline C14-C15-C16-C35 & $-100.9(5)$ & N3-C13-C16-C31 & $-124.6(4)$ \\
\hline Ru1-C13-C16-C31 & $55.3(5)$ & N3-C13-C16-C15 & $-7.0(5)$ \\
\hline Ru1-C13-C16-C15 & $172.9(3)$ & N3-C13-C16-C35 & $113.0(4)$ \\
\hline Ru1-C13-C16-C35 & $-67.1(5)$ & C13-N3-C17-C22 & 101.1(6) \\
\hline C14-N3-C17-C22 & $-83.7(5)$ & C13-N3-C17-C18 & $-85.9(6)$ \\
\hline C14-N3-C17-C18 & $89.4(5)$ & C22-C17-C18-C19 & $2.3(7)$ \\
\hline N3-C17-C18-C19 & $-170.8(4)$ & $\mathrm{C} 22-\mathrm{C} 17-\mathrm{C} 18-\mathrm{C} 23$ & $-175.6(5)$ \\
\hline N3-C17-C18-C23 & $11.3(7)$ & C17-C18-C19-C20 & $-1.9(7)$ \\
\hline C23-C18-C19-C20 & $176.2(5)$ & C18-C19-C20-C21 & $0.1(8)$ \\
\hline C19-C20-C21-C22 & $1.4(9)$ & $\mathrm{C} 20-\mathrm{C} 21-\mathrm{C} 22-\mathrm{C} 17$ & $-1.0(8)$ \\
\hline C20-C21-C22-C26 & $-177.5(5)$ & C18-C17-C22-C21 & $-0.9(7)$ \\
\hline N3-C17-C22-C21 & $171.9(4)$ & $\mathrm{C} 18-\mathrm{C} 17-\mathrm{C} 22-\mathrm{C} 26$ & $175.3(4)$ \\
\hline N3-C17-C22-C26 & $-11.9(7)$ & C19-C18-C23-C25 & $63.0(6)$ \\
\hline C17-C18-C23-C25 & $-119.0(6)$ & C19-C18-C23-C24 & $-56.9(6)$ \\
\hline C17-C18-C23-C24 & $121.1(5)$ & $\mathrm{C} 21-\mathrm{C} 22-\mathrm{C} 26-\mathrm{C} 27$ & $65.8(6)$ \\
\hline C17-C22-C26-C27 & $-110.4(5)$ & C21-C22-C26-C28 & $-55.6(6)$ \\
\hline
\end{tabular}




\begin{tabular}{llll} 
C17-C22-C26-C28 & $128.2(5)$ & C15-C16-C31-C32 & 67.2(6) \\
C13-C16-C31-C32 & $-179.5(4)$ & C35-C16-C31-C32 & $-54.9(5)$ \\
C16-C31-C32-C33 & $58.8(6)$ & C31-C32-C33-C34 & $-58.0(6)$ \\
C32-C33-C34-C35 & $55.7(7)$ & C33-C34-C35-C16 & $-53.3(6)$ \\
C31-C16-C35-C34 & $52.2(5)$ & C15-C16-C35-C34 & $-69.4(5)$ \\
C13-C16-C35-C34 & $175.1(4)$ & C13-Ru1-C36-C37 & $168.2(16)$ \\
N2-Ru1-C36-C37 & $-93.1(16)$ & N1-Ru1-C36-C37 & $-15.8(16)$ \\
C11-Ru1-C36-C37 & $74.8(16)$ & C12-Ru1-C36-C37 & $-13 .(2)$ \\
\hline
\end{tabular}

Table S39. Anisotropic atomic displacement parameters $\left(\AA^{2}\right)$ for $\mathrm{C}_{40} \mathrm{H}_{51} \mathrm{Cl}_{8} \mathrm{~N}_{3} \mathrm{Ru}$

\begin{tabular}{|c|c|c|c|c|c|c|}
\hline \multicolumn{7}{|c|}{ The anisotropic atomic displacement factor exponent takes the form: $-2 \pi^{2}\left[\mathrm{~h}^{2} \mathrm{a}^{* 2} \mathrm{U}_{11}+\ldots+2 \mathrm{~h} \mathrm{k} \mathrm{a} \mathrm{b}^{*} \mathrm{U}_{12}\right]$} \\
\hline & $\mathbf{U}_{11}$ & $\mathbf{U}_{22}$ & $\mathbf{U}_{33}$ & $\mathbf{U}_{23}$ & $\mathbf{U}_{13}$ & $\mathbf{U}_{12}$ \\
\hline Ru1 & $0.01433(19)$ & $0.02178(18)$ & $0.01941(19)$ & $0.00314(17)$ & $0.00517(14)$ & $0.00034(16)$ \\
\hline $\mathrm{C} 11$ & $0.0164(6)$ & $0.0357(7)$ & $0.0320(7)$ & $0.0043(5)$ & $0.0062(5)$ & $0.0033(5)$ \\
\hline $\mathrm{Cl} 2$ & $0.0290(7)$ & $0.0295(6)$ & $0.0238(6)$ & $0.0020(5)$ & $0.0063(5)$ & $-0.0024(5)$ \\
\hline N1 & $0.017(2)$ & $0.023(2)$ & $0.017(2)$ & $0.0007(15)$ & $0.0076(17)$ & $0.0013(15)$ \\
\hline $\mathrm{C} 1$ & $0.022(3)$ & $0.032(3)$ & $0.022(3)$ & $0.004(2)$ & $0.009(2)$ & $0.005(2)$ \\
\hline $\mathrm{C} 2$ & $0.036(3)$ & $0.023(3)$ & $0.031(3)$ & $0.009(2)$ & $0.008(3)$ & $0.006(2)$ \\
\hline $\mathrm{C} 3$ & $0.045(3)$ & $0.024(3)$ & $0.030(3)$ & $0.001(2)$ & $0.013(3)$ & $-0.004(2)$ \\
\hline $\mathrm{C} 4$ & $0.028(3)$ & $0.025(3)$ & $0.025(3)$ & $-0.002(2)$ & $0.009(2)$ & $-0.007(2)$ \\
\hline $\mathrm{C} 5$ & $0.032(3)$ & $0.034(3)$ & $0.039(3)$ & $-0.001(2)$ & $0.015(3)$ & $-0.015(2)$ \\
\hline $\mathrm{C} 6$ & $0.018(3)$ & $0.045(3)$ & $0.039(3)$ & $0.000(3)$ & $0.013(2)$ & $-0.010(2)$ \\
\hline $\mathrm{C} 7$ & $0.015(2)$ & $0.033(3)$ & $0.022(3)$ & $-0.002(2)$ & $0.004(2)$ & $0.000(2)$ \\
\hline $\mathrm{C} 8$ & $0.015(3)$ & $0.051(3)$ & $0.029(3)$ & $0.000(2)$ & $0.004(2)$ & $0.004(2)$ \\
\hline C9 & $0.020(3)$ & $0.034(3)$ & $0.037(3)$ & $0.002(2)$ & $0.006(2)$ & $0.007(2)$ \\
\hline $\mathrm{C} 10$ & $0.024(3)$ & $0.029(3)$ & $0.026(3)$ & $0.006(2)$ & $0.007(2)$ & $0.004(2)$ \\
\hline $\mathrm{N} 2$ & $0.015(2)$ & $0.023(2)$ & $0.019(2)$ & $0.0019(15)$ & $0.0035(17)$ & $-0.0008(15)$ \\
\hline $\mathrm{C} 11$ & $0.019(3)$ & $0.023(2)$ & $0.021(3)$ & $0.0014(19)$ & $0.007(2)$ & $-0.0023(18)$ \\
\hline $\mathrm{C} 12$ & $0.020(3)$ & $0.025(2)$ & $0.018(2)$ & $-0.0011(19)$ & $0.004(2)$ & $-0.0026(19)$ \\
\hline $\mathrm{C} 13$ & $0.014(2)$ & $0.025(2)$ & $0.019(2)$ & $0.0020(18)$ & $0.004(2)$ & $0.0006(18)$ \\
\hline N3 & $0.020(2)$ & $0.0185(19)$ & $0.023(2)$ & $0.0040(16)$ & $0.0021(18)$ & $-0.0001(15)$ \\
\hline $\mathrm{C} 14$ & $0.037(3)$ & $0.022(3)$ & $0.020(2)$ & $0.005(2)$ & $0.003(2)$ & $-0.003(2)$ \\
\hline $\mathrm{C} 15$ & $0.031(3)$ & $0.024(3)$ & $0.027(3)$ & $0.003(2)$ & $0.007(2)$ & $-0.006(2)$ \\
\hline $\mathrm{C} 16$ & $0.024(3)$ & $0.023(3)$ & $0.017(2)$ & $0.0042(18)$ & $-0.001(2)$ & $-0.0044(19)$ \\
\hline
\end{tabular}




\begin{tabular}{|c|c|c|c|c|c|c|}
\hline & $\mathbf{U}_{11}$ & $\mathbf{U}_{22}$ & $\mathbf{U}_{33}$ & $\mathbf{U}_{23}$ & $\mathbf{U}_{13}$ & $\mathbf{U}_{12}$ \\
\hline $\mathrm{C} 17$ & $0.026(3)$ & $0.023(2)$ & $0.018(2)$ & $0.0024(19)$ & $0.007(2)$ & $-0.0010(19)$ \\
\hline $\mathrm{C} 18$ & $0.028(3)$ & $0.024(2)$ & $0.025(3)$ & $0.0054(19)$ & $0.010(2)$ & $0.001(2)$ \\
\hline C19 & $0.044(4)$ & $0.036(3)$ & $0.024(3)$ & $0.001(2)$ & $0.015(3)$ & $0.007(2)$ \\
\hline $\mathrm{C} 20$ & $0.055(4)$ & $0.035(3)$ & $0.024(3)$ & $-0.005(2)$ & $0.003(3)$ & $0.006(3)$ \\
\hline $\mathrm{C} 21$ & $0.038(3)$ & $0.035(3)$ & $0.029(3)$ & $-0.003(3)$ & $-0.005(3)$ & $-0.001(3)$ \\
\hline $\mathrm{C} 22$ & $0.027(3)$ & $0.025(3)$ & $0.025(3)$ & $0.007(2)$ & $0.005(2)$ & $-0.001(2)$ \\
\hline $\mathrm{C} 23$ & $0.023(3)$ & $0.039(3)$ & $0.044(3)$ & $-0.008(3)$ & $0.011(3)$ & $0.003(2)$ \\
\hline $\mathrm{C} 24$ & $0.031(3)$ & $0.047(4)$ & $0.038(3)$ & $0.002(3)$ & $0.005(3)$ & $0.014(3)$ \\
\hline $\mathrm{C} 25$ & $0.034(4)$ & $0.057(5)$ & $0.134(8)$ & $0.024(5)$ & $0.033(4)$ & $0.007(3)$ \\
\hline C26 & $0.022(3)$ & $0.036(3)$ & $0.028(3)$ & $0.002(2)$ & $0.000(2)$ & $0.002(2)$ \\
\hline $\mathrm{C} 27$ & $0.026(3)$ & $0.048(3)$ & $0.036(3)$ & $0.008(3)$ & $0.005(3)$ & $-0.006(3)$ \\
\hline $\mathrm{C} 28$ & $0.032(3)$ & $0.044(3)$ & $0.036(3)$ & $0.005(3)$ & $-0.003(3)$ & $0.007(3)$ \\
\hline $\mathrm{C} 29$ & $0.043(3)$ & $0.023(3)$ & $0.046(4)$ & $0.008(2)$ & $-0.004(3)$ & $0.001(2)$ \\
\hline C30 & $0.073(5)$ & $0.034(3)$ & $0.029(3)$ & $0.007(2)$ & $0.017(3)$ & $-0.012(3)$ \\
\hline C31 & $0.028(3)$ & $0.028(3)$ & $0.032(3)$ & $0.006(2)$ & $0.001(2)$ & $0.000(2)$ \\
\hline C32 & $0.047(4)$ & $0.033(3)$ & $0.040(4)$ & $0.002(3)$ & $-0.012(3)$ & $-0.010(3)$ \\
\hline C33 & $0.079(5)$ & $0.036(3)$ & $0.029(3)$ & $-0.005(3)$ & $-0.005(3)$ & $-0.006(3)$ \\
\hline C34 & $0.060(4)$ & $0.032(3)$ & $0.028(3)$ & $-0.003(2)$ & $0.010(3)$ & $0.004(3)$ \\
\hline $\mathrm{C} 35$ & $0.038(3)$ & $0.025(3)$ & $0.026(3)$ & $0.001(2)$ & $0.012(2)$ & $0.000(2)$ \\
\hline C36 & $0.018(3)$ & $0.023(2)$ & $0.026(3)$ & $0.010(2)$ & $0.004(2)$ & $0.0018(19)$ \\
\hline C37 & $0.035(3)$ & $0.026(3)$ & $0.029(3)$ & $-0.001(2)$ & $0.007(2)$ & $0.004(2)$ \\
\hline C38 & $0.057(5)$ & $0.069(5)$ & $0.085(6)$ & $-0.015(4)$ & $0.014(4)$ & $-0.013(4)$ \\
\hline $\mathrm{Cl} 3$ & $0.159(2)$ & $0.0537(12)$ & $0.0778(15)$ & $0.0042(11)$ & $0.0051(15)$ & $-0.0235(13)$ \\
\hline $\mathrm{Cl} 4$ & $0.1040(18)$ & $0.0798(16)$ & $0.1028(18)$ & $0.0053(13)$ & $-0.0165(15)$ & $-0.0279(13)$ \\
\hline C39 & $0.120(7)$ & $0.057(5)$ & $0.070(5)$ & $0.001(4)$ & $0.046(5)$ & $-0.020(5)$ \\
\hline $\mathrm{Cl} 5$ & $0.0967(18)$ & $0.112(2)$ & $0.0911(17)$ & $-0.0033(15)$ & $-0.0093(15)$ & $0.0225(15)$ \\
\hline $\mathrm{Cl} 6$ & $0.0742(14)$ & $0.0958(17)$ & $0.0695(14)$ & $-0.0055(12)$ & $0.0042(12)$ & $-0.0060(11)$ \\
\hline $\mathrm{C} 40$ & $0.034(3)$ & $0.041(3)$ & $0.042(4)$ & $-0.003(3)$ & $0.005(3)$ & $0.003(2)$ \\
\hline $\mathrm{Cl} 7$ & $0.0394(9)$ & $0.0474(9)$ & $0.0613(11)$ & $0.0102(8)$ & $0.0044(8)$ & $-0.0028(7)$ \\
\hline $\mathrm{Cl} 8$ & $0.0491(10)$ & $0.0458(9)$ & $0.0639(11)$ & $0.0043(8)$ & $0.0198(8)$ & $0.0002(7)$ \\
\hline
\end{tabular}

Table S40. Hydrogen atomic coordinates and isotropic atomic displacement parameters $\left(\AA^{2}\right)$ for $\mathrm{C}_{40} \mathrm{H}_{51} \mathrm{Cl}_{8} \mathrm{~N}_{3} \mathrm{Ru}$ 


$\begin{array}{lllll} & \mathbf{x} / \mathbf{a} & \mathbf{y} / \mathbf{b} & \mathbf{z} / \mathbf{c} & \mathbf{U}(\mathbf{e q}) \\ \text { H1 } & 0.7506 & 0.5003 & 0.9188 & 0.03 \\ \text { H2 } & 0.6686 & 0.3915 & 0.9561 & 0.036 \\ \text { H3 } & 0.4550 & 0.3831 & 0.9574 & 0.038 \\ \text { H5 } & 0.2335 & 0.4450 & 0.9325 & 0.041 \\ \text { H6 } & 0.1120 & 0.5488 & 0.9007 & 0.039 \\ \text { H8 } & 0.0959 & 0.6839 & 0.8608 & 0.038 \\ \text { H9 } & 0.2014 & 0.7882 & 0.8326 & 0.036 \\ \text { H10 } & 0.4126 & 0.7794 & 0.8249 & 0.031 \\ \text { H15A } & 0.7204 & 0.9338 & 0.7997 & 0.033 \\ \text { H15B } & 0.8352 & 0.8802 & 0.7893 & 0.033 \\ \text { H19 } & 0.7783 & 0.6168 & 0.6187 & 0.04 \\ \text { H20 } & 0.5764 & 0.5971 & 0.5639 & 0.047 \\ \text { H21 } & 0.4023 & 0.6481 & 0.5952 & 0.043 \\ \text { H23 } & 0.8691 & 0.7149 & 0.7560 & 0.042 \\ \text { H24A } & 0.8635 & 0.5786 & 0.7557 & 0.059 \\ \text { H24B } & 1.0051 & 0.6105 & 0.7604 & 0.059 \\ \text { H24C } & 0.9272 & 0.5789 & 0.6980 & 0.059 \\ \text { H25A } & 0.9775 & 0.6879 & 0.6538 & 0.108 \\ \text { H25B } & 1.0316 & 0.7419 & 0.7093 & 0.108 \\ \text { H25C } & 0.9100 & 0.7674 & 0.6605 & 0.108 \\ \text { H26 } & 0.4017 & 0.7654 & 0.7214 & 0.035 \\ \text { H27A } & 0.2723 & 0.6320 & 0.6690 & 0.055 \\ \text { H27B } & 0.2308 & 0.6824 & 0.7200 & 0.055 \\ \text { H27C } & 0.3591 & 0.6336 & 0.7347 & 0.055 \\ \text { H28A } & 0.3416 & 0.8158 & 0.6214 & 0.058 \\ \text { H28B } & 0.2194 & 0.7961 & 0.6489 & 0.058 \\ \text { H28 } & 0.2621 & 0.7402 & 0.6014 & 0.058 \\ \text { H29 } & 0.4868 & 0.8811 & 0.7538 & 0.059 \\ \text { H29 } & 0.4823 & 0.8717 & 0.6838 & 0.059 \\ \text { H2734 } & 0.9461 & 0.7179 & 0.059 \\ \text { H2 } & 0.9169 & 0.6724 & 0.067 \\ \text { H29 } & 0.8349 & 0.6480 & 0.067 \\ \text { H2 } & 0.8441 & 0.6926 & 0.067\end{array}$




\begin{tabular}{lllll} 
& $\mathbf{x} / \mathbf{a}$ & $\mathbf{y} / \mathbf{b}$ & $\mathbf{z} / \mathbf{c}$ & $\mathbf{U}(\mathbf{e q})$ \\
H31A & 0.9095 & 0.7916 & 0.8628 & 0.036 \\
H31B & 0.8342 & 0.7725 & 0.9148 & 0.036 \\
H32A & 0.9857 & 0.8695 & 0.9475 & 0.051 \\
H32B & 0.9232 & 0.9213 & 0.8917 & 0.051 \\
H33A & 0.7974 & 0.8771 & 0.9861 & 0.06 \\
H33B & 0.8479 & 0.9609 & 0.9772 & 0.06 \\
H34A & 0.6978 & 0.9756 & 0.8885 & 0.048 \\
H34B & 0.6250 & 0.9517 & 0.9403 & 0.048 \\
H35A & 0.5504 & 0.8821 & 0.8506 & 0.035 \\
H35B & 0.6029 & 0.8276 & 0.9060 & 0.035 \\
H37A & 0.5857 & 0.4930 & 0.7630 & 0.036 \\
H37B & 0.5705 & 0.5531 & 0.7070 & 0.036 \\
H38A & 0.3349 & 0.5152 & 0.7539 & 0.084 \\
H38B & 0.2155 & 0.4773 & 0.7747 & 0.084 \\
H39A & -0.0009 & 0.3039 & 0.5093 & 0.095 \\
H39B & 0.0841 & 0.2997 & 0.4592 & 0.095 \\
H40A & -0.0356 & 0.7096 & 0.0464 & 0.047 \\
H40B & -0.0733 & 0.6822 & -0.0217 & 0.047 \\
\hline
\end{tabular}

\section{Computational (DFT) Studies}

Geometry optimizations of all structures were carried out at the level of B3LYP-D3 ${ }^{7}$ with $\mathrm{SDD}^{8}$ basis set used for $\mathrm{Ru}$ and 6-31G(d) basis set for other atoms. The single-point energy in solution were calculated at the $\omega \mathrm{B} 97 \mathrm{X}-\mathrm{D}^{9}$ level of theory with SDD basis set for Ru and $6-311++\mathrm{G}(\mathrm{d}, \mathrm{p})$ for the other atoms. Solvation effects were evaluated using the SMD model ${ }^{10}$ with $\mathrm{CH}_{2} \mathrm{Cl}_{2}$ as the solvent.

\section{Cartesian coordinates for all optimized geometries.}

\subsection{For complexes where $L=P C_{3}$}

Ru-II

Thermal correction to Gibbs free energy $=0.625507$

Sum of electronic and thermal free energies $=-2525.574222$

E_sol $=-2526.1478583$ 


\begin{tabular}{|c|c|c|c|}
\hline $\mathrm{Ru}$ & -1.50595500 & 1.83619800 & -0.00 \\
\hline $\mathrm{Cl}$ & 0.72575200 & 2.58125600 & 0.14323800 \\
\hline $\mathrm{Cl}$ & -2.96295500 & 0.00545800 & 0.34776300 \\
\hline $\mathrm{C}$ & -2.47820600 & 3.38169500 & 0.16950700 \\
\hline $\mathrm{H}$ & -2.92142400 & 3.94809200 & -0.65787600 \\
\hline $\mathrm{C}$ & -2.66976900 & 3.96410800 & 1.48089300 \\
\hline $\mathrm{C}$ & -2.15296400 & 3.28857300 & 2.61492000 \\
\hline $\mathrm{C}$ & -3.33580000 & 5.18882700 & 1.67270600 \\
\hline $\mathrm{C}$ & -2.29664400 & 3.82908500 & 3.89261400 \\
\hline $\mathrm{C}$ & -3.48363500 & 5.73286600 & 2.94466000 \\
\hline $\mathrm{H}$ & -3.73196200 & 5.70595000 & 0.80202600 \\
\hline $\mathrm{C}$ & -2.96090100 & 5.04983600 & 4.04636300 \\
\hline $\mathrm{H}$ & -1.90504100 & 3.31961300 & 4.76397900 \\
\hline $\mathrm{H}$ & -3.99883200 & 6.67884800 & 3.08044000 \\
\hline $\mathrm{H}$ & -3.06908400 & 5.46593900 & 5.04412900 \\
\hline $\mathrm{O}$ & -1.51731600 & 2.11381200 & 2.32084800 \\
\hline $\mathrm{C}$ & -1.09896500 & 1.19742000 & 3.38502800 \\
\hline $\mathrm{H}$ & -0.57970800 & 1.80540500 & 4.13492800 \\
\hline $\mathrm{C}$ & -0.10794500 & 0.22183400 & 2.76890200 \\
\hline $\mathrm{H}$ & -0.60316400 & -0.40287900 & 2.01912900 \\
\hline $\mathrm{H}$ & 0.71997500 & 0.75619600 & 2.29775300 \\
\hline $\mathrm{H}$ & 0.28471300 & -0.43007100 & 3.55720300 \\
\hline $\mathrm{C}$ & -2.32380900 & 0.50769600 & 3.97717700 \\
\hline $\mathrm{H}$ & -2.82390400 & -0.07392300 & 3.19816000 \\
\hline $\mathrm{H}$ & -2.01263400 & -0.16335000 & 4.78566900 \\
\hline $\mathrm{H}$ & -3.03906600 & 1.22803300 & 4.38454300 \\
\hline $\mathrm{P}$ & -1.75550300 & 1.75884800 & -2.29531500 \\
\hline $\mathrm{C}$ & -3.83557000 & 1.08852900 & -4.27674300 \\
\hline $\mathrm{H}$ & -4.63971500 & 0.34142700 & -4.35390600 \\
\hline $\mathrm{H}$ & -2.98992400 & 0.67170100 & -4.83588100 \\
\hline $\mathrm{C}$ & -4.33411500 & 2.38110200 & -4.94746900 \\
\hline $\mathrm{H}$ & -4.64577000 & 2.15376900 & -5.97507300 \\
\hline $\mathrm{H}$ & -3.52056900 & 3.10775500 & -5.02976900 \\
\hline $\mathrm{C}$ & -5.49275200 & 3.02421000 & -4.17209400 \\
\hline $\mathrm{H}$ & -6.36759600 & 2.35824900 & -4.20660500 \\
\hline $\mathrm{H}$ & -5.79241500 & 3.96438100 & -4.65209700 \\
\hline $\mathrm{C}$ & -5.10969200 & 3.25980900 & -2.70417000 \\
\hline $\mathrm{H}$ & -5.96082700 & 3.67712000 & -2.15115900 \\
\hline $\mathrm{H}$ & -4.31018800 & 4.01249600 & -2.64893500 \\
\hline $\mathrm{C}$ & -4.66463100 & 1.95020500 & -2.04072000 \\
\hline
\end{tabular}




\begin{tabular}{|c|c|c|c|}
\hline $\mathrm{H}$ & -4.42359800 & 2.09617800 & -0.98653900 \\
\hline $\mathrm{H}$ & -5.52186000 & 1.26083100 & -2.05617500 \\
\hline $\mathrm{C}$ & -3.50815900 & 1.22232900 & -2.76793700 \\
\hline $\mathrm{H}$ & -3.49160200 & 0.21491500 & -2.34240900 \\
\hline $\mathrm{C}$ & -0.70524200 & 0.43372600 & -3.11620900 \\
\hline $\mathrm{H}$ & -0.81523500 & 0.59798400 & -4.19267800 \\
\hline $\mathrm{C}$ & 0.80300400 & 0.58629400 & -2.75784700 \\
\hline $\mathrm{H}$ & 1.04219200 & 1.60746400 & -2.45262700 \\
\hline $\mathrm{H}$ & 1.39773100 & 0.38101200 & -3.65845400 \\
\hline $\mathrm{C}$ & 1.21415700 & -0.39484600 & -1.65336500 \\
\hline $\mathrm{H}$ & 0.58184900 & -0.22300000 & -0.77395100 \\
\hline $\mathrm{H}$ & 2.23696800 & -0.18382400 & -1.32266500 \\
\hline $\mathrm{C}$ & 1.07529900 & -1.85783000 & -2.12516300 \\
\hline $\mathrm{H}$ & 0.93173200 & -2.50812100 & -1.25322500 \\
\hline $\mathrm{H}$ & 2.00303000 & -2.18738000 & -2.61009200 \\
\hline $\mathrm{C}$ & -0.10755700 & -2.02510100 & -3.10785400 \\
\hline $\mathrm{H}$ & -0.51173700 & -3.04263800 & -3.05288000 \\
\hline $\mathrm{H}$ & 0.23898200 & -1.88698400 & -4.14167600 \\
\hline $\mathrm{C}$ & -1.21425100 & -1.00141500 & -2.81651500 \\
\hline $\mathrm{H}$ & -2.09738300 & -1.21574100 & -3.42845600 \\
\hline $\mathrm{H}$ & -1.52991400 & -1.09701700 & -1.77126400 \\
\hline $\mathrm{C}$ & -0.89924000 & 3.14982900 & -4.70344000 \\
\hline $\mathrm{H}$ & -1.45056900 & 2.36161200 & -5.22260700 \\
\hline $\mathrm{H}$ & 0.14781700 & 2.82353200 & -4.67404600 \\
\hline $\mathrm{C}$ & -1.02329000 & 4.47061000 & -5.50662400 \\
\hline $\mathrm{H}$ & -1.99684800 & 4.50868800 & -6.01116200 \\
\hline $\mathrm{H}$ & -0.26420000 & 4.47194800 & -6.29889300 \\
\hline $\mathrm{C}$ & -0.85134600 & 5.71838400 & -4.61311100 \\
\hline $\mathrm{H}$ & -1.81981400 & 6.00986200 & -4.18270200 \\
\hline $\mathrm{H}$ & -0.51470400 & 6.57124300 & -5.21410000 \\
\hline $\mathrm{C}$ & 0.13340600 & 5.41826200 & -3.47906100 \\
\hline $\mathrm{H}$ & 0.35691700 & 6.32219400 & -2.90092800 \\
\hline $\mathrm{H}$ & 1.08616300 & 5.09446500 & -3.92014300 \\
\hline $\mathrm{C}$ & -0.40449400 & 4.31669600 & -2.53051200 \\
\hline $\mathrm{H}$ & 0.43514000 & 3.76611900 & -2.10685100 \\
\hline $\mathrm{H}$ & -0.91425300 & 4.76976300 & -1.67439800 \\
\hline $\mathrm{C}$ & -1.38116400 & 3.35120500 & -3.25010100 \\
\hline $\mathrm{H}$ & -2.36189000 & 3.83690600 & -3.291420 \\
\hline
\end{tabular}




\section{Allenyl-B(pin) (1)}

Thermal correction to Gibbs free energy $=0.191564$

Sum of electronic and thermal free energies $=-527.186808$

E_sol $=-527.3403362$

$\begin{array}{lrrr}\mathrm{C} & 1.80347500 & 3.10088600 & 0.15198400 \\ \mathrm{C} & 0.73349400 & 2.03497700 & -0.29324100 \\ \mathrm{~B} & 2.81123300 & 1.10996500 & -0.29857000 \\ \mathrm{O} & 1.56193200 & 0.98069000 & -0.85593600 \\ \mathrm{O} & 2.97163400 & 2.27412200 & 0.41527200 \\ \mathrm{C} & -0.02676900 & 1.41433900 & 0.88529400 \\ \mathrm{H} & -0.72835200 & 2.12602900 & 1.33260900 \\ \mathrm{H} & -0.58981300 & 0.54816200 & 0.52527900 \\ \mathrm{H} & 0.66494100 & 1.07021000 & 1.66055400 \\ \mathrm{C} & 1.45777200 & 3.87455700 & 1.42062800 \\ \mathrm{H} & 2.26402000 & 4.57672200 & 1.65500400 \\ \mathrm{H} & 0.53370100 & 4.44759500 & 1.28341500 \\ \mathrm{H} & 1.33310500 & 3.20705100 & 2.27583600 \\ \mathrm{C} & 2.19611800 & 4.06817400 & -0.97142800 \\ \mathrm{H} & 1.39106600 & 4.77623900 & -1.19345000 \\ \mathrm{H} & 3.08031800 & 4.63215500 & -0.65969400 \\ \mathrm{H} & 2.44631500 & 3.52392900 & -1.88739600 \\ \mathrm{C} & -0.24489700 & 2.50852100 & -1.36378700 \\ \mathrm{H} & -0.93532200 & 1.69739000 & -1.61532000 \\ \mathrm{H} & -0.83305300 & 3.35905500 & -1.00072500 \\ \mathrm{H} & 0.27381400 & 2.80455300 & -2.27799100 \\ \mathrm{C} & 3.94870600 & 0.06569600 & -0.44265800 \\ \mathrm{H} & 4.92394600 & 0.28315100 & -0.00277700 \\ \mathrm{C} & 3.78382600 & -1.07293700 & -1.07601200 \\ \mathrm{C} & 3.60374200 & -2.19976800 & -1.70984400 \\ \mathrm{H} & 3.25509100 & -3.09186700 & -1.19360500 \\ \mathrm{H} & 3.78787000 & -2.28524600 & -2.77880500\end{array}$

\section{Allene 2a}

Thermal correction to Gibbs free energy $=0.259206$

Sum of electronic and thermal free energies $=-796.918383$

E_sol $=-797.1477234$

$\begin{array}{llcc}\mathrm{C} & 3.91188400 & 1.25604200 & 0.03939100 \\ \mathrm{H} & 4.89541600 & 0.82460500 & -0.13977700 \\ \mathrm{H} & 3.10059600 & 0.55820400 & 0.24027600 \\ \mathrm{C} & 3.71445200 & 2.54812500 & 0.01224600 \\ \mathrm{C} & 3.53066700 & 3.84381800 & -0.00536900\end{array}$




\begin{tabular}{llll}
$\mathrm{H}$ & 3.25719700 & 4.32077100 & -0.94892100 \\
$\mathrm{C}$ & 3.63465700 & 4.74386600 & 1.20540400 \\
$\mathrm{H}$ & 4.32254200 & 5.57479500 & 1.00014400 \\
$\mathrm{H}$ & 4.03381200 & 4.19332300 & 2.06336900 \\
$\mathrm{C}$ & 2.27635500 & 5.34091100 & 1.58375300 \\
$\mathrm{H}$ & 1.58698400 & 4.52991700 & 1.86254200 \\
$\mathrm{H}$ & 1.83949700 & 5.85548600 & 0.71369700 \\
$\mathrm{O}$ & 2.45750800 & 6.26330100 & 2.64615200 \\
$\mathrm{Si}$ & 1.31388300 & 6.66568600 & 3.80646900 \\
$\mathrm{C}$ & 0.75237400 & 5.11172300 & 4.72054900 \\
$\mathrm{H}$ & -0.02240700 & 5.34845900 & 5.46001700 \\
$\mathrm{H}$ & 0.33630800 & 4.35936300 & 4.04071700 \\
$\mathrm{H}$ & 1.59300900 & 4.65200200 & 5.25306500 \\
$\mathrm{C}$ & 2.22152300 & 7.82982400 & 4.96964900 \\
$\mathrm{H}$ & 2.51416300 & 8.75410600 & 4.45923900 \\
$\mathrm{H}$ & 1.60111200 & 8.10111600 & 5.83156800 \\
$\mathrm{H}$ & 3.13405000 & 7.35443500 & 5.34650800 \\
$\mathrm{C}$ & -0.18738900 & 7.53737600 & 2.99919100 \\
$\mathrm{C}$ & -1.04650200 & 6.53365600 & 2.20075700 \\
$\mathrm{H}$ & -1.90661900 & 7.04575600 & 1.74475700 \\
$\mathrm{H}$ & -0.48359200 & 6.06152400 & 1.38695600 \\
$\mathrm{H}$ & -1.44391100 & 5.73551600 & 2.83937000 \\
$\mathrm{C}$ & -1.06500800 & 8.17315800 & 4.09907200 \\
$\mathrm{H}$ & -0.51919600 & 8.93267700 & 4.67157600 \\
$\mathrm{H}$ & -1.94026000 & 8.66705400 & 3.65217500 \\
$\mathrm{H}$ & -1.44262500 & 7.42497200 & 4.80801000 \\
$\mathrm{C}$ & 0.31385100 & 8.64330000 & 2.04782200 \\
$\mathrm{H}$ & -0.53507300 & 9.16353100 & 1.58025900 \\
$\mathrm{H}$ & 0.90837800 & 9.39780800 & 2.57759800 \\
$\mathrm{H}$ & 0.93859900 & 8.23512700 & 1.24478000 \\
$\mathrm{ts}-\mathbf{i}$ & & & \\
$\mathrm{H}$ & & & \\
\hline
\end{tabular}

Thermal correction to Gibbs free energy $=0.838982$

Sum of electronic and thermal free energies $=-3052.725218$

E_sol $=-3053.4821526$

$\begin{array}{rrrr}\mathrm{Ru} & 1.17659800 & 0.58824300 & -1.51903500 \\ \mathrm{Cl} & -0.40224700 & -1.20491500 & -1.01829300 \\ \mathrm{Cl} & 2.89532000 & 2.02343600 & -2.48552900 \\ \mathrm{C} & 0.54392000 & 2.01875600 & -0.46328500 \\ \mathrm{C} & -0.48747900 & 2.10160000 & 0.56766500 \\ \mathrm{C} & -0.21607300 & 2.91132300 & 1.70888400 \\ \mathrm{C} & -1.72390000 & 1.43383300 & 0.50292400\end{array}$




\begin{tabular}{|c|c|c|c|}
\hline $\mathrm{C}$ & -1.17053700 & 3.03519000 & 2.72575200 \\
\hline $\mathrm{C}$ & -2.67874300 & 1.57821400 & 1.50280600 \\
\hline $\mathrm{H}$ & -1.91551800 & 0.78582800 & -0.34316700 \\
\hline $\mathrm{C}$ & -2.39620100 & 2.37760300 & 2.61229800 \\
\hline $\mathrm{H}$ & -0.96659000 & 3.63386900 & 3.60492300 \\
\hline $\mathrm{H}$ & -3.62887900 & 1.05924500 & 1.42501000 \\
\hline $\mathrm{H}$ & -3.12869000 & 2.48692200 & 3.40768400 \\
\hline $\mathrm{O}$ & 1.01254900 & 3.49249500 & 1.73245900 \\
\hline $\mathrm{C}$ & 1.47366400 & 4.24345700 & 2.87434200 \\
\hline $\mathrm{H}$ & 0.65936900 & 4.88858400 & 3.23007100 \\
\hline $\mathrm{C}$ & 2.60720100 & 5.11514200 & 2.35011000 \\
\hline $\mathrm{H}$ & 3.40620600 & 4.48771000 & 1.94373000 \\
\hline $\mathrm{H}$ & 2.24800300 & 5.77507200 & 1.55483800 \\
\hline $\mathrm{H}$ & 3.01940700 & 5.72792900 & 3.15875000 \\
\hline $\mathrm{C}$ & 1.92292600 & 3.28865100 & 3.97983900 \\
\hline $\mathrm{H}$ & 2.76415800 & 2.68249500 & 3.62782300 \\
\hline $\mathrm{H}$ & 2.24502900 & 3.85311800 & 4.86176500 \\
\hline $\mathrm{H}$ & 1.11653100 & 2.61169200 & 4.27668400 \\
\hline $\mathrm{P}$ & 2.82832500 & -0.62390900 & -0.22333300 \\
\hline $\mathrm{C}$ & 4.34970000 & -3.01726000 & -1.08650100 \\
\hline $\mathrm{H}$ & 4.11239400 & -4.00489500 & -1.50987200 \\
\hline $\mathrm{H}$ & 4.51759700 & -3.18207200 & -0.01536300 \\
\hline $\mathrm{C}$ & 5.63000100 & -2.50466100 & -1.76943600 \\
\hline $\mathrm{H}$ & 6.43451200 & -3.23691700 & -1.62429200 \\
\hline $\mathrm{H}$ & 5.96677400 & -1.57577200 & -1.29724200 \\
\hline $\mathrm{C}$ & 5.40912700 & -2.23460400 & -3.26444300 \\
\hline $\mathrm{H}$ & 5.18002500 & -3.18105800 & -3.77691800 \\
\hline $\mathrm{H}$ & 6.32998900 & -1.84556500 & -3.71630100 \\
\hline $\mathrm{C}$ & 4.25190000 & -1.24840600 & -3.47295300 \\
\hline $\mathrm{H}$ & 4.07463600 & -1.08051600 & -4.54199100 \\
\hline $\mathrm{H}$ & 4.50111600 & -0.26841700 & -3.04934600 \\
\hline $\mathrm{C}$ & 2.97027000 & -1.77990700 & -2.82511000 \\
\hline $\mathrm{H}$ & 2.14448700 & -1.07327300 & -2.99377700 \\
\hline $\mathrm{H}$ & 2.66431900 & -2.70294900 & -3.33948000 \\
\hline $\mathrm{C}$ & 3.11287100 & -2.12349200 & -1.32091400 \\
\hline $\mathrm{H}$ & 2.21583600 & -2.69453800 & -1.05682500 \\
\hline $\mathrm{C}$ & 2.38463700 & -1.29538800 & 1.47623400 \\
\hline $\mathrm{H}$ & 3.35832700 & -1.45319200 & 1.95309100 \\
\hline $\mathrm{C}$ & 1.60540200 & -0.23853300 & 2.32851500 \\
\hline $\mathrm{H}$ & 1.57626800 & 0.73044500 & 1.82655700 \\
\hline
\end{tabular}




$\begin{array}{lrrr}\mathrm{H} & 2.14666400 & -0.07692500 & 3.27119300 \\ \mathrm{C} & 0.16865500 & -0.67916100 & 2.62885700 \\ \mathrm{H} & -0.36220500 & -0.78447800 & 1.67875800 \\ \mathrm{H} & -0.34918300 & 0.11054000 & 3.18603400 \\ \mathrm{C} & 0.12567900 & -2.00891600 & 3.40551100 \\ \mathrm{H} & -0.86477800 & -2.46282500 & 3.27935500 \\ \mathrm{H} & 0.24784700 & -1.82366200 & 4.48085100 \\ \mathrm{C} & 1.22482500 & -2.99014500 & 2.92409600 \\ \mathrm{H} & 0.86839600 & -4.02523700 & 2.98200700 \\ \mathrm{H} & 2.09923400 & -2.92959400 & 3.58821800 \\ \mathrm{C} & 1.67870200 & -2.67119000 & 1.49067100 \\ \mathrm{H} & 2.37096700 & -3.44511900 & 1.13884900 \\ \mathrm{H} & 0.81700900 & -2.67419300 & 0.81788000 \\ \mathrm{C} & 5.46116600 & -0.35990000 & 0.99679900 \\ \mathrm{H} & 5.55904200 & -1.42937000 & 0.79669800 \\ \mathrm{H} & 5.14511200 & -0.27426800 & 2.04423800 \\ \mathrm{C} & 6.82906200 & 0.33947300 & 0.81564100 \\ \mathrm{H} & 7.36305000 & -0.10700000 & -0.03266800 \\ \mathrm{H} & 7.44403000 & 0.15246400 & 1.70510200 \\ \mathrm{H} & -2.56397900 & 2.10592100 & -6.46538800 \\ \mathrm{H} & -1.94766400 & 1.10077000 & -7.50873900 \\ \mathrm{C} & -1.00445600 & 0.78485700 & -5.46168000 \\ \mathrm{H} & 6.66761300 & 1.86095300 & 0.58680500 \\ \mathrm{H} & 7.57698000 & 2.07278500 & -0.48641000 \\ \mathrm{C} & & & \\ \mathrm{H} & 5.41151800 & 2.37845100 & 1.29843700 \\ \mathrm{H} & 5.34966500 & 3.47002100 & 1.21768100 \\ \mathrm{C} & 5.49377500 & 2.15366600 & 2.37133600 \\ \mathrm{H} & 4.12373700 & 1.74038100 & 0.71233200 \\ \mathrm{H} & 3.37090500 & 1.67539900 & 1.50272200 \\ \mathrm{H} & 3.70763000 & 2.37551300 & -0.07026300 \\ \mathrm{H} & 4.40601400 & 0.33463700 & 0.10892700 \\ \mathrm{H} & 4.81930900 & 0.48346200 & -0.89464900 \\ \mathrm{H} & 1.16428400 & 2.91850600 & -0.52282900 \\ \mathrm{H} & -0.11357300 & 0.19782000 & -4.34567300 \\ \mathrm{H} & 0.29839300 & 2.04947300 & -2.52558800 \\ \mathrm{H} & 2.30225800 & 2.11975700 & -2.11600300 \\ \mathrm{H} & & 0.89314700 & -3.22409000 \\ \mathrm{H} & & & \end{array}$




\begin{tabular}{llll}
$\mathrm{O}$ & -1.26578100 & 0.14578400 & -6.65091800 \\
$\mathrm{O}$ & -1.62837400 & 2.01091900 & -5.35899900 \\
$\mathrm{C}$ & -2.96540000 & 0.35009400 & -8.36252100 \\
$\mathrm{H}$ & -3.55726100 & 1.05033800 & -8.96322100 \\
$\mathrm{H}$ & -2.44409900 & -0.32956900 & -9.04406600 \\
$\mathrm{H}$ & -3.64319600 & -0.24499100 & -7.74672100 \\
$\mathrm{C}$ & -3.92872300 & 1.65520100 & -5.92873900 \\
$\mathrm{H}$ & -4.19362200 & 2.27851900 & -5.06911200 \\
$\mathrm{H}$ & -4.71386500 & 1.75360600 & -6.68569000 \\
$\mathrm{H}$ & -3.89249300 & 0.61375500 & -5.59400000 \\
$\mathrm{C}$ & -2.62835300 & 3.55877800 & -6.92716500 \\
$\mathrm{H}$ & -3.26174700 & 3.65427400 & -7.81657800 \\
$\mathrm{H}$ & -3.05792500 & 4.17873500 & -6.13361900 \\
$\mathrm{H}$ & -1.63509500 & 3.94899700 & -7.15908900 \\
$\mathrm{C}$ & -0.87253000 & 1.73946400 & -8.39707700 \\
$\mathrm{H}$ & -0.34669300 & 0.94786800 & -8.93936800 \\
$\mathrm{H}$ & -1.30872600 & 2.43119400 & -9.12539000 \\
$\mathrm{H}$ & -0.13829100 & 2.28205300 & -7.79341100 \\
mcb-i & & & \\
\hline & & &
\end{tabular}

Thermal correction to Gibbs free energy $=0.840509$

Sum of electronic and thermal free energies $=-3052.740632$

E_sol $=-3053.506343$

$\begin{array}{llll}\mathrm{Ru} & 1.24947800 & 0.64005400 & -1.49465600 \\ \mathrm{Cl} & -0.43100400 & -0.99309800 & -0.85226000 \\ \mathrm{Cl} & 3.01387000 & 2.05294300 & -2.38492300 \\ \mathrm{C} & 0.20935800 & 2.31172000 & -1.01938400 \\ \mathrm{C} & -0.72009300 & 2.30787500 & 0.13109200 \\ \mathrm{C} & -0.27994400 & 2.90834400 & 1.34015600 \\ \mathrm{C} & -2.00409900 & 1.74410900 & 0.10606500 \\ \mathrm{C} & -1.12141200 & 2.94912500 & 2.45889100 \\ \mathrm{C} & -2.84215300 & 1.78296400 & 1.21641400 \\ \mathrm{H} & -2.34001500 & 1.24183200 & -0.79290400 \\ \mathrm{C} & -2.39828600 & 2.38985300 & 2.39145600 \\ \mathrm{H} & -0.78772400 & 3.40282700 & 3.38394000 \\ \mathrm{H} & -3.82990600 & 1.33516700 & 1.16618500 \\ \mathrm{H} & -3.03986600 & 2.42460900 & 3.26790200 \\ \mathrm{O} & 0.99486700 & 3.39599700 & 1.32546700 \\ \mathrm{C} & 1.47026200 & 4.26399900 & 2.37214400 \\ \mathrm{H} & 0.63229300 & 4.88095600 & 2.72335800\end{array}$




\begin{tabular}{|c|c|c|c|}
\hline $\mathrm{C}$ & 2.51307400 & 5.16077000 & 1.71505100 \\
\hline $\mathrm{H}$ & 3.30980000 & 4.55024600 & 1.27892800 \\
\hline $\mathrm{H}$ & 2.06004000 & 5.75326400 & 0.91458800 \\
\hline $\mathrm{H}$ & 2.95383100 & 5.84035500 & 2.45255800 \\
\hline $\mathrm{C}$ & 2.04314100 & 3.44619300 & 3.53097500 \\
\hline $\mathrm{H}$ & 2.93769600 & 2.90650700 & 3.20660000 \\
\hline $\mathrm{H}$ & 2.32234400 & 4.10743800 & 4.35909000 \\
\hline $\mathrm{H}$ & 1.31917200 & 2.71427500 & 3.90041600 \\
\hline $\mathrm{P}$ & 2.85425400 & -0.70397600 & -0.27391100 \\
\hline $\mathrm{C}$ & 4.36755300 & -3.09344900 & -1.09121600 \\
\hline $\mathrm{H}$ & 4.14744500 & -4.07571400 & -1.53510600 \\
\hline $\mathrm{H}$ & 4.48906000 & -3.26993600 & -0.01583300 \\
\hline $\mathrm{C}$ & 5.67702300 & -2.57302300 & -1.71226100 \\
\hline $\mathrm{H}$ & 6.47257800 & -3.30916700 & -1.54021100 \\
\hline $\mathrm{H}$ & 5.99513400 & -1.65201700 & -1.21265400 \\
\hline $\mathrm{C}$ & 5.52774500 & -2.28037200 & -3.21209500 \\
\hline $\mathrm{H}$ & 5.33585000 & -3.22055200 & -3.75056100 \\
\hline $\mathrm{H}$ & 6.46663000 & -1.87503400 & -3.60911900 \\
\hline $\mathrm{C}$ & 4.36898600 & -1.30655100 & -3.46476600 \\
\hline $\mathrm{H}$ & 4.24217900 & -1.12922000 & -4.53946800 \\
\hline $\mathrm{H}$ & 4.58122700 & -0.32660000 & -3.01899100 \\
\hline $\mathrm{C}$ & 3.06514400 & -1.86420900 & -2.88676600 \\
\hline $\mathrm{H}$ & 2.23476400 & -1.18080400 & -3.08694900 \\
\hline $\mathrm{H}$ & 2.81843600 & -2.80291100 & -3.40526700 \\
\hline $\mathrm{C}$ & 3.14025800 & -2.19900600 & -1.37738900 \\
\hline $\mathrm{H}$ & 2.23030200 & -2.76258100 & -1.14180500 \\
\hline $\mathrm{C}$ & 2.25236000 & -1.33085000 & 1.39900500 \\
\hline $\mathrm{H}$ & 3.16955500 & -1.42344500 & 1.99098200 \\
\hline $\mathrm{C}$ & 1.33377400 & -0.26705100 & 2.09277400 \\
\hline $\mathrm{H}$ & 0.97000200 & 0.47347200 & 1.37754300 \\
\hline $\mathrm{H}$ & 1.92843800 & 0.28061000 & 2.83526100 \\
\hline $\mathrm{C}$ & 0.11861400 & -0.89792700 & 2.78587900 \\
\hline $\mathrm{H}$ & -0.59882200 & -1.21900100 & 2.02325200 \\
\hline $\mathrm{H}$ & -0.38679400 & -0.12953300 & 3.38354900 \\
\hline $\mathrm{C}$ & 0.54234300 & -2.08089300 & 3.66402700 \\
\hline $\mathrm{H}$ & -0.33025800 & -2.50800500 & 4.17171600 \\
\hline $\mathrm{H}$ & 1.20512300 & -1.70749200 & 4.45716700 \\
\hline $\mathrm{C}$ & 1.27836200 & -3.17527900 & 2.83840400 \\
\hline $\mathrm{H}$ & 0.67172900 & -4.08701500 & 2.78349100 \\
\hline $\mathrm{H}$ & 2.21003800 & -3.45860000 & 3.34621300 \\
\hline
\end{tabular}




\begin{tabular}{|c|c|c|c|}
\hline $\mathrm{C}$ & 1.60773400 & -2.73173600 & 1.39787800 \\
\hline $\mathrm{H}$ & 2.28980800 & -3.46759900 & 0.95699200 \\
\hline $\mathrm{H}$ & 0.70419600 & -2.72196300 & 0.78479500 \\
\hline $\mathrm{C}$ & 5.40985000 & -0.52183100 & 1.09543600 \\
\hline $\mathrm{H}$ & 5.53192800 & -1.57706300 & 0.84342500 \\
\hline $\mathrm{H}$ & 5.01839200 & -0.49798000 & 2.11961300 \\
\hline $\mathrm{C}$ & 6.77952800 & 0.19271800 & 1.05799300 \\
\hline $\mathrm{H}$ & 7.36121400 & -0.17127500 & 0.20162000 \\
\hline $\mathrm{H}$ & 7.34423000 & -0.07974000 & 1.95846500 \\
\hline $\mathrm{C}$ & 6.61929900 & 1.73012100 & 0.96809800 \\
\hline $\mathrm{H}$ & 6.62486300 & 2.04789000 & -0.08269100 \\
\hline $\mathrm{H}$ & 7.46876300 & 2.23032100 & 1.44716200 \\
\hline $\mathrm{C}$ & 5.29366300 & 2.16762900 & 1.60361400 \\
\hline $\mathrm{H}$ & 5.22400400 & 3.26096800 & 1.63650500 \\
\hline $\mathrm{H}$ & 5.26780800 & 1.82905900 & 2.64914000 \\
\hline $\mathrm{C}$ & 4.08708200 & 1.59742800 & 0.81740000 \\
\hline $\mathrm{H}$ & 3.22713200 & 1.51060500 & 1.48661200 \\
\hline $\mathrm{H}$ & 3.79227800 & 2.28722600 & 0.02626300 \\
\hline $\mathrm{C}$ & 4.42062600 & 0.22027400 & 0.16994400 \\
\hline $\mathrm{H}$ & 4.89829100 & 0.40830600 & -0.79786400 \\
\hline $\mathrm{H}$ & 0.90248900 & 3.14987000 & -0.99557700 \\
\hline $\mathrm{C}$ & 0.41488300 & 0.70742500 & -3.17310500 \\
\hline $\mathrm{C}$ & 0.18291800 & 0.24374600 & -4.39915100 \\
\hline $\mathrm{H}$ & 0.73144200 & -0.64326500 & -4.71131800 \\
\hline $\mathrm{C}$ & -0.25900100 & 1.98472200 & -2.45539400 \\
\hline $\mathrm{H}$ & 0.03280700 & 2.80596300 & -3.11226400 \\
\hline $\mathrm{H}$ & -1.32464400 & 1.76626400 & -2.49885000 \\
\hline $\mathrm{C}$ & -2.55101700 & 2.17178800 & -6.12286100 \\
\hline $\mathrm{C}$ & -1.90953300 & 1.36676900 & -7.31322000 \\
\hline B & -0.82114200 & 0.88963600 & -5.36993600 \\
\hline $\mathrm{O}$ & -1.09861100 & 0.38203400 & -6.61832600 \\
\hline $\mathrm{O}$ & -1.54574100 & 2.03167100 & -5.08314800 \\
\hline $\mathrm{C}$ & -2.90540800 & 0.63519400 & -8.20840400 \\
\hline $\mathrm{H}$ & -3.59117500 & 1.34541100 & -8.68458600 \\
\hline $\mathrm{H}$ & -2.36699100 & 0.09885200 & -8.99619400 \\
\hline $\mathrm{H}$ & -3.49036500 & -0.09419400 & -7.64400200 \\
\hline $\mathrm{C}$ & -3.83439400 & 1.52855100 & -5.58228200 \\
\hline $\mathrm{H}$ & -4.10330600 & 2.01421700 & -4.63903400 \\
\hline $\mathrm{H}$ & -4.66915800 & 1.64157400 & -6.28180100 \\
\hline $\mathrm{H}$ & -3.68406500 & 0.46264400 & -5.3844440 \\
\hline
\end{tabular}




$\begin{array}{llll}\mathrm{C} & -2.77202000 & 3.65758000 & -6.38953200 \\ \mathrm{H} & -3.46327300 & 3.80112900 & -7.22786300 \\ \mathrm{H} & -3.20733300 & 4.13044400 & -5.50321700 \\ \mathrm{H} & -1.83326200 & 4.16716600 & -6.61693600 \\ \mathrm{C} & -0.94873300 & 2.21026000 & -8.16088000 \\ \mathrm{H} & -0.38771200 & 1.54570600 & -8.82475600 \\ \mathrm{H} & -1.48592500 & 2.94267700 & -8.77254800 \\ \mathrm{H} & -0.23080900 & 2.74068300 & -7.52742600\end{array}$

\section{vinyl-i}

Thermal correction to Gibbs free energy $=0.630055$

Sum of electronic and thermal free energies $=-2550.125105$

E_sol $=-2550.7161952$

$\begin{array}{llll}\mathrm{Ru} & 0.20091400 & 0.74745200 & -2.14235100 \\ \mathrm{Cl} & 2.24673700 & 1.78437000 & -1.77362200 \\ \mathrm{Cl} & -0.99989000 & -1.18288200 & -1.58453300 \\ \mathrm{P} & 0.00650000 & 0.47982500 & -4.39596300 \\ \mathrm{C} & -1.90881700 & -0.51759400 & -6.37674300 \\ \mathrm{H} & -2.62648800 & -1.34851200 & -6.44190000 \\ \mathrm{H} & -1.00448300 & -0.86658600 & -6.88899500 \\ \mathrm{C} & -2.51581400 & 0.68912200 & -7.11512100 \\ \mathrm{H} & -2.76228100 & 0.39125600 & -8.14222000 \\ \mathrm{H} & -1.78050300 & 1.49449000 & -7.19919600 \\ \mathrm{C} & -3.76187200 & 1.23803200 & -6.40523900 \\ \mathrm{H} & -4.56539800 & 0.48805200 & -6.44718400 \\ \mathrm{H} & -4.13218100 & 2.12652200 & -6.93169800 \\ \mathrm{C} & -3.46056500 & 1.56211800 & -4.93543000 \\ \mathrm{H} & -4.36931200 & 1.90931600 & -4.42814000 \\ \mathrm{H} & -2.74078600 & 2.38833700 & -4.86632500 \\ \mathrm{C} & -2.91486700 & 0.32624500 & -4.21206700 \\ \mathrm{H} & -2.74120400 & 0.52209700 & -3.15400300 \\ \mathrm{H} & -3.68614500 & -0.45759700 & -4.24725800 \\ \mathrm{C} & -1.65492400 & -0.28634600 & -4.86511300 \\ \mathrm{H} & -1.52590900 & -1.26379100 & -4.39073500 \\ \mathrm{C} & 1.24311900 & -0.79049600 & -5.02120200 \\ \mathrm{H} & 1.20576000 & -0.68721100 & -6.11097100 \\ \mathrm{C} & 2.69518800 & -0.47377300 & -4.55458300 \\ \mathrm{H} & 2.82054500 & 0.58381800 & -4.31313900 \\ \mathrm{H} & 3.38047800 & -0.68668600 & -5.38574400 \\ \mathrm{C} & 3.08781400 & -1.33525400 & -3.34857100\end{array}$




\begin{tabular}{|c|c|c|c|}
\hline $\mathrm{H}$ & 2.36362100 & -1.17147700 & -2.53924800 \\
\hline $\mathrm{H}$ & 4.05138300 & -1.00548200 & -2.94627500 \\
\hline $\mathrm{C}$ & 3.12264000 & -2.83305500 & -3.72056800 \\
\hline $\mathrm{H}$ & 2.94574300 & -3.43403000 & -2.82056000 \\
\hline $\mathrm{H}$ & 4.11995200 & -3.10564000 & -4.08783900 \\
\hline $\mathrm{C}$ & 2.06622700 & -3.17291700 & -4.79763100 \\
\hline $\mathrm{H}$ & 1.75398200 & -4.21993400 & -4.71201900 \\
\hline $\mathrm{H}$ & 2.49945500 & -3.06188300 & -5.80137100 \\
\hline $\mathrm{C}$ & 0.84416900 & -2.25059300 & -4.67837200 \\
\hline $\mathrm{H}$ & 0.04958800 & -2.58477500 & -5.35442800 \\
\hline $\mathrm{H}$ & 0.44199400 & -2.32053400 & -3.66106100 \\
\hline $\mathrm{C}$ & 0.80352500 & 1.76632900 & -6.86155800 \\
\hline $\mathrm{H}$ & 0.30487300 & 0.92072100 & -7.34190700 \\
\hline $\mathrm{H}$ & 1.86818800 & 1.50685800 & -6.80910100 \\
\hline $\mathrm{C}$ & 0.61086700 & 3.03559000 & -7.72950500 \\
\hline $\mathrm{H}$ & -0.37590000 & 3.01037300 & -8.20843300 \\
\hline $\mathrm{H}$ & 1.35002600 & 3.01974200 & -8.54002700 \\
\hline $\mathrm{C}$ & 0.75815100 & 4.33373600 & -6.90429100 \\
\hline $\mathrm{H}$ & -0.21312900 & 4.62215500 & -6.48026100 \\
\hline $\mathrm{H}$ & 1.06810300 & 5.16160300 & -7.55261100 \\
\hline $\mathrm{C}$ & 1.75827100 & 4.11720800 & -5.76429200 \\
\hline $\mathrm{H}$ & 1.96518200 & 5.05348100 & -5.23310000 \\
\hline $\mathrm{H}$ & 2.71650600 & 3.79555800 & -6.19490000 \\
\hline $\mathrm{C}$ & 1.24734300 & 3.06184800 & -4.75204000 \\
\hline $\mathrm{H}$ & 2.10539300 & 2.56159900 & -4.30170800 \\
\hline $\mathrm{H}$ & 0.72237000 & 3.55290800 & -3.93134300 \\
\hline $\mathrm{C}$ & 0.30395500 & 2.02880400 & -5.42297700 \\
\hline $\mathrm{H}$ & -0.69724600 & 2.47078300 & -5.47912800 \\
\hline $\mathrm{C}$ & -1.78452300 & 6.74065700 & -1.76517200 \\
\hline $\mathrm{C}$ & -0.50311700 & 6.45580500 & -2.63235200 \\
\hline B & -1.56466700 & 4.50661900 & -2.12469300 \\
\hline $\mathrm{O}$ & -0.33341400 & 5.02114500 & -2.46287400 \\
\hline $\mathrm{O}$ & -2.50041100 & 5.47610200 & -1.85232100 \\
\hline $\mathrm{C}$ & -0.72840600 & 6.70302800 & -4.12915800 \\
\hline $\mathrm{H}$ & -0.82126600 & 7.77098300 & -4.35110800 \\
\hline $\mathrm{H}$ & 0.12274300 & 6.30631200 & -4.68835500 \\
\hline $\mathrm{H}$ & -1.63254100 & 6.19372300 & -4.47838600 \\
\hline $\mathrm{C}$ & -2.69047100 & 7.84936100 & -2.29167600 \\
\hline $\mathrm{H}$ & -3.55400700 & 7.96442300 & -1.62908400 \\
\hline $\mathrm{H}$ & -2.15320100 & 8.80406400 & $-2.322840 c$ \\
\hline
\end{tabular}




$\begin{array}{llll}\mathrm{H} & -3.06215500 & 7.62224900 & -3.29323900 \\ \mathrm{C} & -1.46756700 & 6.96696800 & -0.28194900 \\ \mathrm{H} & -0.98259500 & 7.93478500 & -0.11772800 \\ \mathrm{H} & -2.40169700 & 6.94368700 & 0.28714500 \\ \mathrm{H} & -0.81451800 & 6.17813000 & 0.10424100 \\ \mathrm{C} & 0.77041000 & 7.15153800 & -2.16380100 \\ \mathrm{H} & 1.60233100 & 6.87348800 & -2.81881600 \\ \mathrm{H} & 0.65250200 & 8.24055000 & -2.20101700 \\ \mathrm{H} & 1.03403400 & 6.85868900 & -1.14564700 \\ \mathrm{C} & -1.89844400 & 3.00240300 & -2.06484900 \\ \mathrm{H} & -2.92945100 & 2.68947100 & -1.88658500 \\ \mathrm{C} & -0.98492900 & 2.04671200 & -2.14294900\end{array}$

ts-ii

Thermal correction to Gibbs free energy $=0.915394$

Sum of electronic and thermal free energies $=-3347.018079$

E_sol $=-3347.8738544$

$\begin{array}{lrrr}\mathrm{Ru} & -0.23963400 & 0.89279000 & -0.91574600 \\ \mathrm{Cl} & 0.55130900 & 3.15025800 & -0.61347000 \\ \mathrm{Cl} & -1.23476200 & -1.30874500 & -0.81820900 \\ \mathrm{C} & -2.07795400 & 1.72512100 & -1.05681600 \\ \mathrm{C} & -3.00670600 & 2.08863000 & -0.19172600 \\ \mathrm{H} & -2.86638900 & 1.98059700 & 0.87947800 \\ \mathrm{P} & 1.59749700 & 0.09281700 & 0.46650300 \\ \mathrm{C} & 1.88975900 & -0.40203000 & 3.33610200 \\ \mathrm{H} & 1.69266800 & 0.14493700 & 4.27002500 \\ \mathrm{H} & 2.95419700 & -0.25316100 & 3.11856100 \\ \mathrm{C} & 1.58725000 & -1.88995700 & 3.58384400 \\ \mathrm{H} & 2.19897500 & -2.24554000 & 4.42259800 \\ \mathrm{H} & 1.87747500 & -2.48757800 & 2.71271100 \\ \mathrm{C} & 0.09682600 & -2.12833800 & 3.86071000 \\ \mathrm{H} & -0.18751800 & -1.62101100 & 4.79482800 \\ \mathrm{H} & -0.09119900 & -3.19837100 & 4.01241000 \\ \mathrm{C} & -0.75814500 & -1.59218200 & 2.70535300 \\ \mathrm{H} & -1.82500000 & -1.73589000 & 2.91409400 \\ \mathrm{H} & -0.54994100 & -2.15156600 & 1.78636700 \\ \mathrm{C} & -0.48755000 & -0.10163600 & 2.47270000 \\ \mathrm{H} & -1.11160500 & 0.26433200 & 1.64889200 \\ \mathrm{H} & -0.80197200 & 0.45520100 & 3.36824800 \\ \mathrm{C} & 1.00508800 & 0.24466000 & 2.24361200\end{array}$




\begin{tabular}{|c|c|c|c|}
\hline $\mathrm{H}$ & 1.08141100 & 1.33408100 & 2.34552500 \\
\hline $\mathrm{C}$ & 3.22026400 & 1.04034500 & 0.40381000 \\
\hline $\mathrm{H}$ & 3.95798700 & 0.32060300 & 0.77858900 \\
\hline $\mathrm{C}$ & 3.59233000 & 1.39087800 & -1.07262100 \\
\hline $\mathrm{H}$ & 2.69314600 & 1.52330200 & -1.68001700 \\
\hline $\mathrm{H}$ & 4.15625000 & 0.55129900 & -1.49945000 \\
\hline $\mathrm{C}$ & 4.44007700 & 2.67267600 & -1.16793200 \\
\hline $\mathrm{H}$ & 3.78100600 & 3.54712500 & -1.10451900 \\
\hline $\mathrm{H}$ & 4.92351100 & 2.71401000 & -2.15145300 \\
\hline $\mathrm{C}$ & 5.47940100 & 2.71706600 & -0.04332200 \\
\hline $\mathrm{H}$ & 6.16608000 & 3.56097000 & -0.17658500 \\
\hline $\mathrm{H}$ & 6.09511200 & 1.80806400 & -0.10133800 \\
\hline $\mathrm{C}$ & 4.78797100 & 2.79892400 & 1.34116800 \\
\hline $\mathrm{H}$ & 4.77199700 & 3.83698100 & 1.69465300 \\
\hline $\mathrm{H}$ & 5.37058200 & 2.23059300 & 2.07804300 \\
\hline $\mathrm{C}$ & 3.33158600 & 2.27739000 & 1.31879000 \\
\hline $\mathrm{H}$ & 3.03817000 & 2.03944100 & 2.34582600 \\
\hline $\mathrm{H}$ & 2.64891300 & 3.05154100 & 0.96445700 \\
\hline $\mathrm{C}$ & 3.42520200 & -2.16301600 & 0.56295900 \\
\hline $\mathrm{H}$ & 3.57292500 & -1.89188300 & 1.61159200 \\
\hline $\mathrm{H}$ & 4.24819500 & -1.69629300 & 0.00759700 \\
\hline $\mathrm{C}$ & 3.49785500 & -3.69905200 & 0.40205400 \\
\hline $\mathrm{H}$ & 3.00300000 & -4.18054000 & 1.25522200 \\
\hline $\mathrm{H}$ & 4.55011300 & -4.00786400 & 0.43597200 \\
\hline $\mathrm{C}$ & 2.84162100 & -4.17095600 & -0.91870400 \\
\hline $\mathrm{H}$ & 1.78396400 & -4.41158600 & -0.74792600 \\
\hline $\mathrm{H}$ & 3.31563400 & -5.09485400 & -1.26927200 \\
\hline $\mathrm{C}$ & 2.92885900 & -3.07059800 & -1.98361300 \\
\hline $\mathrm{H}$ & 2.56166800 & -3.43321300 & -2.95052500 \\
\hline $\mathrm{H}$ & 3.98342900 & -2.80225500 & -2.13555900 \\
\hline $\mathrm{C}$ & 2.11101600 & -1.82580900 & -1.56092400 \\
\hline $\mathrm{H}$ & 2.53324600 & -0.93876500 & -2.04451400 \\
\hline $\mathrm{H}$ & 1.08155400 & -1.91438200 & -1.91073000 \\
\hline $\mathrm{C}$ & 2.08850200 & -1.65034400 & -0.01191300 \\
\hline $\mathrm{H}$ & 1.26954300 & -2.26294200 & 0.38014900 \\
\hline $\mathrm{C}$ & -1.32622400 & 2.60886600 & -6.71439100 \\
\hline $\mathrm{C}$ & -0.70897100 & 1.35162800 & -7.42672800 \\
\hline B & -0.09736700 & 1.37354500 & -5.23787500 \\
\hline $\mathrm{O}$ & 0.30270700 & 0.91951500 & -6.47073300 \\
\hline $\mathrm{O}$ & -1.13178000 & 2.28210400 & -5.30690500 \\
\hline
\end{tabular}




\begin{tabular}{|c|c|c|c|}
\hline $\mathrm{C}$ & -1.70886300 & 0.19567400 & -7.56624700 \\
\hline I & -2.45347900 & 0.40506500 & -8.34125600 \\
\hline $\mathrm{H}$ & -1.16281000 & -0.71124200 & -7.84300200 \\
\hline $\mathrm{H}$ & -2.23822800 & 0.00767400 & -6.62693300 \\
\hline $\mathrm{C}$ & -2.81522100 & 2.82475600 & -6.96944400 \\
\hline $\mathrm{H}$ & -3.15157000 & 3.72489500 & -6.44386700 \\
\hline $\mathrm{H}$ & -2.99962000 & 2.97126000 & -8.04003600 \\
\hline $\mathrm{H}$ & -3.41356000 & 1.98180300 & -6.61738400 \\
\hline $\mathrm{C}$ & -0.54009100 & 3.89866900 & -6.97386800 \\
\hline $\mathrm{H}$ & -0.68121000 & 4.25509900 & -7.99949100 \\
\hline $\mathrm{H}$ & -0.89150800 & 4.67230100 & -6.28452600 \\
\hline $\mathrm{H}$ & 0.52975000 & 3.74852700 & -6.79824500 \\
\hline $\mathrm{C}$ & -0.02687000 & 1.63374200 & -8.76118000 \\
\hline $\mathrm{H}$ & 0.37442100 & 0.70253500 & -9.17342700 \\
\hline $\mathrm{H}$ & -0.74405600 & 2.04542200 & -9.48033900 \\
\hline $\mathrm{H}$ & 0.80058200 & 2.33760000 & -8.64766600 \\
\hline $\mathrm{C}$ & 0.03376100 & 0.94968000 & -2.70405000 \\
\hline $\mathrm{C}$ & 0.54723400 & 0.87751800 & -3.92485800 \\
\hline $\mathrm{H}$ & 1.50908300 & 0.36623100 & -3.98352700 \\
\hline $\mathrm{C}$ & -1.78354200 & 1.59674600 & -2.45414700 \\
\hline $\mathrm{H}$ & -1.52349100 & 2.53979600 & -2.93998100 \\
\hline $\mathrm{H}$ & -3.94395900 & 2.52711800 & -0.53011400 \\
\hline $\mathrm{C}$ & -2.65252800 & 0.63779300 & -3.26232200 \\
\hline $\mathrm{H}$ & -2.93101700 & -0.21410100 & -2.64204900 \\
\hline $\mathrm{H}$ & -2.11561500 & 0.25318900 & -4.12797800 \\
\hline $\mathrm{C}$ & -3.88916000 & 1.37408700 & -3.78502100 \\
\hline $\mathrm{H}$ & -3.58723000 & 2.33002700 & -4.22666400 \\
\hline $\mathrm{H}$ & -4.58672200 & 1.58990000 & -2.96283900 \\
\hline $\mathrm{O}$ & -4.50075800 & 0.59826100 & -4.80697500 \\
\hline $\mathrm{Si}$ & -6.00005400 & -0.15907000 & -4.72748500 \\
\hline $\mathrm{C}$ & -7.31608800 & 1.11614200 & -4.26685900 \\
\hline $\mathrm{H}$ & -8.31075600 & 0.65864800 & -4.20419100 \\
\hline $\mathrm{H}$ & -7.10985600 & 1.58576900 & -3.29795900 \\
\hline $\mathrm{H}$ & -7.36007400 & 1.91170000 & -5.02000000 \\
\hline $\mathrm{C}$ & -6.26220700 & -0.79255700 & -6.47953700 \\
\hline $\mathrm{H}$ & -6.20866200 & 0.03687400 & -7.19411100 \\
\hline $\mathrm{H}$ & -5.48942300 & -1.51928200 & -6.75430300 \\
\hline $\mathrm{H}$ & -7.23870900 & -1.27640300 & -6.59607000 \\
\hline $\mathrm{C}$ & -6.00106200 & -1.59861500 & -3.46701200 \\
\hline $\mathrm{C}$ & -5.88342900 & -1.07569200 & -2.01805400 \\
\hline
\end{tabular}




$\begin{array}{llll}\mathrm{H} & -5.91557500 & -1.91554200 & -1.30906800 \\ \mathrm{H} & -4.94173100 & -0.54663700 & -1.83661100 \\ \mathrm{H} & -6.70749300 & -0.39897700 & -1.75942900 \\ \mathrm{C} & -7.33091700 & -2.37495000 & -3.60150500 \\ \mathrm{H} & -7.45649800 & -2.80643000 & -4.60215400 \\ \mathrm{H} & -7.35822300 & -3.20650700 & -2.88247000 \\ \mathrm{H} & -8.20270800 & -1.74057800 & -3.39586000 \\ \mathrm{C} & -4.82897500 & -2.55939800 & -3.75931400 \\ \mathrm{H} & -4.83576500 & -3.39695000 & -3.04702700 \\ \mathrm{H} & -4.89543800 & -2.98653400 & -4.76789200 \\ \mathrm{H} & -3.85755100 & -2.06216200 & -3.67024300\end{array}$

\section{mcb-ii}

Thermal correction to Gibbs free energy $=0.917293$

Sum of electronic and thermal free energies $=-3347.021234$

$\begin{array}{lrrr}\text { E_sol }=-3347.8808132 & & \\ \mathrm{Ru} & 0.07647300 & 0.74654700 & -1.26414700 \\ \mathrm{Cl} & 0.99855400 & 2.97714500 & -1.19583900 \\ \mathrm{Cl} & -0.91090000 & -1.46479800 & -1.22751100 \\ \mathrm{C} & -1.65249800 & 1.58433000 & -1.26010000 \\ \mathrm{C} & -2.63166900 & 2.06052200 & -0.51032600 \\ \mathrm{H} & -2.56263300 & 2.04548700 & 0.57292500 \\ \mathrm{P} & 1.69643000 & -0.00878500 & 0.36105400 \\ \mathrm{C} & 1.63924200 & -0.52767900 & 3.22220600 \\ \mathrm{H} & 1.28847200 & -0.00949100 & 4.12672300 \\ \mathrm{H} & 2.70768900 & -0.29641700 & 3.13911100 \\ \mathrm{C} & 1.42321100 & -2.03729300 & 3.42496400 \\ \mathrm{H} & 1.96089300 & -2.35704000 & 4.32652500 \\ \mathrm{H} & 1.85574100 & -2.59948600 & 2.58986600 \\ \mathrm{C} & -0.06636500 & -2.39054800 & 3.52610000 \\ \mathrm{H} & -0.49405500 & -1.91911500 & 4.42363700 \\ \mathrm{H} & -0.18822400 & -3.47373200 & 3.64945600 \\ \mathrm{C} & -0.81813800 & -1.90202700 & 2.28205000 \\ \mathrm{H} & -1.88839300 & -2.12628200 & 2.36280200 \\ \mathrm{H} & -0.46336500 & -2.43174000 & 1.39082300 \\ \mathrm{C} & -0.63738000 & -0.39256100 & 2.08733900 \\ \mathrm{H} & -1.18824300 & -0.06170200 & 1.20309700 \\ \mathrm{H} & -1.08433800 & 0.12428200 & 2.95038600 \\ \mathrm{C} & 0.84074600 & 0.06812200 & 2.03501200 \\ \mathrm{H} & 0.82412000 & 1.15832200 & 2.15552900 \\ & & & \\ \mathrm{H} & & & \end{array}$




\begin{tabular}{|c|c|c|c|}
\hline $\mathrm{C}$ & 3.27485500 & 0.97630700 & 0.59171900 \\
\hline $\mathrm{H}$ & 3.91835500 & 0.29216700 & 1.15927800 \\
\hline $\mathrm{C}$ & 3.96342900 & 1.25255200 & -0.78247200 \\
\hline $\mathrm{H}$ & 3.21994000 & 1.36136200 & -1.57608300 \\
\hline $\mathrm{H}$ & 4.59527100 & 0.39220000 & -1.03781700 \\
\hline$C$ & 4.82453200 & 2.52900700 & -0.74667100 \\
\hline $\mathrm{H}$ & 4.17718300 & 3.40306100 & -0.88743600 \\
\hline $\mathrm{H}$ & 5.52468900 & 2.51650800 & -1.59047200 \\
\hline $\mathrm{C}$ & 5.57171200 & 2.64077100 & 0.58603900 \\
\hline $\mathrm{H}$ & 6.28345200 & 3.47408500 & 0.56817600 \\
\hline $\mathrm{H}$ & 6.16986200 & 1.72944100 & 0.72969900 \\
\hline $\mathrm{C}$ & 4.57602700 & 2.80974700 & 1.76088000 \\
\hline $\mathrm{H}$ & 4.47738800 & 3.87023000 & 2.02199100 \\
\hline $\mathrm{H}$ & 4.97091400 & 2.30677800 & 2.65330900 \\
\hline $\mathrm{C}$ & 3.16528700 & 2.26177500 & 1.43703700 \\
\hline $\mathrm{H}$ & 2.64525200 & 2.07459300 & 2.38158000 \\
\hline $\mathrm{H}$ & 2.57719200 & 3.00167000 & 0.89155300 \\
\hline $\mathrm{C}$ & 3.58971900 & -2.19071200 & 0.59679800 \\
\hline $\mathrm{H}$ & 3.59536900 & -1.96021500 & 1.66540900 \\
\hline $\mathrm{H}$ & 4.44932800 & -1.66424200 & 0.16531000 \\
\hline $\mathrm{C}$ & 3.75521600 & -3.71372400 & 0.39084600 \\
\hline $\mathrm{H}$ & 3.17969300 & -4.25037400 & 1.15592900 \\
\hline $\mathrm{H}$ & 4.80831600 & -3.97692200 & 0.54968100 \\
\hline $\mathrm{C}$ & 3.29590500 & -4.15941100 & -1.01945800 \\
\hline $\mathrm{H}$ & 2.24160700 & -4.46512000 & -0.99201800 \\
\hline $\mathrm{H}$ & 3.86408000 & -5.03864000 & -1.34356400 \\
\hline $\mathrm{C}$ & 3.44568300 & -3.00841200 & -2.02187100 \\
\hline $\mathrm{H}$ & 3.22504400 & -3.34607400 & -3.04085300 \\
\hline $\mathrm{H}$ & 4.49061200 & -2.66923200 & -2.02856100 \\
\hline $\mathrm{C}$ & 2.50017600 & -1.83906000 & -1.65842500 \\
\hline $\mathrm{H}$ & 2.89948700 & -0.91087700 & -2.08126100 \\
\hline $\mathrm{H}$ & 1.52055900 & -1.99798800 & -2.11276500 \\
\hline $\mathrm{C}$ & 2.30549500 & -1.71477000 & -0.11360300 \\
\hline $\mathrm{H}$ & 1.47468200 & -2.36950300 & 0.16944400 \\
\hline $\mathrm{C}$ & -0.82347600 & 0.90893300 & -7.80585500 \\
\hline $\mathrm{C}$ & -1.44028100 & 2.19207500 & -7.13973600 \\
\hline B & -0.10045400 & 1.08096600 & -5.65841900 \\
\hline $\mathrm{O}$ & -1.15830100 & 1.96339600 & -5.73009600 \\
\hline $\mathrm{O}$ & 0.24947000 & 0.56382400 & -6.88227500 \\
\hline $\mathrm{C}$ & -0.71195600 & 3.48365200 & -7.52781100 \\
\hline
\end{tabular}




\begin{tabular}{|c|c|c|c|}
\hline $\mathrm{H}$ & -0.92399500 & 3.76857100 & -8.56362100 \\
\hline $\mathrm{H}$ & -1.04710200 & 4.29033900 & -6.86889000 \\
\hline $\mathrm{H}$ & 0.37046400 & 3.37514600 & -7.40744100 \\
\hline $\mathrm{C}$ & -0.22376500 & 1.12289200 & -9.19153500 \\
\hline $\mathrm{H}$ & 0.18429900 & 0.17872200 & -9.56594200 \\
\hline $\mathrm{H}$ & -0.99121700 & 1.46696700 & -9.89423300 \\
\hline $\mathrm{H}$ & 0.58581000 & 1.85562000 & -9.16874900 \\
\hline $\mathrm{C}$ & -1.79396800 & -0.27967100 & -7.81713700 \\
\hline $\mathrm{H}$ & -2.59334500 & -0.13662200 & -8.55145800 \\
\hline $\mathrm{H}$ & -1.23938700 & -1.18663400 & -8.07591400 \\
\hline $\mathrm{H}$ & -2.25254700 & -0.42729200 & -6.83417700 \\
\hline $\mathrm{C}$ & -2.94834900 & 2.34909200 & -7.31850800 \\
\hline $\mathrm{H}$ & -3.27858400 & 3.28533400 & -6.85592800 \\
\hline $\mathrm{H}$ & -3.20502200 & 2.39095500 & -8.38337300 \\
\hline $\mathrm{H}$ & -3.49977800 & 1.53164600 & -6.84872600 \\
\hline $\mathrm{C}$ & -0.00283300 & 0.86672100 & -3.14557000 \\
\hline $\mathrm{C}$ & 0.58011000 & 0.68530600 & -4.32803500 \\
\hline $\mathrm{H}$ & 1.55509500 & 0.20044000 & -4.34014500 \\
\hline $\mathrm{C}$ & -1.44129700 & 1.44138600 & -2.78661000 \\
\hline $\mathrm{H}$ & -1.36732800 & 2.46550000 & -3.17407000 \\
\hline $\mathrm{H}$ & -3.52853600 & 2.49202100 & -0.94787400 \\
\hline $\mathrm{C}$ & -2.55036500 & 0.58440800 & -3.43726100 \\
\hline $\mathrm{H}$ & -2.85062200 & -0.20399500 & -2.74662700 \\
\hline $\mathrm{H}$ & -2.17069900 & 0.09913200 & -4.33595500 \\
\hline $\mathrm{C}$ & -3.74560400 & 1.43872800 & -3.85395000 \\
\hline $\mathrm{H}$ & -3.39486900 & 2.28959800 & -4.44769800 \\
\hline $\mathrm{H}$ & -4.27237000 & 1.83588100 & -2.97538100 \\
\hline $\mathrm{O}$ & -4.61064100 & 0.65966200 & -4.67108100 \\
\hline $\mathrm{Si}$ & -6.17193500 & 0.18131700 & -4.27379000 \\
\hline $\mathrm{C}$ & -7.19219400 & 1.70585500 & -3.81859100 \\
\hline $\mathrm{H}$ & -8.22158400 & 1.43366500 & -3.55630200 \\
\hline $\mathrm{H}$ & -6.76746100 & 2.24400300 & -2.96305900 \\
\hline $\mathrm{H}$ & -7.23524600 & 2.40440900 & -4.66255700 \\
\hline $\mathrm{C}$ & -6.81486200 & -0.60421800 & -5.85777500 \\
\hline $\mathrm{H}$ & -6.22060700 & -1.48327900 & -6.13109700 \\
\hline $\mathrm{H}$ & -7.86002900 & -0.91950300 & -5.75971000 \\
\hline $\mathrm{H}$ & -6.75408400 & 0.11038900 & -6.68662900 \\
\hline $\mathrm{C}$ & -6.18752100 & -1.07624000 & -2.82998400 \\
\hline $\mathrm{C}$ & -5.73886100 & -0.42071200 & -1.50501500 \\
\hline $\mathrm{H}$ & -5.79499900 & -1.15109600 & -0.68459100 \\
\hline
\end{tabular}




$\begin{array}{llll}\mathrm{H} & -4.70398000 & -0.06425200 & -1.53906700 \\ \mathrm{H} & -6.37960500 & 0.42669800 & -1.23002400 \\ \mathrm{C} & -7.62909200 & -1.60477600 & -2.65054100 \\ \mathrm{H} & -7.99565300 & -2.11550800 & -3.54945400 \\ \mathrm{H} & -7.66599400 & -2.33117200 & -1.82567800 \\ \mathrm{H} & -8.33649200 & -0.80190800 & -2.40656000 \\ \mathrm{C} & -5.25653300 & -2.26471600 & -3.15088100 \\ \mathrm{H} & -5.27658000 & -2.99711000 & -2.33090500 \\ \mathrm{H} & -5.56566600 & -2.78770800 & -4.06461600 \\ \mathrm{H} & -4.21624000 & -1.94938400 & -3.28230300\end{array}$

\section{ts-iii}

Thermal correction to Gibbs free energy $=0.914393$

Sum of electronic and thermal free energies $=-3347.009548$

E_sol $=-3347.8646702$

$\begin{array}{llll}\mathrm{Ru} & 0.33860400 & 0.97007300 & -2.01378200 \\ \mathrm{Cl} & 1.73346300 & 2.94833300 & -2.12748200 \\ \mathrm{Cl} & -0.86884000 & -1.12489500 & -2.02302200 \\ \mathrm{C} & -1.08573400 & 2.07213700 & -1.84382300 \\ \mathrm{C} & -2.00899800 & 2.92399900 & -1.44411200 \\ \mathrm{H} & -2.08051700 & 3.15411300 & -0.38401800 \\ \mathrm{P} & 1.38987000 & 0.22773700 & 0.06554900 \\ \mathrm{C} & 0.56965700 & -0.07995200 & 2.85204900 \\ \mathrm{H} & 0.03227900 & 0.52320200 & 3.59870900 \\ \mathrm{H} & 1.63532800 & 0.09904500 & 3.03520900 \\ \mathrm{C} & 0.21711200 & -1.55776200 & 3.09696100 \\ \mathrm{H} & 0.48904800 & -1.82345700 & 4.12649900 \\ \mathrm{H} & 0.81117300 & -2.20477100 & 2.44274700 \\ \mathrm{C} & -1.26782900 & -1.84285700 & 2.83673100 \\ \mathrm{H} & -1.88015600 & -1.28661400 & 3.56235000 \\ \mathrm{H} & -1.47956300 & -2.90764000 & 2.99519600 \\ \mathrm{C} & -1.65151500 & -1.41979200 & 1.41388500 \\ \mathrm{H} & -2.71958400 & -1.58881000 & 1.23232900 \\ \mathrm{H} & -1.11856900 & -2.03216300 & 0.67753500 \\ \mathrm{C} & -1.33433600 & 0.06084200 & 1.17752200 \\ \mathrm{H} & -1.63357300 & 0.35464100 & 0.17108100 \\ \mathrm{H} & -1.94431200 & 0.65603700 & 1.87520000 \\ \mathrm{C} & 0.13602700 & 0.45324000 & 1.46198000 \\ \mathrm{H} & 0.14966800 & 1.54879700 & 1.51007700 \\ \mathrm{C} & 2.91289000 & 1.12458300 & 0.69099100\end{array}$




\begin{tabular}{|c|c|c|c|}
\hline $\mathrm{H}$ & 3.25933400 & 0.50862900 & 1.53112700 \\
\hline $\mathrm{C}$ & 4.04274300 & 1.13949500 & -0.38626300 \\
\hline $\mathrm{H}$ & 3.62151300 & 1.16348100 & -1.39355400 \\
\hline $\mathrm{H}$ & 4.62743100 & 0.21560500 & -0.29688600 \\
\hline $\mathrm{C}$ & 4.97636300 & 2.35458300 & -0.22639500 \\
\hline $\mathrm{H}$ & 4.51528100 & 3.22683700 & -0.70544600 \\
\hline $\mathrm{H}$ & 5.91530300 & 2.16224100 & -0.75927300 \\
\hline $\mathrm{C}$ & 5.23482500 & 2.64888700 & 1.25452700 \\
\hline $\mathrm{H}$ & 6.00069700 & 3.42400400 & 1.37364700 \\
\hline $\mathrm{H}$ & 5.63939600 & 1.74220100 & 1.72706500 \\
\hline $\mathrm{C}$ & 3.92548600 & 3.07426600 & 1.96355500 \\
\hline $\mathrm{H}$ & 3.85755600 & 4.16817100 & 2.00346700 \\
\hline $\mathrm{H}$ & 3.94139600 & 2.72683100 & 3.00486900 \\
\hline $\mathrm{C}$ & 2.65848100 & 2.53758000 & 1.25507100 \\
\hline $\mathrm{H}$ & 1.83643700 & 2.53589200 & 1.97780600 \\
\hline $\mathrm{H}$ & 2.36166200 & 3.19818000 & 0.43743900 \\
\hline $\mathrm{C}$ & 2.98288900 & -2.04692700 & 0.93076700 \\
\hline $\mathrm{H}$ & 2.74032100 & -1.72770400 & 1.94730700 \\
\hline $\mathrm{H}$ & 3.96336300 & -1.61439700 & 0.70212200 \\
\hline $\mathrm{C}$ & 3.06839600 & -3.58938400 & 0.87442300 \\
\hline $\mathrm{H}$ & 2.28133200 & -4.02309800 & 1.50459400 \\
\hline $\mathrm{H}$ & 4.02533900 & -3.90510000 & 1.30829300 \\
\hline $\mathrm{C}$ & 2.93262500 & -4.12385700 & -0.57284700 \\
\hline $\mathrm{H}$ & 1.88166100 & -4.35661300 & -0.79024400 \\
\hline $\mathrm{H}$ & 3.48600800 & -5.06321000 & -0.68448600 \\
\hline $\mathrm{C}$ & 3.42097600 & -3.07671600 & -1.58150900 \\
\hline $\mathrm{H}$ & 3.42977200 & -3.48649600 & -2.59796900 \\
\hline $\mathrm{H}$ & 4.46008500 & -2.80785700 & -1.34784300 \\
\hline $\mathrm{C}$ & 2.51953300 & -1.81952400 & -1.54434000 \\
\hline $\mathrm{H}$ & 3.09234300 & -0.96000800 & -1.91072800 \\
\hline $\mathrm{H}$ & 1.67223900 & -1.95263400 & -2.22137500 \\
\hline $\mathrm{C}$ & 1.96119300 & -1.54924900 & -0.11201800 \\
\hline $\mathrm{H}$ & 1.03554400 & -2.12455900 & -0.00419000 \\
\hline $\mathrm{C}$ & 0.10969100 & 1.67135800 & -8.34297600 \\
\hline $\mathrm{C}$ & 1.11863900 & 0.51101500 & -8.68025900 \\
\hline B & 1.00909400 & 0.82034400 & -6.42966900 \\
\hline $\mathrm{O}$ & 1.82964300 & 0.34297900 & -7.42085700 \\
\hline $\mathrm{O}$ & -0.09335200 & 1.49261100 & -6.91383800 \\
\hline $\mathrm{C}$ & 0.42369800 & -0.82477400 & -8.97145700 \\
\hline $\mathrm{H}$ & -0.08794400 & -0.81015000 & -9.9393500 \\
\hline
\end{tabular}




\begin{tabular}{|c|c|c|c|}
\hline $\mathrm{H}$ & 1.17600700 & -1.61904000 & -8.98637400 \\
\hline $\mathrm{H}$ & -0.30706200 & -1.06421100 & -8.19247800 \\
\hline $\mathrm{C}$ & -1.24376500 & 1.57640700 & -9.04051100 \\
\hline $\mathrm{H}$ & -1.87580900 & 2.41578200 & -8.73332700 \\
\hline $\mathrm{H}$ & -1.12167500 & 1.62101200 & -10.12872100 \\
\hline $\mathrm{H}$ & -1.76380800 & 0.65142100 & -8.78242800 \\
\hline $\mathrm{C}$ & 0.71235100 & 3.06900300 & -8.52951400 \\
\hline $\mathrm{H}$ & 0.84293600 & 3.31342200 & -9.58878900 \\
\hline $\mathrm{H}$ & 0.03973000 & 3.80699300 & -8.08201400 \\
\hline $\mathrm{H}$ & 1.68234400 & 3.14765300 & -8.02878400 \\
\hline $\mathrm{C}$ & 2.13286600 & 0.83394400 & -9.77294000 \\
\hline $\mathrm{H}$ & 2.79422400 & -0.02455500 & -9.92669100 \\
\hline $\mathrm{H}$ & 1.62414800 & 1.04843500 & -10.71972400 \\
\hline $\mathrm{H}$ & 2.75173600 & 1.69257300 & -9.50399000 \\
\hline $\mathrm{C}$ & 0.40377500 & 1.07721000 & -4.02697200 \\
\hline $\mathrm{C}$ & 1.27775000 & 0.62419400 & -4.91951000 \\
\hline $\mathrm{H}$ & 2.17763500 & 0.11466400 & -4.57787600 \\
\hline $\mathrm{C}$ & -0.87013200 & 1.69128900 & -3.87144900 \\
\hline $\mathrm{H}$ & -0.85825300 & 2.77512100 & -4.00019300 \\
\hline $\mathrm{H}$ & -2.70048100 & 3.40928900 & -2.12381300 \\
\hline $\mathrm{C}$ & -2.06735400 & 0.91803200 & -4.40758000 \\
\hline $\mathrm{H}$ & -1.89344400 & -0.15129900 & -4.27993600 \\
\hline $\mathrm{H}$ & -2.07146600 & 1.12209000 & -5.48560800 \\
\hline $\mathrm{C}$ & -3.43542700 & 1.25462200 & -3.82745400 \\
\hline $\mathrm{H}$ & -3.66709600 & 2.32297700 & -3.96428600 \\
\hline $\mathrm{H}$ & -3.43852200 & 1.05213100 & -2.75260900 \\
\hline $\mathrm{O}$ & -4.40510300 & 0.47368200 & -4.51113900 \\
\hline $\mathrm{Si}$ & -5.74971600 & -0.19967200 & -3.76149300 \\
\hline $\mathrm{C}$ & -6.65658900 & 1.14427200 & -2.78899100 \\
\hline $\mathrm{H}$ & -7.50453300 & 0.73201400 & -2.22865400 \\
\hline $\mathrm{H}$ & -5.99166300 & 1.63223700 & -2.06626200 \\
\hline $\mathrm{H}$ & -7.04387800 & 1.91796600 & -3.46257400 \\
\hline $\mathrm{C}$ & -6.80435200 & -0.81978700 & -5.19139000 \\
\hline $\mathrm{H}$ & -6.31070200 & -1.64197100 & -5.72185800 \\
\hline $\mathrm{H}$ & -7.78147200 & -1.17818500 & -4.84781800 \\
\hline $\mathrm{H}$ & -6.97398100 & -0.01184100 & -5.91212700 \\
\hline $\mathrm{C}$ & -5.27030200 & -1.64281800 & -2.59542900 \\
\hline $\mathrm{C}$ & -4.62912100 & -1.14060600 & -1.28227600 \\
\hline $\mathrm{H}$ & -4.44036200 & -1.99071200 & -0.60948900 \\
\hline $\mathrm{H}$ & -3.66073500 & -0.65943300 & -1.45249400 \\
\hline
\end{tabular}




$\begin{array}{llll}\mathrm{H} & -5.28054400 & -0.43858700 & -0.74642000 \\ \mathrm{C} & -6.55466300 & -2.42849600 & -2.24531900 \\ \mathrm{H} & -7.02615300 & -2.86486700 & -3.13407400 \\ \mathrm{H} & -6.31651800 & -3.25658400 & -1.56180300 \\ \mathrm{H} & -7.30124600 & -1.79912500 & -1.74281100 \\ \mathrm{C} & -4.27750100 & -2.58525600 & -3.30745600 \\ \mathrm{H} & -4.04481000 & -3.44792700 & -2.66589300 \\ \mathrm{H} & -4.68694200 & -2.97654900 & -4.24773300 \\ \mathrm{H} & -3.33173800 & -2.08072300 & -3.52979300\end{array}$

\section{Cross-metathesis product}

Thermal correction to Gibbs free energy $=0.419441$

Sum of electronic and thermal free energies $=-1207.477733$

\begin{tabular}{|c|c|c|c|}
\hline \multicolumn{4}{|c|}{ E_sol $=-1207.8405907$} \\
\hline $\mathrm{C}$ & -2.73662400 & 9.83793700 & 6.75506800 \\
\hline $\mathrm{H}$ & -2.39309400 & 10.85362300 & 6.51902900 \\
\hline $\mathrm{H}$ & -2.79188700 & 9.75838300 & 7.84560400 \\
\hline $\mathrm{C}$ & -4.14078700 & 9.66489400 & 6.17076700 \\
\hline $\mathrm{H}$ & -4.53231500 & 8.67534700 & 6.45045500 \\
\hline $\mathrm{H}$ & -4.09419400 & 9.69776800 & 5200 \\
\hline $\mathrm{O}$ & -4.97209100 & 10.71155600 & 6.64756000 \\
\hline $\mathrm{Si}$ & -6.63568700 & 10.63164800 & 6.84846900 \\
\hline $\mathrm{C}$ & -7.07374300 & 9.21768100 & 8.02080700 \\
\hline $\mathrm{H}$ & -8.16065500 & 9.11845200 & 8.13012800 \\
\hline $\mathrm{H}$ & -6.69136000 & 8.25381000 & 7.66580800 \\
\hline $\mathrm{H}$ & -6.65260400 & 9.39729900 & 9.01677500 \\
\hline $\mathrm{C}$ & -7.09377200 & 12.28969300 & 7.60588000 \\
\hline $\mathrm{H}$ & -6.89358500 & 13.11342700 & 6.91169300 \\
\hline $\mathrm{H}$ & -8.15464300 & 12.32747400 & 7.87891900 \\
\hline $\mathrm{H}$ & -6.50491800 & 12.46792300 & 8.51264300 \\
\hline $\mathrm{C}$ & -7.50730200 & 10.38646600 & 5.16119400 \\
\hline $\mathrm{C}$ & -7.30191900 & 8.94923300 & 4.63664700 \\
\hline $\mathrm{H}$ & -7.80844000 & 8.82003500 & 3.66876700 \\
\hline $\mathrm{H}$ & -6.24286700 & 8.71211000 & 4.48091600 \\
\hline $\mathrm{H}$ & -7.71472100 & 8.19995800 & 5.32294300 \\
\hline $\mathrm{C}$ & -9.02141200 & 10.64205700 & 5.32242600 \\
\hline $\mathrm{H}$ & -9.23152100 & 11.66712000 & 5.65048700 \\
\hline $\mathrm{H}$ & -9.53884300 & 10.49304200 & 4.36334600 \\
\hline $\mathrm{H}$ & -9.47987300 & 9.95675100 & 6.04711900 \\
\hline $\mathrm{C}$ & -6.93039100 & 11.38614600 & 4.13786600 \\
\hline
\end{tabular}




$\begin{array}{llll}\mathrm{H} & -7.42486700 & 11.26793800 & 3.16241300 \\ \mathrm{H} & -7.07833800 & 12.42554900 & 4.45595700 \\ \mathrm{H} & -5.85481700 & 11.23723400 & 3.98721000 \\ \mathrm{C} & 2.62775700 & 6.64716700 & 9.45292900 \\ \mathrm{C} & 2.51926700 & 8.20062500 & 9.21955000 \\ \mathrm{~B} & 0.71232900 & 7.14560700 & 8.32753500 \\ \mathrm{O} & 1.46496400 & 8.29102100 & 8.22278500 \\ \mathrm{O} & 1.28096400 & 6.19359300 & 9.14098700 \\ \mathrm{C} & 2.02288500 & 8.96340200 & 10.45415400 \\ \mathrm{H} & 2.78572200 & 9.00238700 & 11.23863100 \\ \mathrm{H} & 1.77233600 & 9.98677300 & 10.15943600 \\ \mathrm{H} & 1.12103000 & 8.49921400 & 10.86542100 \\ \mathrm{C} & 2.96496600 & 6.22888600 & 10.88083900 \\ \mathrm{H} & 3.00464300 & 5.13694900 & 10.94514400 \\ \mathrm{H} & 3.94243800 & 6.62522100 & 11.17841600 \\ \mathrm{H} & 2.21281800 & 6.58096100 & 11.58991900 \\ \mathrm{C} & 3.56526400 & 5.95123500 & 8.45863500 \\ \mathrm{H} & 4.61500400 & 6.18763100 & 8.66091200 \\ \mathrm{H} & 3.42923300 & 4.86884000 & 8.54237300 \\ \mathrm{H} & 3.33224500 & 6.24391700 & 7.43011700 \\ \mathrm{C} & 3.77608900 & 8.86000000 & 8.65999400 \\ \mathrm{H} & 3.59798300 & 9.93074300 & 8.51966200 \\ \mathrm{H} & 4.61663000 & 8.74020900 & 9.35301000 \\ \mathrm{H} & 4.05447700 & 8.43558700 & 7.69313200 \\ \mathrm{C} & -0.64586000 & 6.92312300 & 7.61321700 \\ \mathrm{H} & -1.14409000 & 5.95657900 & 7.71430200 \\ \mathrm{C} & -1.21950800 & 7.85972000 & 6.89152200 \\ \mathrm{C} & -1.76835700 & 8.81942500 & 6.19437700 \\ \mathrm{H} & -1.53586500 & 8.89034800 & 5.13013400\end{array}$

\section{vinyl-ii}

Thermal correction to Gibbs free energy $=0.466954$

Sum of electronic and thermal free energies $=-2139.562238$

$\begin{array}{lrrr}\text { E_sol }=-2140.0147044 & & \\ \mathrm{Ru} & -1.34906200 & 2.29959300 & -0.09287500 \\ \mathrm{Cl} & 0.85462800 & 3.02984100 & 0.04715600 \\ \mathrm{Cl} & -2.74298500 & 0.55579000 & 0.59302900 \\ \mathrm{P} & -1.78824100 & 2.08394300 & -2.31389600 \\ \mathrm{C} & -3.98450100 & 1.30177600 & -4.08665000 \\ \mathrm{H} & -4.79736300 & 0.56119300 & -4.06504200\end{array}$




$\begin{array}{llll}\mathrm{H} & -3.17860000 & 0.83960100 & -4.66862400 \\ \mathrm{C} & -4.51194500 & 2.55856600 & -4.80159100 \\ \mathrm{H} & -4.88603500 & 2.27333200 & -5.79303200 \\ \mathrm{H} & -3.69896100 & 3.26969900 & -4.97605700 \\ \mathrm{C} & -5.61593100 & 3.25988200 & -3.99748000 \\ \mathrm{H} & -6.49798000 & 2.60492700 & -3.94390000 \\ \mathrm{H} & -5.93328800 & 4.17460500 & -4.51335200 \\ \mathrm{C} & -5.14450900 & 3.57577100 & -2.57118600 \\ \mathrm{H} & -5.96004500 & 4.03092600 & -1.99505100 \\ \mathrm{H} & -4.33661400 & 4.31894100 & -2.59318300 \\ \mathrm{C} & -4.67220500 & 2.30271900 & -1.85959300 \\ \mathrm{H} & -4.36671800 & 2.50773100 & -0.83260200 \\ \mathrm{H} & -5.52906200 & 1.61705500 & -1.78458500 \\ \mathrm{C} & -3.56618500 & 1.52427500 & -2.61021300 \\ \mathrm{H} & -3.51531400 & 0.54528100 & -2.12402000 \\ \mathrm{C} & -0.77961800 & 0.69149800 & -3.06783500 \\ \mathrm{H} & -0.94464700 & 0.79153700 & -4.14577500 \\ \mathrm{H} & -0.51778400 & 4.49530800 & -6.55536800 \\ \mathrm{C} & -0.91588000 & 5.86411300 & -4.90724100 \\ \mathrm{C} & -1.83728100 & 6.22903400 & -4.43246100 \\ \mathrm{H} & & & \\ \mathrm{H} & & & \end{array}$




$\begin{array}{lrrr}\mathrm{H} & -0.58352600 & 6.66009700 & -5.58332600 \\ \mathrm{C} & 0.13103000 & 5.58774800 & -3.82362200 \\ \mathrm{H} & 0.43446900 & 6.51379100 & -3.32250700 \\ \mathrm{H} & 1.03569900 & 5.18781400 & -4.30146600 \\ \mathrm{C} & -0.39406100 & 4.58174400 & -2.76939800 \\ \mathrm{H} & 0.44839700 & 4.03454300 & -2.34469200 \\ \mathrm{H} & -0.85461400 & 5.11496700 & -1.93303100 \\ \mathrm{C} & -1.44087200 & 3.60631600 & -3.36923400 \\ \mathrm{H} & -2.41035900 & 4.11482300 & -3.36857300 \\ \mathrm{C} & -2.29963600 & 3.78518500 & -0.01212200 \\ \mathrm{C} & -2.98880200 & 4.90641400 & 0.08272100 \\ \mathrm{H} & -2.48552800 & 5.87000300 & 0.02894900 \\ \mathrm{H} & -4.06179300 & 4.89194500 & 0.26178700\end{array}$

ts-iv

Thermal correction to Gibbs free energy $=0.683915$

Sum of electronic and thermal free energies $=-2666.731411$

E_sol $=-2667.3735793$

$\begin{array}{lrrr}\mathrm{Ru} & 0.81185900 & 0.38668700 & -1.18523600 \\ \mathrm{Cl} & 0.72689700 & 2.76943900 & -0.83528800 \\ \mathrm{Cl} & 1.04127400 & -1.94750300 & -1.81706400 \\ \mathrm{C} & -0.89378300 & 0.17883900 & -0.63851900 \\ \mathrm{C} & -2.09271700 & 0.00750700 & -0.12459500 \\ \mathrm{H} & -2.18948500 & -0.21464700 & 0.93466000 \\ \mathrm{P} & 2.39732200 & -0.01269000 & 0.61820100 \\ \mathrm{C} & 2.47768400 & -1.40777200 & 3.18794000 \\ \mathrm{H} & 1.83036800 & -1.48385400 & 4.07408200 \\ \mathrm{H} & 3.21730800 & -0.63458400 & 3.42534700 \\ \mathrm{C} & 3.16349300 & -2.77097400 & 2.98769300 \\ \mathrm{H} & 3.71924500 & -3.02757000 & 3.89868500 \\ \mathrm{H} & 3.90174200 & -2.71175200 & 2.18113300 \\ \mathrm{C} & 2.15631400 & -3.87358100 & 2.63734800 \\ \mathrm{H} & 1.47370900 & -4.02969700 & 3.48624200 \\ \mathrm{H} & 2.68042900 & -4.82364600 & 2.47459100 \\ \mathrm{C} & 1.34542300 & -3.48123900 & 1.39668000 \\ \mathrm{H} & 0.60088500 & -4.25120100 & 1.16131400 \\ \mathrm{H} & 1.99862300 & -3.41356700 & 0.51890100 \\ \mathrm{C} & 0.62943900 & -2.14283300 & 1.60820300 \\ \mathrm{H} & 0.04265900 & -1.88965700 & 0.72512400 \\ \mathrm{H} & -0.08118400 & -2.26342600 & 2.44113600\end{array}$




\begin{tabular}{|c|c|c|c|}
\hline $\mathrm{C}$ & 1.56283200 & -0.97655900 & 2.01227600 \\
\hline $\mathrm{H}$ & 0.90548900 & -0.18104700 & 2.38369400 \\
\hline $\mathrm{C}$ & 3.13662200 & 1.47030300 & 1.49535100 \\
\hline $\mathrm{H}$ & 3.90111600 & 1.04401300 & 2.15820900 \\
\hline $\mathrm{C}$ & 3.85430000 & 2.42866600 & 0.49379800 \\
\hline $\mathrm{H}$ & 3.36682200 & 2.40905500 & -0.48295100 \\
\hline $\mathrm{H}$ & 4.88555800 & 2.08381300 & 0.35195200 \\
\hline $\mathrm{C}$ & 3.86572600 & 3.88593000 & 0.99509400 \\
\hline $\mathrm{H}$ & 2.91003700 & 4.36052800 & 0.74150100 \\
\hline $\mathrm{H}$ & 4.64550600 & 4.44520000 & 0.46430000 \\
\hline $\mathrm{C}$ & 4.08657900 & 3.93713900 & 2.51006700 \\
\hline $\mathrm{H}$ & 4.23077600 & 4.96855200 & 2.85215800 \\
\hline $\mathrm{H}$ & 5.01706800 & 3.40168700 & 2.74782600 \\
\hline $\mathrm{C}$ & 2.89697000 & 3.28515300 & 3.25630000 \\
\hline $\mathrm{H}$ & 2.17967500 & 4.05497000 & 3.56578600 \\
\hline $\mathrm{H}$ & 3.25683600 & 2.80841700 & 4.17742700 \\
\hline $\mathrm{C}$ & 2.14688200 & 2.24502500 & 2.39003600 \\
\hline $\mathrm{H}$ & 1.59954800 & 1.57189900 & 3.05754600 \\
\hline $\mathrm{H}$ & 1.40479800 & 2.73360600 & 1.75378300 \\
\hline $\mathrm{C}$ & 5.17131600 & -0.87650400 & 0.76862500 \\
\hline $\mathrm{H}$ & 4.98082800 & -1.02352200 & 1.83436800 \\
\hline $\mathrm{H}$ & 5.62490000 & 0.11622900 & 0.67615300 \\
\hline $\mathrm{C}$ & 6.16065600 & -1.95003700 & 0.26053400 \\
\hline $\mathrm{H}$ & 5.93397900 & -2.91258000 & 0.73680200 \\
\hline $\mathrm{H}$ & 7.17371100 & -1.67472700 & 0.57932800 \\
\hline $\mathrm{C}$ & 6.10600300 & -2.10427600 & -1.27971600 \\
\hline $\mathrm{H}$ & 5.39287900 & -2.89335400 & -1.55311600 \\
\hline $\mathrm{H}$ & 7.08135100 & -2.42319900 & -1.66452800 \\
\hline $\mathrm{C}$ & 5.66021600 & -0.79219300 & -1.93734400 \\
\hline $\mathrm{H}$ & 5.72364300 & -0.86041800 & -3.02940500 \\
\hline $\mathrm{H}$ & 6.34516700 & 0.01251900 & -1.63769200 \\
\hline $\mathrm{C}$ & 4.20938800 & -0.44116600 & -1.52927000 \\
\hline $\mathrm{H}$ & 4.06581600 & 0.64125300 & -1.62834300 \\
\hline $\mathrm{H}$ & 3.50637100 & -0.92543300 & -2.21201700 \\
\hline $\mathrm{C}$ & 3.88389600 & -0.91855400 & -0.07939000 \\
\hline $\mathrm{H}$ & 3.53658500 & -1.95518700 & -0.14637300 \\
\hline $\mathrm{C}$ & -0.68937100 & 1.73816700 & -7.26478600 \\
\hline $\mathrm{C}$ & 0.75429100 & 1.50484300 & -7.84968200 \\
\hline B & 0.77146500 & 1.25603100 & -5.59050800 \\
\hline $\mathrm{O}$ & 1.58417400 & 1.55694100 & -6.65422300 \\
\hline
\end{tabular}




$\begin{array}{llrl}\mathrm{O} & -0.56398600 & 1.20578100 & -5.91662900 \\ \mathrm{C} & 0.94257900 & 0.10709300 & -8.45186100 \\ \mathrm{H} & 0.39979000 & -0.00103000 & -9.39661700 \\ \mathrm{H} & 2.00780400 & -0.05581000 & -8.64120900 \\ \mathrm{H} & 0.59899200 & -0.66763000 & -7.75926000 \\ \mathrm{C} & -1.80639300 & 0.98744700 & -7.98307800 \\ \mathrm{H} & -2.76467400 & 1.20247400 & -7.49959500 \\ \mathrm{H} & -1.87298000 & 1.30440100 & -9.03009000 \\ \mathrm{H} & -1.64763300 & -0.09256700 & -7.95212400 \\ \mathrm{C} & -1.04907700 & 3.22186000 & -7.11922300 \\ \mathrm{H} & -1.22306800 & 3.69198300 & -8.09265400 \\ \mathrm{H} & -1.96232700 & 3.30768100 & -6.52266900 \\ \mathrm{H} & -0.25466800 & 3.76916700 & -6.60225600 \\ \mathrm{C} & 1.23751600 & 2.57420200 & -8.82445900 \\ \mathrm{H} & 2.24975600 & 2.33267400 & -9.16373800 \\ \mathrm{H} & 0.58347700 & 2.61895900 & -9.70277000 \\ \mathrm{H} & 1.26516800 & 3.56038500 & -8.35621100 \\ \mathrm{C} & 0.43457600 & 0.76308000 & -3.18195800 \\ \mathrm{C} & 1.29895000 & 0.99707500 & -4.15752100 \\ \mathrm{H} & 2.36733000 & 1.00205000 & -3.94667500 \\ \mathrm{C} & -0.87752300 & 0.61282700 & -2.73658600 \\ \mathrm{H} & -1.47438900 & 1.51112800 & -2.59149100 \\ \mathrm{H} & -1.38914300 & -0.32082200 & -2.96161500 \\ \mathrm{H} & -2.99498100 & 0.08411400 & -0.72380600 \\ \text { mcb-iii } & & & \\ & & & \\ & & & \end{array}$

Thermal correction to Gibbs free energy $=0.684083$

Sum of electronic and thermal free energies $=-2666.737824$

E_sol $=-2667.3804231$

$\begin{array}{lrrr}\mathrm{Ru} & 0.35517500 & 0.07476000 & -0.89491000 \\ \mathrm{Cl} & -0.07780600 & 2.34663400 & -0.23768800 \\ \mathrm{Cl} & 0.69967900 & -2.25537900 & -1.48852800 \\ \mathrm{C} & -1.53513200 & -0.26102400 & -0.84064800 \\ \mathrm{C} & -2.57684400 & -0.62823000 & -0.11721400 \\ \mathrm{H} & -2.46951600 & -0.88044100 & 0.93304000 \\ \mathrm{P} & 2.24505300 & -0.08039500 & 0.59823600 \\ \mathrm{C} & 2.70209200 & -1.23745400 & 3.22658500 \\ \mathrm{H} & 2.19925100 & -1.23608400 & 4.20475200 \\ \mathrm{H} & 3.46593200 & -0.45281000 & 3.28078300 \\ \mathrm{C} & 3.35232200 & -2.61757200 & 3.02816900\end{array}$




\begin{tabular}{|c|c|c|c|}
\hline $\mathrm{H}$ & 4.04648100 & -2.80753600 & 3.85655600 \\
\hline $\mathrm{H}$ & 3.95267800 & -2.62780700 & 2.11170700 \\
\hline$C$ & 2.30308800 & -3.73260600 & 2.93119500 \\
\hline & 1.76096900 & -3.80884600 & 3.88567600 \\
\hline & 2.79585200 & -4.69925400 & 2.76953700 \\
\hline & 1.31070100 & -3.43764200 & 1.79979100 \\
\hline & 0.54113300 & -4.21672900 & 1.74623000 \\
\hline & 1.82279800 & -3.45023200 & 0.83102000 \\
\hline & 0.63190900 & -2.07852000 & 2.00308700 \\
\hline & -0.07665000 & -1.89087600 & 1.19239600 \\
\hline & 0.04607600 & -2.11929500 & 2.93411400 \\
\hline & 1.61768400 & -0.89592700 & 2.17322400 \\
\hline & 1.02474700 & -0.05883900 & 2.56198700 \\
\hline & 3.05686800 & 1.50681900 & 1.17798700 \\
\hline & 4.00741800 & 1.16476500 & 1.60683500 \\
\hline & 3.38363000 & 2.43810300 & -0.03217600 \\
\hline & 2.64055200 & 2.32188000 & -0.82525700 \\
\hline $\mathrm{H}$ & 4.35698200 & 2.14672200 & -0.44687600 \\
\hline$C$ & 3.42433900 & 3.92202900 & 0.37922600 \\
\hline & 2.40089300 & 4.31524000 & 0.40809100 \\
\hline & 3.96019100 & 4.49494500 & -0.38688800 \\
\hline & 4.08552200 & 4.08556600 & 1.75142100 \\
\hline & 4.23551600 & 5.14448700 & 1.99115200 \\
\hline & 5.08756200 & 3.63456500 & 1.71359000 \\
\hline & 3.23886600 & 3.40003000 & 2.85275200 \\
\hline & 2.60112400 & 4.13890500 & 3.35263500 \\
\hline & 3.90230000 & 2.99036400 & 3.62561500 \\
\hline & 2.32969000 & 2.27642600 & 2.29951600 \\
\hline & 2.06050500 & 1.61411400 & 3.12803900 \\
\hline & 1.39858300 & 2.68855800 & 1.90628700 \\
\hline & 5.02403000 & -0.87562300 & 0.40676900 \\
\hline & 4.99041800 & -0.94835500 & 1.49698300 \\
\hline & 5.42919500 & 0.11733700 & 0.17840700 \\
\hline & 5.96331800 & -1.96452900 & -0.16028100 \\
\hline & 5.83153600 & -2.89346400 & 0.40916600 \\
\hline & 7.00209100 & -1.64742500 & -0.00565200 \\
\hline & 5.69972600 & -2.22923400 & -1.66320800 \\
\hline & 4.97743700 & -3.04804800 & -1.77951700 \\
\hline & 6.62083800 & -2.55735900 & -2.15799600 \\
\hline & 5.13080100 & -0.97490900 & -2.33778100 \\
\hline
\end{tabular}




$\begin{array}{lrrr}\mathrm{H} & 5.04253000 & -1.11915300 & -3.42056800 \\ \mathrm{H} & 5.82969300 & -0.13957900 & -2.19362700 \\ \mathrm{C} & 3.74048600 & -0.62222400 & -1.75708200 \\ \mathrm{H} & 3.55215700 & 0.44673600 & -1.90398400 \\ \mathrm{H} & 2.96127000 & -1.15889500 & -2.30120300 \\ \mathrm{C} & 3.63337800 & -1.00859800 & -0.24794400 \\ \mathrm{H} & 3.30937200 & -2.05327200 & -0.19572700 \\ \mathrm{C} & -2.07077000 & 1.34997700 & -6.44125000 \\ \mathrm{C} & -0.77330600 & 1.35218400 & -7.33235700 \\ \mathrm{~B} & -0.21796600 & 1.07401400 & -5.14236700 \\ \mathrm{O} & 0.28044800 & 1.51756000 & -6.34401800 \\ \mathrm{O} & -1.57470900 & 0.81912300 & -5.18163800 \\ \mathrm{C} & -0.51802700 & 0.00871100 & -8.02692300 \\ \mathrm{H} & -1.23335600 & -0.16942000 & -8.83657700 \\ \mathrm{H} & 0.49153200 & 0.01526900 & -8.44867000 \\ \mathrm{H} & -0.58195100 & -0.81917700 & -7.31379500 \\ \mathrm{C} & -3.19703800 & 0.44953200 & -6.93933600 \\ \mathrm{H} & -4.04652400 & 0.50690100 & -6.25114600 \\ \mathrm{H} & -3.53807700 & 0.77021300 & -7.93027800 \\ \mathrm{H} & -2.87972500 & -0.59382100 & -6.99756100 \\ \mathrm{C} & -2.60215700 & 2.75796900 & -6.14538800 \\ \mathrm{H} & -3.05462800 & 3.21345000 & -7.03230200 \\ \mathrm{H} & -3.36327400 & 2.69192400 & -5.36185300 \\ \mathrm{H} & -1.80124400 & 3.41121300 & -5.78529900 \\ \mathrm{C} & -0.68260300 & 2.49640400 & -8.33757800 \\ \mathrm{H} & 0.25385800 & 2.41961100 & -8.89906100 \\ \mathrm{H} & -1.51403700 & 2.45108700 & -9.05029800 \\ \mathrm{H} & -1.65242000 & -0.68366900 & -2.95266100 \\ \mathrm{C} & -0.69850000 & 3.46854300 & -7.84023700 \\ \mathrm{C} & 0.11565200 & 0.50304500 & -2.71818900 \\ \mathrm{H} & 0.64371800 & 0.88418500 & -3.87874100 \\ \mathrm{H} & 1.71719000 & 1.06139300 & -3.91550500 \\ \mathrm{H} & -1.36628400 & 0.15952200 & -2.31888700 \\ \mathrm{H} & -1.94091200 & 1.07333700 & -2.49007500 \\ \mathrm{H} & -0.68658300 & -0.55135100 \\ \mathrm{H} & & & \\ \mathrm{H} & -373000 & & \\ \mathrm{H} & & & \end{array}$

Thermal correction to Gibbs free energy $=0.963195$

Sum of electronic and thermal free energies $=-4280.0471569$

E_sol $=-4279.119902$ 


$\begin{array}{lrrr}\mathrm{Ru} & -1.50092200 & 0.78139200 & -0.28088900 \\ \mathrm{Cl} & -1.88960000 & 1.70287900 & -2.40563700 \\ \mathrm{Cl} & -1.23489800 & 1.04030400 & 2.04460400 \\ \mathrm{C} & -0.61737200 & -0.74481200 & -0.60436400 \\ \mathrm{Ru} & 1.40931600 & -0.80837800 & -0.34621100 \\ \mathrm{Cl} & 1.08222000 & -1.69235400 & 1.88060600 \\ \mathrm{Cl} & 1.60914900 & 0.12043400 & -2.58438200 \\ \mathrm{P} & 3.42386700 & 0.36822500 & 0.30708400 \\ \mathrm{C} & 5.17744800 & 0.66408500 & 2.64545800 \\ \mathrm{H} & 5.05320400 & 0.50784500 & 3.72728700 \\ \mathrm{H} & 4.99177000 & 1.72966800 & 2.47771300 \\ \mathrm{C} & 6.63786100 & 0.33315000 & 2.29732400 \\ \mathrm{H} & 7.29830900 & 0.93716300 & 2.93266900 \\ \mathrm{H} & 6.86485200 & 0.62360600 & 1.26281500 \\ \mathrm{C} & 6.93392800 & -1.15967200 & 2.48343900 \\ \mathrm{H} & 6.81867600 & -1.41980800 & 3.54617500 \\ \mathrm{H} & 7.97457100 & -1.37986700 & 2.21415800 \\ \mathrm{C} & 5.96987300 & -2.00835800 & 1.64528500 \\ \mathrm{H} & 1.13604800 & 3.89993200 & 2.96961900 \\ \mathrm{H} & 2.35723500 & 4.51663800 & 1.86510300 \\ \mathrm{H} & 2.24840600 & 2.36255900 & 1.92977300 \\ \mathrm{H} & 6.16684000 & -3.07639300 & 1.80351400 \\ \mathrm{C} & 4.50232700 & -1.71805400 & 1.99652500 \\ \mathrm{H} & 3.82190400 & -2.30964200 & 1.37750400 \\ \mathrm{H} & & & \\ \mathrm{H} & 4.32766500 & -2.06048100 & 3.02592600 \\ \mathrm{C} & 4.09901400 & -0.22002800 & 1.96011000 \\ \mathrm{H} & 3.18597800 & -0.14608500 & 2.55811100 \\ \mathrm{C} & 3.05371000 & 2.18897600 & 0.61136100 \\ \mathrm{H} & 4.03701900 & 2.66217700 & 0.73284300 \\ \mathrm{H} & 2.32193400 & 2.88590300 & -0.57462400 \\ \mathrm{H} & 2.48772500 & 2.37309200 & -1.51821700 \\ \mathrm{H} & 2.73430500 & 3.89796800 & -0.68466700 \\ \mathrm{H} & 0.81756300 & 3.00832100 & -0.33246600 \\ \mathrm{H} & 0.42473800 & 1.98096900 & -0.14864400 \\ \mathrm{H} & & & \end{array}$




\begin{tabular}{|c|c|c|c|}
\hline $\mathrm{H}$ & 2.90517800 & 2.23549300 & 2.79526700 \\
\hline $\mathrm{H}$ & 1.47061500 & 1.59480700 & 2.01599500 \\
\hline $\mathrm{C}$ & 4.94520900 & 1.35179600 & -1.98319800 \\
\hline $\mathrm{H}$ & 5.27963700 & 2.27690500 & -1.49728800 \\
\hline $\mathrm{H}$ & 3.96856300 & 1.54525300 & -2.42457900 \\
\hline $\mathrm{C}$ & 5.91902400 & 0.95561200 & -3.12668800 \\
\hline $\mathrm{H}$ & 6.41720900 & 1.85179000 & -3.51428400 \\
\hline $\mathrm{H}$ & 5.34004900 & 0.53782200 & -3.96062300 \\
\hline $\mathrm{C}$ & 6.94502100 & -0.08959900 & -2.67488100 \\
\hline $\mathrm{H}$ & 7.51493100 & 0.30496700 & -1.82165400 \\
\hline $\mathrm{H}$ & 7.67792200 & -0.28259500 & -3.46707200 \\
\hline $\mathrm{C}$ & 6.23370200 & -1.40197200 & -2.27533100 \\
\hline $\mathrm{H}$ & 6.87413600 & -1.97031500 & -1.58892000 \\
\hline $\mathrm{H}$ & 6.09425100 & -2.03150500 & -3.16304700 \\
\hline $\mathrm{C}$ & 4.84943800 & -1.14307600 & 56400 \\
\hline $\mathrm{H}$ & 4.05945300 & -1.13707200 & -2.38468500 \\
\hline $\mathrm{H}$ & 4.60178700 & -1.95478100 & -0.93706700 \\
\hline $\mathrm{C}$ & 4.83517700 & 0.23013500 & -0.92256400 \\
\hline $\mathrm{H}$ & 5.72003900 & 0.28901500 & -0.28227100 \\
\hline $\mathrm{P}$ & -3.59203100 & -0.13091400 & -0.12624900 \\
\hline $\mathrm{C}$ & -5.62098900 & -1.55692400 & -1.64418300 \\
\hline $\mathrm{H}$ & -6.12470000 & -1.40423000 & -2.60957900 \\
\hline $\mathrm{H}$ & -6.26245500 & -1.07729500 & -0.89418100 \\
\hline $\mathrm{C}$ & -5.54636700 & -3.07407300 & -1.38784000 \\
\hline $\mathrm{H}$ & -6.55670300 & -3.49623000 & -1.45746800 \\
\hline $\mathrm{H}$ & -5.19932500 & -3.27684600 & -0.36690900 \\
\hline $\mathrm{C}$ & -4.60105500 & -3.77903500 & -2.37005400 \\
\hline $\mathrm{H}$ & -5.00247000 & -3.69436500 & -3.39060100 \\
\hline $\mathrm{H}$ & -4.54800400 & -4.84982800 & -2.13808000 \\
\hline $\mathrm{C}$ & -3.20489200 & -3.14406000 & -2.32385200 \\
\hline $\mathrm{H}$ & -2.53349100 & -3.64090100 & -3.03603700 \\
\hline $\mathrm{H}$ & -2.77373000 & -3.29730200 & -1.32725700 \\
\hline $\mathrm{C}$ & -3.27888100 & -1.64860900 & -2.64587700 \\
\hline $\mathrm{H}$ & -2.29842000 & -1.17156000 & -2.65535400 \\
\hline $\mathrm{H}$ & -3.66353100 & -1.54748200 & -3.67163900 \\
\hline $\mathrm{C}$ & -4.24567400 & -0.84725400 & -1.74903800 \\
\hline $\mathrm{H}$ & -4.39364000 & 0.09271900 & -2.28435300 \\
\hline $\mathrm{C}$ & -4.85721700 & 1.25366600 & 0.18807100 \\
\hline $\mathrm{H}$ & -5.72963400 & 0.70961100 & 0.57341900 \\
\hline $\mathrm{C}$ & -4.38805400 & 2.25776100 & 1.27865500 \\
\hline
\end{tabular}




\begin{tabular}{|c|c|c|c|}
\hline $\mathrm{H}$ & -3.73810800 & 1.78522800 & 2.01305500 \\
\hline $\mathrm{H}$ & -5.27372400 & 2.60812700 & 1.82520200 \\
\hline $\mathrm{C}$ & -3.67387500 & 3.46103100 & 0.65583500 \\
\hline $\mathrm{H}$ & -2.83228600 & 3.10406100 & 0.04179100 \\
\hline & -3.22317700 & 4.07798500 & 1.44070200 \\
\hline & -4.63402400 & 4.29127200 & -0.22841200 \\
\hline & -4.07498300 & 4.72693200 & -1.06513400 \\
\hline & -5.04599700 & 5.12965500 & 0.34672900 \\
\hline $\mathrm{C}$ & -5.78795700 & 3.42244900 & -0.77318900 \\
\hline $\mathrm{H}$ & -6.21506200 & 3.87974100 & -1.67314300 \\
\hline & -6.60354400 & 3.36528400 & -0.03971000 \\
\hline & -5.28690000 & 2.00820000 & -1.10127400 \\
\hline & -6.06327200 & 1.43827100 & -1.62361300 \\
\hline & -4.44144100 & 2.10889700 & -1.79026900 \\
\hline & -4.31819000 & -0.76486400 & 2.63837700 \\
\hline $\mathrm{H}$ & -5.05608500 & 0.03448700 & 2.54361500 \\
\hline $\mathrm{H}$ & -3.43471200 & -0.32791200 & 3.11204400 \\
\hline $\mathrm{C}$ & -4.92136800 & -1.88391300 & 3.52595000 \\
\hline & -6.00082200 & -1.95875000 & 3.34058400 \\
\hline & -4.80537900 & -1.58891800 & 4.57600300 \\
\hline $\mathrm{C}$ & -4.25529000 & -3.25898200 & 3.28821400 \\
\hline $\mathrm{H}$ & -4.79972900 & -3.81236900 & 2.50924800 \\
\hline $\mathrm{H}$ & -4.31328400 & -3.87168700 & 4.19523800 \\
\hline $\mathrm{C}$ & -2.80362100 & -3.06788800 & 2.84152200 \\
\hline $\mathrm{H}$ & -2.27835000 & -4.02687900 & 2.76953100 \\
\hline $\mathrm{H}$ & -2.26481600 & -2.47976500 & 3.59451700 \\
\hline $\mathrm{C}$ & -2.73001300 & -2.33984900 & 1.47938900 \\
\hline $\mathrm{H}$ & -1.78048600 & -1.80322500 & 1.43627600 \\
\hline$H$ & -2.73519600 & -3.07852500 & 0.66965600 \\
\hline & -3.90928000 & -1.35442600 & 1.27130200 \\
\hline & -4.78097300 & -1.90796900 & 0.90463000 \\
\hline & -0.33135100 & -2.06523000 & -1.02105900 \\
\hline & -0.41713200 & -2.25599600 & -2.08937700 \\
\hline $\mathrm{H}$ & -0.59271000 & -2.87138400 & -0.33936200 \\
\hline & 1.68890000 & -2.47370200 & -1.01096700 \\
\hline & 2.02490100 & -3.66049900 & -1.46839300 \\
\hline & 1.28649300 & -4.38361300 & -1.80214300 \\
\hline & 3.07540600 & -3.93414700 & -1.51417000 \\
\hline
\end{tabular}


mcb-iv

Thermal correction to Gibbs free energy $=0.964347$

Sum of electronic and thermal free energies $=-4279.128742$

E_sol $=-4280.057322$

\begin{tabular}{|c|c|c|c|}
\hline & 1.56262100 & 3.90588100 & 1.01039000 \\
\hline $\mathrm{Cl}$ & 1.25971900 & 6.22916600 & 1.30154000 \\
\hline $\mathrm{Cl}$ & 2.84449600 & 1.91110600 & 0.95396600 \\
\hline $\mathrm{C}$ & 0.35144300 & 3.50640100 & -0.24868700 \\
\hline $\mathrm{Ru}_{2}$ & 0.44505700 & 3.14736400 & -2.09905900 \\
\hline $\mathrm{Cl}$ & 0.52476300 & 0.77177100 & -1.71428400 \\
\hline$C 1$ & 0.27030900 & 5.53426800 & -2.52907800 \\
\hline$P$ & 2.08671500 & 2.99928000 & -3.87301600 \\
\hline $\mathrm{C}$ & 3.69532300 & 1.00129100 & -5.28962900 \\
\hline $\mathrm{H}$ & 4.02505600 & -0.02435800 & -5.06790300 \\
\hline $\mathrm{H}$ & 4.52143500 & 1.64947400 & -4.98222000 \\
\hline $\mathrm{C}$ & 3.49823600 & 1.10374600 & -6.81068900 \\
\hline $\mathrm{H}$ & 4.42909200 & 0.80126300 & -7.30713100 \\
\hline $\mathrm{H}$ & 3.32178900 & 2.14574400 & -7.10963800 \\
\hline $\mathrm{C}$ & 2.33028700 & 0.23104000 & -7.28463000 \\
\hline $\mathrm{H}$ & 2.56847800 & -0.82510200 & -7.08956700 \\
\hline $\mathrm{H}$ & 2.19358400 & 0.33014600 & -8.36867100 \\
\hline $\mathrm{C}$ & 1.04144800 & 0.60641800 & -6.54328500 \\
\hline $\mathrm{H}$ & 0.21676800 & -0.04436400 & -6.86034700 \\
\hline $\mathrm{H}$ & 0.75279000 & 1.62347700 & -6.82760700 \\
\hline $\mathrm{C}$ & 1.20273800 & 0.48881100 & -5.01966200 \\
\hline $\mathrm{H}$ & 0.28585800 & 0.78263100 & -4.50189500 \\
\hline $\mathrm{H}$ & 1.34211200 & -0.57335900 & -4.77558700 \\
\hline $\mathrm{C}$ & 2.42680600 & 1.23307600 & -4.42038200 \\
\hline $\mathrm{H}$ & 2.60690400 & 0.77336700 & -3.44436800 \\
\hline $\mathrm{C}$ & 3.75396100 & 3.60439200 & -3.24277400 \\
\hline $\mathrm{H}$ & 4.36784400 & 3.67602900 & -4.15070900 \\
\hline $\mathrm{C}$ & 3.72296500 & 5.01096900 & -2.56868200 \\
\hline $\mathrm{H}$ & 2.84356000 & 5.58353800 & -2.85308700 \\
\hline $\mathrm{H}$ & 4.59830000 & 5.57407000 & -2.91866900 \\
\hline $\mathrm{C}$ & 3.78570100 & 4.92717600 & -1.04211800 \\
\hline $\mathrm{H}$ & 2.92173300 & 4.31141300 & -0.71116900 \\
\hline $\mathrm{H}$ & 3.62431300 & 5.91308000 & -0.59828100 \\
\hline $\mathrm{C}$ & 5.08793000 & 4.27772300 & -0.54996200 \\
\hline 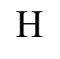 & 4.93048400 & 3.85881800 & 0.44826100 \\
\hline & 586397600 & 5.04847900 & -0.458 \\
\hline
\end{tabular}




\begin{tabular}{|c|c|c|c|}
\hline $\mathrm{C}$ & 5.56381200 & 3.16896600 & -1.51818500 \\
\hline $\mathrm{H}$ & 6.08716700 & 2.38158700 & -0.96485800 \\
\hline $\mathrm{H}$ & 6.28681400 & 3.57844200 & -2.23773000 \\
\hline $\mathrm{C}$ & 4.39073900 & 2.55147100 & -2.29490100 \\
\hline $\mathrm{H}$ & 4.74281000 & 1.68763400 & -2.86549400 \\
\hline $\mathrm{H}$ & 3.65277900 & 2.17177500 & -1.57993300 \\
\hline $\mathrm{C}$ & 2.14879000 & 5.39140100 & -5.54167300 \\
\hline $\mathrm{H}$ & 3.22091500 & 5.35269000 & -5.77305000 \\
\hline $\mathrm{H}$ & 2.03428700 & 5.92271900 & -4.59849500 \\
\hline $\mathrm{C}$ & 1.38837800 & 6.18231800 & -6.63789300 \\
\hline $\mathrm{H}$ & 2.05141300 & 6.94070100 & -7.06997800 \\
\hline $\mathrm{H}$ & 0.55498600 & 6.72573100 & -6.17410900 \\
\hline $\mathrm{C}$ & 0.82782200 & 5.25801000 & -7.72476400 \\
\hline $\mathrm{H}$ & 1.65035100 & 4.67618900 & -8.16498000 \\
\hline $\mathrm{H}$ & 0.39379200 & 5.84044500 & -8.54573400 \\
\hline $\mathrm{C}$ & -0.23428500 & 4.30654000 & -7.12708000 \\
\hline $\mathrm{H}$ & -0.30724000 & 3.40375700 & -7.74638600 \\
\hline $\mathrm{H}$ & -1.22057900 & 4.78557000 & -7.15921800 \\
\hline $\mathrm{C}$ & 0.08118200 & 3.92877100 & -5.65807400 \\
\hline $\mathrm{H}$ & -0.37533500 & 4.64259400 & -4.96833000 \\
\hline $\mathrm{H}$ & -0.35579400 & 2.95252600 & -5.41962100 \\
\hline $\mathrm{C}$ & 1.60831300 & 3.94554800 & -5.41880600 \\
\hline $\mathrm{H}$ & 2.07413100 & 3.35021400 & -6.20913200 \\
\hline $\mathrm{P}$ & 0.47717000 & 3.36141200 & 2.95555700 \\
\hline $\mathrm{C}$ & -1.71799500 & 4.08390900 & 4.72700600 \\
\hline $\mathrm{H}$ & -2.06882100 & 5.01981500 & 5.18544100 \\
\hline $\mathrm{H}$ & -1.03772300 & 3.63047100 & 5.45865500 \\
\hline $\mathrm{C}$ & -2.94546900 & 3.17816700 & 4.51247500 \\
\hline $\mathrm{H}$ & -3.47497300 & 3.06616100 & 5.46692700 \\
\hline $\mathrm{H}$ & -2.63401200 & 2.16881300 & 4.21515900 \\
\hline $\mathrm{C}$ & -3.88982400 & 3.73056000 & 3.43606600 \\
\hline $\mathrm{H}$ & -4.31244900 & 4.68819500 & 3.77394500 \\
\hline $\mathrm{H}$ & -4.73381800 & 3.04572900 & 3.28844300 \\
\hline $\mathrm{C}$ & -3.13267000 & 3.94974300 & 2.11957900 \\
\hline $\mathrm{H}$ & -3.80445800 & 4.34918200 & 1.34919900 \\
\hline $\mathrm{H}$ & -2.77521400 & 2.98057200 & 1.75169100 \\
\hline $\mathrm{C}$ & -1.95814100 & 4.91056000 & 2.32827500 \\
\hline $\mathrm{H}$ & -1.41035100 & 5.12169900 & 1.40974700 \\
\hline $\mathrm{H}$ & -2.37365400 & 5.87818500 & 2.64666300 \\
\hline $\mathrm{C}$ & -0.96788800 & 4.48516400 & 3.42968500 \\
\hline
\end{tabular}




\begin{tabular}{|c|c|c|c|}
\hline $\mathrm{H}$ & -0.39662600 & 5.39081200 & 3.64045100 \\
\hline $\mathrm{C}$ & 1.66885500 & 3.72334300 & 4.39811200 \\
\hline 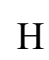 & 1.24121300 & 3.14903000 & 5.23023300 \\
\hline 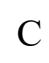 & 3.10751600 & 3.20108200 & 4.13260400 \\
\hline & 3.10793700 & 2.30669600 & 3.51260900 \\
\hline & 3.55249100 & 2.91510500 & 5.09492500 \\
\hline & 3.97402300 & 4.28327000 & 3.48189800 \\
\hline & 3.47976300 & 4.63930200 & 2.56174300 \\
\hline & 4.92735200 & 3.85758600 & 3.15140500 \\
\hline & 4.19021300 & 5.48171500 & 4.43559300 \\
\hline & 4.25544500 & 6.40711500 & 3.85101300 \\
\hline & 5.14514700 & 5.37347800 & 4.96454300 \\
\hline & 3.04110300 & 5.59597600 & 5.45970200 \\
\hline & 2.98619100 & 6.61667500 & 5.85545500 \\
\hline & 3.22867300 & 4.94089400 & 6.32100700 \\
\hline & 1.70222900 & 5.22460900 & 4.80547900 \\
\hline & 0.87057400 & 5.44396200 & 5.48414400 \\
\hline & 1.57881800 & 5.86641400 & 3.92624700 \\
\hline & 1.04181200 & 0.61746300 & 3.80006300 \\
\hline & 1.58100600 & 1.06760900 & 4.63666200 \\
\hline & 1.78104900 & 0.39881200 & 3.02496700 \\
\hline & 0.36690600 & -0.68606200 & 4.29715800 \\
\hline & 0.03126400 & -0.55446000 & 5.33413400 \\
\hline $\mathrm{H}$ & 1.12441300 & -1.47921500 & 4.31350700 \\
\hline & -0.82754700 & -1.11482000 & 3.41268600 \\
\hline & -1.76190500 & -0.69358100 & 3.81153800 \\
\hline & -0.94819000 & -2.20400800 & 3.43781400 \\
\hline $\mathrm{C}$ & -0.62823200 & -0.61255000 & 1.98036400 \\
\hline $\mathrm{H}$ & -1.40619800 & -0.99384000 & 1.30968700 \\
\hline $\mathrm{H}$ & 0.32482500 & -0.98821400 & 1.58845800 \\
\hline $\mathrm{C}$ & -0.62457600 & 0.93156600 & 1.93132200 \\
\hline & -0.04901600 & 1.23770700 & 1.05590200 \\
\hline & -1.65344900 & 1.28970100 & 1.80336300 \\
\hline & -0.02780200 & 1.56504100 & 3.21612000 \\
\hline & -0.81289000 & 1.64363100 & 3.97611500 \\
\hline & -1.18448800 & 3.37141600 & -0.62111100 \\
\hline 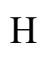 & -1.60731500 & 4.35188000 & -0.39343000 \\
\hline & -1.56376900 & 2.54690700 & -0.01805500 \\
\hline & -1.46551500 & 3.06971000 & -2.11204400 \\
\hline & -2.58602300 & 2.89153700 & -2.78876800 \\
\hline
\end{tabular}




$\begin{array}{llll}\mathrm{H} & -3.55743200 & 2.93352400 & -2.29944700 \\ \mathrm{H} & -2.56744800 & 2.69972200 & -3.85717500\end{array}$

ts-vi

Thermal correction to Gibbs free energy $=0.963675$

Sum of electronic and thermal free energies $=-4279.125819$

E_sol $=-4280.0522742$

\begin{tabular}{|c|c|c|c|}
\hline $\mathrm{Ru}$ & -1.52992200 & 0.95924800 & -0.28117000 \\
\hline $\mathrm{Cl}$ & -1.95654300 & 1.59360800 & -2.51997400 \\
\hline $\mathrm{Cl}$ & -1.22576900 & 1.27298900 & 2.05976800 \\
\hline $\mathrm{C}$ & -0.36178400 & -0.36988900 & -0.47335400 \\
\hline $\mathrm{Ru}$ & 1.36689700 & -0.89729500 & -0.38147600 \\
\hline $\mathrm{Cl}$ & 1.11947700 & -1.76640500 & 1.85012900 \\
\hline $\mathrm{Cl}$ & 1.77434900 & -0.20315300 & -2.67125600 \\
\hline $\mathrm{P}$ & 3.37569800 & 0.36715300 & 0.29822800 \\
\hline $\mathrm{C}$ & 5.04270500 & 0.89769600 & 2.65567100 \\
\hline $\mathrm{H}$ & 4.88614400 & 0.82216800 & 3.74182000 \\
\hline $\mathrm{H}$ & 4.82871600 & 1.93926500 & 2.39844500 \\
\hline $\mathrm{C}$ & 6.52580300 & 0.59903800 & 2.38212300 \\
\hline $\mathrm{H}$ & 7.13847900 & 1.27705400 & 2.98997800 \\
\hline $\mathrm{H}$ & 6.77895000 & 0.81902300 & 1.33631300 \\
\hline $\mathrm{C}$ & 6.87202200 & -0.86117200 & 2.69342100 \\
\hline $\mathrm{H}$ & 6.72756400 & -1.04486000 & 3.76844100 \\
\hline $\mathrm{H}$ & 7.92929800 & -1.05890400 & 2.47704600 \\
\hline $\mathrm{C}$ & 5.97198100 & -1.80714700 & 1.88999500 \\
\hline $\mathrm{H}$ & 6.20047500 & -2.85187900 & 2.13622100 \\
\hline $\mathrm{H}$ & 6.19720900 & -1.68867200 & 0.82508400 \\
\hline $\mathrm{C}$ & 4.48239200 & -1.55106800 & 2.16711900 \\
\hline $\mathrm{H}$ & 3.85183300 & -2.21707800 & 1.57207900 \\
\hline $\mathrm{H}$ & 4.28219500 & -1.82586100 & 3.21200400 \\
\hline $\mathrm{C}$ & 4.01712200 & -0.07739900 & 2.00942700 \\
\hline $\mathrm{H}$ & 3.08422000 & 0.00120800 & 2.57601900 \\
\hline $\mathrm{C}$ & 2.95523900 & 2.19882900 & 0.43474400 \\
\hline $\mathrm{H}$ & 3.93293100 & 2.69224600 & 0.51636400 \\
\hline $\mathrm{C}$ & 2.22130900 & 2.78512600 & -0.81446200 \\
\hline $\mathrm{H}$ & 2.29495700 & 2.12941400 & -1.68003800 \\
\hline $\mathrm{H}$ & 2.71134300 & 3.72859600 & -1.08863100 \\
\hline $\mathrm{C}$ & 0.74156200 & 3.07688200 & -0.55117700 \\
\hline $\mathrm{H}$ & 0.27083200 & 2.10814000 & -0.26805000 \\
\hline H & 0.24559300 & 3.38607500 & -1.47484400 \\
\hline
\end{tabular}




\begin{tabular}{|c|c|c|c|}
\hline $\mathrm{C}$ & 0.52409500 & 4.08247400 & 0.58796200 \\
\hline$H$ & -0.47955900 & 3.94623200 & 1.00236400 \\
\hline H & 0.56976100 & 5.10158900 & 0.18295600 \\
\hline $\mathrm{C}$ & 1.58263000 & 3.91032600 & 1.70250300 \\
\hline $\mathrm{H}$ & 1.14572100 & 4.14675600 & 2.67881800 \\
\hline $\mathrm{H}$ & 2.41013900 & 4.61780800 & 1.55072000 \\
\hline $\mathrm{C}$ & 2.15477100 & 2.48484200 & 1.73134000 \\
\hline $\mathrm{H}$ & 2.79429500 & 2.36954200 & 2.61017600 \\
\hline $\mathrm{H}$ & 1.33433500 & 1.76971200 & 1.84895400 \\
\hline $\mathrm{C}$ & 4.95880600 & 1.22596500 & -1.99585200 \\
\hline $\mathrm{H}$ & 5.32141500 & 2.16710400 & -1.56315500 \\
\hline $\mathrm{H}$ & 3.98745700 & 1.42409700 & -2.44595400 \\
\hline $\mathrm{C}$ & 5.92044600 & 0.74225300 & -3.11156600 \\
\hline $\mathrm{H}$ & 6.38643600 & 1.60829100 & -3.59576100 \\
\hline $\mathrm{H}$ & 5.33925500 & 0.22771300 & -3.88778500 \\
\hline $\mathrm{C}$ & 6.98197300 & -0.22225700 & -2.57012600 \\
\hline $\mathrm{H}$ & 7.53718600 & 0.27030000 & -1.75906800 \\
\hline $\mathrm{H}$ & 7.72118900 & -0.46256700 & -3.34308500 \\
\hline $\mathrm{C}$ & 6.31746500 & -1.51704600 & -2.04710200 \\
\hline $\mathrm{H}$ & 6.96252700 & -1.97953900 & -1.28943800 \\
\hline $\mathrm{H}$ & 6.22707800 & -2.24391700 & -2.86369900 \\
\hline $\mathrm{C}$ & 4.90698200 & -1.25231300 & -1.46464900 \\
\hline $\mathrm{H}$ & 4.14722000 & -1.33181400 & -2.24582400 \\
\hline $\mathrm{H}$ & 4.66112300 & -2.01711100 & -0.71912400 \\
\hline $\mathrm{C}$ & 4.83258800 & 0.17119900 & -0.86757300 \\
\hline $\mathrm{H}$ & 5.69129400 & 0.29597700 & -0.20191100 \\
\hline$P$ & -3.57996600 & -0.07295900 & -0.03073000 \\
\hline $\mathrm{C}$ & -5.71355000 & -1.50476600 & -1.39874100 \\
\hline $\mathrm{H}$ & -6.24611200 & -1.36686500 & -2.35082000 \\
\hline $\mathrm{H}$ & -6.30605000 & -0.97045500 & -0.64605300 \\
\hline $\mathrm{C}$ & -5.69928200 & -3.01273300 & -1.08468900 \\
\hline $\mathrm{H}$ & -6.72913800 & -3.39012000 & -1.11464600 \\
\hline $\mathrm{H}$ & -5.33809100 & -3.19179200 & -0.06395600 \\
\hline $\mathrm{C}$ & -4.81017800 & -3.79746300 & -2.05852200 \\
\hline $\mathrm{H}$ & -5.22948700 & -3.73298100 & -3.07334900 \\
\hline $\mathrm{H}$ & -4.79966500 & -4.85990700 & -1.78627400 \\
\hline $\mathrm{C}$ & -3.38690000 & -3.22448900 & -2.06192800 \\
\hline $\mathrm{H}$ & -2.74561400 & -3.78310300 & -2.75518200 \\
\hline $\mathrm{H}$ & -2.95419800 & -3.35333400 & -1.06249700 \\
\hline $\mathrm{C}$ & -3.40485500 & -1.74422300 & -2.4555750 \\
\hline
\end{tabular}




\begin{tabular}{|c|c|c|c|}
\hline $\mathrm{H}$ & -2.41026900 & -1.30167100 & -2.50232700 \\
\hline $\mathrm{H}$ & -3.80061400 & -1.67818200 & -3.47983300 \\
\hline $\mathrm{C}$ & -4.31454600 & -0.85818800 & -1.58416100 \\
\hline H & -4.44303400 & 0.05277200 & -2.17111200 \\
\hline $\mathrm{C}$ & -4.84771300 & 1.32285300 & 0.25762600 \\
\hline $\mathrm{H}$ & -5.70845700 & 0.79589800 & 0.68884600 \\
\hline $\mathrm{C}$ & -4.35950900 & 2.38041500 & 1.28312500 \\
\hline & -3.72538100 & 1.94196900 & 2.05079000 \\
\hline & -5.23781600 & 2.79049400 & 1.79898900 \\
\hline $\mathrm{C}$ & -3.61643300 & 3.51969100 & 0.58007200 \\
\hline $\mathrm{H}$ & -2.79413200 & 3.09849000 & -0.02701900 \\
\hline $\mathrm{H}$ & -3.12580800 & 4.16619600 & 1.31498000 \\
\hline $\mathrm{C}$ & -4.56125200 & 4.32299500 & -0.34404300 \\
\hline $\mathrm{H}$ & -4.00321900 & 4.67895300 & -1.21820900 \\
\hline $\mathrm{H}$ & -4.93018300 & 5.21310800 & 0.18024100 \\
\hline $\mathrm{C}$ & -5.75504800 & 3.46117600 & -0.80688900 \\
\hline $\mathrm{H}$ & -6.18849500 & 3.87607900 & -1.72412600 \\
\hline $\mathrm{H}$ & -6.55413300 & 3.47638800 & -0.05382600 \\
\hline $\mathrm{C}$ & -5.30350500 & 2.01532800 & -1.06025200 \\
\hline $\mathrm{H}$ & -6.11015000 & 1.43724400 & -1.52396500 \\
\hline $\mathrm{H}$ & -4.48003900 & 2.05436700 & -1.78163600 \\
\hline$c$ & -4.21638900 & -0.60028800 & 2.77203700 \\
\hline $\mathrm{H}$ & -5.00532100 & 0.14663300 & 2.65467200 \\
\hline $\mathrm{H}$ & -3.34819900 & -0.08466100 & 3.19035600 \\
\hline $\mathrm{C}$ & -4.71987500 & -1.69769100 & 3.74277200 \\
\hline $\mathrm{H}$ & -5.79227100 & -1.86831300 & 3.58094800 \\
\hline $\mathrm{H}$ & -4.61603600 & -1.32101700 & 4.76770200 \\
\hline$C$ & -3.94933300 & -3.03074500 & 3.58805100 \\
\hline $\mathrm{H}$ & -4.47538700 & -3.68931000 & 2.88156800 \\
\hline $\mathrm{H}$ & -3.92138000 & -3.56732600 & 4.54342400 \\
\hline $\mathrm{C}$ & -2.53715100 & -2.76566100 & 3.05962200 \\
\hline $\mathrm{H}$ & -1.93556100 & -3.68094900 & 3.04215100 \\
\hline $\mathrm{H}$ & -2.01622400 & -2.07013700 & 3.72877300 \\
\hline $\mathrm{C}$ & -2.58563500 & -2.16421600 & 1.63867100 \\
\hline $\mathrm{H}$ & -1.65939500 & -1.61063100 & 1.47737300 \\
\hline $\mathrm{H}$ & -2.62332800 & -2.98063200 & 0.90718500 \\
\hline $\mathrm{C}$ & -3.81616700 & -1.24152600 & 1.42601300 \\
\hline $\mathrm{H}$ & -4.67064300 & -1.85054800 & 1.11134500 \\
\hline$C$ & -0.28426400 & -2.16553300 & -1.17642000 \\
\hline & -0.60374400 & -1.91490200 & -2.18486700 \\
\hline
\end{tabular}




$\begin{array}{lrrr}\mathrm{H} & -1.03288100 & -2.56843800 & -0.50286700 \\ \mathrm{C} & 1.03507700 & -2.72982700 & -1.07048300 \\ \mathrm{C} & 1.75022600 & -3.82000500 & -1.27669800 \\ \mathrm{H} & 1.29497700 & -4.71924700 & -1.68814600 \\ \mathrm{H} & 2.81142300 & -3.85390300 & -1.04842300\end{array}$

\section{Ru-carbide}

Thermal correction to Gibbs free energy $=0.907307$

Sum of electronic and thermal free energies $=-4162.547775$

E_sol $=-4163.4297531$

$\begin{array}{lccc}\mathrm{Cl} & 2.06248500 & 0.16060500 & 0.72805400 \\ \mathrm{Cl} & -0.01168800 & 0.55959800 & -3.22236000 \\ \mathrm{Ru} & 0.43122600 & 0.58123400 & -0.92804600 \\ \mathrm{C} & -0.43363200 & 2.01298700 & -0.37419200 \\ \mathrm{Ru} & -1.21822000 & 3.57786000 & -0.18931800 \\ \mathrm{Cl} & -2.60034800 & 3.39812300 & 1.70996700 \\ \mathrm{Cl} & -0.93477500 & 4.47201900 & -2.34028500 \\ \mathrm{P} & -1.12274900 & -0.97808300 & -0.32283100 \\ \mathrm{C} & -1.25082900 & -3.84404500 & -0.89898600 \\ \mathrm{H} & -1.12509300 & -4.49740200 & -1.77494500 \\ \mathrm{H} & -2.33174300 & -3.69719100 & -0.78838500 \\ \mathrm{C} & -0.67251700 & -4.57366300 & 0.32623600 \\ \mathrm{H} & -1.16386100 & -5.54980200 & 0.42492300 \\ \mathrm{H} & -0.90290100 & -4.02160600 & 1.24312800 \\ \mathrm{C} & 0.84973100 & -4.75029100 & 0.22312800 \\ \mathrm{H} & 1.07998500 & -5.42831100 & -0.61205000 \\ \mathrm{H} & 1.23428200 & -5.22873200 & 1.13219700 \\ \mathrm{C} & 1.55173000 & -3.40550100 & -0.01482800 \\ \mathrm{H} & 2.63191100 & -3.55314000 & -0.13135100 \\ \mathrm{H} & 1.42878300 & -2.74712700 & 0.85376300 \\ \mathrm{C} & 0.99610900 & -2.72146200 & -1.26808700 \\ \mathrm{H} & 1.52686700 & -1.77963000 & -1.45505200 \\ \mathrm{H} & 1.21022200 & -3.35572900 & -2.14083700 \\ \mathrm{C} & -0.53767900 & -2.51391500 & -1.24212800 \\ \mathrm{H} & -0.82356900 & -2.22422000 & -2.25931500 \\ \mathrm{C} & -2.89735800 & -0.79125300 & -0.89207300 \\ \mathrm{H} & -3.40135400 & -1.61908300 & -0.38034800 \\ \mathrm{C} & -3.54774100 & 0.53455500 & -0.40146000 \\ \mathrm{H} & -3.02754700 & 0.95204500 & 0.46130000 \\ \mathrm{H} & -4.56502500 & 0.31250600 & -0.05292800\end{array}$




\begin{tabular}{|c|c|c|c|}
\hline $\mathrm{C}$ & -3.62343700 & 1.56953500 & -1.53056200 \\
\hline $\mathrm{H}$ & -2.61790900 & 1.74381200 & -1.93249000 \\
\hline $\mathrm{H}$ & -3.98387700 & 2.52611700 & -1.13257300 \\
\hline $\mathrm{C}$ & -4.54163500 & 1.08491100 & -2.67463200 \\
\hline $\mathrm{H}$ & -4.23057500 & 1.56283000 & -3.61063800 \\
\hline $\mathrm{H}$ & -5.57618900 & 1.39856200 & -2.48763600 \\
\hline $\mathrm{C}$ & -4.48894100 & -0.45378100 & -2.82354700 \\
\hline $\mathrm{H}$ & -4.71202000 & -0.74536000 & -3.85620000 \\
\hline $\mathrm{H}$ & -5.26218300 & -0.92088900 & -2.19744500 \\
\hline $\mathrm{C}$ & -3.11419400 & -1.00255800 & -2.41226800 \\
\hline $\mathrm{H}$ & -3.04758100 & -2.07038000 & -2.65051800 \\
\hline $\mathrm{H}$ & -2.33895000 & -0.49478100 & -2.9925750 \\
\hline $\mathrm{C}$ & -2.51353600 & -2.01231500 & 1.99080500 \\
\hline $\mathrm{H}$ & -2.76283300 & -2.86471200 & 1.35191000 \\
\hline $\mathrm{H}$ & -3.36007500 & -1.31854200 & 1.92633200 \\
\hline $\mathrm{C}$ & -2.34303000 & -2.49933100 & 3.44872700 \\
\hline $\mathrm{H}$ & -1.84235600 & -3.47596000 & 3.45182400 \\
\hline $\mathrm{H}$ & -3.33917100 & -2.65922500 & 3.87934800 \\
\hline $\mathrm{C}$ & -1.54061500 & -1.49589900 & 4.31291100 \\
\hline $\mathrm{H}$ & -0.47545700 & -1.76589800 & 4.30776300 \\
\hline $\mathrm{H}$ & -1.86737100 & -1.54937600 & 5.35764200 \\
\hline $\mathrm{C}$ & -1.68783000 & -0.07557700 & 3.75708600 \\
\hline $\mathrm{H}$ & -1.21261300 & 0.65878700 & 4.41791200 \\
\hline $\mathrm{H}$ & -2.75091600 & 0.19601000 & 3.71683900 \\
\hline $\mathrm{C}$ & -1.05680000 & 0.02894400 & 2.35333700 \\
\hline $\mathrm{H}$ & -1.48466600 & 0.89011300 & 1.83873400 \\
\hline $\mathrm{H}$ & 0.01285700 & 0.22381600 & 2.43850900 \\
\hline $\mathrm{C}$ & -1.23783700 & -1.27647000 & 1.52700000 \\
\hline $\mathrm{H}$ & -0.37479000 & -1.91995000 & 1.72868800 \\
\hline$P$ & 0.65302700 & 4.57582600 & 0.67770200 \\
\hline $\mathrm{C}$ & 3.56943800 & 4.59655000 & 0.41053400 \\
\hline $\mathrm{H}$ & 4.36186300 & 3.83442500 & 0.39636700 \\
\hline $\mathrm{H}$ & 3.52960400 & 4.96007200 & 1.44364800 \\
\hline $\mathrm{C}$ & 3.98503600 & 5.72920900 & -0.54417800 \\
\hline $\mathrm{H}$ & 4.95969800 & 6.12156800 & -0.22685500 \\
\hline $\mathrm{H}$ & 3.28011200 & 6.56428900 & -0.47726300 \\
\hline $\mathrm{C}$ & 4.04006800 & 5.25884200 & -2.00297800 \\
\hline $\mathrm{H}$ & 4.83255100 & 4.50407200 & -2.11520200 \\
\hline $\mathrm{H}$ & 4.30552800 & 6.09676800 & -2.65960100 \\
\hline $\mathrm{C}$ & 2.69700300 & 4.64783200 & -2.4181580 \\
\hline
\end{tabular}




\begin{tabular}{|c|c|c|c|}
\hline $\mathrm{H}$ & 2.74026700 & 4.27725300 & -3.44896900 \\
\hline $\mathrm{H}$ & 1.90863100 & 5.41085900 & -2.40232700 \\
\hline $\mathrm{C}$ & 2.31115900 & 3.49067200 & -1.49010800 \\
\hline $\mathrm{H}$ & 1.36795900 & 3.06106500 & -1.83320900 \\
\hline $\mathrm{H}$ & 3.07133300 & 2.70066500 & -1.58373700 \\
\hline $\mathrm{C}$ & 2.26413800 & 3.85613800 & 0.01381800 \\
\hline & 2.26022900 & 2.90416000 & 0.55527300 \\
\hline & 0.85466700 & 4.38853100 & 2.52929300 \\
\hline & 1.86058200 & 4.78226100 & 2.72988500 \\
\hline $\mathrm{C}$ & -0.17025100 & 5.20548600 & 3.37656300 \\
\hline $\mathrm{H}$ & -1.09697300 & 5.37027100 & 2.82501800 \\
\hline & 0.25769500 & 6.18889200 & 3.59750200 \\
\hline & -0.51334300 & 4.50326200 & 4.70637200 \\
\hline & -1.27481300 & 3.73420200 & 4.52794400 \\
\hline & -0.96188400 & 5.23342700 & 5.39071900 \\
\hline & 0.73742000 & 3.86845000 & 5.32510200 \\
\hline $\mathrm{H}$ & 0.52461200 & 3.48133300 & 6.32830200 \\
\hline $\mathrm{H}$ & 1.49931800 & 4.65059000 & 5.45338600 \\
\hline & 1.28479400 & 2.74231600 & 4.41249400 \\
\hline & 0.94253700 & 1.76261100 & 4.76888500 \\
\hline & 2.38101100 & 2.72025100 & 4.45959300 \\
\hline & 0.83023000 & 2.90674100 & 2.94872300 \\
\hline $\mathrm{H}$ & 1.45275100 & 2.28108200 & 2.30617900 \\
\hline$H$ & -0.19231300 & 2.54567400 & 2.82922600 \\
\hline $\mathrm{C}$ & 1.39077000 & 7.33428900 & 1.18841300 \\
\hline $\mathrm{H}$ & 2.38247700 & 6.92453400 & 1.39274000 \\
\hline $\mathrm{H}$ & 0.90081900 & 7.44487800 & 2.15953900 \\
\hline $\mathrm{C}$ & 1.51846900 & 8.72029600 & 0.51522800 \\
\hline $\mathrm{H}$ & 2.35280400 & 8.71112200 & -0.19730000 \\
\hline & 1.77286800 & 9.46020000 & 1.28425300 \\
\hline & 0.21436700 & 9.13054400 & -0.21256700 \\
\hline & 0.25789300 & 8.81988000 & -1.26518900 \\
\hline & 0.10958700 & 10.22132500 & -0.21791700 \\
\hline & -1.00189800 & 8.46663200 & 0.44503400 \\
\hline & -1.93536400 & 8.83759700 & 0.00703700 \\
\hline $\mathrm{H}$ & -1.03055700 & 8.73799100 & 1.50886800 \\
\hline & -0.94017100 & 6.92816200 & 0.28762800 \\
\hline & -1.53516500 & 6.46293400 & 1.08109300 \\
\hline & -1.39644800 & 6.64133000 & -0.66433500 \\
\hline & 0.53047000 & 6.40778100 & 0.30237700 \\
\hline
\end{tabular}




\begin{tabular}{|c|c|c|c|}
\hline \multicolumn{4}{|c|}{ Allen } \\
\hline \multicolumn{4}{|c|}{ Thermal correction to Gibbs free energy $=0.031344$} \\
\hline \multicolumn{4}{|c|}{ Sum of electronic and thermal free energies $=-1046.7$} \\
\hline \multicolumn{4}{|c|}{ E_sol $=-116.6476259$} \\
\hline $\mathrm{C}$ & -0.98170400 & -2.37119800 & -0.00049800 \\
\hline $\mathrm{C}$ & 0.32513300 & -2.37218200 & 0.00026600 \\
\hline $\mathrm{H}$ & 0.89518200 & -1.44575900 & -0.03064800 \\
\hline $\mathrm{H}$ & 0.89382300 & -3.29940800 & 0.03204900 \\
\hline $\mathrm{C}$ & -2.28854000 & -2.37080000 & -0.00121000 \\
\hline $\mathrm{H}$ & -2.85837800 & -2.33924700 & 0.92531800 \\
\hline $\mathrm{H}$ & -2.85739600 & -2.40203000 & -0.9283490 \\
\hline
\end{tabular}

\section{2-Isopropoxystyrene}

Thermal correction to Gibbs free energy $=0.185293$

Sum of electronic and thermal free energies $=-502.641071$

E_sol $=-502.7804248$

$\begin{array}{lrrr}\mathrm{C} & -3.41129100 & -1.10614700 & -0.49030500 \\ \mathrm{C} & -2.10376800 & -1.03084600 & -0.96524400 \\ \mathrm{C} & -1.21141400 & -0.04128900 & -0.52786200 \\ \mathrm{C} & -1.68367700 & 0.90830600 & 0.41305000 \\ \mathrm{C} & -2.99944300 & 0.84120600 & 0.88720600 \\ \mathrm{C} & -3.85516900 & -0.16601500 & 0.43859400 \\ \mathrm{H} & -4.07980700 & -1.88149900 & -0.85295800 \\ \mathrm{H} & -1.76521300 & -1.74208500 & -1.71313300 \\ \mathrm{H} & -3.36316600 & 1.56415100 & 1.60758600 \\ \mathrm{H} & -4.87392900 & -0.20389200 & 0.81514100 \\ \mathrm{C} & 0.17162800 & 0.04903000 & -1.02350500 \\ \mathrm{H} & 0.64567300 & 1.01854200 & -0.89550500 \\ \mathrm{C} & 0.87590900 & -0.94172800 & -1.58597400 \\ \mathrm{H} & 0.48359200 & -1.94908100 & -1.70184300 \\ \mathrm{H} & 1.88814300 & -0.77503100 & -1.94240400 \\ \mathrm{O} & -0.76665500 & 1.83599500 & 0.82230300 \\ \mathrm{C} & -1.15439700 & 2.96661400 & 1.62186900 \\ \mathrm{H} & -1.77853700 & 2.62022000 & 2.45741400 \\ \mathrm{C} & 0.14826000 & 3.52584600 & 2.18062200 \\ \mathrm{H} & 0.80269600 & 3.84576400 & 1.36254000 \\ \mathrm{H} & -0.05207000 & 4.38833900 & 2.82512200 \\ \mathrm{H} & 0.67281700 & 2.76323100 & 2.76393300 \\ \mathrm{C} & -1.91741800 & 3.98714000 & 0.77775500 \\ \mathrm{H} & -1.27867700 & 4.34356000 & -0.03769300 \\ \mathrm{H} & -2.81901600 & 3.55014400 & 0.33918700\end{array}$


$\mathrm{PCy}_{3}$

Thermal correction to Gibbs free energy $=0.44006$

Sum of electronic and thermal free energies $=-1046.767468$

E_sol $=-1047.1709269$

$\mathrm{P} \quad \quad-0.82547600 \quad 5.25921100 \quad-1.34308900$

$\begin{array}{llll}\text { C } & -0.77075900 & 2.27389900 & -1.13487000\end{array}$

$\mathrm{H} \quad-1.26845400 \quad 1.50616100 \quad-0.52145100$

$\mathrm{H} \quad 0.21556600 \quad 2.42823700 \quad-0.68049700$

C $\quad-0.62005500 \quad 1.70624100 \quad-2.55672500$

$\mathrm{H} \quad-0.09500600 \quad 0.74333000 \quad-2.50812700$

$\mathrm{H} \quad 0.00650500 \quad 2.36531000 \quad-3.16628500$

C $\quad-1.98047900 \quad 1.53239900 \quad-3.24733400$

$\mathrm{H} \quad-2.56337300 \quad 0.76843400 \quad-2.71102700$

$\mathrm{H} \quad-1.84184900 \quad 1.16094200 \quad-4.27073900$

C $\quad-2.77287200 \quad 2.84749700 \quad-3.25366100$

$\mathrm{H} \quad \begin{array}{llll}\mathrm{H} & -3.75761100 & 2.69461500 & -3.71372400\end{array}$

$\mathrm{H} \quad-2.25494500 \quad 3.58794500 \quad-3.87812300$

C $\quad-2.95100200 \quad 3.38817000 \quad-1.82742000$

$\mathrm{H} \quad-3.50550100 \quad 4.33424900 \quad-1.84110000$

$\mathrm{H} \quad \begin{array}{llll}\mathrm{H} & -3.57379300 & 2.66882100 & -1.27322300\end{array}$

C $\quad-1.62368200 \quad 3.56209700 \quad-1.04788300$

$\mathrm{H} \quad-1.91226400 \quad 3.68262800 \quad 0.00453200$

C $\quad 0.51257600 \quad 5.24896500 \quad-0.00630500$

$\mathrm{H} \quad 1.21163300 \quad 4.42805400 \quad-0.20347400$

C $\quad \begin{array}{llll}\text { C } & 1.31953100 & 6.58117000 & -0.02966900\end{array}$

$\mathrm{H} \quad \begin{array}{llll}\mathrm{H} & 1.38443600 & 6.98779500 & -1.04356700\end{array}$

$\begin{array}{llll}\mathrm{H} & 2.35555300 & 6.37862400 & 0.27662700\end{array}$

$\begin{array}{llll}\text { C } & 0.71059800 & 7.62088700 & 0.91936900\end{array}$

$\mathrm{H} \quad-0.34141800 \quad 7.76739400 \quad 0.64298700$

$\begin{array}{llll}\mathrm{H} & 1.20169500 & 8.59290200 & 0.79184900\end{array}$

$\begin{array}{llll}\text { C } & 0.81509400 & 7.15412600 & 2.39019300\end{array}$

$\begin{array}{llll}\mathrm{H} & -0.00208100 & 7.59777200 & 2.97281300\end{array}$

$\mathrm{H} \quad 1.74823700 \quad 7.52110300 \quad 2.83618100$

$\begin{array}{llll}\mathrm{C} & 0.76393500 & 5.61300100 & 2.50348700\end{array}$

$\begin{array}{llll}\mathrm{H} & 0.39084700 & 5.31520700 & 3.49084900\end{array}$

$\mathrm{H} \quad 1.77661500 \quad 5.19554700 \quad 2.41987700$

$\begin{array}{llll}\text { C } & -0.11111000 & 5.01001800 & 1.39571000\end{array}$

$\begin{array}{llll}\mathrm{H} & -0.24893600 & 3.93544500 & 1.56153100\end{array}$

$\mathrm{H} \quad-1.11139800 \quad 5.46383600 \quad 1.45467500$

C $\quad \begin{array}{llll}\text { C } & 1.60544600 & 4.52180000 & -2.86914300\end{array}$

$\mathrm{H} \quad 1.62335700 \quad 3.60714400 \quad-2.26852100$ 


$\begin{array}{llll}\mathrm{H} & 2.27261600 & 5.22933600 & -2.36234500 \\ \mathrm{C} & 2.14409300 & 4.21890100 & -4.28907800 \\ \mathrm{H} & 1.84070000 & 3.20792300 & -4.59044800 \\ \mathrm{H} & 3.24134500 & 4.21665200 & -4.26209800 \\ \mathrm{C} & 1.64272800 & 5.24128300 & -5.33731700 \\ \mathrm{H} & 0.70551800 & 4.88639800 & -5.78800100 \\ \mathrm{H} & 2.36367200 & 5.32836800 & -6.15881300 \\ \mathrm{C} & 1.38794300 & 6.60414900 & -4.68267700 \\ \mathrm{H} & 1.15187000 & 7.36475300 & -5.43665000 \\ \mathrm{H} & 2.31071000 & 6.93852900 & -4.18851700 \\ \mathrm{C} & 0.24097500 & 6.51177700 & -3.64922500 \\ \mathrm{H} & 0.34696400 & 7.31993000 & -2.91556100 \\ \mathrm{H} & -0.72355200 & 6.68535000 & -4.14285000 \\ \mathrm{C} & 0.19016500 & 5.12547500 & -2.94228500 \\ \mathrm{H} & -0.40794200 & 4.46416100 & -3.57813200\end{array}$

\section{Ru-vinyl-1}

Thermal correction to Gibbs free energy $=1.100738$

Sum of electronic and thermal free energies $=-3596.922946$

E_sol $=-3597.9448691$

$\begin{array}{llll}\mathrm{Ru} & 0.12308300 & 0.75954200 & -1.83352200 \\ \mathrm{Cl} & 2.32518300 & 1.65887900 & -1.85653200 \\ \mathrm{Cl} & -1.16544600 & -1.25057800 & -1.61116500 \\ \mathrm{P} & -0.02101400 & 0.49195700 & -4.28388700 \\ \mathrm{C} & -2.00470300 & -0.42312700 & -6.27113600 \\ \mathrm{H} & -2.75586700 & -1.22555700 & -6.32704300 \\ \mathrm{H} & -1.12220600 & -0.80647000 & -6.79655900 \\ \mathrm{C} & -2.57627700 & 0.80311800 & -7.00607600 \\ \mathrm{H} & -2.85302400 & 0.51139000 & -8.02761600 \\ \mathrm{H} & -1.81158200 & 1.57817000 & -7.10658600 \\ \mathrm{C} & -3.78756400 & 1.40380300 & -6.27819200 \\ \mathrm{H} & -4.62000700 & 0.68475100 & -6.30177000 \\ \mathrm{H} & -4.13321000 & 2.30354500 & -6.80300100 \\ \mathrm{C} & -3.44827600 & 1.72268800 & -4.81551200 \\ \mathrm{H} & -4.33441100 & 2.10790600 & -4.29470700 \\ \mathrm{H} & -2.69622900 & 2.52058700 & -4.76351400 \\ \mathrm{C} & -2.93812300 & 0.46965400 & -4.09541400 \\ \mathrm{H} & -2.73416800 & 0.67155900 & -3.04434000 \\ \mathrm{H} & -3.74505000 & -0.27896200 & -4.10497000 \\ \mathrm{C} & -1.71521700 & -0.19952700 & -4.76560200 \\ \mathrm{H} & -1.63482200 & -1.18417200 & -4.29738900\end{array}$




\begin{tabular}{|c|c|c|c|}
\hline $\mathrm{C}$ & 1.14142900 & -0.81690600 & -4.98149000 \\
\hline $\mathrm{H}$ & 1.07680900 & -0.71902500 & -6.07004900 \\
\hline $\mathrm{C}$ & 2.61848300 & -0.55716200 & -4.56280300 \\
\hline $\mathrm{H}$ & 2.79210100 & 0.49631700 & -4.33608000 \\
\hline $\mathrm{H}$ & 3.27130100 & -0.80562400 & -5.41093700 \\
\hline $\mathrm{C}$ & 3.01280300 & -1.42108200 & -3.35919600 \\
\hline $\mathrm{H}$ & 2.31697600 & -1.21885900 & -2.53717600 \\
\hline $\mathrm{H}$ & 3.99930000 & -1.12493600 & -2.98548400 \\
\hline $\mathrm{C}$ & 2.98101600 & -2.92319500 & -3.71445000 \\
\hline $\mathrm{H}$ & 2.79391600 & -3.50725700 & -2.80410700 \\
\hline $\mathrm{H}$ & 3.95946700 & -3.24348900 & -4.09458400 \\
\hline $\mathrm{C}$ & 1.89157200 & -3.22892600 & -4.76836900 \\
\hline $\mathrm{H}$ & 1.54715000 & -4.26554700 & -4.67434900 \\
\hline $\mathrm{H}$ & 2.30867600 & -3.13357100 & -5.78062100 \\
\hline $\mathrm{C}$ & 0.70646400 & -2.26356800 & -4.62300700 \\
\hline $\mathrm{H}$ & -0.11802000 & -2.57355600 & -5.27462400 \\
\hline $\mathrm{H}$ & 0.32919700 & -2.31654900 & -3.59613300 \\
\hline $\mathrm{C}$ & 0.77739900 & 1.75513800 & -6.78507100 \\
\hline $\mathrm{H}$ & 0.25500700 & 0.92037900 & -7.25893800 \\
\hline $\mathrm{H}$ & 1.83612800 & 1.46950300 & -6.74419700 \\
\hline $\mathrm{C}$ & 0.60701400 & 3.02567800 & -7.65664200 \\
\hline $\mathrm{H}$ & -0.38175700 & 3.01975000 & -8.13238100 \\
\hline $\mathrm{H}$ & 1.34240100 & 2.99495500 & -8.47062900 \\
\hline $\mathrm{C}$ & 0.78298800 & 4.32385700 & -6.83692000 \\
\hline $\mathrm{H}$ & -0.17966800 & 4.63074300 & -6.40582900 \\
\hline $\mathrm{H}$ & 1.10334100 & 5.14477100 & -7.48963200 \\
\hline $\mathrm{C}$ & 1.78582600 & 4.08805900 & -5.70388700 \\
\hline $\mathrm{H}$ & 2.02619700 & 5.02132200 & -5.18103900 \\
\hline $\mathrm{H}$ & 2.73108700 & 3.73785300 & -6.14160900 \\
\hline $\mathrm{C}$ & 1.25623800 & 3.04904200 & -4.68471100 \\
\hline $\mathrm{H}$ & 2.10633900 & 2.53934700 & -4.23348400 \\
\hline $\mathrm{H}$ & 0.74126900 & 3.55204000 & -3.86280500 \\
\hline $\mathrm{C}$ & 0.29611900 & 2.02462400 & -5.34289600 \\
\hline $\mathrm{H}$ & -0.69690800 & 2.48424700 & -5.39717200 \\
\hline $\mathrm{P}$ & 0.10289900 & 0.91505800 & 0.60627300 \\
\hline $\mathrm{C}$ & -1.79039900 & 1.28856600 & 2.84094200 \\
\hline $\mathrm{H}$ & -2.58870100 & 2.00436900 & 3.08867200 \\
\hline $\mathrm{H}$ & -0.89861000 & 1.64855300 & 3.36589200 \\
\hline $\mathrm{C}$ & -2.21067300 & -0.08695500 & 3.38648800 \\
\hline $\mathrm{H}$ & -2.38812000 & -0.00434600 & 4.466715 \\
\hline
\end{tabular}




\begin{tabular}{|c|c|c|c|}
\hline $\mathrm{H}$ & -1.39872300 & -0.81084500 & 3.26133600 \\
\hline $\mathrm{C}$ & -3.45687800 & -0.62556400 & 2.67312400 \\
\hline $\mathrm{H}$ & -4.31390400 & 0.03052400 & 2.88800300 \\
\hline & -3.71458300 & -1.61961300 & 3.06007900 \\
\hline & -3.22023200 & -0.67694300 & 1.15938100 \\
\hline & -4.11791200 & -1.03389100 & 0.63926700 \\
\hline & -2.42683500 & -1.39670800 & 0.92743100 \\
\hline & -2.83815700 & 0.70411800 & 0.61322500 \\
\hline & -2.69896000 & 0.65405500 & -0.46612100 \\
\hline & -3.68340600 & 1.38751400 & 0.79136500 \\
\hline $\mathrm{C}$ & -1.60969000 & 1.34671900 & 1.30092900 \\
\hline $\mathrm{H}$ & -1.63365000 & 2.40454500 & 1.01472300 \\
\hline & 1.10526500 & 2.31615000 & 1.36575700 \\
\hline & 0.72366300 & 2.40649000 & 2.39083400 \\
\hline & 2.64454800 & 2.06242100 & 1.46114700 \\
\hline & 2.96906800 & 1.28762800 & 0.76640300 \\
\hline & 2.87287600 & 1.70658800 & 2.47241800 \\
\hline & 3.46362500 & 3.33861100 & 1.20114200 \\
\hline $\mathrm{H}$ & 3.49328700 & 3.54309900 & 0.12453200 \\
\hline & 4.50029400 & 3.17158800 & 1.51921500 \\
\hline & 2.85198300 & 4.52825900 & 1.94952200 \\
\hline & 3.49303600 & 5.41389900 & 1.86672100 \\
\hline & 2.80809000 & 4.28025900 & 3.01962800 \\
\hline & 1.42740900 & 4.83975200 & 1.42136000 \\
\hline & 1.45966000 & 5.70308800 & 0.74652900 \\
\hline & 0.77430800 & 5.12483700 & 2.25689400 \\
\hline & 0.80654600 & 3.65476200 & 0.65412300 \\
\hline & -0.27081900 & 3.82030800 & 0.55493200 \\
\hline & 1.19464400 & 3.61773100 & -0.36527200 \\
\hline & 1.21413100 & -0.66516100 & 2.81005200 \\
\hline & 0.59916300 & -0.03584300 & 3.45821200 \\
\hline & 2.21554500 & -0.22681700 & 2.82227500 \\
\hline & 1.27812700 & -2.10443300 & 3.37193600 \\
\hline & 0.29792700 & -2.39033300 & 3.77446100 \\
\hline & 1.97867500 & -2.12281200 & 4.21641700 \\
\hline & 1.71566800 & -3.12599400 & 2.29228600 \\
\hline & 0.83105200 & -3.55341400 & 1.80072900 \\
\hline & 2.24583400 & -3.96587400 & 2.75616700 \\
\hline & 2.58603100 & -2.44695100 & 1.22704800 \\
\hline & 2.98889800 & -3.18384500 & 0.52201400 \\
\hline
\end{tabular}




$\begin{array}{lrrr}\mathrm{H} & 3.45363000 & -1.98111800 & 1.71384500 \\ \mathrm{C} & 1.77416400 & -1.38364400 & 0.45128500 \\ \mathrm{H} & 2.45712900 & -0.64693500 & 0.01648200 \\ \mathrm{H} & 1.25609400 & -1.85998500 & -0.38640300 \\ \mathrm{C} & 0.70566500 & -0.69664000 & 1.35469400 \\ \mathrm{H} & -0.19703200 & -1.31791000 & 1.31904000 \\ \mathrm{C} & -1.68652600 & 6.85470600 & -1.73573900 \\ \mathrm{C} & -0.36638000 & 6.52061000 & -2.51930100 \\ \mathrm{~B} & -1.48336600 & 4.60371900 & -2.01017100 \\ \mathrm{O} & -0.21922800 & 5.09585800 & -2.26980600 \\ \mathrm{O} & -2.41707800 & 5.60266200 & -1.82750700 \\ \mathrm{C} & -0.51735800 & 6.69734400 & -4.03550800 \\ \mathrm{H} & -0.58548600 & 7.75415600 & -4.31324700 \\ \mathrm{H} & 0.35290500 & 6.26380100 & -4.53312400 \\ \mathrm{H} & -1.41022800 & 6.18094700 & -4.40236000 \\ \mathrm{C} & -2.53929100 & 7.96354200 & -2.34492700 \\ \mathrm{H} & -3.43508800 & 8.11657600 & -1.73468100 \\ \mathrm{H} & -1.98171600 & 8.90661600 & -2.37715300 \\ \mathrm{H} & -2.86099400 & 7.71030800 & -3.35740200 \\ \mathrm{C} & -1.44774900 & 7.12231000 & -0.24418200 \\ \mathrm{H} & -0.95654400 & 8.08727300 & -0.08073400 \\ \mathrm{H} & -2.41249500 & 7.13067100 & 0.27218500 \\ \mathrm{H} & -0.83005700 & 6.33664100 & 0.20085500 \\ \mathrm{C} & 0.88695200 & 7.23499400 & -2.02658400 \\ \mathrm{H} & 1.75026700 & 6.90773000 & -2.61480100 \\ \mathrm{H} & 0.78361700 & 8.32013300 & -2.14085300 \\ \mathrm{H} & 1.09104100 & 7.01051300 & -0.97814900 \\ \mathrm{C} & -1.85761200 & 3.12084500 & -1.92241500 \\ \mathrm{H} & -2.90880400 & 2.84883100 & -1.80750300 \\ \mathrm{C} & -0.98202400 & 2.12242500 & -1.91372700\end{array}$

\section{Ru-vinyl-2}

Thermal correction to Gibbs free energy $=0.934881$

Sum of electronic and thermal free energies $=-3187.2366046$

E_sol $=-3186.356356$

$\begin{array}{lrrr}\mathrm{Ru} & -1.47981600 & 2.21783200 & -1.13301200 \\ \mathrm{Cl} & 0.75315300 & 3.04074800 & -1.21357900 \\ \mathrm{Cl} & -2.83915100 & 0.25457800 & -0.95593400 \\ \mathrm{P} & -1.72350200 & 2.08553600 & -3.58205600 \\ \mathrm{C} & -3.77724400 & 1.27814600 & -5.54003900\end{array}$




\begin{tabular}{|c|c|c|c|}
\hline $\mathrm{H}$ & -4.54454400 & 0.49145700 & -5.60057900 \\
\hline 1 & -2.91559600 & 0.89503600 & -6.09895900 \\
\hline$S_{0}$ & -4.34314900 & 2.53401700 & -6.22659300 \\
\hline [ & -4.65132900 & 2.27516900 & -7.24793400 \\
\hline $\mathrm{H}$ & -3.56675000 & 3.29787900 & -6.32708500 \\
\hline & -5.52639900 & 3.13105200 & -5.45117800 \\
\hline & -6.36788400 & 2.42251000 & -5.47198000 \\
\hline & -5.87458000 & 4.04864400 & -5.94221500 \\
\hline & -5.14634600 & 3.40734300 & -3.98964700 \\
\hline $\mathrm{H}$ & -6.01696900 & 3.78552200 & -3.43777900 \\
\hline $\mathrm{H}$ & -4.38702800 & 4.19822600 & -3.93821100 \\
\hline & -4.63005700 & 2.13319900 & -3.31038000 \\
\hline & -4.38101500 & 2.31683400 & -2.26447700 \\
\hline & -5.45082100 & 1.40019500 & -3.29854300 \\
\hline & -3.44527400 & 1.45129400 & -4.03626000 \\
\hline & -3.37881100 & 0.45138600 & -3.59936000 \\
\hline & -0.61493900 & 0.80149900 & -4.39932200 \\
\hline & -0.73511900 & 0.94668300 & -5.47766100 \\
\hline & 0.88601000 & 1.02539400 & -4.05014400 \\
\hline & 1.08272400 & 2.06324700 & -3.77323700 \\
\hline & 1.48742100 & 0.82211100 & -4.94691800 \\
\hline & 1.33911200 & 0.08893900 & -2.92343000 \\
\hline & 0.69502200 & 0.24811500 & -2.05135300 \\
\hline & 2.34940200 & 0.35405000 & -2.59239300 \\
\hline & 1.26929200 & -1.38898400 & -3.36303600 \\
\hline & 1.14355200 & -2.02488500 & -2.47725200 \\
\hline & 2.21523900 & -1.69025900 & -3.83087000 \\
\hline & 0.10433400 & -1.62713400 & -4.35207 \\
\hline & -0.25052100 & -2.66233200 & -4.28541800 \\
\hline & 0.45222100 & -1.48675900 & -5.38505500 \\
\hline & -1.05158600 & -0.65370400 & -4.08021700 \\
\hline & -1.92262400 & -0.92058000 & -4.68871200 \\
\hline & -1.36232600 & -0.74999800 & -3.03403900 \\
\hline & -0.94760900 & 3.49215700 & -6.01467400 \\
\hline & -1.44726200 & 2.66547800 & -6.52687200 \\
\hline & 0.11802000 & 3.23232500 & -5.98093000 \\
\hline & -1.15272000 & 4.79513700 & -6.83141700 \\
\hline & -2.11878000 & 4.76022400 & -7.35040300 \\
\hline & -0.38384300 & 4.84582200 & -7.61271300 \\
\hline & -1.08480500 & 6.05942000 & -5.94723900 \\
\hline
\end{tabular}




\begin{tabular}{|c|c|c|c|}
\hline $\mathrm{H}$ & -2.07583300 & 6.27757400 & -5.52538900 \\
\hline $\mathrm{H}$ & -0.81129800 & 6.93096800 & -6.55371500 \\
\hline C & -0.08739200 & 5.84709900 & -4.80419100 \\
\hline $\mathrm{H}$ & 0.05823900 & 6.77125800 & -4.23255800 \\
\hline $\mathrm{H}$ & 0.89138300 & 5.59852700 & -5.23739700 \\
\hline & -0.54131900 & 4.71140000 & -3.85136200 \\
\hline & 0.33837900 & 4.22531700 & -3.43042300 \\
\hline & -1.08927900 & 5.12336100 & -2.99883100 \\
\hline & -1.44609500 & 3.67655300 & -4.56577400 \\
\hline $\mathrm{H}$ & -2.45481600 & 4.09812800 & -4.61007000 \\
\hline $\mathrm{C}$ & -2.50465900 & 3.65761800 & -1.13101800 \\
\hline ( & -3.26330300 & 4.73905400 & -1.12645900 \\
\hline & -2.81441500 & 5.73004000 & -1.13107700 \\
\hline & -4.34805300 & 4.66144600 & -1.12258100 \\
\hline & -1.41056900 & 2.26444700 & 1.30570400 \\
\hline & -3.21030300 & 2.60478500 & 3.62189500 \\
\hline & -3.96661000 & 3.34067300 & 3.93429400 \\
\hline & -2.28649500 & 2.89689300 & 4.13361100 \\
\hline & -3.67549600 & 1.22190200 & 4.10912300 \\
\hline & -3.81382600 & 1.25377900 & 5.19771500 \\
\hline & -2.90236900 & 0.47038000 & 3.91786900 \\
\hline & -4.96774400 & 0.77847100 & 3.41216400 \\
\hline & -5.78613900 & 1.45955600 & 3.69061600 \\
\hline & -5.25861200 & -0.22242600 & 3.75549200 \\
\hline & -4.78241200 & 0.79740600 & 1.89041100 \\
\hline & -5.71165700 & 0.50820500 & 1.38380400 \\
\hline & -4.03063000 & 0.05658300 & 1.59515700 \\
\hline & -4.35508100 & 2.18770800 & 1.40389900 \\
\hline & -4.25262500 & 2.19298800 & 0.31876600 \\
\hline & -5.16194600 & 2.89664100 & 1.64828800 \\
\hline & -3.07672900 & 2.73639900 & 2.08182100 \\
\hline & -3.05934400 & 3.80789900 & 1.85134500 \\
\hline & -0.32350300 & 3.58697400 & 2.09041400 \\
\hline & -0.66946100 & 3.65299600 & 3.13002000 \\
\hline & 1.20532300 & 3.26565300 & 2.12489400 \\
\hline & 1.47601800 & 2.51848600 & 1.37845800 \\
\hline & 1.44677000 & 2.84374400 & 3.10710400 \\
\hline & 2.07129300 & 4.51986100 & 1.90915100 \\
\hline & 2.09132700 & 4.77339700 & 0.84262600 \\
\hline & 3.10581500 & 4.29613400 & 2.19780900 \\
\hline
\end{tabular}




$\begin{array}{lrrr}\mathrm{C} & 1.52438500 & 5.69832800 & 2.72325100 \\ \mathrm{H} & 2.20054000 & 6.55942500 & 2.66630100 \\ \mathrm{H} & 1.49232400 & 5.40310800 & 3.78172400 \\ \mathrm{C} & 0.10281000 & 6.09260600 & 2.24312900 \\ \mathrm{H} & 0.15083700 & 6.98660400 & 1.60873400 \\ \mathrm{H} & -0.51939700 & 6.36078200 & 3.10707500 \\ \mathrm{C} & -0.58161900 & 4.96605900 & 1.44216100 \\ \mathrm{H} & -1.65266000 & 5.17854300 & 1.36618700 \\ \mathrm{H} & -0.20468200 & 4.94555600 & 0.41692900 \\ \mathrm{C} & -0.29709800 & 0.53131700 & 3.39361800 \\ \mathrm{H} & -0.86704600 & 1.14855800 & 4.09247800 \\ \mathrm{H} & 0.72107000 & 0.92969600 & 3.39699100 \\ \mathrm{C} & -0.27430900 & -0.93698600 & 3.87798600 \\ \mathrm{H} & -1.25358200 & -1.20599800 & 4.29416800 \\ \mathrm{H} & 0.44815300 & -1.02684900 & 4.69908100 \\ \mathrm{C} & 0.09188400 & -1.91594600 & 2.73409900 \\ \mathrm{H} & -0.82246600 & -2.28423700 & 2.24914200 \\ \mathrm{H} & 0.60284400 & -2.79792000 & 3.13742700 \\ \mathrm{C} & 0.95618800 & -1.21485200 & 1.67823900 \\ \mathrm{H} & 1.30894400 & -1.92831400 & 0.92398500 \\ \mathrm{H} & 1.85530600 & -0.80799100 & 2.16057000 \\ \mathrm{C} & 0.16386900 & -0.08228000 & 0.98431100 \\ \mathrm{H} & 0.86247100 & 0.65015100 & 0.56735600 \\ \mathrm{H} & -0.39630300 & -0.49388800 & 0.13919500 \\ \mathrm{C} & -0.84965900 & 0.59599600 & 1.95569500 \\ \mathrm{H} & -1.77680000 & 0.01181200 & 1.91678200\end{array}$

\subsection{For complexes where $\mathrm{L}=\mathrm{NHC}$}

\section{Ru-IV}

Thermal correction to Gibbs free energy $=0.552313$

Sum of electronic and thermal free energies $=-2403.891028$

E_sol $=-2404.3363098$

$\begin{array}{crrr}\mathrm{Ru} & 0.16701700 & 0.35937600 & -0.04977900 \\ \mathrm{C} & 0.18848500 & -1.62220900 & 0.01519100 \\ \mathrm{~N} & -0.78438600 & -2.56366400 & 0.05667000 \\ \mathrm{C} & -0.27247800 & -3.94443100 & 0.08523100 \\ \mathrm{C} & 1.25471600 & -3.74382000 & 0.05776800 \\ \mathrm{~N} & 1.38007100 & -2.28298200 & 0.01334400\end{array}$




\begin{tabular}{|c|c|c|c|}
\hline $\mathrm{H}$ & 1.72559000 & -4.19213200 & -0.82439300 \\
\hline $\mathrm{H}$ & 1.75051300 & -4.14047300 & 0.95098200 \\
\hline $\mathrm{H}$ & -0.64279200 & -4.50312800 & -0.78128400 \\
\hline $\mathrm{H}$ & -0.61728400 & -4.45720000 & 0.98983200 \\
\hline $\mathrm{C}$ & 2.65748600 & -1.63382600 & -0.01284500 \\
\hline C & 3.29294600 & -1.31355500 & 1.20341800 \\
\hline $\mathrm{C}$ & 4.47926000 & -0.57685200 & 1.15159900 \\
\hline $\mathrm{C}$ & 5.05638600 & -0.19222400 & -0.06307300 \\
\hline $\mathrm{C}$ & 4.45665600 & -0.62226700 & -1.25020500 \\
\hline $\mathrm{C}$ & 3.26952700 & -1.36070900 & -1.25165600 \\
\hline $\mathrm{H}$ & 4.96224900 & -0.29699200 & 2.08548300 \\
\hline $\mathrm{H}$ & 4.92138600 & -0.37846800 & -2.20329100 \\
\hline $\mathrm{C}$ & 2.73160900 & -1.91560700 & -2.54763600 \\
\hline $\mathrm{H}$ & 3.27510600 & -2.83518800 & -2.80842000 \\
\hline 11 & 1.66755600 & -2.14372400 & -2.48695100 \\
\hline $\mathrm{H}$ & 2.86302400 & -1.20230000 & -3.36489500 \\
\hline $\mathrm{C}$ & 2.77485700 & -1.81681300 & 2.52840500 \\
\hline $\mathrm{H}$ & 2.97091500 & -1.09564000 & 3.32563000 \\
\hline $\mathrm{H}$ & 3.28051300 & -2.75755500 & 2.79032900 \\
\hline $\mathrm{H}$ & 1.69923400 & -1.99253600 & 2.50915000 \\
\hline $\mathrm{C}$ & 6.29205400 & 0.67583600 & -0.09094300 \\
\hline $\mathrm{H}$ & 6.89791600 & 0.48697700 & -0.98374700 \\
\hline $\mathrm{H}$ & 6.92087700 & 0.51154400 & 0.79084600 \\
\hline $\mathrm{H}$ & 6.01716300 & 1.73910900 & -0.10211100 \\
\hline $\mathrm{C}$ & -2.19184600 & -2.32622500 & 0.08487800 \\
\hline $\mathrm{C}$ & -2.82693000 & -2.15878900 & 1.32621000 \\
\hline $\mathrm{C}$ & -4.19854400 & -1.88718600 & 1.32906600 \\
\hline $\mathrm{H}$ & -4.70383800 & -1.73895600 & 2.28103000 \\
\hline $\mathrm{C}$ & -4.26034000 & -1.95478800 & -1.07683800 \\
\hline $\mathrm{C}$ & -2.88985600 & -2.22810600 & -1.12947600 \\
\hline $\mathrm{H}$ & -4.81398800 & -1.85970500 & -2.00847600 \\
\hline $\mathrm{C}$ & -4.93033400 & -1.78223200 & 0.14012900 \\
\hline & -2.15942300 & -2.32535500 & -2.44601900 \\
\hline 11 & -2.85989200 & -2.28957800 & -3.28583400 \\
\hline $\mathrm{H}$ & -1.58538800 & -3.25643400 & -2.52695000 \\
\hline $\mathrm{H}$ & -1.44736800 & -1.49816500 & -2.55468000 \\
\hline $\mathrm{H}$ & -1.45126600 & -3.10859400 & 2.71140300 \\
\hline $\mathrm{C}$ & -2.02923000 & -2.18227700 & 2.60688700 \\
\hline 11 & -2.68542500 & -2.09696900 & 3.47827400 \\
\hline & -1.31313800 & -1.35174900 & 2.63181700 \\
\hline
\end{tabular}




$\begin{array}{lrrr}\mathrm{C} & -6.41687600 & -1.51170500 & 0.17111000 \\ \mathrm{H} & -6.69763700 & -0.91275300 & 1.04417800 \\ \mathrm{H} & -6.98645200 & -2.44930100 & 0.22264000 \\ \mathrm{H} & -6.74699600 & -0.97999700 & -0.72781300 \\ \mathrm{Cl} & 0.64387600 & 0.62395500 & 2.27003100 \\ \mathrm{Cl} & 0.51980000 & 0.46322400 & -2.40087500 \\ \mathrm{C} & -1.62486100 & 0.75319100 & -0.03386000 \\ \mathrm{H} & -2.43342900 & 0.02963200 & 0.04937200 \\ \mathrm{C} & -2.04512000 & 2.13266900 & -0.15653600 \\ \mathrm{C} & -1.05751500 & 3.14587500 & -0.22226700 \\ \mathrm{C} & -1.41274700 & 4.48585500 & -0.36297300 \\ \mathrm{C} & -2.76903200 & 4.82128600 & -0.43713700 \\ \mathrm{C} & -3.76410600 & 3.84133600 & -0.36989000 \\ \mathrm{C} & -3.39943400 & 2.50520700 & -0.23008700 \\ \mathrm{H} & -4.15351700 & 1.72339300 & -0.17966800 \\ \mathrm{H} & -4.81156400 & 4.12170600 & -0.42704900 \\ \mathrm{H} & -3.04498900 & 5.86649100 & -0.54742200 \\ \mathrm{H} & -0.66096300 & 5.26385400 & -0.41430500 \\ \mathrm{O} & 0.21822900 & 2.66371700 & -0.15052000 \\ \mathrm{C} & 1.37362200 & 3.55029600 & -0.01686000 \\ \mathrm{H} & 1.25837300 & 4.33185000 & -0.77743600 \\ \mathrm{C} & 2.60499000 & 2.71481000 & -0.33976200 \\ \mathrm{H} & 3.48696500 & 3.36577800 & -0.33829900 \\ \mathrm{H} & 2.50944400 & 2.24293600 & -1.32066200 \\ \mathrm{H} & 2.75396400 & 1.93487300 & 0.41433000 \\ \mathrm{C} & 1.40874800 & 4.14027600 & 1.38925400 \\ \mathrm{H} & 2.27169800 & 4.80895700 & 1.48580400 \\ \mathrm{H} & 1.49311800 & 3.32952900 & 2.11788800 \\ \mathrm{H} & 0.50348600 & 4.71186800 & 1.61320400\end{array}$

ts-i

Thermal correction to Gibbs free energy $=0.768438$

Sum of electronic and thermal free energies $=-2931.050456$

$\begin{array}{lrrr}\text { E_sol }=-2931.6809702 & & \\ \mathrm{Ru} & 0.81031200 & 0.12556900 & -1.35021100 \\ \mathrm{Cl} & -0.17616000 & -1.174866800 & 0.45234200 \\ \mathrm{Cl} & 1.75338300 & 1.03466000 & -3.41399400 \\ \mathrm{C} & 0.30559900 & 1.91240900 & -0.93713200 \\ \mathrm{C} & -0.15234300 & 2.61333100 & 0.25628200 \\ \mathrm{C} & -0.04344000 & 4.03973700 & 0.26805400\end{array}$




\begin{tabular}{|c|c|c|c|}
\hline $\mathrm{C}$ & -0.66171900 & 1.98145800 & 1.40642700 \\
\hline $\mathrm{C}$ & -0.44171500 & 4.76445600 & 1.39943000 \\
\hline $\mathrm{C}$ & -1.05951200 & 2.70595300 & 2.52385200 \\
\hline $\mathrm{H}$ & -0.72898200 & 0.90090700 & 1.40131200 \\
\hline $\mathrm{C}$ & -0.94426200 & 4.09757300 & 2.51610200 \\
\hline $\mathrm{H}$ & -0.36126200 & 5.84399500 & 1.41799200 \\
\hline $\mathrm{H}$ & -1.45214100 & 2.18956600 & 3.39457700 \\
\hline $\mathrm{H}$ & -1.24768900 & 4.67757300 & 3.38430500 \\
\hline $\mathrm{O}$ & 0.47267200 & 4.61011800 & -0.84794500 \\
\hline $\mathrm{C}$ & 0.68611800 & 6.02934100 & -0.95654600 \\
\hline $\mathrm{H}$ & 1.09235000 & 6.40021900 & -0.00590400 \\
\hline $\mathrm{C}$ & -0.62505100 & 6.73769100 & -1.29542100 \\
\hline $\mathrm{H}$ & -1.00561900 & 6.36897900 & -2.25398700 \\
\hline $\mathrm{H}$ & -1.38938800 & 6.55824700 & -0.53378800 \\
\hline $\mathrm{H}$ & -0.46435900 & 7.81844600 & -1.37706500 \\
\hline $\mathrm{C}$ & 1.73798200 & 6.18992900 & -2.04746900 \\
\hline $\mathrm{H}$ & 1.36556300 & 5.78264400 & -2.99321300 \\
\hline $\mathrm{H}$ & 1.98033900 & 7.24844600 & -2.19082400 \\
\hline $\mathrm{H}$ & 2.64851400 & 5.64761900 & -1.77879600 \\
\hline $\mathrm{H}$ & 0.56901800 & 2.60372900 & -1.73684600 \\
\hline $\mathrm{C}$ & -0.80434100 & -0.37959700 & -2.46927000 \\
\hline $\mathrm{C}$ & -0.97274300 & -1.47804600 & -3.19590700 \\
\hline $\mathrm{H}$ & -0.14947600 & -2.18569900 & -3.25418000 \\
\hline $\mathrm{C}$ & -1.29089500 & 0.83632200 & -1.96603100 \\
\hline $\mathrm{H}$ & -1.37010800 & 1.67326000 & -2.65743900 \\
\hline $\mathrm{H}$ & -1.98812100 & 0.81464000 & -1.13136700 \\
\hline $\mathrm{C}$ & -3.70117300 & -2.85491300 & -5.37548800 \\
\hline $\mathrm{C}$ & -4.46591900 & -1.73435600 & -4.57636700 \\
\hline B & -2.28207200 & -1.79741900 & -3.93680200 \\
\hline $\mathrm{O}$ & -3.37570900 & -0.96350500 & -4.00574200 \\
\hline $\mathrm{O}$ & -2.47096000 & -2.98595000 & -4.61482600 \\
\hline $\mathrm{C}$ & -5.32601600 & -0.80646200 & -5.42925000 \\
\hline $\mathrm{H}$ & -6.11177400 & -1.37144900 & -5.94390000 \\
\hline $\mathrm{H}$ & -5.80546800 & -0.05848000 & -4.78972500 \\
\hline $\mathrm{H}$ & -4.72680800 & -0.27851100 & -6.17415200 \\
\hline $\mathrm{C}$ & -3.29941900 & -2.41903300 & -6.79026100 \\
\hline $\mathrm{H}$ & -2.59875100 & -3.15280400 & -7.20045700 \\
\hline $\mathrm{H}$ & -4.16686400 & -2.35518800 & -7.45558200 \\
\hline $\mathrm{H}$ & -2.79841300 & -1.44617500 & -6.77156500 \\
\hline $\mathrm{C}$ & -4.39740200 & -4.21201000 & -5.41466200 \\
\hline
\end{tabular}




\begin{tabular}{|c|c|c|c|}
\hline $\mathrm{H}$ & -5.37279000 & -4.13263800 & -5.90827100 \\
\hline $\mathrm{H}$ & -3.78591600 & -4.92352100 & -5.97914900 \\
\hline $\mathrm{H}$ & -4.54345800 & -4.61555000 & -4.41040200 \\
\hline $\mathrm{C}$ & -5.28008300 & -2.28509600 & -3.39851800 \\
\hline $\mathrm{H}$ & -5.60471000 & -1.44850600 & -2.77245900 \\
\hline $\mathrm{H}$ & -6.16618400 & -2.83127600 & -3.73889600 \\
\hline & -4.67113100 & -2.95259100 & -2.78081700 \\
\hline & 2.69841400 & -0.47295200 & -0.75737300 \\
\hline & 3.57046100 & 0.20338000 & 0.01120100 \\
\hline & 3.24790700 & -1.65008000 & -1.10610200 \\
\hline & 4.81314100 & -0.55661700 & 0.26306600 \\
\hline $\mathrm{H}$ & 4.90053700 & -0.78531000 & 1.33067500 \\
\hline $\mathrm{H}$ & 5.68442100 & 0.03560200 & -0.03225400 \\
\hline$C$ & 4.62123200 & -1.81881700 & -0.60190300 \\
\hline & 4.70070900 & -2.74726300 & -0.02768200 \\
\hline & 5.32335100 & -1.86779600 & -1.44179200 \\
\hline & 3.36913500 & 1.49398400 & 0.60650500 \\
\hline & 3.80456400 & 2.63563500 & -0.08683500 \\
\hline C & 2.79466800 & 1.57902000 & 1.88727000 \\
\hline $\mathrm{C}$ & 3.63077400 & 3.88086100 & 0.52671200 \\
\hline $\mathrm{C}$ & 2.63751500 & 2.84629200 & 2.45366900 \\
\hline & 3.04806400 & 4.00675300 & 1.79009700 \\
\hline & 3.96550400 & 4.77336100 & 0.00307600 \\
\hline $\mathrm{H}$ & 2.16854100 & 2.92895200 & 3.43094400 \\
\hline C & 2.61421500 & -2.60803800 & -1.96826800 \\
\hline $\mathrm{C}$ & 1.81888800 & -3.62251900 & -1.40334100 \\
\hline$C$ & 2.81656100 & -2.52001900 & -3.36016200 \\
\hline $\mathrm{C}$ & 1.12605300 & -4.47242000 & -2.27162500 \\
\hline $\mathrm{C}$ & 2.10320700 & -3.39581700 & -4.18270500 \\
\hline $\mathrm{C}$ & 1.22662800 & -4.35431600 & -3.66099900 \\
\hline & 0.47594900 & -5.23423500 & -1.84684700 \\
\hline & 2.22018600 & -3.31148000 & -5.26080200 \\
\hline & 2.89030200 & 5.35901600 & 2.44465500 \\
\hline & 1.91896300 & 5.44239600 & 2.94310700 \\
\hline & 3.66627600 & 5.52584500 & 3.20340000 \\
\hline & 2.96751700 & 6.17077200 & 1.71295400 \\
\hline $\mathrm{C}$ & 4.42723200 & 2.52815300 & -1.45561500 \\
\hline$H$ & 5.28652000 & 1.84567500 & -1.45877600 \\
\hline & 3.70467900 & 2.14372500 & -2.18391400 \\
\hline & 4.77837400 & 3.50571300 & -1.80068300 \\
\hline
\end{tabular}




$\begin{array}{lrrr}\mathrm{C} & 2.34528000 & 0.34284700 & 2.62434800 \\ \mathrm{H} & 1.59466100 & -0.21119500 & 2.05193800 \\ \mathrm{H} & 3.18286800 & -0.34140900 & 2.81275200 \\ \mathrm{H} & 1.90977100 & 0.60674700 & 3.59267700 \\ \mathrm{C} & 1.74228900 & -3.85315400 & 0.08610600 \\ \mathrm{H} & 2.36612300 & -4.71513100 & 0.36174200 \\ \mathrm{H} & 2.07208500 & -2.98445300 & 0.65595300 \\ \mathrm{H} & 0.71542300 & -4.06508100 & 0.39436800 \\ \mathrm{C} & 3.81611200 & -1.56299400 & -3.96213200 \\ \mathrm{H} & 3.93698100 & -0.66108200 & -3.36212300 \\ \mathrm{H} & 4.79427800 & -2.05549800 & -4.06231500 \\ \mathrm{H} & 3.49782200 & -1.24326900 & -4.95763700 \\ \mathrm{C} & 0.36608800 & -5.19467400 & -4.57347800 \\ \mathrm{H} & 0.84690100 & -5.35639500 & -5.54458500 \\ \mathrm{H} & 0.14641600 & -6.17291400 & -4.13157900 \\ \mathrm{H} & -0.59129800 & -4.68746900 & -4.75325100\end{array}$

\section{mcb-i}

Thermal correction to Gibbs free energy $=0.769173$

Sum of electronic and thermal free energies $=-2931.067039$

$\begin{array}{lrrr}\text { E_sol }=-2931.7026622 & & \\ \mathrm{Ru} & 1.15637500 & 0.67579900 & -0.99612300 \\ \mathrm{Cl} & 0.06515200 & 0.35947900 & 1.12939500 \\ \mathrm{Cl} & 2.34011800 & 0.94906700 & -3.11060000 \\ \mathrm{C} & 0.67634800 & 2.66812400 & -1.22511200 \\ \mathrm{C} & 0.65066900 & 3.64363600 & -0.12138400 \\ \mathrm{C} & 1.61457500 & 4.68564100 & -0.14580000 \\ \mathrm{C} & -0.29763400 & 3.65091500 & 0.91580800 \\ \mathrm{C} & 1.59322300 & 5.69923900 & 0.81863300 \\ \mathrm{C} & -0.31622400 & 4.65560300 & 1.87761800 \\ \mathrm{H} & -1.01794300 & 2.84440500 & 0.97046600 \\ \mathrm{C} & 0.62733600 & 5.68497200 & 1.82378200 \\ \mathrm{H} & 2.34159700 & 6.48345200 & 0.79458600 \\ \mathrm{H} & -1.06225300 & 4.63525600 & 2.66641700 \\ \mathrm{H} & 0.62130900 & 6.47511100 & 2.57034800 \\ \mathrm{O} & 2.55878100 & 4.62945200 & -1.12993300 \\ \mathrm{C} & 3.03119200 & 5.83920000 & -1.76274700 \\ \mathrm{H} & 2.32838100 & 6.65065800 & -1.53248600 \\ \mathrm{C} & 3.02176900 & 5.55899900 & -3.26221100 \\ \mathrm{H} & 3.65734300 & 4.69571700 & -3.48570600\end{array}$




\begin{tabular}{|c|c|c|c|}
\hline $\mathrm{H}$ & 2.00729900 & 5.32859500 & -3.60159000 \\
\hline$H$ & 3.39278700 & 6.42563300 & -3.82085900 \\
\hline C & 4.42327800 & 6.18869400 & -1.24163400 \\
\hline I & 5.11386500 & 5.36315300 & -1.44358600 \\
\hline $\mathrm{H}$ & 4.80296200 & 7.08806300 & -1.73939300 \\
\hline $\mathrm{H}$ & 4.41323500 & 6.37020600 & -0.16250200 \\
\hline & 1.26520900 & 3.03482900 & -2.05969900 \\
\hline & -0.41734600 & 0.33778800 & -1.92680000 \\
\hline & -1.22224600 & -0.52219100 & -2.54987800 \\
\hline $\mathrm{H}$ & -0.89257700 & -1.55632000 & -2.56514200 \\
\hline $\mathrm{C}$ & -0.58788500 & 1.91918200 & -1.70486400 \\
\hline $\mathrm{H}$ & -0.79788100 & 2.25538200 & -2.72330200 \\
\hline & -1.43758300 & 2.05280600 & -1.03615500 \\
\hline & -4.35358200 & 1.03119100 & -3.94330800 \\
\hline & -4.28817000 & -0.35114000 & -4.69355000 \\
\hline B & -2.51963300 & -0.14647500 & -3.26952300 \\
\hline $\mathrm{O}$ & -3.31124300 & -1.08151100 & -3.90745900 \\
\hline $\mathrm{O}$ & -3.01917200 & 1.13817800 & -3.38080100 \\
\hline $\mathrm{C}$ & -5.59372100 & -1.14100400 & -4.70790500 \\
\hline $\mathrm{H}$ & -6.38114100 & -0.57887000 & -5.22309700 \\
\hline & -5.44553200 & -2.08677700 & -5.23909800 \\
\hline & -5.93193500 & -1.37287000 & -3.69567200 \\
\hline & -5.33155900 & 1.02824300 & -2.76149600 \\
\hline & -5.16979600 & 1.93248100 & -2.16669700 \\
\hline & -6.37353200 & 1.01405500 & -3.09812500 \\
\hline $\mathrm{H}$ & -5.15938900 & 0.16220100 & -2.11482600 \\
\hline $\mathrm{C}$ & -4.60430100 & 2.24151600 & -4.83795300 \\
\hline & -5.57003600 & 2.15201200 & -5.34858600 \\
\hline $\mathrm{H}$ & -4.62256900 & 3.15172200 & -4.22978700 \\
\hline & -3.81887200 & 2.35320600 & -5.58859000 \\
\hline & -3.71695900 & -0.23771700 & -6.11299000 \\
\hline & -3.50292200 & -1.24310000 & -6.48835600 \\
\hline & -4.42206700 & 0.24725600 & -6.79628300 \\
\hline & -2.78086700 & 0.32949300 & -6.11282600 \\
\hline $\mathrm{C}$ & 2.68988600 & -0.56754100 & -0.47367700 \\
\hline & 3.73462000 & -0.21140700 & 0.28811300 \\
\hline & 2.84372000 & -1.83571000 & -0.88626500 \\
\hline & 4.75419200 & -1.27505000 & 0.37723000 \\
\hline & 4.96809300 & -1.50750100 & 1.42378100 \\
\hline & 5.68170200 & -0.93808900 & -0.09858100 \\
\hline
\end{tabular}




\begin{tabular}{|c|c|c|c|}
\hline $\mathrm{C}$ & 4.08724000 & -2.44516600 & -0.37537700 \\
\hline $\mathrm{H}$ & 3.84823200 & -3.29227200 & 0.27696600 \\
\hline $\mathrm{H}$ & 4.69080300 & -2.81133800 & -1.21093000 \\
\hline $\mathrm{C}$ & 3.82423500 & 1.00365900 & 1.05011100 \\
\hline $\mathrm{C}$ & 4.37160900 & 2.15633400 & 0.46651300 \\
\hline $\mathrm{C}$ & 3.38249900 & 0.98504200 & 2.38935000 \\
\hline $\mathrm{C}$ & 4.42510300 & 3.31884900 & 1.24593900 \\
\hline $\mathrm{C}$ & 3.44676100 & 2.17290900 & 3.11571300 \\
\hline $\mathrm{C}$ & 3.95326500 & 3.35313200 & 2.55736900 \\
\hline $\mathrm{H}$ & 4.84152100 & 4.21937100 & 0.80542000 \\
\hline $\mathrm{H}$ & 3.08955400 & 2.17992000 & 4.14311400 \\
\hline $\mathrm{C}$ & 1.89570000 & -2.58500800 & -1.66394700 \\
\hline $\mathrm{C}$ & 0.81537200 & -3.20061300 & -1.00314000 \\
\hline $\mathrm{C}$ & 2.08571700 & -2.71773100 & -3.04985800 \\
\hline $\mathrm{C}$ & -0.13438600 & -3.86823800 & -1.78127300 \\
\hline $\mathrm{C}$ & 1.09574100 & -3.37875400 & -3.78476100 \\
\hline $\mathrm{C}$ & -0.03527900 & -3.92930300 & -3.17602900 \\
\hline $\mathrm{H}$ & -0.99129600 & -4.32087200 & -1.28734900 \\
\hline $\mathrm{H}$ & 1.20686200 & -3.44743500 & -4.86464600 \\
\hline $\mathrm{C}$ & 3.96348600 & 4.63348600 & 3.35676700 \\
\hline $\mathrm{H}$ & 4.65111000 & 5.37058900 & 2.92806800 \\
\hline $\mathrm{H}$ & 2.96238500 & 5.08155000 & 3.36676000 \\
\hline $\mathrm{H}$ & 4.25795900 & 4.45679800 & 4.39763000 \\
\hline $\mathrm{C}$ & 4.87345600 & 2.18024000 & -0.95528600 \\
\hline $\mathrm{H}$ & 5.20845200 & 1.19663400 & -1.29528800 \\
\hline $\mathrm{H}$ & 4.07872900 & 2.49290000 & -1.63896800 \\
\hline $\mathrm{H}$ & 5.70628900 & 2.88506700 & -1.05380300 \\
\hline $\mathrm{C}$ & 2.88100300 & -0.28285500 & 3.03609900 \\
\hline $\mathrm{H}$ & 3.71062200 & -0.96451400 & 3.27121000 \\
\hline $\mathrm{H}$ & 2.36606800 & -0.05864100 & 3.97435600 \\
\hline $\mathrm{H}$ & 2.17340800 & -0.80782800 & 2.39110700 \\
\hline $\mathrm{C}$ & 0.66088700 & -3.12996500 & 0.49564700 \\
\hline $\mathrm{H}$ & 1.61106400 & -3.30559300 & 1.01364800 \\
\hline $\mathrm{H}$ & 0.30000300 & -2.14443800 & 0.80889600 \\
\hline $\mathrm{H}$ & -0.05502900 & -3.88087500 & 0.84371500 \\
\hline $\mathrm{C}$ & 3.33214700 & -2.22012700 & -3.73878900 \\
\hline $\mathrm{H}$ & 3.77921500 & -1.37567500 & -3.21400500 \\
\hline $\mathrm{H}$ & 4.07331500 & -3.02942800 & -3.81039100 \\
\hline $\mathrm{H}$ & 3.10690900 & -1.88516100 & -4.75504700 \\
\hline $\mathrm{C}$ & -1.16004700 & -4.50765400 & -4.0006420 \\
\hline
\end{tabular}




$\begin{array}{llll}\mathrm{H} & -1.92073300 & -3.73612500 & -4.18486400 \\ \mathrm{H} & -0.80729300 & -4.86628200 & -4.97356800 \\ \mathrm{H} & -1.65372800 & -5.33913900 & -3.48537700\end{array}$

\section{vinyl-i}

Thermal correction to Gibbs free energy $=0.556904$

Sum of electronic and thermal free energies $=-2428.441199$

E_sol $=-2428.9048487$

$\mathrm{Ru}$

Ru

$2.12414400 \quad 2.28236500-1.14382100$

$\mathrm{Cl}$

$2.43842700 \quad 4.60341700-1.14118000$

$\mathrm{Cl}$

$\begin{array}{llll}2.67070500 & 0.12696100 & -1.90965600\end{array}$

$\mathrm{C}$

$-3.32084000 \quad 5.07871100-1.95733000$

C $\quad \begin{array}{llll}\text { C } & -2.00675200 & 5.76583600 & -1.41631100\end{array}$

B $\quad-1.67261200 \quad 3.53144000 \quad-1.65339400$

$\begin{array}{llll}\mathrm{O} & -1.23210500 & 4.63091000 & -0.95557100\end{array}$

$\begin{array}{llll}\mathrm{O} & -2.85465200 & 3.74864400 & -2.32197000\end{array}$

C $\quad \begin{array}{lllll}-1.17507000 & 6.44279300 & -2.51285200\end{array}$

$\mathrm{H} \quad-1.65179200 \quad 7.35915800 \quad-2.87682900$

$\mathrm{H} \quad-0.19153000 \quad 6.68877600 \quad-2.10263400$

$\mathrm{H} \quad-1.02002400 \quad 5.76637200 \quad-3.35913700$

$\begin{array}{llll}\text { C } & -3.92443800 & 5.73445700 & -3.19629100\end{array}$

$\mathrm{H} \quad-4.81677800 \quad 5.18229400 \quad-3.50812900$

$\mathrm{H} \quad-4.21826700 \quad 6.76819600 \quad-2.98106800$

$\mathrm{H} \quad-3.22037700 \quad 5.73659900 \quad-4.03075200$

$\begin{array}{lllll}\text { C } & -4.39636500 & 4.89005800 & -0.88208100\end{array}$

$\mathrm{H} \quad-4.84330100 \quad 5.84463800 \quad-0.58562300$

$\mathrm{H} \quad \quad-5.18600000 \quad 4.24454100 \quad-1.27897500$

$\mathrm{H} \quad \begin{array}{llll}\mathrm{H} & -3.97921400 & 4.40618900 & 0.00438600\end{array}$

C $\quad \begin{array}{llll}-2.20989500 & 6.71760100 & -0.24100400\end{array}$

$\mathrm{H} \quad \begin{array}{llll}\mathrm{H} & -1.24088600 & 7.11721400 & 0.07389900\end{array}$

$\mathrm{H} \quad-2.85288900 \quad 7.55790500 \quad-0.52631700$

$\mathrm{H} \quad \begin{array}{llll}\mathrm{H} & -2.65847100 & 6.20723600 & 0.61464600\end{array}$

$\begin{array}{lllll}\text { C } & -0.88849500 & 2.20459400 & -1.71738900\end{array}$

$\mathrm{H} \quad-1.33213600 \quad 1.27914800 \quad-2.08375400$

C $\quad 0.37797800 \quad 2.19505200 \quad-1.34380400$

$\begin{array}{llll}\mathrm{C} & 2.27688000 & 1.87250700 & 0.75959600\end{array}$

$\begin{array}{llll}\mathrm{N} & 3.49100400 & 1.85655500 & 1.36499000\end{array}$

$\begin{array}{llll}\mathrm{N} & 1.34748500 & 1.51100200 & 1.66892100\end{array}$

$\begin{array}{lllll}\text { C } & 3.43498900 & 1.39358500 & 2.75815400\end{array}$

$\begin{array}{llll}\mathrm{H} & 3.92196000 & 2.11628100 & 3.41977900\end{array}$ 


\begin{tabular}{|c|c|c|c|}
\hline l & 3.95447500 & 0.43282200 & 2.85311700 \\
\hline $\mathrm{C}$ & 1.92015100 & 1.27655300 & 3.00657500 \\
\hline $\mathrm{H}$ & 1.54637900 & 2.03018900 & 3.70916700 \\
\hline $\mathrm{H}$ & 1.62276100 & 0.29049400 & 3.37528700 \\
\hline $\mathrm{C}$ & -0.07271700 & 1.52070700 & 1.50168500 \\
\hline $\mathrm{C}$ & -0.77157600 & 2.72009700 & 1.70348200 \\
\hline $\mathrm{C}$ & -0.71863600 & 0.32383400 & 1.15653700 \\
\hline $\mathrm{C}$ & -2.16012700 & 2.69375300 & 1.55528000 \\
\hline $\mathrm{C}$ & -2.10566500 & 0.35253400 & 1.00005300 \\
\hline $\mathrm{C}$ & -2.84114000 & 1.52926300 & 1.18571600 \\
\hline $\mathrm{H}$ & -2.71803300 & 3.61380300 & 1.70914700 \\
\hline $\mathrm{H}$ & -2.62341900 & -0.55973400 & 0.71171300 \\
\hline $\mathrm{C}$ & -0.04289700 & 4.01390600 & 1.96764500 \\
\hline $\mathrm{H}$ & -0.74033800 & 4.79733900 & 2.27760800 \\
\hline $\mathrm{H}$ & 0.72427900 & 3.91371900 & 2.74388600 \\
\hline $\mathrm{H}$ & 0.45122900 & 4.35461500 & 1.05054800 \\
\hline $\mathrm{C}$ & 0.07941000 & -0.92901900 & 0.89162700 \\
\hline $\mathrm{H}$ & 0.76680000 & -0.78150000 & 0.04973500 \\
\hline $\mathrm{H}$ & 0.69014300 & -1.21614100 & 1.75687800 \\
\hline $\mathrm{H}$ & -0.57903700 & -1.76998800 & 0.65448600 \\
\hline $\mathrm{C}$ & -4.33064400 & 1.55562300 & 0.93677300 \\
\hline $\mathrm{H}$ & -4.53772800 & 1.85552600 & -0.09875000 \\
\hline $\mathrm{H}$ & -4.78477600 & 0.57161800 & 1.09384300 \\
\hline $\mathrm{H}$ & -4.83677700 & 2.27475000 & 1.59018000 \\
\hline $\mathrm{C}$ & 4.72050600 & 2.25873400 & 0.74731300 \\
\hline $\mathrm{C}$ & 5.10642200 & 3.61220300 & 0.84612300 \\
\hline $\mathrm{C}$ & 5.53520800 & 1.30339800 & 0.11563200 \\
\hline $\mathrm{C}$ & 6.27959500 & 4.00743500 & 0.20564000 \\
\hline $\mathrm{C}$ & 6.70134000 & 1.75348500 & -0.51648600 \\
\hline $\mathrm{C}$ & 7.07719700 & 3.09765800 & -0.50146500 \\
\hline $\mathrm{H}$ & 6.57903100 & 5.05185100 & 0.25540700 \\
\hline $\mathrm{H}$ & 7.33053000 & 1.02745100 & -1.02701800 \\
\hline${ }_{1}$ & 8.31025200 & 3.56941000 & -1.23499900 \\
\hline 11 & 8.04221700 & 3.98823500 & -2.21367800 \\
\hline $\mathrm{H}$ & 8.83210600 & 4.35570000 & -0.67804800 \\
\hline $\mathrm{H}$ & 9.01430600 & 2.74930300 & -1.41000000 \\
\hline $\mathrm{C}$ & 4.30936100 & 4.59265500 & 1.67170700 \\
\hline 11 & 3.23649400 & 4.49417500 & 1.49908200 \\
\hline$\Pi$ & 4.49976600 & 4.43794900 & 2.74336700 \\
\hline I & 4.58966500 & 5.62145800 & 1.43036100 \\
\hline
\end{tabular}




$\begin{array}{llll}\mathrm{C} & 5.23792600 & -0.17515300 & 0.16998800 \\ \mathrm{H} & 5.90543800 & -0.66208700 & 0.89433200 \\ \mathrm{H} & 4.20528700 & -0.37984200 & 0.45337800 \\ \mathrm{H} & 5.40071100 & -0.64342200 & -0.80440500 \\ & & & \\ \text { ts-ii } & & & \end{array}$

Thermal correction to Gibbs free energy $=0.845242$

\section{Sum of electronic and thermal free energies $=-3225.34358$}

E_sol $=-3226.069263$

$\begin{array}{lrrr}\mathrm{Ru} & -1.47200300 & 0.93845200 & -2.12634100 \\ \mathrm{Cl} & 0.66834500 & 1.96750100 & -2.60455600 \\ \mathrm{Cl} & -3.65722200 & 0.11769300 & -1.51975000 \\ \mathrm{C} & -2.29810000 & 2.60237900 & -2.94972700 \\ \mathrm{C} & -2.64834200 & 3.75950600 & -2.41997500 \\ \mathrm{H} & -2.50500000 & 3.96954600 & -1.36379700 \\ \mathrm{C} & -2.01449600 & -1.35693800 & -8.24717100 \\ \mathrm{C} & -1.73420800 & 0.19050600 & -8.23808200 \\ \mathrm{~B} & -1.40388500 & -0.69948900 & -6.15921500 \\ \mathrm{O} & -1.68725500 & 0.48030100 & -6.81225500 \\ \mathrm{O} & -1.40330100 & -1.79137200 & -6.99912700 \\ \mathrm{C} & -0.35880400 & 0.55810300 & -8.80503300 \\ \mathrm{H} & -0.32592000 & 0.43324000 & -9.89242300 \\ \mathrm{H} & -0.14704700 & 1.60503400 & -8.56790000 \\ \mathrm{H} & 0.42741500 & -0.05678600 & -8.35589300 \\ \mathrm{C} & -1.37414600 & -2.12498900 & -9.39834000 \\ \mathrm{H} & -1.61788500 & -3.18868700 & -9.31157800 \\ \mathrm{H} & -1.75404800 & -1.76432100 & -10.36094700 \\ \mathrm{H} & -0.28652300 & -2.02528800 & -9.39017700 \\ \mathrm{C} & -3.50839700 & -1.69001900 & -8.14129800 \\ \mathrm{H} & -4.04117800 & -1.45678500 & -9.06878300 \\ \mathrm{H} & -3.62126100 & -2.75795000 & -7.93183700 \\ \mathrm{H} & -3.97775800 & -1.13262800 & -7.32525600 \\ \mathrm{C} & -2.82771400 & 1.04716400 & -8.87135900 \\ \mathrm{H} & -2.52504900 & 2.09951800 & -8.85846100 \\ \mathrm{H} & -2.98769200 & 0.75549400 & -9.91577400 \\ \mathrm{H} & -3.77171000 & 0.96635400 & -8.32757000 \\ \mathrm{C} & -1.37796700 & 0.04144900 & -3.68946500 \\ & -1.14788500 & -0.83661600 & -4.65003000 \\ \mathrm{H} & -0.75520500 & -1.78414500 & -4.28410800 \\ -2.29433200 & 1.78468300 & -4.11560500\end{array}$




\begin{tabular}{|c|c|c|c|}
\hline $\mathrm{H}$ & -1.50702800 & 2.01009500 & -4.83724700 \\
\hline $\mathrm{H}$ & -3.08085000 & 4.54567200 & -3.03712300 \\
\hline $\mathrm{C}$ & -3.63777300 & 1.35570600 & -4.68926600 \\
\hline $\mathrm{H}$ & -4.35034300 & 1.21115600 & -3.87768300 \\
\hline $\mathrm{H}$ & -3.54867400 & 0.41305400 & -5.22447500 \\
\hline $\mathrm{C}$ & -4.14478300 & 2.40185300 & -5.68570900 \\
\hline $\mathrm{H}$ & -3.36683900 & 2.60787700 & -6.42803300 \\
\hline $\mathrm{H}$ & -4.37561000 & 3.34424300 & -5.16714600 \\
\hline $\mathrm{O}$ & -5.27533800 & 1.91618500 & -6.39581400 \\
\hline $\mathrm{Si}$ & -6.85996400 & 1.82459000 & -5.83876500 \\
\hline $\mathrm{C}$ & -7.00018600 & 2.51867400 & -4.08750300 \\
\hline$H$ & -8.05459500 & 2.57093500 & -3.78992100 \\
\hline & -6.47431400 & 1.90850200 & -3.34539500 \\
\hline $\mathrm{H}$ & -6.59456500 & 3.53592900 & -4.02797300 \\
\hline $\mathrm{C}$ & -7.91207400 & 2.85547300 & -7.01707500 \\
\hline $\mathrm{H}$ & -7.63405000 & 3.91428500 & -6.95278000 \\
\hline $\mathrm{H}$ & -7.76675800 & 2.53692100 & -8.05543500 \\
\hline $\mathrm{H}$ & -8.98107400 & 2.77536900 & -6.78553900 \\
\hline $\mathrm{C}$ & -7.39043700 & -0.00928400 & -5.89823100 \\
\hline $\mathrm{C}$ & -6.46718400 & -0.86710400 & -5.00813200 \\
\hline $\mathrm{H}$ & -6.79922800 & -1.91573100 & -5.01563000 \\
\hline $\mathrm{H}$ & -5.43169400 & -0.84921700 & -5.36262000 \\
\hline $\mathrm{H}$ & -6.46197300 & -0.53260200 & -3.96382000 \\
\hline $\mathrm{C}$ & -8.84300900 & -0.14368100 & -5.39297400 \\
\hline $\mathrm{H}$ & -9.54705900 & 0.44132300 & -5.99839400 \\
\hline $\mathrm{H}$ & -9.16770900 & -1.19342100 & -5.44028100 \\
\hline $\mathrm{H}$ & -8.94444600 & 0.18083200 & -4.34980600 \\
\hline $\mathrm{C}$ & -7.30641800 & -0.52433200 & -7.35078000 \\
\hline $\mathrm{H}$ & -7.57206300 & -1.59094900 & -7.39574500 \\
\hline $\mathrm{H}$ & -7.99421100 & 0.01228800 & -8.01609800 \\
\hline & -6.29394800 & -0.41777600 & -7.75729100 \\
\hline & -0.66528500 & 0.06771800 & -0.41665100 \\
\hline $\mathrm{N}$ & -0.09386500 & -1.14287800 & -0.31405600 \\
\hline N & -0.64493300 & 0.66958400 & 0.78505100 \\
\hline $\mathrm{C}$ & 0.41244600 & -1.41130500 & 1.04937600 \\
\hline & 1.50569000 & -1.46916400 & 1.03507900 \\
\hline & 0.02152300 & -2.36479200 & 1.41530600 \\
\hline & -0.10493100 & -0.19875700 & 1.84797400 \\
\hline & 0.68426900 & 0.31910300 & 2.40006300 \\
\hline & -0.90397700 & -0.46414100 & 2.54946500 \\
\hline
\end{tabular}




\begin{tabular}{|c|c|c|c|}
\hline $\mathrm{C}$ & -1.27275800 & 1.93347700 & 1.05727600 \\
\hline $\mathrm{C}$ & -0.49509100 & 3.10588800 & 0.97867600 \\
\hline $\mathrm{C}$ & -2.62928300 & 1.96937900 & 1.42878900 \\
\hline $\mathrm{C}$ & -1.13835100 & 4.33140500 & 1.16786600 \\
\hline $\mathrm{C}$ & -3.22633700 & 3.22275400 & 1.60202500 \\
\hline $\mathrm{C}$ & -2.50708400 & 4.41126000 & 1.45257800 \\
\hline $\mathrm{H}$ & -0.55476300 & 5.24601700 & 1.09251100 \\
\hline $\mathrm{H}$ & -4.28140100 & 3.26564400 & 1.86325400 \\
\hline $\mathrm{C}$ & 0.99075800 & 3.04201000 & 0.73155600 \\
\hline $\mathrm{H}$ & 1.48843600 & 2.40741600 & 1.47606600 \\
\hline $\mathrm{H}$ & 1.20910400 & 2.63107100 & -0.25778400 \\
\hline $\mathrm{H}$ & 1.43544500 & 4.03966100 & 0.79278900 \\
\hline $\mathrm{C}$ & -3.42027100 & 0.71769200 & 1.72064500 \\
\hline $\mathrm{H}$ & -3.02273100 & -0.15650800 & 1.20534700 \\
\hline $\mathrm{H}$ & -3.41994100 & 0.52115400 & 2.802 \\
\hline $\mathrm{H}$ & -4.45838300 & 0.83023300 & 1.39855900 \\
\hline $\mathrm{C}$ & -3.19008900 & 5.75198900 & 1.58526300 \\
\hline $\mathrm{H}$ & -2.50601800 & 6.51549700 & 1.97106300 \\
\hline $\mathrm{H}$ & -3.55196900 & 6.10162800 & 0.60926400 \\
\hline $\mathrm{H}$ & -4.05609700 & 5.69846500 & 2.25346900 \\
\hline $\mathrm{C}$ & 0.07975200 & -2.09582300 & -1.376 \\
\hline $\mathrm{C}$ & 1.26880300 & -2.08584800 & -2.12685200 \\
\hline $\mathrm{C}$ & -0.93369300 & -3.04326600 & -1.60536300 \\
\hline $\mathrm{C}$ & 1.38881800 & -3.00527100 & -3.17385800 \\
\hline $\mathrm{C}$ & -0.76562300 & -3.94040300 & -2.66498700 \\
\hline $\mathrm{C}$ & 0.37367400 & -3.91915700 & -3.47678400 \\
\hline $\mathrm{H}$ & 2.29013600 & -2.98966900 & -3.78218200 \\
\hline $\mathrm{H}$ & -1.55423000 & -4.66024700 & -2.87171800 \\
\hline $\mathrm{C}$ & 0.47272900 & -4.81072500 & -4.69160800 \\
\hline $\mathrm{H}$ & 0.03220900 & -4.30810800 & -5.56374300 \\
\hline $\mathrm{H}$ & 1.51388900 & -5.04563200 & -4.93744900 \\
\hline $\mathrm{H}$ & -0.06764000 & -5.75253100 & -4.54728900 \\
\hline $\mathrm{C}$ & 2.37655300 & -1.10670300 & -1.83148900 \\
\hline $\mathrm{H}$ & 2.02755200 & -0.07549000 & -1.94247600 \\
\hline $\mathrm{H}$ & 2.75806200 & -1.22416700 & -0.80914800 \\
\hline $\mathrm{H}$ & 3.21713800 & -1.25606800 & -2.51542000 \\
\hline $\mathrm{C}$ & -2.17623200 & -3.08332800 & -0.75224700 \\
\hline $\mathrm{H}$ & -1.93335800 & -3.10958300 & 0.31738200 \\
\hline $\mathrm{H}$ & -2.79688800 & -2.19780400 & -0.92471500 \\
\hline $\mathrm{H}$ & -2.77407500 & -3.97073400 & -0.980641 \\
\hline
\end{tabular}




\section{mcb-ii}

Thermal correction to Gibbs free energy $=0.846414$

Sum of electronic and thermal free energies $=-3225.34924$

E_sol $=-3226.0607676$

$\mathrm{Ru}$

$\begin{array}{lll}-1.12537600 & 0.38794500 & -2.31198700\end{array}$

$\mathrm{Cl}$

$\begin{array}{lll}0.00688200 & 2.52792700 & -2.38976600\end{array}$

$\mathrm{Cl}$

$-2.27114500 \quad-1.72252700 \quad-2.17023500$

$\mathrm{C}$

$-2.78781200 \quad 1.32800100-2.51067800$

$\mathrm{C}$

$-3.78650800 \quad 1.91600800-1.87552900$

$\mathrm{H}$

$\begin{array}{lll}-3.80016900 & 1.95748000 & -0.79096600\end{array}$

$\mathrm{C}$

$\begin{array}{lll}-1.24288100 & 0.07390800 & -8.93417700\end{array}$

$\begin{array}{llll}\text { C } & -2.00360400 & 1.35760600 & -8.43556600\end{array}$

B $\quad-0.85308700 \quad 0.38453900 \quad-6.71555700$

$\begin{array}{llll}\mathrm{O} & -1.91731600 & 1.21779400 & -6.99193700\end{array}$

$\begin{array}{llll}\mathrm{O} & -0.30399600 & -0.17382000 & -7.84893000\end{array}$

C $\quad-1.28395600 \quad 2.66232400 \quad-8.79507500$

$\mathrm{H} \quad-1.35884000 \quad 2.88184200 \quad-9.86532700$

$\mathrm{H} \quad-1.74333700 \quad 3.48427800 \quad-8.23773100$

$\mathrm{H} \quad-0.22612800 \quad 2.61499200 \quad-8.51822600$

C $\quad-0.45715400 \quad 0.24841000 \quad-10.22956100$

$\mathrm{H} \quad 0.04136300 \quad-0.69155400 \quad-10.48720900$

$\mathrm{H} \quad-1.12721400 \quad 0.51754000 \quad-11.05403100$

$\mathrm{H} \quad 0.30836200 \quad 1.02139700 \quad-10.13258200$

C $\quad-2.15135500 \quad-1.15969900 \quad-9.01816400$

$\mathrm{H} \quad-2.85041300 \quad-1.08820800 \quad-9.85771500$

$\mathrm{H} \quad-1.52919600 \quad-2.04926700 \quad-9.15538300$

$\mathrm{H} \quad-2.72817900 \quad-1.28656300 \quad-8.09651000$

C $\quad \begin{array}{llll}\text { C } & -3.47847700 & 1.42913700 & -8.82555200\end{array}$

$\mathrm{H} \quad-3.90507200 \quad 2.37734900 \quad-8.48190400$

$\mathrm{H} \quad-3.59375400 \quad 1.38164100 \quad-9.91452900$

$\mathrm{H} \quad-4.05544000 \quad 0.62331800 \quad-8.36628000$

$\begin{array}{lllll}\text { C } & -1.05758100 & 0.38079800 & -4.19923600\end{array}$

$\begin{array}{llll}\text { C } & -0.36073300 & 0.07895200 & -5.29044600\end{array}$

$\mathrm{H} \quad 0.58545000 \quad-0.43318900 \quad-5.14180300$

C $\quad \begin{array}{llll}-2.47286000 & 1.05824100 & -4.00913000\end{array}$

$\mathrm{H} \quad-2.31301500 \quad 2.04544000 \quad-4.46061900$

$\mathrm{H} \quad-4.61227700 \quad 2.37695100 \quad-2.41261400$

C $\quad-3.58321600 \quad 0.21994900 \quad-4.68291500$

$\mathrm{H} \quad-4.00186400 \quad-0.47559600 \quad-3.95491200$ 


\begin{tabular}{|c|c|c|c|}
\hline $\mathrm{H}$ & -3.16523100 & -0.37266400 & -5.49669100 \\
\hline $\mathrm{C}$ & -4.66987200 & 1.11124900 & -5.27750400 \\
\hline $\mathrm{H}$ & -4.21003000 & 1.83865800 & -5.95538400 \\
\hline $\mathrm{H}$ & -5.19425200 & 1.67012900 & -4.48975000 \\
\hline $\mathrm{O}$ & -5.57004800 & 0.31133400 & -6.03530000 \\
\hline $\mathrm{Si}$ & -7.18701700 & 0.04515100 & -5.67242300 \\
\hline $\mathrm{C}$ & -8.06373500 & 1.70915700 & -5.47866500 \\
\hline $\mathrm{H}$ & -9.12744000 & 1.57657000 & -5.24726100 \\
\hline $\mathrm{H}$ & -7.62604200 & 2.31290500 & -4.67505700 \\
\hline $\mathrm{H}$ & -7.99264600 & 2.28936800 & -6.40630100 \\
\hline $\mathrm{C}$ & -7.84125000 & -0.87803800 & -7.17599000 \\
\hline $\mathrm{H}$ & -7.33959000 & -1.84488800 & -7.29490600 \\
\hline $\mathrm{H}$ & -8.91909400 & -1.06295600 & -7.10177600 \\
\hline $\mathrm{H}$ & -7.66056100 & -0.29439900 & -8.08595600 \\
\hline $\mathrm{C}$ & -7.40720700 & -0.99932100 & -4.08186100 \\
\hline $\mathrm{C}$ & -6.95714600 & -0.21937800 & -2.82667400 \\
\hline $\mathrm{H}$ & -7.13484900 & -0.82226000 & -1.92383300 \\
\hline $\mathrm{H}$ & -5.88913000 & 0.02149600 & -2.83826500 \\
\hline $\mathrm{H}$ & -7.51436500 & 0.71847700 & -2.70581300 \\
\hline $\mathrm{C}$ & -8.90397400 & -1.35371700 & -3.92777600 \\
\hline $\mathrm{H}$ & -9.27722700 & -1.94324100 & -4.77421600 \\
\hline $\mathrm{H}$ & -9.05929700 & -1.95408400 & -3.01943100 \\
\hline $\mathrm{H}$ & -9.53350800 & -0.45934500 & -3.83614800 \\
\hline $\mathrm{C}$ & -6.59329300 & -2.30641900 & -4.18609700 \\
\hline $\mathrm{H}$ & -6.73464400 & -2.91670100 & -3.28222000 \\
\hline $\mathrm{H}$ & -6.90808700 & -2.91403900 & -5.04386700 \\
\hline $\mathrm{H}$ & -5.51987500 & -2.11594500 & -4.28726200 \\
\hline $\mathrm{C}$ & 0.14423100 & -0.16619500 & -0.81090400 \\
\hline $\mathrm{N}$ & 1.36139500 & -0.69514800 & -1.00033300 \\
\hline $\mathrm{N}$ & -0.09926800 & -0.03457900 & 0.50067200 \\
\hline $\mathrm{C}$ & 2.08870400 & -0.87438700 & 0.27254000 \\
\hline & 2.91732400 & -0.15967300 & 0.32508800 \\
\hline & 2.49563900 & -1.88712400 & 0.33721700 \\
\hline & 0.99517600 & -0.59016800 & 1.32284300 \\
\hline $\mathrm{H}$ & 1.30488200 & 0.13430600 & 2.08073000 \\
\hline $\mathrm{H}$ & 0.65035300 & -1.49944400 & 1.82758100 \\
\hline $\mathrm{C}$ & -1.36757700 & 0.35460500 & 1.05652200 \\
\hline & -1.58995300 & 1.71327500 & 1.34499300 \\
\hline$c$ & -2.33742500 & -0.62986300 & 1.31976400 \\
\hline & -2.84923800 & 2.08077700 & 1.82923800 \\
\hline
\end{tabular}




\begin{tabular}{|c|c|c|c|}
\hline $\mathrm{C}$ & -3.58183500 & -0.20806400 & 1.79660900 \\
\hline $\mathrm{C}$ & -3.86358000 & 1.13986900 & 2.03739400 \\
\hline $\mathrm{H}$ & -3.04072300 & 3.12937700 & 2.04554800 \\
\hline $\mathrm{H}$ & -4.35015600 & -0.95538000 & 1.98206400 \\
\hline $\mathrm{C}$ & -0.50854300 & 2.74469200 & 1.14707000 \\
\hline $\mathrm{H}$ & -0.79618500 & 3.69755700 & 1.60148000 \\
\hline $\mathrm{H}$ & 0.43749200 & 2.42588800 & 1.60182600 \\
\hline $\mathrm{H}$ & -0.31475000 & 2.91389200 & 0.08289800 \\
\hline $\mathrm{C}$ & -2.05128800 & -2.10374100 & 1.16516500 \\
\hline $\mathrm{H}$ & -1.31258500 & -2.30370600 & 0.38852200 \\
\hline $\mathrm{H}$ & -1.68728400 & -2.52111200 & 2.11501300 \\
\hline $\mathrm{H}$ & -2.95834000 & -2.64660000 & 0.88753500 \\
\hline $\mathrm{C}$ & -5.23743600 & 1.57049600 & 2.49413700 \\
\hline$\Pi$ & -5.90108400 & 1.72440800 & 1.63298300 \\
\hline $\mathrm{H}$ & -5.70505300 & 0.81298700 & 3.13243600 \\
\hline $\mathrm{H}$ & -5.20025900 & 2.51243800 & 3.05151800 \\
\hline $\mathrm{C}$ & 1.95879800 & -0.93724600 & -2.28662400 \\
\hline $\mathrm{C}$ & 2.76980500 & 0.04809900 & -2.87667200 \\
\hline $\mathrm{C}$ & 1.72579200 & -2.17792700 & -2.91025900 \\
\hline$C$ & 3.25026500 & -0.19120600 & -4.16851400 \\
\hline $\mathrm{C}$ & 2.23682200 & -2.36963400 & -4.19654800 \\
\hline $\mathrm{C}$ & 2.96861200 & -1.37534500 & -4.85615500 \\
\hline $\mathrm{H}$ & 3.84538300 & 0.57880800 & -4.65402700 \\
\hline $\mathrm{H}$ & 2.03973500 & -3.31149000 & -4.70331100 \\
\hline $\mathrm{C}$ & 3.38629700 & -1.55611300 & -6.29587200 \\
\hline $\mathrm{H}$ & 2.57233100 & -1.24629400 & -6.96594700 \\
\hline $\mathrm{H}$ & 4.26527700 & -0.95098300 & -6.54176600 \\
\hline $\mathrm{H}$ & 3.61506100 & -2.60345200 & -6.52153400 \\
\hline $\mathrm{C}$ & 3.16813000 & 1.30360500 & -2.14122800 \\
\hline $\mathrm{H}$ & 2.39713700 & 1.63446200 & -1.44475000 \\
\hline $\mathrm{H}$ & 4.10176700 & 1.13666600 & -1.58499000 \\
\hline $\mathrm{H}$ & 3.34075000 & 2.12542700 & -2.84119800 \\
\hline $\mathrm{C}$ & 0.94769000 & -3.26991300 & -2.22041700 \\
\hline $\mathrm{H}$ & 1.28747100 & -3.41575200 & -1.18765200 \\
\hline $\mathrm{H}$ & -0.11968300 & -3.02899500 & -2.18314900 \\
\hline $\mathrm{H}$ & 1.06541200 & -4.21987700 & -2.7503780 \\
\hline
\end{tabular}




\section{ts-iii}

Thermal correction to Gibbs free energy $=0.845462$

Sum of electronic and thermal free energies $=-3225.336281$

E_sol $=-3226.0607676$

$\begin{array}{llll}\mathrm{Ru} & 0.78264800 & 0.55569900 & -2.32927400\end{array}$

$\begin{array}{llll}\mathrm{Cl} & 2.53460300 & 2.22705200 & -2.14582400\end{array}$

$\mathrm{Cl} \quad-0.86019600 \quad-1.19778500 \quad-2.43724900$

$\begin{array}{lllll}\text { C } & -0.35640500 & 1.89081900 & -2.77048100\end{array}$

C $\quad \begin{array}{llll}\text { C } & -1.15380300 & 2.93492000 & -2.84928700\end{array}$

$\mathrm{H} \quad-1.52451000 \quad 3.37019600 \quad-1.92544900$

$\begin{array}{llll}\text { C } & 2.88835500 & -0.37803400 & -8.32032200\end{array}$

$\begin{array}{llll}\text { C } & 3.79371000 & -1.60334900 & -7.92175500\end{array}$

B $\quad 2.88420600 \quad-0.65731400 \quad-6.05934800$

O $\quad 3.95378100 \quad-1.41346800 \quad-6.48824100$

$\begin{array}{llll}\mathrm{O} & 2.14190900 & -0.13667200 & -7.09650500\end{array}$

C $\quad 3.09397000 \quad-2.95521400 \quad-8.10842400$

$\mathrm{H} \quad 2.98684900 \quad-3.21168300 \quad-9.16746500$

$\mathrm{H} \quad 3.68998600 \quad-3.73234800 \quad-7.62021400$

$\mathrm{H} \quad 2.10147200 \quad-2.94881200 \quad-7.64705300$

C $\quad 1.89676900 \quad-0.64712300 \quad-9.44809000$

$\mathrm{H} \quad 1.30929400 \quad 0.25546900 \quad-9.64428200$

$\mathrm{H} \quad 2.42360400 \quad-0.91951300 \quad-10.36976100$

$\mathrm{H} \quad \begin{array}{llll}\mathrm{H} & 1.20320600 & -1.44965100 & -9.18825400\end{array}$

C $\quad 3.69146600 \quad 0.89701000 \quad-8.60506800$

$\mathrm{H} \quad 4.23931100 \quad 0.82673100 \quad-9.55055100$

$\mathrm{H} \quad 3.00066300 \quad 1.74343900 \quad-8.66469600$

$\mathrm{H} \quad 4.40466000 \quad 1.09848500 \quad-7.79964400$

C $\quad 5.17538200 \quad-1.62410000 \quad-8.56771800$

$\mathrm{H} \quad 5.73054200 \quad-2.50203500 \quad-8.22184600$

$\mathrm{H} \quad 5.09064200 \quad-1.68123500 \quad-9.65888000$

$\mathrm{H} \quad 5.75304300 \quad-0.73500500 \quad-8.30609100$

$\begin{array}{llll}\text { C } & 1.51598200 & 0.28796500 & -4.18846100\end{array}$

$\begin{array}{llll}\text { C } & 2.55523200 & -0.44532700 & -4.57046200\end{array}$

$\mathrm{H} \quad 3.16738800 \quad-0.92075300 \quad-3.80927800$

C $\quad \begin{array}{llll}0.39635400 & 1.05665200 & -4.58330500\end{array}$

$\mathrm{H} \quad 0.62624500 \quad 2.08837000 \quad-4.85324200$

$\mathrm{H} \quad-1.46402300 \quad 3.36574400 \quad-3.79510900$

C $\quad-0.70702000 \quad 0.34893700 \quad-5.36048200$

$\mathrm{H} \quad-0.72730800 \quad-0.70555200 \quad-5.08115500$

$\mathrm{H} \quad-0.40465800 \quad 0.40270200 \quad-6.41464000$ 


\begin{tabular}{|c|c|c|c|}
\hline $\mathrm{C}$ & -2.11131400 & 0.91232800 & -5.18566000 \\
\hline $\mathrm{H}$ & -2.15863900 & 1.95723500 & -5.53221200 \\
\hline $\mathrm{H}$ & -2.36350200 & 0.90325500 & -4.12331300 \\
\hline $\mathrm{O}$ & -3.02148200 & 0.11812800 & -5.93654500 \\
\hline $\mathrm{Si}$ & -4.65497700 & 0.04791700 & -5.54884200 \\
\hline $\mathrm{C}$ & -5.32693900 & 1.80842200 & -5.38355200 \\
\hline $\mathrm{H}$ & -6.38227900 & 1.80990500 & -5.08502000 \\
\hline $\mathrm{H}$ & -4.77045200 & 2.37807500 & -4.62960300 \\
\hline $\mathrm{H}$ & -5.24698700 & 2.34677100 & -6.33553400 \\
\hline $\mathrm{C}$ & -5.43858000 & -0.83243400 & -7.01656000 \\
\hline $\mathrm{H}$ & -5.09776800 & -1.87180400 & -7.08387700 \\
\hline $\mathrm{H}$ & -6.53252400 & -0.83916200 & -6.94929700 \\
\hline $\mathrm{H}$ & -5.15936300 & -0.32906600 & -7.9491310 \\
\hline $\mathrm{C}$ & -4.97533100 & -0.92701000 & -3.92794900 \\
\hline $\mathrm{C}$ & -4.56378900 & -0.13217400 & -2.66765700 \\
\hline $\mathrm{H}$ & -4.85832800 & -0.68980500 & -1.76461100 \\
\hline $\mathrm{H}$ & -3.48205500 & 0.01842400 & -2.60340400 \\
\hline $\mathrm{H}$ & -5.05431100 & 0.84832000 & -2.61606200 \\
\hline $\mathrm{C}$ & -6.48870400 & -1.23061800 & -3.83963400 \\
\hline $\mathrm{H}$ & -6.83719600 & -1.84400000 & -4.67922700 \\
\hline $\mathrm{H}$ & -6.70879400 & -1.78681400 & -2.91643300 \\
\hline $\mathrm{H}$ & -7.09442600 & -0.31479300 & -3.81737400 \\
\hline $\mathrm{C}$ & -4.19186200 & -2.25583700 & -3.96055000 \\
\hline $\mathrm{H}$ & -4.39919300 & -2.84301900 & -3.05335200 \\
\hline $\mathrm{H}$ & -4.47476100 & -2.87451700 & -4.82204200 \\
\hline $\mathrm{H}$ & -3.11177400 & -2.08444600 & -4.00170900 \\
\hline $\mathrm{C}$ & 1.13230100 & 0.05794200 & -0.33647000 \\
\hline $\mathrm{N}$ & 2.11633000 & -0.75722100 & 0.07779100 \\
\hline $\mathrm{N}$ & 0.42344100 & 0.47132600 & 0.72490400 \\
\hline $\mathrm{C}$ & 2.17702000 & -0.86831200 & 1.54841800 \\
\hline $\mathrm{H}$ & 3.08300100 & -0.37514100 & 1.91818000 \\
\hline $\mathrm{H}$ & 2.20470400 & -1.91885600 & 1.85059200 \\
\hline $\mathrm{C}$ & 0.88743600 & -0.14508100 & 1.98504900 \\
\hline $\mathrm{H}$ & 1.06148400 & 0.62527100 & 2.74148100 \\
\hline $\mathrm{H}$ & 0.12312400 & -0.83495600 & 2.36015700 \\
\hline $\mathrm{C}$ & -0.77112500 & 1.26630200 & 0.67131700 \\
\hline $\mathrm{C}$ & -0.64701100 & 2.66543200 & 0.73705100 \\
\hline $\mathrm{C}$ & -2.02418100 & 0.63533000 & 0.58402900 \\
\hline $\mathrm{C}$ & -1.81421900 & 3.43170500 & 0.66089700 \\
\hline $\mathrm{C}$ & -3.15916700 & 1.44723600 & 0.48802800 \\
\hline
\end{tabular}




$\begin{array}{lrrr}\mathrm{C} & -3.07492700 & 2.84167500 & 0.51132200 \\ \mathrm{H} & -1.73348800 & 4.51573500 & 0.70644700 \\ \mathrm{H} & -4.13261600 & 0.97234500 & 0.38795700 \\ \mathrm{C} & 0.70514500 & 3.32047900 & 0.86539000 \\ \mathrm{H} & 0.60083900 & 4.39448100 & 1.04674300 \\ \mathrm{H} & 1.28296800 & 2.89286800 & 1.69424300 \\ \mathrm{H} & 1.29816100 & 3.18044300 & -0.04539700 \\ \mathrm{C} & -2.18280500 & -0.86452200 & 0.64072000 \\ \mathrm{H} & -1.27817100 & -1.38908800 & 0.33300100 \\ \mathrm{H} & -2.44641400 & -1.18031900 & 1.66009800 \\ \mathrm{H} & -2.98235500 & -1.19186300 & -0.02930500 \\ \mathrm{C} & -4.31394200 & 3.69002400 & 0.34748100 \\ \mathrm{H} & -5.18862700 & 3.21773400 & 0.80768700 \\ \mathrm{H} & -4.18639400 & 4.68211800 & 0.79339300 \\ \mathrm{H} & -4.54608000 & 3.83478600 & -0.71612900 \\ \mathrm{C} & 3.08407600 & -1.36465600 & -0.79504400 \\ \mathrm{C} & 4.30517700 & -0.71386400 & -1.04666900 \\ \mathrm{C} & 2.78601500 & -2.62107700 & -1.35975300 \\ \mathrm{C} & 5.16914700 & -1.28743800 & -1.98601500 \\ \mathrm{C} & 3.68630400 & -3.15356700 & -2.28587700 \\ \mathrm{C} & 4.86295300 & -2.48375300 & -2.64231300 \\ \mathrm{H} & 6.09710100 & -0.77206100 & -2.22293900 \\ \mathrm{H} & 3.45375000 & -4.10738200 & -2.75374500 \\ \mathrm{C} & 5.74453600 & -3.01201100 & -3.74809600 \\ \mathrm{H} & 5.38438300 & -2.64462400 & -4.71865400 \\ \mathrm{H} & 6.78215000 & -2.68172800 & -3.63024100 \\ \mathrm{H} & 5.64013900 & -2.88791300 & -1.34699200 \\ \mathrm{H} & & & \end{array}$




\section{vinyl-ii}

Thermal correction to Gibbs free energy $=0.391256$

Sum of electronic and thermal free energies $=-2017.87707$

E_sol $=-2018.2008373$

\begin{tabular}{|c|c|c|c|}
\hline 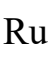 & 2.14966300 & 2.44651200 & -1.03084000 \\
\hline $\mathrm{Cl}$ & 2.38937100 & 4.76758700 & -0.77679200 \\
\hline $\mathrm{Cl}$ & 2.75781500 & 0.38831200 & -1.98146000 \\
\hline $\mathrm{C}$ & 0.39896400 & 2.35982200 & -1.23796400 \\
\hline $\mathrm{C}$ & 2.32943700 & 1.92696200 & 0.84263200 \\
\hline $\mathrm{N}$ & 3.55346400 & 1.86928000 & 1.42411900 \\
\hline $\mathbf{N}$ & 1.40883400 & 1.57167600 & 1.76275500 \\
\hline $\mathrm{C}$ & 3.50774700 & 1.47365900 & 2.83774500 \\
\hline $\mathrm{H}$ & 3.91175900 & 2.27578500 & 3.46516100 \\
\hline $\mathrm{H}$ & 4.11146600 & 0.57370900 & 2.99595900 \\
\hline $\mathrm{C}$ & 2.00328200 & 1.23503700 & 3.06889000 \\
\hline $\mathrm{H}$ & 1.58431200 & 1.87960500 & 3.84839900 \\
\hline $\mathrm{H}$ & 1.77331900 & 0.19620400 & 3.32796700 \\
\hline $\mathrm{C}$ & -0.00858900 & 1.49968900 & 1.59144400 \\
\hline $\mathrm{C}$ & -0.77842200 & 2.64177200 & 1.85667700 \\
\hline $\mathrm{C}$ & -0.58192900 & 0.28764800 & 1.18065100 \\
\hline $\mathrm{C}$ & -2.16350500 & 2.54399100 & 1.70679700 \\
\hline $\mathrm{C}$ & -1.97136100 & 0.23729300 & 1.04403200 \\
\hline $\mathrm{C}$ & -2.77596900 & 1.35375900 & 1.29785900 \\
\hline $\mathrm{H}$ & -2.77825700 & 3.42016900 & 1.90212200 \\
\hline $\mathrm{H}$ & -2.43570200 & -0.69199600 & 0.72074900 \\
\hline $\mathrm{C}$ & -0.11615700 & 3.94759500 & 2.22263800 \\
\hline $\mathrm{H}$ & -0.86196300 & 4.71811700 & 2.43936100 \\
\hline $\mathrm{H}$ & 0.52841700 & 3.84947900 & 3.10504500 \\
\hline $\mathrm{H}$ & 0.51705200 & 4.30501200 & 1.40111500 \\
\hline $\mathrm{C}$ & 0.28628600 & -0.89687000 & 0.83334000 \\
\hline $\mathrm{H}$ & 0.94425700 & -0.66186100 & -0.01220100 \\
\hline $\mathrm{H}$ & 0.93163900 & -1.19384300 & 1.66941500 \\
\hline $\mathrm{H}$ & -0.32401400 & -1.76316300 & 0.56133100 \\
\hline $\mathrm{C}$ & -4.27244700 & 1.28699300 & 1.09943600 \\
\hline $\mathrm{H}$ & -4.54483700 & 1.58416700 & 0.07795500 \\
\hline $\mathrm{H}$ & -4.65459000 & 0.27265700 & 1.25610100 \\
\hline $\mathrm{H}$ & -4.79959400 & 1.95956200 & 1.78465600 \\
\hline $\mathrm{C}$ & 4.78593200 & 2.21771100 & 0.77967800 \\
\hline $\mathrm{C}$ & 5.25009500 & 3.54603900 & 0.86261800 \\
\hline & & 14 & 0.13434 \\
\hline
\end{tabular}




$\begin{array}{lrrr}\mathrm{C} & 6.42791200 & 3.87196000 & 0.18730700 \\ \mathrm{C} & 6.70580400 & 1.59399600 & -0.52685400 \\ \mathrm{C} & 7.15554300 & 2.91731400 & -0.53176200 \\ \mathrm{H} & 6.78543100 & 4.89868600 & 0.22470500 \\ \mathrm{H} & 7.28148700 & 0.83188900 & -1.04773900 \\ \mathrm{C} & 8.39297700 & 3.31174800 & -1.30263800 \\ \mathrm{H} & 8.12908800 & 3.64133400 & -2.31601800 \\ \mathrm{H} & 8.92147700 & 4.14004900 & -0.81832000 \\ \mathrm{H} & 9.08963300 & 2.47267200 & -1.40286000 \\ \mathrm{C} & 4.55711900 & 4.58184700 & 1.71446000 \\ \mathrm{H} & 3.49453700 & 4.37101900 & 1.83954800 \\ \mathrm{H} & 5.02367100 & 4.62335100 & 2.70908300 \\ \mathrm{H} & 4.63905100 & 5.57444600 & 1.26444500 \\ \mathrm{C} & 5.15348600 & -0.24443400 & 0.20686300 \\ \mathrm{H} & 5.77780000 & -0.75540300 & 0.95319800 \\ \mathrm{H} & 4.10595700 & -0.38587900 & 0.47413200 \\ \mathrm{H} & 5.30996300 & -0.73704900 & -0.75623500 \\ \mathrm{C} & -0.86522700 & 2.36113100 & -1.60344200 \\ \mathrm{H} & -1.33384500 & 1.45377900 & -1.97682800 \\ \mathrm{H} & -1.47175400 & 3.25633100 & -1.48832600\end{array}$


ts-iv

Thermal correction to Gibbs free energy $=0.611865$

Sum of electronic and thermal free energies $=-2545.053191$

E_sol $=-2545.5621274$

$\mathrm{Ru} \quad-0.48162100 \quad-0.24514200 \quad-1.60554200$

$\mathrm{Cl} \quad-0.89150600 \quad 2.05154700 \quad-0.96324400$

Cl $\quad-0.00777500 \quad-2.45567900 \quad-2.45986600$

$\begin{array}{llll}\text { C } & -2.13985100 & -0.74097700 & -1.08189700\end{array}$

C $\quad-3.27811200 \quad-1.13794300 \quad-0.55862800$

$\mathrm{H} \quad-3.28249900 \quad-1.47562400 \quad 0.47445600$

C $\quad \begin{array}{llll}-1.89162200 & 1.61294300 & -7.65412100\end{array}$

$\begin{array}{llll}\text { C } & -0.37577200 & 1.62218400 & -8.08121600\end{array}$

B $\quad-0.57191400 \quad 1.12565600 \quad-5.86648600$

$\begin{array}{llll}\mathrm{O} & 0.30375600 & 1.64622200 & -6.79535900\end{array}$

O $\quad-1.84355500 \quad 0.95379400 \quad-6.35868800$

C $\quad 0.06137100 \quad 0.33073800 \quad-8.78341300$

$\mathrm{H} \quad-0.35761200 \quad 0.25830700 \quad-9.79240900$

$\mathrm{H} \quad \begin{array}{llll}\mathrm{H} & 1.15310300 & 0.32070800 & -8.85819600\end{array}$

$\mathrm{H} \quad \begin{array}{llll}0.24726700 & -0.54959900 & -8.21107100\end{array}$

$\begin{array}{llll}\text { C } & -2.81523700 & 0.81731900 & -8.57135900\end{array}$

$\mathrm{H} \quad-3.83977200 \quad 0.86007500 \quad-8.18843100$

$\mathrm{H} \quad-2.81019800 \quad 1.23788700 \quad-9.58347100$

$\mathrm{H} \quad-2.51908500 \quad-0.23247200 \quad-8.62498400$

C $\quad \begin{array}{llll}-2.45755200 & 3.01773100 & -7.41233500\end{array}$

$\mathrm{H} \quad-2.58729100 \quad 3.56748300 \quad-8.35040200$

$\mathrm{H} \quad-3.43226500 \quad 2.92672600 \quad-6.92372900$

$\mathrm{H} \quad-1.80199900 \quad 3.59621200 \quad-6.75388300$

$\begin{array}{llll}\text { C } & 0.06272700 & 2.84133000 & -8.88651500\end{array}$

$\mathrm{H} \quad 1.12863200 \quad 2.76218400 \quad-9.12360500$

$\mathrm{H} \quad-0.49338100 \quad 2.90226200 \quad-9.82892700$

$\mathrm{H} \quad-0.09068200 \quad 3.76679400 \quad-8.32743800$

C $\quad-0.96322600 \quad 0.29917700 \quad-3.52253900$

$\begin{array}{llll}\text { C } & -0.12765000 & 0.77478800 & -4.43230600\end{array}$

$\mathrm{H} \quad 0.91646400 \quad 0.90957500 \quad-4.15936400$

C $\quad-2.23084000 \quad-0.10937200 \quad-3.09223500$

$\mathrm{H} \quad-2.96127600 \quad 0.66557000 \quad-2.86721900$

$\mathrm{H} \quad-2.59518500 \quad-1.07537000 \quad-3.43675200$

$\mathrm{H} \quad-4.20692300 \quad-1.14030700 \quad-1.12097600$

C $\quad 1.02760500 \quad-0.41919700 \quad-0.19816100$ 


\begin{tabular}{|c|c|c|c|}
\hline $\mathrm{N}$ & 2.30363000 & -0.08000500 & -0.45739500 \\
\hline $\mathrm{N}$ & 0.94154400 & -0.87682700 & 1.06087300 \\
\hline $\mathrm{C}$ & 3.21690900 & -0.41046800 & 0.65170300 \\
\hline $\mathrm{H}$ & 3.83335000 & 0.45447500 & 0.91165500 \\
\hline $\mathrm{H}$ & 3.87795300 & -1.23254400 & 0.35373100 \\
\hline $\mathrm{C}$ & 2.23718500 & -0.81272500 & 1.77119700 \\
\hline $\mathrm{H}$ & 2.18527300 & -0.06734100 & 2.57207300 \\
\hline $\mathrm{H}$ & 2.47112200 & -1.78273900 & 2.21834300 \\
\hline $\mathrm{C}$ & -0.24726500 & -1.32577900 & 1.73020000 \\
\hline $\mathrm{C}$ & -1.02729000 & -0.40065700 & 2.44576900 \\
\hline $\mathrm{C}$ & -0.56788300 & -2.69389700 & 1.68900100 \\
\hline $\mathrm{C}$ & -2.17356500 & -0.87392500 & 3.09246600 \\
\hline $\mathrm{C}$ & -1.72382400 & -3.11666600 & 2.35262500 \\
\hline $\mathrm{C}$ & -2.54019700 & -2.22353400 & 3.05470000 \\
\hline $\mathrm{H}$ & -2.79506900 & -0.16757300 & 3.63822100 \\
\hline $\mathrm{H}$ & -1.99187400 & -4.17023200 & 2.31781300 \\
\hline $\mathrm{C}$ & -0.65495300 & 1.05897500 & 2.50688000 \\
\hline $\mathrm{H}$ & -1.34621900 & 1.60819900 & 3.15291900 \\
\hline $\mathrm{H}$ & 0.35683700 & 1.20261900 & 2.90629200 \\
\hline $\mathrm{H}$ & -0.68375200 & 1.51334400 & 1.51132900 \\
\hline $\mathrm{C}$ & 0.29543300 & -3.67634200 & 0.93914800 \\
\hline $\mathrm{H}$ & 0.25755900 & -3.48418600 & -0.13847100 \\
\hline $\mathrm{H}$ & 1.34662900 & -3.60952400 & 1.24662600 \\
\hline $\mathrm{H}$ & -0.03951200 & -4.70251300 & 1.11765500 \\
\hline $\mathrm{C}$ & -3.76878300 & -2.71155900 & 3.78691300 \\
\hline $\mathrm{H}$ & -4.20917000 & -3.58449200 & 3.29337300 \\
\hline $\mathrm{H}$ & -3.52209800 & -3.00797200 & 4.81522400 \\
\hline $\mathrm{H}$ & -4.53532700 & -1.93171100 & 3.84790100 \\
\hline $\mathrm{C}$ & 2.76304100 & 0.35298300 & -1.74914700 \\
\hline $\mathrm{C}$ & 2.83661300 & 1.73621600 & -2.01136000 \\
\hline $\mathrm{C}$ & 3.15436900 & -0.60098700 & -2.70702700 \\
\hline $\mathrm{C}$ & 3.19012600 & 2.14051500 & -3.30058400 \\
\hline $\mathrm{C}$ & 3.49448200 & -0.14263000 & -3.98484200 \\
\hline $\mathrm{C}$ & 3.48600600 & 1.21633800 & -4.31129600 \\
\hline $\mathrm{H}$ & 3.21963200 & 3.20380800 & -3.52649100 \\
\hline $\mathrm{H}$ & 3.75963100 & -0.87259300 & -4.74634600 \\
\hline $\mathrm{C}$ & 3.73603300 & 1.67571300 & -5.72743700 \\
\hline $\mathrm{H}$ & 2.78390500 & 1.74414600 & -6.27047200 \\
\hline $\mathrm{H}$ & 4.20508500 & 2.66549200 & -5.75260200 \\
\hline
\end{tabular}




$\begin{array}{llll}\mathrm{H} & 4.38088500 & 0.97567900 & -6.26979700 \\ \mathrm{C} & 2.55980700 & 2.74927300 & -0.92952700 \\ \mathrm{H} & 3.20123800 & 2.58038800 & -0.05506000 \\ \mathrm{H} & 2.75049800 & 3.76338700 & -1.29279800 \\ \mathrm{H} & 1.51909200 & 2.69616200 & -0.59830100 \\ \mathrm{C} & 3.28260500 & -2.06833000 & -2.37904100 \\ \mathrm{H} & 4.32902800 & -2.30554800 & -2.13962000 \\ \mathrm{H} & 2.65484500 & -2.36433200 & -1.53864900 \\ \mathrm{H} & 2.98208500 & -2.68463000 & -3.22981400\end{array}$

mcb-iii

Thermal correction to Gibbs free energy $=0.612458$

Sum of electronic and thermal free energies $=-2545.066378$

E_sol $=-2545.5784457$

$\begin{array}{lrrr}\mathrm{Ru} & -0.61858600 & -0.47433600 & -1.41188200 \\ \mathrm{Cl} & -1.29621700 & 1.68070600 & -0.57096900 \\ \mathrm{Cl} & 0.00972500 & -2.65175300 & -2.24736200 \\ \mathrm{C} & -2.45486800 & -1.02397600 & -1.37508800 \\ \mathrm{C} & -3.43162300 & -1.56781300 & -0.67328600 \\ \mathrm{H} & -3.26049400 & -1.89489700 & 0.34755500 \\ \mathrm{C} & -2.98539500 & 1.03789900 & -7.01956700 \\ \mathrm{C} & -1.62594300 & 1.21358300 & -7.79476800 \\ \mathrm{~B} & -1.22757500 & 0.82734800 & -5.58560200 \\ \mathrm{O} & -0.67537300 & 1.40011900 & -6.71178500 \\ \mathrm{O} & -2.54756200 & 0.46415100 & -5.75782900 \\ \mathrm{C} & -1.19403900 & -0.05199600 & -8.54628000 \\ \mathrm{H} & -1.82104200 & -0.23437600 & -9.42527100 \\ \mathrm{H} & -0.15784700 & 0.06963000 & -8.87644000 \\ \mathrm{H} & -1.24069300 & -0.92929300 & -7.89349400 \\ \mathrm{C} & -3.98129500 & 0.08235500 & -7.66966400 \\ \mathrm{H} & -4.88512400 & 0.01834700 & -7.05531000 \\ \mathrm{H} & -4.26877400 & 0.44175600 & -8.66439000 \\ \mathrm{H} & -3.56681300 & -0.92349300 & -7.76543500 \\ \mathrm{C} & -3.66431300 & 2.37180300 & -6.68440400 \\ \mathrm{H} & -4.08484900 & 2.84672300 & -7.57695200 \\ \mathrm{H} & -4.47524000 & 2.18731400 & -5.97321500 \\ \mathrm{H} & -2.95657500 & 3.06445900 & -6.21833300 \\ \mathrm{C} & -1.56009500 & 2.42775300 & -8.71642100 \\ \mathrm{H} & -0.57831100 & 2.47069300 & -9.19887100 \\ \mathrm{H} & -2.32367600 & 2.36054600 & -9.49982200 \\ & & & \end{array}$




\begin{tabular}{|c|c|c|c|}
\hline $\mathrm{H}$ & -1.70480400 & 3.35830800 & -8.16341200 \\
\hline $\mathrm{C}$ & -0.94729900 & 0.08106900 & -3.18994400 \\
\hline $\mathrm{C}$ & -0.43146800 & 0.61534800 & -4.29086200 \\
\hline $\mathrm{H}$ & 0.61739100 & 0.89348800 & -4.25582400 \\
\hline $\mathrm{C}$ & -2.36829700 & -0.45772600 & -2.82212600 \\
\hline $\mathrm{H}$ & -3.05226100 & 0.39127600 & -2.90230800 \\
\hline & -2.57541400 & -1.27274900 & -3.52066600 \\
\hline $\mathrm{H}$ & -4.42548800 & -1.70180300 & -1.09666100 \\
\hline C & 1.00747500 & -0.49674700 & -0.17704700 \\
\hline $\mathrm{N}$ & 2.23925000 & -0.10780900 & -0.53676100 \\
\hline$N$ & 1.01516000 & -0.92677400 & 1.09242700 \\
\hline $\mathrm{C}$ & 3.23046000 & -0.35360900 & 0.53015100 \\
\hline & 3.81234100 & 0.55186400 & 0.72237300 \\
\hline & 3.91591300 & -1.14922500 & 0.21874300 \\
\hline C & 2.34263200 & -0.76928600 & 1.72183500 \\
\hline $\mathrm{H}$ & 2.29403600 & 0.00096000 & 2.49881900 \\
\hline $\mathrm{H}$ & 2.65600400 & -1.70970700 & 2.18292300 \\
\hline $\mathrm{C}$ & -0.15877100 & -1.30015500 & 1.83558600 \\
\hline $\mathrm{C}$ & -0.86174100 & -0.31560500 & 2.55494200 \\
\hline & -0.55119200 & -2.65004300 & 1.84515600 \\
\hline $\mathrm{C}$ & -2.03072600 & -0.70163700 & 3.21567200 \\
\hline $\mathrm{C}$ & -1.72564500 & -2.98429200 & 2.52784100 \\
\hline $\mathrm{C}$ & -2.48922500 & -2.02303300 & 3.19739800 \\
\hline $\mathrm{H}$ & -2.59714300 & 0.05188700 & 3.75818100 \\
\hline $\mathrm{H}$ & -2.04915500 & -4.02263800 & 2.53375100 \\
\hline & -0.35537400 & 1.10106500 & 2.67138700 \\
\hline $\mathrm{H}$ & -1.16913100 & 1.78225900 & 2.93360900 \\
\hline $\mathrm{H}$ & 0.40593900 & 1.17386800 & 3.46107300 \\
\hline $\mathrm{H}$ & 0.07645400 & 1.45991800 & 1.73601200 \\
\hline$C$ & 0.26614700 & -3.71066600 & 1.15266100 \\
\hline & 0.20177700 & -3.61090700 & 0.06432600 \\
\hline & 1.32693300 & -3.63988200 & 1.42323200 \\
\hline $\mathrm{H}$ & -0.08480300 & -4.70950300 & 1.42862100 \\
\hline $\mathrm{C}$ & -3.78691100 & -2.39652500 & 3.87434700 \\
\hline$U$ & -4.64071600 & -2.19923200 & 3.21277300 \\
\hline & -3.81370700 & -3.45946800 & 4.13613300 \\
\hline & -3.94501400 & -1.81459800 & 4.78899100 \\
\hline & 2.59989800 & 0.37450100 & -1.84351700 \\
\hline & 2.49992100 & 1.75495900 & -2.10022500 \\
\hline
\end{tabular}




$\begin{array}{lrrr}\mathrm{C} & 3.06158300 & -0.52883400 & -2.81736700 \\ \mathrm{C} & 2.76781400 & 2.20004900 & -3.39772700 \\ \mathrm{C} & 3.30498500 & -0.03320200 & -4.10250600 \\ \mathrm{C} & 3.13082000 & 1.31701900 & -4.42121900 \\ \mathrm{H} & 2.66281000 & 3.25940800 & -3.61985900 \\ \mathrm{H} & 3.62115900 & -0.72744100 & -4.87766400 \\ \mathrm{C} & 3.26112900 & 1.79992100 & -5.84584100 \\ \mathrm{H} & 2.28617700 & 1.74465300 & -6.35012600 \\ \mathrm{H} & 3.59554700 & 2.84206400 & -5.89049400 \\ \mathrm{H} & 3.96553100 & 1.18709800 & -6.41854100 \\ \mathrm{C} & 2.10565700 & 2.72726500 & -1.01740100 \\ \mathrm{H} & 1.04323800 & 2.62916800 & -0.77067400 \\ \mathrm{H} & 2.67504500 & 2.55717000 & -0.09526800 \\ \mathrm{H} & 2.28821700 & 3.75676900 & -1.33941800 \\ \mathrm{C} & 3.34173500 & -1.97632100 & -2.49757500 \\ \mathrm{H} & 4.37127200 & -2.09194700 & -2.12960200 \\ \mathrm{H} & 2.65487700 & -2.37247700 & -1.74883200 \\ \mathrm{H} & 3.23618400 & -2.59818800 & -3.39041800\end{array}$

ts-v

Thermal correction to Gibbs free energy $=0.820656$

Sum of electronic and thermal free energies $=-4035.758022$

E_sol $=-4036.4280007$

$\begin{array}{lrrr}\mathrm{Ru} & 1.62973400 & 0.00773000 & -0.03461600 \\ \mathrm{Cl} & 1.87586100 & -0.53836700 & -2.30769600 \\ \mathrm{Cl} & 1.89117500 & -0.34521100 & 2.27897300 \\ \mathrm{C} & 0.00328400 & 0.75699600 & -0.14728800 \\ \mathrm{C} & 2.88129200 & 1.53563200 & 0.07952500 \\ \mathrm{~N} & 4.22484500 & 1.29559400 & 0.01586000 \\ \mathrm{~N} & 2.72428800 & 2.84723100 & 0.37226800 \\ \mathrm{C} & 5.03433300 & 2.51634700 & 0.15219800 \\ \mathrm{H} & 5.46788600 & 2.78396900 & -0.81945100 \\ \mathrm{H} & 5.84915800 & 2.35708200 & 0.86307900 \\ \mathrm{C} & 4.00236600 & 3.53530400 & 0.63539500 \\ \mathrm{H} & 4.04004200 & 4.48360400 & 0.09229500 \\ \mathrm{H} & 4.08627100 & 3.74854400 & 1.70792300 \\ \mathrm{C} & 1.53855200 & 3.63964700 & 0.45717500 \\ \mathrm{C} & 1.11981200 & 4.30209900 & -0.71080400 \\ \mathrm{C} & 0.88742900 & 3.81955500 & 1.68861800\end{array}$




\begin{tabular}{|c|c|c|c|}
\hline $\mathrm{C}$ & 0.01866100 & 5.15821100 & -0.62884500 \\
\hline $\mathrm{C}$ & -0.21292700 & 4.68613300 & 1.71920900 \\
\hline $\mathrm{C}$ & -0.66265300 & 5.35903500 & 0.57786300 \\
\hline $\mathrm{H}$ & -0.32570500 & 5.66423300 & -1.52793200 \\
\hline $\mathrm{H}$ & -0.74434000 & 4.81870800 & 2.65889400 \\
\hline $\mathrm{C}$ & 1.79880900 & 4.02524500 & -2.03099500 \\
\hline $\mathrm{H}$ & 1.35808500 & 4.62474800 & -2.83276800 \\
\hline $\mathrm{H}$ & 2.87270600 & 4.24638900 & -1.99755700 \\
\hline $\mathrm{H}$ & 1.70161300 & 2.96652700 & -2.30327100 \\
\hline $\mathrm{C}$ & 1.26784100 & 3.04089100 & 2.92050300 \\
\hline $\mathrm{H}$ & 2.31489900 & 2.73035600 & 2.91677800 \\
\hline $\mathrm{H}$ & 1.07097000 & 3.62369900 & 3.82632200 \\
\hline $\mathrm{H}$ & 0.67479200 & 2.11937100 & 2.97013600 \\
\hline $\mathrm{C}$ & -1.88935700 & 6.23821300 & 0.64401800 \\
\hline $\mathrm{H}$ & -1.88070200 & 6.87095300 & 1.53865700 \\
\hline $\mathrm{H}$ & -1.96906800 & 6.88802900 & -0.23340600 \\
\hline $\mathrm{H}$ & -2.79939000 & 5.62595500 & 0.69062700 \\
\hline $\mathrm{C}$ & 4.86733700 & 0.10125700 & -0.45890200 \\
\hline $\mathrm{C}$ & 5.05360300 & -0.07756500 & -1.84284300 \\
\hline $\mathrm{C}$ & 5.41598200 & -0.79664100 & 0.47714700 \\
\hline $\mathrm{C}$ & 5.68182300 & -1.24788300 & -2.27557100 \\
\hline $\mathrm{C}$ & 6.04300700 & -1.94823600 & -0.00757700 \\
\hline $\mathrm{C}$ & 6.15810500 & -2.20625000 & -1.37696000 \\
\hline $\mathrm{H}$ & 5.80147200 & -1.41172200 & -3.34427800 \\
\hline $\mathrm{H}$ & 6.45673100 & -2.65773400 & 0.70529400 \\
\hline $\mathrm{C}$ & 6.77736300 & -3.49221700 & -1.87090200 \\
\hline $\mathrm{H}$ & 6.01275300 & -4.27215800 & -1.98567100 \\
\hline $\mathrm{H}$ & 7.25564300 & -3.36055000 & -2.84740200 \\
\hline $\mathrm{H}$ & 7.52846000 & -3.87299600 & -1.17024100 \\
\hline $\mathrm{C}$ & 4.66752900 & 0.97978200 & -2.84925100 \\
\hline $\mathrm{H}$ & 3.83347200 & 1.59319500 & -2.50723400 \\
\hline $\mathrm{H}$ & 5.52467200 & 1.63776400 & -3.05304500 \\
\hline $\mathrm{H}$ & 4.36399100 & 0.52221300 & -3.79381100 \\
\hline $\mathrm{C}$ & 5.39931000 & -0.49882800 & 1.95573000 \\
\hline $\mathrm{H}$ & 6.01021300 & 0.38548000 & 2.18210500 \\
\hline $\mathrm{H}$ & 4.38777800 & -0.30136200 & 2.31795100 \\
\hline $\mathrm{H}$ & 5.81540700 & -1.33634400 & 2.52405300 \\
\hline $\mathrm{Ru}$ & -1.78508100 & -0.06412200 & -0.06647300 \\
\hline $\mathrm{C}$ & -2.74509600 & -1.86627500 & 0.2097540 \\
\hline
\end{tabular}




\begin{tabular}{|c|c|c|c|}
\hline $\mathrm{N}$ & -4.05699800 & -2.09462800 & 0.02564600 \\
\hline $\mathrm{C}$ & -4.41928800 & -3.49688900 & 0.32279000 \\
\hline $\mathrm{C}$ & -3.04405400 & -4.17287500 & 0.44020600 \\
\hline $\mathrm{N}$ & -2.13542900 & -3.01353000 & 0.53503200 \\
\hline $\mathrm{H}$ & -2.78528200 & -4.76308600 & -0.44717300 \\
\hline $\mathrm{H}$ & -2.94732900 & -4.80946500 & 1.32280500 \\
\hline $\mathrm{H}$ & -5.03345300 & -3.90871100 & -0.48111200 \\
\hline $\mathrm{H}$ & -4.99191000 & -3.53976900 & 1.25679700 \\
\hline $\mathrm{C}$ & -0.72583800 & -3.18387400 & 0.75092300 \\
\hline $\mathrm{C}$ & -0.27334900 & -3.30131300 & 2.08142000 \\
\hline $\mathrm{C}$ & 1.08205900 & -3.53225600 & 2.29112800 \\
\hline $\mathrm{C}$ & 1.99364100 & -3.60244000 & 1.22982300 \\
\hline $\mathrm{C}$ & 1.51402500 & -3.47706800 & -0.07293000 \\
\hline $\mathrm{C}$ & 0.14277100 & -3.31540600 & -0.34543900 \\
\hline $\mathrm{H}$ & 1.45030700 & -3.60978900 & 3.31091100 \\
\hline $\mathrm{H}$ & 2.20419500 & -3.53074500 & -0.91129800 \\
\hline $\mathrm{C}$ & -0.33017700 & -3.37624800 & -1.77552200 \\
\hline $\mathrm{H}$ & -0.03666700 & -4.33680100 & -2.21805700 \\
\hline $\mathrm{H}$ & -1.41013700 & -3.26194700 & -1.86974500 \\
\hline $\mathrm{H}$ & 0.12573900 & -2.57704700 & -2.36615000 \\
\hline $\mathrm{C}$ & -1.21938900 & -3.13805400 & 3.24355000 \\
\hline $\mathrm{H}$ & -0.70927600 & -3.34482000 & 4.18887700 \\
\hline $\mathrm{H}$ & -2.08471800 & -3.80909900 & 3.17521100 \\
\hline $\mathrm{H}$ & -1.59733900 & -2.11050300 & 3.27447800 \\
\hline $\mathrm{C}$ & 3.46434300 & -3.76243900 & 1.51483000 \\
\hline $\mathrm{H}$ & 3.65229300 & -4.61157700 & 2.18338500 \\
\hline$\Pi$ & 3.84339000 & -2.86110500 & 2.00975100 \\
\hline П & 4.04243700 & -3.90625700 & 0.59791200 \\
\hline $\mathrm{C}$ & -5.06258700 & -1.12129800 & -0.30044500 \\
\hline $\mathrm{C}$ & -5.62676400 & -0.34216600 & 0.72479400 \\
\hline $\mathrm{C}$ & -6.59890800 & 0.60086000 & 0.37085900 \\
\hline $\mathrm{H}$ & -7.03714500 & 1.21706700 & 1.15292400 \\
\hline$c$ & -6.47657800 & -0.06780600 & -1.93316900 \\
\hline $\mathrm{C}$ & -5.50852300 & -1.02902700 & -1.63223100 \\
\hline $\mathrm{H}$ & -6.81643000 & 0.02766600 & -2.96197500 \\
\hline $\mathrm{C}$ & -7.02020700 & 0.76747300 & -0.95135300 \\
\hline $\mathrm{C}$ & -5.00176700 & -1.96499600 & -2.70101000 \\
\hline $\mathrm{H}$ & -5.27439600 & -1.60048200 & -3.69555200 \\
\hline $\mathrm{H}$ & -5.44211000 & -2.96569700 & -2.58647700 \\
\hline
\end{tabular}




\begin{tabular}{lrrr}
$\mathrm{H}$ & -3.91461500 & -2.05725600 & -2.66611200 \\
$\mathrm{H}$ & -5.16885200 & -1.56482100 & 2.45579900 \\
$\mathrm{C}$ & -5.21538700 & -0.50874800 & 2.16553100 \\
$\mathrm{H}$ & -5.92800100 & -0.00947000 & 2.82922200 \\
$\mathrm{H}$ & -4.22234500 & -0.08555700 & 2.34755100 \\
$\mathrm{C}$ & -8.02978800 & 1.83031500 & -1.31677900 \\
$\mathrm{H}$ & -8.65444600 & 2.10437900 & -0.46000000 \\
$\mathrm{H}$ & -8.68795800 & 1.49773100 & -2.12706200 \\
$\mathrm{H}$ & -7.52701300 & 2.74346000 & -1.66218200 \\
$\mathrm{Cl}$ & -1.76802500 & 0.35034800 & 2.32463200 \\
$\mathrm{Cl}$ & -1.87421800 & -0.45110800 & -2.43703800 \\
$\mathrm{C}$ & -2.56016400 & 1.60458800 & -0.37653000 \\
$\mathrm{C}$ & -0.85776900 & 1.93350400 & -0.44138500 \\
$\mathrm{H}$ & -0.69175900 & 2.27972200 & -1.46229400 \\
$\mathrm{H}$ & -0.77877100 & 2.67388200 & 0.34792700 \\
$\mathrm{C}$ & -3.50796400 & 2.50555200 & -0.54915600 \\
$\mathrm{H}$ & -4.55114500 & 2.20786500 & -0.50830100 \\
$\mathrm{H}$ & -3.26666900 & 3.54758800 & -0.74523500 \\
mcb-iv & & & \\
\hline
\end{tabular}

Thermal correction to Gibbs free energy $=0.821726$

Sum of electronic and thermal free energies $=-4035.765996$

E_sol $=-4036.4382293$

$\begin{array}{lrrr}\mathrm{Ru} & 1.26887900 & 2.92229000 & 0.45527600 \\ \mathrm{Cl} & 0.56360500 & 5.09987400 & 1.01836000 \\ \mathrm{Cl} & 2.76513500 & 1.10420900 & 0.28208600 \\ \mathrm{C} & 0.40844000 & 2.83332700 & -1.11671100 \\ \mathrm{C} & 0.19907100 & 1.88254900 & 1.75663700 \\ \mathrm{~N} & 0.57096000 & 1.91798400 & 3.07168900 \\ \mathrm{~N} & -0.78806800 & 0.96420800 & 1.64748500 \\ \mathrm{C} & -0.27457400 & 1.07577500 & 3.93169400 \\ \mathrm{H} & -0.93177900 & 1.70922800 & 4.54040800 \\ \mathrm{H} & 0.34284000 & 0.47230200 & 4.60201700 \\ \mathrm{C} & -1.04586200 & 0.24265200 & 2.90811100 \\ \mathrm{H} & -2.12050800 & 0.19740900 & 3.10484100 \\ \mathrm{H} & -0.66693900 & -0.78307300 & 2.82380200 \\ \mathrm{C} & -1.59752000 & 0.61084600 & 0.52505900 \\ \mathrm{C} & -2.81188200 & 1.30186500 & 0.36137000 \\ \mathrm{C} & -1.22588000 & -0.45063800 & -0.31683500 \\ \mathrm{C} & -3.65820100 & 0.91579900 & -0.68098200\end{array}$




\begin{tabular}{|c|c|c|c|}
\hline $\mathrm{C}$ & -2.10817900 & -0.79955300 & -1.34774600 \\
\hline $\mathrm{C}$ & -3.31954500 & -0.12966400 & -1.54892300 \\
\hline $\mathrm{H}$ & -4.59154900 & 1.45371500 & -0.8309460 \\
\hline $\mathrm{H}$ & -1.82454900 & -1.60148900 & -2.02549400 \\
\hline $\mathrm{C}$ & -3.14419700 & 2.48349300 & 1.24129900 \\
\hline $\mathrm{H}$ & -4.12560600 & 2.89611300 & 0.99017600 \\
\hline $\mathrm{H}$ & -3.15416400 & 2.21653600 & 2.30520500 \\
\hline $\mathrm{H}$ & -2.39858900 & 3.27998100 & 1.12189000 \\
\hline $\mathrm{C}$ & 0.11227500 & -1.13368500 & -0.20728600 \\
\hline $\mathrm{H}$ & 0.53202200 & -1.08020800 & 0.79944200 \\
\hline $\mathrm{H}$ & 0.03915000 & -2.18365900 & -0.50933800 \\
\hline $\mathrm{H}$ & 0.83411200 & -0.63684500 & -0.86707400 \\
\hline $\mathrm{C}$ & -4.21002800 & -0.49591100 & -2.71271900 \\
\hline $\mathrm{H}$ & -4.28912000 & -1.58248100 & -2.8303650 \\
\hline $\mathrm{H}$ & -5.21985400 & -0.09038100 & -2.5928770 \\
\hline $\mathrm{H}$ & -3.79864400 & -0.09826400 & -3.6495880 \\
\hline $\mathrm{C}$ & 1.43359100 & 2.88251700 & 3.69687500 \\
\hline $\mathrm{C}$ & 0.90332200 & 4.12341400 & 4.09891700 \\
\hline $\mathrm{C}$ & 2.75435000 & 2.51154900 & 4.01638700 \\
\hline $\mathrm{C}$ & 1.76471500 & 5.04114600 & 7000 \\
\hline $\mathrm{C}$ & 3.57260900 & 3.46297800 & 4.63244100 \\
\hline $\mathrm{C}$ & 3.10630500 & 4.74102900 & 4.95529400 \\
\hline $\mathrm{H}$ & 1.37320500 & 6.01536500 & 4.98937600 \\
\hline $\mathrm{H}$ & 4.59890800 & 3.19384700 & 4.87109200 \\
\hline $\mathrm{C}$ & 4.03268200 & 5.77196500 & 5.55538500 \\
\hline $\mathrm{H}$ & 4.52225200 & 6.35941000 & 4.76711900 \\
\hline $\mathrm{H}$ & 3.49139100 & 6.47629800 & 6.19611700 \\
\hline $\mathrm{H}$ & 4.82361600 & 5.30442800 & 6.15198800 \\
\hline $\mathrm{C}$ & -0.56428800 & 4.45209500 & 3.96573200 \\
\hline $\mathrm{H}$ & -1.03387100 & 3.92443500 & 3.13522600 \\
\hline $\mathrm{H}$ & -1.09512900 & 4.18895700 & 4.89211200 \\
\hline $\mathrm{H}$ & -0.70675800 & 5.52079700 & 3.78917400 \\
\hline $\mathrm{C}$ & 3.25881100 & 1.11153200 & 3.77125000 \\
\hline $\mathrm{H}$ & 2.69548200 & 0.38256200 & 4.36894300 \\
\hline $\mathrm{H}$ & 3.15707600 & 0.82315400 & 2.72225200 \\
\hline $\mathrm{H}$ & 4.31154600 & 1.02429200 & 4.05703900 \\
\hline $\mathrm{Ru}$ & 0.80779900 & 3.32666200 & -2.91229100 \\
\hline $\mathrm{C}$ & 2.34430300 & 4.12445300 & -4.02267800 \\
\hline $\mathrm{N}$ & 2.25915700 & 4.52397400 & -5.3014310 \\
\hline
\end{tabular}




\begin{tabular}{|c|c|c|c|}
\hline $\mathrm{C}$ & 3.53177700 & 5.09314800 & -5.79146400 \\
\hline $\mathrm{C}$ & 4.37778400 & 5.16859900 & -4.50773500 \\
\hline $\mathrm{N}$ & 3.56324800 & 4.39958300 & -3.54546800 \\
\hline $\mathrm{H}$ & 4.50002300 & 6.19367400 & -4.13904100 \\
\hline $\mathrm{H}$ & 5.36746200 & 4.71780200 & -4.61499100 \\
\hline $\mathrm{H}$ & 3.35383300 & 6.07143000 & -6.24538300 \\
\hline $\mathrm{H}$ & 3.96643000 & 4.43056100 & -6.54853100 \\
\hline $\mathrm{C}$ & 4.02315600 & 4.15657100 & -2.20643500 \\
\hline $\mathrm{C}$ & 4.85334300 & 3.03789700 & -1.98837700 \\
\hline $\mathrm{C}$ & 5.36042600 & 2.84214400 & -0.70807600 \\
\hline $\mathrm{C}$ & 5.02879200 & 3.68989000 & 0.35628100 \\
\hline $\mathrm{C}$ & 4.19572000 & 4.78011000 & 0.10854100 \\
\hline$C$ & 3.71473700 & 5.06696700 & -1.18231500 \\
\hline $\mathrm{H}$ & 5.99006300 & 1.97655800 & -0.51890000 \\
\hline $\mathrm{H}$ & 3.92630600 & 5.45087900 & 0.92077800 \\
\hline $\mathrm{C}$ & 2.96356900 & 6.35295600 & -1.41443000 \\
\hline $\mathrm{H}$ & 3.57350600 & 7.20180900 & -1.08064100 \\
\hline $\mathrm{H}$ & 2.69545600 & 6.50239900 & -2.46060000 \\
\hline $\mathrm{H}$ & 2.03007700 & 6.36123900 & -0.84476400 \\
\hline$C$ & 5.14292100 & 2.05463200 & -3.09352900 \\
\hline $\mathrm{H}$ & 5.87805400 & 1.31281100 & -2.76818100 \\
\hline $\mathrm{H}$ & 5.53320800 & 2.54135000 & -3.99592800 \\
\hline $\mathrm{H}$ & 4.22371600 & 1.52784300 & -3.37257100 \\
\hline $\mathrm{C}$ & 5.53845000 & 3.38684900 & 1.74142400 \\
\hline $\mathrm{H}$ & 6.63001000 & 3.27716300 & 1.74999000 \\
\hline $\mathrm{H}$ & 5.10874300 & 2.44308900 & 2.09574000 \\
\hline $\mathrm{H}$ & 5.26175600 & 4.16735700 & 2.45529700 \\
\hline $\mathrm{C}$ & 1.10913800 & 4.41495500 & -6.15543300 \\
\hline $\mathrm{C}$ & 0.87639400 & 3.20854400 & -6.84125500 \\
\hline $\mathrm{C}$ & -0.26177100 & 3.12452700 & -7.64969100 \\
\hline $\mathrm{H}$ & -0.46001700 & 2.19430500 & -8.17759800 \\
\hline $\mathrm{C}$ & -0.84420700 & 5.40050300 & -7.14806300 \\
\hline $\mathrm{C}$ & 0.28434400 & 5.53998600 & -6.33498100 \\
\hline $\mathrm{H}$ & -1.50317800 & 6.25630700 & -7.27732900 \\
\hline $\mathrm{C}$ & -1.14438100 & 4.19954700 & -7.79740800 \\
\hline $\mathrm{C}$ & 0.62181200 & 6.88511900 & -5.74030600 \\
\hline $\mathrm{H}$ & -0.28788700 & 7.44911500 & -5.51682900 \\
\hline $\mathrm{H}$ & 1.21437300 & 7.47771800 & -6.45248500 \\
\hline $\mathrm{H}$ & 1.17978500 & 6.79227600 & -4.80845800 \\
\hline
\end{tabular}




\begin{tabular}{llll}
$\mathrm{H}$ & 2.86752300 & 2.35329500 & -6.82321300 \\
$\mathrm{C}$ & 1.82142900 & 2.03935100 & -6.72741600 \\
$\mathrm{H}$ & 1.61823600 & 1.30310900 & -7.51112400 \\
$\mathrm{H}$ & 1.72436500 & 1.54302300 & -5.75659900 \\
$\mathrm{C}$ & -2.39895200 & 4.06256900 & -8.62772800 \\
$\mathrm{H}$ & -3.24745400 & 3.75381100 & -8.00273600 \\
$\mathrm{H}$ & -2.28122300 & 3.30918100 & -9.41399300 \\
$\mathrm{H}$ & -2.67357200 & 5.01131800 & -9.10142300 \\
$\mathrm{Cl}$ & 1.61400400 & 1.07724800 & -3.31131300 \\
$\mathrm{Cl}$ & -0.05769400 & 5.55792800 & -2.70119200 \\
$\mathrm{C}$ & -0.97288100 & 2.78798300 & -3.35242900 \\
$\mathrm{C}$ & -0.95857400 & 2.54349200 & -1.83197200 \\
$\mathrm{H}$ & -1.67945400 & 3.20666400 & -1.34747900 \\
$\mathrm{H}$ & -1.12690300 & 1.48037200 & -1.68823800 \\
$\mathrm{C}$ & -1.90405300 & 2.62352400 & -4.27417100 \\
$\mathrm{H}$ & -1.71006500 & 2.87610600 & -5.31134500 \\
$\mathrm{H}$ & -2.88939300 & 2.24371000 & -4.00934000 \\
ts-vi & & & \\
\hline
\end{tabular}

Thermal correction to Gibbs free energy $=0.821055$

Sum of electronic and thermal free energies $=-4035.760665$

E_sol $=-4036.4324463$

$\begin{array}{rrrr}\mathrm{Ru} & 1.40006500 & 3.02826300 & 0.12924600 \\ \mathrm{Cl} & 0.64522100 & 5.19178800 & 0.70433200 \\ \mathrm{Cl} & 2.83833000 & 1.16199200 & -0.14570300 \\ \mathrm{C} & 0.62148700 & 3.01922100 & -1.47502700 \\ \mathrm{C} & 0.31805100 & 1.92750500 & 1.37852300 \\ \mathrm{~N} & 0.72872400 & 1.92633300 & 2.68280900 \\ \mathrm{~N} & -0.66795300 & 1.01091000 & 1.27019500 \\ \mathrm{C} & -0.09724000 & 1.06625200 & 3.54333300 \\ \mathrm{H} & -0.75158900 & 1.68581000 & 4.16959200 \\ \mathrm{H} & 0.53344500 & 0.45735400 & 4.19580200 \\ \mathrm{C} & -0.87400800 & 0.24506200 & 2.51589700 \\ \mathrm{H} & -1.94124500 & 0.16518900 & 2.73782100 \\ \mathrm{H} & -0.46960600 & -0.76638700 & 2.38829000 \\ \mathrm{C} & -1.52057800 & 0.68419000 & 0.17132500 \\ \mathrm{C} & -2.75678700 & 1.35373100 & 0.09626300 \\ \mathrm{C} & -1.17313600 & -0.34041700 & -0.72397700 \\ \mathrm{C} & -3.64670100 & 0.98730600 & -0.91504000 \\ \mathrm{C} & -2.09939100 & -0.66839000 & -1.72349700\end{array}$




\begin{tabular}{|c|c|c|c|}
\hline $\mathrm{C}$ & -3.32995100 & -0.01625000 & -1.83997300 \\
\hline $\mathrm{H}$ & -4.59671700 & 1.51030300 & -0.99 \\
\hline $\mathrm{H}$ & -1.83402200 & -1.43839400 & -2.44408600 \\
\hline $\mathrm{C}$ & -3.06733600 & 2.49518700 & 1.03501900 \\
\hline $\mathrm{H}$ & -4.06526700 & 2.89994200 & 0.84327900 \\
\hline $\mathrm{H}$ & -3.02605900 & 2.18953600 & 2.08781500 \\
\hline $\mathrm{H}$ & -2.34059100 & 3.30826700 & 0.91252600 \\
\hline $\mathrm{C}$ & 0.17494000 & -1.01163100 & -0.69869200 \\
\hline $\mathrm{H}$ & 0.64983900 & -0.96853300 & 0.28350500 \\
\hline $\mathrm{H}$ & 0.09321200 & -2.05767800 & -1.01241700 \\
\hline $\mathrm{H}$ & 0.85306100 & -0.49897400 & -1.39133400 \\
\hline $\mathrm{C}$ & -4.26637300 & -0.34471800 & -2.97844900 \\
\hline $\mathrm{H}$ & -3.95632900 & 0.18248900 & -3.89037500 \\
\hline $\mathrm{H}$ & -4.26156600 & -1.41612500 & -3.20668400 \\
\hline $\mathrm{H}$ & -5.29583400 & -0.04568300 & -2.7553300 \\
\hline $\mathrm{C}$ & 1.61363600 & 2.87721300 & 3.29788000 \\
\hline $\mathrm{C}$ & 1.10194300 & 4.11505500 & 3.73388700 \\
\hline $\mathrm{C}$ & 2.94151800 & 2.49410900 & 3.57529600 \\
\hline $\mathrm{C}$ & 1.98786100 & 5.02283800 & 4.31901300 \\
\hline $\mathrm{C}$ & 3.78375400 & 3.43531500 & 4.17423900 \\
\hline $\mathrm{C}$ & 3.33556800 & 4.71376000 & 4.52095700 \\
\hline $\mathrm{H}$ & 1.61080000 & 5.99561000 & 4.62658200 \\
\hline $\mathrm{H}$ & 4.81368600 & 3.15671900 & 4.38329300 \\
\hline $\mathrm{C}$ & 4.28725000 & 5.73449000 & 5.09819500 \\
\hline $\mathrm{H}$ & 5.09549000 & 5.25771200 & 5.66346100 \\
\hline $\mathrm{H}$ & 4.75283300 & 6.32599900 & 4.29848300 \\
\hline $\mathrm{H}$ & 3.77199100 & 6.43626900 & 5.76269100 \\
\hline $\mathrm{C}$ & -0.36891400 & 4.44679800 & 3.66109300 \\
\hline $\mathrm{H}$ & -0.86850800 & 3.93971800 & 2.83540400 \\
\hline $\mathrm{H}$ & -0.86514200 & 4.15985400 & 4.59955700 \\
\hline $\mathrm{H}$ & -0.51689600 & 5.51931000 & 3.51607500 \\
\hline $\mathrm{C}$ & 3.42752700 & 1.09197600 & 3.30732300 \\
\hline $\mathrm{H}$ & 2.84915000 & 0.35960900 & 3.88564400 \\
\hline $\mathrm{H}$ & 3.32887000 & 0.82597500 & 2.25184700 \\
\hline $\mathrm{H}$ & 4.47638700 & 0.98421900 & 3.60005400 \\
\hline $\mathrm{Ru}$ & 0.70953400 & 3.42514700 & -3.23135400 \\
\hline $\mathrm{C}$ & 2.34837500 & 4.27784500 & -4.23046000 \\
\hline $\mathrm{N}$ & 2.31870900 & 4.70744500 & -5.50430400 \\
\hline $\mathrm{C}$ & 3.54192200 & 5.43761900 & -5.89121100 \\
\hline
\end{tabular}




\begin{tabular}{|c|c|c|c|}
\hline $\mathrm{C}$ & 4.35157800 & 5.44565000 & -4.58146300 \\
\hline $\mathrm{N}$ & 3.50585100 & 4.64529600 & -3.66932700 \\
\hline $\mathrm{H}$ & 4.48383900 & 6.45100900 & -4.16942400 \\
\hline $\mathrm{H}$ & 5.33688700 & 4.98072800 & -4.68143100 \\
\hline $\mathrm{H}$ & 3.28206300 & 6.44227300 & -6.23782300 \\
\hline $\mathrm{H}$ & 4.05036100 & 4.91151600 & -6.70636800 \\
\hline $\mathrm{C}$ & 4.00960300 & 4.27519300 & -2.37640800 \\
\hline $\mathrm{C}$ & 4.81388700 & 3.12407300 & -2.28860000 \\
\hline $\mathrm{C}$ & 5.43108200 & 2.85009100 & -1.06961800 \\
\hline $\mathrm{C}$ & 5.22257400 & 3.64796900 & 0.05906500 \\
\hline $\mathrm{C}$ & 4.38456400 & 4.76034900 & -0.05205400 \\
\hline $\mathrm{C}$ & 3.79825900 & 5.12466200 & -1.27658700 \\
\hline $\mathrm{H}$ & 6.05197300 & 1.96193500 & -0.98384200 \\
\hline $\mathrm{H}$ & 4.20260300 & 5.38715800 & 0.81738500 \\
\hline $\mathrm{C}$ & 3.05093700 & 6.42579900 & -1.41265200 \\
\hline $\mathrm{H}$ & 3.64478200 & 7.14696300 & -1.99268800 \\
\hline $\mathrm{H}$ & 2.08994000 & 6.29886400 & -1.91464400 \\
\hline $\mathrm{H}$ & 2.85243500 & 6.85920100 & -0.43033400 \\
\hline $\mathrm{C}$ & 4.95608100 & 2.17946400 & -3.45462700 \\
\hline $\mathrm{H}$ & 5.74099300 & 1.44167800 & -3.26431400 \\
\hline $\mathrm{H}$ & 5.19591600 & 2.69661100 & -4.39137700 \\
\hline $\mathrm{H}$ & 4.01240800 & 1.64310400 & -3.61067400 \\
\hline $\mathrm{C}$ & 5.86223700 & 3.27293300 & 1.37127600 \\
\hline $\mathrm{H}$ & 6.93979700 & 3.10468700 & 1.25474600 \\
\hline $\mathrm{H}$ & 5.42306400 & 2.34283300 & 1.74967200 \\
\hline $\mathrm{H}$ & 5.71221700 & 4.04573600 & 2.13014100 \\
\hline $\mathrm{C}$ & 1.26491000 & 4.45954400 & -6.44746200 \\
\hline $\mathrm{C}$ & 1.26666900 & 3.23634000 & -7.15007000 \\
\hline $\mathrm{C}$ & 0.21820200 & 2.98797200 & -8.03760500 \\
\hline $\mathrm{H}$ & 0.19868800 & 2.04201700 & -8.57406700 \\
\hline $\mathrm{C}$ & -0.72401100 & 5.15469500 & -7.60274100 \\
\hline $\mathrm{C}$ & 0.30624400 & 5.45444000 & -6.70254100 \\
\hline $\mathrm{H}$ & -1.48526500 & 5.90884800 & -7.79096100 \\
\hline $\mathrm{C}$ & -0.79850400 & 3.92547700 & -8.26064000 \\
\hline $\mathrm{C}$ & 0.38711800 & 6.83732400 & -6.10415500 \\
\hline $\mathrm{H}$ & -0.59394800 & 7.16259700 & -5.74704000 \\
\hline $\mathrm{H}$ & 0.72411600 & 7.55437700 & -6.86580000 \\
\hline $\mathrm{H}$ & 1.06213700 & 6.88034800 & -5.25027000 \\
\hline $\mathrm{H}$ & 2.37706000 & 1.80327100 & -5.97556900 \\
\hline
\end{tabular}




$\begin{array}{llll}\mathrm{C} & 2.38481800 & 2.23854500 & -6.97848300 \\ \mathrm{H} & 3.36480300 & 2.70725200 & -7.13280600 \\ \mathrm{H} & 2.28703200 & 1.42313600 & -7.70136600 \\ \mathrm{C} & -1.95046000 & 3.60529100 & -9.18391400 \\ \mathrm{H} & -1.62653400 & 2.99232800 & -10.03234000 \\ \mathrm{H} & -2.41674200 & 4.51471000 & -9.57710300 \\ \mathrm{H} & -2.72865200 & 3.04017900 & -8.65397600 \\ \mathrm{Cl} & 1.36598500 & 1.16700500 & -3.78696000 \\ \mathrm{Cl} & -0.09260600 & 5.68646400 & -3.02986400 \\ \mathrm{C} & -1.19853500 & 2.94441500 & -3.63171200 \\ \mathrm{C} & -1.92465900 & 2.89157800 & -4.73117900 \\ \mathrm{H} & -1.51217500 & 3.15285700 & -5.70037400 \\ \mathrm{H} & -2.97096200 & 2.59213600 & -4.68579400 \\ \mathrm{C} & -1.16657100 & 2.73043200 & -2.22419300 \\ \mathrm{H} & -1.65561300 & 3.46574900 & -1.58826600 \\ \mathrm{H} & -1.19909500 & 1.69581200 & -1.92102100\end{array}$

\section{Ru-carbide}

Thermal correction to Gibbs free energy $=0.771101$

Sum of electronic and thermal free energies $=-3919.180629$

E_sol $=-3919.8010054$

$\mathrm{C}$

$\begin{array}{lll}-1.41263300 & -0.48190800 & 0.09889200\end{array}$

$\mathrm{N} \quad-2.74439000 \quad-0.29658100 \quad 0.20222700$

C $\quad-3.50976900 \quad-1.51660300 \quad-0.12246200$

C $\quad-2.42212800 \quad-2.59946600 \quad-0.13036300$

$\mathrm{N} \quad-1.19035200 \quad-1.79709200 \quad-0.14881100$

$\mathrm{H} \quad-2.46723800 \quad-3.24773700 \quad-1.00980400$

$\mathrm{H} \quad-2.44385200 \quad-3.22722600 \quad 0.76913000$

$\mathrm{H} \quad \quad-3.99212800 \quad-1.39873200 \quad-1.09999600$

$\mathrm{H} \quad-4.28629900 \quad-1.69097100 \quad 0.62578200$

$\begin{array}{llll}\text { C } & 0.09778900 & -2.40787900 & -0.31011000\end{array}$

$\begin{array}{llll}\text { C } & 0.81643700 & -2.84712300 & 0.81746900\end{array}$

$\begin{array}{llll}\text { C } & 2.10626100 & -3.35642700 & 0.61577600\end{array}$

$\begin{array}{llll}\text { C } & 2.65927300 & -3.48250200 & -0.65918900\end{array}$

C $\quad \begin{array}{llll}\text { C } & 1.87174700 & -3.13397000 & -1.76375900\end{array}$

C $\quad 0.58795800 \quad-2.60825600 \quad-1.61726700$

$\mathrm{H} \quad 2.68097800 \quad-3.67511500 \quad 1.48314900$

$\mathrm{H} \quad 2.27182200 \quad-3.26649500 \quad-2.76661300$

C $\quad-0.26457800 \quad-2.33393200 \quad-2.83153300$

$\mathrm{H} \quad-0.86508900 \quad-3.22124800 \quad-3.07996800$ 


\begin{tabular}{|c|c|c|c|}
\hline $\mathrm{H}$ & -0.93734700 & -1.49065400 & -2.67409200 \\
\hline $\mathrm{H}$ & 0.35807700 & -2.09944800 & -3.69889100 \\
\hline $\mathrm{C}$ & 0.21658100 & -2.89072900 & 2.20203500 \\
\hline $\mathrm{H}$ & 0.90588900 & -2.48171100 & 2.94534100 \\
\hline $\mathrm{H}$ & 0.00562600 & -3.93435500 & 2.47301700 \\
\hline $\mathrm{H}$ & -0.70389400 & -2.31353700 & 2.27942900 \\
\hline $\mathrm{C}$ & 4.07754200 & -3.96500000 & -0.84720200 \\
\hline 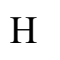 & 4.18759900 & -4.54327800 & -1.77111000 \\
\hline $\mathrm{H}$ & 4.40610200 & -4.59012700 & -0.01021800 \\
\hline $\mathrm{H}$ & 4.76782800 & -3.11319900 & -0.91175800 \\
\hline $\mathrm{C}$ & -3.43519500 & 0.94380600 & 0.36701500 \\
\hline $\mathrm{C}$ & -4.03641700 & 1.22835900 & 1.59939000 \\
\hline $\mathrm{C}$ & -4.76928400 & 2.41445300 & 1.71685700 \\
\hline $\mathrm{H}$ & -5.22815800 & 2.65237200 & 2.67260200 \\
\hline $\mathrm{C}$ & -4.18318500 & 3.05146700 & -0.52954500 \\
\hline $\mathrm{C}$ & -3.53168000 & 1.81754300 & -0.73401700 \\
\hline $\mathrm{H}$ & -4.26401300 & 3.73898100 & -1.36712400 \\
\hline $\mathrm{C}$ & -4.83110200 & 3.34743800 & 0.68280900 \\
\hline $\mathrm{C}$ & -3.04960800 & 1.39493100 & -2.10076500 \\
\hline $\mathrm{H}$ & -3.29718400 & 2.14917900 & -2.84798400 \\
\hline $\mathrm{H}$ & -3.52510200 & 0.44877800 & -2.39021800 \\
\hline $\mathrm{H}$ & -1.96635400 & 1.25078700 & -2.13144300 \\
\hline $\mathrm{H}$ & -4.11902100 & -0.70360500 & 2.60382200 \\
\hline $\mathrm{C}$ & -3.81652500 & 0.33379900 & 2.79189500 \\
\hline 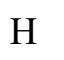 & -4.37572600 & 0.69575800 & 3.65916700 \\
\hline $\mathrm{H}$ & -2.75002400 & 0.32413900 & 3.04681800 \\
\hline $\mathrm{C}$ & -5.47214800 & 4.69299800 & 0.90427400 \\
\hline $\mathrm{H}$ & -4.77341100 & 5.33980200 & 1.45013500 \\
\hline $\mathrm{H}$ & -6.38636600 & 4.60996900 & 1.50275700 \\
\hline$H$ & -5.71712800 & 5.18962100 & -0.04035100 \\
\hline$C$ & 0.02809300 & 0.34933800 & 2.70328000 \\
\hline $\mathrm{C}$ & 0.68339700 & 0.68604100 & -1.97245100 \\
\hline $\mathrm{Ru}$ & 0.12897200 & 0.75122400 & 0.35010700 \\
\hline $\mathrm{C}$ & -0.70582300 & 2.29573500 & 0.24768400 \\
\hline $\mathrm{Ru}$ & -1.54060100 & 3.84023600 & 0.14528600 \\
\hline $\mathrm{Cl}$ & -2.09502000 & 3.90532600 & 2.46785200 \\
\hline $\mathrm{Cl}$ & -1.43960000 & 4.24224400 & -2.20786900 \\
\hline$C$ & 0.00104300 & 5.07333300 & 0.39656300 \\
\hline & 1.01055900 & 7.19085000 & 0.62610100 \\
\hline
\end{tabular}




\begin{tabular}{|c|c|c|c|}
\hline $\mathrm{H}$ & 1.05568700 & 7.83890700 & 1.50569800 \\
\hline $\mathrm{H}$ & 1.03223700 & 7.81882400 & -0.27324100 \\
\hline $\mathrm{C}$ & 2.09820000 & 6.10798500 & 0.61791400 \\
\hline $\mathrm{H}$ & 2.58068500 & 5.98997700 & 1.59536800 \\
\hline $\mathrm{H}$ & 2.87463400 & 6.28246300 & -0.13040300 \\
\hline $\mathrm{N}$ & -0.22123100 & 6.38847300 & 0.64441000 \\
\hline & 1.33279200 & 4.88799800 & 0.29314400 \\
\hline $\mathrm{C}$ & -1.50934300 & 6.99927400 & 0.80587600 \\
\hline $\mathrm{C}$ & -2.22803200 & 7.43864500 & -0.32162100 \\
\hline $\mathrm{C}$ & -1.99942500 & 7.19954500 & 2.11308200 \\
\hline $\mathrm{C}$ & -3.51779500 & 7.94806200 & -0.11981000 \\
\hline $\mathrm{C}$ & -3.28314600 & 7.72539300 & 2.25969100 \\
\hline $\mathrm{C}$ & -4.07070200 & 8.07411400 & 1.15520000 \\
\hline $\mathrm{H}$ & -4.09253100 & 8.26687000 & -0.98712500 \\
\hline $\mathrm{H}$ & -3.68315000 & 7.85787900 & 3.26257900 \\
\hline $\mathrm{C}$ & 2.02357300 & 3.64759300 & 0.12834000 \\
\hline $\mathrm{C}$ & 2.12003200 & 2.77385300 & 1.22936500 \\
\hline $\mathrm{C}$ & 2.62484200 & 3.36305300 & -1.10402100 \\
\hline $\mathrm{C}$ & 2.77152800 & 1.53992000 & 1.02490000 \\
\hline $\mathrm{C}$ & 3.35770000 & 2.17695300 & -1.22147600 \\
\hline $\mathrm{C}$ & 3.41947400 & 1.24395300 & -0.18743600 \\
\hline $\mathrm{H}$ & 2.85233100 & 0.85240500 & 1.86248000 \\
\hline $\mathrm{H}$ & 3.81660100 & 1.93903700 & -2.17720800 \\
\hline $\mathrm{C}$ & -1.62828900 & 7.48222900 & -1.70623600 \\
\hline $\mathrm{H}$ & -2.31755000 & 7.07293600 & -2.44943900 \\
\hline $\mathrm{H}$ & -1.41761400 & 8.52586900 & -1.97737600 \\
\hline $\mathrm{H}$ & -0.70768500 & 6.90524600 & -1.78363100 \\
\hline $\mathrm{C}$ & -5.48888800 & 8.55680800 & 1.34335100 \\
\hline $\mathrm{H}$ & -5.59880500 & 9.13498100 & 2.26734100 \\
\hline $\mathrm{H}$ & -5.81739100 & 9.18209900 & 0.50646600 \\
\hline $\mathrm{H}$ & -6.17930800 & 7.70511100 & 1.40782800 \\
\hline $\mathrm{C}$ & -1.14688100 & 6.92501200 & 3.32729500 \\
\hline $\mathrm{H}$ & -1.76952400 & 6.69005700 & 4.19453300 \\
\hline $\mathrm{H}$ & -0.47389600 & 6.08195300 & 3.16960100 \\
\hline $\mathrm{H}$ & -0.54660500 & 7.81239000 & 3.57607300 \\
\hline $\mathrm{C}$ & 2.40501200 & 4.25763100 & -2.29652600 \\
\hline $\mathrm{H}$ & 1.33850900 & 4.26740800 & -2.55143000 \\
\hline $\mathrm{H}$ & 2.70763100 & 5.29500200 & -2.10846300 \\
\hline $\mathrm{H}$ & 2.96416000 & 3.89561000 & -3.16380500 \\
\hline
\end{tabular}




$\begin{array}{lrrr}\mathrm{C} & 1.63798300 & 3.19647100 & 2.59611700 \\ \mathrm{H} & 2.11352000 & 4.14260400 & 2.88557300 \\ \mathrm{H} & 0.55473500 & 3.34065000 & 2.62681100 \\ \mathrm{H} & 1.88553600 & 2.44220700 & 3.34332800 \\ \mathrm{C} & 4.06048700 & -0.10162400 & -0.40890400 \\ \mathrm{H} & 4.97461400 & -0.01864200 & -1.00752900 \\ \mathrm{H} & 4.30560100 & -0.59818900 & 0.53571800 \\ \mathrm{H} & 3.36166300 & -0.74845600 & -0.95462300\end{array}$

\section{Ru-vinyl-4}

Thermal correction to Gibbs free energy $=0.962202$

Sum of electronic and thermal free energies $=-3353.539264$

E_sol $=-3354.3115942$

$\begin{array}{llll}\mathrm{Ru} & 1.16264600 & 1.33595800 & -2.09545100 \\ \mathrm{Cl} & 1.19377700 & 3.68613400 & -2.55997000 \\ \mathrm{Cl} & 1.75936200 & -0.99578700 & -1.82976700 \\ \mathrm{C} & -4.46965700 & 3.91816600 & -0.88839000 \\ \mathrm{C} & -3.15843100 & 4.77365700 & -1.13523400 \\ \mathrm{~B} & -2.63328800 & 2.54786600 & -1.10194400 \\ \mathrm{O} & -2.10135500 & 3.80901900 & -0.94764400 \\ \mathrm{O} & -4.01931700 & 2.55977100 & -1.11614000 \\ \mathrm{C} & -3.02330600 & 5.28854900 & -2.57332900 \\ \mathrm{H} & -3.75322300 & 6.07353000 & -2.79855600 \\ \mathrm{H} & -2.01433100 & 5.68941100 & -2.70647800 \\ \mathrm{H} & -3.14891300 & 4.47173100 & -3.28760600 \\ \mathrm{C} & -5.61497800 & 4.21355300 & -1.85767300 \\ \mathrm{H} & -6.46324200 & 3.55876200 & -1.63344700 \\ \mathrm{H} & -5.94934400 & 5.25257200 & -1.75947600 \\ \mathrm{H} & -5.32145900 & 4.03749600 & -2.89390100 \\ \mathrm{C} & -4.99410200 & 3.98792600 & 0.54978600 \\ \mathrm{H} & -5.39698400 & 4.97849300 & 0.78566400 \\ \mathrm{H} & -5.79289100 & 3.24980200 & 0.67249500 \\ \mathrm{H} & -4.20668800 & 3.74911200 & 1.26556900 \\ \mathrm{C} & -2.93964700 & 5.91917500 & -0.14870100 \\ \mathrm{H} & -1.99287900 & 6.41736400 & -0.37946300 \\ \mathrm{H} & -3.74542500 & 6.65865500 & -0.21963900 \\ \mathrm{H} & -2.88533800 & 5.55638600 & 0.88031300 \\ \mathrm{C} & -1.80952400 & 1.27295700 & -1.29037900 \\ \mathrm{H} & -2.30632500 & 0.30409300 & -1.27283900 \\ \mathrm{C} & -0.53045700 & 1.27930900 & -1.62497000 \\ & & & \\ & & & \end{array}$




$\begin{array}{lrrr}\mathrm{C} & 1.90627700 & 1.53591300 & -0.11172300 \\ \mathrm{~N} & 3.21205200 & 1.81952600 & 0.11667500 \\ \mathrm{~N} & 1.38183400 & 1.10950500 & 1.06249000 \\ \mathrm{C} & 3.66229400 & 1.47267900 & 1.47770300 \\ \mathrm{H} & 4.19838200 & 2.31467900 & 1.92585100 \\ \mathrm{H} & 4.34091300 & 0.61363200 & 1.43563100 \\ \mathrm{C} & 2.34381500 & 1.14527500 & 2.18239100 \\ \mathrm{H} & 2.04404700 & 1.91495900 & 2.90412500 \\ \mathrm{H} & 2.35939400 & 0.18123400 & 2.69775100 \\ \mathrm{C} & -0.00537000 & 1.02418900 & 1.41402300 \\ \mathrm{C} & -0.70180300 & 2.21750600 & 1.66859500 \\ \mathrm{C} & -0.60484000 & -0.23149100 & 1.59137500 \\ \mathrm{C} & -2.03561600 & 2.12690400 & 2.06600700 \\ \mathrm{C} & -1.95689700 & -0.26848700 & 1.94815400 \\ \mathrm{C} & -2.69340000 & 0.89758200 & 2.17189200 \\ \mathrm{H} & -2.57861400 & 3.04412000 & 2.27551900 \\ \mathrm{H} & -2.44537400 & -1.23518300 & 2.05210400 \\ \mathrm{H} & -0.06219700 & 3.56256500 & 1.43824300 \\ \mathrm{C} & -0.68267800 & 4.36378500 & 1.85031000 \\ \mathrm{H} & & & \end{array}$




\begin{tabular}{|c|c|c|c|}
\hline $\mathrm{H}$ & 7.20322100 & 5.65107300 & -2.73198500 \\
\hline $\mathrm{H}$ & 8.05491900 & 4.12960400 & -3.05710000 \\
\hline $\mathrm{C}$ & 3.15346400 & 4.68464000 & 0.09779500 \\
\hline $\mathrm{H}$ & 2.13260800 & 4.53383300 & -0.25871100 \\
\hline $\mathrm{H}$ & 3.19049700 & 4.37415400 & 1.14967100 \\
\hline $\mathrm{H}$ & 3.37442500 & 5.75564900 & 0.05708400 \\
\hline & 5.19964100 & 0.26470200 & -1.34106000 \\
\hline $\mathrm{H}$ & 5.67759700 & -0.04489300 & -0.40115700 \\
\hline $\mathrm{H}$ & 4.21318300 & -0.20364200 & -1.37957000 \\
\hline $\mathrm{H}$ & 5.80153000 & -0.13666700 & -2.16103500 \\
\hline & 0.89909700 & 0.81530500 & -4.17907500 \\
\hline & -0.20461100 & 0.33893300 & -4.79769300 \\
\hline & 1.92103400 & 0.68743400 & -5.06425600 \\
\hline & 0.01695400 & -0.07125800 & -6.19787200 \\
\hline 1 & -0.51534500 & 0.61165800 & -6.87112200 \\
\hline $\mathrm{H}$ & -0.37067800 & -1.08037100 & -6.36233900 \\
\hline $\mathrm{C}$ & 1.53376300 & 0.02439200 & -6.32795800 \\
\hline $\mathrm{H}$ & 1.86149200 & 0.62554700 & -7.18148900 \\
\hline $\mathrm{H}$ & 2.01675300 & -0.95669500 & -6.39269800 \\
\hline & 3.29337900 & 1.10384400 & -4.99795700 \\
\hline & 3.61111500 & 2.40888400 & -5.42750700 \\
\hline & 4.29928300 & 0.13856800 & -4.79857100 \\
\hline $\mathrm{C}$ & 4.95785400 & 2.74053900 & -5.60180300 \\
\hline$C$ & 5.63057000 & 0.52030300 & -4.99403500 \\
\hline & 5.98054700 & 1.80875000 & -5.40343100 \\
\hline & 5.20865200 & 3.74180900 & -5.94485500 \\
\hline $\mathrm{H}$ & 6.41165200 & -0.22499900 & -4.85251900 \\
\hline & 2.52818200 & 3.39451600 & -5.78494800 \\
\hline & 2.96005800 & 4.31736300 & -6.18426300 \\
\hline & 1.85359200 & 2.98132000 & -6.54658300 \\
\hline & 1.91909900 & 3.64530700 & -4.91266100 \\
\hline & 3.98555400 & -1.30529300 & -4.48876100 \\
\hline & 2.98287600 & -1.43198500 & -4.08207900 \\
\hline & 4.08264200 & -1.91940200 & -5.39493300 \\
\hline $\mathrm{H}$ & 4.68889100 & -1.70517000 & -3.75120600 \\
\hline & 7.42775200 & 2.16676900 & -5.64614600 \\
\hline & 7.98600700 & 2.24037300 & -4.70462500 \\
\hline & 7.92662500 & 1.40548200 & -6.25735500 \\
\hline & 7.52437600 & 3.12942600 & -6.15824800 \\
\hline
\end{tabular}




$\begin{array}{lrrr}\text { C } & -1.57044900 & 0.41061000 & -4.37967300 \\ \mathrm{C} & -2.27319800 & 1.61055600 & -4.57616400 \\ \mathrm{C} & -2.19396000 & -0.74651900 & -3.89366200 \\ \mathrm{C} & -3.62846900 & 1.63414400 & -4.24572200 \\ \mathrm{C} & -3.55124700 & -0.66960100 & -3.56424900 \\ \mathrm{C} & -4.27973600 & 0.51306900 & -3.71941700 \\ \mathrm{H} & -4.18919400 & 2.55537600 & -4.38363000 \\ \mathrm{H} & -4.04587000 & -1.55077900 & -3.16063300 \\ \mathrm{C} & -5.71455100 & 0.60911600 & -3.26448800 \\ \mathrm{H} & -5.74379300 & 1.06657900 & -2.26831800 \\ \mathrm{H} & -6.31007100 & 1.24034200 & -3.93425500 \\ \mathrm{H} & -6.19144600 & -0.37517600 & -3.20709800 \\ \mathrm{C} & -1.56389400 & 2.85157900 & -5.05800000 \\ \mathrm{H} & -0.92164100 & 3.25818600 & -4.26682800 \\ \mathrm{H} & -0.91411400 & 2.65275200 & -5.91782500 \\ \mathrm{H} & -2.28075100 & 3.62449300 & -5.35129000 \\ \mathrm{C} & -1.40798700 & -2.01669000 & -3.67964000 \\ \mathrm{H} & -1.02857000 & -2.42694400 & -4.62479000 \\ \mathrm{H} & -0.53726500 & -1.83718000 & -3.03960400 \\ \mathrm{H} & -2.03135100 & -2.78651900 & -3.21404800\end{array}$

\section{Derivative of Ru-vinyl-4 with $R=H$}

Thermal correction to Gibbs free energy $=0.794738$

Sum of electronic and thermal free energies $=-2942.976417$

E_sol $=-2943.6127016$

$\begin{array}{lrrr}\mathrm{Ru} & 1.09274900 & 1.33264500 & -2.12995800 \\ \mathrm{Cl} & 0.64596100 & 3.67884600 & -2.58809800 \\ \mathrm{Cl} & 1.87386000 & -0.94266300 & -1.77382700 \\ \mathrm{C} & -1.79980600 & 0.72185800 & -1.23938200 \\ \mathrm{H} & -2.55710900 & 0.43393500 & -1.95660900 \\ \mathrm{C} & -0.56688000 & 0.98229100 & -1.61924400 \\ \mathrm{C} & 1.91259900 & 1.72053300 & -0.20630900 \\ \mathrm{~N} & 3.19735700 & 2.10475600 & -0.01398700 \\ \mathrm{~N} & 1.40513200 & 1.42360300 & 1.01429100 \\ \mathrm{C} & 3.65697400 & 1.92861700 & 1.37807500 \\ \mathrm{H} & 4.19948300 & 2.81685600 & 1.71298000 \\ \mathrm{H} & 4.33057100 & 1.06547900 & 1.43419900 \\ \mathrm{C} & 2.34447400 & 1.69954100 & 2.12037400 \\ \mathrm{H} & 2.00919800 & 2.58486700 & 2.67597900 \\ \mathrm{H} & 2.37825300 & 0.85388100 & 2.81130600\end{array}$




\begin{tabular}{|c|c|c|c|}
\hline $\mathrm{C}$ & 0.04044900 & 1.22730200 & 1.39929900 \\
\hline C & -0.80181600 & 2.34858800 & 1.51151000 \\
\hline $\mathrm{C}$ & -0.37461100 & -0.05386600 & 1.80020400 \\
\hline $\mathrm{C}$ & -2.08640900 & 2.15582900 & 2.02916000 \\
\hline $\mathrm{C}$ & -1.66965000 & -0.19616700 & 2.30714300 \\
\hline $\mathrm{C}$ & -2.53886400 & 0.89375700 & 2.42921500 \\
\hline $\mathrm{H}$ & -2.75174700 & 3.01234600 & 2.11347900 \\
\hline $\mathrm{H}$ & -2.00843200 & -1.18435200 & 2.61060500 \\
\hline $\mathrm{C}$ & -0.35605700 & 3.71068400 & 1.04382300 \\
\hline $\mathrm{H}$ & -1.07142300 & 4.48061800 & 1.34838700 \\
\hline $\mathrm{H}$ & 0.62628200 & 3.98456100 & 1.44514900 \\
\hline $\mathrm{H}$ & -0.26918200 & 3.73518900 & -0.04861200 \\
\hline $\mathrm{C}$ & 0.54183900 & -1.24283600 & 1.66477700 \\
\hline $\mathrm{H}$ & 0.90190800 & -1.33892600 & 0.63543500 \\
\hline $\mathrm{H}$ & 1.42671300 & -1.14934900 & 2.30798100 \\
\hline $\mathrm{H}$ & 0.02538100 & -2.16571300 & 1.94608400 \\
\hline $\mathrm{C}$ & -3.94633400 & 0.70552000 & 2.94564500 \\
\hline $\mathrm{H}$ & -4.63752400 & 0.47477500 & 2.12409700 \\
\hline $\mathrm{H}$ & -4.00454000 & -0.12163000 & 3.66148400 \\
\hline $\mathrm{H}$ & -4.31777000 & 1.61040400 & 3.43871100 \\
\hline$C$ & 4.12372100 & 2.72165500 & -0.91765600 \\
\hline $\mathrm{C}$ & 4.08945600 & 4.12791700 & -1.03362100 \\
\hline $\mathrm{C}$ & 5.17589200 & 1.96499400 & -1.45582600 \\
\hline $\mathrm{C}$ & 5.10577000 & 4.75420700 & -1.75577900 \\
\hline $\mathrm{C}$ & 6.19128900 & 2.64685000 & -2.13664600 \\
\hline $\mathrm{C}$ & 6.16873000 & 4.03033300 & -2.30989800 \\
\hline $\mathrm{H}$ & 5.08532900 & 5.83698700 & -1.86097600 \\
\hline $\mathrm{H}$ & 7.01687500 & 2.07300400 & -2.54824600 \\
\hline $\mathrm{C}$ & 7.24906400 & 4.73396700 & -3.09668800 \\
\hline $\mathrm{H}$ & 6.89712000 & 4.99060500 & -4.10540600 \\
\hline $\mathrm{H}$ & 7.55210700 & 5.67139700 & -2.61633800 \\
\hline $\mathrm{H}$ & 8.13900900 & 4.10588000 & -3.20974900 \\
\hline$c$ & 3.02526300 & 4.93934000 & -0.33944100 \\
\hline $\mathrm{H}$ & 2.03259900 & 4.69658100 & -0.72654400 \\
\hline $\mathrm{H}$ & 3.01904300 & 4.74333100 & 0.74104000 \\
\hline $\mathrm{H}$ & 3.19828000 & 6.01015600 & -0.48356800 \\
\hline $\mathrm{C}$ & 5.25720700 & 0.46896700 & -1.27696200 \\
\hline 11 & 5.82801700 & 0.21148700 & -0.37345900 \\
\hline $\mathrm{H}$ & 4.26877500 & 0.01085300 & -1.19744300 \\
\hline
\end{tabular}




\begin{tabular}{|c|c|c|c|}
\hline $\mathrm{H}$ & 5.76657900 & 0.01152600 & -2.13061800 \\
\hline $\mathrm{C}$ & 0.81675100 & 1.05908500 & -4.22098700 \\
\hline $\mathrm{N}$ & -0.33624500 & 1.00572800 & -4.93054600 \\
\hline $\mathrm{N}$ & 1.82590400 & 1.15846700 & -5.11937000 \\
\hline $\mathrm{C}$ & -0.12186000 & 0.97212600 & -6.39165100 \\
\hline $\mathrm{H}$ & -0.78283600 & 1.68507900 & -6.89009900 \\
\hline $\mathrm{H}$ & -0.34741200 & -0.03182800 & -6.77386400 \\
\hline & 1.35578000 & 1.33153300 & -6.50829000 \\
\hline & 1.51479400 & 2.37003000 & -6.82149300 \\
\hline & 1.91357900 & 0.67493700 & -7.18147700 \\
\hline $\mathrm{C}$ & 3.24257700 & 1.03518800 & -4.93674700 \\
\hline $\mathrm{C}$ & 4.04876500 & 2.18335500 & -4.96955700 \\
\hline $\mathrm{C}$ & 3.79965900 & -0.26089100 & -4.98139600 \\
\hline $\mathrm{C}$ & 5.43630000 & 2.00780200 & -5.02768300 \\
\hline & 5.18967800 & -0.38020100 & -4.99890900 \\
\hline & 6.02467100 & 0.74350900 & -5.02837300 \\
\hline $\mathrm{H}$ & 6.07032200 & 2.88923500 & -5.06485900 \\
\hline $\mathrm{H}$ & 5.63163900 & -1.37417000 & -5.02319900 \\
\hline $\mathrm{C}$ & 3.46008200 & 3.57246000 & -4.99123300 \\
\hline $\mathrm{H}$ & 4.14116500 & 4.28131800 & -4.51057800 \\
\hline $\mathrm{H}$ & 3.29881600 & 3.91536800 & -6.02314200 \\
\hline $\mathrm{H}$ & 2.50503800 & 3.62142000 & -4.46333200 \\
\hline $\mathrm{C}$ & 2.92061500 & -1.48176400 & -5.07854300 \\
\hline & 2.28650000 & -1.57945300 & -4.19404500 \\
\hline $\mathrm{H}$ & 2.26302800 & -1.42771700 & -5.95635500 \\
\hline $\mathrm{H}$ & 3.52553500 & -2.38899400 & -5.16987300 \\
\hline & 7.52658800 & 0.58393800 & -5.03566500 \\
\hline $\mathrm{H}$ & 7.90003600 & 0.33033600 & -4.03395400 \\
\hline $\mathrm{H}$ & 7.84318900 & -0.22193300 & -5.70774700 \\
\hline $\mathrm{H}$ & 8.02810400 & 1.50564200 & -5.34904500 \\
\hline $\mathrm{C}$ & -1.64792400 & 0.63719500 & -4.49137400 \\
\hline$r$ & -2.65287000 & 1.61844000 & -4.45557400 \\
\hline & -1.92909800 & -0.72131900 & -4.25687100 \\
\hline & -3.95779800 & 1.21187500 & -4.16146700 \\
\hline & -3.25047000 & -1.07871000 & -3.97182600 \\
\hline & -4.27627300 & -0.12863500 & -3.91707500 \\
\hline & -4.74391700 & 1.96257400 & -4.11881200 \\
\hline & -3.48114600 & -2.12430600 & -3.77962100 \\
\hline & .687980 & -0.53616 & -3.5647 \\
\hline
\end{tabular}




$\begin{array}{lrrr}\mathrm{H} & -5.84607200 & -0.50225800 & -2.47859900 \\ \mathrm{H} & -6.42539700 & 0.13250100 & -4.02192800 \\ \mathrm{H} & -5.90462800 & -1.55860500 & -3.89291300 \\ \mathrm{C} & -2.32822400 & 3.06923400 & -4.70394200 \\ \mathrm{H} & -1.53378800 & 3.40890300 & -4.03173000 \\ \mathrm{H} & -1.97533200 & 3.23673700 & -5.73005500 \\ \mathrm{H} & -3.21181200 & 3.69678400 & -4.55225500 \\ \mathrm{C} & -0.83446400 & -1.75787400 & -4.26320200 \\ \mathrm{H} & -0.21119300 & -1.69525800 & -5.16252900 \\ \mathrm{H} & -0.16638600 & -1.62006300 & -3.40517200 \\ \mathrm{H} & -1.25346300 & -2.76742800 & -4.21299200 \\ \mathrm{H} & -2.09036800 & 0.78867000 & -0.19921500\end{array}$

\subsection{For complexes where $L=C A A C$}

\section{Ru-Va}

Thermal correction to Gibbs free energy $=0.673732$

Sum of electronic and thermal free energies $=-2430.664036$

E_sol $=-2431.2401049$ 


$\begin{array}{lrrr}\mathrm{Ru} & 0.68212800 & 0.20664300 & 0.02226400 \\ \mathrm{C} & 0.14854600 & -1.65909600 & -0.01178800 \\ \mathrm{C} & 0.61671500 & -3.94107100 & 0.61000500 \\ \mathrm{C} & -0.90901200 & -3.77195500 & 0.48409500 \\ \mathrm{~N} & -1.01726300 & -2.28104500 & 0.20643900 \\ \mathrm{H} & 0.90108000 & -3.90866500 & 1.66793900 \\ \mathrm{H} & 0.94044400 & -4.90713000 & 0.21490000 \\ \mathrm{C} & -2.32850100 & -1.67487900 & 0.07795400 \\ \mathrm{C} & -2.89375800 & -1.57731000 & -1.21893800 \\ \mathrm{C} & -4.19221200 & -1.06617400 & -1.33745800 \\ \mathrm{C} & -4.91202300 & -0.64969400 & -0.22296000 \\ \mathrm{C} & -4.31744300 & -0.69679700 & 1.03232000 \\ \mathrm{C} & -3.01589500 & -1.18516600 & 1.21470200 \\ \mathrm{H} & -4.63784500 & -0.98135700 & -2.32388800 \\ \mathrm{H} & -4.86295800 & -0.32311600 & 1.89332300 \\ \mathrm{C} & -2.39305700 & -1.03230100 & 2.59973200 \\ \mathrm{H} & -1.37278500 & -1.41910500 & 2.56806600 \\ \mathrm{C} & -2.13447200 & -1.89486900 & -2.50466100 \\ \mathrm{H} & -1.65713000 & 2.41933800 & 0.63728100 \\ \mathrm{H} & 3.64649100 & 1.66101400 & -0.62336300 \\ \mathrm{H} & 3.50378300 & 1.13962800 & 1.07388900 \\ \mathrm{Cl} & -1.17405800 & -2.33513600 & -2.24085100 \\ \mathrm{Cl} & 1.00705700 & 0.10283300 & 2.37545400 \\ \mathrm{C} & -0.90719300 & 1.09761400 & -0.19801800 \\ \mathrm{H} & -1.86229100 & 0.60426000 & -0.36968500 \\ \mathrm{H} & -0.95278500 & 2.54263400 & -0.20307800 \\ \mathrm{C} & 0.23862200 & 3.27425800 & 0.02608400 \\ \mathrm{C} & 0.23081800 & 4.66906600 & 0.01407200 \\ \mathrm{H} & -0.97293600 & 5.33984900 & -0.22399200 \\ \mathrm{H} & -2.16316500 & 4.64007100 & -0.44558500 \\ \mathrm{C} & -2.14783400 & 3.24906100 & -0.43411300 \\ \mathrm{C} & -3.05903600 & 2.68029800 & -0.60315100 \\ \mathrm{H} & -3.08896000 & 5.17811600 & -0.62503000 \\ \mathrm{H} & & & \\ \mathrm{H} & & & \end{array}$




\begin{tabular}{|c|c|c|c|}
\hline $\mathrm{C}$ & 2.52006100 & 3.48939400 & 2.12040800 \\
\hline $\mathrm{H}$ & 3.47387300 & 3.92744000 & 2.43532800 \\
\hline $\mathrm{H}$ & 2.30171700 & 2.61496500 & 2.73940600 \\
\hline $\mathrm{H}$ & 1.73181200 & 4.23087500 & 2.27974300 \\
\hline $\mathrm{C}$ & -3.18351600 & -1.76966600 & 3.69918100 \\
\hline $\mathrm{H}$ & -2.65344600 & -1.68737800 & 4.65506600 \\
\hline $\mathrm{H}$ & -4.17623800 & -1.32510700 & 3.83721700 \\
\hline $\mathrm{H}$ & -3.32617500 & -2.83083000 & 3.48051100 \\
\hline $\mathrm{C}$ & -2.28782200 & 0.46164600 & 2.97533400 \\
\hline $\mathrm{H}$ & -3.28144300 & 0.91560700 & 3.07396500 \\
\hline $\mathrm{H}$ & -1.76638700 & 0.56638300 & 3.93213900 \\
\hline $\mathrm{H}$ & -1.72035000 & 1.02100400 & 2.23019500 \\
\hline $\mathrm{C}$ & -2.88259800 & -2.89343700 & -3.40811900 \\
\hline $\mathrm{H}$ & -3.81022100 & -2.46175900 & -3.80103600 \\
\hline $\mathrm{H}$ & -2.25678900 & -3.15779600 & -4.26840900 \\
\hline $\mathrm{H}$ & -3.14252600 & -3.81539600 & -2.87957000 \\
\hline $\mathrm{C}$ & -1.82627800 & -0.60089200 & -3.28621300 \\
\hline $\mathrm{H}$ & -1.26753900 & -0.83948300 & -4.19828600 \\
\hline $\mathrm{H}$ & -2.75074200 & -0.08809500 & -3.57843900 \\
\hline $\mathrm{H}$ & -1.21263200 & 0.08419900 & -2.69810100 \\
\hline $\mathrm{C}$ & -1.53164900 & -4.61174600 & -0.64206500 \\
\hline $\mathrm{H}$ & -1.44366000 & -5.67267200 & -0.38505800 \\
\hline $\mathrm{H}$ & -2.59474100 & -4.37890300 & -0.75123500 \\
\hline $\mathrm{H}$ & -1.04352700 & -4.45923500 & -1.60473700 \\
\hline $\mathrm{C}$ & -1.61196500 & -4.14394600 & 1.79248200 \\
\hline $\mathrm{H}$ & -2.69071200 & -3.97703600 & 1.72740600 \\
\hline $\mathrm{H}$ & -1.44561500 & -5.20878600 & 1.98988900 \\
\hline $\mathrm{H}$ & -1.21413100 & -3.57677000 & 2.63590700 \\
\hline $\mathrm{H}$ & -5.92249600 & -0.26563900 & -0.33598800 \\
\hline $\mathrm{C}$ & 1.24898100 & -2.74012900 & -0.12394200 \\
\hline $\mathrm{C}$ & 1.49614500 & -3.04774500 & -1.63441600 \\
\hline $\mathrm{C}$ & 2.58964300 & -2.30060500 & 0.51008400 \\
\hline $\mathrm{C}$ & 2.62707600 & -4.06457900 & -1.85727100 \\
\hline $\mathrm{H}$ & 1.74969700 & -2.11346300 & -2.14066500 \\
\hline $\mathrm{H}$ & 0.57853800 & -3.41434800 & -2.10242400 \\
\hline $\mathrm{C}$ & 3.70658900 & -3.33271200 & 0.29869400 \\
\hline $\mathrm{H}$ & 2.90981500 & -1.35352000 & 0.04792400 \\
\hline $\mathrm{H}$ & 2.44562800 & -2.09755000 & 1.57436300 \\
\hline $\mathrm{C}$ & 3.93241400 & -3.61002900 & -1.19255400 \\
\hline $\mathrm{H}$ & 2.77238100 & -4.19907600 & -2.93651100 \\
\hline
\end{tabular}




$\begin{array}{llrr}\mathrm{H} & 2.34214400 & -5.04935300 & -1.45948900 \\ \mathrm{H} & 4.62917700 & -2.96948800 & 0.76801900 \\ \mathrm{H} & 3.44384500 & -4.26968800 & 0.81171600 \\ \mathrm{H} & 4.71708800 & -4.36438700 & -1.33166700 \\ \mathrm{H} & 4.28260100 & -2.68879000 & -1.68009100\end{array}$

ts-i

Thermal correction to Gibbs free energy $=0.886987$

Sum of electronic and thermal free energies $=-2957.804396$

E_sol $=-2958.566948$

$\mathrm{Ru} \quad 0.95076000 \quad-0.21537200 \quad-1.34470500$

$\mathrm{Cl} \quad-0.35115900 \quad-1.40002200 \quad 0.32703000$

$\mathrm{Cl} \quad 2.18333900 \quad 0.45431200 \quad-3.35556400$

C $\quad 0.46791900 \quad 1.59640300 \quad-1.07760600$

$\begin{array}{llll}\text { C } & -0.16586900 & 2.37072300 & -0.02757100\end{array}$

$\begin{array}{llll}\text { C } & -0.37940400 & 3.76956300 & -0.24460800\end{array}$

$\begin{array}{llll}\text { C } & -0.56191600 & 1.83011600 & 1.21116800\end{array}$

$\begin{array}{lllll}\text { C } & -0.93730400 & 4.55856700 & 0.76895900\end{array}$

C $\quad-1.12738500 \quad 2.61434200 \quad 2.20810100$

$\mathrm{H} \quad-0.42302100 \quad 0.77036500 \quad 1.36005800$

$\begin{array}{llll}\mathrm{C} & -1.30347500 & 3.98122000 & 1.98478100\end{array}$

$\mathrm{H} \quad-1.09389300 \quad 5.61932200 \quad 0.61516700$

$\mathrm{H} \quad-1.42216900 \quad 2.16488800 \quad 3.15134400$

$\mathrm{H} \quad-1.73582000 \quad 4.61120300 \quad 2.75825600$

O $\quad-0.00485100 \quad 4.26315400 \quad-1.45258200$

C $\quad-0.48217400 \quad 5.54092800 \quad-1.92645100$

$\mathrm{H} \quad-0.28307500 \quad 6.30422300 \quad-1.16209600$

C $\quad-1.97759600 \quad 5.46775200 \quad-2.23171300$

$\mathrm{H} \quad-2.15936400 \quad 4.71622700 \quad-3.00783300$

$\mathrm{H} \quad-2.55725900 \quad 5.19394200 \quad-1.34557900$

$\mathrm{H} \quad \begin{array}{llll}\mathrm{H} & -2.33767600 & 6.43673700 & -2.59411800\end{array}$

C $\quad 0.35108600 \quad 5.84889100 \quad-3.16335700$

$\mathrm{H} \quad 0.21683300 \quad 5.06305800 \quad-3.91407900$

$\mathrm{H} \quad \begin{array}{llll}0.04100600 & 6.80560600 & -3.59672700\end{array}$

$\mathrm{H} \quad 1.41304300 \quad 5.90291500 \quad-2.91191100$

$\mathrm{H} \quad \begin{array}{llll}\mathrm{H} & 0.75694300 & 2.22110300 & -1.91990900\end{array}$

$\begin{array}{llll}\text { C } & -0.57092200 & -0.81979300 & -2.70887200\end{array}$

C $\quad-0.66027800 \quad-1.92491000 \quad-3.43867100$

$\mathrm{H} \quad 0.13881600 \quad-2.66107700 \quad-3.42244900$ 


\begin{tabular}{|c|c|c|c|}
\hline $\mathrm{C}$ & -1.10996500 & 0.38882300 & -2.32759900 \\
\hline $\mathrm{H}$ & -1.06037200 & 1.23079000 & -3.01431700 \\
\hline $\mathrm{H}$ & -1.85101400 & 0.42631700 & -1.53329600 \\
\hline $\mathrm{C}$ & -3.28197000 & -3.06863400 & -5.88636 \\
\hline $\mathrm{C}$ & -4.02577400 & -1.90951800 & -5.12326000 \\
\hline B & -1.91531100 & -2.15826700 & -4.31048000 \\
\hline $\mathrm{O}$ & -2.93222500 & -1.23846800 & -4.44339300 \\
\hline $\mathrm{O}$ & -2.12964800 & -3.30846500 & -5.03357900 \\
\hline $\mathrm{C}$ & -4.73768000 & -0.89905000 & -6.0180090 \\
\hline $\mathrm{H}$ & -5.51804300 & -1.38971000 & -6.61103100 \\
\hline $\mathrm{H}$ & -5.21134600 & -0.12982100 & -5.3995610 \\
\hline $\mathrm{H}$ & -4.03972100 & -0.40372100 & -6.69647600 \\
\hline $\mathrm{C}$ & -2.73495600 & -2.63625200 & -7.25269100 \\
\hline $\mathrm{H}$ & -2.05898500 & -3.41223500 & -7.62433800 \\
\hline $\mathrm{H}$ & -3.53857400 & -2.49404600 & -7.98 \\
\hline $\mathrm{H}$ & -2.16717700 & -1.70429300 & -7.1691090 \\
\hline $\mathrm{C}$ & -4.07156900 & -4.36773400 & -6.01719200 \\
\hline $\mathrm{H}$ & -4.99391000 & -4.20347200 & -6.5861980 \\
\hline $\mathrm{H}$ & -3.47002700 & -5.11242900 & -6.5481100 \\
\hline $\mathrm{H}$ & -4.33044400 & -4.77893000 & -5.03914600 \\
\hline $\mathrm{C}$ & -4.97638100 & -2.41919400 & -4.0324140 \\
\hline $\mathrm{H}$ & -5.29004400 & -1.57301600 & -3.41359900 \\
\hline $\mathrm{H}$ & -5.86830300 & -2.88890300 & -4.46033100 \\
\hline $\mathrm{H}$ & -4.47446600 & -3.14394300 & -3.38385400 \\
\hline $\mathrm{C}$ & 2.77020200 & -0.62319000 & -0.56771700 \\
\hline $\mathrm{N}$ & 3.71578700 & 0.13736700 & -0.02427300 \\
\hline $\mathrm{C}$ & 5.10395800 & -0.51713400 & 0.00620800 \\
\hline $\mathrm{C}$ & 4.84700600 & -1.78748400 & -0.82609400 \\
\hline $\mathrm{H}$ & 5.43230300 & -2.62939400 & -0.44794600 \\
\hline $\mathrm{H}$ & 5.15240800 & -1.61320800 & -1.86387900 \\
\hline $\mathrm{C}$ & 3.40995400 & 1.43868600 & 0.57488700 \\
\hline $\mathrm{C}$ & 3.53487600 & 2.64707300 & -0.16268900 \\
\hline $\mathrm{C}$ & 2.90558900 & 1.44899100 & 1.90239500 \\
\hline $\mathrm{C}$ & 3.10858100 & 3.83360500 & 0.45113300 \\
\hline $\mathrm{C}$ & 2.48866300 & 2.66876800 & 2.44877600 \\
\hline $\mathrm{C}$ & 2.57591800 & 3.85367300 & 1.73242600 \\
\hline $\mathrm{H}$ & 3.18471600 & 4.76272100 & -0.10372600 \\
\hline $\mathrm{H}$ & 2.07892300 & 2.68164200 & 3.45360300 \\
\hline $\mathrm{C}$ & 4.13484400 & 2.78349700 & -1.56177300 \\
\hline $\mathrm{H}$ & 4.25025800 & 1.78709100 & -1.9886060 \\
\hline
\end{tabular}




\begin{tabular}{|c|c|c|c|}
\hline $\mathrm{C}$ & 2.83596000 & 0.22657100 & 2.81729300 \\
\hline $\mathrm{H}$ & 3.20451200 & -0.63495100 & 2.26648700 \\
\hline $\mathrm{C}$ & 3.32631900 & -2.03448300 & -0.78612400 \\
\hline $\mathrm{C}$ & 2.88806500 & -2.90810100 & 0.43331200 \\
\hline $\mathrm{C}$ & 2.79311600 & -2.70824400 & -2.06920600 \\
\hline $\mathrm{C}$ & 3.34676800 & -4.36920200 & 0.30330600 \\
\hline $\mathrm{H}$ & 1.79689900 & -2.87429500 & 0.50479300 \\
\hline $\mathrm{H}$ & 3.26859100 & -2.48256900 & 1.36587200 \\
\hline $\mathrm{C}$ & 3.27310800 & -4.15922900 & -2.21035600 \\
\hline $\mathrm{H}$ & 1.69446200 & -2.72016400 & -2.01981800 \\
\hline $\mathrm{H}$ & 3.06359600 & -2.11265000 & -2.94472700 \\
\hline $\mathrm{C}$ & 2.84878400 & -5.00351700 & -1.00188300 \\
\hline $\mathrm{H}$ & 2.97689600 & -4.93282700 & 1.16895400 \\
\hline $\mathrm{H}$ & 4.44405600 & -4.42983900 & 0.34130800 \\
\hline $\mathrm{H}$ & 2.87112700 & -4.58513300 & -3.13778200 \\
\hline $\mathrm{H}$ & 4.36860700 & -4.17663600 & -2.30846500 \\
\hline $\mathrm{H}$ & 3.22321500 & -6.03061000 & -1.09605100 \\
\hline $\mathrm{H}$ & 1.75121600 & -5.06386000 & -0.97557800 \\
\hline $\mathrm{H}$ & 2.22812200 & 4.78583000 & 2.16843100 \\
\hline v & 5.59541300 & -0.81737100 & 1.42999500 \\
\hline $\mathrm{H}$ & 6.61087200 & -1.22270800 & 1.36798800 \\
\hline $\mathrm{H}$ & 5.63818600 & 0.09797000 & 2.02428800 \\
\hline $\mathrm{H}$ & 4.98142400 & -1.54916600 & 1.95497700 \\
\hline $\mathrm{C}$ & 6.16303200 & 0.36039700 & -0.66071000 \\
\hline $\mathrm{H}$ & 6.36074800 & 1.25741000 & -0.07096700 \\
\hline $\mathrm{H}$ & 7.09525300 & -0.21211900 & -0.72152500 \\
\hline $\mathrm{H}$ & 5.87876500 & 0.64915300 & -1.67307700 \\
\hline $\mathrm{C}$ & 5.50261600 & 3.50382700 & -1.49737400 \\
\hline $\mathrm{H}$ & 5.36235900 & 4.55704800 & -1.22605700 \\
\hline $\mathrm{H}$ & 6.18651000 & 3.07257900 & -0.76462300 \\
\hline $\mathrm{H}$ & 5.99082400 & 3.47605100 & -2.47880800 \\
\hline $\mathrm{C}$ & 3.24802800 & 3.57749300 & -2.53970900 \\
\hline $\mathrm{H}$ & 2.24224500 & 3.17288900 & -2.60916100 \\
\hline $\mathrm{H}$ & 3.18086800 & 4.63214900 & -2.24969300 \\
\hline $\mathrm{H}$ & 3.68957200 & 3.53546300 & -3.54132300 \\
\hline $\mathrm{C}$ & 3.73652000 & 0.42911100 & 4.05596400 \\
\hline $\mathrm{H}$ & 4.74306300 & 0.76763200 & 3.79262700 \\
\hline $\mathrm{H}$ & 3.30664300 & 1.17652600 & 4.73262900 \\
\hline$\Pi$ & 3.82545200 & -0.50962600 & 4.61540100 \\
\hline $\mathrm{C}$ & 1.41146400 & -0.13172000 & 3.27499300 \\
\hline
\end{tabular}




\begin{tabular}{|c|c|c|c|}
\hline & 1.45793800 & -0.93289800 & 4.02284200 \\
\hline & 0.90549800 & 0.72425500 & 3.73571800 \\
\hline & 0.80779500 & -0.49210 & \\
\hline
\end{tabular}

\section{mcb-i}

Thermal correction to Gibbs free energy $=0.892638$

Sum of electronic and thermal free energies $=-2957.827474$

E_sol $=-2958.5968492$

\begin{tabular}{|c|c|c|c|}
\hline $\mathrm{Ru}$ & 1.20234800 & 0.66610500 & -1.18621900 \\
\hline $\mathrm{Cl}$ & -0.03768700 & 0.10479700 & 0.82420900 \\
\hline $\mathrm{Cl}$ & 2.37825700 & 1.03785900 & -3.28256500 \\
\hline $\mathrm{C}$ & 0.53120500 & 2.60806300 & -1.36887300 \\
\hline $\mathrm{C}$ & 0.21813000 & 3.45964400 & -0.20767400 \\
\hline $\mathrm{C}$ & 1.07425500 & 4.55953700 & 0.06941400 \\
\hline $\mathrm{C}$ & -0.89350200 & 3.26370500 & 0.62967400 \\
\hline $\mathrm{C}$ & 0.82467200 & 5.38850000 & 49200 \\
\hline $\mathrm{C}$ & -1.15233000 & 4.09899300 & 1.710 \\
\hline $\mathrm{H}$ & -1.54295100 & 2.41711200 & 0.44712300 \\
\hline $\mathrm{C}$ & -0.28352300 & 5.15669900 & 1.98477900 \\
\hline $\mathrm{H}$ & 1.49502100 & 6.20699200 & 1.40378600 \\
\hline $\mathrm{H}$ & -2.01467700 & 3.91487100 & 2.34372200 \\
\hline $\mathrm{H}$ & -0.46135600 & 5.80588600 & 2.83804300 \\
\hline $\mathrm{O}$ & 2.12313300 & 4.74085800 & -0.78243100 \\
\hline $\mathrm{C}$ & 2.75756200 & 6.03394000 & -0.89293500 \\
\hline $\mathrm{H}$ & 2.03504500 & 6.80210800 & -0.58863500 \\
\hline $\mathrm{C}$ & 3.08765500 & 6.22361500 & -2.36954100 \\
\hline $\mathrm{H}$ & 3.77506900 & 5.44515500 & -2.71190600 \\
\hline $\mathrm{H}$ & 2.17701600 & 6.16937700 & -2.97403600 \\
\hline $\mathrm{H}$ & 3.55780700 & 7.20044800 & -2.52921700 \\
\hline $\mathrm{C}$ & 3.99253900 & 6.08042700 & 0.00246900 \\
\hline $\mathrm{H}$ & 4.70912700 & 5.31919300 & -0.31650600 \\
\hline $\mathrm{H}$ & 4.47026600 & 7.06523400 & -0.05562400 \\
\hline $\mathrm{H}$ & 3.73662100 & 5.87418400 & 1.04591800 \\
\hline $\mathrm{H}$ & 1.18784100 & 3.10452500 & -2.07741700 \\
\hline $\mathrm{C}$ & -0.30066100 & 0.22313400 & -2.22667500 \\
\hline $\mathrm{C}$ & -1.03665000 & -0.67543000 & -2.88359800 \\
\hline $\mathrm{H}$ & -0.68571600 & -1.70557100 & -2.89996900 \\
\hline $\mathrm{C}$ & -0.57008200 & 1.78400300 & -2.07957500 \\
\hline $\mathrm{H}$ & -0.61599000 & 2.12144100 & -3.11725100 \\
\hline $\mathrm{H}$ & -1.53183700 & 1.87032900 & -1.57651700 \\
\hline
\end{tabular}




\begin{tabular}{|c|c|c|c|}
\hline $\mathrm{C}$ & -4.20086200 & 0.84084800 & -4.24673000 \\
\hline $\mathrm{C}$ & -4.12086800 & -0.52447300 & -5.02480700 \\
\hline B & -2.34117300 & -0.32121600 & -3.61699500 \\
\hline $\mathrm{O}$ & -3.11917600 & -1.25307500 & -4.26684400 \\
\hline $\mathrm{O}$ & -2.86147300 & 0.95757700 & -3.69702700 \\
\hline $\mathrm{C}$ & -5.41198600 & -1.33779700 & -5.03646800 \\
\hline $\mathrm{H}$ & -6.21649300 & -0.77916500 & -5.52857800 \\
\hline $\mathrm{H}$ & -5.25483700 & -2.26955600 & -5.58907300 \\
\hline $\mathrm{H}$ & -5.73123600 & -1.59632700 & -4.02454000 \\
\hline $\mathrm{C}$ & -5.16382400 & 0.79891500 & -3.05336100 \\
\hline $\mathrm{H}$ & -5.01088200 & 1.69497700 & -2.44386900 \\
\hline $\mathrm{H}$ & -6.20931600 & 0.77295500 & -3.37790800 \\
\hline $\mathrm{H}$ & -4.96951000 & -0.07585300 & -2.42495700 \\
\hline $\mathrm{C}$ & -4.48047400 & 2.06530700 & -5.11292400 \\
\hline $\mathrm{H}$ & -5.45094400 & 1.97128600 & -5.61356400 \\
\hline $\mathrm{H}$ & -4.50508900 & 2.96250000 & -4.48595400 \\
\hline $\mathrm{H}$ & -3.70626000 & 2.20439500 & -5.87051400 \\
\hline $\mathrm{C}$ & -3.57246500 & -0.37296000 & -6.44955100 \\
\hline $\mathrm{H}$ & -3.34680400 & -1.36653900 & -6.84835100 \\
\hline $\mathrm{H}$ & -4.29639000 & 0.11312800 & -7.11202000 \\
\hline $\mathrm{H}$ & -2.64666300 & 0.21092500 & -6.45216100 \\
\hline $\mathrm{C}$ & 2.68885300 & -0.47466500 & -0.49059500 \\
\hline $\mathrm{N}$ & 3.73876700 & -0.18370400 & 0.25428400 \\
\hline $\mathrm{C}$ & 4.60316400 & -1.39815400 & 0.61748500 \\
\hline $\mathrm{C}$ & 3.70069600 & -2.55671900 & 0.17657900 \\
\hline $\mathrm{H}$ & 3.17562700 & -2.96380400 & 1.04718500 \\
\hline $\mathrm{H}$ & 4.28952200 & -3.36919600 & -0.25416800 \\
\hline $\mathrm{C}$ & 4.20188600 & 1.12781100 & 0.71526500 \\
\hline $\mathrm{C}$ & 5.14278300 & 1.84531200 & -0.06971900 \\
\hline $\mathrm{C}$ & 5.81091800 & 2.92000300 & 0.53131000 \\
\hline $\mathrm{C}$ & 4.53178900 & 2.67478100 & 2.54279000 \\
\hline $\mathrm{C}$ & 5.53335800 & 3.32092600 & 1.83163900 \\
\hline $\mathrm{H}$ & 6.54937000 & 3.46591100 & -0.04732200 \\
\hline $\mathrm{H}$ & 4.27150000 & 3.02542000 & 3.53614300 \\
\hline $\mathrm{C}$ & 5.38202400 & 1.62997400 & -1.56261000 \\
\hline $\mathrm{H}$ & 4.78958700 & 0.77802500 & -1.90261000 \\
\hline $\mathrm{C}$ & 2.71167400 & 0.99343800 & 2.85431900 \\
\hline $\mathrm{H}$ & 2.29608200 & 0.12224000 & 2.34248100 \\
\hline $\mathrm{C}$ & 3.14049100 & 0.58438300 & 4.27862000 \\
\hline $\mathrm{H}$ & 3.41135100 & 1.46079400 & 4.87864500 \\
\hline
\end{tabular}




\begin{tabular}{|c|c|c|c|}
\hline $\mathrm{H}$ & 2.30174400 & 0.09292600 & 4.78476900 \\
\hline $\mathrm{H}$ & 3.99427700 & -0.09756700 & 4.29318200 \\
\hline $\mathrm{C}$ & 1.58366300 & 2.04099100 & 2.95570500 \\
\hline $\mathrm{H}$ & 0.70568900 & 1.60342500 & 3.44019100 \\
\hline $\mathrm{H}$ & 1.90921000 & 2.90959900 & 3.54107900 \\
\hline $\mathrm{H}$ & 1.27490700 & 2.38739100 & 1.97140800 \\
\hline $\mathrm{C}$ & 6.85637100 & 1.40234600 & -1.94987700 \\
\hline & 7.30399800 & 0.53545300 & -1.45945400 \\
\hline & 6.92662600 & 1.25002800 & -3.03297700 \\
\hline $\mathrm{H}$ & 7.47231600 & 2.27539900 & -1.70457800 \\
\hline $\mathrm{C}$ & 4.86177000 & 2.87469100 & -2.31608600 \\
\hline $\mathrm{H}$ & 4.85182200 & 2.68670600 & -3.39350500 \\
\hline $\mathrm{H}$ & 3.84517100 & 3.12627800 & -2.01158900 \\
\hline $\mathrm{H}$ & 5.51054300 & 3.73812300 & -2.11969300 \\
\hline & 6.07074100 & 4.15539100 & 2.27448300 \\
\hline $\mathrm{C}$ & 3.84727900 & 1.57101100 & 2.01525900 \\
\hline $\mathrm{C}$ & 5.93055200 & -1.35647500 & -0.14431900 \\
\hline $\mathrm{H}$ & 6.53371200 & -0.50530900 & 0.17846900 \\
\hline $\mathrm{H}$ & 6.49260000 & -2.27091500 & 0.07313400 \\
\hline $\mathrm{H}$ & 5.78168600 & -1.29406000 & -1.22393100 \\
\hline & 4.88779400 & -1.46203100 & 2.11744100 \\
\hline $\mathrm{H}$ & 5.47243000 & -0.60500500 & 2.46241700 \\
\hline $\mathrm{H}$ & 3.96124800 & -1.51597300 & 2.69062900 \\
\hline $\mathrm{H}$ & 5.46440100 & -2.37144400 & 2.31978700 \\
\hline $\mathrm{C}$ & 2.66559600 & -1.97947800 & -0.81781800 \\
\hline $\mathrm{C}$ & 1.28176800 & -2.64101300 & -0.60012300 \\
\hline $\mathrm{C}$ & 3.09033200 & -2.17819200 & -2.30566800 \\
\hline $\mathrm{C}$ & 1.29288200 & -4.12283800 & -1.00105100 \\
\hline $\mathrm{H}$ & 0.52706800 & -2.12000500 & -1.19260900 \\
\hline $\mathrm{H}$ & 0.97556100 & -2.51361700 & 0.44174100 \\
\hline $\mathrm{C}$ & 3.07704700 & -3.65536600 & -2.72636100 \\
\hline 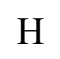 & 2.39929900 & -1.61896200 & -2.94120500 \\
\hline & 4.07738100 & -1.73688100 & $-2.4813470 \mathrm{c}$ \\
\hline & 1.70762800 & -4.29806800 & -2.46819200 \\
\hline $\mathrm{H}$ & 0.29528200 & -4.54551000 & -0.83089300 \\
\hline $\mathrm{H}$ & 1.98299600 & -4.68572500 & $-0.3552680 \mathrm{c}$ \\
\hline $\mathrm{H}$ & 3.33724700 & -3.71894100 & -3.79014100 \\
\hline 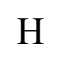 & 3.85081900 & -4.21949800 & -2.18611100 \\
\hline 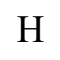 & 1.72470200 & -5.36051600 & -2.74154900 \\
\hline & 0.95816600 & -3.81563300 & -3.11278500 \\
\hline
\end{tabular}




\section{vinyl-i}

Thermal correction to Gibbs free energy $=0.675365$

Sum of electronic and thermal free energies $=-2455.209146$

E_sol $=-2455.8004637$

$\begin{array}{lrrr}\mathrm{Ru} & 2.86594300 & 1.53190300 & -1.49856300 \\ \mathrm{Cl} & 3.93830300 & 3.50125300 & -2.11587100 \\ \mathrm{Cl} & 2.92188200 & -0.81417100 & -1.60054300 \\ \mathrm{C} & -2.79141200 & -0.02106500 & -3.63568000 \\ \mathrm{C} & -1.90113100 & -1.05303900 & -2.84109100 \\ \mathrm{~B} & -0.98990900 & 1.03363600 & -2.73627700 \\ \mathrm{O} & -0.68142700 & -0.29874200 & -2.60844400 \\ \mathrm{O} & -2.26879500 & 1.26055600 & -3.18322200 \\ \mathrm{C} & -2.47076200 & -1.41224700 & -1.46443200 \\ \mathrm{H} & -3.37061500 & -2.03007900 & -1.54923200 \\ \mathrm{H} & -1.71404900 & -1.97324200 & -0.90860200 \\ \mathrm{H} & -2.71598000 & -0.51290700 & -0.89176700 \\ \mathrm{C} & -4.28260300 & -0.07452900 & -3.31734600 \\ \mathrm{H} & -4.81074400 & 0.68544800 & -3.90174900 \\ \mathrm{H} & -4.69749300 & -1.05533400 & -3.57591300 \\ \mathrm{H} & -4.47397600 & 0.12008800 & -2.25981300 \\ \mathrm{C} & -2.56747500 & -0.07119100 & -5.15193300 \\ \mathrm{H} & -2.98305900 & -0.98413200 & -5.59067600 \\ \mathrm{H} & -3.05886400 & 0.79196700 & -5.61092300 \\ \mathrm{H} & -1.50097400 & -0.02143200 & -5.39271500 \\ \mathrm{C} & -1.54054400 & -2.32250800 & -3.60752200 \\ \mathrm{C} & -0.90303900 & -2.95602200 & -2.98323200 \\ \mathrm{H} & -2.44287900 & -2.88807000 & -3.86709200 \\ \mathrm{H} & -0.99066500 & -2.09480500 & -4.52274200 \\ \mathrm{H} & 0.00524600 & 2.17377700 & -2.42631300 \\ \mathrm{C} & -0.25479700 & 3.21663200 & -2.61575100 \\ \mathrm{C} & 1.21563900 & 1.91002400 & -1.96480700 \\ \mathrm{C} & 2.64980900 & 1.52951400 & 0.41792800 \\ \mathrm{C} & 3.72563600 & 1.56281600 & 1.21704300 \\ \mathrm{H} & 3.48062800 & 0.88624200 & 2.55062400 \\ \mathrm{H} & 1.96754500 & 1.11089300 & 2.70478300 \\ \mathrm{H} & 2.99242600 & 3.64761800 & 1.21422200\end{array}$




\begin{tabular}{|c|c|c|c|}
\hline $\mathrm{C}$ & 6.05726100 & 1.61461700 & 0.30812300 \\
\hline $\mathrm{C}$ & 6.15166000 & 4.36101600 & 0.90108900 \\
\hline $\mathrm{C}$ & 7.20073200 & 2.37857300 & 0.03489800 \\
\hline $\mathrm{C}$ & 7.25087700 & 3.73627600 & 0.31949200 \\
\hline $\mathrm{H}$ & 6.19148500 & 5.42584100 & 1.10802400 \\
\hline $\mathrm{H}$ & 8.05778100 & 1.89711700 & -0.42576700 \\
\hline $\mathrm{C}$ & 3.82392400 & 4.39442200 & 1.85283200 \\
\hline $\mathrm{H}$ & 3.10636500 & 3.65717000 & 2.21752700 \\
\hline $\mathrm{C}$ & 6.06295200 & 0.14321400 & -0.08159900 \\
\hline $\mathrm{H}$ & 5.07208700 & -0.27232800 & 0.10798800 \\
\hline $\mathrm{C}$ & 4.26442300 & 5.23562700 & 3.06686800 \\
\hline $\mathrm{H}$ & 4.87971800 & 6.08989700 & 2.76403600 \\
\hline $\mathrm{H}$ & 3.38436200 & 5.63611600 & 3.58341200 \\
\hline $\mathrm{H}$ & 4.84660700 & 4.64646400 & 3.78384700 \\
\hline $\mathrm{C}$ & 3.08158300 & 5.27676000 & 0.83152900 \\
\hline $\mathrm{H}$ & 2.25469000 & 5.80499500 & 1.32280300 \\
\hline $\mathrm{H}$ & 3.75660600 & 6.02441900 & 0.40036800 \\
\hline $\mathrm{H}$ & 2.68225800 & 4.68233700 & 0.00774100 \\
\hline $\mathrm{C}$ & 7.08888900 & -0.65097800 & 0.74999700 \\
\hline $\mathrm{H}$ & 7.02070400 & -1.72042000 & 0.51936100 \\
\hline $\mathrm{H}$ & 8.11159900 & -0.32431300 & 0.52808100 \\
\hline $\mathrm{H}$ & 6.93242900 & -0.52096500 & 1.82670300 \\
\hline $\mathrm{C}$ & 6.34446800 & -0.04186300 & -1.58625000 \\
\hline $\mathrm{H}$ & 7.37302600 & 0.24097800 & -1.83842300 \\
\hline $\mathrm{H}$ & 6.19574100 & -1.08902700 & -1.86845200 \\
\hline $\mathrm{H}$ & 5.67385400 & 0.56610700 & -2.20140900 \\
\hline $\mathrm{C}$ & 4.31011700 & 1.49199300 & 3.68146600 \\
\hline $\mathrm{H}$ & 5.38174300 & 1.40858900 & 3.47572200 \\
\hline $\mathrm{H}$ & 4.07155900 & 2.54249300 & 3.85370100 \\
\hline $\mathrm{H}$ & 4.09770600 & 0.94160400 & 4.60425000 \\
\hline $\mathrm{C}$ & 3.80413600 & -0.61474400 & 2.42467500 \\
\hline $\mathrm{H}$ & 3.31121400 & -1.05773000 & 1.55519300 \\
\hline $\mathrm{H}$ & 4.87822700 & -0.78504100 & 2.33337600 \\
\hline $\mathrm{H}$ & 3.45885800 & -1.13288300 & 3.32653400 \\
\hline $\mathrm{H}$ & 8.14280800 & 4.31062000 & 0.08430800 \\
\hline $\mathrm{C}$ & 1.39179600 & 1.26755100 & 1.26489600 \\
\hline $\mathrm{C}$ & 0.47494600 & 2.52466300 & 1.19192000 \\
\hline $\mathrm{C}$ & 0.59008500 & 0.01921700 & 0.81083800 \\
\hline $\mathrm{C}$ & -0.81988700 & 2.33838300 & 1.99639500 \\
\hline $\mathrm{H}$ & 0.21351900 & 2.71548600 & 0.149769 \\
\hline
\end{tabular}




$\begin{array}{lrrr}\mathrm{H} & 1.02455900 & 3.40264700 & 1.54858200 \\ \mathrm{C} & -0.71910300 & -0.14866000 & 1.59710900 \\ \mathrm{H} & 0.35552700 & 0.10419200 & -0.25287100 \\ \mathrm{H} & 1.21097400 & -0.87623000 & 0.90757400 \\ \mathrm{C} & -1.59466100 & 1.10789300 & 1.50695500 \\ \mathrm{H} & -1.43233800 & 3.24375400 & 1.90245400 \\ \mathrm{H} & -0.59247800 & 2.22529300 & 3.06648200 \\ \mathrm{H} & -1.25773200 & -1.02000800 & 1.20848700 \\ \mathrm{H} & -0.50907900 & -0.36410900 & 2.65471100 \\ \mathrm{H} & -2.51736300 & 0.97728600 & 2.08618100 \\ \mathrm{H} & -1.89265700 & 1.26860900 & 0.46057900\end{array}$

\section{ts-ii}

Thermal correction to Gibbs free energy $=0.968381$

Sum of electronic and thermal free energies $=-3252.093016$

E_sol $=-3252.9490046$

$\begin{array}{lrrr}\mathrm{Ru} & -0.93998800 & -0.65351100 & -0.65929100 \\ \mathrm{Cl} & -1.83436900 & 0.22876700 & -3.04270500 \\ \mathrm{Cl} & -0.19200700 & -2.04066100 & 1.17818700 \\ \mathrm{C} & 0.64711100 & -1.38504100 & -1.84205800 \\ \mathrm{C} & 0.82101400 & -2.45719900 & -2.59357900 \\ \mathrm{H} & 0.03592900 & -3.19256900 & -2.73812700 \\ \mathrm{C} & 3.75569200 & 3.43119000 & -1.11454600 \\ \mathrm{C} & 3.67704700 & 3.95915400 & 0.36390800 \\ \mathrm{~B} & 1.82124600 & 2.73555000 & -0.11367600 \\ \mathrm{O} & 2.27391400 & 3.75105600 & 0.69408200 \\ \mathrm{O} & 2.70022000 & 2.42796600 & -1.13172900 \\ \mathrm{C} & 4.49336900 & 3.10550400 & 1.34390800 \\ \mathrm{H} & 5.56823800 & 3.27260400 & 1.21879500 \\ \mathrm{H} & 4.21376700 & 3.37675600 & 2.36637000 \\ \mathrm{H} & 4.29485000 & 2.03811900 & 1.20445200 \\ \mathrm{C} & 5.07463500 & 2.76089700 & -1.49010100 \\ \mathrm{H} & 5.04053500 & 2.44511200 & -2.53829600 \\ \mathrm{H} & 5.90644900 & 3.46583000 & -1.37778700 \\ \mathrm{H} & 5.27117400 & 1.87824900 & -0.87739200 \\ \mathrm{C} & 3.36927600 & 4.48719300 & -2.15595600 \\ \mathrm{H} & 4.14368200 & 5.25464400 & -2.25747400 \\ \mathrm{H} & 3.23629600 & 3.99646500 & -3.12489300 \\ \mathrm{H} & 2.42536800 & 4.97322500 & -1.88998700\end{array}$




\begin{tabular}{|c|c|c|c|}
\hline $\mathrm{C}$ & 4.00558200 & 5.43780700 & 0.54061200 \\
\hline $\mathrm{H}$ & 3.91730300 & 5.71126400 & 1.59686000 \\
\hline $\mathrm{H}$ & 5.03219300 & 5.64470700 & 0.21775900 \\
\hline $\mathrm{H}$ & 3.32301900 & 6.07081800 & -0.03054700 \\
\hline $\mathrm{C}$ & 0.13095100 & 0.81159800 & -0.34384700 \\
\hline $\mathrm{C}$ & 0.48393800 & 2.00544500 & 0.13285500 \\
\hline $\mathrm{H}$ & -0.25972200 & 2.47941300 & 0.77637400 \\
\hline $\mathrm{C}$ & 1.38570600 & -0.22666700 & -1.35076200 \\
\hline $\mathrm{H}$ & 1.50843400 & 0.56149700 & -2.09545100 \\
\hline $\mathrm{H}$ & 1.76217900 & -2.62369900 & -3.11612800 \\
\hline $\mathrm{C}$ & 2.55090600 & -0.49478900 & -0.40931300 \\
\hline $\mathrm{H}$ & 2.32025900 & -1.35953000 & 0.21370600 \\
\hline $\mathrm{H}$ & 2.71678400 & 0.35491100 & 0.25062100 \\
\hline $\mathrm{C}$ & 3.84549200 & -0.70757200 & -1.19879800 \\
\hline $\mathrm{H}$ & 3.95538300 & 0.08948900 & -1.94296000 \\
\hline $\mathrm{H}$ & 3.82253900 & -1.66712300 & -1.73542000 \\
\hline $\mathrm{O}$ & 4.95003100 & -0.62531500 & -0.30931200 \\
\hline $\mathrm{S}$ & 5.94685000 & -1.88928100 & 0.17846500 \\
\hline $\mathrm{C}$ & 6.72329500 & -2.69156400 & -1.34589300 \\
\hline $\mathrm{H}$ & 7.37013300 & -3.53196700 & -1.06693300 \\
\hline $\mathrm{H}$ & 5.96583000 & -3.07696000 & -2.03823900 \\
\hline $\mathrm{H}$ & 7.33546000 & -1.96462100 & -1.89258000 \\
\hline $\mathrm{C}$ & 7.25447300 & -1.03215200 & 1.22525600 \\
\hline $\mathrm{H}$ & 8.01097500 & -1.73666500 & 1.58957100 \\
\hline $\mathrm{H}$ & 7.76555300 & -0.26123800 & 0.63720100 \\
\hline $\mathrm{H}$ & 6.80097100 & -0.54303500 & 2.09464700 \\
\hline $\mathrm{C}$ & 5.00056400 & -3.20100800 & 1.20518600 \\
\hline C & 3.97797300 & -3.96531800 & 0.33578100 \\
\hline $\mathrm{H}$ & 3.47377300 & -4.73735300 & 0.93460500 \\
\hline $\mathrm{H}$ & 3.19269100 & -3.31419000 & -0.06190900 \\
\hline $\mathrm{H}$ & 4.45945600 & -4.47151000 & -0.51047900 \\
\hline $\mathrm{C}$ & 6.02225100 & -4.21735800 & 1.76458300 \\
\hline $\mathrm{H}$ & 6.76352800 & -3.73997800 & 2.41714600 \\
\hline $\mathrm{H}$ & 5.50624800 & -4.98200000 & 2.36298500 \\
\hline $\mathrm{H}$ & 6.56287700 & -4.74151600 & 0.96614000 \\
\hline $\mathrm{C}$ & 4.26992400 & -2.52832500 & 2.38653700 \\
\hline $\mathrm{H}$ & 3.73959700 & -3.28195700 & 2.98605600 \\
\hline $\mathrm{H}$ & 4.97028600 & -2.01119600 & 3.05456800 \\
\hline $\mathrm{H}$ & 3.52646300 & -1.79769500 & 2.05185500 \\
\hline & -2.92157400 & -0.78567900 & -0.19582900 \\
\hline
\end{tabular}




\begin{tabular}{|c|c|c|c|}
\hline $\mathrm{N}$ & -3.67334600 & 0.15050500 & 0.35312000 \\
\hline $\mathrm{C}$ & -5.15502300 & -0.22262400 & 0.44751100 \\
\hline $\mathrm{C}$ & -5.20316600 & -1.47170700 & -0.44773900 \\
\hline $\mathrm{H}$ & -5.52190400 & -1.18547400 & -1.45591800 \\
\hline $\mathrm{H}$ & -5.92501700 & -2.19916400 & -0.07037500 \\
\hline $\mathrm{C}$ & -3.21416200 & 1.40677900 & 0.95346900 \\
\hline $\mathrm{C}$ & -3.32035400 & 2.61920400 & 0.22342300 \\
\hline $\mathrm{C}$ & -2.75861800 & 1.39829600 & 2.29812900 \\
\hline $\mathrm{C}$ & -3.08125300 & 3.81745200 & 0.91008300 \\
\hline $\mathrm{C}$ & -2.54463400 & 2.63451000 & 2.92355700 \\
\hline $\mathrm{C}$ & -2.72873300 & 3.83606600 & 2.25277500 \\
\hline $\mathrm{H}$ & -3.16062000 & 4.75396700 & 0.36795600 \\
\hline $\mathrm{H}$ & -2.20920500 & 2.64804200 & 3.95529900 \\
\hline $\mathrm{C}$ & -3.56635500 & 2.72479400 & -1.27965100 \\
\hline $\mathrm{H}$ & -3.72275000 & 1.72384000 & -1.68787400 \\
\hline $\mathrm{C}$ & -2.36836400 & 0.15249900 & 3.09201500 \\
\hline $\mathrm{H}$ & -2.51187600 & -0.73310500 & 2.46856900 \\
\hline $\mathrm{H}$ & -2.56276000 & 4.78050900 & 2.76394300 \\
\hline $\mathrm{C}$ & -3.76320000 & -2.02836300 & -0.51298400 \\
\hline $\mathrm{C}$ & -3.43496700 & -2.64284300 & -1.89581700 \\
\hline $\mathrm{C}$ & -3.48514900 & -3.10877800 & 0.57999000 \\
\hline $\mathrm{C}$ & -4.24183500 & -3.92243700 & -2.15376700 \\
\hline $\mathrm{H}$ & -2.36387200 & -2.88991700 & -1.93246600 \\
\hline $\mathrm{H}$ & -3.59586700 & -1.89793800 & -2.67918300 \\
\hline $\mathrm{C}$ & -4.26875900 & -4.40606600 & 0.32687700 \\
\hline $\mathrm{H}$ & -2.41454900 & -3.33046900 & 0.58461700 \\
\hline $\mathrm{H}$ & -3.71389100 & -2.71693100 & 1.57603300 \\
\hline $\mathrm{C}$ & -3.97914100 & -4.97554400 & -1.06883000 \\
\hline $\mathrm{H}$ & -3.98441800 & -4.31749200 & -3.14384900 \\
\hline $\mathrm{H}$ & -5.31515300 & -3.68411800 & -2.18450300 \\
\hline $\mathrm{H}$ & -3.99999700 & -5.13479400 & 1.10148300 \\
\hline $\mathrm{H}$ & -5.34870900 & -4.22970400 & 0.43181100 \\
\hline $\mathrm{H}$ & -4.58216700 & -5.87432200 & -1.24800100 \\
\hline $\mathrm{H}$ & -2.92466200 & -5.28435400 & -1.11770300 \\
\hline $\mathrm{C}$ & -6.05520800 & 0.88407900 & -0.09763000 \\
\hline $\mathrm{H}$ & -7.09494900 & 0.54397600 & -0.03717500 \\
\hline $\mathrm{H}$ & -5.96418900 & 1.80255700 & 0.48790900 \\
\hline $\mathrm{H}$ & -5.83012300 & 1.10125500 & -1.14260300 \\
\hline $\mathrm{C}$ & -5.53899100 & -0.49847400 & 1.90543900 \\
\hline $\mathrm{H}$ & -5.44170200 & 0.41055400 & 2.50456700 \\
\hline
\end{tabular}




$\begin{array}{lrrr}\mathrm{H} & -6.58483300 & -0.82069100 & 1.94417400 \\ \mathrm{H} & -4.92362900 & -1.27882300 & 2.35477800 \\ \mathrm{C} & -3.17022800 & -0.02572400 & 4.39710200 \\ \mathrm{H} & -2.98383500 & 0.80302400 & 5.08989700 \\ \mathrm{H} & -4.24869400 & -0.08228900 & 4.23462600 \\ \mathrm{H} & -2.85359000 & -0.94765900 & 4.89836000 \\ \mathrm{C} & -0.86397600 & 0.22472300 & 3.44102100 \\ \mathrm{H} & -0.67352200 & 1.01277300 & 4.17947600 \\ \mathrm{H} & -0.53293000 & -0.72905700 & 3.86183200 \\ \mathrm{H} & -0.25656000 & 0.42298800 & 2.55681100 \\ \mathrm{C} & -4.76784200 & 3.62232200 & -1.64350300 \\ \mathrm{H} & -4.57138100 & 4.66908500 & -1.38397700 \\ \mathrm{H} & -4.93739300 & 3.58366000 & -2.72559700 \\ \mathrm{H} & -5.69463500 & 3.33110800 & -1.14367700 \\ \mathrm{C} & -2.30435600 & 3.29401100 & -1.96583200 \\ \mathrm{H} & -2.42946500 & 3.26330200 & -3.05241900 \\ \mathrm{H} & -2.13414900 & 4.33528800 & -1.66375800 \\ \mathrm{H} & -1.41951200 & 2.70798700 & -1.72035600\end{array}$

\section{mcb-ii}

Thermal correction to Gibbs free energy $=0.967146$

Sum of electronic and thermal free energies $=-3252.112135$

E_sol $=-3252.9728176$

$\begin{array}{lrrr}\mathrm{Ru} & -0.90335500 & -0.60016900 & -0.59488000 \\ \mathrm{Cl} & -1.59656800 & 0.13942600 & -2.79952800 \\ \mathrm{Cl} & -0.22643500 & -1.76978100 & 1.39825300 \\ \mathrm{C} & 0.57257300 & -1.45349700 & -1.51925800 \\ \mathrm{C} & 0.96902900 & -2.53507300 & -2.16456900 \\ \mathrm{H} & 0.29784000 & -3.37380000 & -2.31950300 \\ \mathrm{C} & 3.78128500 & 3.50086700 & -1.04130400 \\ \mathrm{C} & 3.59447300 & 4.13454000 & 0.38503500 \\ \mathrm{~B} & 1.78461900 & 2.85988600 & -0.13039200 \\ \mathrm{O} & 2.17196800 & 3.93839500 & 0.62906800 \\ \mathrm{O} & 2.73573400 & 2.48953600 & -1.05972300 \\ \mathrm{C} & 4.34054800 & 3.36135300 & 1.48075500 \\ \mathrm{H} & 5.42170600 & 3.52274200 & 1.41921300 \\ \mathrm{H} & 3.99023400 & 3.70666200 & 2.45812300 \\ \mathrm{H} & 4.14902900 & 2.28602200 & 1.40836300 \\ \mathrm{C} & 5.12714800 & 2.81793800 & -1.27195600 \\ \mathrm{H} & 5.17763500 & 2.44142600 & -2.29924300\end{array}$




\begin{tabular}{|c|c|c|c|}
\hline $\mathrm{H}$ & 5.94755000 & 3.53179900 & -1.13423400 \\
\hline $\mathrm{H}$ & 5.27223700 & 1.97062100 & -0.59810400 \\
\hline $\mathrm{C}$ & 3.46664100 & 4.47642500 & -2.18098500 \\
\hline $\mathrm{H}$ & 4.24431300 & 5.24061500 & -2.28270400 \\
\hline $\mathrm{H}$ & 3.40384800 & 3.91610200 & -3.11875400 \\
\hline $\mathrm{H}$ & 2.50493700 & 4.97280800 & -2.01744300 \\
\hline $\mathrm{C}$ & 3.90377700 & 5.62505000 & 0.47766800 \\
\hline $\mathrm{H}$ & 3.73765700 & 5.97244000 & 1.50234800 \\
\hline $\mathrm{H}$ & 4.95029500 & 5.81851000 & 0.21587600 \\
\hline $\mathrm{H}$ & 3.26187000 & 6.20916400 & -0.18543100 \\
\hline $\mathrm{C}$ & 0.27098400 & 0.85808400 & -0.38623000 \\
\hline $\mathrm{C}$ & 0.45206700 & 2.10290200 & 0.05754700 \\
\hline $\mathrm{H}$ & -0.37255300 & 2.56832900 & 0.59599000 \\
\hline $\mathrm{C}$ & 1.27324700 & -0.13558800 & -1.10856600 \\
\hline $\mathrm{H}$ & 1.49807200 & 0.39426300 & -2.04236500 \\
\hline $\mathrm{H}$ & 1.97438600 & -2.62040300 & -2.57032800 \\
\hline $\mathrm{C}$ & 2.50955800 & -0.40270700 & -0.21933900 \\
\hline $\mathrm{H}$ & 2.31670900 & -1.26793600 & 0.41568600 \\
\hline $\mathrm{H}$ & 2.68519100 & 0.44855500 & 0.43797400 \\
\hline $\mathrm{C}$ & 3.77388600 & -0.59712700 & -1.05221900 \\
\hline $\mathrm{H}$ & 3.86877600 & 0.22865800 & -1.76580600 \\
\hline $\mathrm{H}$ & 3.72964100 & -1.53358300 & -1.62546300 \\
\hline $\mathrm{O}$ & 4.90747600 & -0.56551400 & -0.19334200 \\
\hline $\mathrm{Si}$ & 5.93513900 & -1.85424500 & 0.13258100 \\
\hline $\mathrm{C}$ & 6.59637700 & -2.54852600 & -1.49637000 \\
\hline $\mathrm{H}$ & 7.27307000 & -3.39435900 & -1.32549800 \\
\hline $\mathrm{H}$ & 5.78942700 & -2.90117000 & -2.14927800 \\
\hline $\mathrm{H}$ & 7.15383900 & -1.77870300 & -2.04294700 \\
\hline $\mathrm{C}$ & 7.32186100 & -1.07508900 & 1.13753600 \\
\hline $\mathrm{H}$ & 8.10878600 & -1.80031500 & 1.37451700 \\
\hline $\mathrm{H}$ & 7.77902000 & -0.25180800 & 0.57664100 \\
\hline $\mathrm{H}$ & 6.94345300 & -0.66609500 & 2.08104000 \\
\hline $\mathrm{C}$ & 5.05742200 & -3.23372800 & 1.12856200 \\
\hline $\mathrm{C}$ & 3.97681700 & -3.94024300 & 0.28008300 \\
\hline $\mathrm{H}$ & 3.51342900 & -4.75252300 & 0.85881400 \\
\hline $\mathrm{H}$ & 3.16718900 & -3.26660600 & -0.01978600 \\
\hline $\mathrm{H}$ & 4.39881100 & -4.38803000 & -0.62861200 \\
\hline $\mathrm{C}$ & 6.11000100 & -4.28310900 & 1.55339900 \\
\hline $\mathrm{H}$ & 6.89002900 & -3.84926700 & 2.19109300 \\
\hline $\mathrm{H}$ & 5.63057700 & -5.08904200 & 2.12787800 \\
\hline
\end{tabular}




\begin{tabular}{|c|c|c|c|}
\hline $\mathrm{H}$ & 6.60109500 & -4.74943300 & 0.68970600 \\
\hline$C$ & 4.40432800 & -2.64122400 & 2.39528200 \\
\hline & 3.91300900 & -3.43416400 & 2.97743900 \\
\hline & 5.14553300 & -2.16551000 & 3.04980700 \\
\hline & 3.64240500 & -1.89284300 & 2.15405600 \\
\hline & -2.84622700 & -0.86149600 & -0.21605000 \\
\hline & -3.68093900 & 0.03908300 & 0.26139300 \\
\hline & -5.13433000 & -0.44752600 & 0.31345000 \\
\hline & -5.04904900 & -1.72845200 & -0.53376700 \\
\hline & -5.35530100 & -1.50714600 & -1.56206400 \\
\hline & -5.72322600 & -2.49733300 & -0.15083000 \\
\hline & -3.35077300 & 1.35610300 & 0.82028500 \\
\hline & -3.52357800 & 2.52339400 & 0.02901400 \\
\hline & -2.98808200 & 1.45158700 & 2.19096200 \\
\hline & -3.43429700 & 3.76744600 & 0.66784400 \\
\hline & -2.92844900 & 2.72924500 & 2.76501000 \\
\hline & -3.17296600 & 3.87812400 & 2.02646400 \\
\hline & -3.56485800 & 4.66789800 & 0.07695700 \\
\hline & -2.66536800 & 2.81876200 & 3.81367600 \\
\hline & -3.69422700 & 2.54867300 & -1.48776800 \\
\hline & -3.74216200 & 1.52237600 & -1.85724900 \\
\hline & -2.53744100 & 0.29024500 & 3.07537500 \\
\hline & -2.55976000 & -0.63598100 & 2.49708800 \\
\hline & -3.12651300 & 4.85532000 & 2.49942100 \\
\hline & -3.56680100 & -2.17115900 & -0.52913400 \\
\hline & -3.13444400 & -2.78102300 & -1.88535200 \\
\hline & -3.25641000 & -3.20793600 & 0.59619300 \\
\hline & -3.82671400 & -4.12466600 & -2.15045500 \\
\hline & -2.04766600 & -2.93720100 & -1.87595100 \\
\hline & -3.32655100 & -2.06635300 & -2.68986700 \\
\hline & -3.92723800 & -4.56530300 & 0.33535500 \\
\hline & -2.17380700 & -3.34617800 & 0.65119200 \\
\hline & -3.55737600 & -2.81516600 & 1.57251600 \\
\hline & -3.52942800 & -5.13406900 & -1.03344300 \\
\hline & -3.49569500 & -4.51506600 & -3.12033900 \\
\hline & -4.91330700 & -3.97396000 & -2.23099400 \\
\hline & -3.63987100 & -5.25728600 & 1.13643700 \\
\hline & -5.02137500 & -4.47210200 & 0.38696100 \\
\hline & -4.04932400 & -6.08186500 & -1.22019900 \\
\hline & -2.45234400 & -5.35663800 & -1.02941000 \\
\hline
\end{tabular}




$\begin{array}{lrrr}\mathrm{C} & -6.09292000 & 0.56429400 & -0.30941600 \\ \mathrm{H} & -7.10422700 & 0.14376400 & -0.28113800 \\ \mathrm{H} & -6.10212800 & 1.50720100 & 0.24360300 \\ \mathrm{H} & -5.83805500 & 0.76278500 & -1.35126200 \\ \mathrm{C} & -5.55318100 & -0.70229100 & 1.76449400 \\ \mathrm{H} & -5.55393300 & 0.23227600 & 2.33083600 \\ \mathrm{H} & -6.57030500 & -1.10785700 & 1.77726100 \\ \mathrm{H} & -4.89435000 & -1.41278300 & 2.26550400 \\ \mathrm{C} & -3.39407700 & 0.10546600 & 4.34418700 \\ \mathrm{H} & -3.33263800 & 0.98592000 & 4.99413300 \\ \mathrm{H} & -4.45007400 & -0.07144700 & 4.12972100 \\ \mathrm{H} & -3.01726500 & -0.74969300 & 4.91696900 \\ \mathrm{C} & -1.06961000 & 0.52787600 & 3.50164200 \\ \mathrm{H} & -1.00149200 & 1.36394900 & 4.20802100 \\ \mathrm{H} & -0.67355300 & -0.36783900 & 3.98832300 \\ \mathrm{H} & -0.43240900 & 0.74500500 & 2.64265300 \\ \mathrm{C} & -4.94812500 & 3.31790100 & -1.95463800 \\ \mathrm{H} & -4.86411500 & 4.38674100 & -1.72713500 \\ \mathrm{H} & -5.04874200 & 3.22580300 & -3.04219700 \\ \mathrm{H} & -5.87266000 & 2.95930900 & -1.49642000 \\ \mathrm{C} & -2.45009500 & 3.20577800 & -2.12849800 \\ \mathrm{H} & -2.49160000 & 3.09602700 & -3.21679100 \\ \mathrm{H} & -2.41251200 & 4.27622700 & -1.89084200 \\ \mathrm{H} & -1.52706100 & 2.73921600 & -1.78417600\end{array}$

\section{ts-iii}

Thermal correction to Gibbs free energy $=0.96321$

Sum of electronic and thermal free energies $=-3252.09638$

$\begin{array}{lrrr}\text { E_sol }=-3252.9522764 & & \\ \mathrm{Ru} & -1.07166800 & -0.64332000 & -0.98888400 \\ \mathrm{Cl} & -1.90276800 & 0.62303400 & -2.88223500 \\ \mathrm{Cl} & -0.28820300 & -2.07827300 & 0.79975900 \\ \mathrm{C} & -0.87238500 & -2.04119600 & -2.10468400 \\ \mathrm{C} & -0.80707200 & -3.08544700 & -2.89688800 \\ \mathrm{H} & -1.65239000 & -3.76569400 & -2.96612000 \\ \mathrm{C} & 4.17761000 & 3.15180300 & -1.42611900 \\ \mathrm{C} & 3.96452500 & 3.64524900 & 0.05444900 \\ \mathrm{~B} & 2.28944000 & 2.22759400 & -0.54700300 \\ \mathrm{O} & 2.58777000 & 3.25086000 & 0.31783800\end{array}$




$\begin{array}{lrrr}\mathrm{O} & 3.24712200 & 2.03638500 & -1.51503300 \\ \mathrm{C} & 4.83726800 & 2.89904000 & 1.07120000 \\ \mathrm{H} & 5.88944400 & 3.18998400 & 0.98759400 \\ \mathrm{H} & 4.48810100 & 3.13739300 & 2.08028100 \\ \mathrm{H} & 4.76426100 & 1.81578000 & 0.93776300 \\ \mathrm{C} & 5.57836700 & 2.63748000 & -1.74330400 \\ \mathrm{H} & 5.61958200 & 2.30137400 & -2.78405300 \\ \mathrm{H} & 6.32148000 & 3.43163600 & -1.60965000 \\ \mathrm{H} & 5.85020800 & 1.79209600 & -1.10724300 \\ \mathrm{C} & 3.73050800 & 4.17606100 & -2.47541700 \\ \mathrm{H} & 4.41275500 & 5.03121800 & -2.51970900 \\ \mathrm{H} & 3.71241200 & 3.69249800 & -3.45660900 \\ \mathrm{H} & 2.72167100 & 4.54184400 & -2.26005300 \\ \mathrm{C} & 4.08474800 & 5.15246300 & 0.25670700 \\ \mathrm{H} & 3.90892400 & 5.39710500 & 1.30899700 \\ \mathrm{H} & 5.08896100 & 5.49904800 & -0.01234900 \\ \mathrm{H} & 3.35251600 & 5.69671900 & -0.34346800 \\ \mathrm{H} & 3.27774400 & -0.01014900 & 1.06846000 \\ \mathrm{C} & 0.76062000 & 0.30428100 & -1.16168200 \\ \mathrm{C} & 1.01021300 & 1.37051500 & -0.40862400 \\ \mathrm{H} & & & \\ \mathrm{H} & & & \end{array}$




\begin{tabular}{|c|c|c|c|}
\hline & 5.87781900 & -4.59093500 & 0.48252300 \\
\hline $\mathrm{H}$ & 5.88693600 & -5.64032300 & 0.81290400 \\
\hline & 5.71916500 & -4.58363400 & -0.60210800 \\
\hline & 6.87492900 & -4.17763300 & 0.68085800 \\
\hline & 5.05135700 & -3.82639000 & 2.73601700 \\
\hline & 4.27069000 & -3.30307500 & 3.30215000 \\
\hline & 5.08258500 & -4.86220700 & 3.10457700 \\
\hline & 6.01570400 & -3.36410600 & 2.98435500 \\
\hline & 3.40779400 & -4.46987200 & 0.93863800 \\
\hline & 3.40926900 & -5.50998900 & 1.29687700 \\
\hline & 2.58211800 & -3.95026300 & 1.43973100 \\
\hline & 3.18554700 & -4.48569000 & -0.13417000 \\
\hline & -2.89768500 & -0.45147800 & -0.10328700 \\
\hline & -3.27210400 & 0.49522000 & 0.74076100 \\
\hline & -4.72568800 & 0.37313800 & 1.21485700 \\
\hline & -5.26772800 & -0.71432800 & 0.27565600 \\
\hline & -5.86689700 & -0.25138600 & -0.51535500 \\
\hline & -5.92453500 & -1.40143700 & 0.81375900 \\
\hline & -2.45997600 & 1.59275000 & 1.28704600 \\
\hline & -2.49103800 & 2.86375200 & 0.64729700 \\
\hline & -1.77183600 & 1.42259200 & 2.52222800 \\
\hline & -1.91691000 & 3.95394800 & 1.31477900 \\
\hline & -1.23205900 & 2.56263600 & 3.13483200 \\
\hline & -1.31964500 & 3.82132800 & 2.55943100 \\
\hline & -1.93689000 & 4.92774900 & 0.83732800 \\
\hline & -0.71354000 & 2.44926000 & 4.08082000 \\
\hline & -3.01353200 & 3.14103700 & -0.76112200 \\
\hline & -3.40522000 & 2.21483600 & -1.18759400 \\
\hline & -1.44531400 & 0.08948300 & 3.19739600 \\
\hline & -1.84505000 & -0.72641100 & 2.59194900 \\
\hline & -0.89650200 & 4.68668500 & 3.06212400 \\
\hline & -4.04572900 & -1.42737300 & -0.35202000 \\
\hline & -4.28672800 & -1.69613600 & -1.85843700 \\
\hline & -3.70855900 & -2.77371400 & 0.35766800 \\
\hline & -5.38691600 & -2.74376500 & -2.07717800 \\
\hline & -3.36239300 & -2.05283300 & -2.31858300 \\
\hline & -4.52276500 & -0.75496900 & -2.36534800 \\
\hline & -4.78032300 & -3.84692800 & 0.11459700 \\
\hline & -2.74410900 & -3.13088100 & -0.01509000 \\
\hline & -3.56882700 & -2.60763300 & 1.43192900 \\
\hline
\end{tabular}




$\begin{array}{lrrr}\mathrm{C} & -5.03067300 & -4.06620900 & -1.38382900 \\ \mathrm{H} & -5.52585000 & -2.89966700 & -3.15390000 \\ \mathrm{H} & -6.34838100 & -2.37541600 & -1.69015700 \\ \mathrm{H} & -4.45945700 & -4.78103800 & 0.59181200 \\ \mathrm{H} & -5.72486700 & -3.56206000 & 0.59985000 \\ \mathrm{H} & -5.82406600 & -4.80884800 & -1.53446000 \\ \mathrm{H} & -4.11949500 & -4.47771300 & -1.84351200 \\ \mathrm{C} & -5.48818700 & 1.68466600 & 1.04058600 \\ \mathrm{H} & -6.52446100 & 1.52935700 & 1.36057600 \\ \mathrm{H} & -5.06245900 & 2.48982300 & 1.64562400 \\ \mathrm{H} & -5.50079300 & 1.99255100 & -0.00595500 \\ \mathrm{C} & -4.76944000 & -0.04523100 & 2.68546300 \\ \mathrm{H} & -4.36394200 & 0.74382600 & 3.32160200 \\ \mathrm{H} & -5.81176900 & -0.21182600 & 2.97743000 \\ \mathrm{H} & -4.21050800 & -0.96651600 & 2.86401700 \\ \mathrm{C} & -1.98218700 & -0.03809700 & 4.63844300 \\ \mathrm{H} & -1.54820400 & 0.72820500 & 5.29105800 \\ \mathrm{H} & -3.06766100 & 0.04624000 & 4.71005300 \\ \mathrm{H} & -1.69397900 & -1.01389500 & 5.04607400 \\ \mathrm{C} & 0.09268800 & -0.08123900 & 3.24947200 \\ \mathrm{H} & 0.53483500 & 0.60305400 & 3.98360600 \\ \mathrm{H} & 0.34362400 & -1.10501900 & 3.54153700 \\ \mathrm{H} & 0.55687500 & 0.10479900 & 2.28071900 \\ \mathrm{C} & -4.11048800 & 4.22649700 & -0.80832300 \\ \mathrm{H} & -3.70944100 & 5.20513200 & -0.52060300 \\ \mathrm{H} & -4.48794100 & 4.31918300 & -1.83315800 \\ \mathrm{H} & -4.95723400 & 4.01701500 & -0.15163900 \\ \mathrm{H} & -1.84036200 & 3.60326700 & -1.65714900 \\ \mathrm{H} & -2.16472400 & 3.64802900 & -2.70105900 \\ \mathrm{H} & -1.49651000 & 4.60140700 & -1.35907200 \\ \mathrm{H} & & & \end{array}$

\section{vinyl-ii}

Thermal correction to Gibbs free energy $=0.515164$

Sum of electronic and thermal free energies $=-2044.649574$

$\begin{array}{lrrr}\text { E_sol }=-2045.104888 & & \\ \mathrm{Ru} & 0.81163400 & -0.16082500 & -0.83888200 \\ \mathrm{Cl} & -0.89066000 & -1.13946300 & 0.42409900 \\ \mathrm{Cl} & 1.69147900 & 0.10756300 & -2.99208600 \\ \mathrm{C} & 0.81928900 & 1.46809500 & -0.14991500\end{array}$




$\begin{array}{lrrr}\mathrm{C} & 0.64596700 & 2.68192100 & 0.32993200 \\ \mathrm{H} & 1.15568700 & 3.54031600 & -0.09793200 \\ \mathrm{C} & 2.41146800 & -0.91101600 & -0.09043900 \\ \mathrm{~N} & 3.49243300 & -0.36003200 & 0.45985700 \\ \mathrm{C} & 4.67330300 & -1.31083000 & 0.60182900 \\ \mathrm{C} & 4.17040600 & -2.53541700 & -0.18756400 \\ \mathrm{H} & 4.52358400 & -3.46636000 & 0.26174300 \\ \mathrm{H} & 4.56230900 & -2.49671100 & -1.21014700 \\ \mathrm{C} & 3.57121600 & 0.98756600 & 0.99428800 \\ \mathrm{C} & 4.08814300 & 2.04294500 & 0.20736900 \\ \mathrm{C} & 3.11938000 & 1.20406400 & 2.31860200 \\ \mathrm{C} & 4.23224400 & 3.29673100 & 0.81621900 \\ \mathrm{C} & 3.27720200 & 2.48217100 & 2.86514300 \\ \mathrm{C} & 3.84670600 & 3.51775000 & 2.13285100 \\ \mathrm{H} & 4.63578200 & 4.12030000 & 0.23523400 \\ \mathrm{H} & 2.93387900 & 2.67016000 & 3.87787900 \\ \mathrm{C} & 4.40023800 & 1.94042300 & -1.28403000 \\ \mathrm{H} & 2.39196900 & 0.15392700 & 3.15539000 \\ \mathrm{C} & 2.41837200 & -0.79584000 & 2.62169400 \\ \mathrm{H} & 2.63029300 & -2.43165000 & -0.22914800 \\ \mathrm{H} & & & \\ \mathrm{H} & & & \end{array}$




$\begin{array}{lrrr}\mathrm{H} & 6.25861000 & 0.18160200 & 0.48090300 \\ \mathrm{H} & 6.73694300 & -1.46262100 & 0.02705300 \\ \mathrm{H} & 5.77380300 & -0.48913600 & -1.09393500 \\ \mathrm{C} & 5.86181300 & 2.30735200 & -1.61448500 \\ \mathrm{H} & 6.05340000 & 3.36809200 & -1.41517000 \\ \mathrm{H} & 6.58747800 & 1.72962300 & -1.03721800 \\ \mathrm{H} & 6.05742100 & 2.13493300 & -2.67908800 \\ \mathrm{C} & 3.46474900 & 2.85852600 & -2.09929700 \\ \mathrm{H} & 3.62550200 & 3.91292500 & -1.84420500 \\ \mathrm{H} & 3.66257400 & 2.73354100 & -3.16890500 \\ \mathrm{H} & 2.41581200 & 2.61253400 & -1.93441200 \\ \mathrm{C} & 3.04086700 & -0.05991300 & 4.53653400 \\ \mathrm{H} & 2.54893800 & -0.89087500 & 5.05528900 \\ \mathrm{H} & 4.10824500 & -0.28798300 & 4.46348400 \\ \mathrm{H} & 2.93343600 & 0.82791500 & 5.16976500 \\ \mathrm{C} & 0.90355000 & 0.51999200 & 3.32669400 \\ \mathrm{H} & 0.39946400 & -0.24020200 & 3.93456500 \\ \mathrm{H} & 0.79171700 & 1.48665200 & 3.83207900 \\ \mathrm{H} & 0.39128100 & 0.56567900 & 2.36475100 \\ \mathrm{H} & 3.96858000 & 4.50076300 & 2.58005600 \\ \mathrm{H} & 4.20162800 & 0.91777300 & -1.61409900 \\ \mathrm{H} & 0.02127900 & 2.83121800 & 1.20795600\end{array}$

ts-iv

Thermal correction to Gibbs free energy $=0.731361$

Sum of electronic and thermal free energies $=-2571.804661$

E_sol $=-2572.4519192$

$\begin{array}{llll}\mathrm{Ru} & 0.92635900 & 2.31401200 & -2.07513500 \\ \mathrm{Cl} & 2.39415800 & 3.98318800 & -1.10745000 \\ \mathrm{Cl} & -0.67239000 & 0.79911400 & -3.06750000 \\ \mathrm{C} & -0.46125700 & 3.40423700 & -1.76496000 \\ \mathrm{C} & -1.47993700 & 4.20947200 & -1.57517100 \\ \mathrm{H} & -2.06483300 & 4.14934800 & -0.66126900 \\ \mathrm{C} & 3.78000500 & 3.48870700 & -7.81094600 \\ \mathrm{C} & 4.43021600 & 2.05478600 & -7.82007800 \\ \mathrm{~B} & 3.19098500 & 2.34664800 & -5.93944800 \\ \mathrm{O} & 4.24202300 & 1.62222500 & -6.44197900 \\ \mathrm{O} & 2.77923300 & 3.36591000 & -6.76089000 \\ \mathrm{C} & 3.67094300 & 1.05348600 & -8.69890900\end{array}$




\begin{tabular}{|c|c|c|c|}
\hline $\mathrm{H}$ & 3.80962200 & 1.26614900 & -9.76395900 \\
\hline $\mathrm{H}$ & 4.04572100 & 0.04644300 & -8.49253200 \\
\hline $\mathrm{H}$ & 2.59975300 & 1.06913400 & -8.47487600 \\
\hline $\mathrm{C}$ & 3.08101200 & 3.88941800 & -9.10618900 \\
\hline $\mathrm{H}$ & 2.65178500 & 4.89041600 & -8.99791200 \\
\hline $\mathrm{H}$ & 3.79446800 & 3.91076000 & -9.93790000 \\
\hline & 2.27139600 & 3.20010100 & -9.35491300 \\
\hline & 4.75091400 & 4.58841400 & -7.36388100 \\
\hline & 5.50906100 & 4.79114200 & -8.12739700 \\
\hline $\mathrm{H}$ & 4.18506800 & 5.50688900 & -7.18146000 \\
\hline $\mathrm{H}$ & 5.25562200 & 4.31153700 & -6.43296400 \\
\hline C & 5.92139700 & 2.02125800 & -8.14007600 \\
\hline & 6.28100200 & 0.98794600 & -8.10730400 \\
\hline & 6.10955500 & 2.41755600 & -9.14448200 \\
\hline & 6.49940900 & 2.60297500 & -7.41885300 \\
\hline 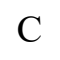 & 1.59308500 & 2.79727100 & -4.06887900 \\
\hline C & 2.54583200 & 2.02847700 & -4.56576800 \\
\hline $\mathrm{H}$ & 2.90234900 & 1.16496100 & -4.00129500 \\
\hline $\mathrm{H}$ & -1.76155900 & 4.94646600 & -2.32280300 \\
\hline & 1.22722700 & 1.11338400 & -0.47278400 \\
\hline & 2.13161100 & 0.14813600 & -0.38178400 \\
\hline C & 2.15665400 & -0.56806900 & 0.97325200 \\
\hline & 1.20275400 & 0.29983000 & 1.80657900 \\
\hline & 1.77953400 & 0.92697100 & 2.49411200 \\
\hline $\mathrm{H}$ & 0.54491400 & -0.32092700 & 2.41907700 \\
\hline & 3.07345500 & -0.30995500 & -1.41405100 \\
\hline 4 & 4.38313900 & 0.24675200 & -1.45429000 \\
\hline $\mathrm{C}$ & 2.73011500 & -1.40694400 & -2.25629600 \\
\hline & 5.34225300 & -0.37342800 & -2.26646000 \\
\hline & 3.74778600 & -1.98270200 & -3.03055300 \\
\hline & 5.04632000 & -1.49594400 & -3.02496200 \\
\hline & 6.34373600 & 0.04166000 & -2.30213700 \\
\hline & 3.50348900 & -2.82589100 & -3.66771800 \\
\hline & 4.82917400 & 1.52943300 & -0.75430000 \\
\hline & 3.99455800 & 1.93393500 & -0.17756200 \\
\hline 0 & 1.32202400 & -1.95274100 & -2.50562000 \\
\hline & 0.60513500 & -1.37426100 & -1.91883600 \\
\hline & 5.81380400 & -1.96872300 & -3.63172400 \\
\hline & 0.41875300 & 1.19853200 & 0.81962000 \\
\hline & 0.31394300 & 2.63904900 & 1.37699700 \\
\hline
\end{tabular}




\begin{tabular}{|c|c|c|c|}
\hline $\mathrm{C}$ & -1.01057800 & 0.65006100 & 0.52417200 \\
\hline $\mathrm{C}$ & -0.59110900 & 2.70170100 & 2.61533300 \\
\hline $\mathrm{H}$ & -0.08787200 & 3.30531900 & 0.61136400 \\
\hline $\mathrm{H}$ & 1.31751300 & 3.01849400 & 1.59607200 \\
\hline $\mathrm{C}$ & -1.93854100 & 0.74770100 & 1.74391500 \\
\hline $\mathrm{H}$ & -1.43739100 & 1.21792800 & -0.30811700 \\
\hline $\mathrm{H}$ & -0.94712900 & -0.38713300 & 0.17460300 \\
\hline $\mathrm{C}$ & -1.99904500 & 2.17844200 & 2.29596600 \\
\hline $\mathrm{H}$ & -0.63866000 & 3.73712700 & 2.97390900 \\
\hline $\mathrm{H}$ & -0.15976300 & 2.10882000 & 3.43529300 \\
\hline $\mathrm{H}$ & -2.93954300 & 0.40323400 & 1.45640900 \\
\hline $\mathrm{H}$ & -1.59645900 & 0.06983900 & 2.53901900 \\
\hline $\mathrm{H}$ & -2.63766300 & 2.21769100 & 3.18728500 \\
\hline $\mathrm{H}$ & -2.46422700 & 2.83415400 & 1.54460600 \\
\hline $\mathrm{C}$ & 3.56076700 & -0.57858600 & 1.57463300 \\
\hline $\mathrm{H}$ & 3.51544400 & -1.06371200 & 2.55605600 \\
\hline $\mathrm{H}$ & 4.26845200 & -1.13411200 & 0.95321100 \\
\hline $\mathrm{H}$ & 3.93154800 & 0.43772700 & 1.71668600 \\
\hline $\mathrm{C}$ & 1.65766900 & -2.00599600 & 0.82999200 \\
\hline $\mathrm{H}$ & 2.34840100 & -2.59639100 & 0.22526000 \\
\hline $\mathrm{H}$ & 1.60390000 & -2.46387100 & 1.82345000 \\
\hline $\mathrm{H}$ & 0.66420300 & -2.04663100 & 0.37778600 \\
\hline $\mathrm{C}$ & 1.15575700 & -3.45293800 & -2.18310000 \\
\hline $\mathrm{H}$ & 1.82526200 & -4.06441500 & -2.79897100 \\
\hline $\mathrm{H}$ & 1.35034700 & -3.70371900 & -1.13959500 \\
\hline $\mathrm{H}$ & 0.12951800 & -3.75994700 & -2.41581400 \\
\hline $\mathrm{C}$ & 0.96877000 & -1.75741900 & -4.00103100 \\
\hline $\mathrm{H}$ & 1.52820400 & -2.46410600 & -4.62609900 \\
\hline $\mathrm{H}$ & -0.09932700 & -1.93477200 & -4.15634900 \\
\hline $\mathrm{H}$ & 1.18448800 & -0.74426100 & -4.34149000 \\
\hline $\mathrm{C}$ & 6.04239100 & 1.33204700 & 0.18008000 \\
\hline $\mathrm{H}$ & 6.93897500 & 1.06122300 & -0.38917000 \\
\hline $\mathrm{H}$ & 6.25921400 & 2.27308700 & 0.69849000 \\
\hline $\mathrm{H}$ & 5.88964600 & 0.55673800 & 0.93351400 \\
\hline $\mathrm{C}$ & 5.21114500 & 2.58489000 & -1.81824000 \\
\hline $\mathrm{H}$ & 5.38443600 & 3.55201100 & -1.33731700 \\
\hline $\mathrm{H}$ & 6.12761000 & 2.29033600 & -2.34408200 \\
\hline $\mathrm{H}$ & 4.41846000 & 2.72440800 & -2.55388300 \\
\hline $\mathrm{C}$ & 0.67296300 & 3.78883600 & -3.94128000 \\
\hline $\mathrm{H}$ & -0.30753400 & 3.67073700 & -4.39711700 \\
\hline
\end{tabular}




\section{mcb-iii}

Thermal correction to Gibbs free energy $=0.733806$

Sum of electronic and thermal free energies $=-2571.828598$

E_sol $=-2572.4780954$

$\begin{array}{llll}\mathrm{Ru} & 1.25104300 & 1.75412300 & -1.94183600 \\ \mathrm{Cl} & 2.65157600 & 3.59281400 & -1.21988800 \\ \mathrm{Cl} & -0.47682400 & 0.18829400 & -2.54380200 \\ \mathrm{C} & 0.25578300 & 3.06389600 & -2.96613800 \\ \mathrm{C} & -0.71059200 & 3.96157600 & -2.96269700 \\ \mathrm{H} & -1.29002900 & 4.16297500 & -2.06746500 \\ \mathrm{C} & 3.56474400 & 1.97911100 & -7.68944800 \\ \mathrm{C} & 4.39832100 & 0.64399700 & -7.70431700 \\ \mathrm{~B} & 3.51972700 & 0.99415800 & -5.63332300 \\ \mathrm{O} & 4.54802300 & 0.35684100 & -6.28746700 \\ \mathrm{O} & 2.81515100 & 1.85732200 & -6.45053200 \\ \mathrm{C} & 3.63321400 & -0.53744000 & -8.31439600 \\ \mathrm{H} & 3.53112800 & -0.43530900 & -9.39987300 \\ \mathrm{H} & 4.18069200 & -1.46043900 & -8.10099300 \\ \mathrm{H} & 2.63470600 & -0.62650300 & -7.87496600 \\ \mathrm{C} & 2.57809400 & 2.13943300 & -8.84184000 \\ \mathrm{H} & 2.03796500 & 3.08568700 & -8.73537800 \\ \mathrm{H} & 3.10482400 & 2.15294300 & -9.80286000 \\ \mathrm{H} & 1.84394100 & 1.33114500 & -8.85441600 \\ \mathrm{C} & 4.43691200 & 3.23366200 & -7.55375800 \\ \mathrm{H} & 4.99741200 & 3.43769900 & -8.47199900 \\ \mathrm{H} & 3.79164500 & 4.09191700 & -7.34230400 \\ \mathrm{H} & 5.14569600 & 3.13073300 & -6.72613200 \\ \mathrm{C} & 5.78619400 & 0.75667000 & -8.32814700 \\ \mathrm{H} & 6.29117700 & -0.21329400 & -8.27934500 \\ \mathrm{H} & 5.71409400 & 1.05282700 & -9.38096600 \\ \mathrm{H} & 6.40462800 & 1.48529500 & -7.79958600 \\ \mathrm{C} & 2.21728800 & 1.45613800 & -3.53380100 \\ \mathrm{H} & 3.18488600 & 0.77328600 & -4.14713200 \\ \mathrm{H} & 3.73828700 & 0.04269700 & -3.55884000 \\ \mathrm{H} & 1.395868600 & 4.52820100 & -3.85890300 \\ \mathrm{H} & & 1.04872200 & -0.08338800 \\ \mathrm{H} & 0.32192800 & 0.24056900 & 0.39020100\end{array}$




\begin{tabular}{|c|c|c|c|}
\hline $\mathrm{C}$ & 2.22209500 & 0.02319000 & 1.90547100 \\
\hline $\mathrm{C}$ & 1.20603300 & 1.11095800 & 2.29601100 \\
\hline $\mathrm{H}$ & 1.73975700 & 1.99767400 & 2.65530800 \\
\hline $\mathrm{H}$ & 0.55825300 & 0.76996600 & 3.10640800 \\
\hline $\mathrm{C}$ & 3.32994600 & -0.50654800 & -0.37196600 \\
\hline $\mathrm{C}$ & 4.65982900 & -0.01431300 & -0.45618600 \\
\hline $\mathrm{C}$ & 2.98757100 & -1.77857900 & -0.90484100 \\
\hline $\mathrm{C}$ & 5.64224600 & -0.86569100 & -0.97927800 \\
\hline $\mathrm{C}$ & 4.02499200 & -2.58015000 & -1.40158100 \\
\hline $\mathrm{C}$ & 5.34349300 & -2.14798100 & -1.41835900 \\
\hline $\mathrm{H}$ & 6.66276700 & -0.50430900 & -1.04958400 \\
\hline $\mathrm{H}$ & 3.78280900 & -3.55972800 & -1.80024500 \\
\hline $\mathrm{C}$ & 5.09890100 & 1.40927900 & -0.12401900 \\
\hline $\mathrm{H}$ & 4.24312300 & 1.96354000 & 0.26672300 \\
\hline $\mathrm{C}$ & 1.56834300 & -2.31016200 & -1.09803900 \\
\hline $\mathrm{H}$ & 0.85179400 & -1.56660300 & -0.74232000 \\
\hline $\mathrm{H}$ & 6.12814300 & -2.79380900 & -1.80311600 \\
\hline $\mathrm{C}$ & 0.42225600 & 1.47455800 & 1.01327500 \\
\hline $\mathrm{C}$ & 0.06584500 & 2.98009200 & 0.95178400 \\
\hline $\mathrm{C}$ & -0.89608600 & 0.65087400 & 0.86561000 \\
\hline $\mathrm{C}$ & -0.96309600 & 3.36430700 & 2.02376500 \\
\hline $\mathrm{H}$ & -0.35092400 & 3.20815300 & -0.03799600 \\
\hline $\mathrm{H}$ & 0.97555900 & 3.57994300 & 1.03857200 \\
\hline $\mathrm{C}$ & -1.94126400 & 1.03667800 & 1.92344800 \\
\hline $\mathrm{H}$ & -1.30770300 & 0.82942000 & -0.13107700 \\
\hline $\mathrm{H}$ & -0.68304900 & -0.42168500 & 0.91333700 \\
\hline $\mathrm{C}$ & -2.24958500 & 2.53954700 & 1.88443200 \\
\hline $\mathrm{H}$ & -1.18264600 & 4.43578000 & 1.94346100 \\
\hline $\mathrm{H}$ & -0.53300300 & 3.21058300 & 3.02452500 \\
\hline $\mathrm{H}$ & -2.85256000 & 0.45292400 & 1.74513900 \\
\hline $\mathrm{H}$ & -1.59115800 & 0.76287900 & 2.92889500 \\
\hline $\mathrm{H}$ & -2.96529500 & 2.80439500 & 2.67250200 \\
\hline $\mathrm{H}$ & -2.72840900 & 2.78186100 & 0.92442000 \\
\hline $\mathrm{C}$ & 3.56567400 & 0.24830800 & 2.59464400 \\
\hline $\mathrm{H}$ & 3.42441300 & 0.12577100 & 3.67415200 \\
\hline $\mathrm{H}$ & 4.31582600 & -0.47570200 & 2.26579100 \\
\hline $\mathrm{H}$ & 3.93948300 & 1.25700300 & 2.41403000 \\
\hline $\mathrm{C}$ & 1.73873400 & -1.39824900 & 2.20706800 \\
\hline $\mathrm{H}$ & 2.47406500 & -2.13165800 & 1.86721600 \\
\hline $\mathrm{H}$ & 1.62049500 & -1.51336800 & 3.28966300 \\
\hline
\end{tabular}




$\begin{array}{llrr}\mathrm{H} & 0.78120200 & -1.61838600 & 1.73296200 \\ \mathrm{C} & 1.30054500 & -3.64678500 & -0.37699000 \\ \mathrm{H} & 1.95174000 & -4.44012600 & -0.76170400 \\ \mathrm{H} & 1.45165600 & -3.59297600 & 0.70302200 \\ \mathrm{H} & 0.26535200 & -3.95771300 & -0.55865300 \\ \mathrm{C} & 1.30975400 & -2.50629000 & -2.61028400 \\ \mathrm{H} & 1.88948200 & -3.35364600 & -2.99607100 \\ \mathrm{H} & 0.24877500 & -2.70813200 & -2.78302100 \\ \mathrm{H} & 1.57237400 & -1.61408400 & -3.18112200 \\ \mathrm{C} & 6.25543900 & 1.47527000 & 0.89569500 \\ \mathrm{H} & 7.17868700 & 1.06111000 & 0.47467300 \\ \mathrm{H} & 6.45770500 & 2.52186800 & 1.15086200 \\ \mathrm{H} & 6.04768600 & 0.93334400 & 1.82125400 \\ \mathrm{C} & 5.54699600 & 2.11901900 & -1.42197900 \\ \mathrm{H} & 5.72645100 & 3.18000800 & -1.22169600 \\ \mathrm{H} & 6.47571500 & 1.67743400 & -1.80435200 \\ \mathrm{H} & 4.78505900 & 2.05408500 & -2.19912600 \\ \mathrm{C} & 1.23206700 & 2.56386000 & -4.05451000 \\ \mathrm{H} & 0.65429800 & 2.10767000 & -4.86243700 \\ \mathrm{H} & 1.85593800 & 3.40141100 & -4.37699300\end{array}$

ts-v

Thermal correction to Gibbs free energy $=1.0564$

Sum of electronic and thermal free energies $=-4090.206089$

E_sol $=-4089.281755$

$\begin{array}{llll}\mathrm{Ru} & -1.46374500 & 0.01593600 & 0.61508000 \\ \mathrm{Cl} & -2.20780500 & -0.47104400 & 2.74641400 \\ \mathrm{Cl} & -0.59875700 & -0.82649700 & -1.38818300 \\ \mathrm{C} & -0.47660500 & 1.46625700 & 0.83684800 \\ \mathrm{C} & -2.94634700 & 0.81342000 & -0.36632700 \\ \mathrm{~N} & -4.05229000 & 0.09842700 & -0.59899400 \\ \mathrm{C} & -4.78008600 & 0.53268600 & -1.86016100 \\ \mathrm{C} & -4.35927200 & 2.00884700 & -1.93141000 \\ \mathrm{H} & -5.11788800 & 2.63178800 & -1.44534000 \\ \mathrm{H} & -4.28194700 & 2.34718300 & -2.96708600 \\ \mathrm{C} & -4.57953300 & -0.88885400 & 0.33061600 \\ \mathrm{C} & -5.35098100 & -0.38872800 & 1.40827700 \\ \mathrm{C} & -4.31596900 & -2.26497200 & 0.18257200 \\ \mathrm{C} & -5.81217200 & -1.30175700 & 2.35937900\end{array}$




\begin{tabular}{|c|c|c|c|}
\hline $\mathrm{C}$ & -4.81773100 & -3.13449500 & 1.16111400 \\
\hline $\mathrm{C}$ & -5.54615600 & -2.66285000 & 2.24500500 \\
\hline $\mathrm{H}$ & -6.37966200 & -0.93883100 & 3.21062300 \\
\hline $\mathrm{H}$ & -4.62081700 & -4.19830900 & 1.07128000 \\
\hline $\mathrm{C}$ & -5.70036200 & 1.08735000 & 1.58312300 \\
\hline $\mathrm{H}$ & -5.42694100 & 1.61370800 & 0.66688000 \\
\hline $\mathrm{C}$ & -3.53057700 & -2.85467600 & -0.97957600 \\
\hline $\mathrm{H}$ & -3.11473500 & -2.03498400 & -1.56557000 \\
\hline $\mathrm{Ru}$ & 1.84063900 & 1.14443400 & 0.61521700 \\
\hline $\mathrm{C}$ & 3.53371500 & 0.06887000 & 0.24223900 \\
\hline $\mathrm{C}$ & 5.85545300 & -0.54142100 & -0.04674900 \\
\hline $\mathrm{C}$ & 5.01551000 & -1.80729600 & 0.12462500 \\
\hline $\mathrm{N}$ & 3.61930000 & -1.21042700 & -0.10447100 \\
\hline $\mathrm{H}$ & 6.81082100 & -0.61307500 & 0.47727000 \\
\hline $\mathrm{H}$ & 6.07588200 & -0.39381000 & -1.11043400 \\
\hline $\mathrm{C}$ & 2.63288000 & -2.00376900 & -0.82584600 \\
\hline $\mathrm{C}$ & 2.51606200 & -1.77412500 & -2.22330100 \\
\hline $\mathrm{C}$ & 1.78969200 & -2.70520700 & -2.97500100 \\
\hline $\mathrm{C}$ & 1.16159300 & -3.79415300 & -2.38339900 \\
\hline $\mathrm{C}$ & 1.22839700 & -3.95656000 & -1.00677400 \\
\hline $\mathrm{C}$ & 1.96459400 & -3.08020400 & -0.19954900 \\
\hline $\mathrm{H}$ & 1.70458200 & -2.56182500 & -4.04703700 \\
\hline $\mathrm{H}$ & 0.70148000 & -4.78255900 & -0.53974900 \\
\hline $\mathrm{C}$ & 1.97372800 & -3.34798000 & 1.30013900 \\
\hline $\mathrm{H}$ & 2.57372600 & -2.57996500 & 1.78648000 \\
\hline $\mathrm{C}$ & 3.09121800 & -0.56630400 & -2.96697200 \\
\hline $\mathrm{H}$ & 3.37597500 & 0.19249800 & -2.23799800 \\
\hline $\mathrm{Cl}$ & 1.82774200 & 2.35252200 & -1.47604800 \\
\hline $\mathrm{Cl}$ & 1.66305400 & -0.11917000 & 2.66405600 \\
\hline $\mathrm{C}$ & 2.47871200 & 2.58204100 & 1.46118600 \\
\hline $\mathrm{H}$ & 0.60306800 & -4.50047000 & -2.99234900 \\
\hline $\mathrm{C}$ & 2.55339500 & -4.73971900 & 1.63008700 \\
\hline $\mathrm{H}$ & 3.51558500 & -4.92379000 & 1.14069800 \\
\hline $\mathrm{H}$ & 2.69310600 & -4.84506100 & 2.71223300 \\
\hline $\mathrm{H}$ & 1.87256900 & -5.53556900 & 1.30636200 \\
\hline $\mathrm{C}$ & 0.55831700 & -3.23838100 & 1.89665300 \\
\hline $\mathrm{H}$ & 0.10025100 & -2.27853100 & 1.65568800 \\
\hline $\mathrm{H}$ & -0.09238000 & -4.04038500 & 1.53105200 \\
\hline $\mathrm{H}$ & 0.60290800 & -3.30446200 & 2.98892900 \\
\hline $\mathrm{C}$ & 2.02546700 & 0.08649800 & -3.87266000 \\
\hline
\end{tabular}




\begin{tabular}{|c|c|c|c|}
\hline $\mathrm{H}$ & 2.39457500 & 1.05505700 & -4.22415500 \\
\hline $\mathrm{H}$ & 1.81134300 & -0.53223900 & -4.75297300 \\
\hline $\mathrm{H}$ & 1.09812000 & 0.26037100 & -3.32583600 \\
\hline $\mathrm{C}$ & 4.32920800 & -0.91008800 & -3.81871600 \\
\hline $\mathrm{H}$ & 5.19543800 & -1.18946500 & -3.21278600 \\
\hline $\mathrm{H}$ & 4.11606700 & -1.73918700 & -4.50446800 \\
\hline & 4.61794700 & -0.04102300 & -4.42143500 \\
\hline & 5.36323500 & -2.91384400 & -0.86952800 \\
\hline & 4.69989700 & -3.77571200 & -0.75612300 \\
\hline $\mathrm{H}$ & 5.30700700 & -2.57920300 & -1.90453400 \\
\hline $\mathrm{H}$ & 6.38834600 & -3.24504300 & -0.66905600 \\
\hline & 5.13390800 & -2.37451400 & 1.55707800 \\
\hline & 4.60867700 & -1.75964000 & 2.28903500 \\
\hline & 4.73775900 & -3.38633800 & 1.62226500 \\
\hline & 6.19304800 & -2.41875200 & 1.83338300 \\
\hline & -6.28993900 & 0.32396900 & -1.76118500 \\
\hline $\mathrm{H}$ & -6.74925600 & 0.63653900 & -2.70500000 \\
\hline $\mathrm{H}$ & -6.53385000 & -0.73063300 & -1.59858700 \\
\hline & -6.73747900 & 0.91118800 & -0.95800300 \\
\hline & -4.23186600 & -0.24651100 & -3.07173000 \\
\hline & -4.55989300 & -1.28691900 & -3.06566300 \\
\hline & -4.61208800 & 0.21594600 & -3.98955300 \\
\hline & -3.13920900 & -0.22805900 & -3.09810600 \\
\hline & -4.91108000 & 1.74460100 & 2.73064000 \\
\hline & -5.21217400 & 2.79439500 & 2.83748900 \\
\hline & -5.10500300 & 1.23452000 & 3.68056100 \\
\hline & -3.83564700 & 1.70129600 & 2.55078100 \\
\hline & -7.21438900 & 1.29538000 & 1.78844900 \\
\hline & -7.80539800 & 0.79305800 & 1.01483200 \\
\hline & -7.54556700 & 0.91053600 & 2.75903800 \\
\hline & -7.45204700 & 2.36528900 & 1.76379700 \\
\hline & -4.45072000 & -3.69367800 & -1.88841000 \\
\hline & -5.32708000 & -3.12674400 & -2.22249600 \\
\hline & -3.90384400 & -4.03536900 & -2.77513500 \\
\hline & -4.81932700 & -4.57958600 & -1.35809500 \\
\hline & -2.33732400 & -3.70040000 & -0.50436900 \\
\hline & -1.69307200 & -3.13369900 & 0.16919700 \\
\hline & -2.66481000 & -4.60716500 & 0.01795100 \\
\hline & -1.72623600 & -3.99836700 & -1.36205200 \\
\hline & -5.90985200 & -3.35423000 & 3.00023100 \\
\hline
\end{tabular}




\begin{tabular}{|c|c|c|c|}
\hline $\mathrm{C}$ & 4.96123600 & 0.61903600 & 0.46272900 \\
\hline $\mathrm{C}$ & 5.16980000 & 1.88257600 & -0.41661500 \\
\hline $\mathrm{C}$ & 5.25397000 & 0.97796300 & 1.94618400 \\
\hline $\mathrm{C}$ & 6.57779000 & 2.46853900 & -0.24323500 \\
\hline $\mathrm{H}$ & 4.42944700 & 2.64265200 & -0.15591500 \\
\hline $\mathrm{H}$ & 4.98089600 & 1.63325600 & -1.46666800 \\
\hline $\mathrm{C}$ & 6.63187500 & 1.63283400 & 2.13983900 \\
\hline $\mathrm{H}$ & 4.47682900 & 1.65302100 & 2.30705800 \\
\hline $\mathrm{H}$ & 5.17726100 & 0.09191600 & 2.57842100 \\
\hline $\mathrm{C}$ & 6.83643200 & 2.84539800 & 1.22140100 \\
\hline $\mathrm{H}$ & 6.68277400 & 3.34794900 & -0.89040400 \\
\hline $\mathrm{H}$ & 7.33661800 & 1.74484700 & -0.57502000 \\
\hline $\mathrm{H}$ & 6.74017800 & 1.92509500 & 3.19186600 \\
\hline $\mathrm{H}$ & 7.42666000 & 0.89914000 & 1.94368800 \\
\hline $\mathrm{H}$ & 7.84854500 & 3.25077200 & 1.34538600 \\
\hline $\mathrm{H}$ & 6.13931100 & 3.64540400 & 1.51076000 \\
\hline $\mathrm{C}$ & -3.00244800 & 2.12001500 & -1.17400800 \\
\hline $\mathrm{C}$ & -3.06209500 & 3.31750800 & -0.18001700 \\
\hline $\mathrm{C}$ & -1.79881100 & 2.28933200 & -2.13585700 \\
\hline $\mathrm{C}$ & -3.06199600 & 4.66744600 & -0.91295200 \\
\hline $\mathrm{H}$ & -2.20461200 & 3.27785800 & 0.49196500 \\
\hline $\mathrm{H}$ & -3.95346400 & 3.22679200 & 0.45057000 \\
\hline $\mathrm{C}$ & -1.77784600 & 3.65492700 & -2.83539600 \\
\hline $\mathrm{H}$ & -0.86497700 & 2.17070100 & -1.58358700 \\
\hline $\mathrm{H}$ & -1.80341100 & 1.48346100 & -2.87527800 \\
\hline $\mathrm{C}$ & -1.83533200 & 4.80654300 & -1.82403300 \\
\hline $\mathrm{H}$ & -3.08883400 & 5.47593700 & -0.17148900 \\
\hline $\mathrm{H}$ & -3.97653200 & 4.76878200 & -1.51567200 \\
\hline $\mathrm{H}$ & -0.86358300 & 3.72084500 & -3.43595100 \\
\hline $\mathrm{H}$ & -2.62308700 & 3.74499800 & -3.53380900 \\
\hline $\mathrm{H}$ & -1.85202400 & 5.77330300 & -2.34232900 \\
\hline $\mathrm{H}$ & -0.92083300 & 4.79037300 & -1.21420400 \\
\hline $\mathrm{C}$ & 0.05142900 & 2.65183400 & 1.22710200 \\
\hline $\mathrm{H}$ & 0.18609000 & 3.46622000 & 0.51973500 \\
\hline $\mathrm{H}$ & 0.03047600 & 2.87652400 & 2.29357500 \\
\hline $\mathrm{C}$ & 2.84825000 & 3.69433400 & 2.06090600 \\
\hline $\mathrm{H}$ & 2.11194200 & 4.44644200 & 2.33301900 \\
\hline $\mathrm{H}$ & 3.89057300 & 3.89198900 & 2.288504 \\
\hline
\end{tabular}

mcb-iv 
Thermal correction to Gibbs free energy $=1.058768$

Sum of electronic and thermal free energies $=-4089.302898$

E_sol $=-4090.229031$

\begin{tabular}{|c|c|c|c|}
\hline $\mathrm{Ru}$ & -2.26346500 & 1.00159900 & -0.47928500 \\
\hline $\mathrm{Cl}$ & -2.79275800 & -0.06046100 & -2.50135500 \\
\hline $\mathrm{Cl}$ & -1.99115600 & 3.01190800 & 0.73220100 \\
\hline $\mathrm{C}$ & -0.69552500 & 0.15301100 & -0.28156800 \\
\hline $\mathrm{C}$ & -3.34650600 & 0.25954100 & 0.95335800 \\
\hline $\mathrm{N}$ & -4.67883200 & 0.36796700 & 0.83143600 \\
\hline $\mathrm{C}$ & -5.39885600 & 0.41332400 & 2.16315300 \\
\hline $\mathrm{C}$ & -4.42109500 & -0.39688800 & 3.02165800 \\
\hline $\mathrm{H}$ & -4.70351000 & -1.45537300 & 2.99118100 \\
\hline $\mathrm{H}$ & -4.45755500 & -0.08548200 & 4.06724700 \\
\hline $\mathrm{C}$ & -5.39779400 & 0.31055300 & -0.42831100 \\
\hline $\mathrm{C}$ & -5.69497200 & -0.98142300 & -0.92534900 \\
\hline $\mathrm{C}$ & -5.77833000 & 1.47824400 & -1.11649700 \\
\hline $\mathrm{C}$ & -6.38361600 & -1.07047800 & -2.13696700 \\
\hline $\mathrm{C}$ & -6.48370200 & 1.32711800 & -2.31822400 \\
\hline $\mathrm{C}$ & -6.78229500 & 0.07060500 & -2.82797600 \\
\hline $\mathrm{H}$ & -6.60362500 & -2.04844200 & -2.55323600 \\
\hline $\mathrm{H}$ & -6.78272800 & 2.21337500 & -2.86977300 \\
\hline $\mathrm{C}$ & -5.27141600 & -2.26612000 & -0.21574800 \\
\hline $\mathrm{H}$ & -4.98621900 & -2.01350400 & 0.80732400 \\
\hline $\mathrm{C}$ & -5.41259300 & 2.88362500 & -0.65768400 \\
\hline $\mathrm{H}$ & -4.80856800 & 2.80976700 & 81300 \\
\hline $\mathrm{Ru}$ & 1.16597600 & 0.35434800 & -0.25932900 \\
\hline $\mathrm{C}$ & 2.66382500 & 1.73814000 & -0.32722000 \\
\hline $\mathrm{C}$ & 4.89899200 & 2.62325400 & -0.38804400 \\
\hline $\mathrm{C}$ & 3.91287700 & 3.67708400 & -0.89906400 \\
\hline $\mathrm{N}$ & 2.58925800 & 3.04216200 & -0.44520900 \\
\hline $\mathrm{H}$ & 5.80712300 & 2.60044300 & -0.99367300 \\
\hline $\mathrm{H}$ & 5.19377400 & 2.87055600 & 0.63858700 \\
\hline $\mathrm{C}$ & 1.47667100 & 3.92008600 & -0.09875700 \\
\hline $\mathrm{C}$ & 1.35496900 & 4.29289100 & 1.26345400 \\
\hline $\mathrm{C}$ & 0.52676600 & 5.38056900 & 1.56289200 \\
\hline $\mathrm{C}$ & -0.18987200 & 6.04490200 & 0.57546000 \\
\hline $\mathrm{C}$ & -0.13622000 & 5.58798400 & -0.73497000 \\
\hline $\mathrm{C}$ & 0.68989400 & 4.52052600 & -1.10588900 \\
\hline $\mathrm{H}$ & 0.42868400 & 5.69678800 & 2.59657200 \\
\hline H & -0.75918000 & 6.05720600 & -1.489693 \\
\hline
\end{tabular}




\begin{tabular}{|c|c|c|c|}
\hline $\mathrm{C}$ & 0.60745100 & 4.01334500 & -2.53970600 \\
\hline $\mathrm{H}$ & 1.37481200 & 3.25569200 & -2.69279300 \\
\hline $\mathrm{C}$ & 1.98518400 & 3.53566300 & 2.43252700 \\
\hline $\mathrm{H}$ & 2.45089800 & 2.62336100 & 2.05584400 \\
\hline $\mathrm{Cl}$ & 1.36861600 & 0.03664600 & 2.11207200 \\
\hline $\mathrm{Cl}$ & 1.26855200 & 0.58732600 & -2.67189100 \\
\hline $\mathrm{C}$ & 1.27507100 & -1.56506600 & -0.51853400 \\
\hline $\mathrm{H}$ & -0.82405600 & 6.88824600 & 0.83490400 \\
\hline $\mathrm{C}$ & 0.79247100 & 5.12720000 & -3.58802400 \\
\hline $\mathrm{H}$ & 1.69064500 & 5.72748500 & -3.40543700 \\
\hline $\mathrm{H}$ & 0.87109800 & 4.68788000 & -4.58872200 \\
\hline $\mathrm{H}$ & -0.06077300 & 5.81512100 & -3.59879400 \\
\hline $\mathrm{C}$ & -0.74541000 & 3.31144900 & -2.76570300 \\
\hline $\mathrm{H}$ & -0.85650700 & 2.47222700 & -2.07289400 \\
\hline $\mathrm{H}$ & -1.58222800 & 3.99998000 & -2.60973000 \\
\hline $\mathrm{H}$ & -0.80254800 & 2.89534200 & -3.77624600 \\
\hline $\mathrm{C}$ & 0.87636600 & 3.08821500 & 3.40949000 \\
\hline $\mathrm{H}$ & 1.27682300 & 2.35105300 & 4.11240200 \\
\hline $\mathrm{H}$ & 0.48897800 & 3.93767100 & 3.98519700 \\
\hline $\mathrm{H}$ & 0.04584900 & 2.63243500 & 2.87049600 \\
\hline $\mathrm{C}$ & 3.05032900 & 4.33813400 & 3.20271600 \\
\hline $\mathrm{H}$ & 3.94834500 & 4.53003900 & 2.60802400 \\
\hline $\mathrm{H}$ & 2.65559200 & 5.30551800 & 3.53506500 \\
\hline $\mathrm{H}$ & 3.36054000 & 3.77979600 & 4.09365100 \\
\hline $\mathrm{C}$ & 4.13392400 & 5.06407800 & -0.30136500 \\
\hline $\mathrm{H}$ & 3.37556100 & 5.77289700 & -0.64562600 \\
\hline $\mathrm{H}$ & 4.12011800 & 5.05014400 & 0.78766400 \\
\hline $\mathrm{H}$ & 5.11470900 & 5.42674900 & -0.62810800 \\
\hline $\mathrm{C}$ & 3.93483700 & 3.78328600 & -2.43383500 \\
\hline $\mathrm{H}$ & 3.62143100 & 2.85484300 & -2.91448800 \\
\hline $\mathrm{H}$ & 3.28661500 & 4.58681300 & -2.78299500 \\
\hline $\mathrm{H}$ & 4.95633700 & 4.01687100 & -2.75359600 \\
\hline $\mathrm{C}$ & -6.80147100 & -0.19125700 & 2.09954300 \\
\hline $\mathrm{H}$ & -7.25644500 & -0.11716700 & 3.09317200 \\
\hline $\mathrm{H}$ & -7.43739200 & 0.35172300 & 1.39371100 \\
\hline $\mathrm{H}$ & -6.78825900 & -1.24410100 & 1.81348200 \\
\hline $\mathrm{C}$ & -5.49714500 & 1.87353700 & 2.64453200 \\
\hline $\mathrm{H}$ & -6.22056400 & 2.43794500 & 2.05296200 \\
\hline $\mathrm{H}$ & -5.83523600 & 1.88563100 & 3.68689400 \\
\hline $\mathrm{H}$ & -4.53210900 & 2.38197500 & 2.5838390 \\
\hline
\end{tabular}




$\begin{array}{lrrr}\mathrm{C} & -4.03318100 & -2.90282000 & -0.87512500 \\ \mathrm{H} & -3.76132100 & -3.82657900 & -0.34844500 \\ \mathrm{H} & -4.23198400 & -3.14896800 & -1.92391800 \\ \mathrm{H} & -3.18288800 & -2.21905400 & -0.85755000 \\ \mathrm{C} & -6.41727700 & -3.29176700 & -0.12416600 \\ \mathrm{H} & -7.32989400 & -2.84861600 & 0.28951500 \\ \mathrm{H} & -6.66660300 & -3.70887700 & -1.10600900 \\ \mathrm{H} & -6.11923200 & -4.12888600 & 0.51769700 \\ \mathrm{C} & -6.66378400 & 3.72187300 & -0.33586600 \\ \mathrm{H} & -7.31137000 & 3.22613400 & 0.39566200 \\ \mathrm{H} & -6.37418400 & 4.69882400 & 0.06837600 \\ \mathrm{H} & -7.26337400 & 3.89633600 & -1.23693200 \\ \mathrm{C} & -4.54669000 & 3.60409100 & -1.71132000 \\ \mathrm{H} & -3.69842500 & 2.98870800 & -2.02637200 \\ \mathrm{H} & -5.12752800 & 3.84138100 & -2.61037300 \\ \mathrm{H} & -4.14756400 & 4.53554900 & -1.29656400 \\ \mathrm{H} & -7.31732500 & -0.02280700 & -3.76913700 \\ \mathrm{C} & 4.13234900 & 1.27143500 & -0.39445200 \\ \mathrm{C} & -1.27161200 & -1.36629700 & 1.88848800 \\ \mathrm{C} & -2.78363600 & -2.28221500 & 1.74525200 \\ \mathrm{C} & -1.78989600 & 0.30587800 & 4.58960100 \\ \mathrm{C} & & & \\ \mathrm{C} & & & \end{array}$




$\begin{array}{lrrr}\mathrm{H} & -1.20872100 & 0.94477900 & 2.61217800 \\ \mathrm{H} & -2.61599200 & 1.78093800 & 3.22919600 \\ \mathrm{C} & -1.14712300 & -1.08880300 & 4.58336900 \\ \mathrm{H} & -1.45632500 & -3.04763600 & 3.69363600 \\ \mathrm{H} & -2.93367000 & -2.29329200 & 4.27915200 \\ \mathrm{H} & -1.10822800 & 1.02976500 & 5.05299800 \\ \mathrm{H} & -2.69387000 & 0.29187600 & 5.21587300 \\ \mathrm{H} & -1.01503800 & -1.45095200 & 5.61103800 \\ \mathrm{H} & -0.15128700 & -1.01742400 & 4.13229300 \\ \mathrm{C} & -0.22446500 & -1.37773200 & -0.57452300 \\ \mathrm{H} & -0.72151800 & -1.97661700 & 0.18786400 \\ \mathrm{H} & -0.61280400 & -1.53988700 & -1.58651900 \\ \mathrm{C} & 2.08621300 & -2.60598400 & -0.59294900 \\ \mathrm{H} & 1.69614400 & -3.61178600 & -0.73997700 \\ \mathrm{H} & 3.16328200 & -2.49521300 & -0.51631700\end{array}$

\section{ts-vi}

Thermal correction to Gibbs free energy $=1.058347$

Sum of electronic and thermal free energies $=-4089.301037$

E_sol $=-4090.223772$

$\begin{array}{lrrr}\mathrm{Ru} & -2.11561500 & 0.81932800 & -0.42110500 \\ \mathrm{Cl} & -2.58935200 & -0.28905300 & -2.43533800 \\ \mathrm{Cl} & -2.03003600 & 2.83666300 & 0.80899900 \\ \mathrm{C} & -0.47272700 & 0.16569500 & -0.21836700 \\ \mathrm{C} & -3.10956000 & -0.03040800 & 1.01833500 \\ \mathrm{~N} & -4.44775500 & -0.02813600 & 0.91636800 \\ \mathrm{C} & -5.14490100 & -0.04787600 & 2.26186600 \\ \mathrm{C} & -4.09436100 & -0.79093300 & 3.09390900 \\ \mathrm{H} & -4.29731100 & -1.86716400 & 3.05618400 \\ \mathrm{H} & -4.13536100 & -0.49376700 & 4.14334200 \\ \mathrm{C} & -5.18308300 & -0.13321400 & -0.33181700 \\ \mathrm{C} & -5.38746300 & -1.44122000 & -0.83427000 \\ \mathrm{C} & -5.66823800 & 1.00510800 & -1.00373800 \\ \mathrm{C} & -6.08488400 & -1.57604000 & -2.03656800 \\ \mathrm{C} & -6.37703100 & 0.80671400 & -2.19660300 \\ \mathrm{C} & -6.58172600 & -0.46552600 & -2.71330800 \\ \mathrm{H} & -6.23369600 & -2.56579000 & -2.45660000 \\ \mathrm{H} & -6.75540600 & 1.67027700 & -2.73521700 \\ \mathrm{C} & -4.85928300 & -2.69556300 & -0.14124300 \\ \mathrm{H} & -4.56300300 & -2.42618500 & 0.87429700\end{array}$




\begin{tabular}{|c|c|c|c|}
\hline $\mathrm{C}$ & -5.41973500 & 2.43227900 & -0.53442400 \\
\hline $\mathrm{H}$ & -4.79388600 & 2.40286500 & 0.35938700 \\
\hline $\mathrm{Ru}$ & 1.33860000 & 0.20939500 & -0.25893600 \\
\hline $\mathrm{C}$ & 2.78578000 & 1.70762700 & -0.31618000 \\
\hline $\mathrm{C}$ & 4.96310200 & 2.74113600 & -0.42913200 \\
\hline $\mathrm{C}$ & 3.90152900 & 3.71909000 & -0.93697900 \\
\hline $\mathrm{N}$ & 2.63100800 & 3.00288500 & -0.45283000 \\
\hline $\mathrm{H}$ & 5.85902600 & 2.76568300 & -1.05274100 \\
\hline $\mathrm{H}$ & 5.26190800 & 3.02561700 & 0.58666400 \\
\hline $\mathrm{C}$ & 1.47671600 & 3.81553900 & -0.09011600 \\
\hline $\mathrm{C}$ & 1.35918700 & 4.18300300 & 1.27326800 \\
\hline $\mathrm{C}$ & 0.48696100 & 5.23159700 & 1.58636300 \\
\hline $\mathrm{C}$ & -0.27555500 & 5.86050500 & 0.60986300 \\
\hline $\mathrm{C}$ & -0.22597300 & 5.40124400 & -0.70024600 \\
\hline $\mathrm{C}$ & 0.64253300 & 4.37243200 & -1.08316800 \\
\hline $\mathrm{H}$ & 0.39188200 & 5.54464900 & 2.62141200 \\
\hline $\mathrm{H}$ & -0.88585200 & 5.83691100 & -1.44368300 \\
\hline $\mathrm{C}$ & 0.55729400 & 3.84747900 & -2.51042800 \\
\hline $\mathrm{H}$ & 1.36583600 & 3.13652800 & -2.67583200 \\
\hline $\mathrm{C}$ & 2.04651200 & 3.45310000 & 2.42697200 \\
\hline $\mathrm{H}$ & 2.53627800 & 2.55889300 & 2.03844500 \\
\hline $\mathrm{Cl}$ & 1.63171600 & -0.10170800 & 2.10452100 \\
\hline $\mathrm{Cl}$ & 1.47149200 & 0.45481900 & -2.66295000 \\
\hline $\mathrm{C}$ & 1.50685500 & -1.77130000 & -0.50337300 \\
\hline $\mathrm{H}$ & -0.94350000 & 6.67429500 & 0.87869200 \\
\hline $\mathrm{C}$ & 0.64719200 & 4.95826200 & -3.57398600 \\
\hline $\mathrm{H}$ & 1.51213000 & 5.61289600 & -3.41989500 \\
\hline $\mathrm{H}$ & 0.72700600 & 4.51349700 & -4.57215900 \\
\hline $\mathrm{H}$ & -0.24599200 & 5.59355800 & -3.56913600 \\
\hline $\mathrm{C}$ & -0.75390800 & 3.06056200 & -2.69557200 \\
\hline $\mathrm{H}$ & -0.78471600 & 2.21714700 & -1.99749500 \\
\hline $\mathrm{H}$ & -1.62865300 & 3.69337200 & -2.51495600 \\
\hline $\mathrm{H}$ & -0.81619000 & 2.63266400 & -3.70072000 \\
\hline $\mathrm{C}$ & 0.98144100 & 2.96091100 & 3.42953100 \\
\hline $\mathrm{H}$ & 1.42697600 & 2.23654900 & 4.11863900 \\
\hline $\mathrm{H}$ & 0.57450700 & 3.79179700 & 4.01872200 \\
\hline $\mathrm{H}$ & 0.15612800 & 2.47589900 & 2.90819100 \\
\hline $\mathrm{C}$ & 3.10113500 & 4.29602100 & 3.16723400 \\
\hline $\mathrm{H}$ & 3.97562500 & 4.51585700 & 2.54729500 \\
\hline $\mathrm{H}$ & 2.68089800 & 5.25059100 & 3.50559600 \\
\hline
\end{tabular}




\begin{tabular}{|c|c|c|c|}
\hline $\mathrm{H}$ & 3.45454800 & 3.75349800 & 4.05189600 \\
\hline $\mathrm{C}$ & 4.04071600 & 5.12737300 & -0.36338100 \\
\hline $\mathrm{H}$ & 3.23401700 & 5.78058300 & -0.70774100 \\
\hline $\mathrm{H}$ & 4.04138700 & 5.13083400 & 0.72609600 \\
\hline $\mathrm{H}$ & 4.99181400 & 5.54714100 & -0.70911400 \\
\hline $\mathrm{C}$ & 3.89263300 & 3.80112700 & -2.47361500 \\
\hline $\mathrm{H}$ & 3.63087800 & 2.84616900 & -2.93334700 \\
\hline $\mathrm{H}$ & 3.18960100 & 4.55606000 & -2.82548400 \\
\hline $\mathrm{H}$ & 4.89203400 & 4.09313700 & -2.81456000 \\
\hline $\mathrm{C}$ & -6.49980700 & -0.75347900 & 2.21720600 \\
\hline $\mathrm{H}$ & -6.94010900 & -0.72184300 & 3.21965600 \\
\hline $\mathrm{H}$ & -7.18745800 & -0.25275800 & 1.52883900 \\
\hline $\mathrm{H}$ & -6.41412100 & -1.79983600 & 1.92053600 \\
\hline $\mathrm{C}$ & -5.33960800 & 1.39702200 & 2.75975200 \\
\hline $\mathrm{H}$ & -6.11393800 & 1.91235300 & 2.18831700 \\
\hline $\mathrm{H}$ & -5.65670900 & 1.37485800 & 3.80853000 \\
\hline $\mathrm{H}$ & -4.41554200 & 1.97507900 & 2.68513900 \\
\hline $\mathrm{C}$ & -3.60152200 & -3.24727100 & -0.83851200 \\
\hline $\mathrm{H}$ & -3.24640100 & -4.14526700 & -0.31702700 \\
\hline $\mathrm{H}$ & -3.81735800 & -3.51546500 & -1.87860100 \\
\hline $\mathrm{H}$ & -2.80308000 & -2.50403200 & -0.85448200 \\
\hline $\mathrm{C}$ & -5.93153900 & -3.79563800 & -0.02279900 \\
\hline $\mathrm{H}$ & -6.85966000 & -3.41641700 & 0.41879300 \\
\hline $\mathrm{H}$ & -6.18049400 & -4.22413800 & -0.99981100 \\
\hline $\mathrm{H}$ & -5.56019300 & -4.61362200 & 0.60538400 \\
\hline $\mathrm{C}$ & -6.73549000 & 3.14934400 & -0.17816900 \\
\hline $\mathrm{H}$ & -7.32058100 & 2.58797600 & 0.55860700 \\
\hline $\mathrm{H}$ & -6.52804200 & 4.14380200 & 0.23380200 \\
\hline $\mathrm{H}$ & -7.36626800 & 3.27869000 & -1.06546300 \\
\hline $\mathrm{C}$ & -4.64414500 & 3.24133100 & -1.59398200 \\
\hline $\mathrm{H}$ & -3.74846600 & 2.71069000 & -1.93121200 \\
\hline $\mathrm{H}$ & -5.26103700 & 3.43424400 & -2.47946800 \\
\hline $\mathrm{H}$ & -4.32553200 & 4.20115600 & -1.17430700 \\
\hline $\mathrm{H}$ & -7.12127600 & -0.59430300 & -3.64767800 \\
\hline $\mathrm{C}$ & 4.28382300 & 1.34389500 & -0.39734400 \\
\hline $\mathrm{C}$ & 4.70138300 & 0.56956800 & 0.88502500 \\
\hline $\mathrm{C}$ & 4.63777200 & 0.46058100 & -1.62862500 \\
\hline $\mathrm{C}$ & 6.19379900 & 0.21066600 & 0.86963100 \\
\hline $\mathrm{H}$ & 4.11620700 & -0.35086500 & 0.96397800 \\
\hline $\mathrm{H}$ & 4.44978300 & 1.15822700 & 1.77381100 \\
\hline
\end{tabular}




\begin{tabular}{|c|c|c|c|}
\hline $\mathrm{C}$ & 6.11687500 & 0.04408200 & -1.64826300 \\
\hline $\mathrm{H}$ & 4.01330300 & -0.43760500 & -1.60729000 \\
\hline $\mathrm{H}$ & 4.37517800 & 0.97225000 & -2.55617700 \\
\hline $\mathrm{C}$ & 6.53351000 & -0.65730200 & -0.34883900 \\
\hline $\mathrm{H}$ & 6.44351900 & -0.31563200 & 1.79899300 \\
\hline $\mathrm{H}$ & 6.80667700 & 1.12351900 & 0.85426500 \\
\hline $\mathrm{H}$ & 6.28484900 & -0.61351300 & -2.51001000 \\
\hline $\mathrm{H}$ & 6.75702800 & 0.92381700 & -1.80506900 \\
\hline$\Pi$ & 7.60384200 & -0.89735100 & -0.36809000 \\
\hline $\mathrm{H}$ & 5.99649300 & -1.61410100 & -0.26660100 \\
\hline $\mathrm{C}$ & -2.71548600 & -0.48497200 & 2.43954500 \\
\hline $\mathrm{C}$ & -1.85069000 & -1.76983800 & 2.36902000 \\
\hline $\mathrm{C}$ & -1.89390400 & 0.56884600 & 3.22382300 \\
\hline $\mathrm{C}$ & -1.53439000 & -2.31246800 & 3.77218600 \\
\hline $\mathrm{H}$ & -0.90257300 & -1.51498800 & 1.89075100 \\
\hline $\mathrm{H}$ & -2.35033300 & -2.53361800 & 1.75937800 \\
\hline $\mathrm{C}$ & -1.50124000 & 0.07995600 & 4.62825400 \\
\hline $\mathrm{H}$ & -0.98939600 & 0.77534000 & 2.64903400 \\
\hline $\mathrm{H}$ & -2.43921400 & 1.51242600 & 3.29432400 \\
\hline $\mathrm{C}$ & -0.76967500 & -1.27033200 & 4.60112800 \\
\hline $\mathrm{H}$ & -0.93761900 & -3.22788400 & 3.67415100 \\
\hline $\mathrm{H}$ & -2.46280400 & -2.60059400 & 4.28579200 \\
\hline $\mathrm{H}$ & -0.86199800 & 0.84147500 & 5.09179000 \\
\hline $\mathrm{H}$ & -2.39502000 & 0.00285100 & 5.26428900 \\
\hline $\mathrm{H}$ & -0.61432400 & -1.63776800 & 5.62369800 \\
\hline $\mathrm{H}$ & 0.21878900 & -1.12800300 & 4.15255800 \\
\hline $\mathrm{C}$ & 0.08683100 & -1.63519700 & -0.64916100 \\
\hline $\mathrm{H}$ & -0.52554700 & -2.11747000 & 0.10611200 \\
\hline $\mathrm{H}$ & -0.31903100 & -1.64376100 & -1.66108800 \\
\hline $\mathrm{C}$ & 2.49082200 & -2.65281000 & -0.47960000 \\
\hline $\mathrm{H}$ & 2.29544000 & -3.70644900 & -0.67175900 \\
\hline $\mathrm{H}$ & 3.51789700 & -2.36556200 & -0.278718 \\
\hline
\end{tabular}

\section{Ru-carbide}

Thermal correction to Gibbs free energy $=0.998716$

Sum of electronic and thermal free energies $=-3972.708779$

$$
\begin{array}{lrrl}
\text { E_sol }=-3973.5904165 & & \\
\text { C } & -1.44721800 & -0.42097900 & 0.12872400 \\
\text { C } & -3.54711100 & -1.56845000 & -0.22053100 \\
\text { C } & -2.48334300 & -2.55301400 & 0.28637200
\end{array}
$$




\begin{tabular}{|c|c|c|c|}
\hline $\mathrm{N}$ & -1.22561100 & -1.73877000 & 0.04162700 \\
\hline $\mathrm{H}$ & -3.74821600 & -1.76013300 & -1.28009700 \\
\hline $\mathrm{H}$ & -4.49084200 & -1.69422600 & 0.31436600 \\
\hline $\mathrm{C}$ & 0.02967600 & -2.34806700 & -0.36332200 \\
\hline $\mathrm{C}$ & 0.98901100 & -2.76107300 & 0.58107100 \\
\hline $\mathrm{C}$ & 2.17319900 & -3.33644400 & 0.09968100 \\
\hline $\mathrm{C}$ & 2.40693900 & -3.47792400 & -1.26162100 \\
\hline$C$ & 1.44798600 & -3.05305900 & -2.17673500 \\
\hline $\mathrm{C}$ & 0.24049600 & -2.49058800 & -1.75655000 \\
\hline $\mathrm{H}$ & 2.92875900 & -3.66025300 & 0.80900500 \\
\hline $\mathrm{H}$ & 1.64594400 & -3.15030000 & -3.23949500 \\
\hline $\mathrm{C}$ & -0.76847700 & -2.04338300 & -2.81272700 \\
\hline $\mathrm{H}$ & -1.71840600 & -1.84293400 & -2.31392300 \\
\hline $\mathrm{C}$ & 0.82988900 & -2.57749900 & 2.08427400 \\
\hline $\mathrm{H}$ & -0.09859700 & -2.03570300 & 2.27545600 \\
\hline $\mathrm{Cl}$ & -0.57349900 & 0.69963600 & 2.94570500 \\
\hline $\mathrm{Cl}$ & 1.67208000 & 0.46845100 & -0.96401100 \\
\hline $\mathrm{Ru}$ & 0.01212200 & 0.74047400 & 0.65823600 \\
\hline $\mathrm{C}$ & -0.70636300 & 2.29411200 & 0.24906900 \\
\hline $\mathrm{Ru}$ & -1.42487100 & 3.84773900 & -0.16008900 \\
\hline $\mathrm{Cl}$ & -3.08475200 & 4.11946700 & 1.46230800 \\
\hline $\mathrm{Cl}$ & -0.83910800 & 3.88888800 & -2.44751800 \\
\hline $\mathrm{C}$ & 0.03441900 & 5.00916700 & 0.36958900 \\
\hline $\mathrm{C}$ & 1.07049400 & 7.14123300 & 0.21203200 \\
\hline C & 2.13430100 & 6.15665000 & 0.71881200 \\
\hline$H$ & 2.33547100 & 6.34827600 & 1.77837600 \\
\hline $\mathrm{H}$ & 3.07799800 & 6.28248200 & 0.18387000 \\
\hline $\mathrm{N}$ & -0.18724200 & 6.32694200 & 0.45681700 \\
\hline $\mathrm{C}$ & -1.44251300 & 6.93620500 & 0.86189100 \\
\hline $\mathrm{C}$ & -2.40188900 & 7.34925800 & -0.08245200 \\
\hline 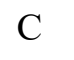 & -1.65329900 & 7.07859900 & 2.25513000 \\
\hline C & -3.58608300 & 7.92454200 & 0.39900200 \\
\hline & -2.86082400 & 7.64097100 & 2.67538100 \\
\hline $\mathrm{C}$ & -3.81980700 & 8.06587900 & 1.76032800 \\
\hline $\mathrm{H}$ & -4.34167300 & 8.24838400 & -0.31027500 \\
\hline $\mathrm{H}$ & -3.05877900 & 7.73809100 & 3.73815200 \\
\hline 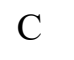 & -2.24279700 & 7.16578000 & -1.58567500 \\
\hline 11 & -1.31428400 & 6.62405200 & -1.77691600 \\
\hline & -0.64428800 & 6.63135500 & 3.31125100 \\
\hline & 0.30563500 & 6.43099100 & 2.81241000 \\
\hline
\end{tabular}




\begin{tabular}{|c|c|c|c|}
\hline $\mathrm{H}$ & -4.75176100 & 8.50083700 & 2.11121800 \\
\hline$H$ & 3.33887700 & -3.91296200 & -1.61245600 \\
\hline$S_{0}$ & 1.06784500 & 8.47717600 & 0.95312500 \\
\hline I & 1.99090600 & 9.01199600 & 0.70498700 \\
\hline $\mathrm{H}$ & 0.22218700 & 9.10041800 & 0.64663000 \\
\hline & 1.03257400 & 8.35296900 & 2.03596700 \\
\hline & 1.21337400 & 7.39976500 & -1.29993100 \\
\hline & 0.46998300 & 8.11856300 & -1.65017500 \\
\hline & 2.20573300 & 7.81874400 & -1.50102800 \\
\hline & 1.09907000 & 6.47950900 & -1.87790400 \\
\hline $\mathrm{C}$ & -2.16785700 & 8.52176100 & -2.31322300 \\
\hline & -1.96997800 & 8.37152600 & -3.38080400 \\
\hline & -3.11259100 & 9.07023100 & -2.22036000 \\
\hline & -1.37825000 & 9.16165400 & -1.90420300 \\
\hline & -3.38702400 & 6.31391100 & -2.17249500 \\
\hline & -3.17156900 & 6.06374900 & -3.21601600 \\
\hline & -3.51399200 & 5.37366900 & -1.62562800 \\
\hline $\mathrm{H}$ & -4.34321700 & 6.84835800 & -2.13255300 \\
\hline $\mathrm{C}$ & -0.38163400 & 7.72990700 & 4.36076600 \\
\hline $\mathrm{H}$ & -1.25100600 & 7.88259100 & 5.00964800 \\
\hline & 0.45611200 & 7.43827300 & 5.00458500 \\
\hline & -0.14172700 & 8.69314100 & 3.89715200 \\
\hline & -1.06724000 & 5.32289500 & 4.00728800 \\
\hline & -0.33682700 & 5.06181100 & 4.78316600 \\
\hline & -2.04740900 & 5.43496100 & 4.48399000 \\
\hline 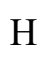 & -1.13880500 & 4.49247300 & 3.30310800 \\
\hline $\mathrm{C}$ & 0.75485300 & -3.93343100 & 2.81190400 \\
\hline $\mathrm{H}$ & 1.69957400 & -4.48193900 & 2.71914200 \\
\hline $\mathrm{H}$ & 0.55690200 & -3.78311800 & 3.87946100 \\
\hline $\mathrm{H}$ & -0.03474800 & -4.57332800 & 2.40288000 \\
\hline $\mathrm{C}$ & 1.97415000 & -1.72565600 & 2.67108800 \\
\hline & 2.10119900 & -0.78546500 & 2.12415800 \\
\hline & 1.75865800 & -1.47541500 & 3.71457800 \\
\hline & 2.93030600 & -2.26017700 & 2.63123000 \\
\hline $\mathrm{C}$ & -1.03114300 & -3.14204600 & -3.86213100 \\
\hline & -1.86889600 & -2.85048100 & -4.50597300 \\
\hline & -0.16177700 & -3.29479800 & -4.51100700 \\
\hline & -1.27104600 & -4.10523000 & -3.39841200 \\
\hline & -0.34544100 & -0.73502300 & -3.50887900 \\
\hline & 0.63472700 & -0.84717200 & -3.98556000 \\
\hline
\end{tabular}




\begin{tabular}{|c|c|c|c|}
\hline $\mathrm{H}$ & -1.07583700 & -0.47396700 & -4.28478200 \\
\hline $\mathrm{H}$ & -0.27383000 & 0.09546200 & -2.80477600 \\
\hline $\mathrm{C}$ & -2.48067200 & -3.88899800 & -0.45466200 \\
\hline $\mathrm{H}$ & -2.44535000 & -3.76484800 & -1.53751000 \\
\hline H & -1.63502800 & -4.51222300 & -0.14809500 \\
\hline $\mathrm{H}$ & -3.40374800 & -4.42379400 & -0.20653400 \\
\hline $\mathrm{C}$ & -2.62631100 & -2.81150700 & 1.79834300 \\
\hline & -3.61864200 & -3.23057400 & 1.99938300 \\
\hline $\mathrm{H}$ & -1.88286600 & -3.53021300 & 2.14866400 \\
\hline$\Pi$ & -2.51214100 & -1.89121900 & 2.37629300 \\
\hline $\mathrm{C}$ & 1.53604600 & 4.73049200 & 0.52880900 \\
\hline $\mathrm{C}$ & 1.74399900 & 3.88354400 & 1.81524100 \\
\hline $\mathrm{C}$ & 2.16120200 & 3.99394100 & -0.68090100 \\
\hline $\mathrm{C}$ & 3.22577500 & 3.57073100 & 2.05921100 \\
\hline $\mathrm{H}$ & 1.19402200 & 2.93988500 & 1.72487400 \\
\hline$\Pi$ & 1.30708600 & 4.39807100 & 2.67804700 \\
\hline C & 3.63175900 & 3.61269000 & -0.44182400 \\
\hline $\mathrm{H}$ & 1.58770500 & 3.09183400 & -0.88809200 \\
\hline $\mathrm{H}$ & 2.07635800 & 4.61373300 & -1.57841600 \\
\hline $\mathrm{C}$ & 3.83285300 & 2.82802300 & 0.86294000 \\
\hline $\mathrm{H}$ & 3.31654000 & 2.97022000 & 2.97286900 \\
\hline $\mathrm{H}$ & 3.77977800 & 4.50322800 & 2.24161900 \\
\hline $\mathrm{H}$ & 3.97602900 & 3.01545100 & -1.29398200 \\
\hline $\mathrm{H}$ & 4.25888200 & 4.51641000 & -0.41964400 \\
\hline $\mathrm{H}$ & 4.90132300 & 2.64273700 & 1.03172800 \\
\hline $\mathrm{H}$ & 3.35054100 & 1.84830300 & 0.76469300 \\
\hline $\mathrm{C}$ & -2.94884700 & -0.14228200 & -0.03057700 \\
\hline $\mathrm{C}$ & -3.57402200 & 0.59426800 & 1.17912800 \\
\hline $\mathrm{C}$ & -3.15680000 & 0.70459800 & -1.31703300 \\
\hline $\mathrm{C}$ & -5.04456000 & 0.97557000 & 0.94002300 \\
\hline $\mathrm{H}$ & -3.00050900 & 1.49634800 & 1.38637800 \\
\hline $\mathrm{H}$ & -3.48924000 & -0.02556300 & 2.07662400 \\
\hline & -4.63857800 & 1.01739400 & -1.56101900 \\
\hline $\mathrm{H}$ & -2.60684400 & 1.64827400 & -1.22670500 \\
\hline $\mathrm{H}$ & -2.71988000 & 0.19003300 & -2.17981200 \\
\hline $\mathrm{C}$ & -5.24563400 & 1.76017600 & -0.36478100 \\
\hline$H$ & -5.38879400 & 1.57288300 & 1.79214300 \\
\hline 11 & -5.67172300 & 0.07187700 & 0.91789600 \\
\hline 11 & -4.72936000 & 1.61784100 & -2.47471700 \\
\hline & -5.19258700 & 0.08488500 & -1.74335100 \\
\hline
\end{tabular}


Gonsales, et. al., Supporting Information, pg 210

$\begin{array}{llll}\mathrm{H} & -6.31409900 & 1.94548900 & -0.53356900 \\ \mathrm{H} & -4.76329700 & 2.73989000 & -0.26658100\end{array}$

8. NMR Spectra

8.1. Allenyl substrates

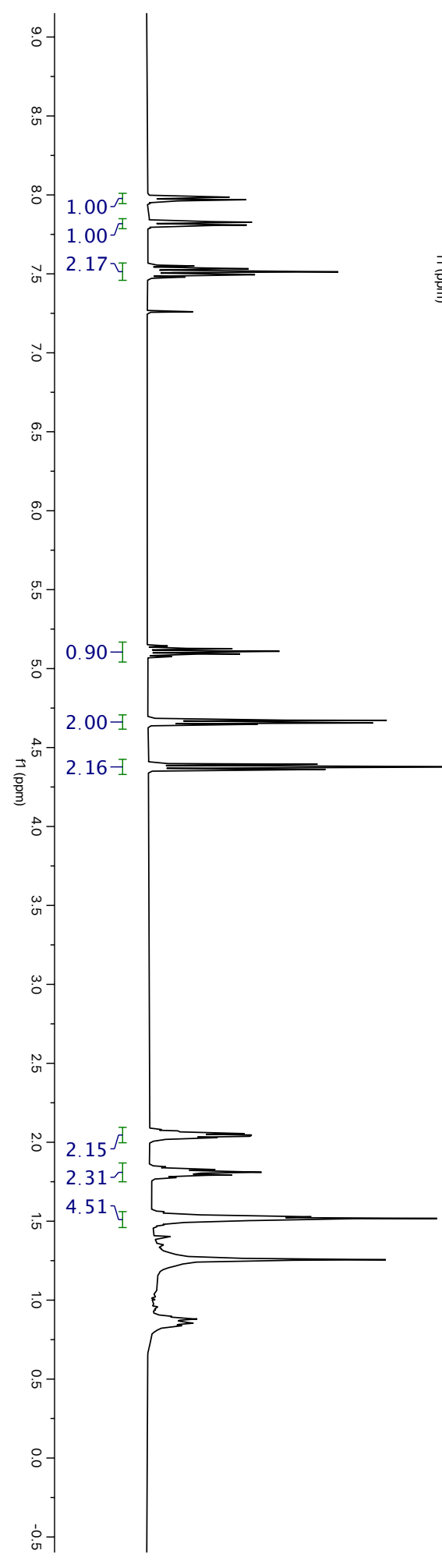

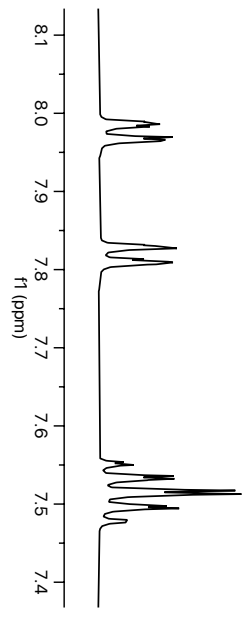

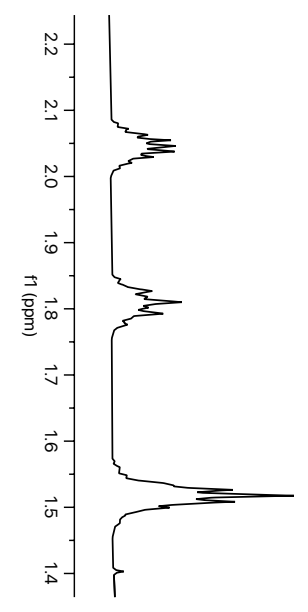

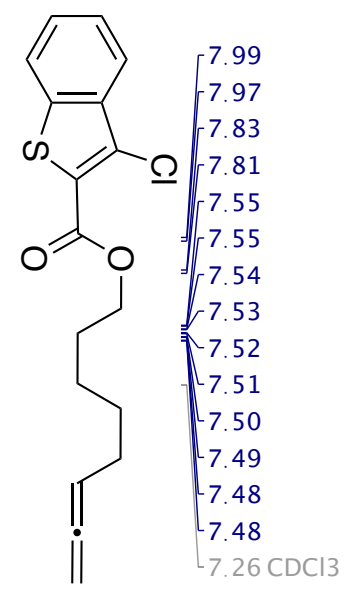

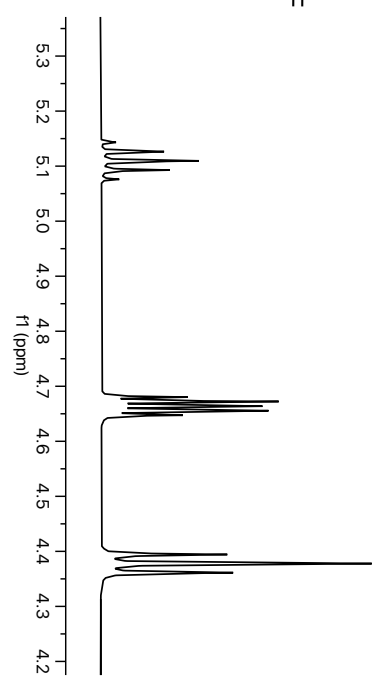




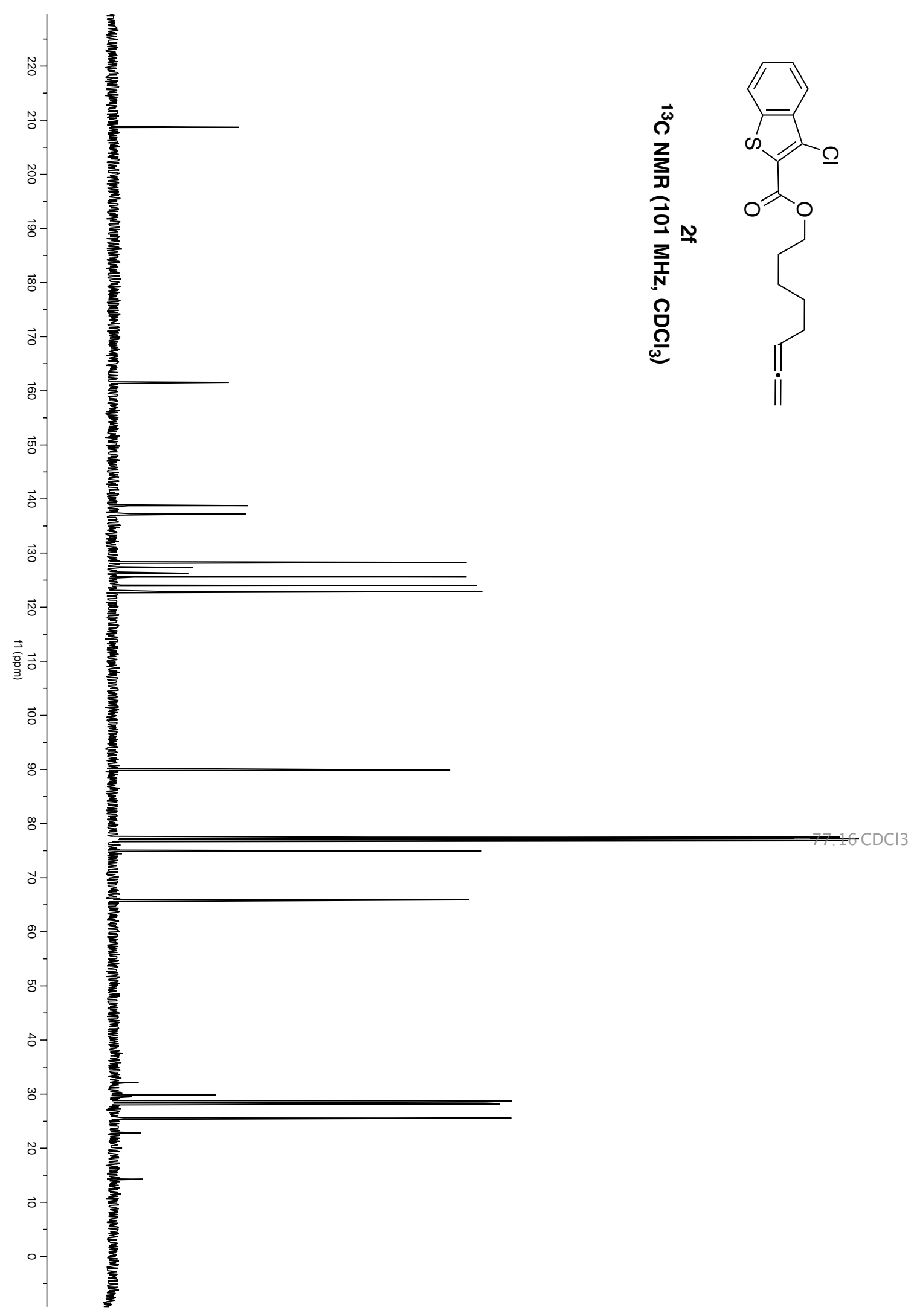




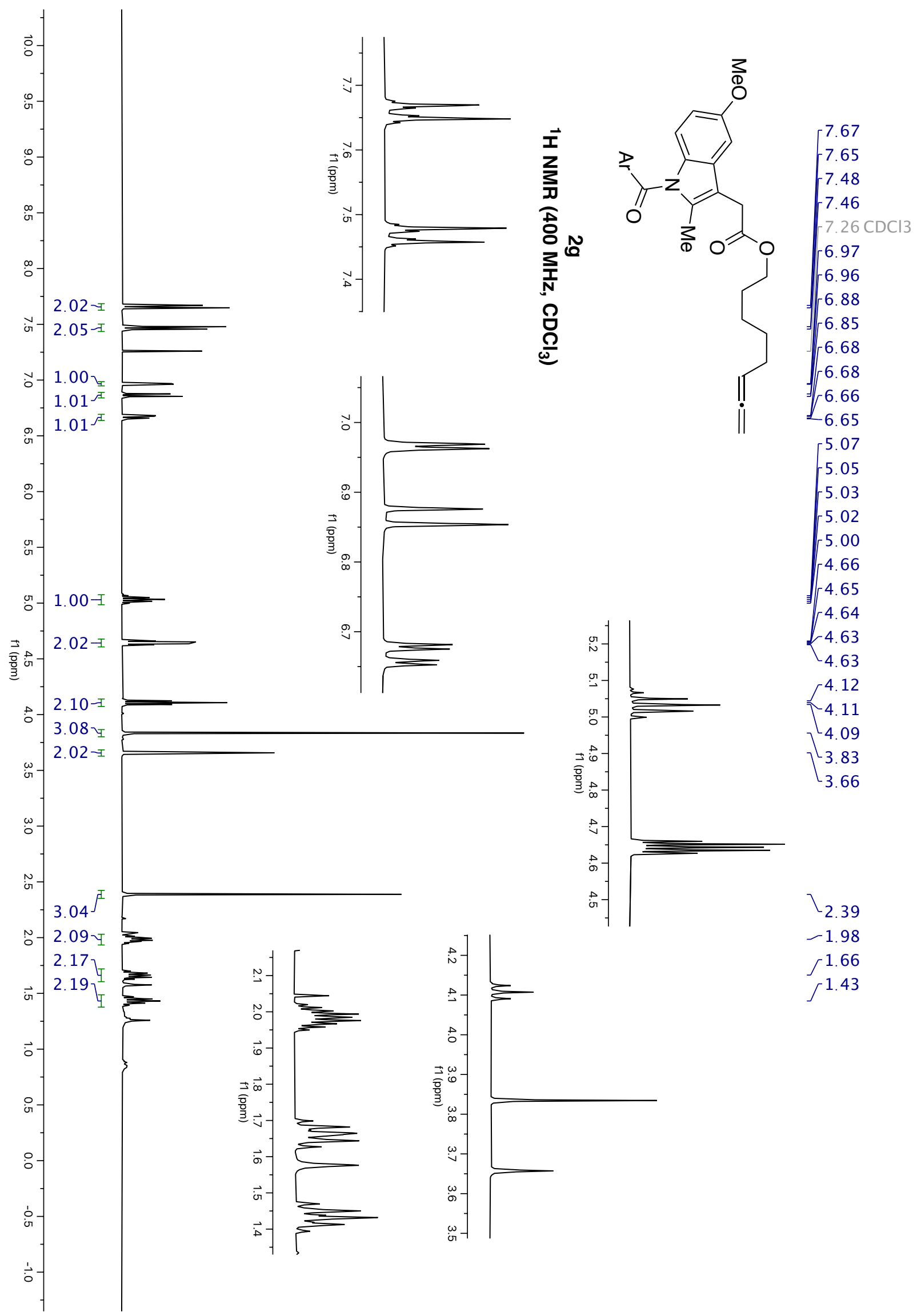




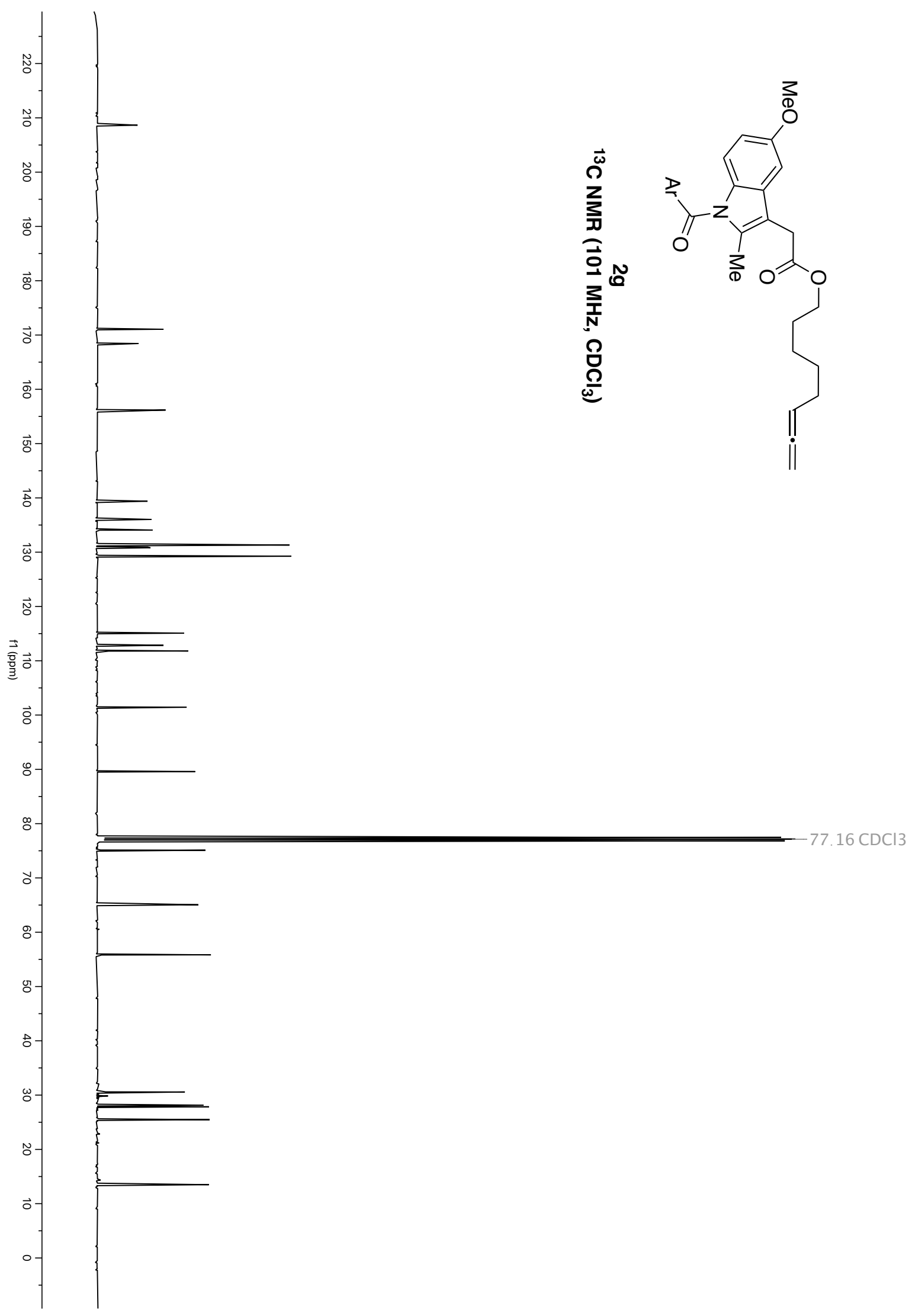




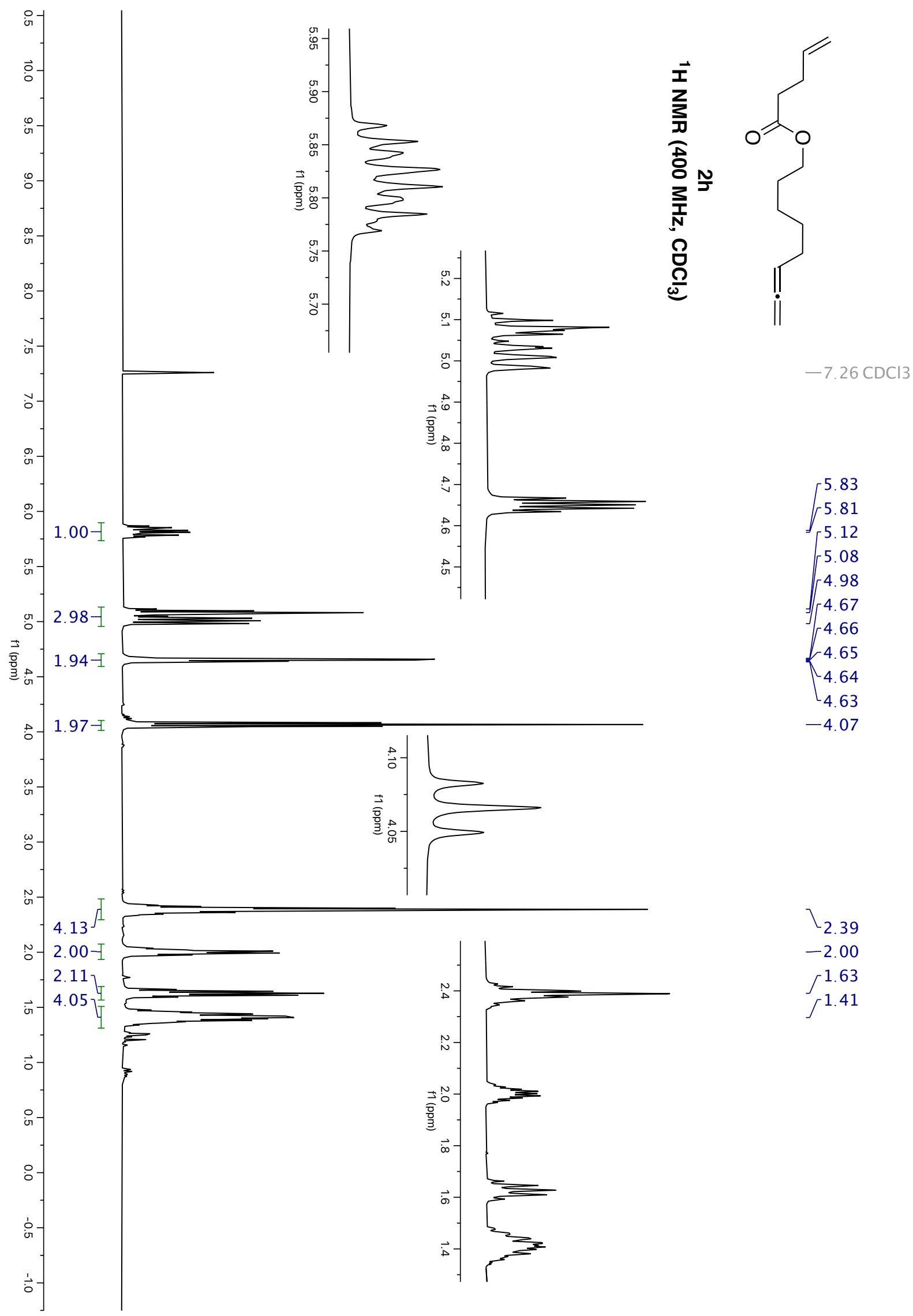




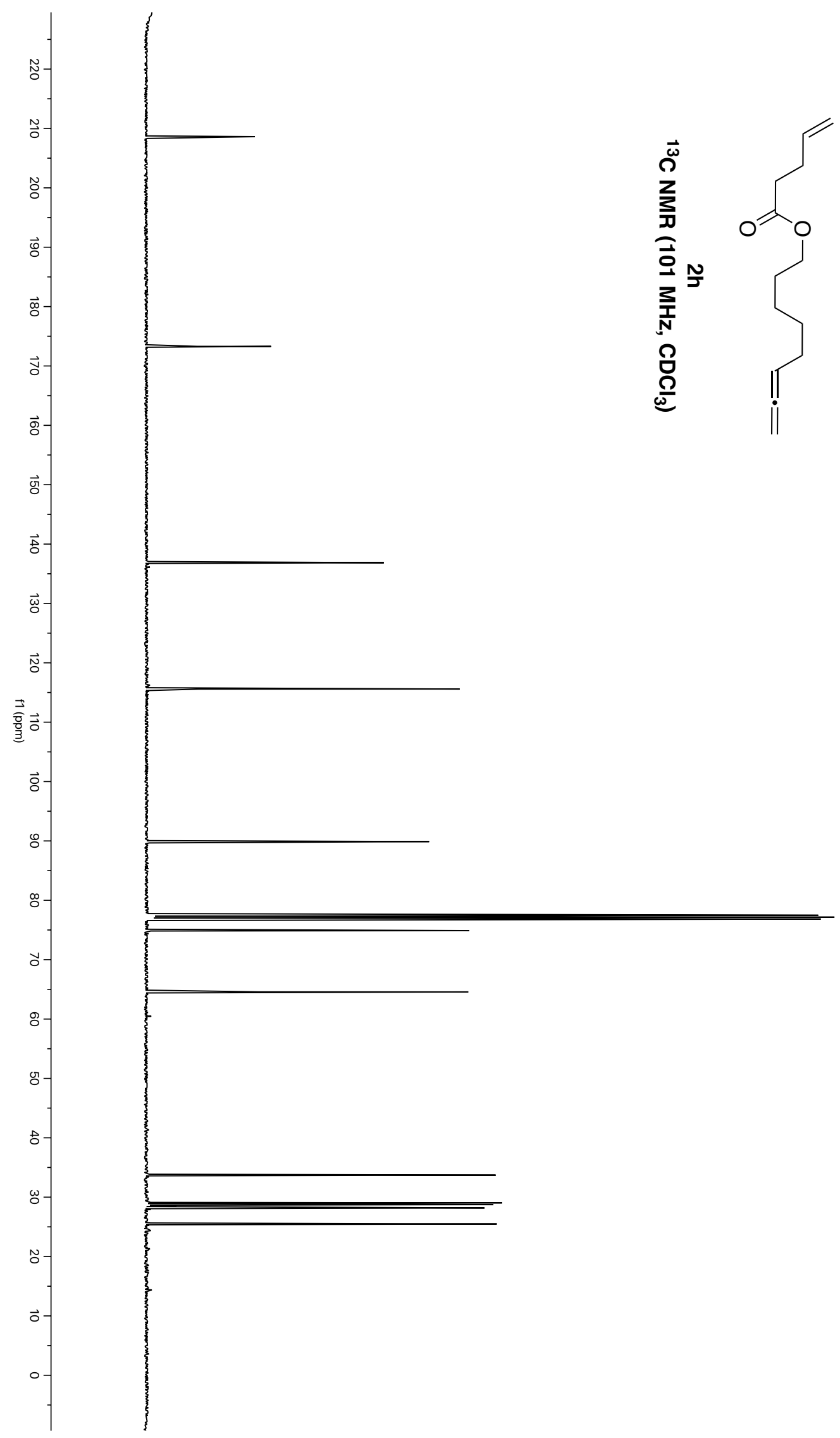

$-77.16 \mathrm{CDCl} 3$ 


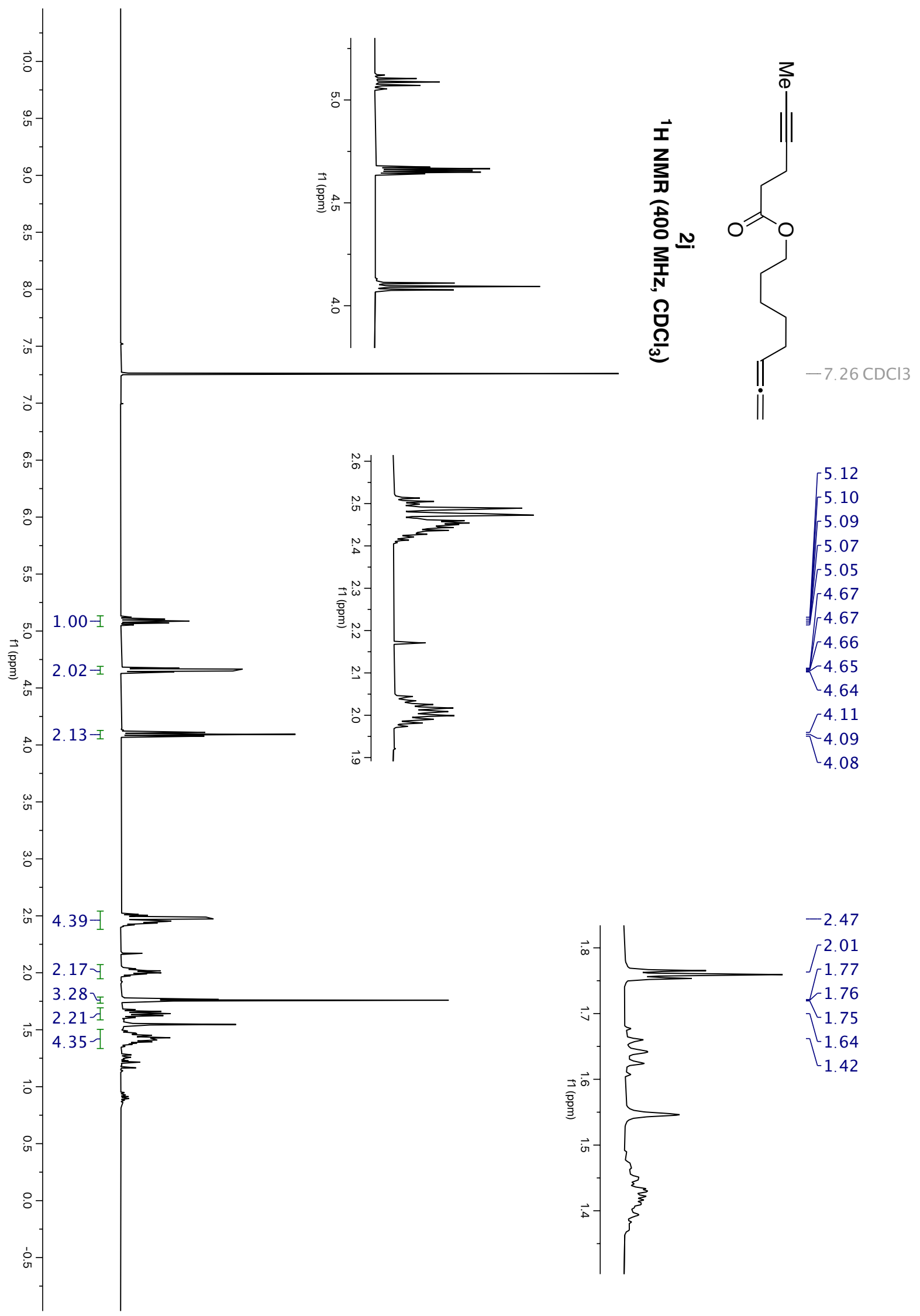


Gonsales, et. al., Supporting Information, pg 217

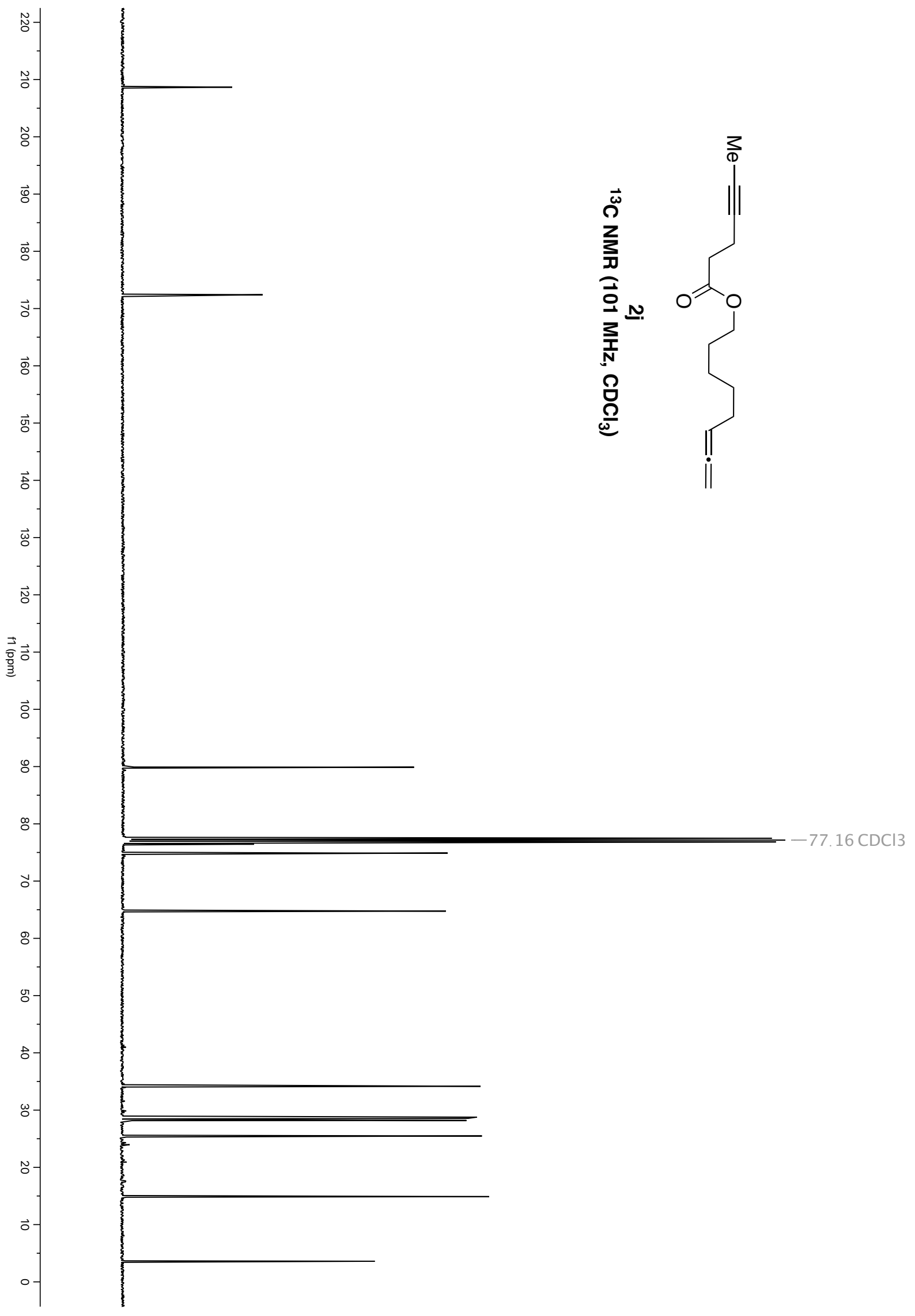




\subsection{Cross-metathesis products}

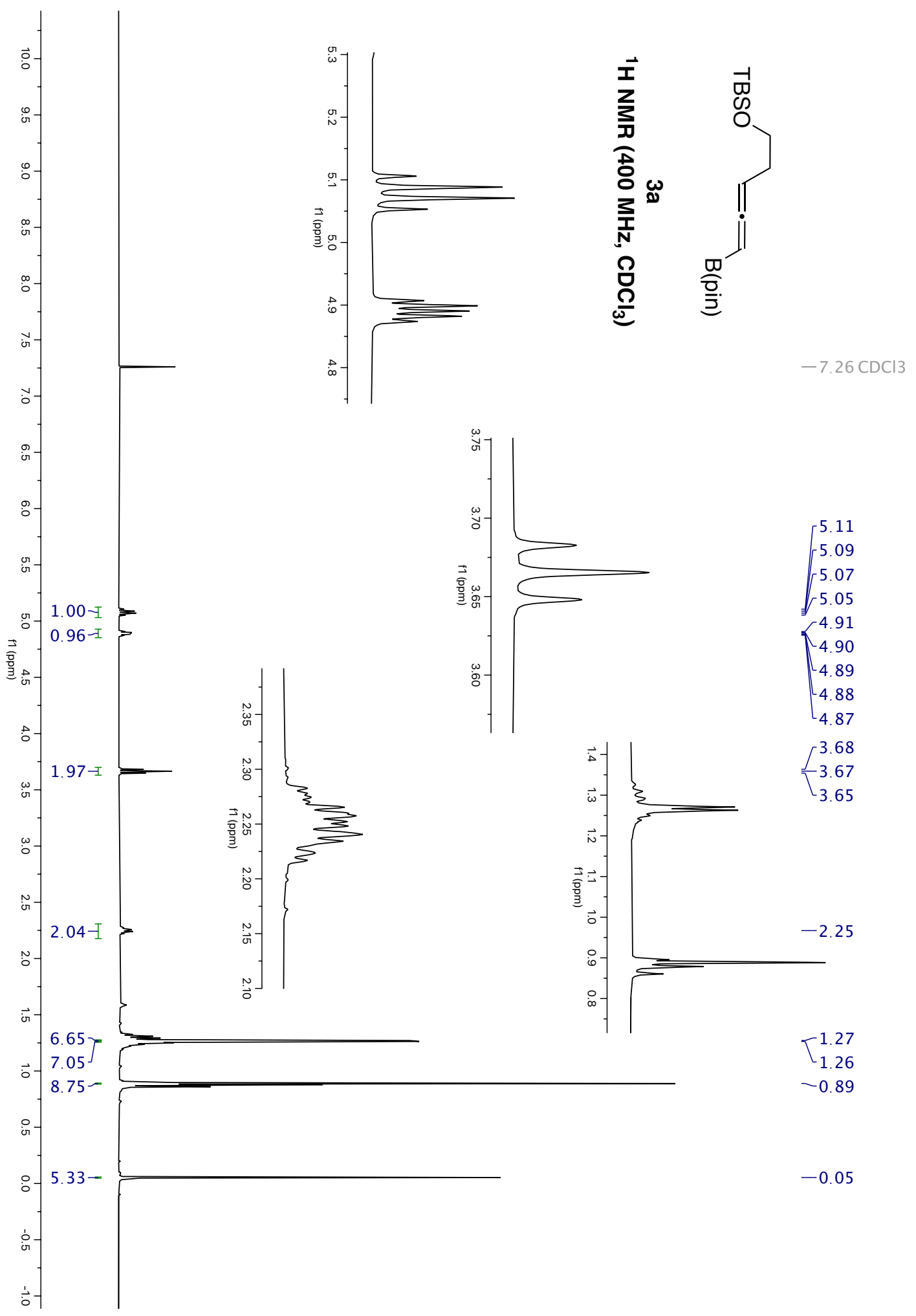




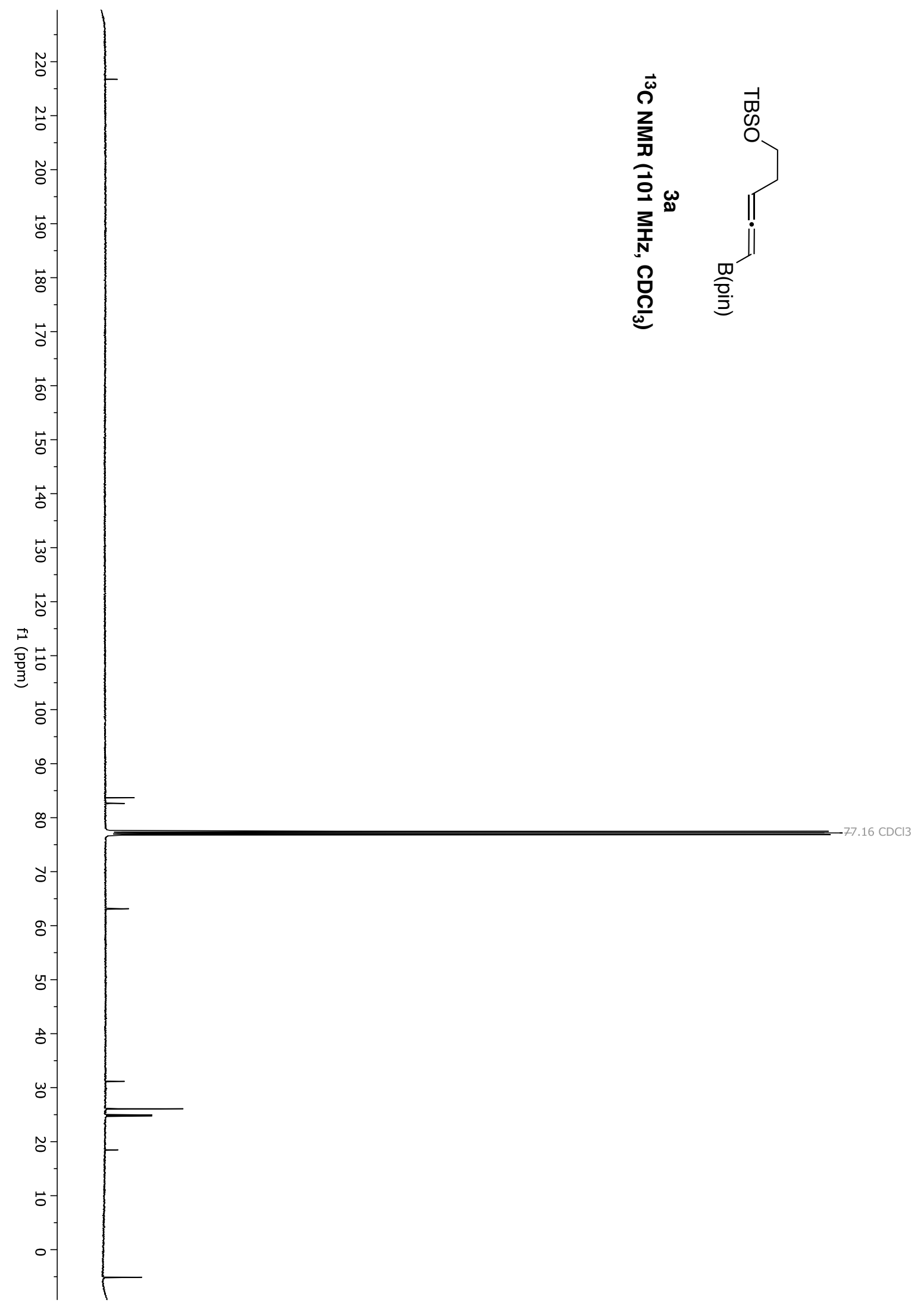




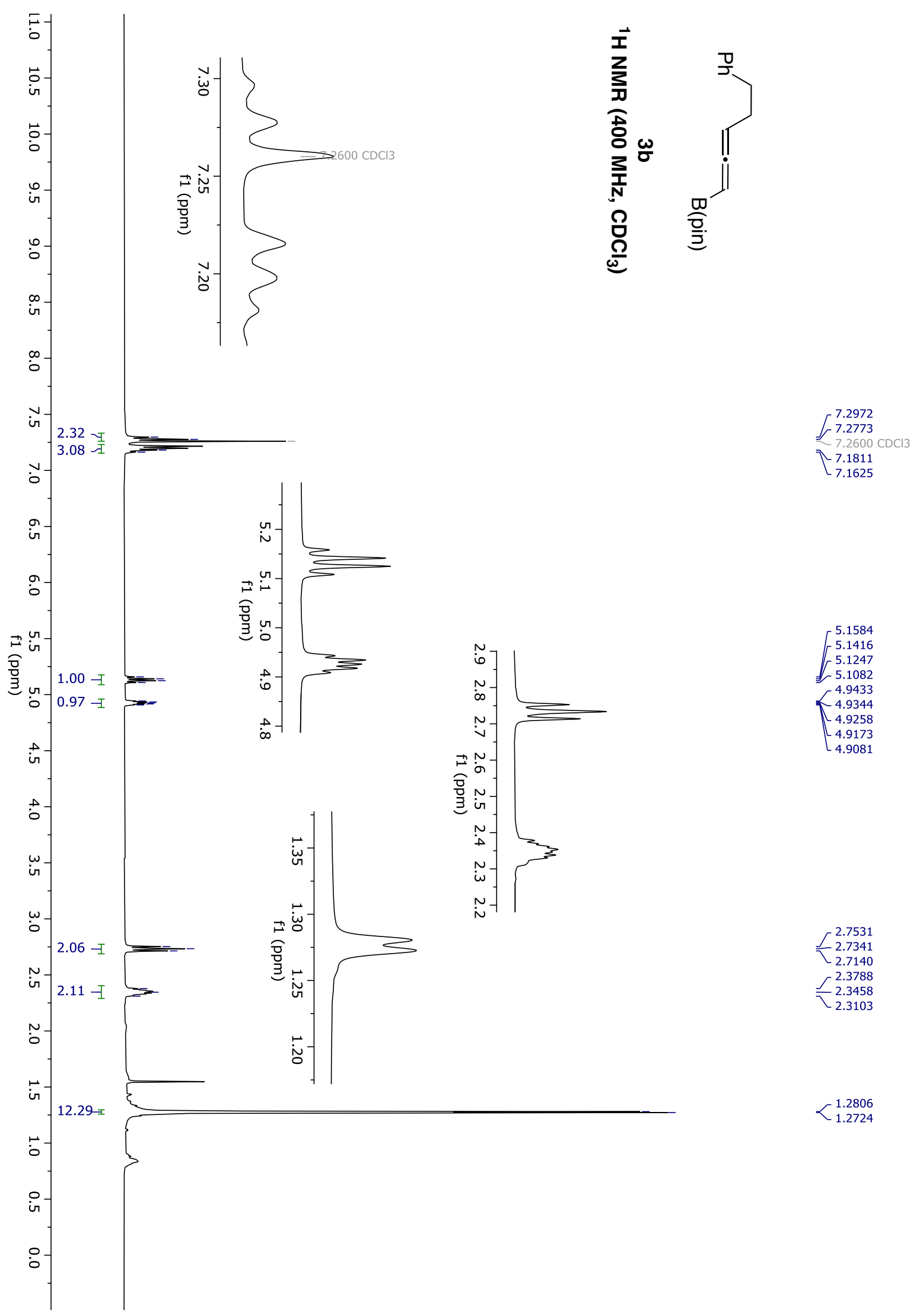




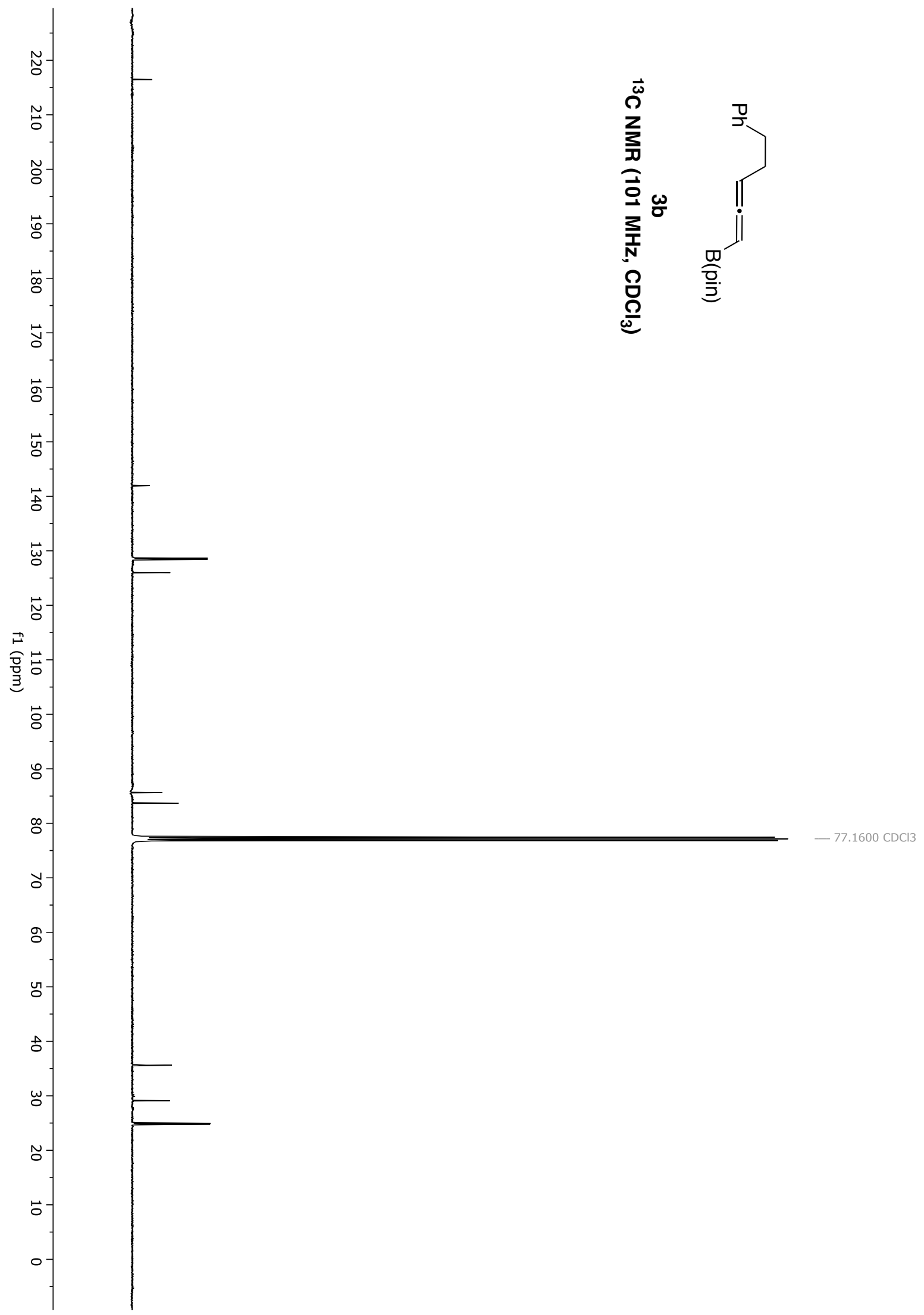




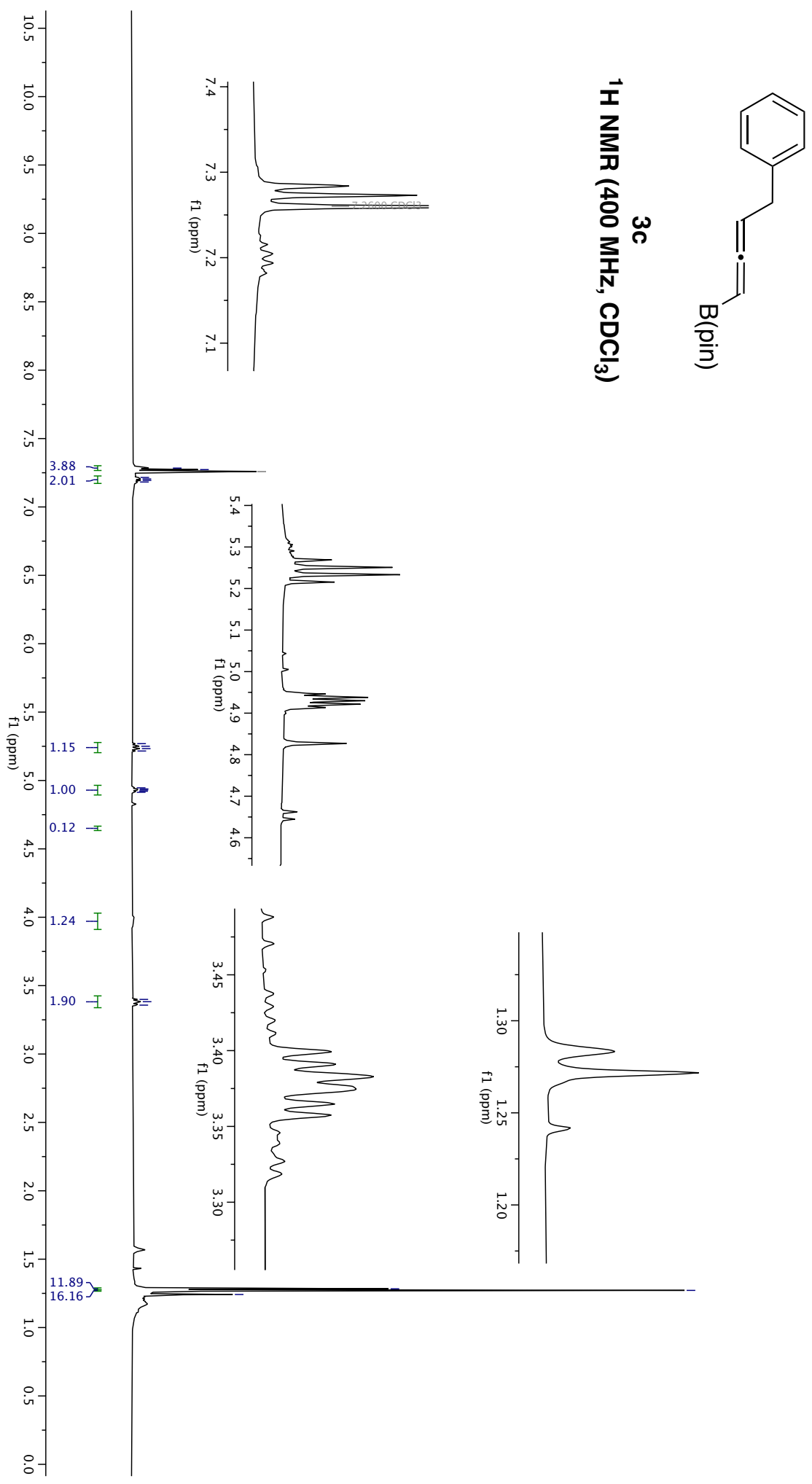

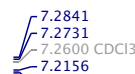
7.2046
7.1939

$\int_{-5.2503}^{5.2693}$

$\begin{array}{r}5.2329 \\ -5.2155 \\ \hline\end{array}$

4.938
-4.9298

4.9216
4.9136

$\tau_{-3.3575}^{3.3993}$ 


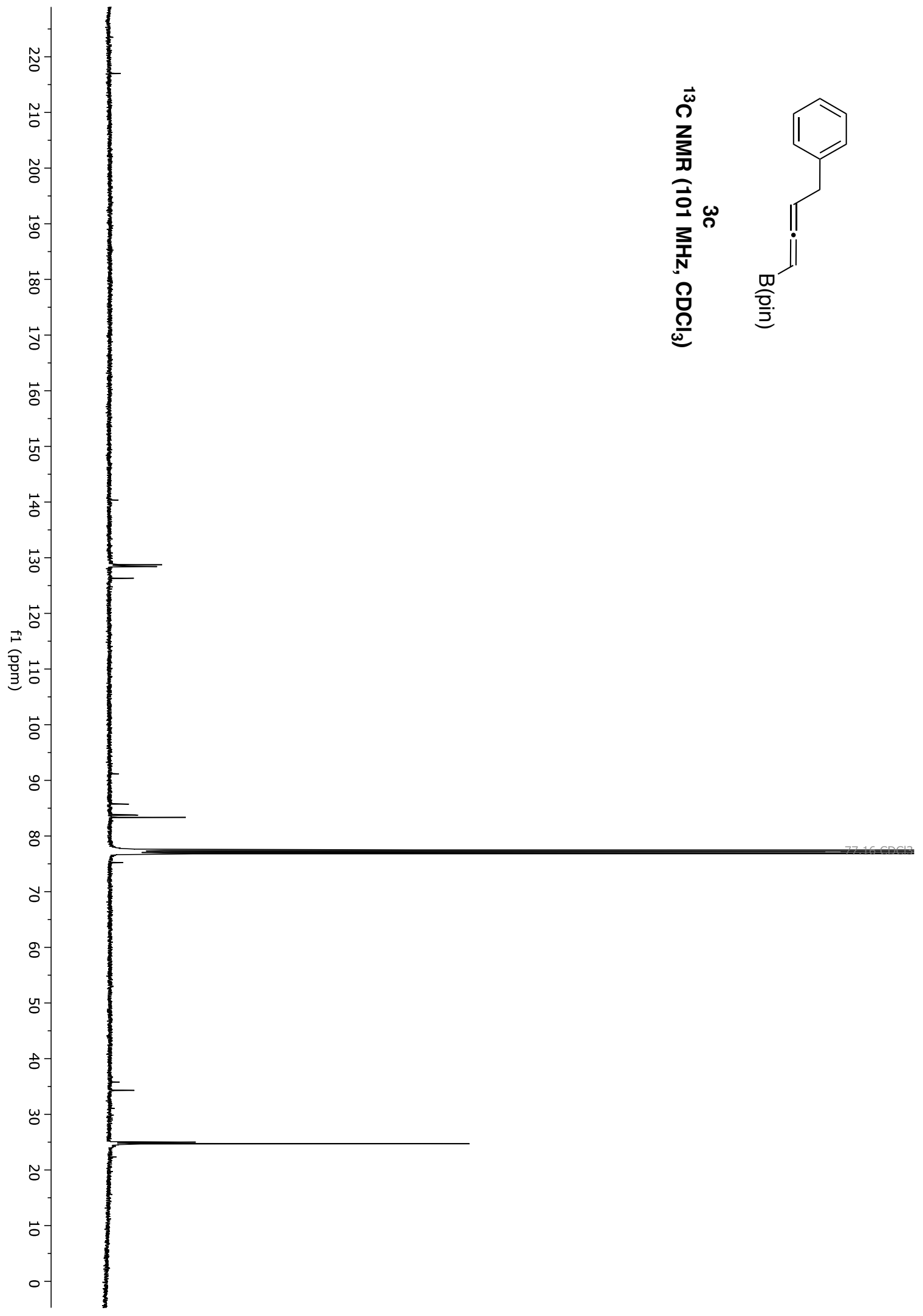




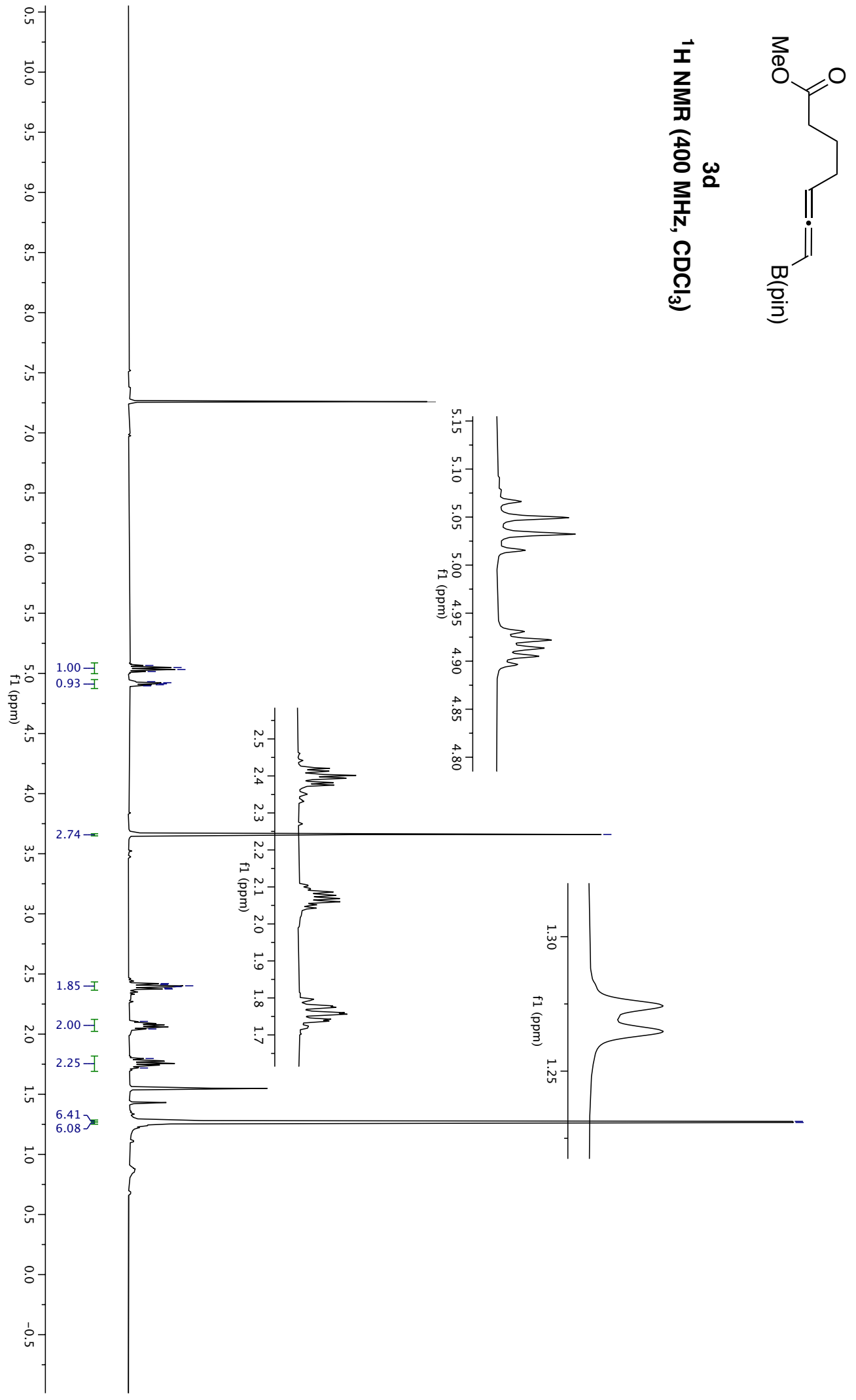

- $7.2600 \mathrm{CDCl} 3$

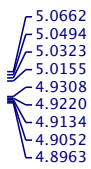

$-3.6604$

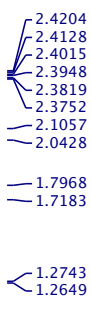


Gonsales, et. al., Supporting Information, pg 225

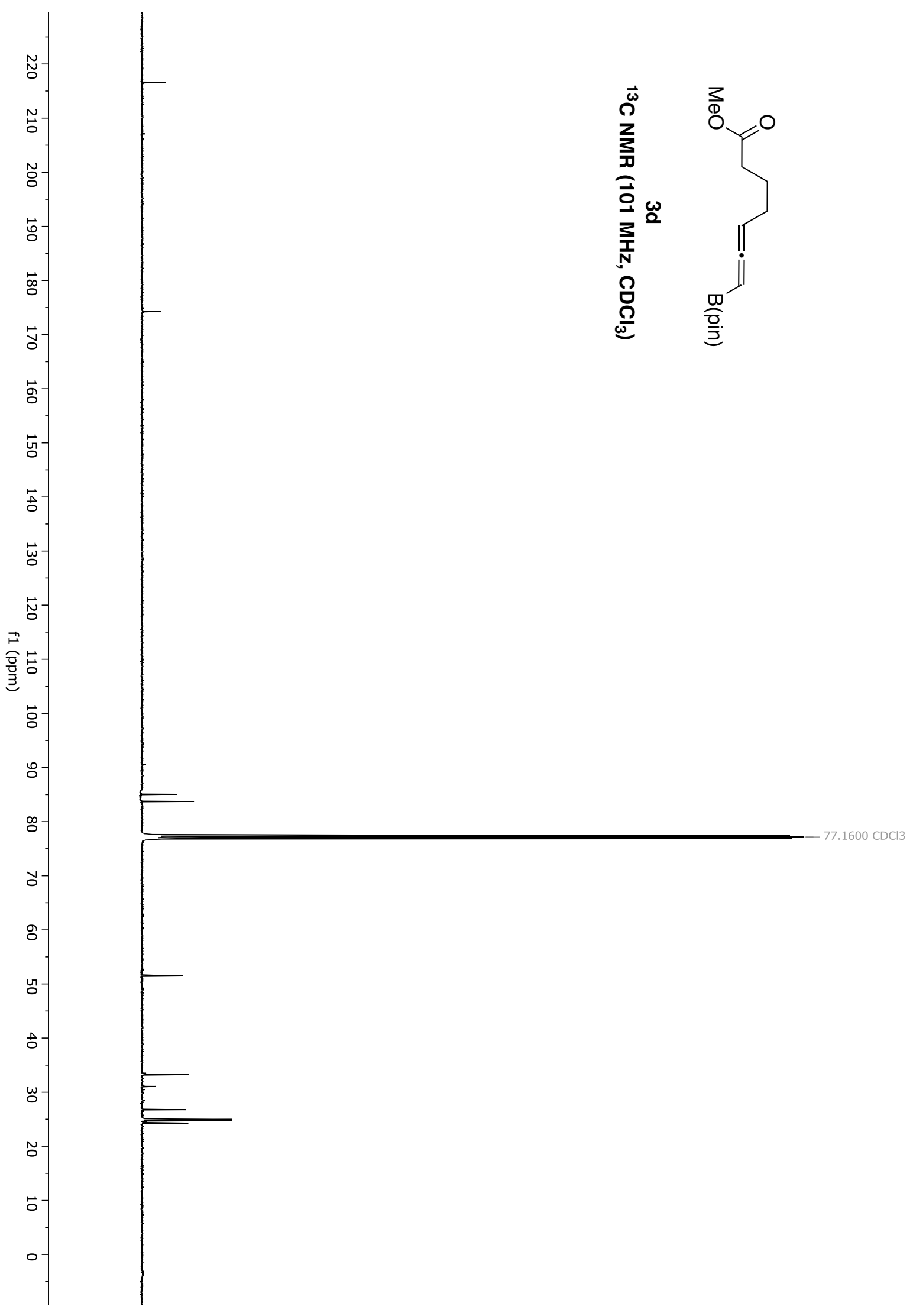




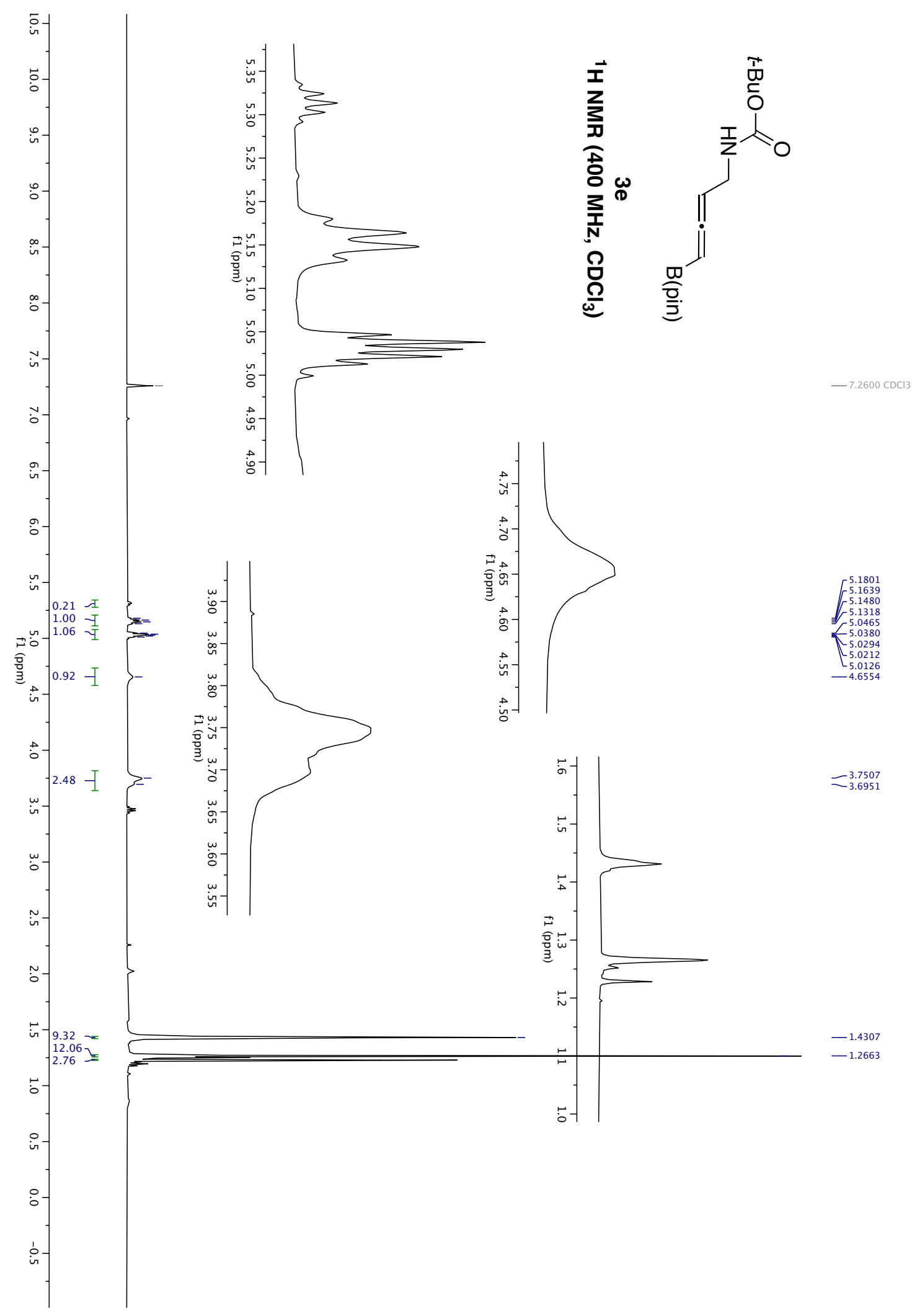




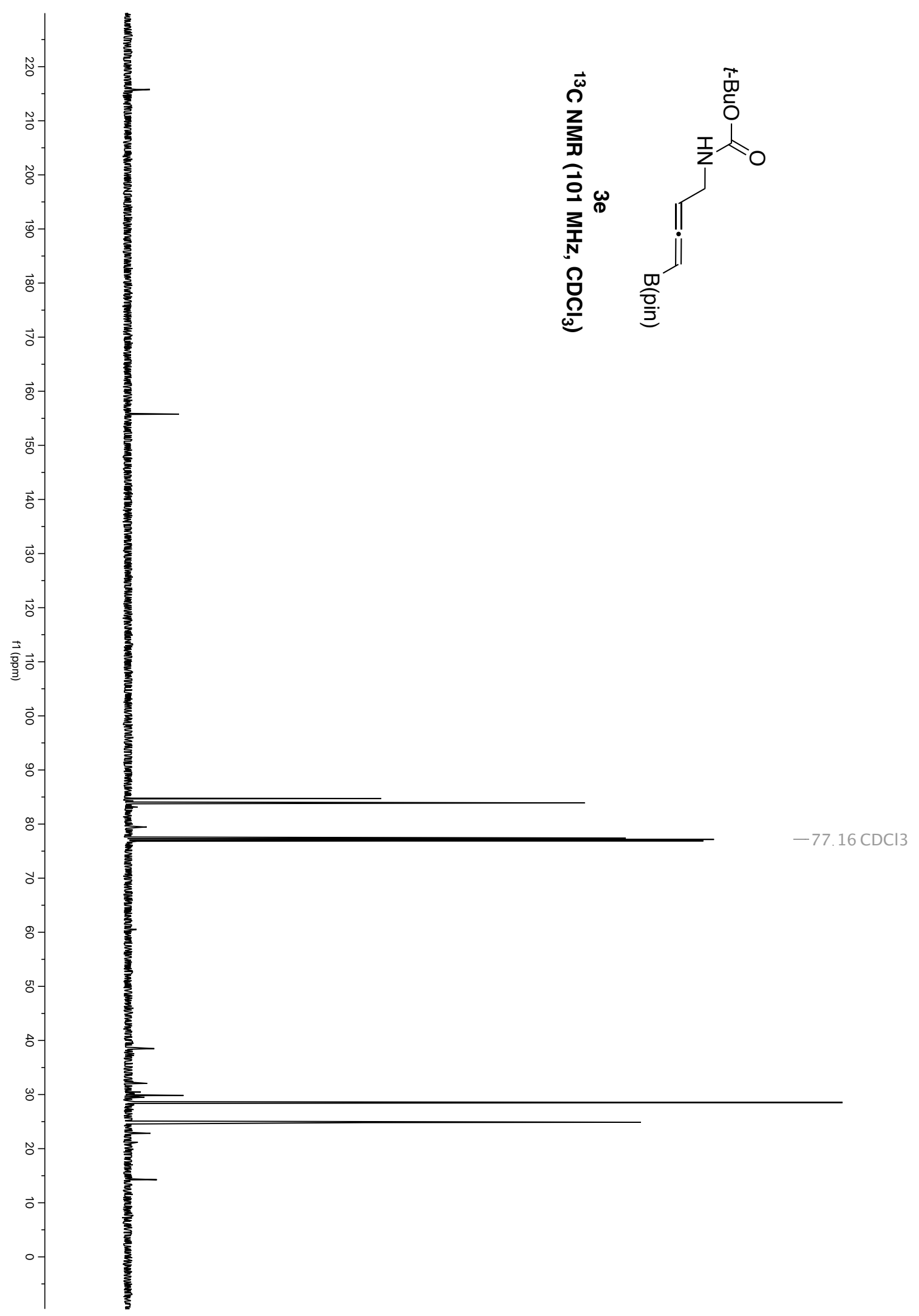




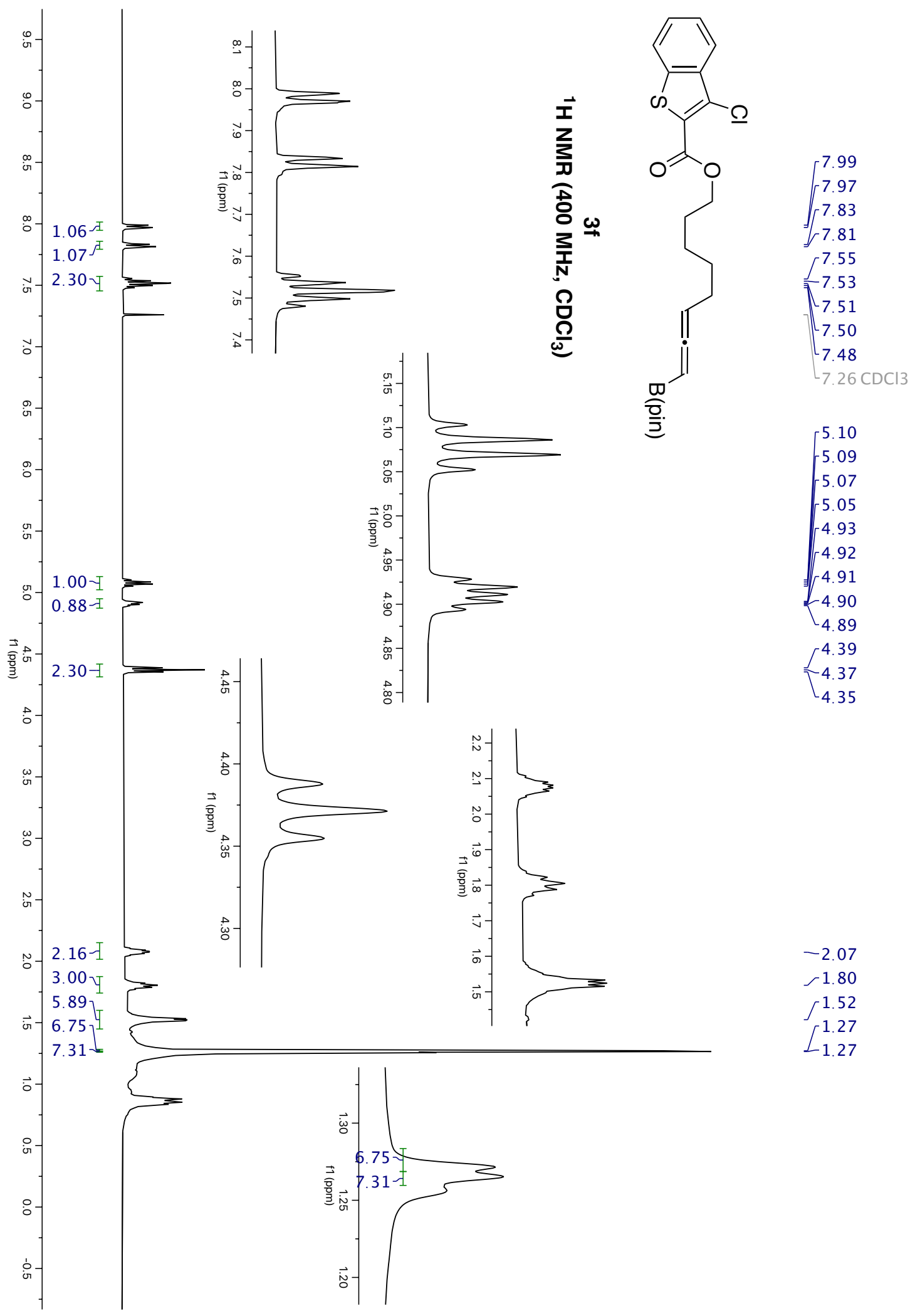




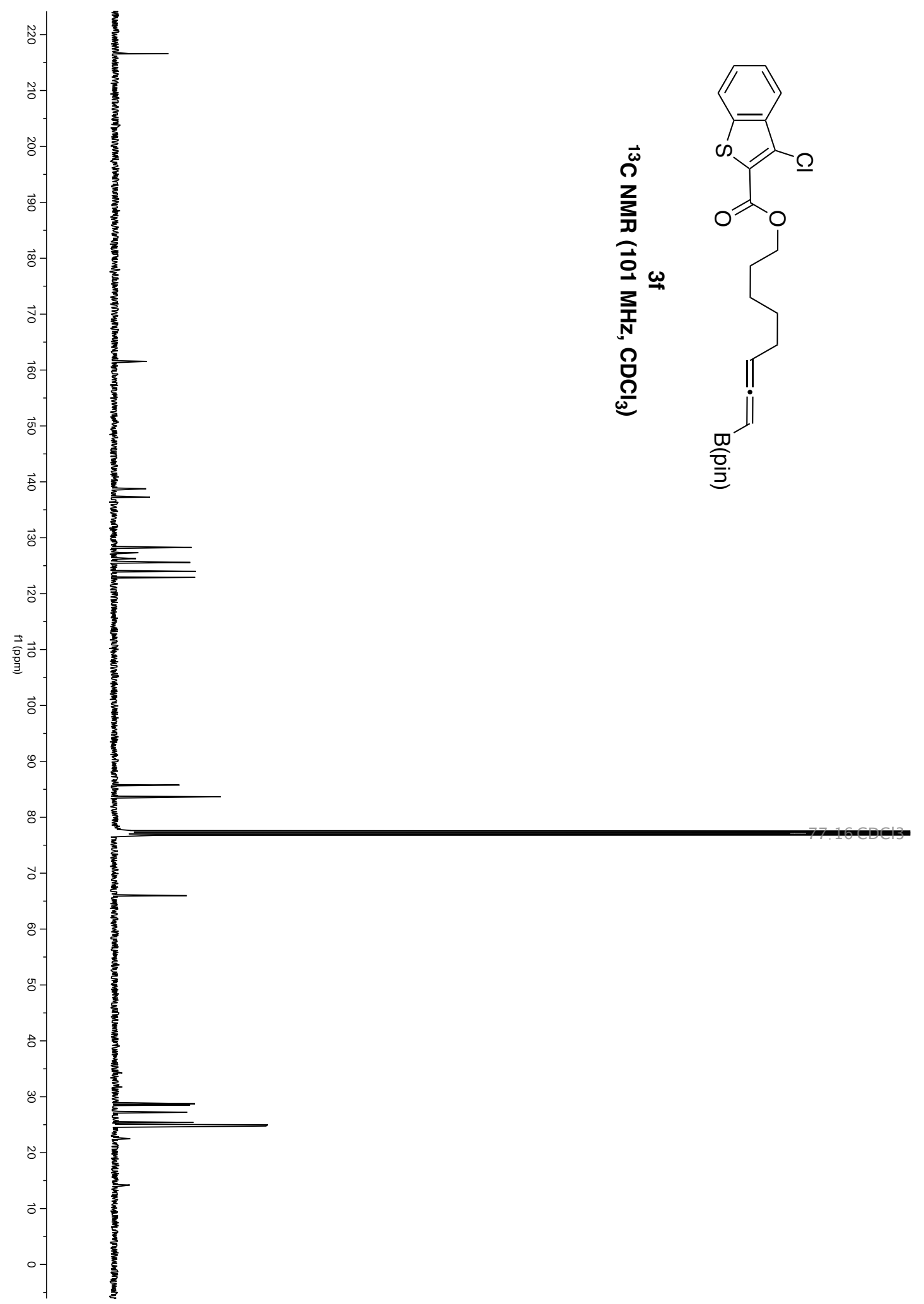



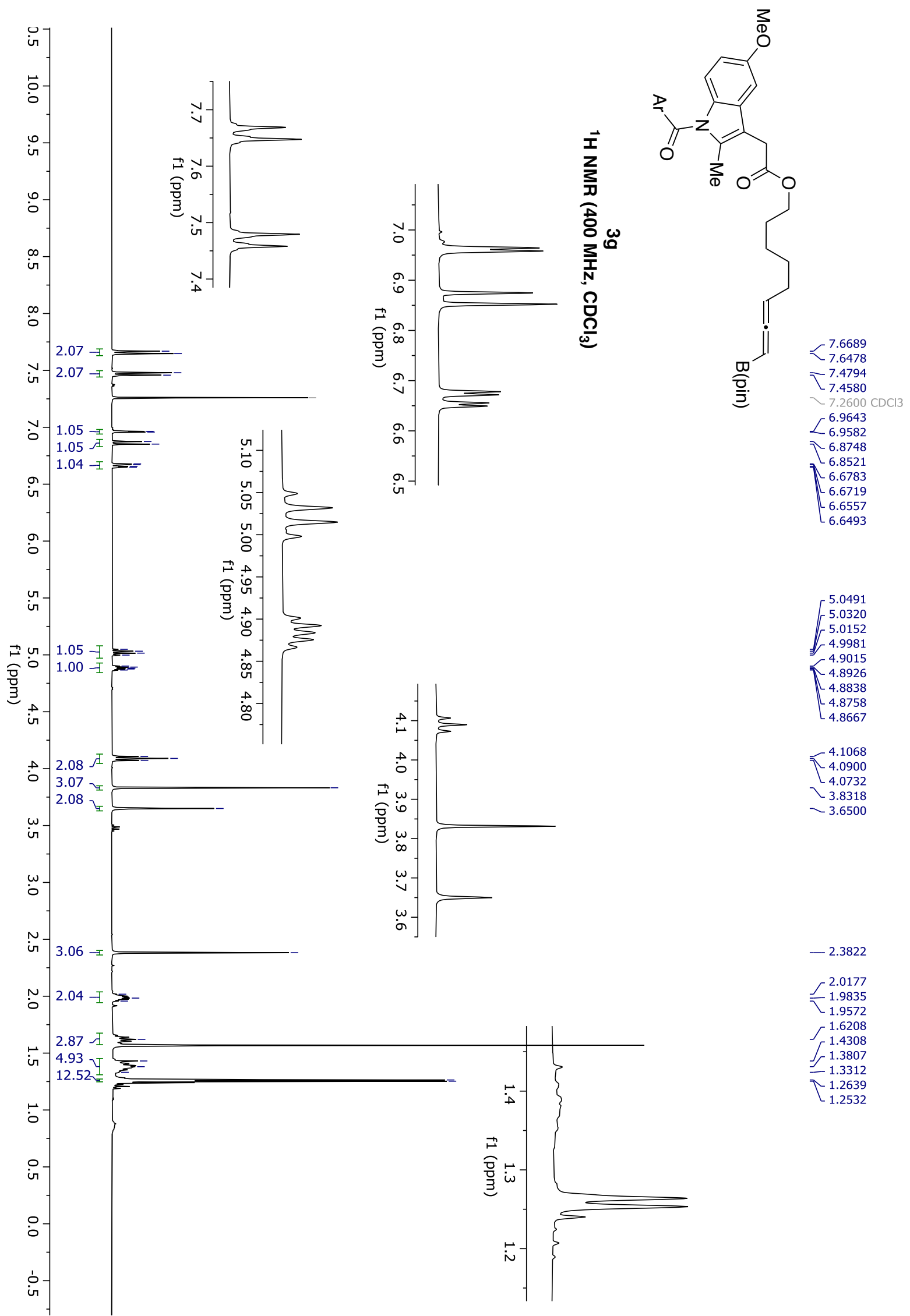

$-2.3822$

2.0177

$-1.9835$

1.9572

1.6208
1.4308

r 1.3807

- 1.3312

$\uparrow 1.2639$

1.2532 


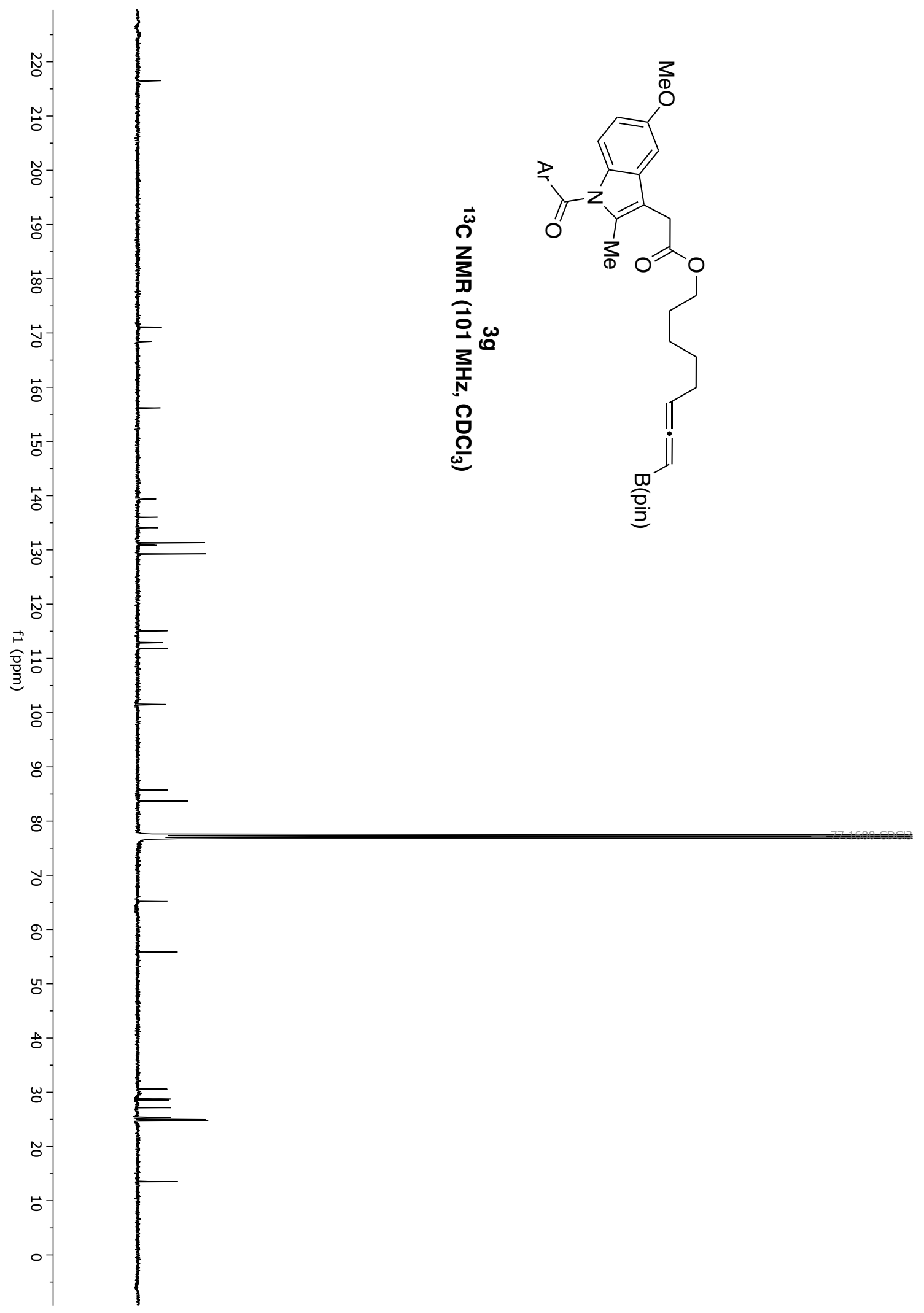




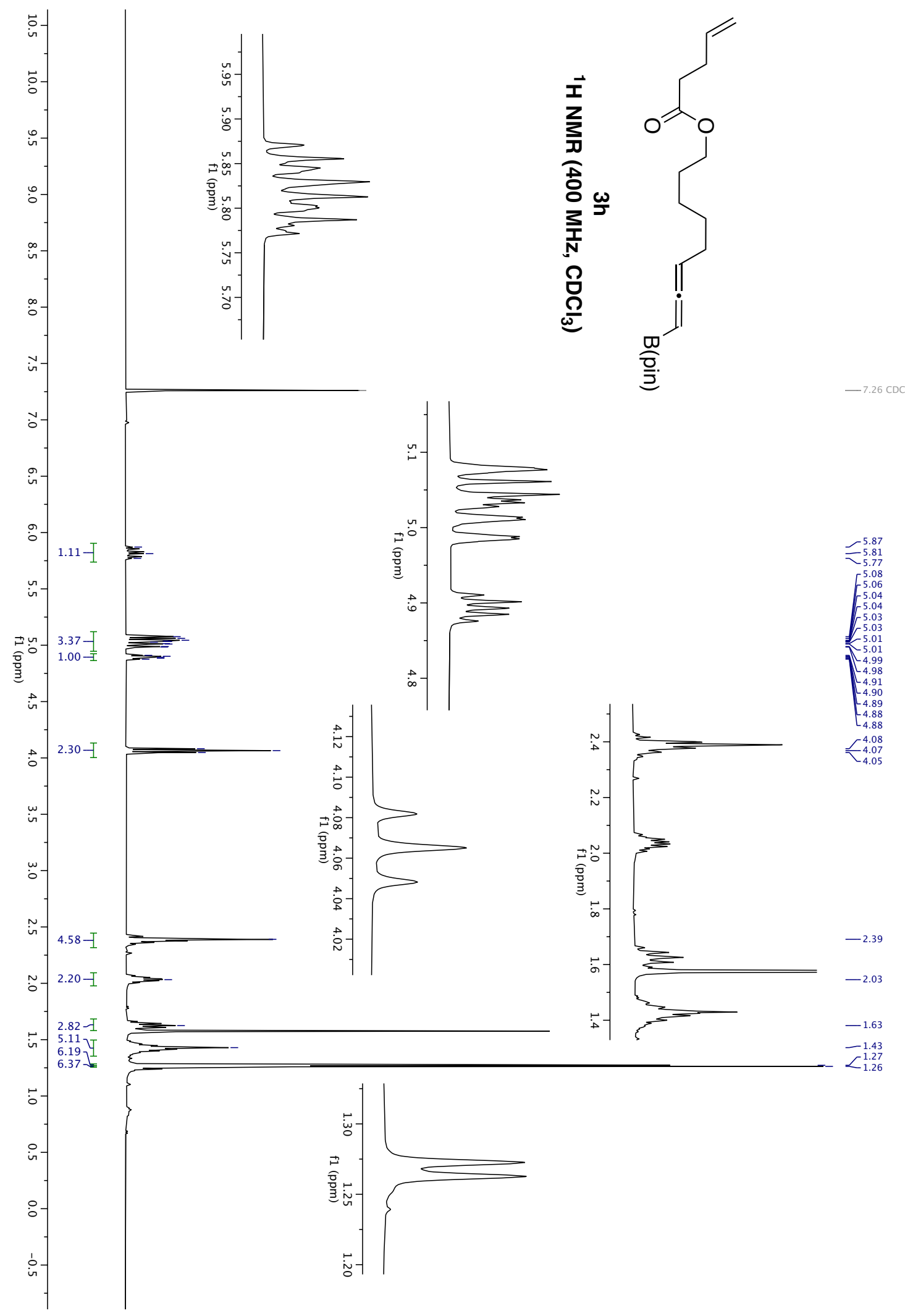


Gonsales, et. al., Supporting Information, pg 233

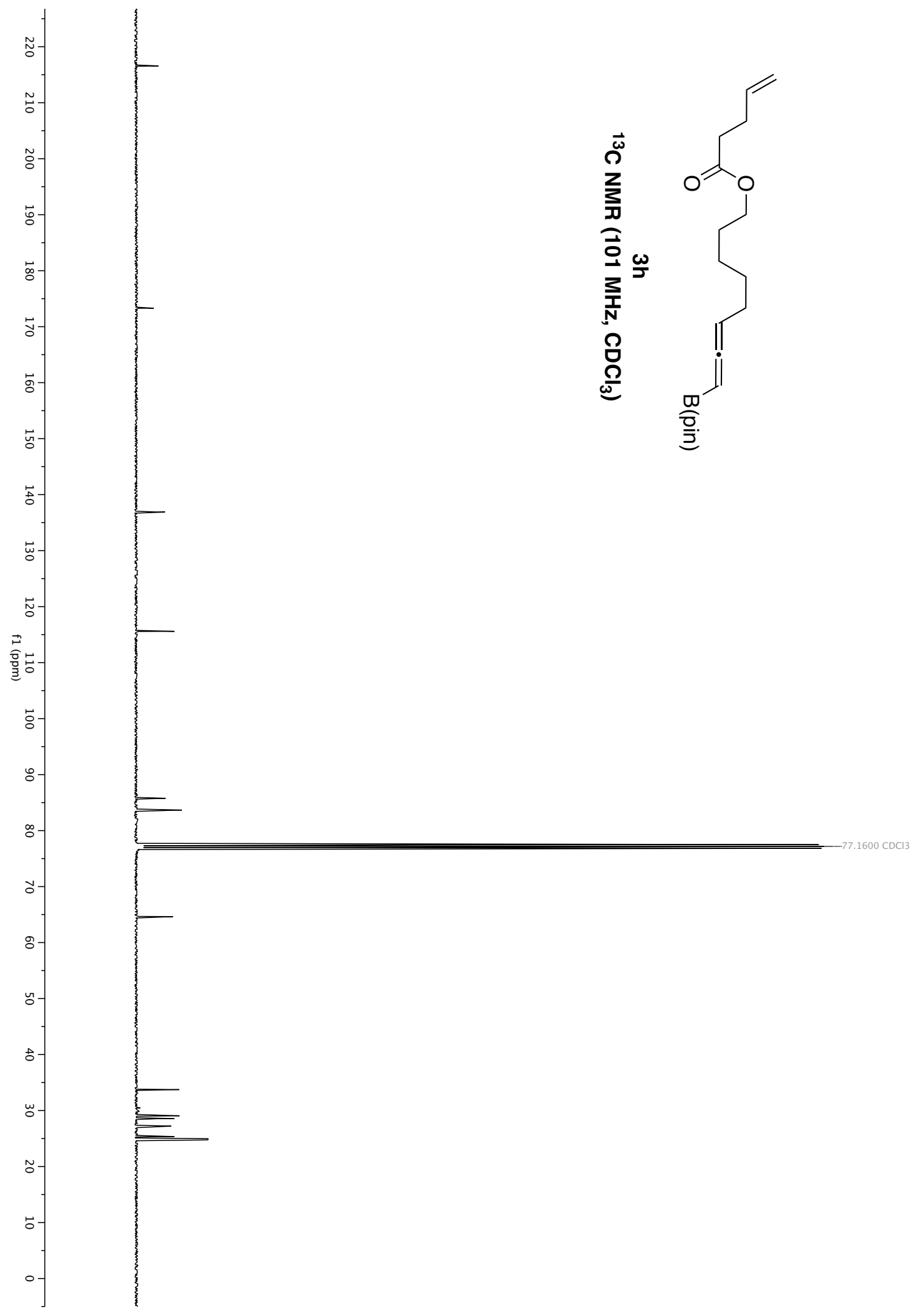




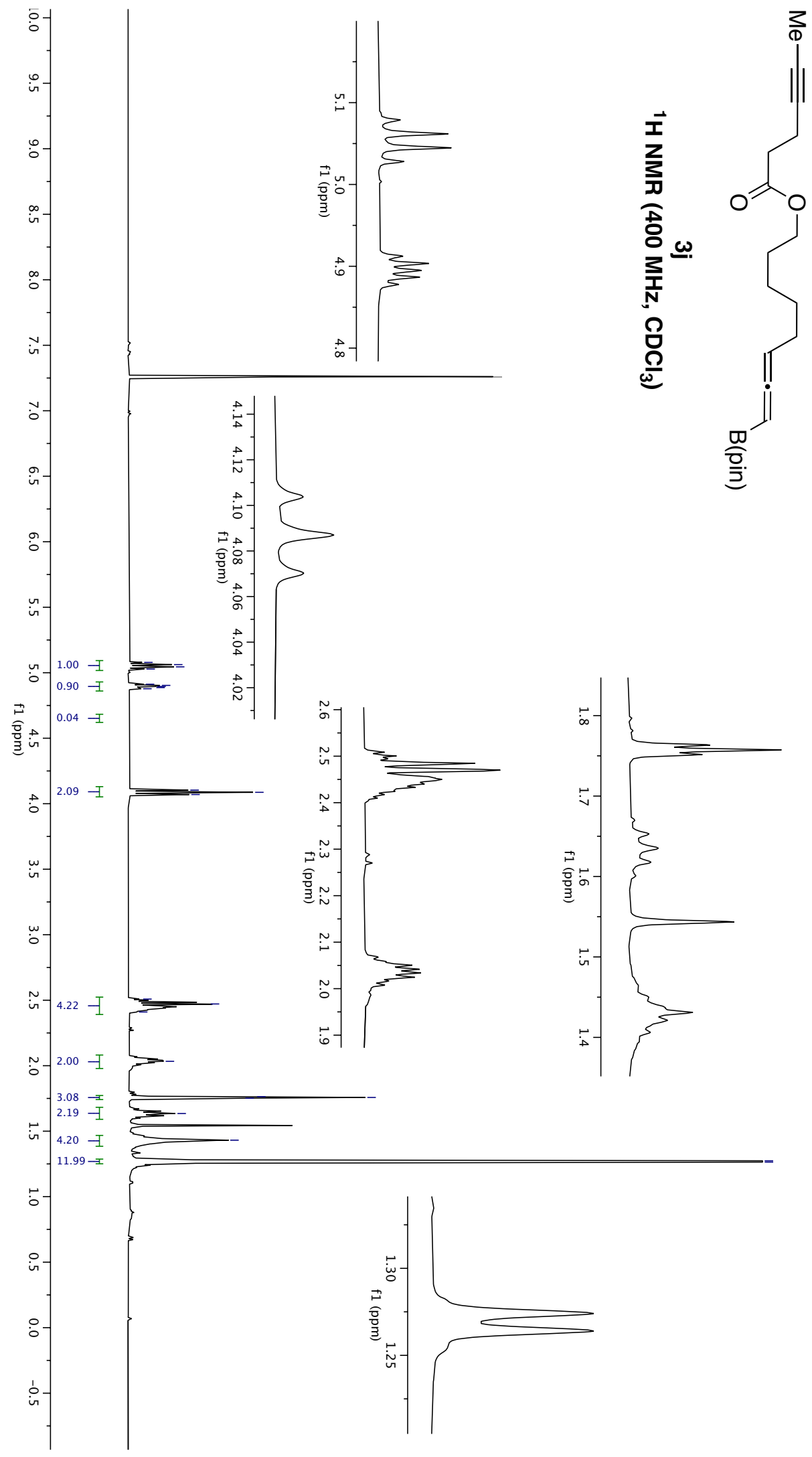




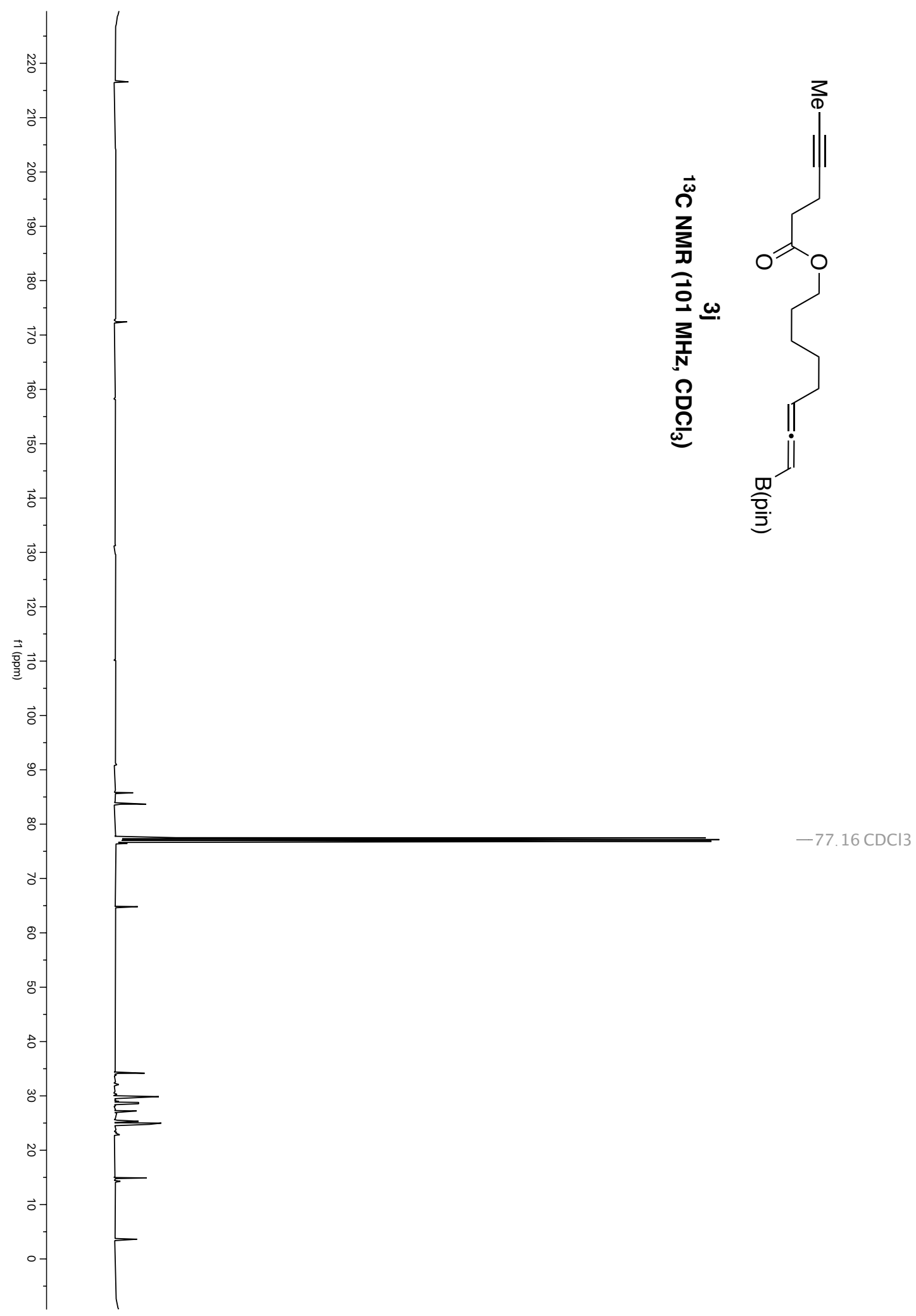




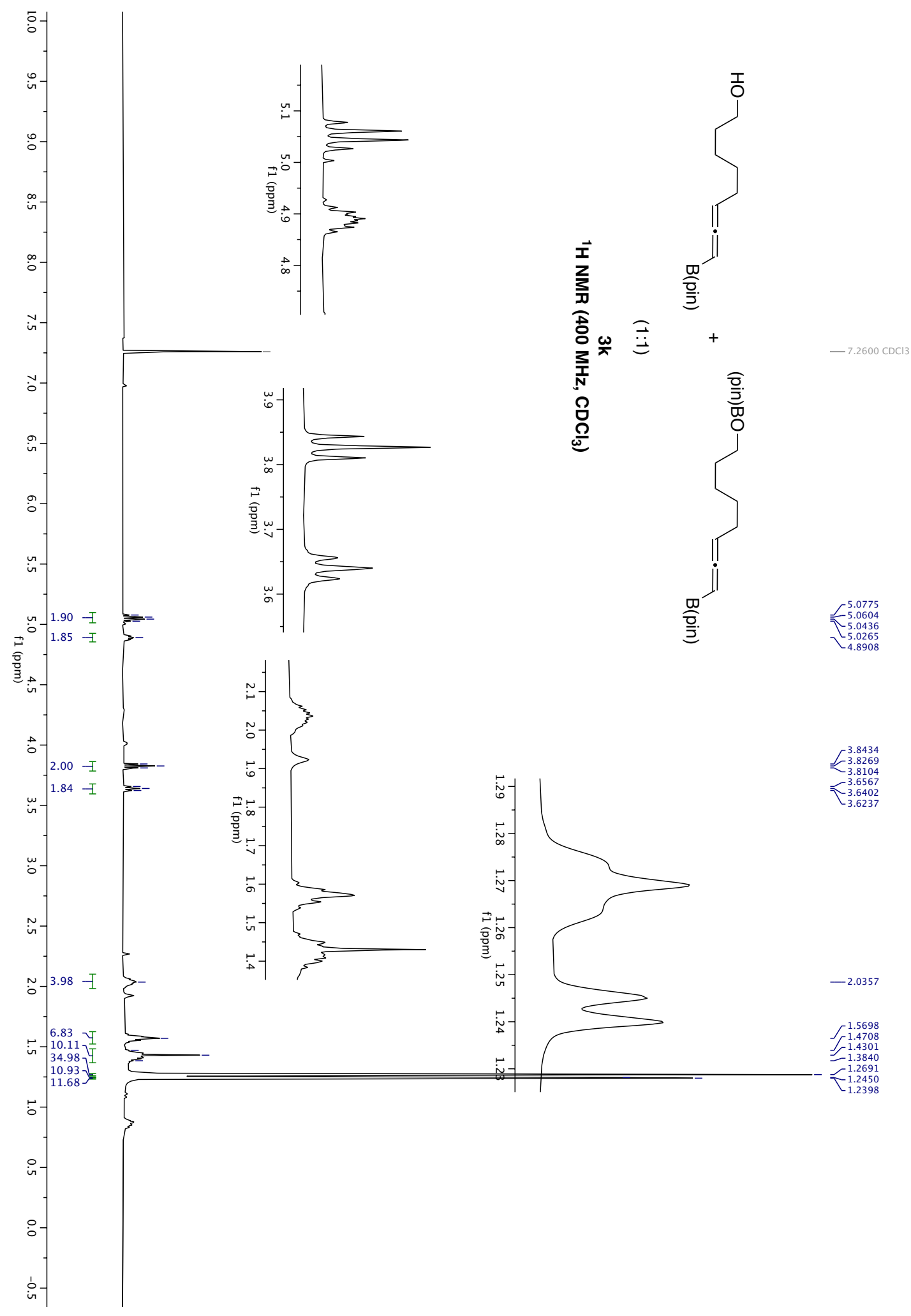




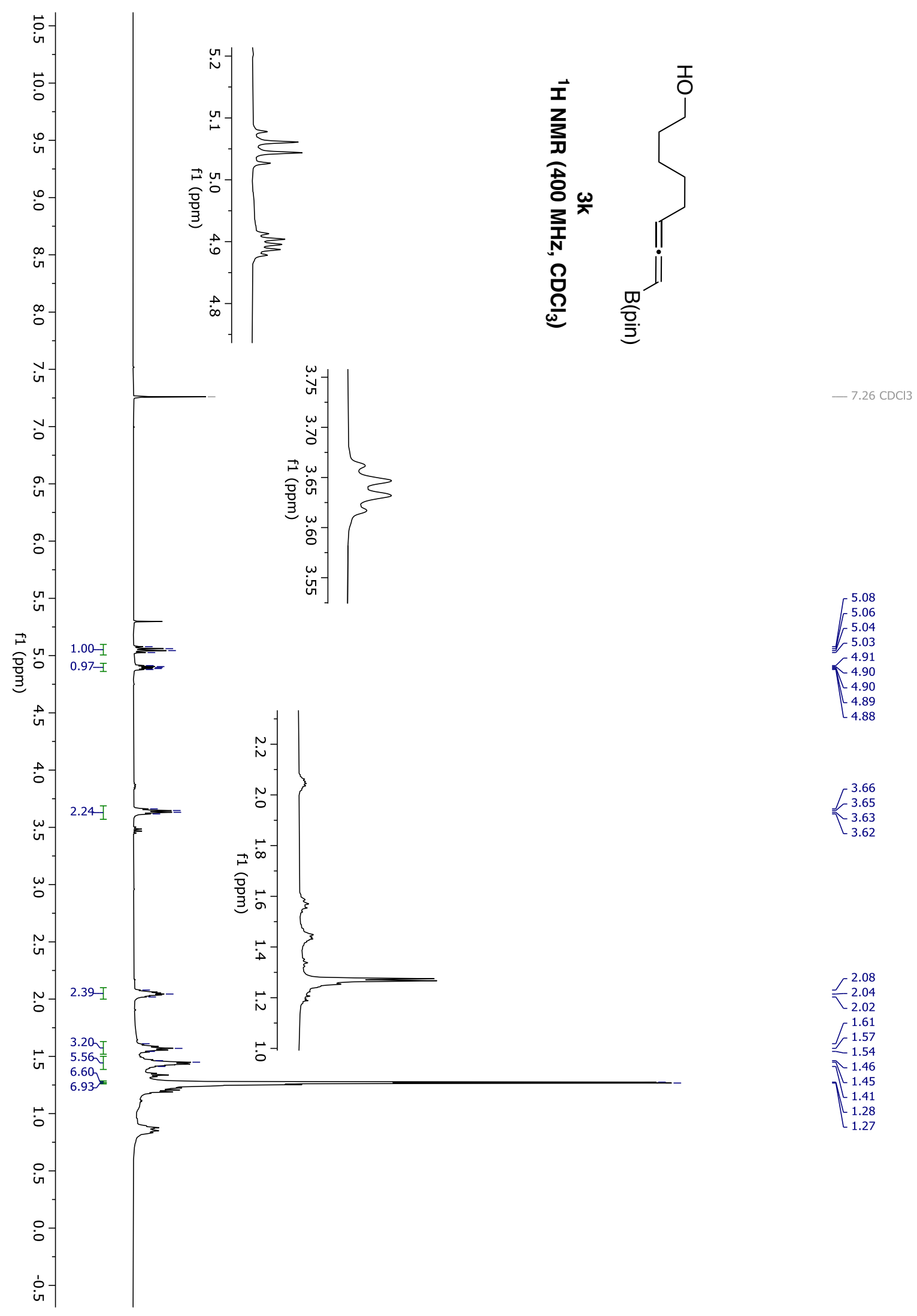




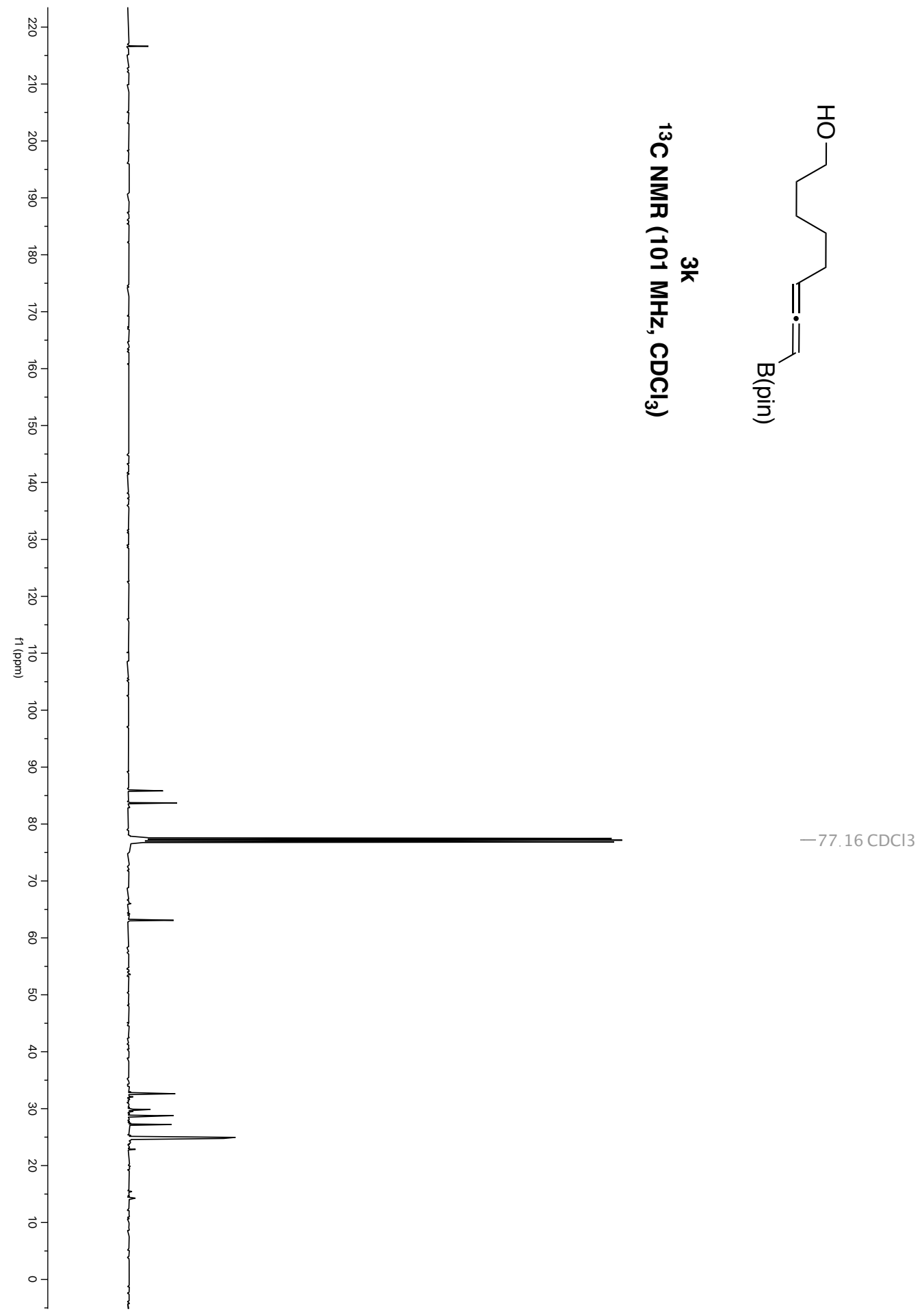


Gonsales, et. al., Supporting Information, pg 239

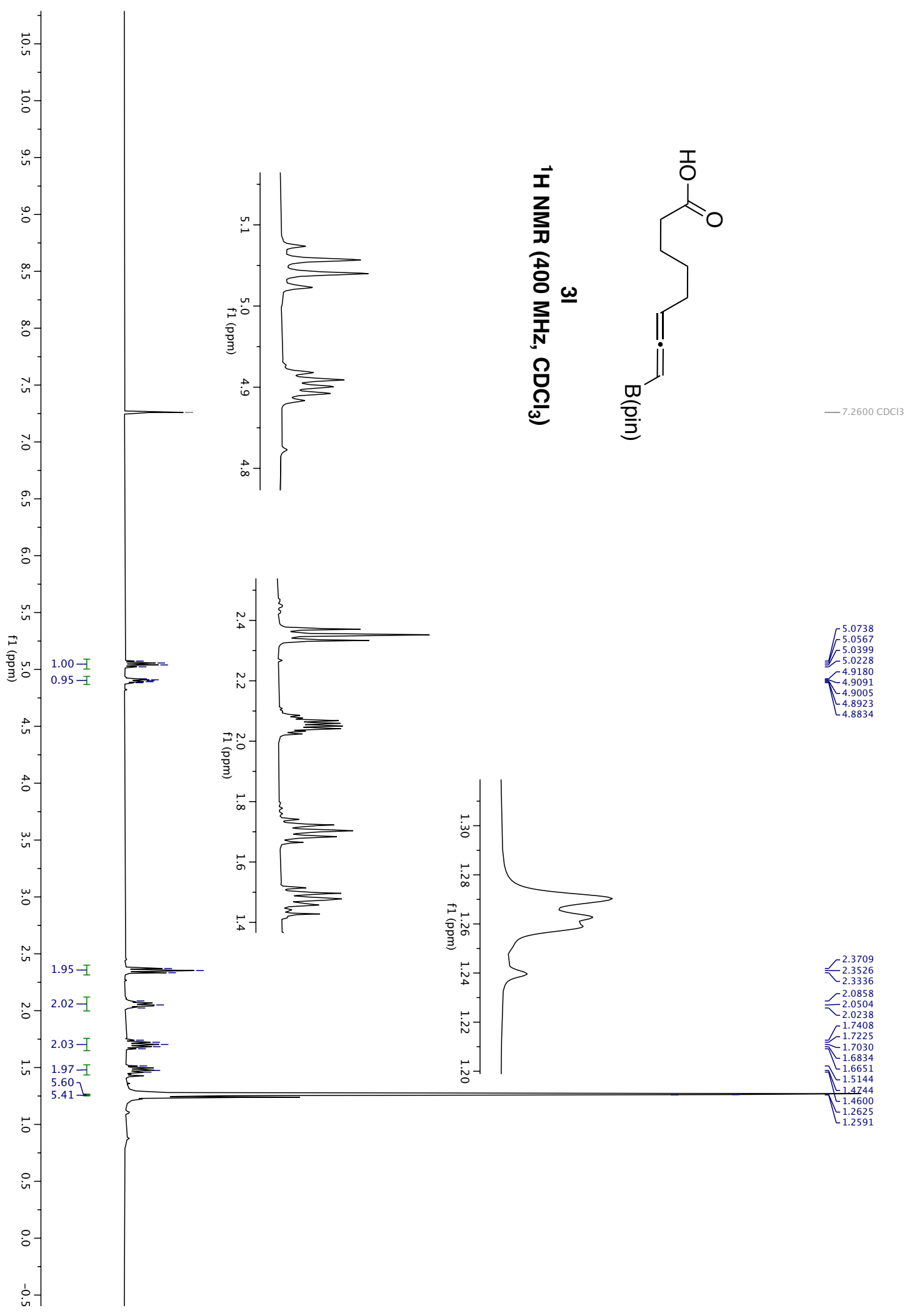




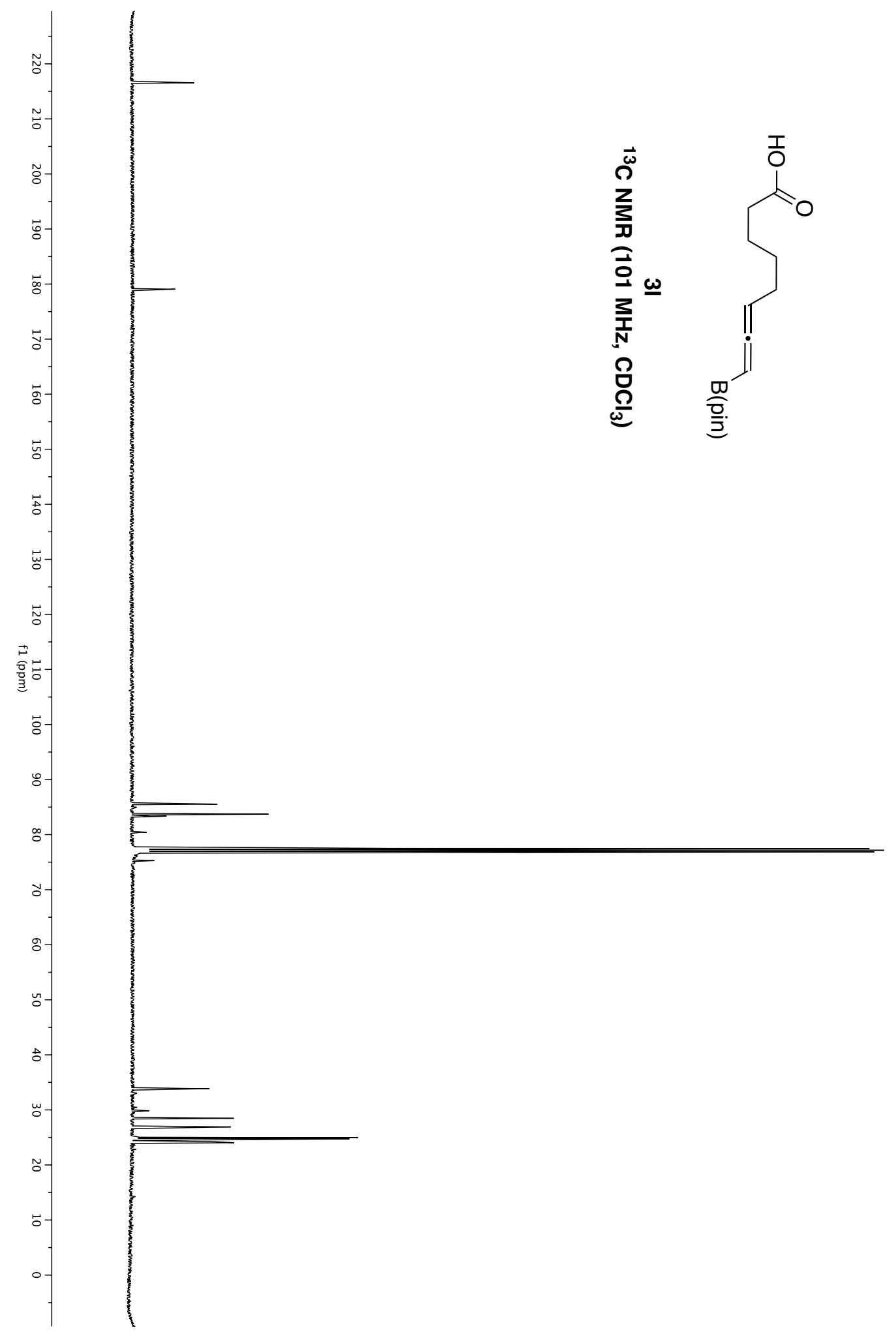




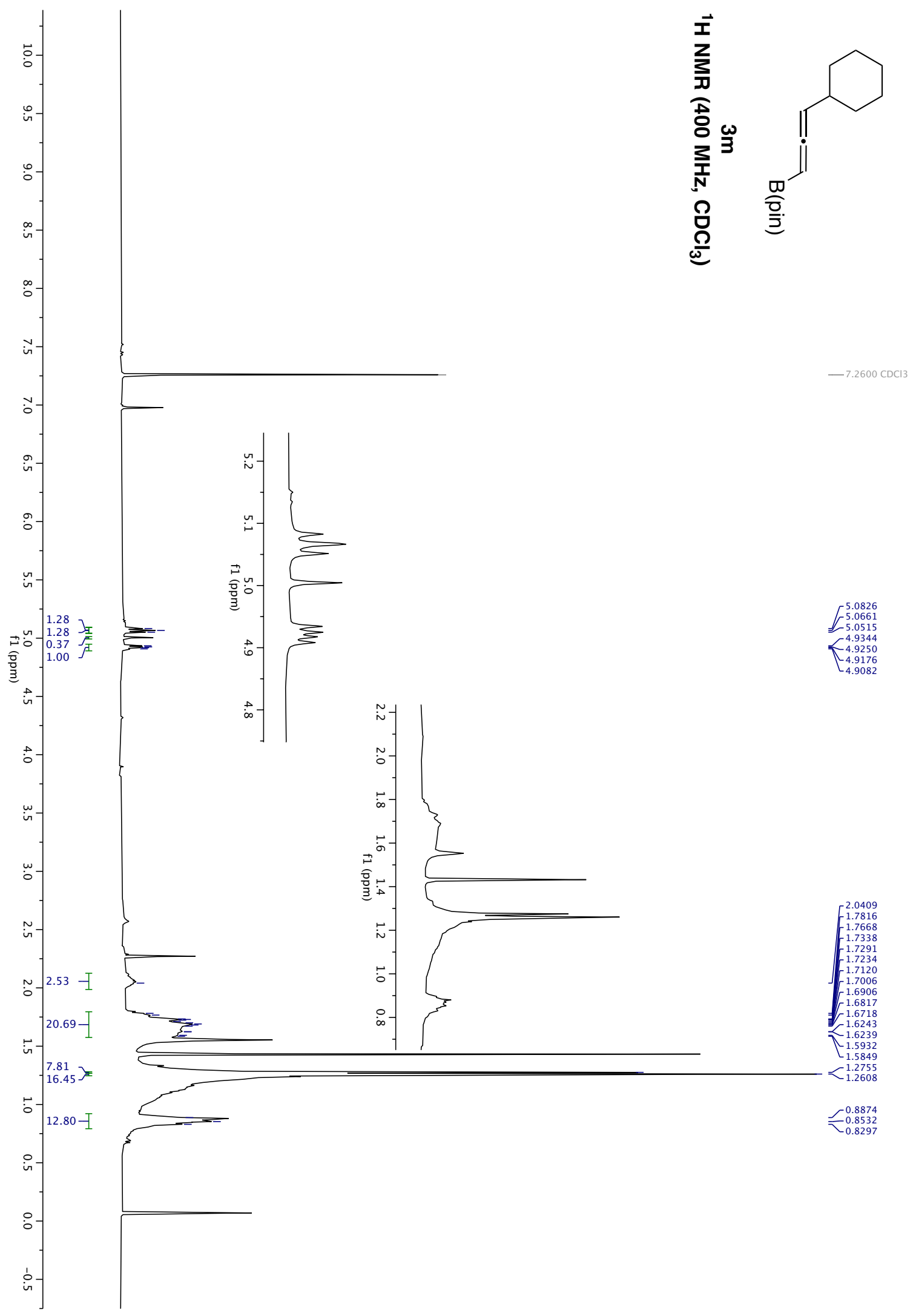




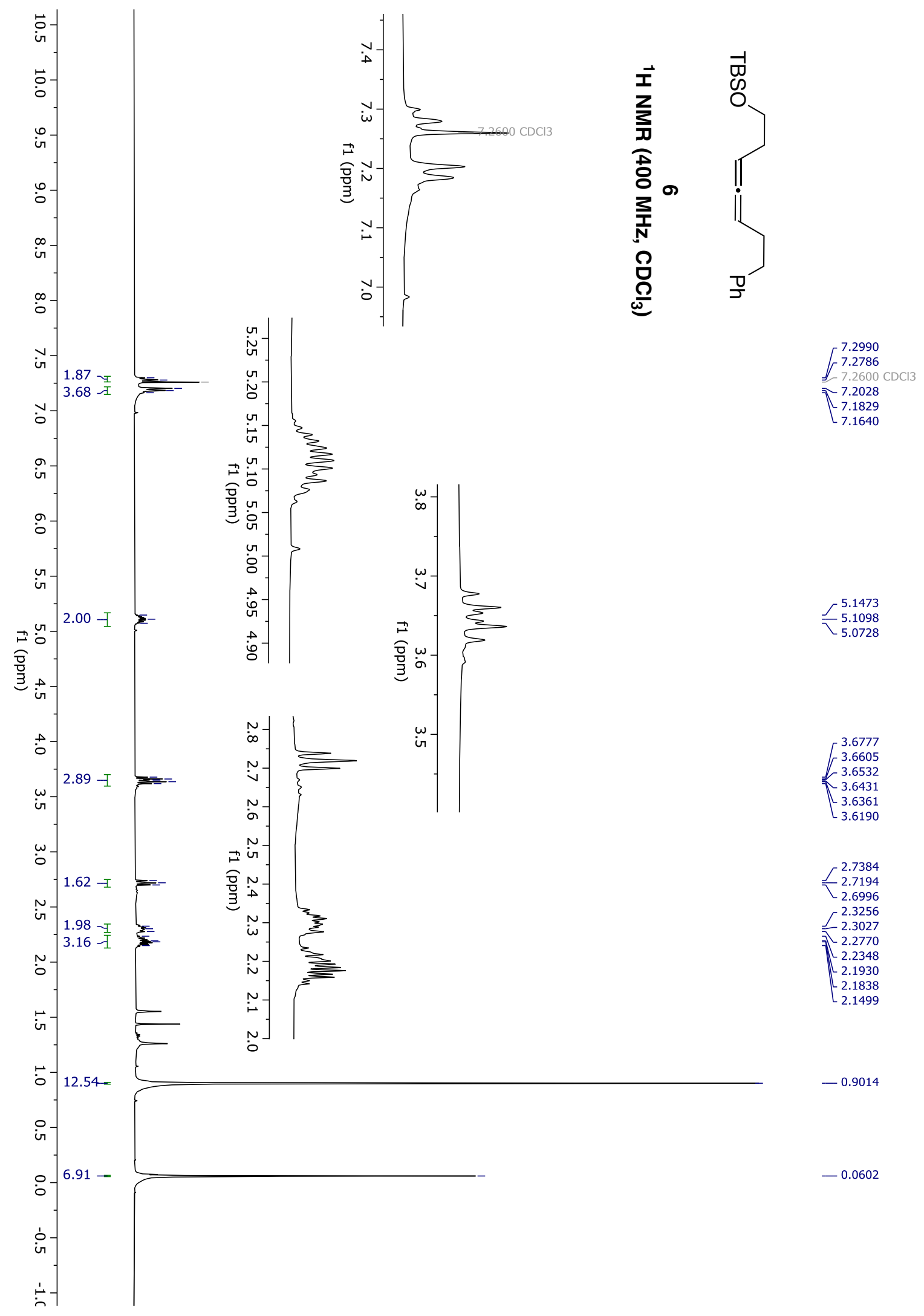



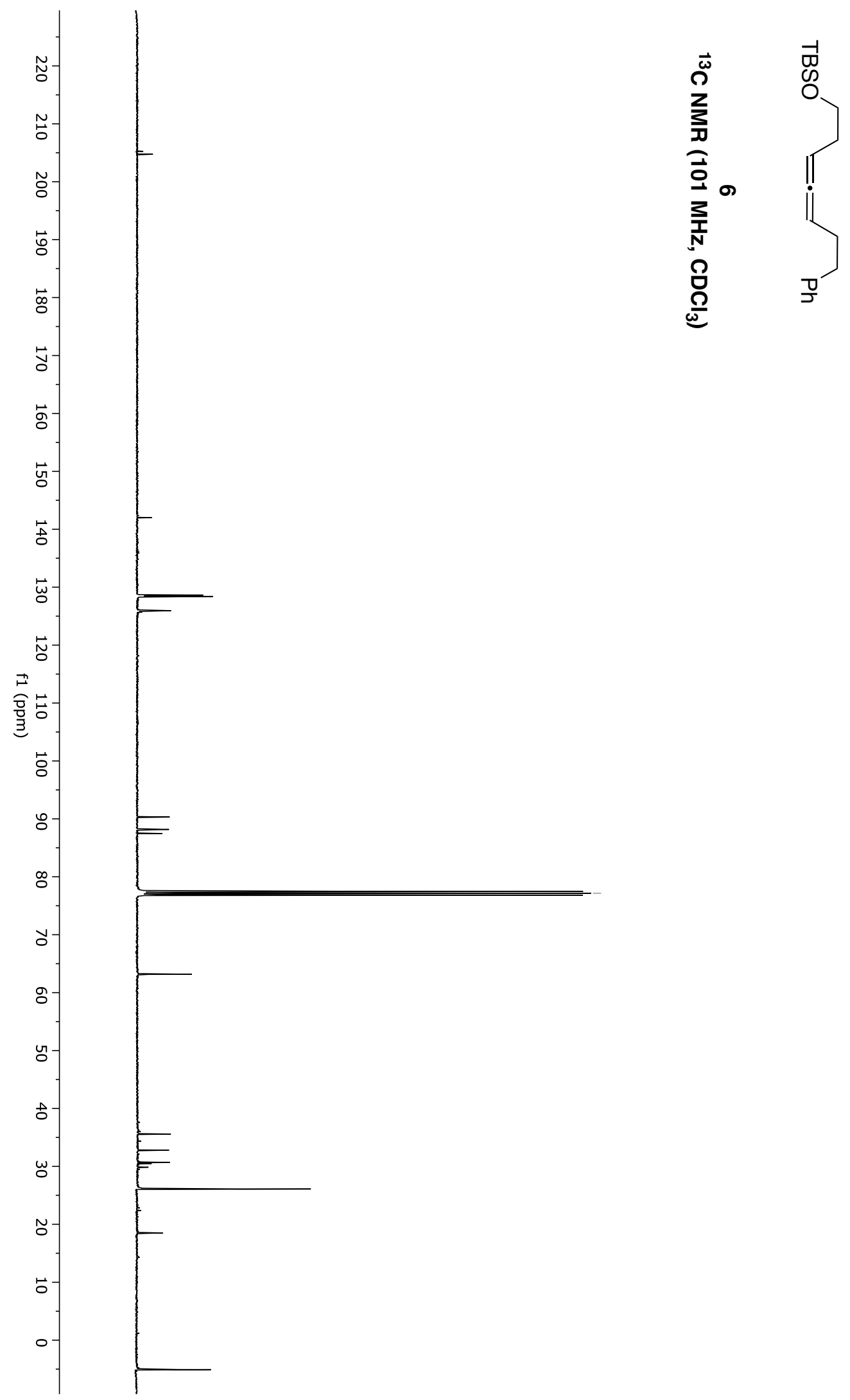


\subsection{NMR spectra of $R u$ complexes}

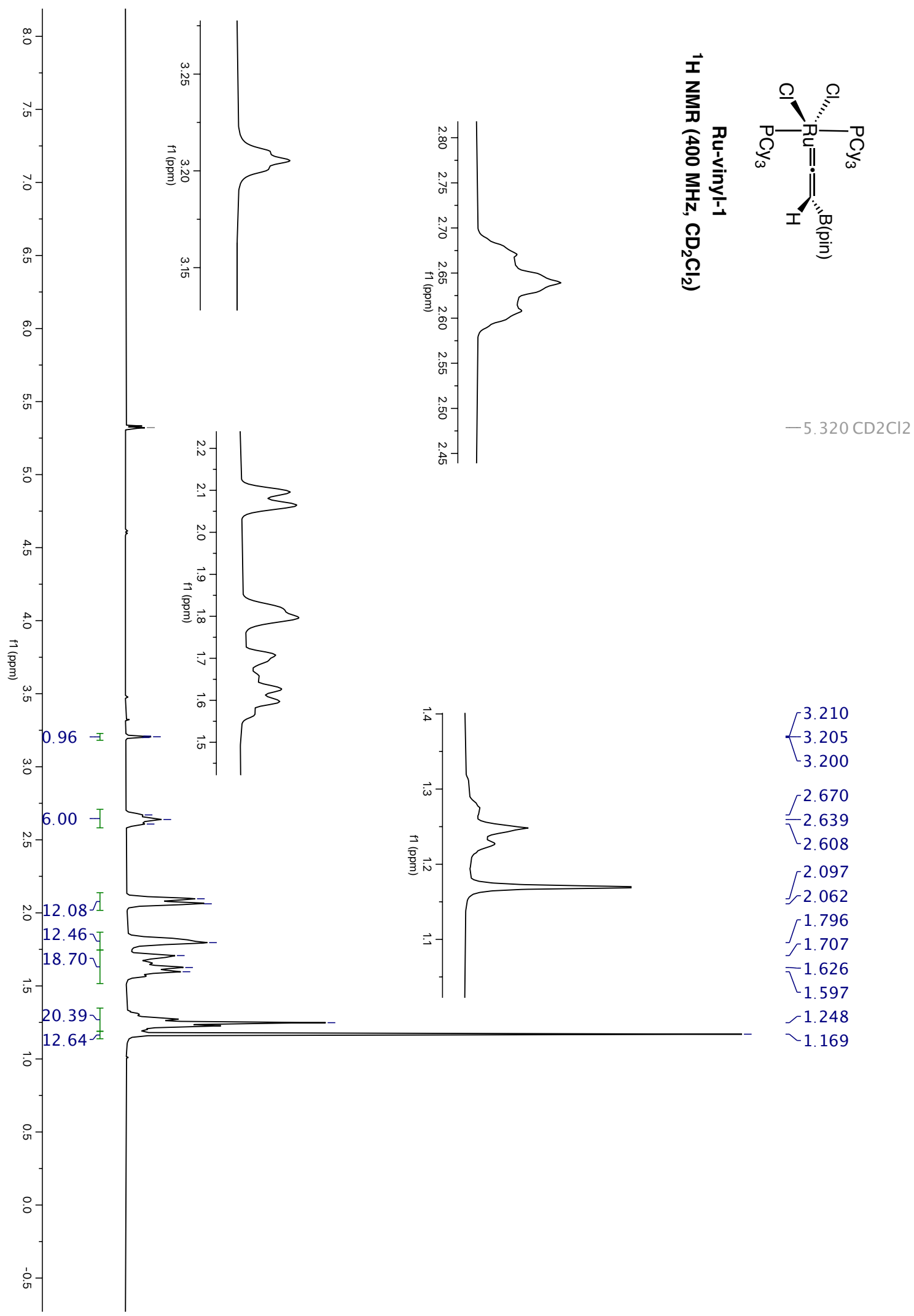




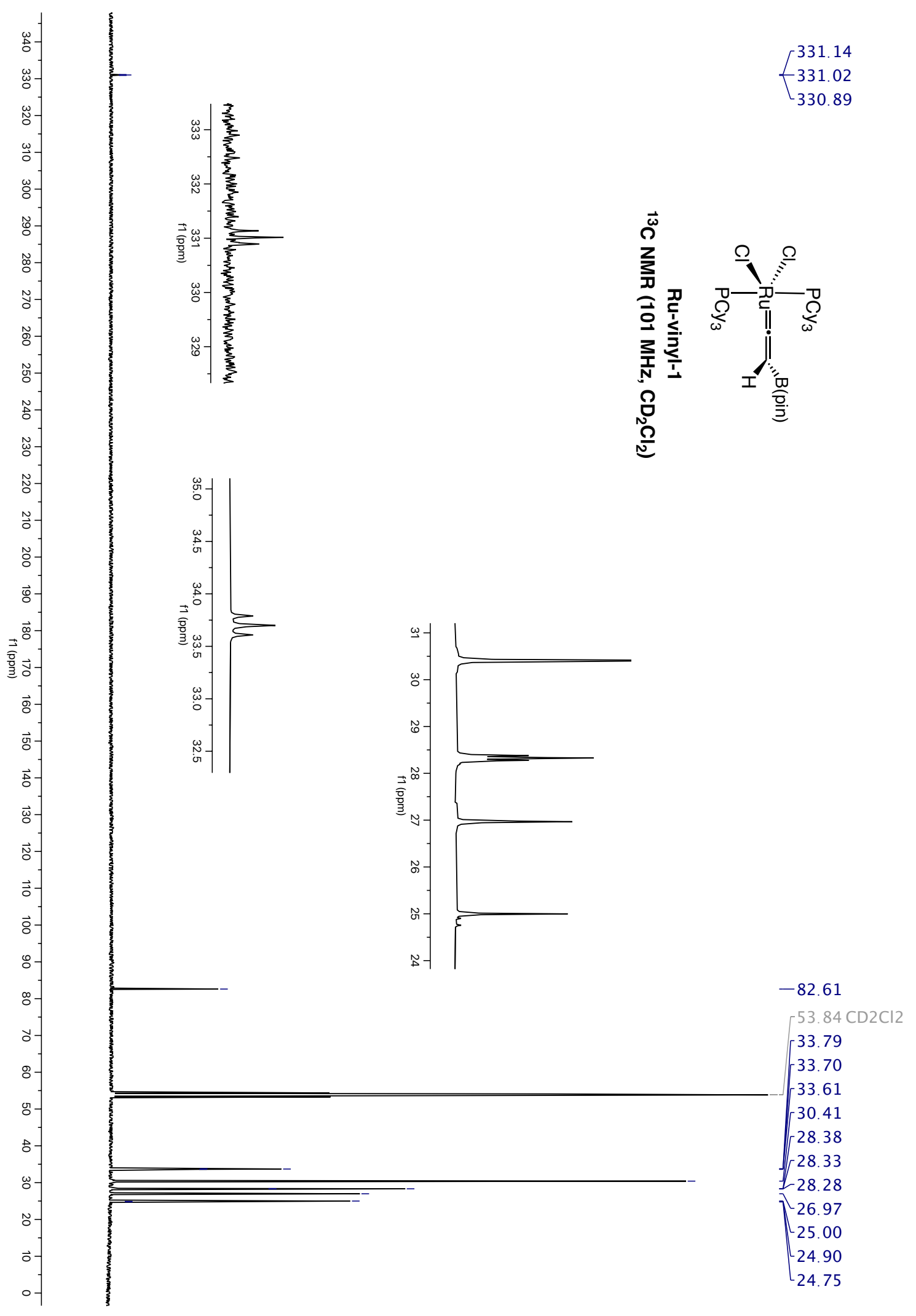




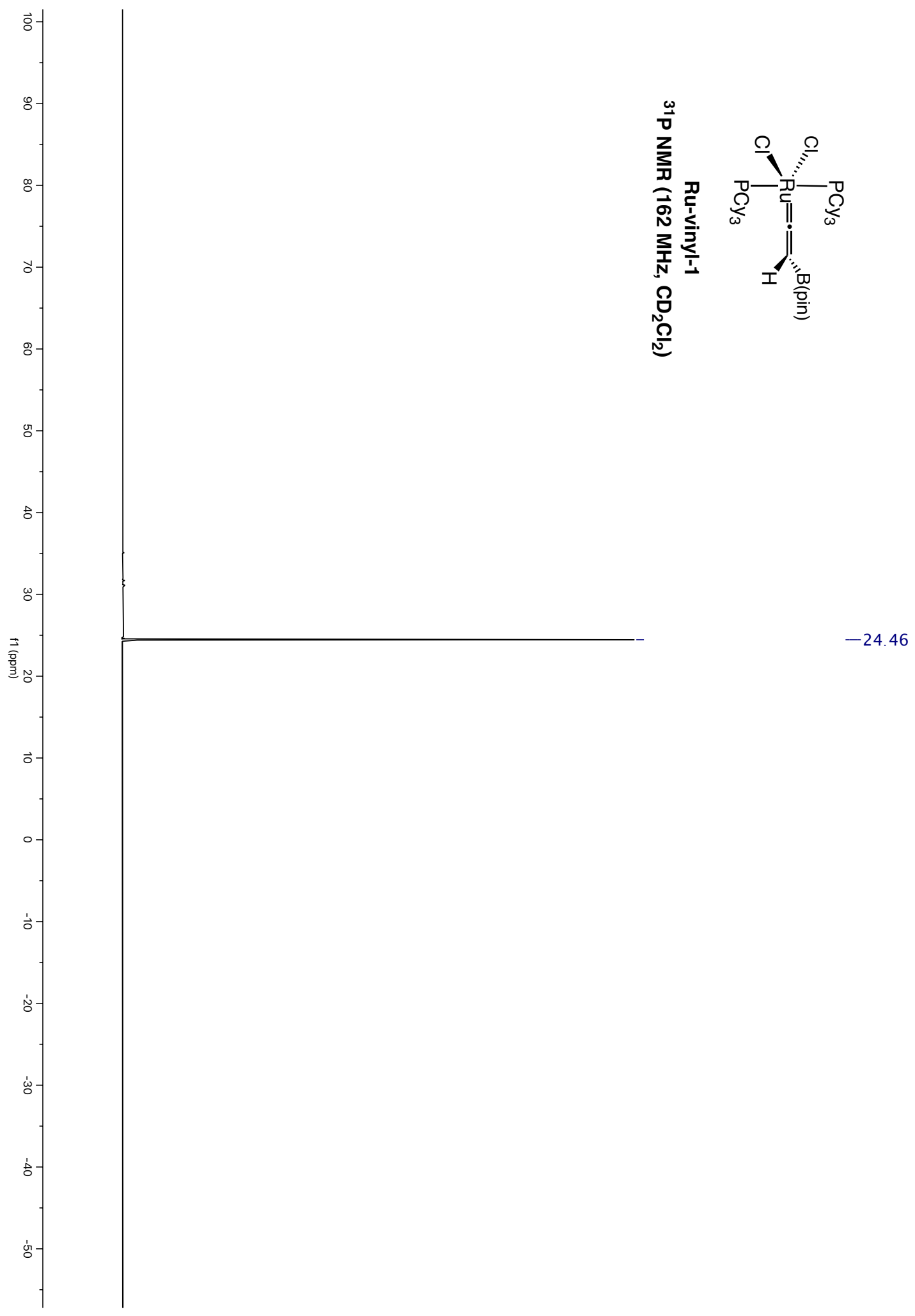




$$
\|^{\prime \prime}
$$




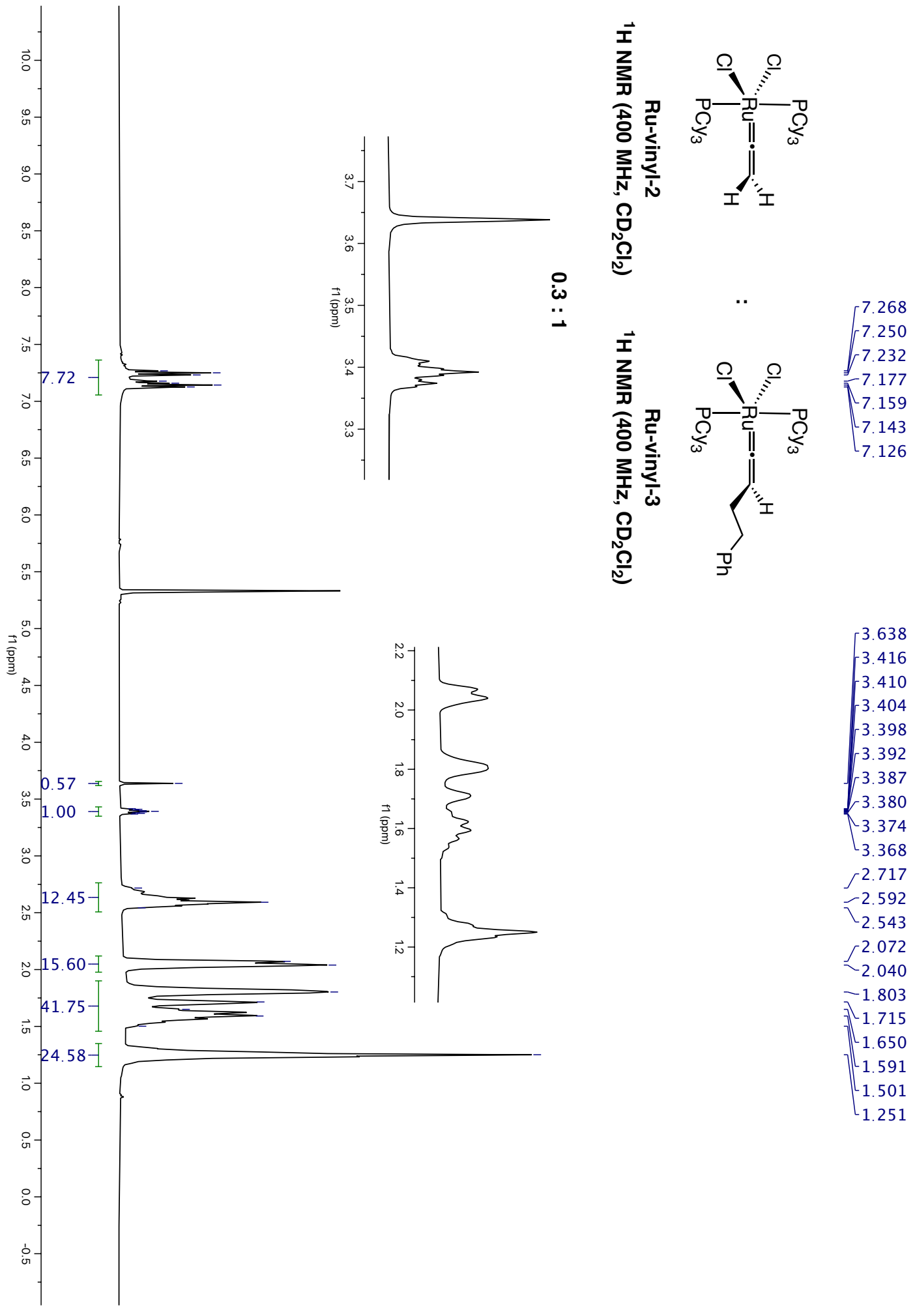


Gonsales, et. al., Supporting Information, pg 249

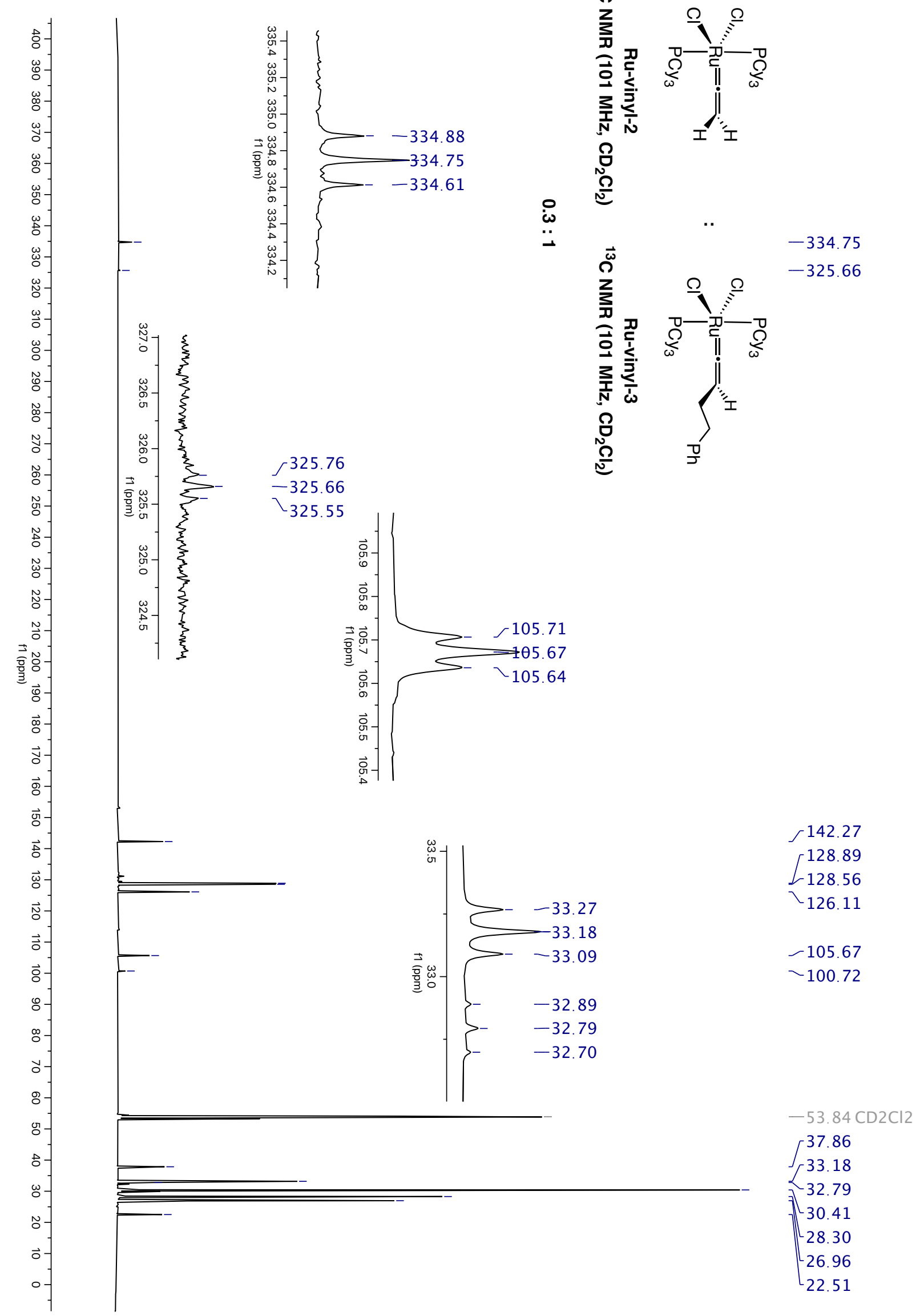




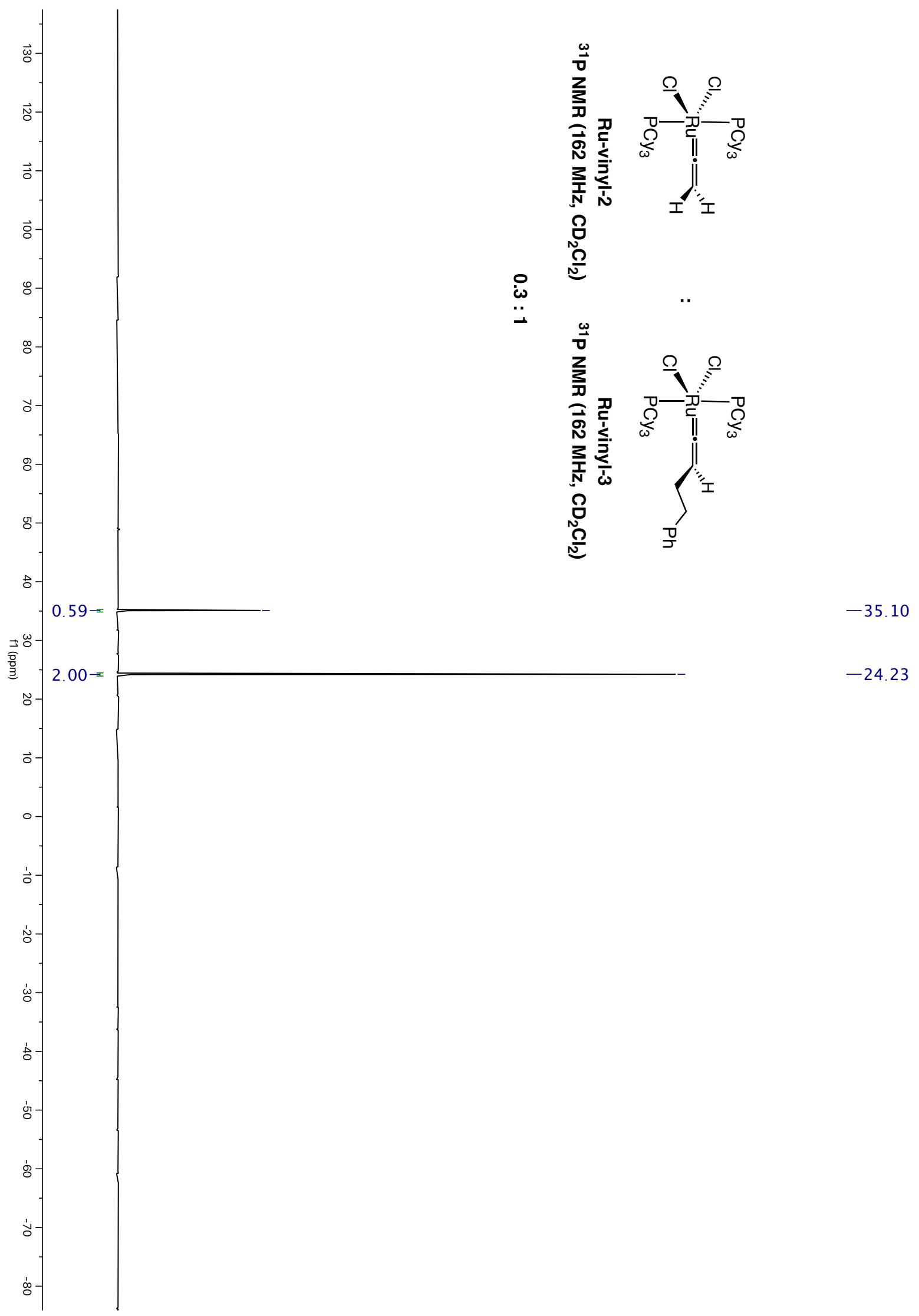




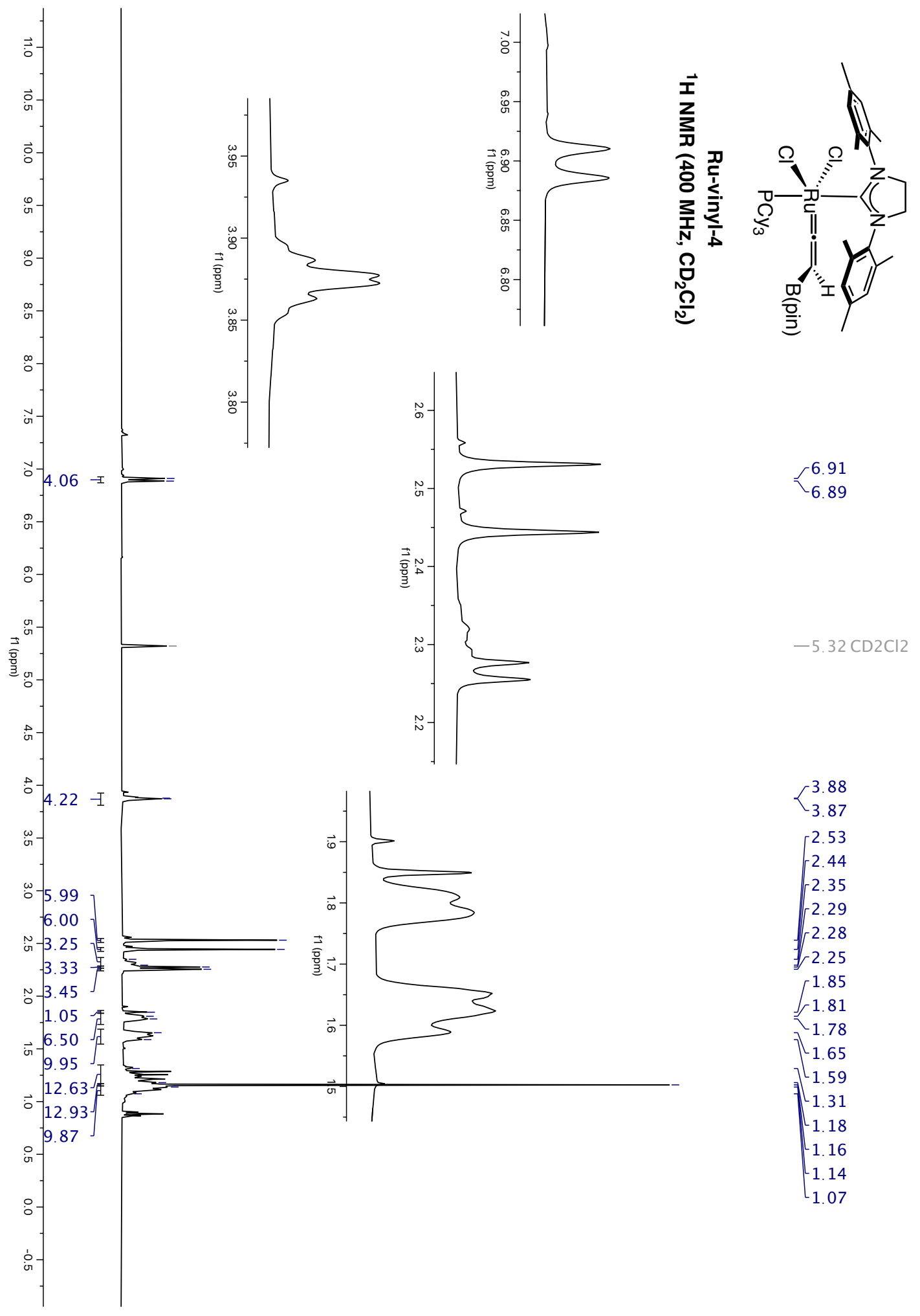




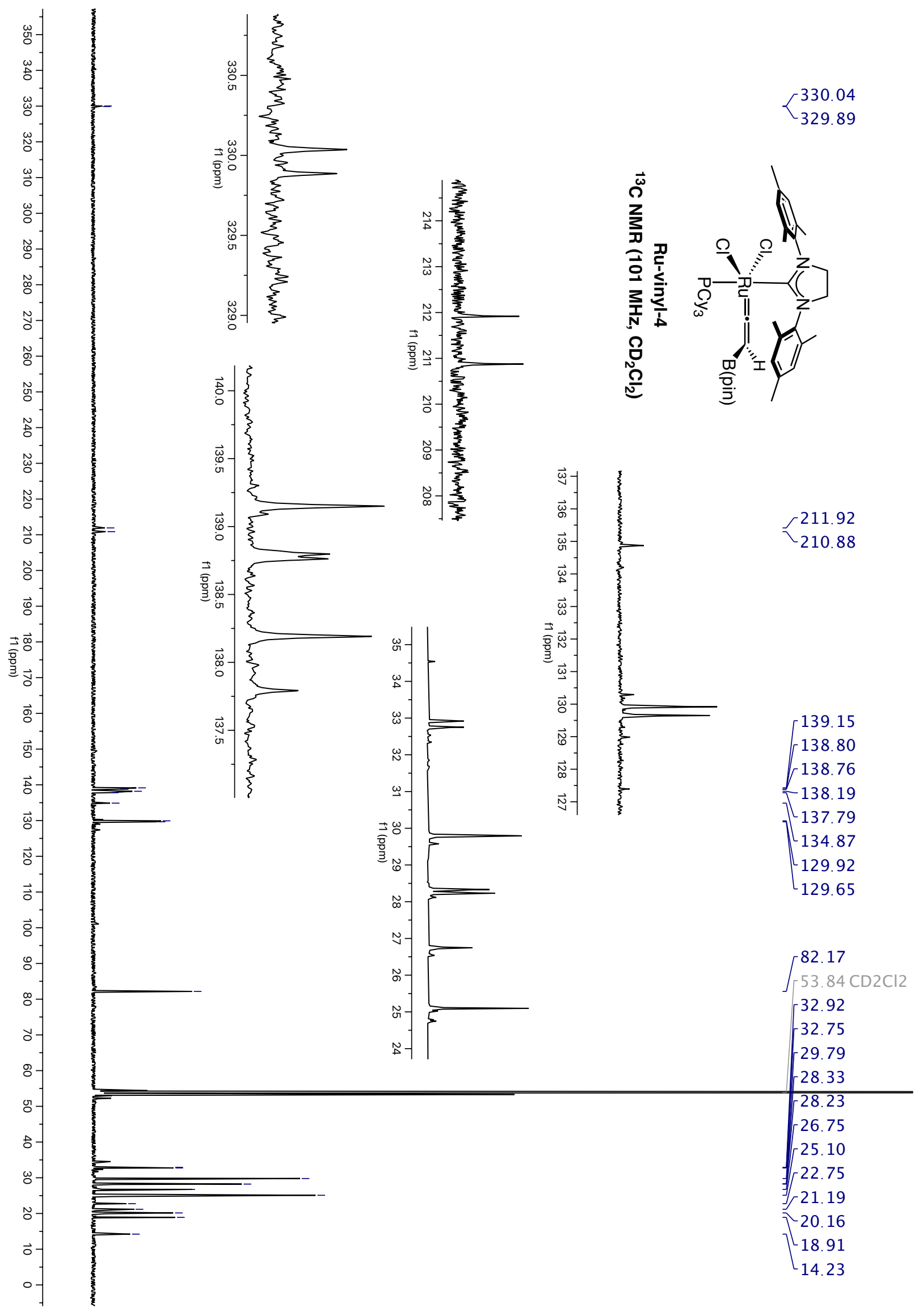


Gonsales, et. al., Supporting Information, pg 253

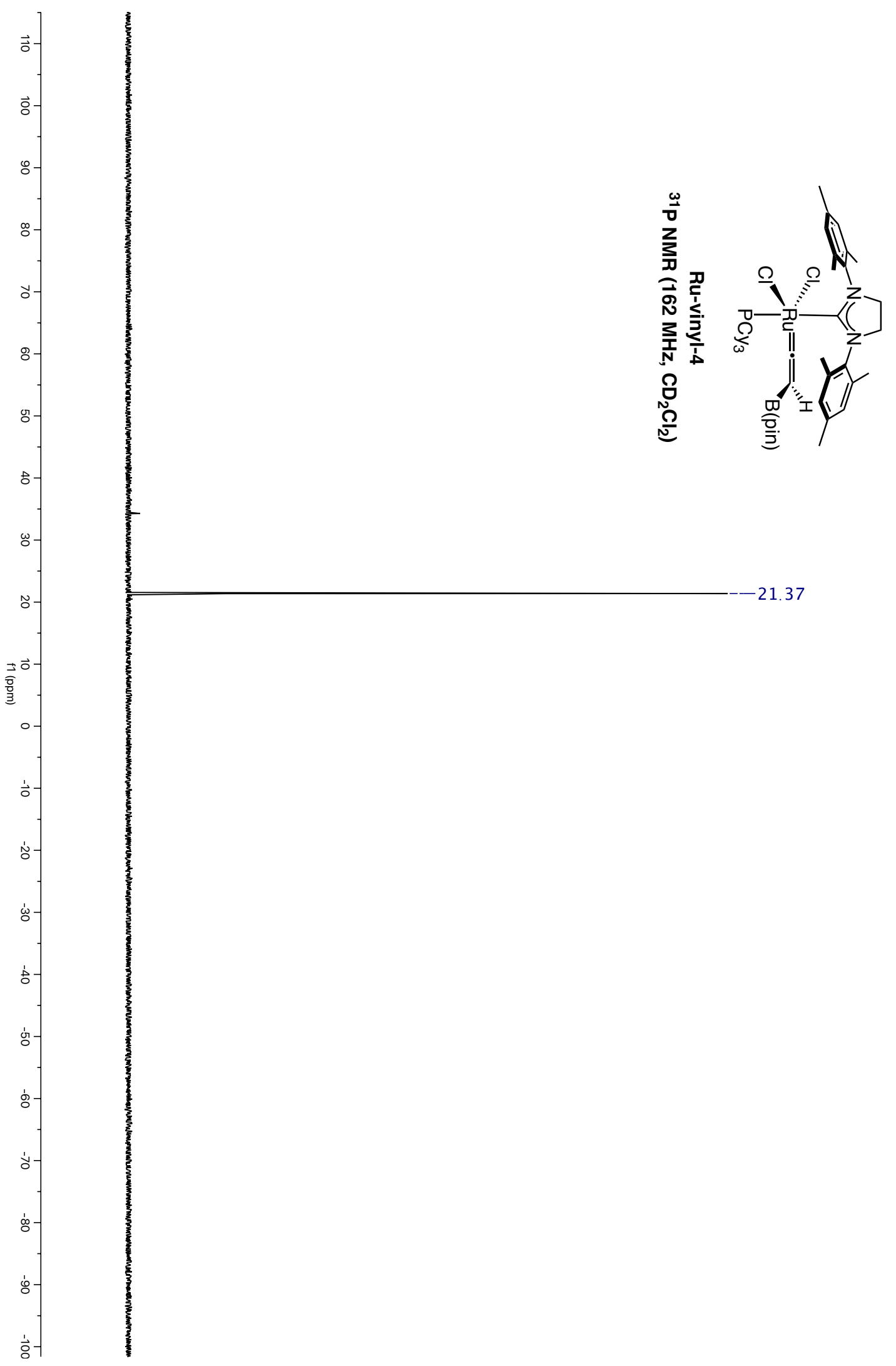



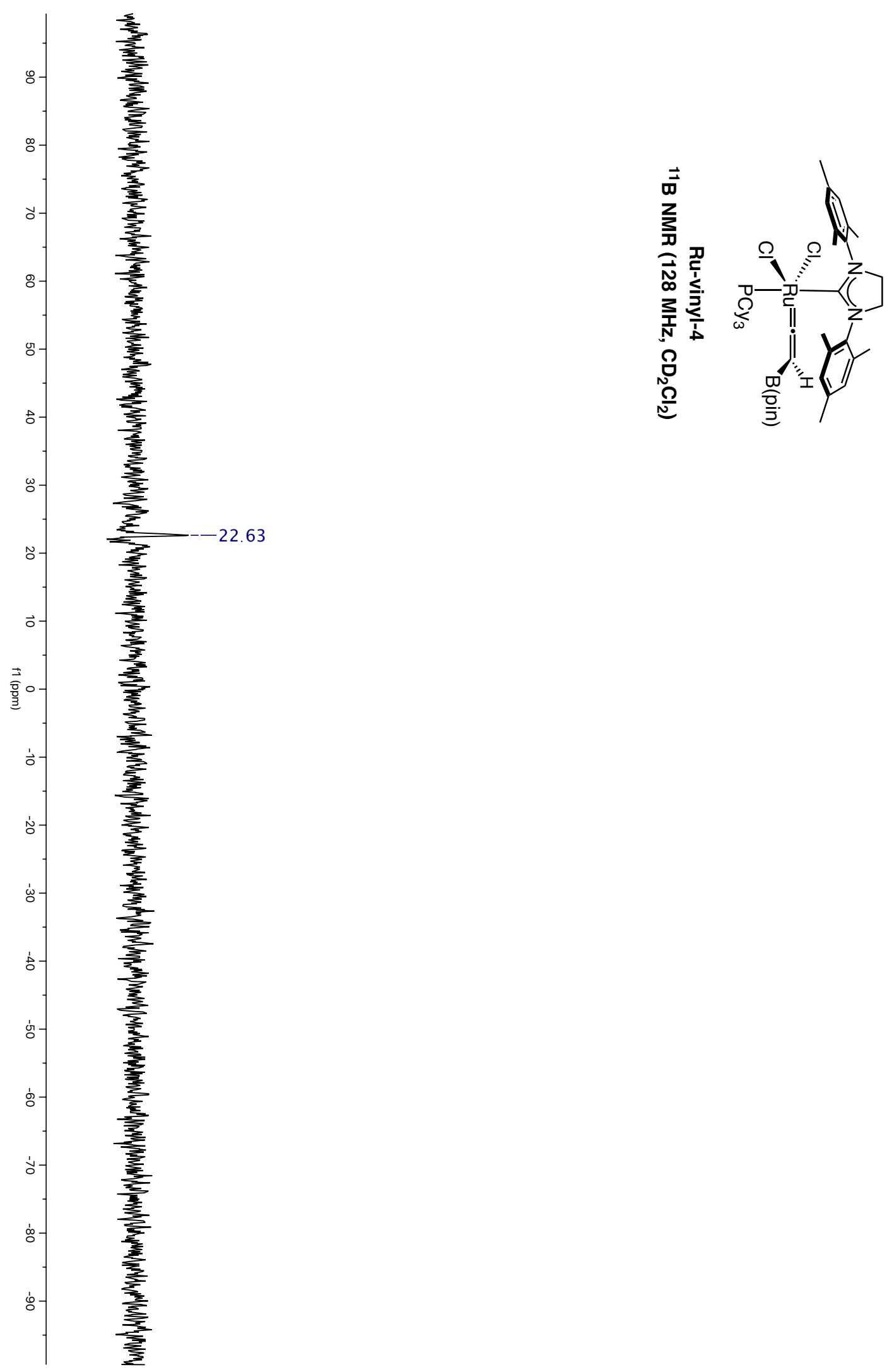


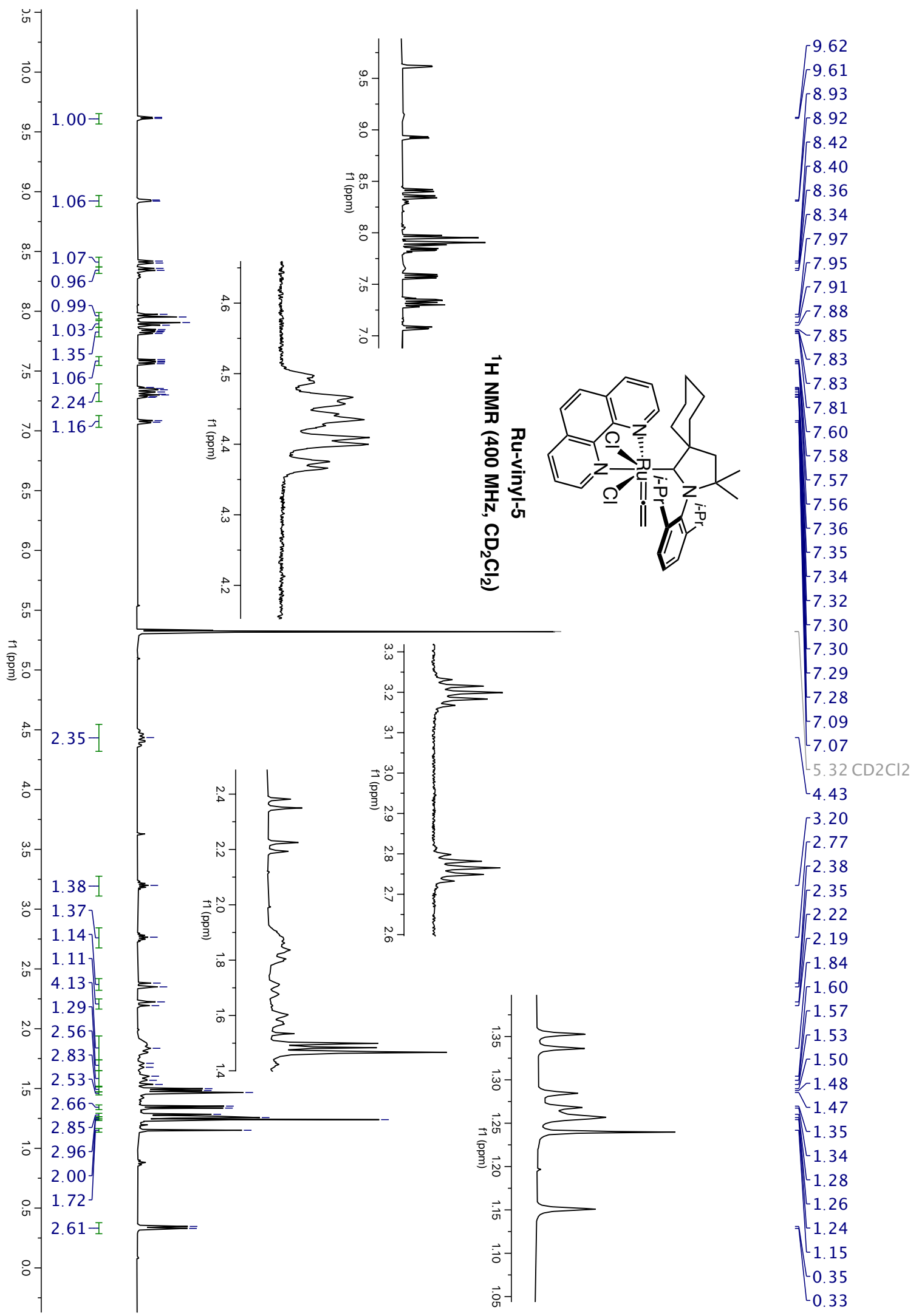


Gonsales, et. al., Supporting Information, pg 256

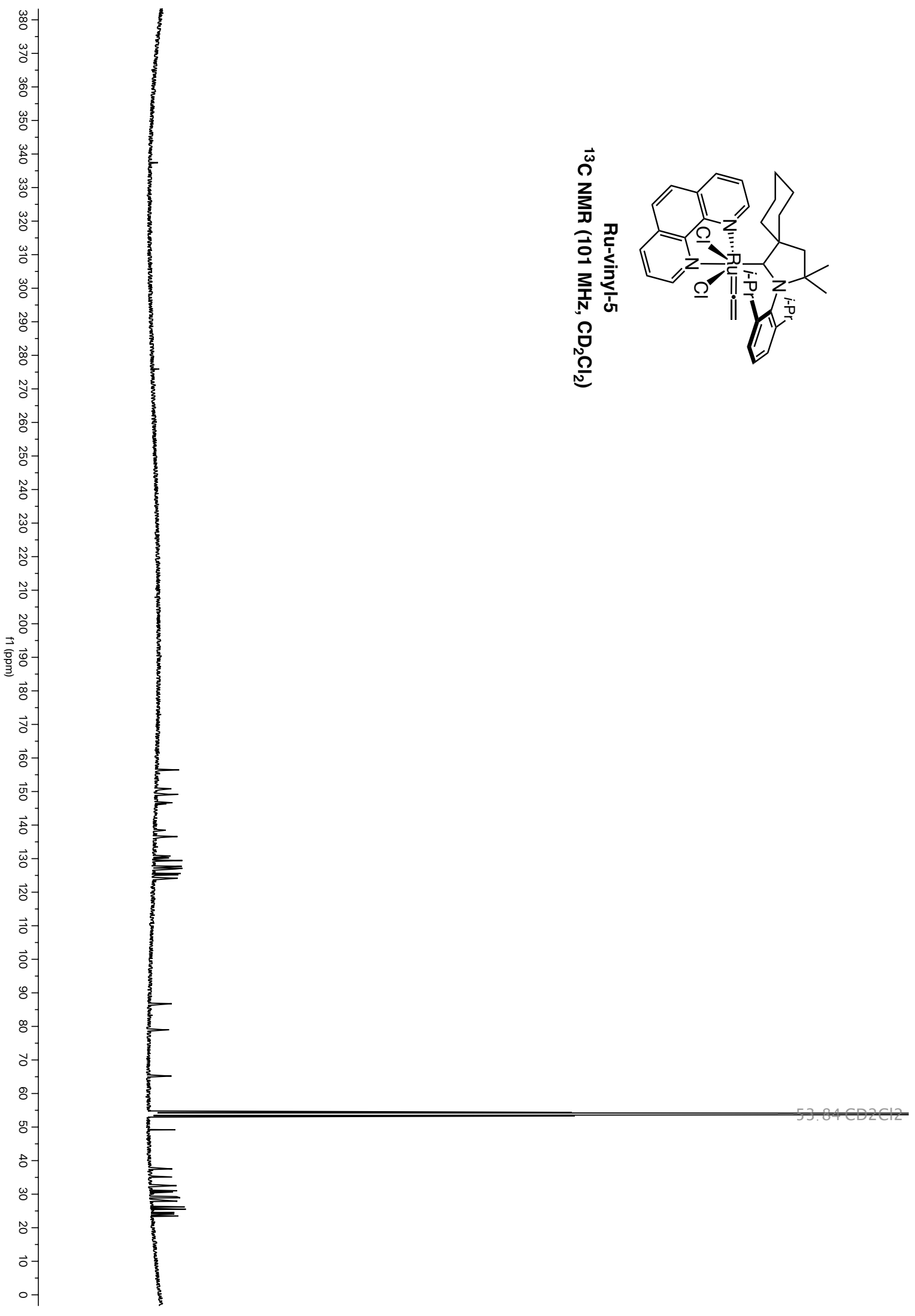




\section{References}

1. Kuang, J.; Ma, S. An efficient synthesis of terminal allenes from terminal 1-alkynes. J. Org. Chem. 2009, 74, $1763-1765$.

2. Anderson, D. R.; Lavallo, V.; O'Leary, D. J.; Bertrand, G.; Grubbs, R. H. Synthesis and reactivity of olefin metathesis catalysts bearing cyclic (alkyl)(amino)carbenes. Angew. Chem., Int. Ed. 2007, 46, 7262-7265.

3. Ishizawa, K.; Majima, S.; Wei, X.-F.; Mitsunuma, H.; Shimizu, Y.; Kanai, M. Copper(I)-catalyzed stereodivergent propargylation of $\mathrm{N}$-acetyl mannosamine for protecting group minimal synthesis of C3-substituted sialic acids. J. Org. Chem. 2019, 84, 10615-10628.

4. “M86-EXX229V1 APEX3 User Manual”, Bruker AXS Inc., Madison, USA, 2016.

5. Sheldrick, G. M. Acta Cryst. 2015, A71, 3-8.

6. Sheldrick, G. M. Acta Cryst. 2015, C71, 3-8.

7. (a) Becke, A. D. Density-functional thermochemistry. III. The role of exact exchange. J. Chem. Phys. 1993, 98, 5648-5652. (b) Lee, C.; Yang, W.; Parr, R. G. Development of the ColleSalvetti correlation-energy formula into functional of the electron density. Phys. Rev. B: Condens. Matter Mater. Phys. 1988, 37, 785-789. (c) Grimme, S.; Ehrlich, S.; Goerigk, L. Effect of the damping function in dispersion corrected density functional theory. J. Comput. Chem. 2011, 32, 1456-1465.

8. (a) von Szentpály, L.; Fuentealba, P.; Preuss, H.; Stoll, H. Pseudopotential calculations on $\mathrm{Rb}^{+}{ }_{2}, \mathrm{Cs}^{+}{ }_{2}, \mathrm{RbH}^{+}$, $\mathrm{CsH}^{+}$and the mixed alkali dimer ions. Chem. Phys. Lett. 1982, 93, 555-559. (b) Dolg, M.; Wedig, U.; Stoll, H.; Preuss, H. Energy-adjusted ab initio pseudopotentials for the first-row transition elements. J. Chem. Phys. 1987, 86, 866-872. (c) Schwerdtfeger, P.; Dolg, M.; Schwarz, W. E.; Bowmaker, G. A.; Boyd, P. D. Relativistic effects in gold chemistry. I. diatomic gold compounds. J. Chem. Phys. 1989, 91, 1762-1774.

9. Chai, J.-D.; Head-Gordon, M. Long-range corrected hybrid density functionals with damped atom-atom dispersion corrections. Phys. Chem. Chem. Phys. 2008, 10, 6615-6620.

10. Marenich, A. V.; Cramer, C. J.; Truhlar, D. G. Universal solvation model based on solute electron density and on a continuum model of the solvent defined by the bulk dielectric constant and atomic surface tensions. J. Phys. Chem. B 2009, 113, 6378-6396. 